\title{
STREAMFLOW CHARACTERISTICS OF STREAMS IN THE UPPER RED RIVER OF THE NORTH BASIN, NORTH DAKOTA, MINNESOTA, AND SOUTH DAKOTA
}

By Gregg J. Wiche and Tara Williams-Sether

U.S. GEOLOGICAL SURVEY

Open-File Report 97-416

Prepared in cooperation with the SOUTHEAST CASS WATER RESOURCE DISTRICT and the CASS COUNTY JOINT WATER RESOURCE DISTRICT 


\title{
U.S. DEPARTMENT OF THE INTERIOR BRUCE BABBITT, Secretary
}

\author{
U.S. GEOLOGICAL SURVEY \\ GORDON P. EATON, Director
}

Any use of trade, product, or firm names is for descriptive purposes only and does not imply endorsement by the U.S. Government.

For additional information write to:

District Chief U.S. Geological Survey Water Resources Division 821 East Interstate Avenue Bismarck, ND 58501-1199
Copies of this report can be purchased from:

U.S. Geological Survey

Branch of Information Services

Box 25286

Denver, CO 80225-0286 


\section{CONTENTS}

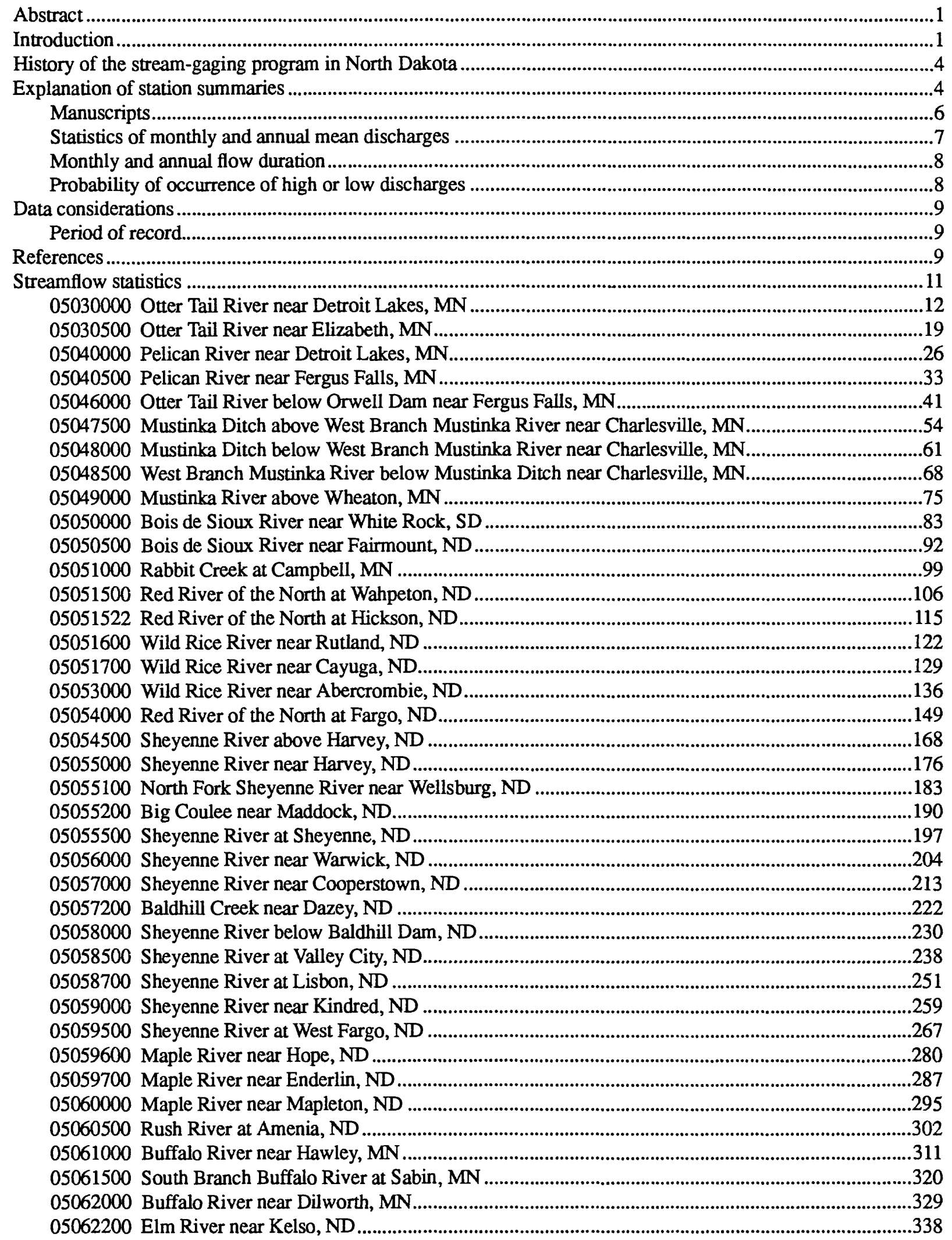


05062500 Wild Rice River at Twin Valley, MN

\section{FIGURES}

1. Map showing location of streamflow-gaging stations for which streamflow statistics

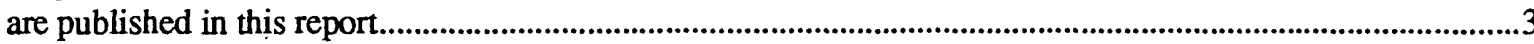

2. Graph showing number of streamflow-gaging stations in North Dakota, 1901-94 ............................................5

\section{TABLES}

1. List of streamflow-gaging stations for which streamflow statistics are published in this report .2 


\section{DEFINITION OF TERMS}

Climatic year is the 12-month period April 1 through March 31. The climatic year is designated by the calendar year in which it begins.

Cubic foot per second $\left(\mathrm{ft}^{3} / \mathrm{s}\right)$ is the rate of discharge representing a volume of 1 cubic foot passing a given point during 1 second and is equivalent to 7.48 gallons per second or 448.8 gallons per minute or 0.02832 cubic meters per second.

Discharge is the volume of water in a stream at a given point and for a given period of time. Discharge is often used interchangeable with the term "streamflow".

Drainage area is the area, measured in a horizontal plane, enclosed by a topographic divide from which direct surface runoff from precipitation normally drains by gravity into the stream upstream from the station. Figures of drainage area given herein include all closed basins, or noncontributing areas, within the area unless otherwise specified.

Drainage basin is a part of the surface of the earth that is occupied by a drainage system, which consists of a surface stream or a body of impounded surface water together with all tributary surface streams and bodies of impounded surface water.

Gage height is the water-surface elevation referred to some arbitrary gage datum. Gage height is often used interchangeable with the more general term "stage", although gage height is more appropriate when used with a reading on a gage.

Gaging station is a particular site on a stream, canal, lake, or reservoir where systematic observations of hydrologic data are obtained.

Ground water is the water in the ground that is in the zone of saturation, from which wells, springs, and ground-water runoff are supplied.

Hydrologic unit is a geographic area representing part or all of a surface drainage basin or distinct hydrologic feature as delineated by the Office of Water Data Coordination on the State Hydrologic Unit Maps; each hydrologic unit is identified by an eight-digit number.

Instantaneous discharge is the discharge at a particular instant of time.

Mean is the arithmetic average of a list of values.

Mean discharge is the arithmetic mean of individual discharges during a specific period.

Period of record is the time during which a stream-gaging station is in operation and for which the records are published.

Probability of occurrence is the likelihood that an event will occur. Probabilities are generally expressed as a decimal number between 0 and 1 . If the probability is 0 , the event will not occur, if the probability is 1 , the event will occur absolutely. Probability also can be expressed as a percent, where 0 percent corresponds to 0 probability and 100 percent corresponds to a probability of 1 .

Recurrence interval is the average time interval between occurrences of a hydrologic event of a given or greater magnitude, usually expressed in years. 
Regulation is the artificial manipulation of the flow of a stream.

Stage see "gage height"

Standard deviation is a measure of the variability of the values in a list of values.

Stream-gaging station is a particular site on a stream where a record of discharge is obtained.

Streamflow see "discharge"

Surface water is the water on the surface of the earth.

Water year is the 12 -month period October 1 through September 30 . The water year is designated by the calendar year in which it ends and which includes 9 of the 12 months. 


\title{
STREAMFLOW CHARACTERISTICS OF STREAMS IN THE UPPER RED RIVER OF THE NORTH BASIN, NORTH DAKOTA, MINNESOTA, AND SOUTH DAKOTA
}

\author{
By Gregg J. Wiche and Tara Williams-Sether
}

\section{ABSTRACT}

Statistical summaries of streamflow data for all active and inactive gaging stations for the Red River Basin upstream of and including Halstad, Minnesota, are presented in this report. The summaries for each streamflow-gaging station include (1) manuscript (station description), (2) graph of the annual mean discharge for the period of record, (3) statistics of monthly and annual mean discharges, (4) graph of the annual flow duration, (5) monthly and annual flow duration, (6) probability of annual high discharges, (7) probability of annual low discharges, (8) probability of seasonal low discharges, (9) annual peak discharge and corresponding gage height for the period of record, and (10) monthly and annual mean discharges for the period of record.

\section{INTRODUCTION}

A part of the mission of the United States Geological Survey is the collection of systematic data to determine the quantity as well as the quality and use of surface and ground water. A total of 7,292 streamflow-gaging stations (as of 1994) were operated by the U. S. Geological Survey in the United States, Puerto Rico, and the Trust Territories of the Pacific Islands (Wahl and others, 1995). Of the 7,292 streamflow-gaging stations, 60 were operated in the Red River Basin upstream of Emerson, Manitoba, excluding the Devils Lake Basin.

At streamflow-gaging stations, the water level in the river is monitored continually. A relation between water level and discharge is developed by making periodic discharge measurements throughout the range in water level. This relation is referred to as a station rating. A continuous record of streamflow is computed for each gaging station by using the water level record and the station rating.

Knowledge of the magnitude and time distribution of streamflow is essential for all aspects of water management and environmental planning. Federal, State, and local agencies responsible for the development and management of North Dakota's surface-water resources use this knowledge for making safe, economical, and environmentally sound water-resource planning decisions.

Streamflow statistics published in annual state water reports by the U. S. Geological Survey include records of daily mean discharge, annual high and low discharge, and annual mean discharge. Other statistics can be retrieved from U.S. Geological Survey computer files. Water resource managers must go to various sources to obtain the necessary statistics. These sources may only include active gaging stations listed in the most recent annual report and, thus, overlook information available for many inactive gaging stations. 
The purpose of this report is to provide a comprehensive publication summarizing streamflow characteristics for all active and inactive gaging stations for the Red River Basin upstream of and including Halstad, Minnesota, excluding the Devils Lake Basin. Active and discontinued gaging stations that have a least 10 years of record are listed in table 1 and their locations are shown in figure 1.

Table 1. List of streamflow-gaging stations for which streamflow statistics are published in this report

\begin{tabular}{|c|c|}
\hline $\begin{array}{l}\text { Station } \\
\text { number }\end{array}$ & Station name \\
\hline 05030000 & Otter Tail River near Detroit Lakes, MN \\
\hline 05030500 & Otter Tail River near Elizabeth, MN \\
\hline 05040000 & Pelican River near Detroit Lakes, MN \\
\hline 05040500 & Pelican River near Fergus Falls, MN \\
\hline 05046000 & Otter Tail River below Orwell Dam near Fergus Falls, MN \\
\hline 05047500 & Mustinka ditch above West Branch Mustinka River near Charlesville, MN \\
\hline 05048000 & Mustinka ditch below West Branch Mustinka River near Charlesville, MN \\
\hline 05048500 & West Branch Mustinka River below Mustinka Ditch near Charlesville, MN \\
\hline 05049000 & Mustinka River above Wheaton, MN \\
\hline 05050000 & Bois de Sioux River near White Rock, SD \\
\hline 05050500 & Bois de Sioux River near Fairmont, ND \\
\hline 05051000 & Rabbit Creek at Campbell, $\mathrm{MN}$ \\
\hline 05051500 & Red River of the North at Wahpeton, ND \\
\hline 05051522 & Red River of the North at Hickson, ND \\
\hline 05051600 & Wild Rice River near Rutland, ND \\
\hline 05051700 & Wild Rice River near Cayuga, ND \\
\hline 05053000 & Wild Rice River near Abercrombie, ND \\
\hline 05054000 & Red River of the North at Fargo, ND \\
\hline 05054500 & Sheyenne River above Harvey, ND \\
\hline 05055000 & Sheyenne River near Harvey, ND \\
\hline 05055100 & North Fork Sheyenne River near Wellsburg, ND \\
\hline 05055200 & Big Coulee near Maddock, ND \\
\hline 05055500 & Sheyenne River at Sheyenne, ND \\
\hline 05056000 & Sheyenne River near Warwick, ND \\
\hline 05057000 & Sheyenne River near Cooperstown, ND \\
\hline 05057200 & Baldhill Creek near Dazey, ND \\
\hline 05058000 & Sheyenne River below Baldhill Dam, ND \\
\hline 05058500 & Sheyenne River at Valley City, ND \\
\hline 05058700 & Sheyenne River at Lisbon, ND \\
\hline 05059000 & Sheyenne River near Kindred, ND \\
\hline 05059500 & Sheyenne River at West Fargo, ND \\
\hline 05059600 & Maple River near Hope, ND \\
\hline 05059700 & Maple River near Enderlin, ND \\
\hline 05060000 & Maple River near Mapleton, ND \\
\hline 05060500 & Rush River at Amenia, ND \\
\hline 05061000 & Buffalo River near Hawley, MN \\
\hline 05061500 & South Branch Buffalo River at Sabin, MN \\
\hline 05062000 & Buffalo River near Dilworth, $\mathrm{MN}$ \\
\hline 05062200 & Elm River near Kelso, ND \\
\hline 05062500 & Wild Rice River at Twin Valley, MN \\
\hline 05064000 & Wild Rice River at Hendrum, MN \\
\hline 05064500 & Red River of the North at Halstad, MN \\
\hline
\end{tabular}




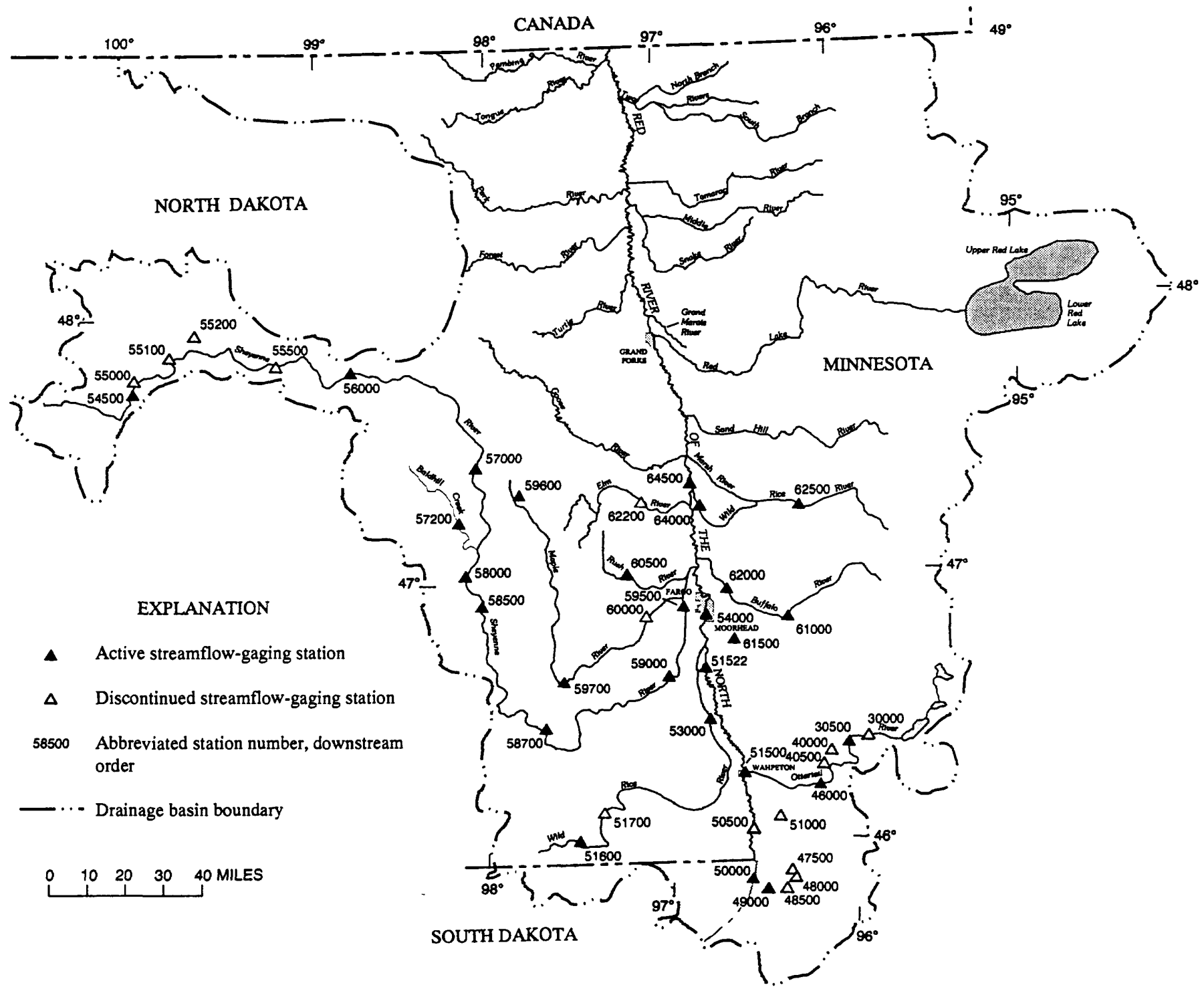

Figure 1. Location of streamflow-gaging stations for which streamflow statistics are published in this report. 


\section{HISTORY OF THE STREAM-GAGING PROGRAM IN NORTH DAKOTA}

Much of the history of the stream-gaging program in North Dakota outlined in this report was written by Crosby (1970). However, the number of streamflow-gaging stations given in figure 2 may differ from the number given by Crosby (1970) because the type of gaging stations included may differ. The collection of systematic streamflow data began in 1882 when a gaging station was established on the Red River of the North at Grand Forks. This gaging station was a stage station; however, infrequent discharge measurements were made for navigational purposes. Stage data were obtained on the Missouri River at Bismarck in 1881-82 and in 1886-89 by the Missouri River Commission. As result of the National Reclamation Act of 1902 and the disastrous flood in 1897 in the Red River of the North Basin, the U.S. Geological Survey, in cooperation with the state of North Dakota, established and operated streamflowgaging stations from 1901-09 (fig. 2). Additional interest was created as problems with Canada concerning the division of waters along the international boundary resulted in the formation of the International Joint Commission in 1912. Eight streamflow-gaging stations were in operation in 1925 when State cooperation was discontinued (fig. 2). Only five federally operated gaging stations were continued. State cooperation resumed in 1931, but funds were limited from 1934-38. However, the Rivers and Harbors Act of 1927 and the Flood Control Acts of 1928 and 1936 resulted in the U.S. Army Corps of Engineers supporting a large expansion of the stream-gaging program. Forty-one gaging stations were in operation when the North Dakota-South Dakota U.S. Geological Survey Office was created on October 16, 1944. Plans for the coordinated development of the waters of the Missouri River Basin, with respect to flood control, navigation, power, and irrigation, were formulated in 1943-44 by the U.S. Army Corps of Engineers, the Bureau of Reclamation, and the States in the Basin. These plans resulted in a rapid increase in the stream-gaging program, and, by 1947, 64 gaging stations were in operation. The number of gaging stations grew steadily from the late 1940's until the late 1960's, and, by 1969, 109 gaging stations were in operation.

During 1969-76, the number of gaging stations in operation remained relatively stable. During the 1970's, the U.S. Geological Survey established 25 additional gaging stations to monitor the quantity and quality of streamflow in drainage basins underlain by strippable lignite deposits (Haffield, 1981). By 1979, about 145 gaging stations were in operation in North Dakota. During 1981-83, the number of gaging stations in operation declined rapidly, and, during 1984-87, the number declined slowly to about 110. Since 1987, the number of gaging stations in operation has been relatively stable at about 105 to 110 .

\section{EXPLANATION OF STATION SUMMARIES}

Station summaries are presented so that each station description and tables of streamflow statistics and probabilities of occurrence are presented in the same order and format for each gaging station, including the same relative placement of the pages. Because the tables were created by "data retrievals" or statistical program results, significant figures were not rounded to U.S. Geological Survey standards. The order of presentation is as follows:

1. manuscript (station description),

2. graph of the annual mean discharge for the period of record,

3. table of statistics of monthly and annual mean discharges,

4. graph of the annual flow duration,

5. table of monthly and annual flow duration,

6. table of probability of annual high discharges,

7. table of probability of annual low discharges,

8. table of probability of seasonal low discharges,

9. table of annual peak discharge and corresponding gage height for the period of record, and

10. table of monthly and annual mean discharges for the period of record. 


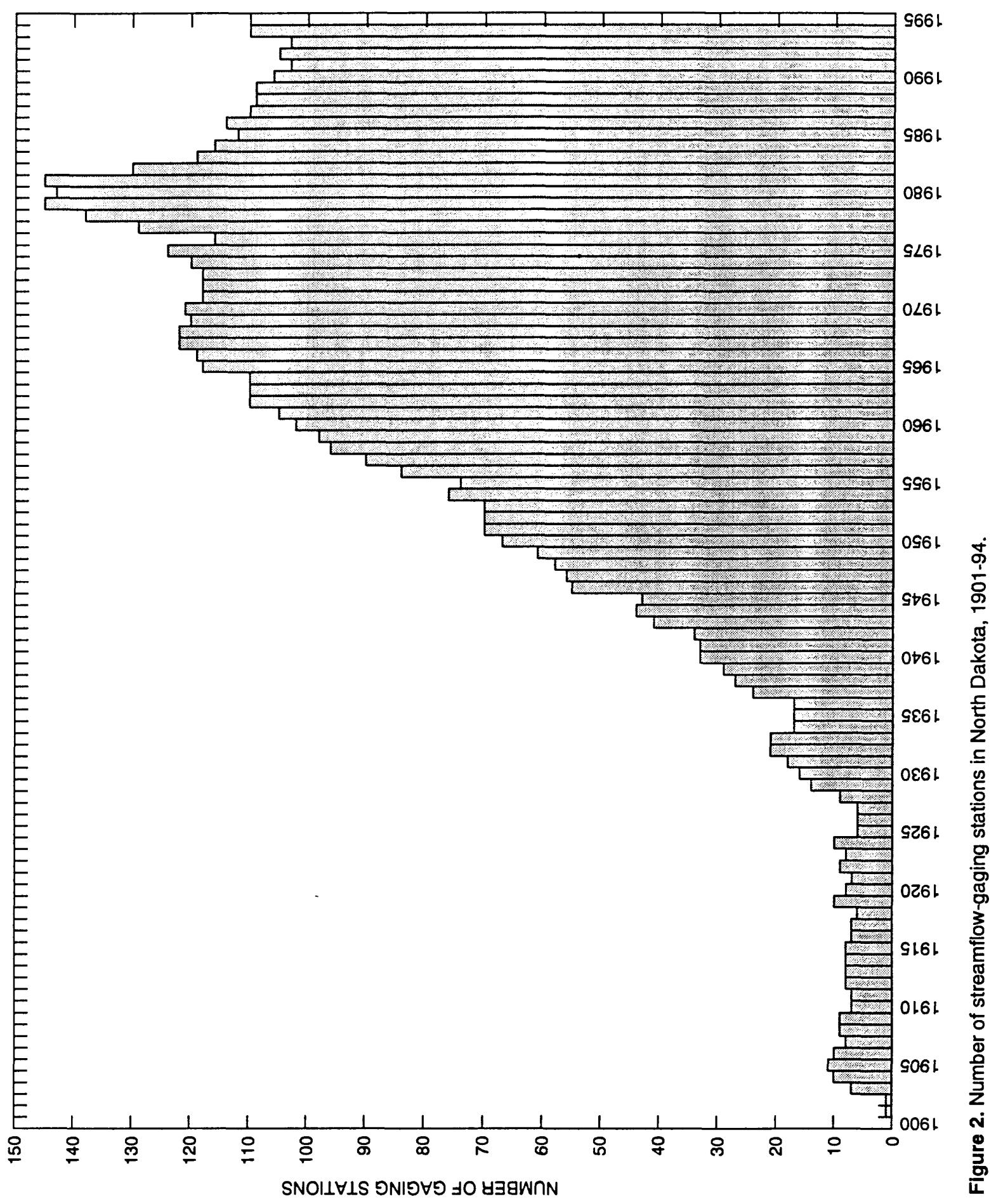


Where both pre-regulation and post-regulation statistics are presented for a gaging station, the manuscript, graph of annual mean discharges, table of annual peak discharges and corresponding gage heights, and table of monthly and annual mean discharges are presented with the pre- and post-regulation data. The respective tables for the post-regulation data are presented in the same relative page formats.

\section{Manuscripts}

The location, drainage area, period of record, and other information about each streamflow-gaging station included in this report are presented in manuscript form. This information is compiled from records maintained by the U.S. Geological Survey and generally is presented in the same format as published in the annual state water report. The following comments clarify information presented under the various headings of the station manuscript.

LOCATION.--Information on gaging station location is obtained from the most accurate maps available and is furnished with respect to cultural and physical features in the vicinity of the gaging station and the community or landmark included in the gaging station name. In the case of discontinued gaging stations, the location is furnished using features in the vicinity at the time the gaging station was in operation. In many instances, the identifying features have been altered since the gaging station was discontinued.

DRAINAGE AREA.--Drainage area is measured using U.S. Geological Survey 7.5-minute topographic quadrangle maps. However, 7.5-minute topographic maps for drainage area computations were not available when some gaging stations were installed; therefore, the accuracy of drainage areas also varies. Drainage areas of discontinued gaging stations are those determined while the gaging station was in operation.

PERIOD OF RECORD.--The period of record is the period for which there are published records for the gaging station or for an equivalent gaging station. An equivalent gaging station is a gaging station that was in operation prior to the subject gaging station, and whose location is such that records from it can reasonably be considered equivalent with records from the subject gaging station. This situation arises when a gaging station is relocated upstream or downstream and given a new gaging station number and name, but the changes in drainage area and other basin characteristics are not significantly different.

GAGE.--The type of gage or recorder that is or was used to collect data, the datum of the gage referred to sea level, and a condensed history of the types, locations, and datums of previous gages are given under this heading.

EXTREMES FOR PERIOD OF RECORD.--Extremes may include maximum and minimum discharges and maximum and minimum gage heights. Unless otherwise qualified, the maximum discharge is the instantaneous maximum corresponding to the highest gage height that occurred. If the maximum gage height did not occur on the same day as the maximum discharge, it is listed separately. Similarly, the minimum discharge is the instantaneous minimum corresponding to the lowest gage height that occurred, unless qualified and listed otherwise.

EXTREMES OUTSIDE PERIOD OF RECORD.--Included is any information available concerning major floods or unusually low flows that occurred outside the stated period of record. The information may not have been obtained by the U.S. Geological Survey. 


\section{Statistics of Monthly and Annual Mean Discharges}

Statistics of monthly and annual mean discharges presented for each gaging station include (1) the maximum, minimum, and mean monthly discharges and (2) the maximum, minimum, and mean annual discharges. The water years (October 1 through September 30 ) in which the maximum and minimum discharges occurred are listed with the respective values, and the standard deviation and coefficient of variation of the monthly and annual mean discharges are listed with the respective values. Also, the percentage of the annual discharge that is comprised by each monthly mean discharge is listed in the table.

Each of the statistics is explained in the following paragraphs. As an aid to the readers' understanding of how the monthly mean and annual mean discharges are determined, data for the gaging station Otter Tail River near Detroit Lakes, MN (05053000) are used as an example. Each monthly value is the mean of the daily values for the month. Months or years for which all daily values are not available are not included in the compilation of statistics.

The maximum monthly mean discharge is the maximum value of all the monthly mean values. The maximum mean value for October is 138 cubic feet per second $\left(\mathrm{ft}^{3} / \mathrm{s}\right)$, which occurred during water year 1945. Similarly, the minimum monthly mean discharge is the minimum value of all the monthly mean values. The minimum mean value for October is $2.16 \mathrm{ft}^{3} / \mathrm{s}$, which occurred during water year 1957. The maximum and minimum monthly mean values can be found in the statistics of monthly and annual mean discharges table or by searching the monthly and annual mean discharges table.

The mean monthly discharge is the mean of all the monthly mean discharges for a given month, and the standard deviation is a measure of the variability of the values. The mean monthly discharge for October is $29.2 \mathrm{ft}^{3} / \mathrm{s}$, and the standard deviation is $28.9 \mathrm{ft}^{3} / \mathrm{s}$. The monthly mean discharge for October (mean of the mean monthly values) is the same as the mean of all October daily values for the period of record used. However, the standard deviation is smaller than the standard deviation obtained using all daily values. The standard deviation is smaller because the monthly values have less variability than the daily values.

The coefficient of variation is the ratio of the standard deviation to the mean, and it provides a comparison of the standard deviation in units of the mean. The coefficient of variation is dimensionless. Because monthly mean discharges are much greater in spring than in winter, the standard deviations also are much greater in spring than in winter. However, dividing the standard deviation by the mean monthly discharge tends to equalize the measures for all months so a more meaningful comparison among months can be made.

The percentage of the annual discharge is the percent of the annual discharge that occurred during each month. It is calculated by dividing the mean discharge for the month by the total of the 12 monthly mean discharges and multiplying by 100 . Because of rounding of the monthly percentage, the sum of the 12 percentages may not equal 100 percent.

The maximum, minimum, and mean annual discharges are selected or computed from the annual mean discharges for the period of record. The water years of occurrence of the maximum and minimum values are listed with the respective values, and the standard deviation of the mean of the annual mean values is listed with the mean value. The minimum annual mean discharge of $9.82 \mathrm{ft}^{3} / \mathrm{s}$ occurred in 1940 , and the maximum annual mean discharge of $98.4 \mathrm{ft}^{3} / \mathrm{s}$ occurred in 1966. The mean annual discharge for the period of record is $53.7 \mathrm{ft}^{3} / \mathrm{s}$. 


\section{Monthly and Annual Flow Duration}

The monthly and annual flow duration table is a magnitude and frequency analysis of daily discharge values. It is computed by tabulating the number of daily discharge values that fall within preselected class limits, computing the percentage of values within each class, and interpolating discharge values for the percentages shown in the table. Monthly values are calculated from daily values in all complete months in the record, and annual figures are calculated for all complete water years. For example, if the 90 -percent flow duration value for October is $2.80 \mathrm{ft}^{3} / \mathrm{s}$, then 90 percent of all October daily discharge values for the period of record were equal to or greater than $2.80 \mathrm{ft}^{3} / \mathrm{s}$.

\section{Probability of Occurrence of High or Low Discharges}

The probabilities of occurrence of annual high discharges, annual low discharges, and seasonal low discharges are presented in three tables for each gaging station. Probability of occurrence is an estimate of the likelihood that a particular discharge in a stream will be equaled or exceeded in 1 year or, in the case of low flows, the likelihood that the discharge will not be equaled or exceeded during the year. The probability of occurrence of a high flow is called the exceedance probability, and the probability of occurrence of low flow is called the nonexceedance probability. For example, if the discharge for the 0.20 exceedance probability is listed as $255 \mathrm{ft}^{3} / \mathrm{s}$, then a 20 percent chance exists that a discharge equal to or greater than $255 \mathrm{ft}^{3} / \mathrm{s}$ will occur once during the year.

Recurrence interval is another way of expressing annual probability and it is the reciprocal of probability of occurrence. The recurrence interval for an exceedance probability of 0.20 is 5 years ( 1 divided by 0.20 ). For a long discharge record the annual maximum discharge can be expected to equal or exceed $255 \mathrm{ft}^{3} / \mathrm{s}$ on average once every 5 years.

The table of probability of annual high discharges for each gaging station lists the maximum instantaneous discharge and the maximum mean discharge for 3,7,15, and 30 consecutive-day periods for selected exceedance probabilities and recurrence intervals. Values for the maximum instantaneous discharge are computed from the streamflow record according to the guidelines established by the Hydrology Subcommittee of the Interagency Advisory Committee on Water Data (1982). According to the guidelines, adjustments are made for length of record and regional skew.

Values for the maximum mean discharges for 3,7,15, and 30 consecutive-day periods are computed from the annual high mean values of the corresponding periods. The computations are based on the logPearson Type III distribution using values obtained for the water year.

The table of probability of annual low discharges for each gaging station lists the minimum mean discharge for $1,3,7,14,30,60,90,120$, and 183 consecutive-day periods for selected nonexceedance probabilities and recurrence intervals. Values for the minimum mean discharges are computed from the annual low discharge values of the corresponding periods using the log-Pearson Type III distribution. If the log-Pearson Type III distribution curve fails to fit the data at the lower end, a graphical interpretation is made. Probabilities of annual low discharges are computed using values obtained for the climatic year (April 1 through March 31).

The table of probability of seasonal low discharges for each gaging station lists the minimum mean discharge for $1,7,14$, and 30 consecutive-day periods for selected probabilities and recurrence intervals. These values are computed from the seasonal low mean values of the corresponding periods using the logPearson Type III distribution. 
The annual low discharge and the seasonal low discharges that occur in any given year are sensitive to natural-channel processes, such as evapotranspiration and human-induced hydrologic modifications, such as the operation of many small water-storage reservoirs; the effects of surface-water withdrawal for agricultural, municipal, and industrial use; and the effects of return flow to the river. Thus, the statistics in tables are given for recurrence intervals that generally are within twice the period of record.

\section{DATA CONSIDERATIONS}

\section{Perlod of Record}

The reliability of statistical data is related to the length of record for a stream. The Hydrology Subcommittee of the Interagency Advisory committee on Water Data (1982) recommends that at least 10 years of record be used for computing flood frequency estimates. Therefore, the length of record criterion for inclusion of a gaging station in this report is at least 10 years. Even with this criterion, the lengths of record for each gaging station varies substantially. Subsequently, extreme high or low flows may be included in the streamflow record of one gaging station and not in another, resulting in inconsistencies in the streamflow statistics when comparing gaging station data. Also, longer record lengths for many of the gaging stations in this report may result in different streamflow statistics when comparing data in this report with data in previous publications.

Differences in statistical data for pre- and post-regulation periods are not caused solely by regulation. Differences can be attributed to the length of record and climatic variability as expressed by hydrologic variability. By comparing a statistic that easily can be affected by regulation, such as the 7-day low flow, and a statistic that generally is unaffected by regulation, such as the mean annual discharge, a determination can be made about the effect of regulation. As an example, the annual 7-day low flow with a 10-year recurrence interval for the Red River of the North at Fargo is zero for the pre-regulation period $(1901-41)$ and $17.9 \mathrm{ft}^{3} / \mathrm{s}$ for the post-regulation period (1942-94). The effect of regulation on the mean annual discharge of the Red River of the North can be assumed to be negligible; however, the mean annual discharge is $403 \mathrm{ft}^{3} / \mathrm{s}$ for the pre-regulation period and $741 \mathrm{ft}^{3} / \mathrm{s}$ for the post-regulation period. Although annual 7-day low flow for a 10-year recurrence interval is much greater for the regulation period, the mean annual discharge for the regulated period also is much greater, indicating that regulation may happen to correspond to a relatively wet period in the Red River of the North Basin.

\section{REFERENCES}

Crosby, O.A, 1970, A proposed streamflow data program for North Dakota: U.S. Geological Survey Open-File Report, 68 p.

Haffield, N.D., 1981, Statistical summaries of streamflow and water-quality data for streams of western North Dakota, 1977-80: U.S. Geological Survey Open-File Report 81-1066, 78 p.

Hydrology Subcommittee of the Interagency Advisory Committee on Water Data, 1982, Guidelines for determining flood flow frequency: Hydrology Subcommittee Bulletin 17B, 28 p., 14 appendices.

Wahl, K.L., Thomas, W.O., Jr., and Hirsch, R.M., 1995, Stream-gaging program of the U.S. Geological Survey: U.S. Geological Survey Circular 1123, 22 p. 


\section{STREAMFLOW STATISTICS}




\section{OTTER TAIL RIVER NEAR DETROIT LAKES, MN}

\section{Station Description}

LOCATION.--Lat $46^{\circ} 50^{\prime} 12^{\prime \prime}$, long $95^{\circ} 41^{\prime} 57^{\prime \prime}$, in $\mathrm{NE}^{1} / \mathrm{SWW}^{1} / 4$ sec.23, T.139 N., R.40 W., Becker County, Hydrologic Unit 09020103, on right bank $10 \mathrm{ft}$ upstream from highway bridge, 5 mi downstream from Height of Land Lake, and 7.5 mi east of city of Detroit Lakes.

DRAINAGE AREA.--270 $\mathrm{mi}^{2}$.

PERIOD OF RECORD.--March 1937 to September 1971.

GAGE.--Water-stage recorder and concrete control. Datum of gage is $1,409.49 \mathrm{ft}$ above sea level.

EXTREMES FOR PERIOD OF RECORD.--Maximum discharge, $371 \mathrm{ft}^{3} / \mathrm{s}$, June 26, 1943 (gage height, $4.78 \mathrm{ft}$, from graph based on partial record); maximum gage height, $6.96 \mathrm{ft}$, Jan. 27, 1950 (backwater from ice); minimum daily discharge, $0.10 \mathrm{ft}^{3} / \mathrm{s}, \mathrm{Mar} .23,1940$.

Annual mean discharge

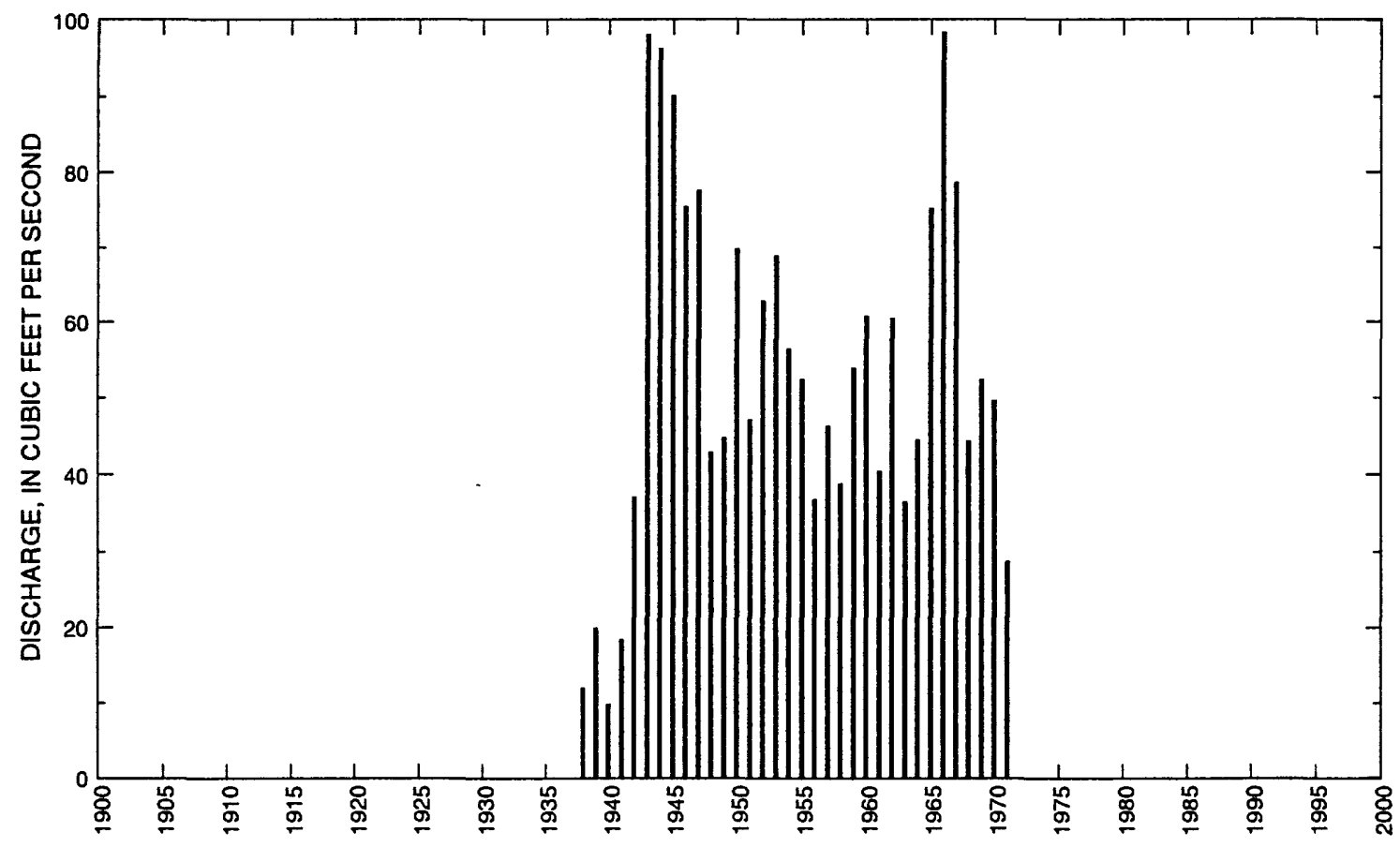


05030000 OTTER TAIL RIVER NEAR DETROIT LAKES, MN--Continued

Statistics of monthly and annual mean discharges

\begin{tabular}{|c|c|c|c|c|c|c|c|c|}
\hline \multirow[b]{2}{*}{ Month } & \multicolumn{2}{|c|}{ Maximum } & \multicolumn{2}{|c|}{ Minimum } & \multicolumn{4}{|c|}{ Mean } \\
\hline & $\begin{array}{c}\text { Discharge } \\
\left(\mathrm{ft}^{3} / \mathrm{s}\right)\end{array}$ & $\begin{array}{l}\text { Water year } \\
\text { of } \\
\text { occurrence }\end{array}$ & $\begin{array}{c}\text { Discharge } \\
\left(\mathrm{ft}^{3} / \mathrm{s}\right)\end{array}$ & $\begin{array}{c}\text { Water year } \\
\text { of } \\
\text { occurrence }\end{array}$ & $\begin{array}{c}\text { Discharge } \\
\left(\mathrm{ft}^{3} / \mathrm{s}\right)\end{array}$ & $\begin{array}{c}\text { Standard } \\
\text { deviation } \\
\left(\mathrm{ft}^{3} / \mathrm{s}\right)\end{array}$ & $\begin{array}{l}\text { Coeffi- } \\
\text { cient of } \\
\text { variation }\end{array}$ & $\begin{array}{l}\text { Percentage } \\
\text { of annual } \\
\text { discharge }\end{array}$ \\
\hline October & 138 & 1945 & 2.16 & 1957 & 29.2 & 28.9 & 0.99 & 4.61 \\
\hline November & 105 & 1945 & 4.94 & 1958 & 25.2 & 22.6 & 0.90 & 3.98 \\
\hline December & 77.6 & 1945 & 4.37 & 1938 & 26.3 & 20.0 & 0.76 & 4.15 \\
\hline January & 57.0 & 1966 & 3.23 & 1938 & 24.4 & 17.4 & 0.71 & 3.85 \\
\hline February & 60.1 & 1969 & 0.793 & 1940 & 24.5 & 15.7 & 0.64 & 3.87 \\
\hline March & 83.2 & 1969 & 0.371 & 1940 & 33.9 & 19.6 & 0.58 & 5.36 \\
\hline April & 190 & 1966 & 1.15 & 1938 & 77.8 & 48.3 & 0.62 & 12.3 \\
\hline May & 265 & 1966 & 1.45 & 1940 & 115 & 65.6 & 0.57 & 18.2 \\
\hline June & 284 & 1943 & 2.16 & 1940 & 114 & 71.1 & 0.62 & 18.0 \\
\hline July & 206 & 1944 & 1.52 & 1940 & 77.1 & 51.8 & 0.67 & 12.2 \\
\hline August & 288 & 1944 & 0.510 & 1940 & 49.8 & 57.5 & 1.15 & 7.87 \\
\hline September & 238 & 1944 & 1.40 & 1970 & 35.6 & 42.8 & 1.20 & 5.63 \\
\hline Annual & 98.4 & 1966 & 9.82 & 1940 & 53.7 & 23.6 & 0.44 & 100 \\
\hline
\end{tabular}

Annual flow duration

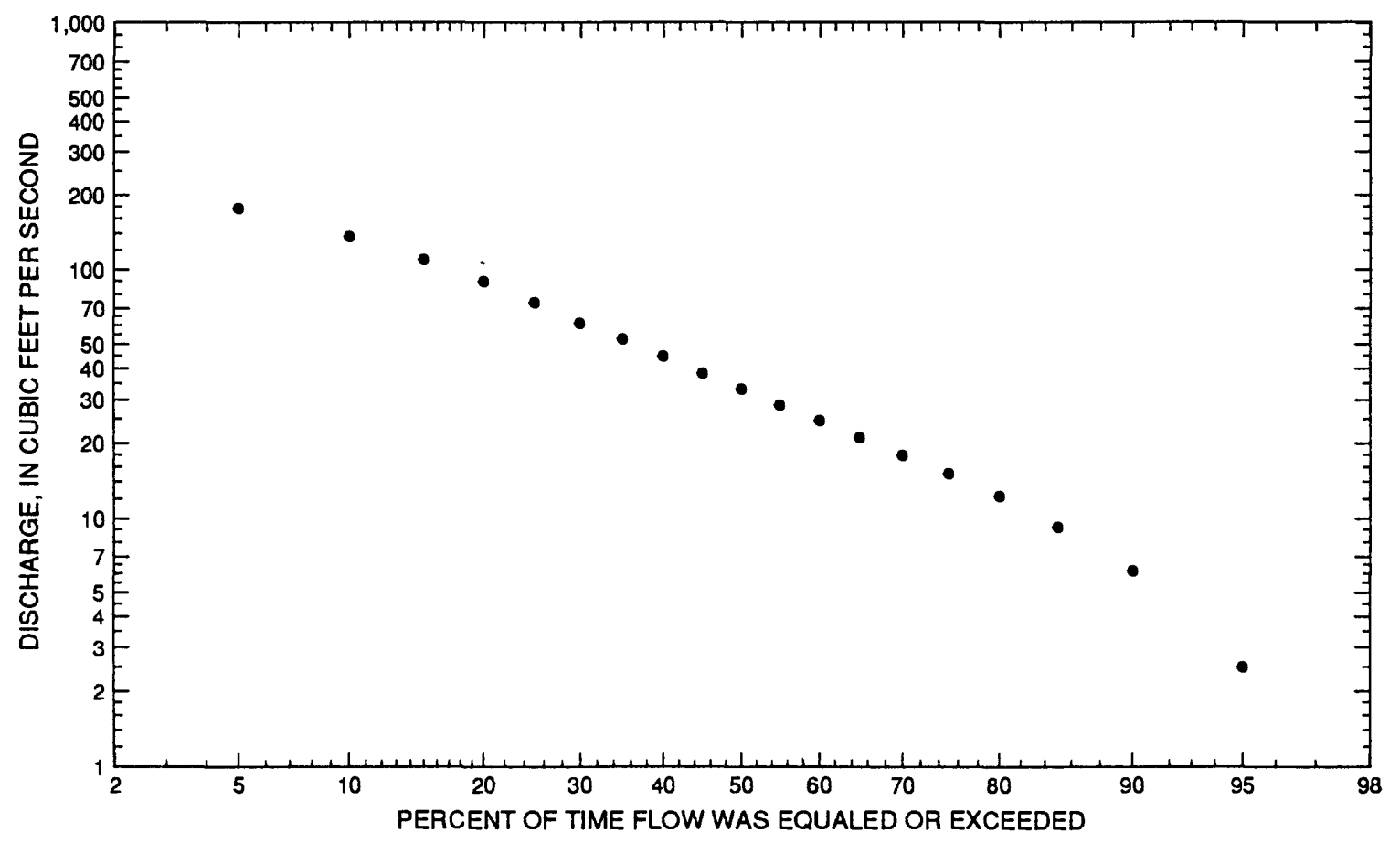




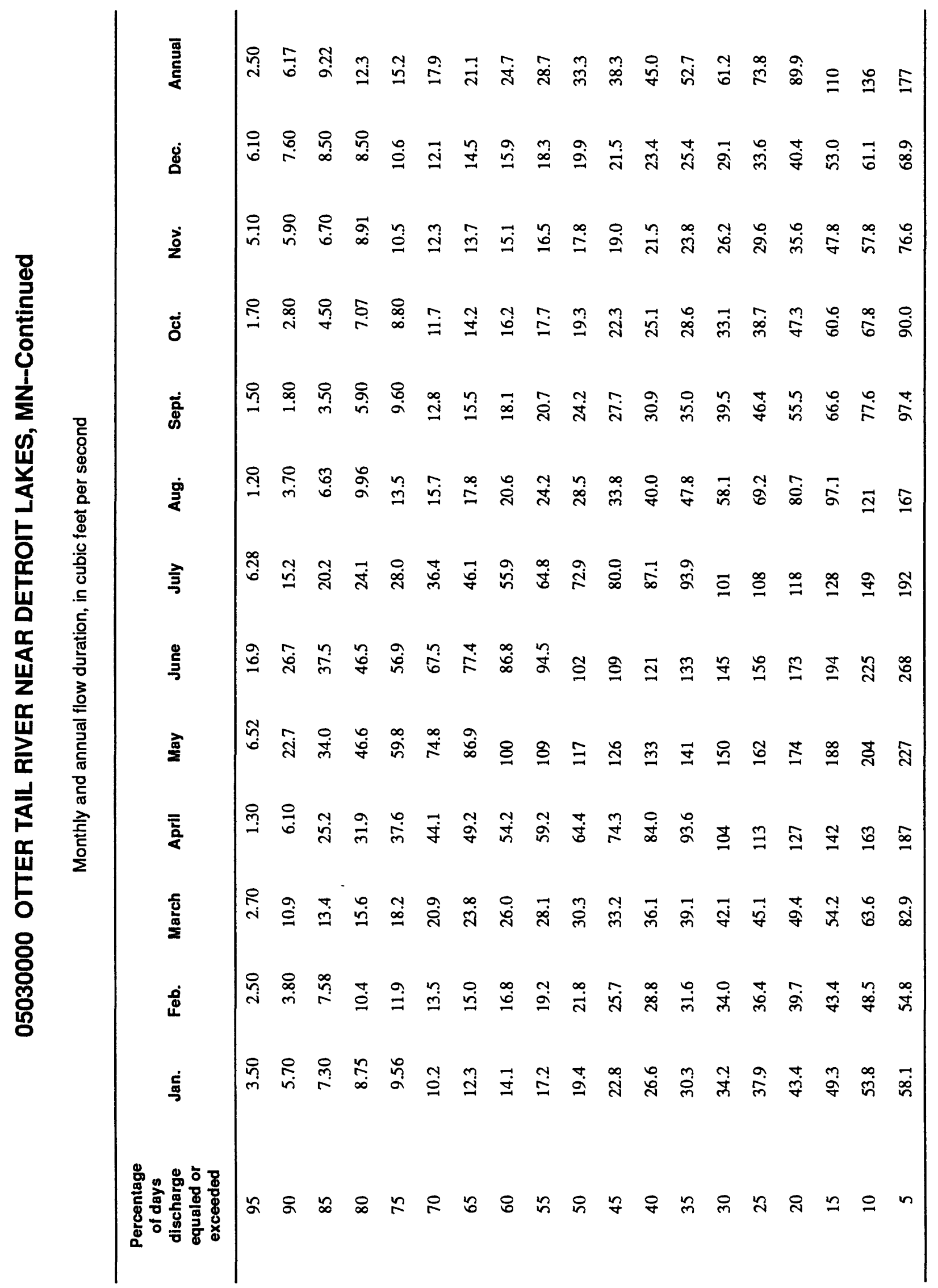




\section{OTTER TAIL RIVER NEAR DETROIT LAKES, MN--Continued}

Probability of annual high discharges

[ng, statistic not given]

\begin{tabular}{|c|c|c|c|c|c|c|}
\hline \multirow[b]{2}{*}{$\begin{array}{l}\text { Exceedance } \\
\text { probability }\end{array}$} & \multirow[b]{2}{*}{$\begin{array}{l}\text { Recurrence } \\
\text { interval } \\
\text { (years) }\end{array}$} & \multirow[b]{2}{*}{$\begin{array}{c}\text { Maximum } \\
\text { instantaneous } \\
\left(\mathrm{ft}^{3} / \mathrm{s}\right)\end{array}$} & \multicolumn{4}{|c|}{$\begin{array}{l}\text { Maximum average discharge } \\
\qquad\left(\mathrm{ft}^{3} / \mathrm{s}\right)\end{array}$} \\
\hline & & & 3-day period & 7-day period & 15-day period & 30-day period \\
\hline 0.99 & 1.01 & 37.6 & 37.4 & 32.7 & 25.9 & 22.1 \\
\hline 0.95 & 1.05 & 62.7 & 61.6 & 56.4 & 48.7 & 43.5 \\
\hline 0.90 & 1.11 & 80.4 & 78.5 & 73.4 & 65.7 & 59.9 \\
\hline 0.80 & 1.25 & 106 & 103 & 98.6 & 91.4 & 84.8 \\
\hline 0.50 & 2 & 171 & 164 & 161 & 155 & 147 \\
\hline 0.20 & 5 & 255 & 242 & 239 & 234 & 223 \\
\hline 0.10 & 10 & 306 & 288 & 284 & 278 & 264 \\
\hline 0.04 & 25 & 363 & 341 & 334 & 324 & 306 \\
\hline 0.02 & 50 & 401 & 375 & 366 & 352 & 331 \\
\hline 0.01 & 100 & 436 & 407 & 394 & 376 & 352 \\
\hline 0.005 & 200 & 467 & 436 & 420 & 397 & 369 \\
\hline 0.002 & 500 & 506 & ng & ng & ng & ng \\
\hline
\end{tabular}

Probability of annual low discharges

\begin{tabular}{|c|c|c|c|c|c|c|c|c|c|c|}
\hline \multirow[b]{3}{*}{$\begin{array}{l}\text { Non- } \\
\text { exceed- } \\
\text { ance } \\
\text { prob- } \\
\text { ability }\end{array}$} & \multirow[b]{3}{*}{$\begin{array}{c}\text { Recur- } \\
\text { rence } \\
\text { inter- } \\
\text { val } \\
\text { (years) }\end{array}$} & \multicolumn{9}{|c|}{ Minimum average discharge $\left(\mathrm{ft}^{3} / \mathrm{s}\right)$} \\
\hline & & \multicolumn{9}{|c|}{ Number of consecutive days } \\
\hline & & 1 & 3 & 7 & 14 & 30 & 60 & 90 & 120 & 183 \\
\hline 0.05 & 20 & 0.222 & 0.275 & 0.317 & 0.432 & 0.691 & 1.15 & 2.32 & 3.11 & 5.96 \\
\hline 0.10 & 10 & 0.430 & 0.506 & 0.583 & 0.799 & 120 & 2.00 & 3.45 & 4.51 & 7.56 \\
\hline 0.20 & 5 & 0.909 & 1.02 & 1.17 & 1.60 & 2.24 & 3.66 & 5.46 & 6.92 & 10.1 \\
\hline 0.50 & 2 & 3.24 & 3.40 & 3.86 & 5.13 & 6.45 & 9.84 & 12.3 & 14.7 & 18.1 \\
\hline
\end{tabular}




\section{OTTER TAIL RIVER NEAR DETROIT LAKES, MN--Continued}

Probability of seasonal low discharges

\begin{tabular}{|c|c|c|c|c|c|c|c|c|c|}
\hline \multirow[b]{3}{*}{$\begin{array}{c}\text { Non- } \\
\text { exceedance } \\
\text { probability }\end{array}$} & \multirow[b]{3}{*}{$\begin{array}{l}\text { Recurrence } \\
\text { Interval } \\
\text { (years) }\end{array}$} & \multicolumn{8}{|c|}{ Minimum average discharge $\left(\mathrm{ft}^{3} / \mathrm{s}\right)$} \\
\hline & & \multicolumn{8}{|c|}{ Number of consecutive days } \\
\hline & & 1 & 7 & 14 & 30 & 1 & 7 & 14 & 30 \\
\hline & & \multicolumn{4}{|c|}{ December-January-February } & \multicolumn{4}{|c|}{ March-April-May } \\
\hline 0.05 & 20 & 1.49 & 1.79 & 1.98 & 2.46 & 0.623 & 1.01 & 1.45 & 2.63 \\
\hline 0.10 & 10 & 2.48 & 2.88 & 3.17 & 3.91 & 1.63 & 2.37 & 3.30 & 5.94 \\
\hline 0.20 & 5 & 4.35 & 4.88 & 5.35 & 6.53 & 4.38 & 5.67 & 7.56 & 13.0 \\
\hline \multirow[t]{2}{*}{0.50} & 2 & 11.0 & 11.7 & 12.7 & 15.1 & 17.4 & 19.4 & 23.2 & 33.7 \\
\hline & & \multicolumn{4}{|c|}{ June-July-August } & \multicolumn{4}{|c|}{ September-October-November } \\
\hline 0.05 & 20 & 0.435 & 0.749 & 1.27 & 2.65 & 0.538 & 0.680 & 1.06 & 1.78 \\
\hline 0.10 & 10 & 0.991 & 1.57 & 2.45 & 4.93 & 0.998 & 1.23 & 1.73 & 2.66 \\
\hline 0.20 & 5 & 2.47 & 3.57 & 5.11 & 9.66 & 2.02 & 2.43 & 3.06 & 4.30 \\
\hline 0.50 & 2 & 11.1 & 41.7 & 17.4 & 27.9 & 6.82 & 7.88 & 8.65 & 10.7 \\
\hline
\end{tabular}




\section{OTTER TAIL RIVER NEAR DETROIT LAKES, MN--Continued}

Annual peak discharge and corresponding gage height, period of record

\begin{tabular}{|c|c|c|c|c|c|c|c|}
\hline $\begin{array}{l}\text { Water } \\
\text { year }\end{array}$ & Date & $\begin{array}{c}\text { Gage } \\
\text { height } \\
\text { (feet) }\end{array}$ & $\begin{array}{c}\text { Peak } \\
\text { discharge } \\
\left(\mathrm{ft}^{3} / \mathrm{s}\right)\end{array}$ & $\begin{array}{l}\text { Water } \\
\text { year }\end{array}$ & Date & $\begin{array}{c}\text { Gage } \\
\text { height } \\
\text { (feet) }\end{array}$ & $\begin{array}{c}\text { Peak } \\
\text { discharge } \\
\left(\mathrm{ft}^{3} / \mathrm{s}\right)\end{array}$ \\
\hline \multicolumn{8}{|c|}{ Annual peak discharge, by year, and corresponding gage beight } \\
\hline 1937 & June 20 & 3.53 & 54.0 & 1955 & August 3 & 4.28 & 195 \\
\hline 1938 & June 3 & 3.67 & 73.0 & 1956 & May 3 & 4.07 & 139 \\
\hline 1939 & March 23 & 4.83 & 239 & 1957 & May 14 & 4.00 & 133 \\
\hline 1940 & September 21 & 3.45 & 42.0 & 1958 & October 16 & 3.66 & 72.0 \\
\hline 1941 & May 15 & 3.66 & 68.0 & 1959 & June 11 & 3.99 & 136 \\
\hline 1942 & June 16 & 3.88 & 112 & 1960 & May 3 & 4.34 & 214 \\
\hline 1943 & June 26 & 4.78 & 371 & 1961 & May 15 & 4.12 & 151 \\
\hline 1944 & August 22 & 4.68 & 336 & 1962 & June 8 & 4.65 & 316 \\
\hline 1945 & May 13 & 4.29 & 198 & 1963 & June 9 & 4.07 & 157 \\
\hline 1946 & July 21 & 4.26 & 190 & 1964 & May 19 & 4.10 & 164 \\
\hline 1947 & May 2 & 4.35 & 212 & 1965 & June 13 & 4.45 & 264 \\
\hline 1948 & April 30 & 3.93 & 119 & 1966 & May 3 & 4.51 & 283 \\
\hline 1949 & July 31 & 4.00 & 133 & 1967 & June 22 & 4.27 & 267 \\
\hline 1950 & May 29 & 4.71 & 332 & 1968 & May 29 & 4.18 & 188 \\
\hline 1951 & June 2 & 4.26 & 190 & 1969 & April 21 & 4.30 & 207 \\
\hline 1952 & April 21 & 4.16 & 167 & 1970 & June 16 & 4.21 & 182 \\
\hline 1953 & June 15 & 4.36 & 218 & 1971 & April 10 & 3.76 & 84.0 \\
\hline 1954 & May 5 & 4.27 & 193 & & & & \\
\hline \multicolumn{8}{|c|}{ Annual peak discharge, from highest to lowest, and corresponding gage height } \\
\hline 1943 & June 26 & 4.78 & 371 & 1970 & June 16 & 4.21 & 182 \\
\hline 1944 & August 22 & 4.68 & 336 & 1952 & April 21 & 4.16 & 167 \\
\hline 1950 & May 29 & 4.71 & 332 & 1964 & May 19 & 4.10 & 164 \\
\hline 1962 & June 8 & 4.65 & 316 & 1963 & June 9 & 4.07 & 157 \\
\hline 1966 & May 3 & 4.51 & 283 & 1961 & May 15 & 4.12 & 151 \\
\hline 1967 & June 22 & 4.27 & 267 & 1956 & May 3 & 4.07 & 139 \\
\hline 1965 & June 13 & 4.45 & 264 & 1959 & June 11 & 3.99 & 136 \\
\hline 1939 & March 23 & 4.83 & 239 & 1949 & July 31 & 4.00 & 133 \\
\hline 1953 & June 15 & 4.36 & 218 & 1957 & May 14 & 4.00 & 133 \\
\hline 1960 & May 3 & 4.34 & 214 & 1948 & April 30 & 3.93 & 119 \\
\hline 1947 & May 2 & 4.35 & 212 & 1942 & June 16 & 3.88 & 112 \\
\hline 1969 & April 21 & 4.30 & 207 & 1971 & April 10 & 3.76 & 84.0 \\
\hline 1945 & May 13 & 4.29 & 198 & 1938 & June 3 & 3.67 & 73.0 \\
\hline 1955 & August 3 & 4.28 & 195 & 1958 & October 16 & 3.66 & 72.0 \\
\hline 1954 & May 5 & 4.27 & 193 & 1941 & May 15 & 3.66 & 68.0 \\
\hline 1946 & July 21 & 4.26 & 190 & 1937 & June 20 & 3.53 & 54.0 \\
\hline 1951 & June 2 & 4.26 & 190 & 1940 & September 21 & 3.45 & 42.0 \\
\hline 1968 & May 29 & 4.18 & 188 & & & & \\
\hline
\end{tabular}




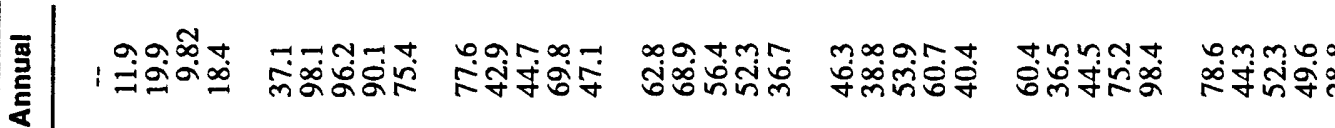

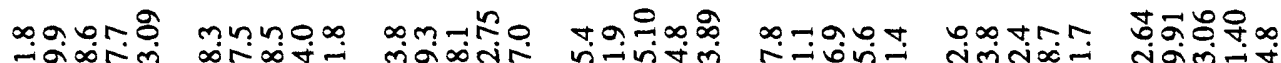

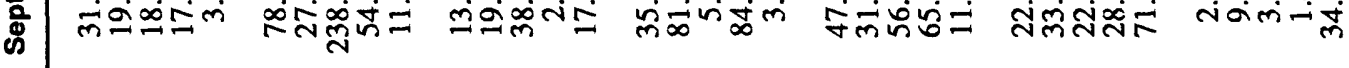

ㅇำ b동ำ

$\operatorname{tin} \infty \pi$

ningong

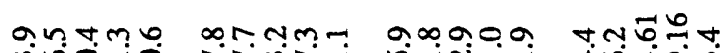

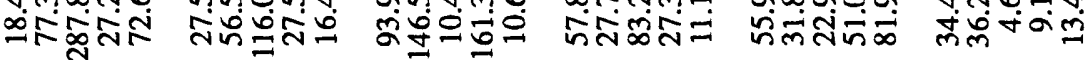

ำำกำ

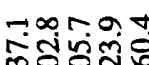

70090

mitun on ono

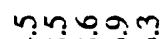

$\infty \pi-N$

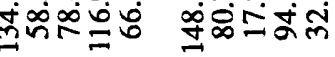

ที่ำำ

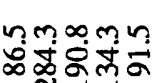

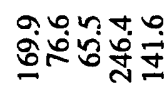

लिळ0.

orang.

aomrt

gmatr. (1) .

สู่ำ่ำ

윰ำㅇํㅇ

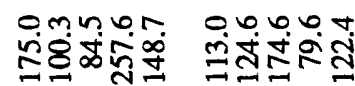

บิํํํํำ

जกำ

mơñ

กุบn สำำ

이의.

mrinom

mmoon

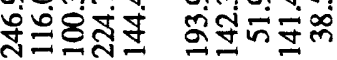

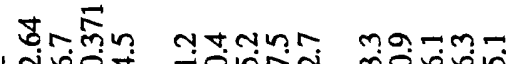

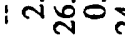
F⿻ำ่ง

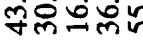

ตำกำ

ㅇm요맘

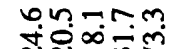

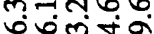

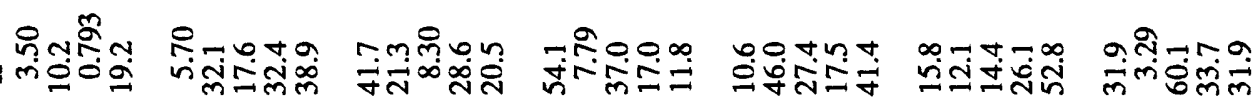

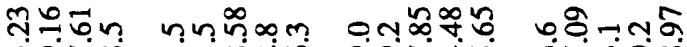

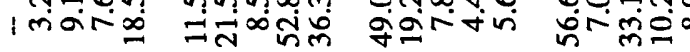

oinmtin

ming.

$\sin \infty \nabla$ (1)

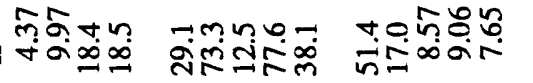

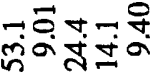

बंक्षित्रें

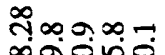

은ำ (1)

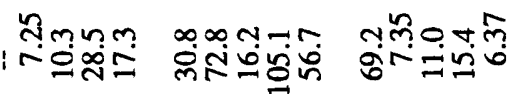

rọmno

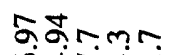
巡 मेंतुल

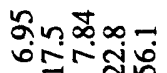

- 000.0

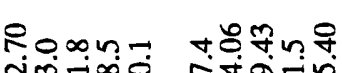

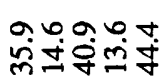

$\rightarrow \infty+n o$

कึna-- âmo

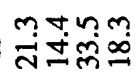
लूूं

केष्तंज

กำ 뉴ल

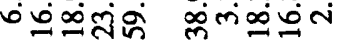

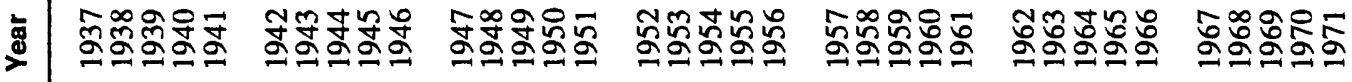




\section{Station Description}

LOCATION.--Lat $46^{\circ} 22^{\prime} 10^{\prime \prime}$, long $96^{\circ} 01^{\prime} 02^{\prime \prime}$, in SW $1 / 4 \mathrm{SE}^{1} / 4 \mathrm{sec} .31$, T.134 N., R.42 W., Ottertail County, Hydrologic Unit 09020103, on right bank, $2.5 \mathrm{mi}$ below Taplin Gorge Dam, $5.0 \mathrm{mi}$ above the Diversion Dam, 5.7 mi east of Elizabeth and 6.6 mi northeast of Fergus Falls.

DRAINAGE AREA.--1,230 $\mathrm{mi}^{2}$, approximately.

PERIOD OF RECORD.--May 1904 to September 1917, monthly discharge only, published as at German Church near Fergus Falls in WSP 1308. July 1992 to current year.

GAGE.--Water-stage recorder. Datum of gage is $1,250 \mathrm{ft}$ above mean sea level, from topographic map. Nonrecording gage at same site November 1913 to September 1917 at datum 1,265 ft from topographic map.

EXTREMES FOR PERIOD OF RECORD.--Maximum discharge, 1,075 $\mathrm{ft}^{3} / \mathrm{s}$, June 23, 1904 (gage height, $4.2 \mathrm{ft}$ ); minimum daily discharge, $16.0 \mathrm{ft}^{3} / \mathrm{s}$, Sept. 13,1910 (minimum gage height, $1.9 \mathrm{ft}$ ).

Annual mean discharge

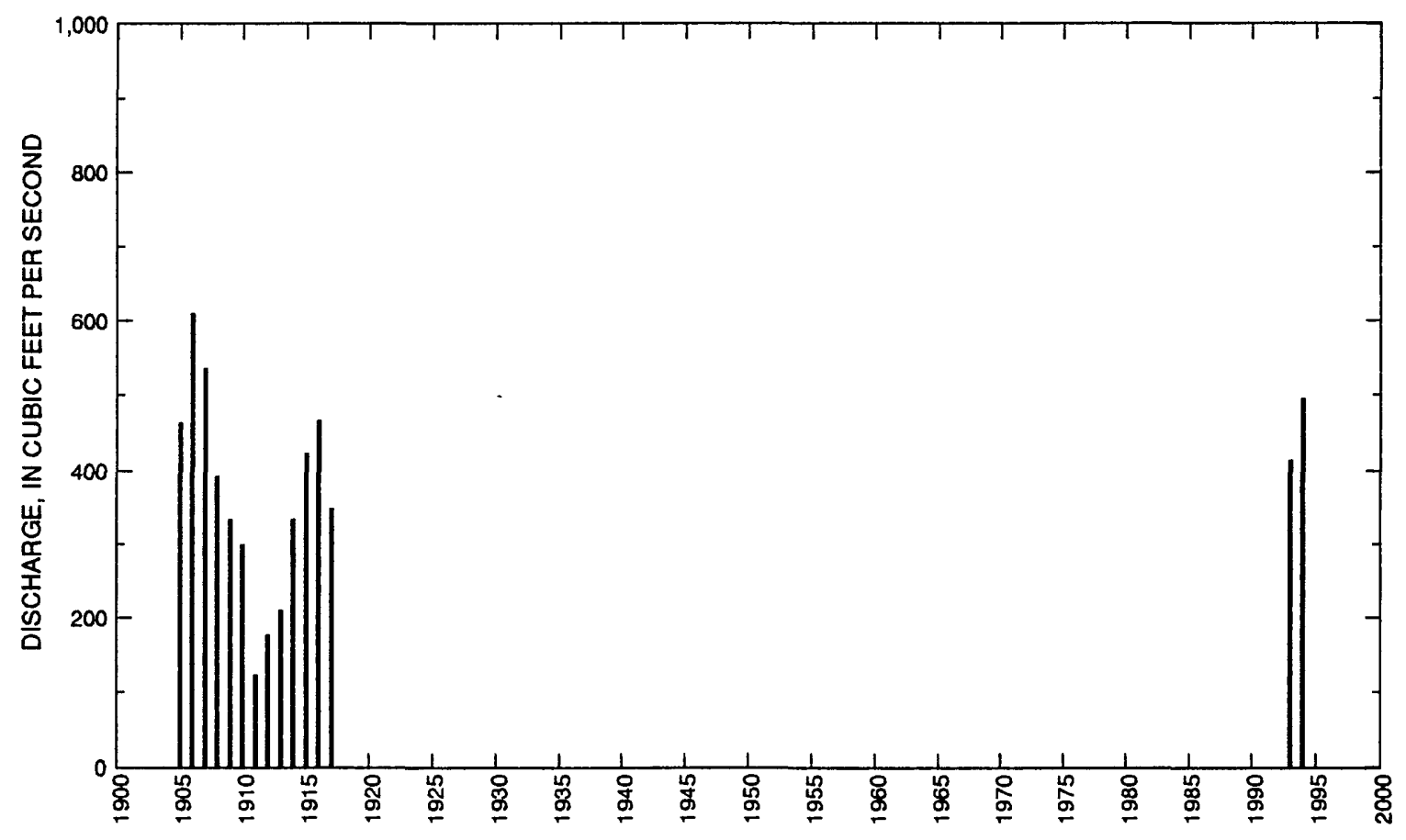




\section{OTTER TAIL RIVER NEAR ELIZABETH, MN--Continued}

Statistics of monthly and annual mean discharges

[m, more than 1 year of occurrence]

\begin{tabular}{|c|c|c|c|c|c|c|c|c|}
\hline \multirow[b]{2}{*}{ Month } & \multicolumn{2}{|c|}{ Maximum } & \multicolumn{2}{|c|}{ Minimum } & \multicolumn{4}{|c|}{ Mean } \\
\hline & $\begin{array}{c}\text { Discharge } \\
\left(\mathrm{ft}^{3} / \mathrm{s}\right)\end{array}$ & $\begin{array}{c}\text { Water year } \\
\text { of } \\
\text { occurrence }\end{array}$ & $\begin{array}{c}\text { Discharge } \\
\left(\mathrm{ft}^{3} / \mathrm{s}\right)\end{array}$ & $\begin{array}{c}\text { Water year } \\
\text { of } \\
\text { occurrence }\end{array}$ & $\begin{array}{c}\text { Discharge } \\
\left(\mathrm{ft}^{3} / \mathrm{s}\right)\end{array}$ & $\begin{array}{c}\text { Standard } \\
\text { deviation } \\
\left(\mathrm{ft}^{3} / \mathrm{s}\right)\end{array}$ & $\begin{array}{l}\text { Coeffi- } \\
\text { cient of } \\
\text { variation }\end{array}$ & $\begin{array}{l}\text { Percentage } \\
\text { of annual } \\
\text { discharge }\end{array}$ \\
\hline October & 740 & 1994 & 48.3 & 1911 & 386 & 207 & 0.54 & 8.63 \\
\hline November & 688 & 1907 & 79.3 & 1911 & 360 & 178 & 0.49 & 8.04 \\
\hline December & 489 & 1994 & 79.9 & 1911 & 303 & 132 & 0.44 & 6.77 \\
\hline January & 400 & 1994 & 80.0 & $\mathrm{~m}$ & 247 & 105 & 0.43 & 5.52 \\
\hline February & 439 & 1994 & 70.0 & 1912 & 221 & 98.6 & 0.45 & 4.94 \\
\hline March & 650 & 1907 & 70.0 & 1912 & 280 & 142 & 0.51 & 6.25 \\
\hline April & 652 & 1907 & 180 & 1913 & 363 & 137 & 0.38 & 8.11 \\
\hline May & 772 & 1906 & 221 & 1911 & 482 & 175 & 0.36 & 10.8 \\
\hline June & 965 & 1906 & 169 & 1911 & 545 & 225 & 0.41 & 12.2 \\
\hline July & 932 & 1906 & 108 & 1911 & 506 & 244 & 0.48 & 11.3 \\
\hline August & 780 & 1905 & 64.8 & 1910 & 400 & 228 & 0.57 & 8.93 \\
\hline September & 817 & 1993 & 32.6 & 1910 & 383 & 236 & 0.62 & 8.57 \\
\hline Annual & 609 & 1906 & 123 & 1911 & 375 & 135 & 0.36 & 100 \\
\hline
\end{tabular}

Annual flow duration

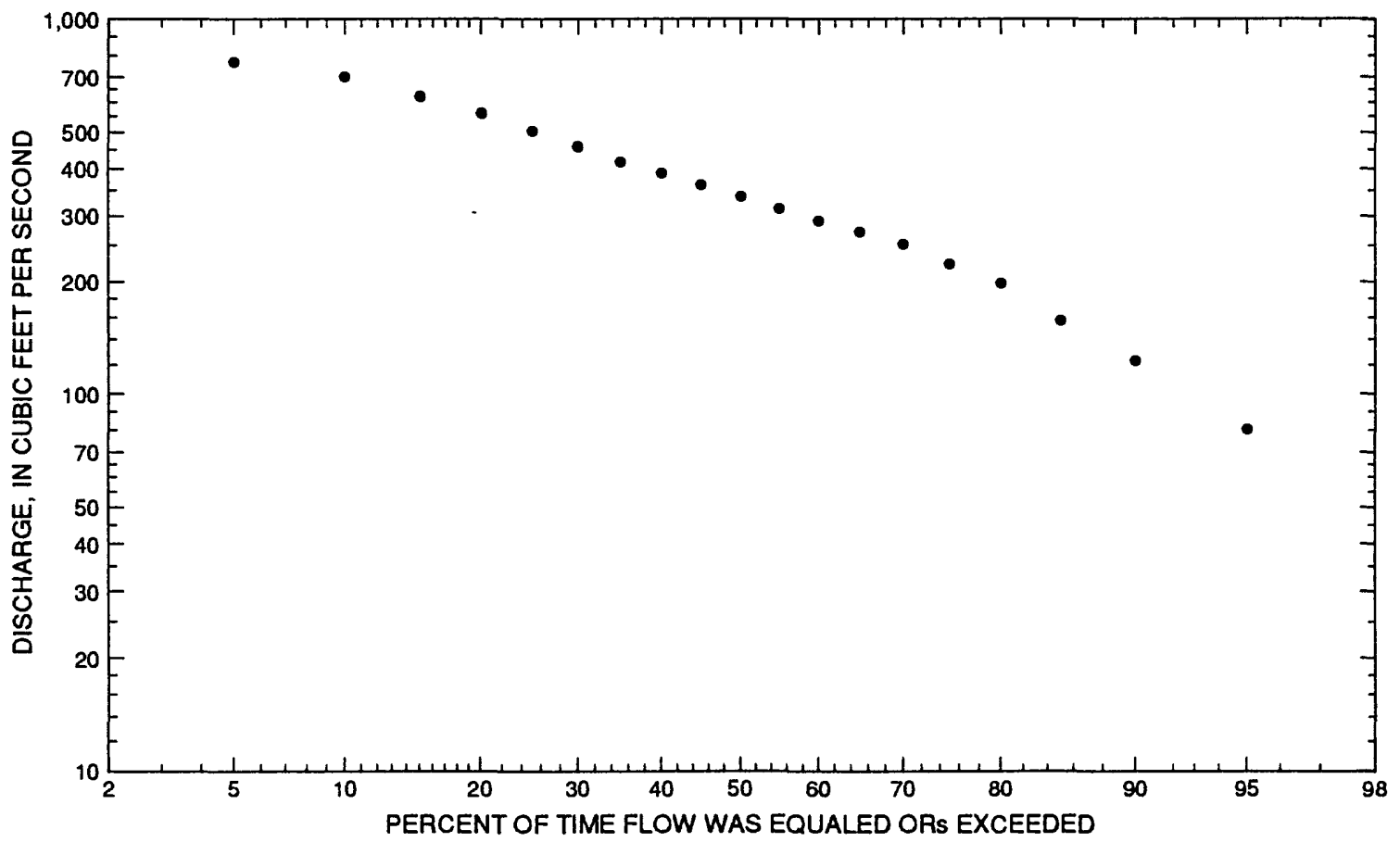




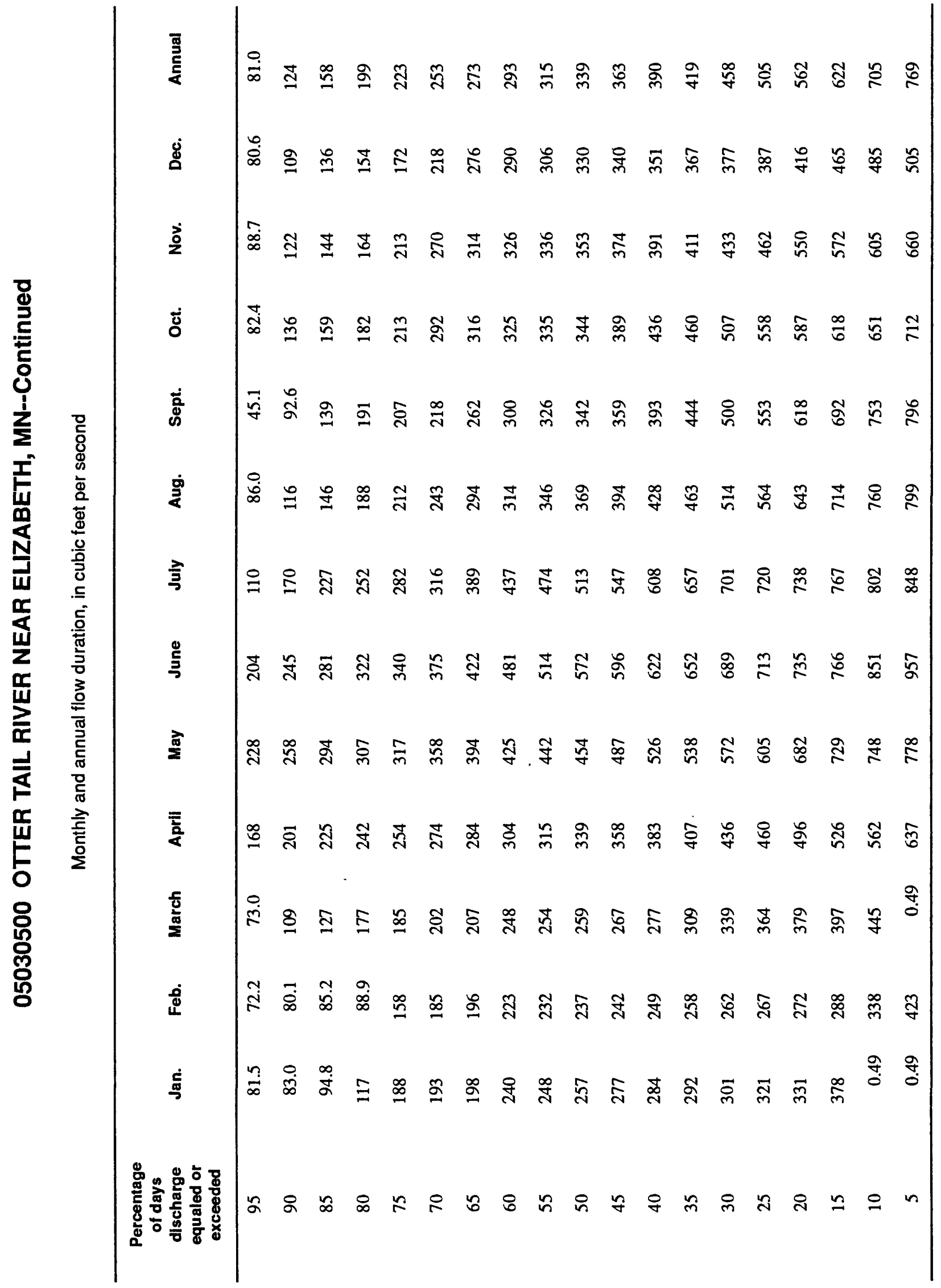




\section{OTTER TAIL RIVER NEAR ELIZABETH, MN--Continued}

Probability of annual high discharges

[ng, statistic not given]

\begin{tabular}{lcccccc}
\hline & & & \multicolumn{5}{c}{$\begin{array}{c}\text { Maximum average discharge } \\
\left(\mathrm{ft}^{3} / \mathbf{s}\right)\end{array}$} \\
\cline { 5 - 7 } $\begin{array}{c}\text { Exceedance } \\
\text { probability }\end{array}$ & $\begin{array}{c}\text { Recurrence } \\
\text { interval } \\
\text { (years) }\end{array}$ & $\begin{array}{c}\text { Maximum } \\
\text { instantaneous } \\
\left(\mathrm{ft}^{3} / \mathbf{s}\right)\end{array}$ & 3-day period & 7-day period & 15-day period & 30-day period \\
\hline 0.99 & 1.01 & $\mathrm{ng}$ & 198 & 187 & 181 & 172 \\
0.95 & 1.05 & $\mathrm{ng}$ & 324 & 307 & 291 & 277 \\
0.90 & 1.11 & 462 & 405 & 385 & 364 & 346 \\
0.80 & 1.25 & 549 & 512 & 489 & 462 & 440 \\
0.50 & 2 & 738 & 726 & 701 & 667 & 640 \\
0.20 & 5 & 947 & 913 & 895 & 868 & 843 \\
0.10 & 10 & 1,060 & 989 & 978 & 961 & 939 \\
0.04 & 25 & 1,180 & 1,050 & 1,050 & 1,050 & 1,030 \\
0.02 & 50 & 1,260 & 1,080 & 1,080 & 1,090 & 1,080 \\
0.01 & 100 & 1,330 & 1,100 & 1,110 & 1,130 & 1,120 \\
0.005 & 200 & 1,390 & 1,120 & 1,130 & 1,150 & 1,150 \\
0.002 & 500 & 1,460 & ng & ng & ng & ng \\
\hline
\end{tabular}

Probability of annual low discharges

Minimum average dlscharge $\left(\mathrm{ft}^{3} / \mathrm{s}\right)$

Number of consecutive days

\begin{tabular}{|c|c|c|c|c|c|c|c|c|c|c|}
\hline $\begin{array}{l}\text { Non- } \\
\text { exceed- } \\
\text { ance } \\
\text { prob- } \\
\text { ability }\end{array}$ & $\begin{array}{l}\text { Recur- } \\
\text { rence } \\
\text { inter- } \\
\text { val } \\
\text { (years) }\end{array}$ & 1 & 3 & 7 & 14 & 30 & 60 & 90 & 120 & 183 \\
\hline 0.05 & 20 & 29.2 & 33.1 & 35.7 & 37.8 & 41.7 & 45.9 & 58.1 & 66.2 & 79.4 \\
\hline 0.10 & 10 & 50.8 & 54.6 & 57.2 & 59.6 & 64.4 & 70.8 & 84.6 & 95.3 & 113 \\
\hline 0.20 & 5 & 88.7 & 91.1 & 93.0 & 95.4 & 101 & 111 & 126 & 141 & 166 \\
\hline 0.50 & 2 & 186 & ${ }^{1} 186$ & ${ }^{1} 187$ & 187 & 194 & 216 & 232 & 254 & 298 \\
\hline
\end{tabular}

${ }^{1}$ Graphical interpretation. 


\section{OTTER TAIL RIVER NEAR ELIZABETH, MN--Continued}

Probability of seasonal low discharges

\begin{tabular}{|c|c|c|c|c|c|c|c|c|c|}
\hline \multirow[b]{3}{*}{$\begin{array}{c}\text { Non- } \\
\text { excoedance } \\
\text { probability }\end{array}$} & \multirow[b]{3}{*}{$\begin{array}{l}\text { Recurrence } \\
\text { Interval } \\
\text { (years) }\end{array}$} & \multicolumn{8}{|c|}{ Minimum average discharge $\left(\mathrm{ft}^{3} / \mathrm{s}\right)$} \\
\hline & & \multicolumn{8}{|c|}{ Number of consecutive days } \\
\hline & & 1 & 7 & 14 & 30 & 1 & 7 & 14 & 30 \\
\hline & & \multicolumn{4}{|c|}{ December-January-February } & \multicolumn{4}{|c|}{ March-April-May } \\
\hline 0.05 & 20 & 64.3 & 66.5 & 68.7 & 70.3 & 86.9 & 86.9 & 87.0 & 90.3 \\
\hline 0.10 & 10 & 85.4 & 87.1 & 89.5 & 91.7 & 109 & 109 & 111 & 117 \\
\hline 0.20 & 5 & 116 & 118 & 120 & 123 & 141 & 143 & 147 & 157 \\
\hline \multirow[t]{2}{*}{0.50} & 2 & 190 & 192 & 193 & 198 & 224 & 229 & 238 & 257 \\
\hline & & \multicolumn{4}{|c|}{ June-July-August } & \multicolumn{4}{|c|}{ September-October-November } \\
\hline 0.05 & 20 & 54.5 & 56.6 & 66.7 & 83.9 & 38.6 & 48.7 & 51.8 & 57.5 \\
\hline 0.10 & 10 & 84.6 & 88.7 & 100 & 122 & 72.5 & 85.3 & 89.7 & 97.1 \\
\hline 0.20 & 5 & 136 & 144 & 156 & 183 & 136 & 150 & 156 & 166 \\
\hline 0.50 & 2 & 287 & 304 & 316 & 351 & 307 & 320 & 333 & 347 \\
\hline
\end{tabular}




\section{OTTER TAIL RIVER NEAR ELIZABETH, MN--Continued}

Annual peak discharge and corresponding gage height, period of record

$$
[--, \text { no data }]
$$

\begin{tabular}{|c|c|c|c|c|c|c|c|}
\hline $\begin{array}{l}\text { Water } \\
\text { year }\end{array}$ & Date & $\begin{array}{c}\text { Gage } \\
\text { height } \\
\text { (feet) }\end{array}$ & $\begin{array}{c}\text { Peak } \\
\text { discharge } \\
\left(\mathrm{f}^{3} / \mathrm{s}\right)\end{array}$ & $\begin{array}{l}\text { Water } \\
\text { year }\end{array}$ & Date & $\begin{array}{c}\text { Gage } \\
\text { height } \\
\text { (feet) }\end{array}$ & $\begin{array}{c}\text { Peak } \\
\text { discharge } \\
\left(\mathrm{tt}^{3} / \mathbf{s}\right)\end{array}$ \\
\hline \multicolumn{8}{|c|}{ Annual peak discharge, by year, and correspon ding gage height } \\
\hline 1904 & June 23 & - & 1,075 & 1913 & July 5 & 3.85 & 758 \\
\hline 1905 & August 7 & 4.00 & 855 & 1914 & June 27 & 2.40 & 584 \\
\hline 1906 & - & 4.25 & 1,020 & 1915 & July 19 & 2.80 & 837 \\
\hline 1908 & June 13 & 4.10 & 921 & 1916 & June 29 & 3.00 & 982 \\
\hline 1909 & September 15 & 3.55 & 580 & 1917 & October 1 & 2.45 & 613 \\
\hline 1910 & -- & 3.30 & 449 & 1993 & September 20 & 8.10 & 842 \\
\hline 1911 & April 19 & 2.90 & 274 & 1994 & October 3 & 8.09 & 840 \\
\hline 1912 & July 8 & 3.20 & 402 & & & & \\
\hline \multicolumn{8}{|c|}{ Annual peak discharge, from highest to lowest, and corresponding gage height } \\
\hline 1904 & June 23 & - & 1,075 & 1913 & July 5 & 3.85 & 758 \\
\hline 1906 & -- & 4.25 & 1,020 & 1917 & October 1 & 2.45 & 613 \\
\hline 1916 & June 29 & 3.00 & 982 & 1914 & June 27 & 2.40 & 584 \\
\hline 1908 & June 13 & 4.10 & 921 & 1909 & September 15 & 3.55 & 580 \\
\hline 1905 & August 7 & 4.00 & 855 & 1910 & - & 3.30 & 449 \\
\hline 1993 & September 20 & 8.10 & 842 & 1912 & July 8 & 3.20 & 402 \\
\hline 1994 & October 3 & 8.09 & 840 & 1911 & April 19 & 2.90 & 274 \\
\hline 1915 & July 19 & 2.80 & 837 & & & & \\
\hline
\end{tabular}




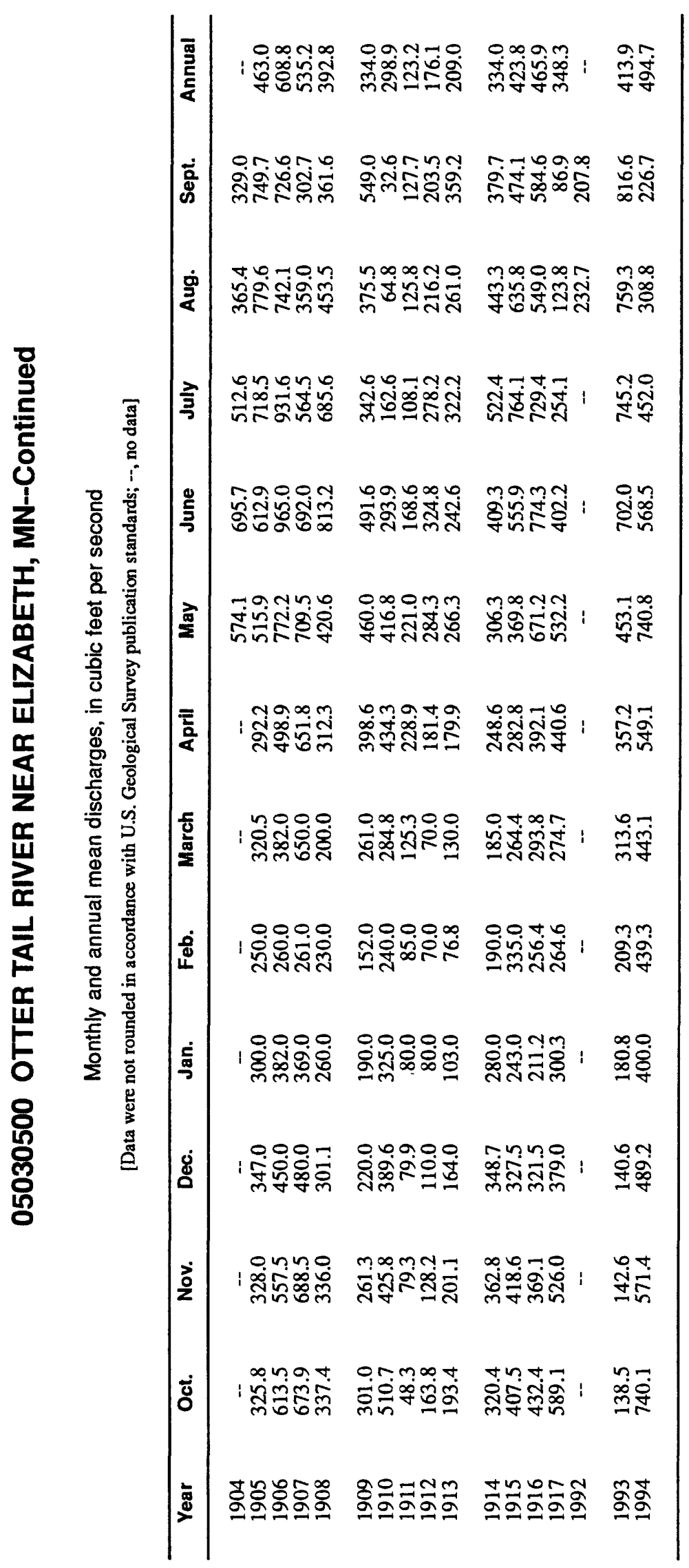




\section{PELICAN RIVER NEAR DETROIT LAKES, MN}

\section{Station Description}

LOCATION.--Lat 46 $43^{\prime} 26^{\prime \prime}$, long 95 $54^{\prime} 56^{\prime \prime}$, in NE $1 / 4 \mathrm{SW}^{1} / 4$ sec.31, T.138 N., R.41 W., Becker County, Hydrologic Unit 09020103, in upstream concrete retaining wall at highway crossing at Buck's Mill, $200 \mathrm{ft}$ downstream from concrete millpond dam, and $6.5 \mathrm{mi}$ southwest of city of Detroit Lakes.

DRAINAGE AREA.--123 $\mathrm{mi}^{2}$.

PERIOD OF RECORD.--July 1942 to November 1953.

GAGE.--Staff gage. Datum of gage is $1,315 \mathrm{ft}$ above sea level from topographic map.

EXTREMES FOR PERIOD OF RECORD.--Maximum discharge, $229 \mathrm{ft}^{3} / \mathrm{s}$, June 20, 1953 (gage height, $4.28 \mathrm{ft}$ ); maximum gage height, $4.40 \mathrm{ft}$, Aug. 15, 1952, affected by backwater from aquatic vegetation; minimum daily discharge, $0.1 \mathrm{ft}^{3} / \mathrm{s}$, Sept. 4,1950 , and many days during 1951 .

Annual mean discharge

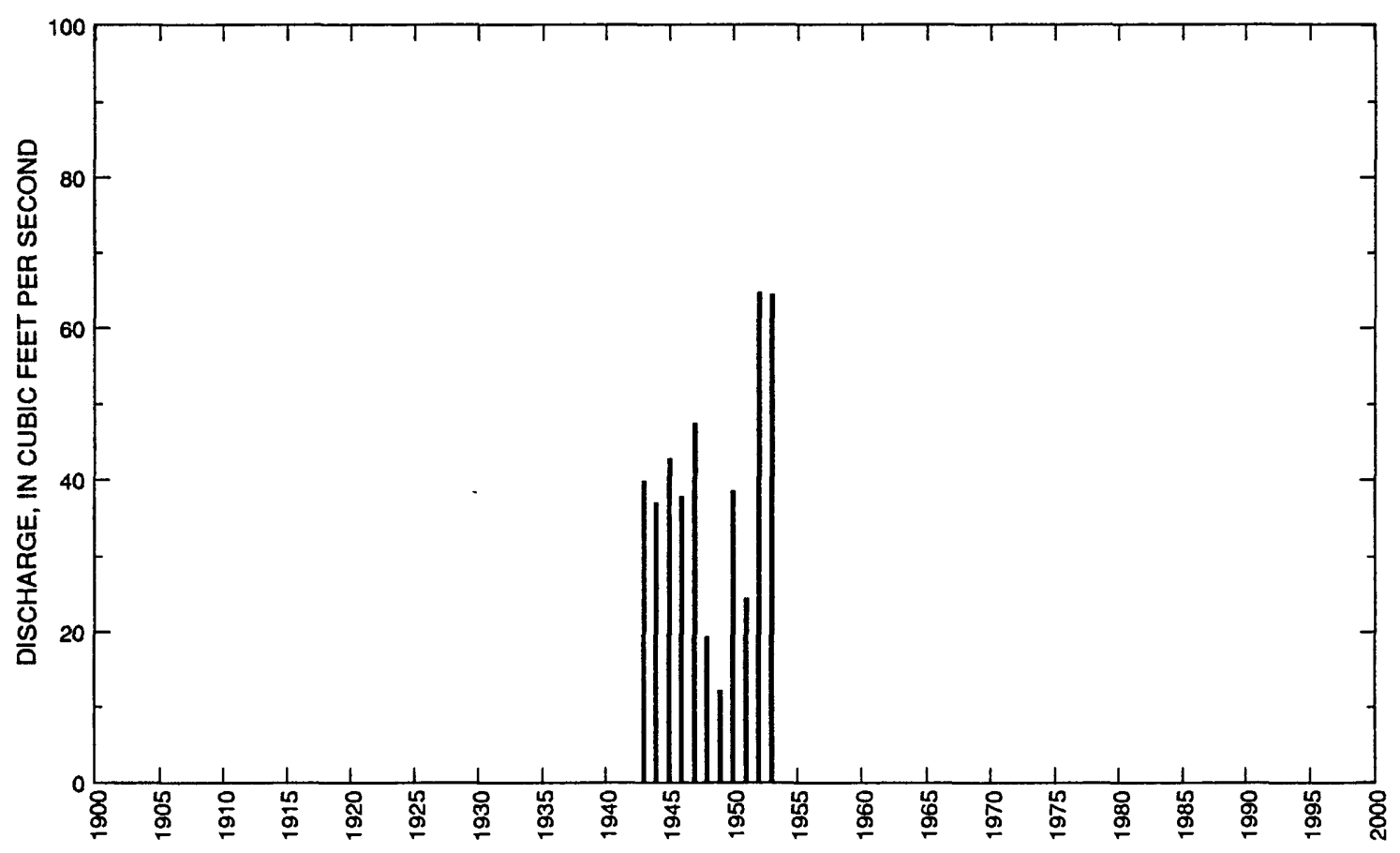


05040000 PELICAN RIVER NEAR DETROIT LAKES, MN-Continued

Statistics of monthly and annual mean discharges

\begin{tabular}{|c|c|c|c|c|c|c|c|c|}
\hline \multirow[b]{2}{*}{ Month } & \multicolumn{2}{|c|}{ Maximum } & \multicolumn{2}{|c|}{ Minimum } & \multicolumn{4}{|c|}{ Mean } \\
\hline & $\begin{array}{c}\text { Discharge } \\
\left(\mathrm{ft}^{3} / \mathbf{s}\right)\end{array}$ & $\begin{array}{c}\text { Water year } \\
\text { of } \\
\text { occurrence }\end{array}$ & $\begin{array}{c}\text { Discharge } \\
\left(\mathrm{ft}^{3} / \mathrm{s}\right)\end{array}$ & $\begin{array}{c}\text { Water year } \\
\text { of } \\
\text { occurrence }\end{array}$ & $\begin{array}{c}\text { Discharge } \\
\left(\mathrm{ft}^{3} / \mathrm{s}\right)\end{array}$ & $\begin{array}{c}\text { Standard } \\
\text { deviatlon } \\
\left(\mathrm{ft}^{3} / \mathrm{s}\right)\end{array}$ & $\begin{array}{l}\text { Coeffl- } \\
\text { clent of } \\
\text { variatlon }\end{array}$ & $\begin{array}{l}\text { Percentage } \\
\text { of annual } \\
\text { dlscharge }\end{array}$ \\
\hline October & 70.0 & 1945 & 0.165 & 1951 & 17.4 & 22.4 & 1.29 & 3.76 \\
\hline November & 46.3 & 1947 & 0.133 & 1951 & 13.7 & 17.6 & 1.29 & 2.98 \\
\hline December & 56.5 & 1952 & 0.235 & 1949 & 18.9 & 22.0 & 1.16 & 4.10 \\
\hline January & 55.4 & 1952 & 0.216 & 1950 & 18.4 & 18.8 & 1.02 & 4.00 \\
\hline February & 41.4 & 1952 & 0.421 & 1949 & 16.6 & 14.2 & 0.85 & 3.61 \\
\hline March & 77.2 & 1953 & 1.05 & 1949 & 28.2 & 21.4 & 0.76 & 6.13 \\
\hline April & 131 & 1953 & 29.8 & 1944 & 77.9 & 31.8 & 0.41 & 16.9 \\
\hline May & 167 & 1950 & 27.9 & 1949 & 82.9 & 38.1 & 0.46 & 18.0 \\
\hline June & 198 & 1953 & 9.06 & 1952 & 71.5 & 57.2 & 0.80 & 15.5 \\
\hline July & 118 & 1946 & 4.25 & 1945 & 53.2 & 37.8 & 0.71 & 11.5 \\
\hline August & 147 & 1952 & 0.326 & 1945 & 35.4 & 43.5 & 1.23 & 7.69 \\
\hline September & 111 & 1944 & 0.197 & 1950 & 26.8 & 37.7 & 1.41 & 5.81 \\
\hline Annual & 64.8 & 1952 & 12.1 & 1949 & 39.0 & 16.5 & 0.42 & 100 \\
\hline
\end{tabular}

Annual flow duration

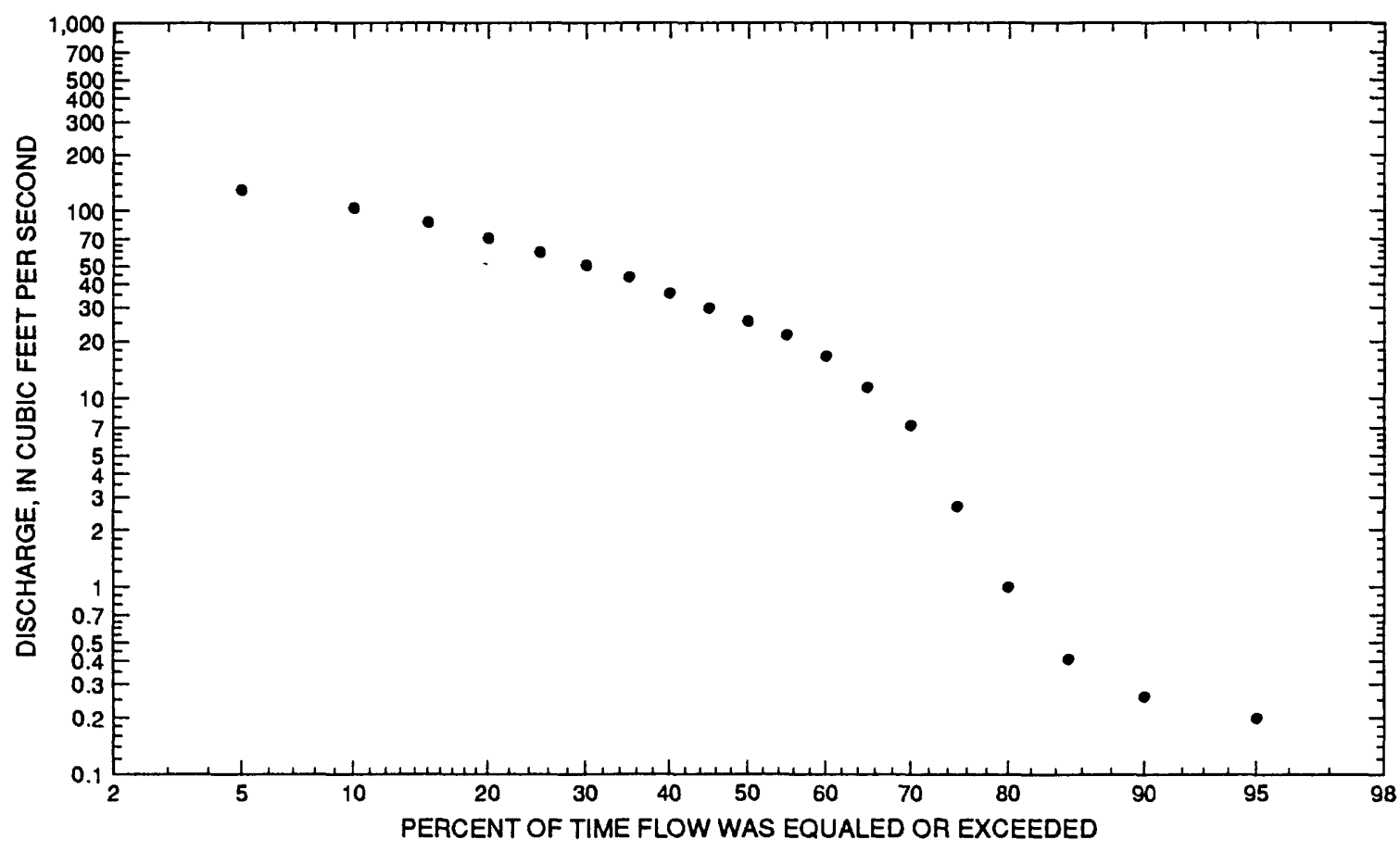




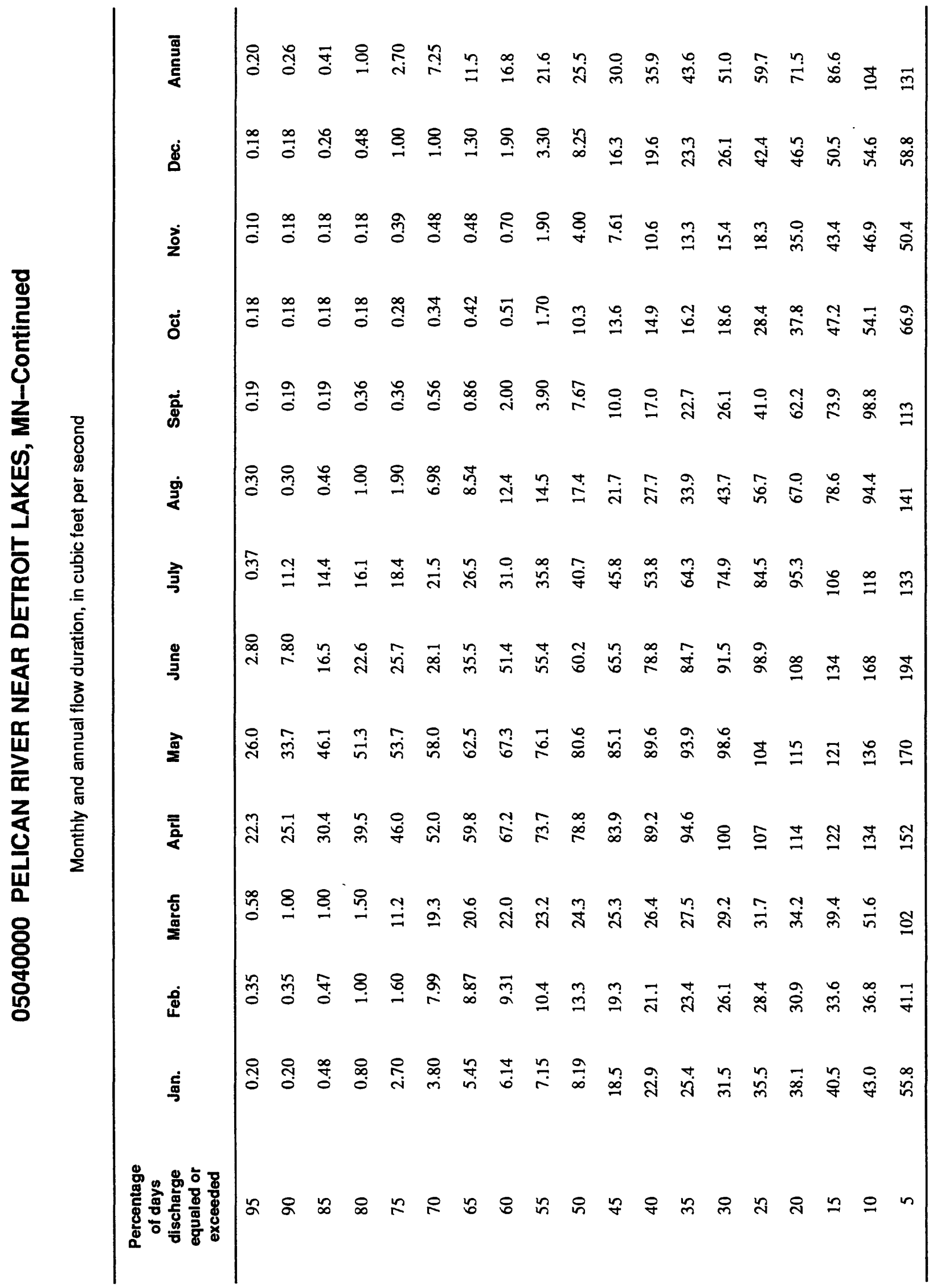


05040000 PELICAN RIVER NEAR DETROIT LAKES, MN-Continued

Probability of annual high discharges

[ng, statistic not given]

\begin{tabular}{lcccccc}
\hline & & & \multicolumn{5}{c}{$\begin{array}{c}\text { Maximum average discharge } \\
\left(\mathbf{f t}^{\mathbf{3}} \mathbf{s}\right)\end{array}$} \\
\cline { 6 - 7 } $\begin{array}{c}\text { Exceedance } \\
\text { probability }\end{array}$ & $\begin{array}{c}\text { Recurrence } \\
\text { interval } \\
\text { (years) }\end{array}$ & $\begin{array}{c}\text { Maximum } \\
\text { instantaneous } \\
\left(\mathbf{f t}^{3} / \mathbf{s}\right)\end{array}$ & 3-day period & 7-day period & 15-day period & 30-day period \\
\hline 0.99 & 1.01 & 57.8 & 61.7 & 58.2 & 51.5 & 40.9 \\
0.95 & 1.05 & 76.2 & 76.1 & 72.5 & 66.4 & 56.6 \\
0.90 & 1.11 & 87.7 & 85.3 & 81.6 & 75.9 & 66.8 \\
0.80 & 1.25 & 104 & 98.2 & 94.3 & 89.1 & 80.7 \\
0.50 & 2 & 140 & 129 & 125 & 120 & 113 \\
0.20 & 5 & 186 & 172 & 167 & 161 & 153 \\
0.10 & 10 & 214 & 201 & 195 & 187 & 177 \\
0.04 & 25 & 246 & 237 & 230 & 220 & 205 \\
0.02 & 50 & 269 & 264 & 256 & 243 & 225 \\
0.01 & 100 & 291 & 291 & 282 & 266 & 243 \\
0.005 & 200 & 312 & 319 & 309 & 288 & 260 \\
0.002 & 500 & 340 & ng & ng & ng & ng \\
\hline
\end{tabular}

Probability of annual low discharges

\begin{tabular}{|c|c|c|c|c|c|c|c|c|c|c|}
\hline \multirow[b]{3}{*}{$\begin{array}{l}\text { Non- } \\
\text { exceed- } \\
\text { ance } \\
\text { prob- } \\
\text { abliity }\end{array}$} & \multirow[b]{3}{*}{$\begin{array}{c}\text { Recur- } \\
\text { rence } \\
\text { Inter- } \\
\text { val } \\
\text { (years) }\end{array}$} & \multicolumn{9}{|c|}{ Minimum average discharge $\left(\mathrm{ft}^{3} / \mathrm{s}\right)$} \\
\hline & & \multicolumn{9}{|c|}{ Number of consecutive days } \\
\hline & & 1 & 3 & 7 & 14 & 30 & 60 & 90 & 120 & 183 \\
\hline 0.05 & 20 & 0.064 & ${ }^{1} 0.064$ & ${ }^{1} 0.065$ & ${ }^{1} 0.066$ & ${ }^{1} 0.067$ & ${ }^{1} 0.068$ & ${ }^{1} 0.069$ & 0.069 & 0.257 \\
\hline 0.10 & 10 & 0.089 & ${ }^{1} 0.089$ & ${ }^{1} 0.090$ & ${ }^{1} 0.090$ & 0.091 & 0.106 & 0.116 & 0.130 & 0.516 \\
\hline 0.20 & 5 & 0.143 & 0.143 & 0.144 & 0.151 & 0.177 & 0.242 & 0.261 & 0.295 & 1.18 \\
\hline 0.50 & 2 & 0.470 & 0.491 & 0.561 & 0.625 & 0.808 & 1.35 & 1.45 & 1.65 & 5.32 \\
\hline
\end{tabular}

${ }^{1}$ Graphical interpretation. 


\section{PELICAN RIVER NEAR DETROIT LAKES, MN-Continued}

Probability of seasonal low discharges

\begin{tabular}{|c|c|c|c|c|c|c|c|c|c|}
\hline \multirow[b]{3}{*}{$\begin{array}{c}\text { Non- } \\
\text { exceedance } \\
\text { probability }\end{array}$} & \multirow[b]{3}{*}{$\begin{array}{l}\text { Recurrence } \\
\text { interval } \\
\text { (years) }\end{array}$} & \multicolumn{8}{|c|}{ Minimum average discharge $\left(\mathrm{ft}^{3} / \mathrm{s}\right)$} \\
\hline & & \multicolumn{8}{|c|}{ Number of consecutive days } \\
\hline & & 1 & 7 & 14 & 30 & 1 & 7 & 14 & 30 \\
\hline & & \multicolumn{4}{|c|}{ December-January-February } & \multicolumn{4}{|c|}{ March-April-May } \\
\hline 0.05 & 20 & 0.054 & 0.058 & 0.064 & 0.115 & 0.329 & 0.422 & 0.474 & 1.27 \\
\hline 0.10 & 10 & 0.125 & 0.151 & 0.164 & 0.273 & 0.820 & 1.01 & 1.12 & 2.90 \\
\hline 0.20 & 5 & 0.341 & 0.456 & 0.486 & 0.743 & 2.20 & 2.60 & 2.85 & 6.85 \\
\hline \multirow[t]{2}{*}{0.50} & 2 & 2.28 & 3.30 & 3.44 & 4.48 & 10.2 & 11.3 & 12.0 & 23.7 \\
\hline & & \multicolumn{4}{|c|}{ June-July-August } & \multicolumn{4}{|c|}{ September-October-November } \\
\hline 0.05 & 20 & 0.108 & 0.111 & 0.176 & 0.323 & 0.054 & ${ }^{1} 0.055$ & 0.055 & 0.065 \\
\hline 0.10 & 10 & 0.279 & 0.292 & 0.421 & 0.770 & 0.098 & 0.099 & 0.107 & 0.133 \\
\hline 0.20 & 5 & 0.813 & 0.869 & 1.14 & 2.02 & 0.206 & 0.223 & 0.248 & 0.319 \\
\hline 0.50 & 2 & 4.92 & 5.47 & 6.28 & 10.0 & 0.945 & 1.16 & 1.35 & 1.80 \\
\hline
\end{tabular}

${ }^{1}$ Graphical interpretation. 


\section{PELICAN RIVER NEAR DETROIT LAKES, MN-Continued}

Annual peak discharge and corresponding gage height, period of record

\begin{tabular}{|c|c|c|c|c|c|c|c|}
\hline $\begin{array}{l}\text { Water } \\
\text { year }\end{array}$ & Date & $\begin{array}{c}\text { Gage } \\
\text { height } \\
\text { (foet) }\end{array}$ & $\begin{array}{c}\text { Peak } \\
\text { discharge } \\
\left(\mathrm{ft}^{3} / \mathrm{s}\right)\end{array}$ & $\begin{array}{l}\text { Water } \\
\text { year }\end{array}$ & Date & $\begin{array}{c}\text { Gage } \\
\text { height } \\
\text { (feet) }\end{array}$ & $\begin{array}{c}\text { Peak } \\
\text { discharge } \\
\left(\mathrm{ft}^{3} / \mathrm{s}\right)\end{array}$ \\
\hline \multicolumn{8}{|c|}{ Annual peak discharge, by year, and corresponding gage height } \\
\hline 1943 & June 26 & 3.84 & 136 & 1949 & April 10 & 2.82 & 75.0 \\
\hline 1944 & September 2 & 3.82 & 136 & 1950 & May 19 & 5.10 & 210 \\
\hline 1945 & April 22 & 3.54 & 115 & 1951 & June 3 & 2.84 & 117 \\
\hline 1946 & July 18 & 4.07 & 145 & 1952 & August 15 & 4.40 & 214 \\
\hline 1947 & May 2 & 3.86 & 133 & 1953 & June 20 & 4.28 & 229 \\
\hline 1948 & May 7 & 3.20 & 94.0 & & & & \\
\hline \multicolumn{8}{|c|}{ Annual peak discharge, from highest to lowest, and corresponding gage height } \\
\hline 1953 & June 20 & 4.28 & 229 & 1947 & May 2 & 3.86 & 133 \\
\hline 1952 & August 15 & 4.40 & 214 & 1951 & June 3 & 2.84 & 117 \\
\hline 1950 & May 19 & 5.10 & 210 & 1945 & April 22 & 3.54 & 115 \\
\hline 1946 & July 18 & 4.07 & 145 & 1948 & May 7 & 3.20 & 94.0 \\
\hline 1943 & June 26 & 3.84 & 136 & 1949 & April 10 & 2.82 & 75.0 \\
\hline 1944 & September 2 & 3.82 & 136 & & & & \\
\hline
\end{tabular}




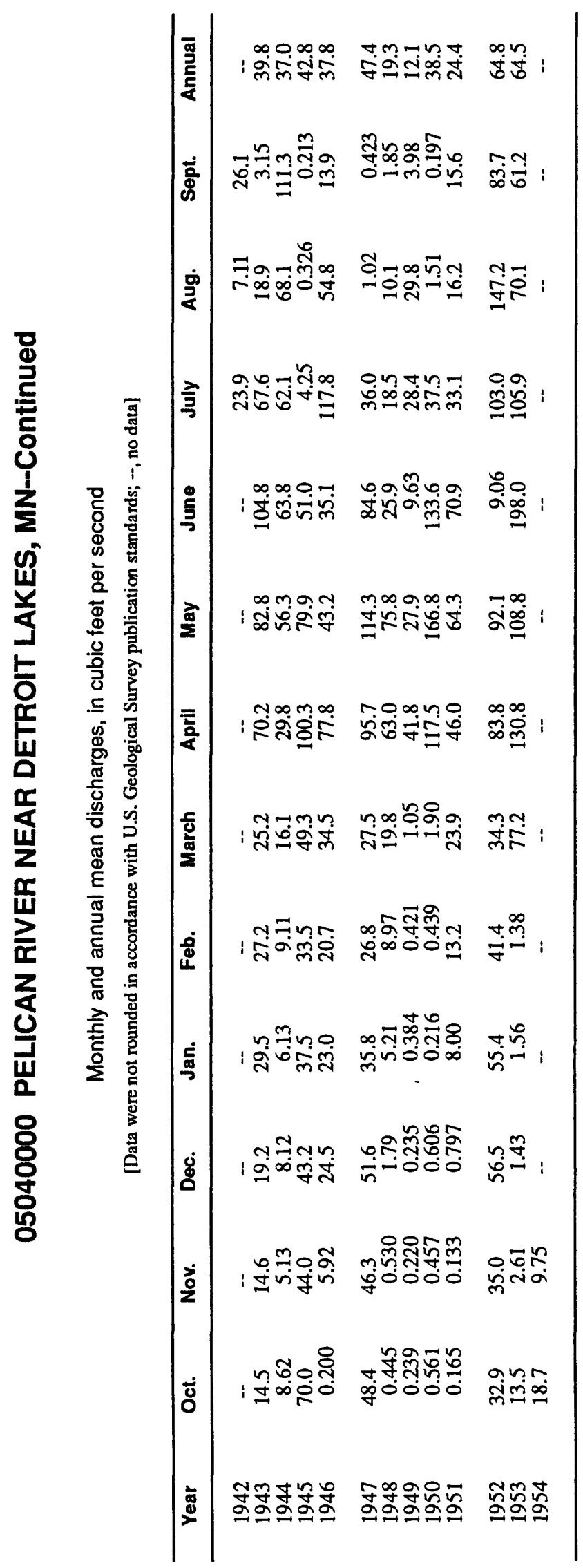




\section{PELICAN RIVER NEAR FERGUS FALLS, MN}

\section{Station Description}

LOCATION.--Lat $46^{\circ} 20^{\prime} 10^{\prime \prime}$, long $96^{\circ} 07^{\prime} 00^{\prime \prime}$, in NE $1 / 4$ sec.17, T.133 N., R.43 W., Otter Tail County, Hydrologic Unit 09020103, on right downstream wingwall of bridge on U.S. Highway 52, $3 \mathrm{mi}$ northwest of Fergus Falls and 7.5 mi upstream from mouth.

DRAINAGE AREA.--482 $\mathrm{mi}^{2}$.

PERIOD OF RECORD.--June 1909 to December 1913, July 1942 to September 1980.

GAGE.--Water-stage recorder. Datum of gage is $1,176.98 \mathrm{ft}$ above sea level, datum of 1929. June 19, 1909, to Dec. 31, 1912, staff gage at site 1 mil downstream at different datum. July 1, 1942, to Nov. 6, 1955 , staff gage and Nov. 7,1955 , to Sept. 30,1963, water-stage recorder, at site $900 \mathrm{ft}$ upstream at datum $3.00 \mathrm{ft}$ higher.

EXTREMES FOR PERIOD OF RECORD.--Maximum discharge, $756 \mathrm{ft}^{3} / \mathrm{s}$, Mar. 29, 1943 (gage height, $8.53 \mathrm{ft}$, present datum); maximum gage height, $8.60 \mathrm{ft}$, Mar. 28, 1950 (backwater from ice, present datum); no flow for many days in 1946, and 1949-50; no flow at times in some years.

Annual mean discharge

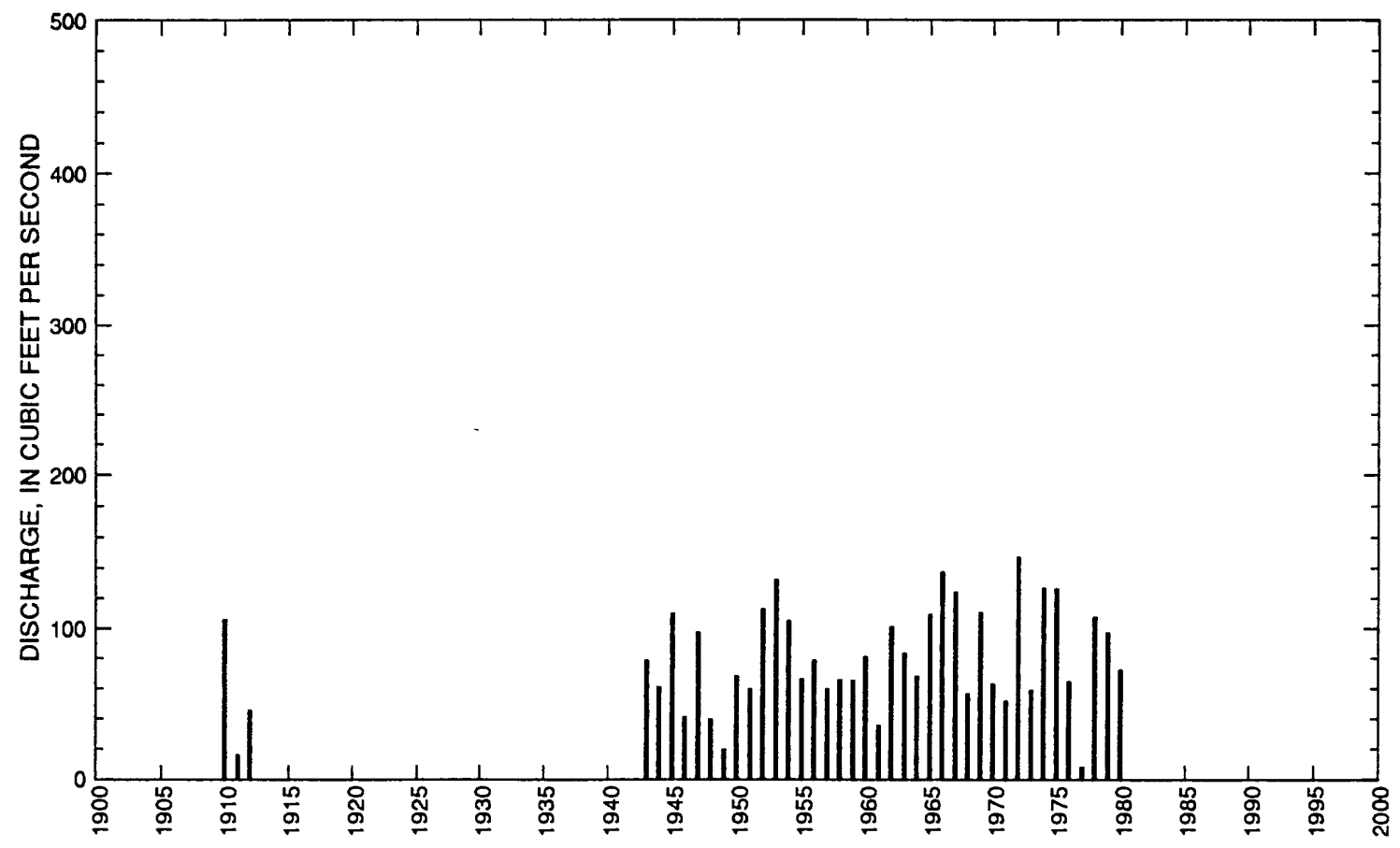




\section{PELICAN RIVER NEAR FERGUS FALLS, MN-Continued}

Statistics of monthly and annual mean discharges

[m, more than 1 year of occurrence]

\begin{tabular}{|c|c|c|c|c|c|c|c|c|}
\hline \multirow[b]{2}{*}{ Month } & \multicolumn{2}{|c|}{ Maximum } & \multicolumn{2}{|c|}{ Minimum } & \multicolumn{4}{|c|}{ Mean } \\
\hline & $\begin{array}{c}\text { Discharge } \\
\left(\mathrm{ft}^{3} / \mathrm{s}\right)\end{array}$ & $\begin{array}{c}\text { Water year } \\
\text { of } \\
\text { occurrence }\end{array}$ & $\begin{array}{c}\text { Dlscharge } \\
\left(\mathrm{ft}^{3} / \mathrm{s}\right)\end{array}$ & $\begin{array}{c}\text { Water year } \\
\text { of } \\
\text { occurrence }\end{array}$ & $\begin{array}{c}\text { Discharge } \\
\left(\mathrm{ft}^{3} / \mathrm{s}\right)\end{array}$ & $\begin{array}{c}\text { Standard } \\
\text { deviation } \\
\left(\mathrm{ft}^{3} / \mathrm{s}\right)\end{array}$ & $\begin{array}{l}\text { Coeffl- } \\
\text { cient of } \\
\text { variation }\end{array}$ & $\begin{array}{l}\text { Percentege } \\
\text { of annual } \\
\text { discharge }\end{array}$ \\
\hline October & 173 & 1910 & 0.083 & 1977 & 52.4 & 39.1 & 0.75 & 5.50 \\
\hline November & 142 & 1910 & 1.25 & 1977 & 49.9 & 38.6 & 0.78 & 5.24 \\
\hline December & 132 & 1945 & 0.172 & 1977 & 42.0 & 37.9 & 0.90 & 4.41 \\
\hline January & 125 & 1910 & 0 & $\mathrm{~m}$ & 40.4 & 37.8 & 0.94 & 4.24 \\
\hline February & 117 & 1945 & 0 & $\mathrm{~m}$ & 39.2 & 34.7 & 0.89 & 4.11 \\
\hline March & 186 & 1945 & 5.28 & 1962 & 68.5 & 49.9 & 0.73 & 7.20 \\
\hline April & 320 & 1966 & 17.1 & 1977 & 156 & 71.7 & 0.46 & 16.4 \\
\hline May & 310 & 1966 & 12.8 & 1977 & 161 & 76.4 & 0.47 & 16.9 \\
\hline June & 356 & 1953 & 7.52 & 1977 & 137 & 83.0 & 0.61 & 14.4 \\
\hline July & 266 & 1953 & 4.14 & 1977 & 88.1 & 61.0 & 0.69 & 9.25 \\
\hline August & 179 & 1953 & 0.328 & 1976 & 62.0 & 47.0 & 0.76 & 6.51 \\
\hline September & 189 & 1909 & 0.003 & 1976 & 55.4 & 43.2 & 0.78 & 5.82 \\
\hline Annual & 147 & 1972 & 7.69 & 1977 & 79.4 & 34.4 & 0.43 & 100 \\
\hline
\end{tabular}

Annual flow duration

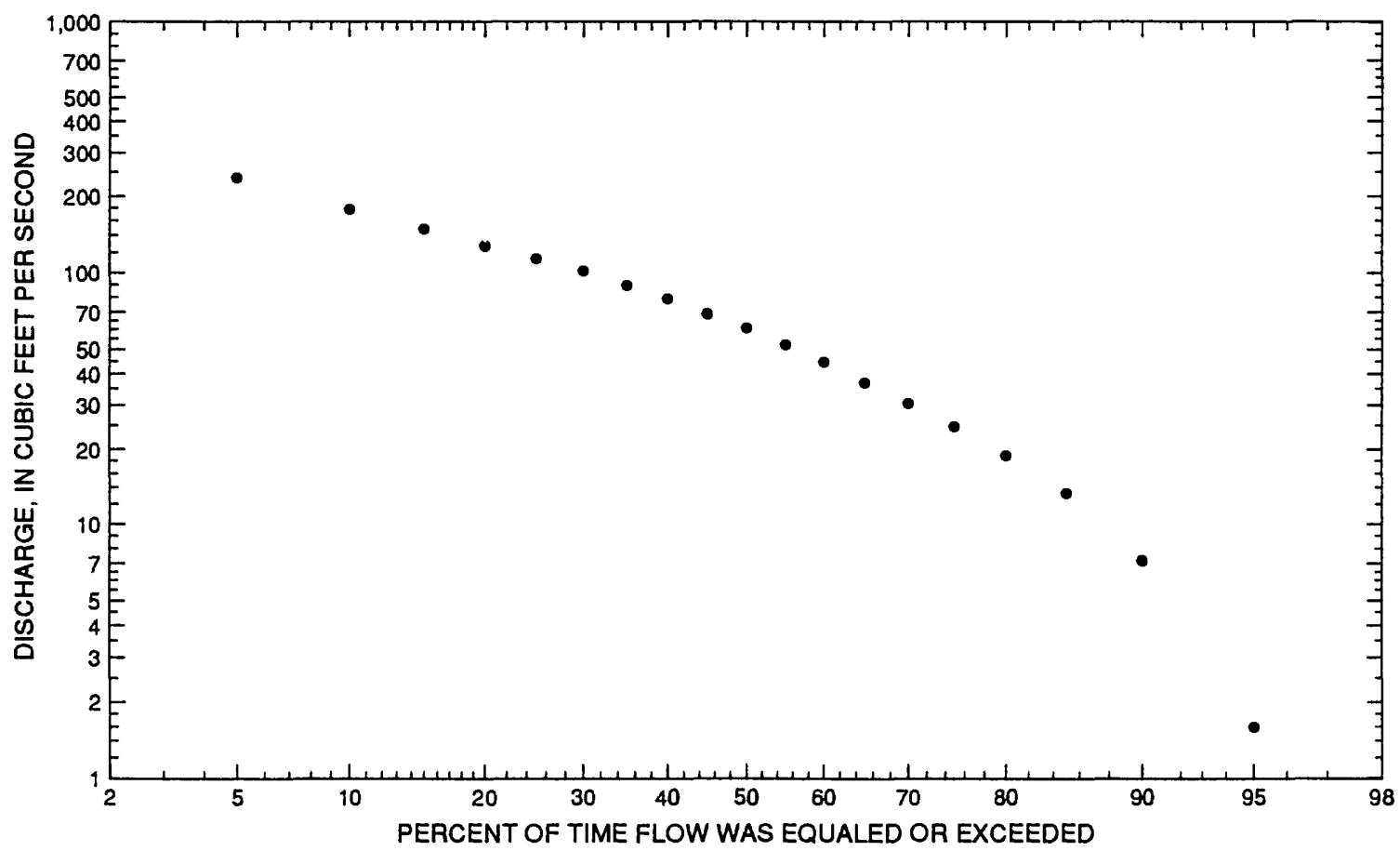




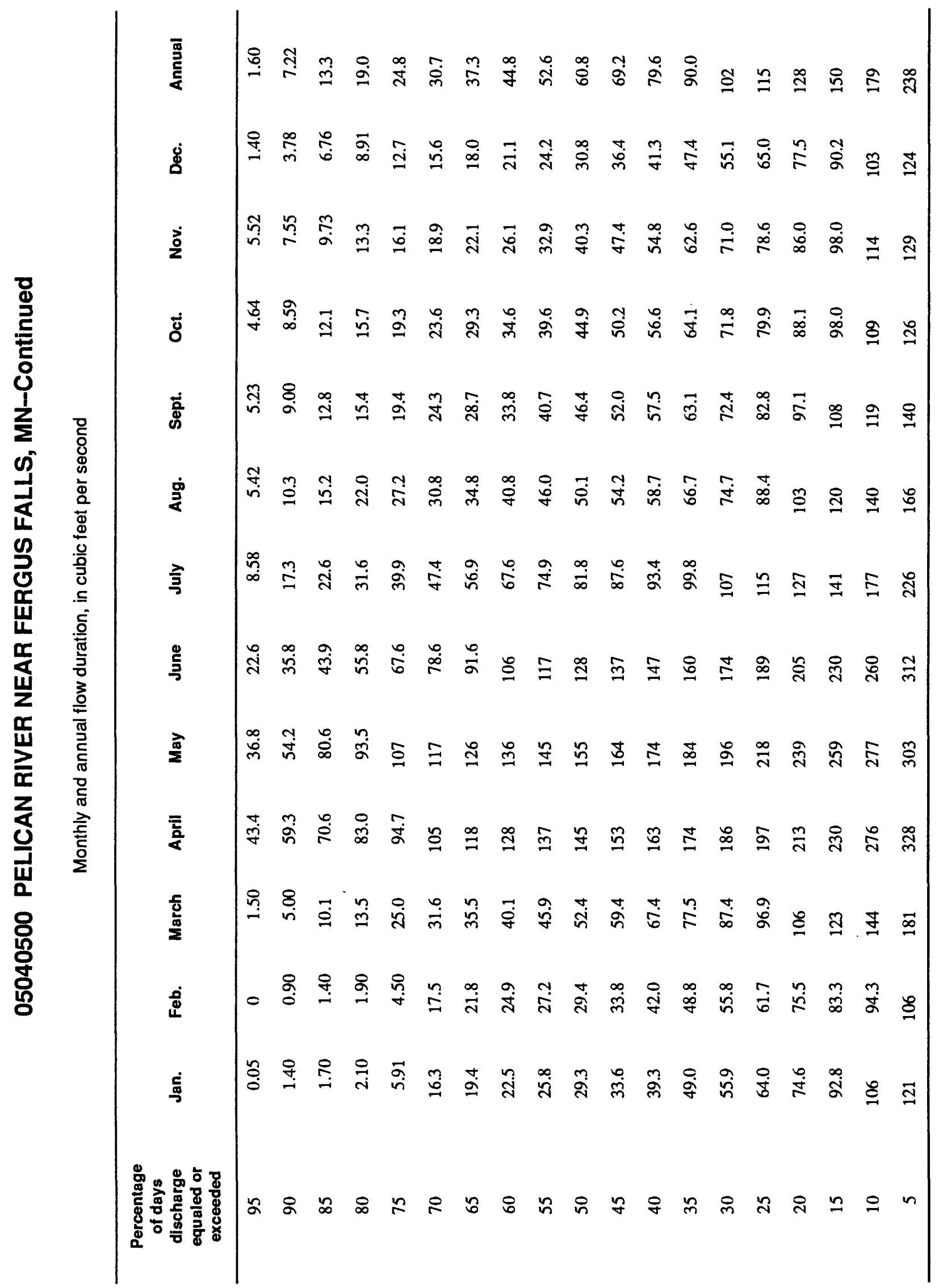




\section{PELICAN RIVER NEAR FERGUS FALLS, MN-Continued}

Probability of annual high discharges

[ng, statistic not given]

\begin{tabular}{lcccccc}
\hline & & & \multicolumn{5}{c}{$\begin{array}{c}\text { Maximum average discharge } \\
\left(\mathrm{ft}^{3} / \mathbf{s}\right)\end{array}$} \\
\cline { 5 - 7 } $\begin{array}{c}\text { Exceedance } \\
\text { probability }\end{array}$ & $\begin{array}{c}\text { Recurrence } \\
\text { interval } \\
\text { (years) }\end{array}$ & $\begin{array}{c}\text { Maximum } \\
\text { instantaneous } \\
\left(\mathrm{ft}^{3} / \mathrm{s}\right)\end{array}$ & 3-day period & 7-day period & 15-day period & 30-day period \\
\hline 0.99 & 1.01 & 84.8 & 63.6 & 55.5 & 38.2 & 28.5 \\
0.95 & 1.05 & 123 & 98.3 & 89.5 & 72.2 & 60.4 \\
0.90 & 1.11 & 149 & 123 & 113 & 96.9 & 84.6 \\
0.80 & 1.25 & 190 & 159 & 148 & 133 & 120 \\
0.50 & 2 & 300 & 253 & 235 & 218 & 201 \\
0.20 & 5 & 475 & 388 & 351 & 311 & 279 \\
0.10 & 10 & 606 & 479 & 422 & 357 & 311 \\
0.04 & 25 & 785 & 593 & 504 & 400 & 337 \\
0.02 & 50 & 928 & 677 & 561 & 425 & 349 \\
0.01 & 100 & 1,080 & 760 & 614 & 443 & 357 \\
0.005 & 200 & 1,240 & 842 & 663 & 458 & 363 \\
0.002 & 500 & 1,470 & $\mathrm{ng}$ & $\mathrm{ng}$ & $\mathrm{ng}$ & $\mathrm{ng}$ \\
\hline
\end{tabular}

Probability of annual low discharges

\begin{tabular}{|c|c|c|c|c|c|c|c|c|c|c|}
\hline \multirow[b]{3}{*}{$\begin{array}{c}\text { Non- } \\
\text { exceed- } \\
\text { ance } \\
\text { prob- } \\
\text { ability }\end{array}$} & \multirow[b]{3}{*}{$\begin{array}{c}\text { Recur. } \\
\text { rence } \\
\text { inter- } \\
\text { vai } \\
\text { (years) }\end{array}$} & \multicolumn{9}{|c|}{ Minimum average discharge $\left(\mathrm{ft}^{3} / \mathrm{s}\right)$} \\
\hline & & \multicolumn{9}{|c|}{ Number of consecutive days } \\
\hline & & 1 & 3 & 7 & 14 & 30 & 60 & 90 & 120 & 183 \\
\hline 0.05 & 20 & 0 & 0 & 0 & 0 & 0 & 0 & 0.124 & 1.35 & 2.14 \\
\hline 0.10 & 10 & 0 & 0 & 0 & 0 & 0 & 0.612 & 0.778 & 2.87 & 4.68 \\
\hline 0.20 & 5 & 1.86 & 2.09 & 2.50 & 2.62 & 2.94 & 3.45 & 3.60 & 6.49 & 10.5 \\
\hline 0.50 & 2 & 11.4 & 12.2 & 13.8 & 14.4 & 15.6 & 18.0 & 22.7 & 22.9 & 33.0 \\
\hline
\end{tabular}




\section{PELICAN RIVER NEAR FERGUS FALLS, MN-Continued}

Probability of seasonal low discharges

\begin{tabular}{|c|c|c|c|c|c|c|c|c|c|}
\hline \multirow[b]{3}{*}{$\begin{array}{c}\text { Non- } \\
\text { exceedance } \\
\text { probability }\end{array}$} & \multirow[b]{3}{*}{$\begin{array}{l}\text { Recurrence } \\
\text { intervai } \\
\text { (years) }\end{array}$} & \multicolumn{8}{|c|}{ Minimum average discharge $\left(\mathrm{ft}^{3} / \mathrm{s}\right)$} \\
\hline & & \multicolumn{8}{|c|}{ Number of consecutive days } \\
\hline & & 1 & 7 & 14 & 30 & 1 & 7 & 14 & 30 \\
\hline & & \multicolumn{4}{|c|}{ December-January-February } & \multicolumn{4}{|c|}{ March-April-May } \\
\hline 0.05 & 20 & 0 & 0 & 0 & 0 & 0 & 0 & 1.00 & 8.15 \\
\hline 0.10 & 10 & 0.059 & 0.059 & 0.063 & 0.081 & 0.141 & 1.37 & 2.69 & 13.0 \\
\hline 0.20 & 5 & 3.02 & 3.07 & 3.20 & 3.58 & 6.57 & 6.85 & 7.53 & 22.0 \\
\hline \multirow[t]{2}{*}{0.50} & 2 & 18.8 & 19.1 & 19.7 & 21.0 & 31.9 & 32.5 & 33.8 & 51.6 \\
\hline & & \multicolumn{4}{|c|}{ June-July-August } & \multicolumn{4}{|c|}{ September-October-November } \\
\hline 0.05 & 20 & 2.34 & 4.07 & 5.56 & ${ }^{1} 6.80$ & 1.32 & 2.39 & 2.90 & 4.30 \\
\hline 0.10 & 10 & 5.33 & 7.78 & 9.84 & ${ }^{1} 11.0$ & 2.88 & 4.56 & 5.37 & 7.42 \\
\hline 0.20 & 5 & 11.3 & 14.3 & 17.0 & 20.5 & 6.12 & 8.58 & 9.81 & 12.7 \\
\hline 0.50 & 2 & 33.1 & 36.1 & 39.5 & 57.7 & 19.6 & 23.4 & 25.6 & 30.2 \\
\hline
\end{tabular}

${ }^{1}$ Graphical interpretation. 


\section{PELICAN RIVER NEAR FERGUS FALLS, MN-Continued}

Annual peak discharge and corresponding gage height, period of record

$$
[-, \text { no data }]
$$

\begin{tabular}{|c|c|c|c|c|c|c|c|}
\hline $\begin{array}{l}\text { Water } \\
\text { year }\end{array}$ & Date & $\begin{array}{c}\text { Gage } \\
\text { height } \\
\text { (feet) }\end{array}$ & $\begin{array}{c}\text { Peak } \\
\text { discharge } \\
\left(\mathrm{ft}^{3} / \mathbf{s}\right)\end{array}$ & $\begin{array}{l}\text { Water } \\
\text { year }\end{array}$ & Date & $\begin{array}{c}\text { Gage } \\
\text { height } \\
\text { (feet) }\end{array}$ & $\begin{array}{c}\text { Peak } \\
\text { discharge } \\
\left(\mathrm{ft}^{3} / \mathrm{s}\right)\end{array}$ \\
\hline \multicolumn{8}{|c|}{ Annual peak discharge, by year, and corresponding gage height } \\
\hline 1910 & October 11 & - & 303 & 1961 & April 24 & 2.33 & 135 \\
\hline 1911 & May 8 & - & 124 & 1962 & May 24 & 3.45 & 365 \\
\hline 1912 & May 28 & - & 230 & 1963 & March 29 & 4.11 & 230 \\
\hline 1943 & March 29 & 5.53 & 756 & 1964 & May 8 & 4.47 & 274 \\
\hline 1944 & June 5 & - & 212 & 1965 & April 9 & 6.34 & 551 \\
\hline 1945 & March 17 & - & 330 & 1966 & April 1 & 4.73 & 560 \\
\hline 1946 & May 16 & -- & 212 & 1967 & March 30 & 6.99 & 655 \\
\hline 1947 & June 7 & - & 218 & 1968 & May 15 & 4.05 & 194 \\
\hline 1948 & - & - & - & 1969 & April 12 & 5.30 & 710 \\
\hline 1949 & April 3 & - & 245 & 1970 & June 20 & 4.38 & 350 \\
\hline 1950 & May 6 & - & 296 & 1971 & July 3 & 4.16 & 251 \\
\hline 1951 & April 7 & -- & 177 & 1972 & May 30 & 4.60 & 420 \\
\hline 1952 & April 14 & - & 388 & 1973 & March 25 & 3.97 & 163 \\
\hline 1953 & June 15 & 4.85 & 680 & 1974 & April 12 & 6.08 & 725 \\
\hline 1954 & July 2 & - & 242 & 1975 & April 19 & 6.72 & 585 \\
\hline 1955 & April 4 & - & 250 & 1976 & March 26 & 5.11 & 186 \\
\hline 1956 & April 13 & - & 315 & 1977 & May 14 & 3.70 & 129 \\
\hline 1957 & April 20 & -- & 216 & 1978 & April 6 & 5.41 & 635 \\
\hline 1958 & July 7 & - & 125 & 1979 & April 18 & 5.08 & 610 \\
\hline 1959 & May 31 & - & 164 & 1980 & April 5 & 5.86 & 495 \\
\hline 1960 & April 27 & - & 206 & & & & \\
\hline \multicolumn{8}{|c|}{ Annual peak discharge, from highest to lowest, and corresponding gage height } \\
\hline 1943 & March 29 & 5.53 & 756 & 1955 & April 4 & - & 250 \\
\hline 1974 & April 12 & 6.08 & 725 & 1949 & April 3 & -- & 245 \\
\hline 1969 & April 12 & 5.30 & 710 & 1954 & July 2 & - & 242 \\
\hline 1953 & June 15 & 4.85 & 680 & 1912 & May 28 & - & 230 \\
\hline 1967 & March 30 & 6.99 & 655 & 1963 & March 29 & 4.11 & 230 \\
\hline 1978 & April 6 & 5.41 & 635 & 1947 & June 7 & - & 218 \\
\hline 1979 & April 18 & 5.08 & 610 & 1957 & April 20 & - & 216 \\
\hline 1975 & April 19 & 6.72 & 585 & 1944 & June 5 & - & 212 \\
\hline 1966 & April 1 & 4.73 & 560 & 1946 & May 16 & - & 212 \\
\hline 1965 & April 9 & 6.34 & 551 & 1960 & April 27 & - & 206 \\
\hline 1980 & April 5 & 5.86 & 495 & 1968 & May 15 & 4.05 & 194 \\
\hline 1972 & May 30 & 4.60 & 420 & 1976 & March 26 & 5.11 & 186 \\
\hline 1952 & April 14 & - & 388 & 1951 & April 7 & - & 177 \\
\hline 1962 & May 24 & 3.45 & 365 & 1959 & May 31 & - & 164 \\
\hline 1970 & June 20 & 4.38 & 350 & 1973 & March 25 & 3.97 & 163 \\
\hline 1945 & March 17 & - & 330 & 1961 & April 24 & 2.33 & 135 \\
\hline 1956 & April 13 & - & 315 & 1977 & May 14 & 3.70 & 129 \\
\hline 1910 & October 11 & - & 303 & 1958 & July 7 & - & 125 \\
\hline 1950 & May 6 & - & 296 & 1911 & May 8 & - & 124 \\
\hline 1964 & May 8 & 4.47 & 274 & 1948 & -- & - & - \\
\hline 1971 & July 3 & 4.16 & 251 & & & & \\
\hline
\end{tabular}




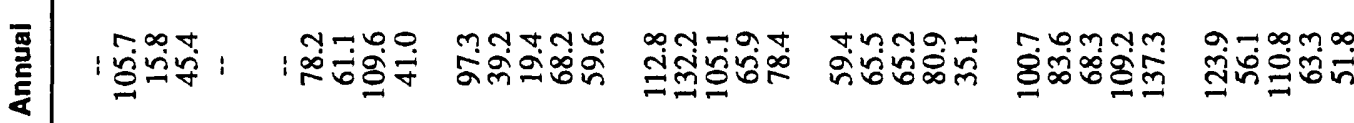

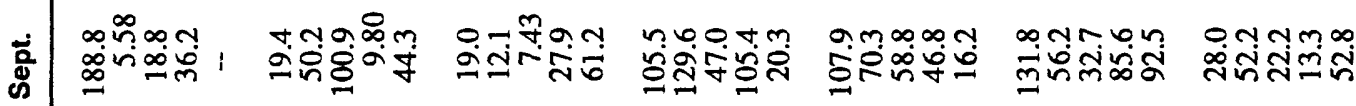

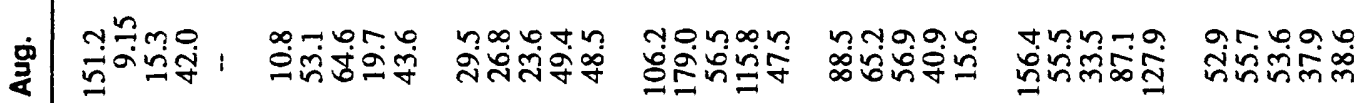

ว

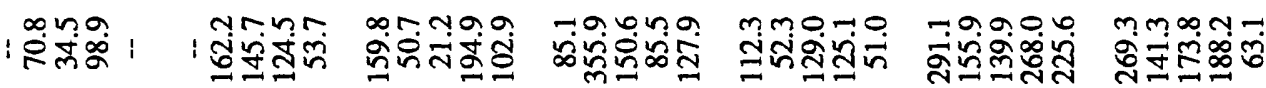

छ |

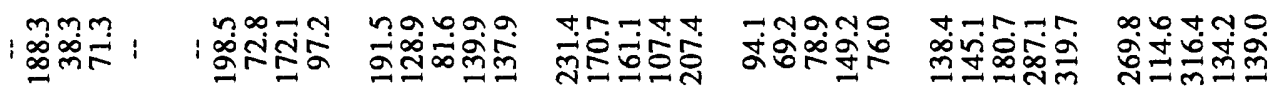

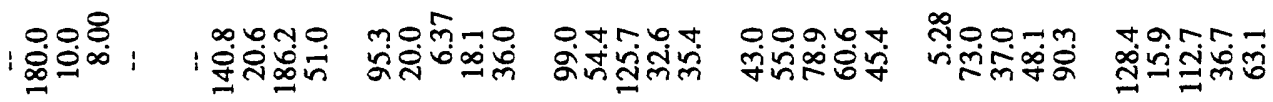

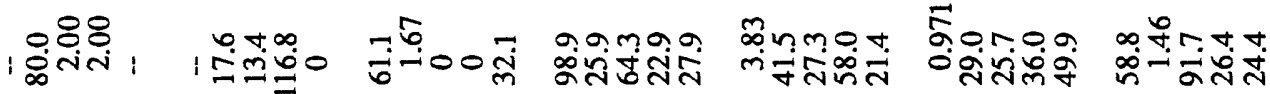
।

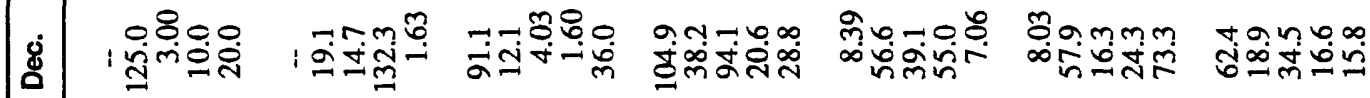

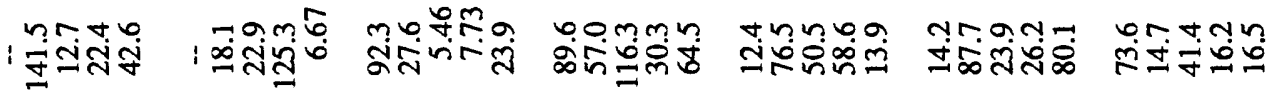

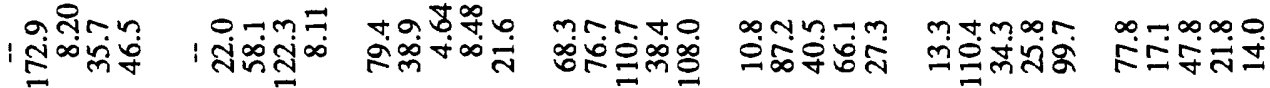

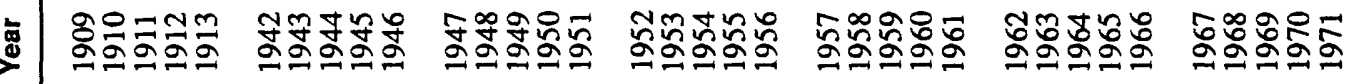




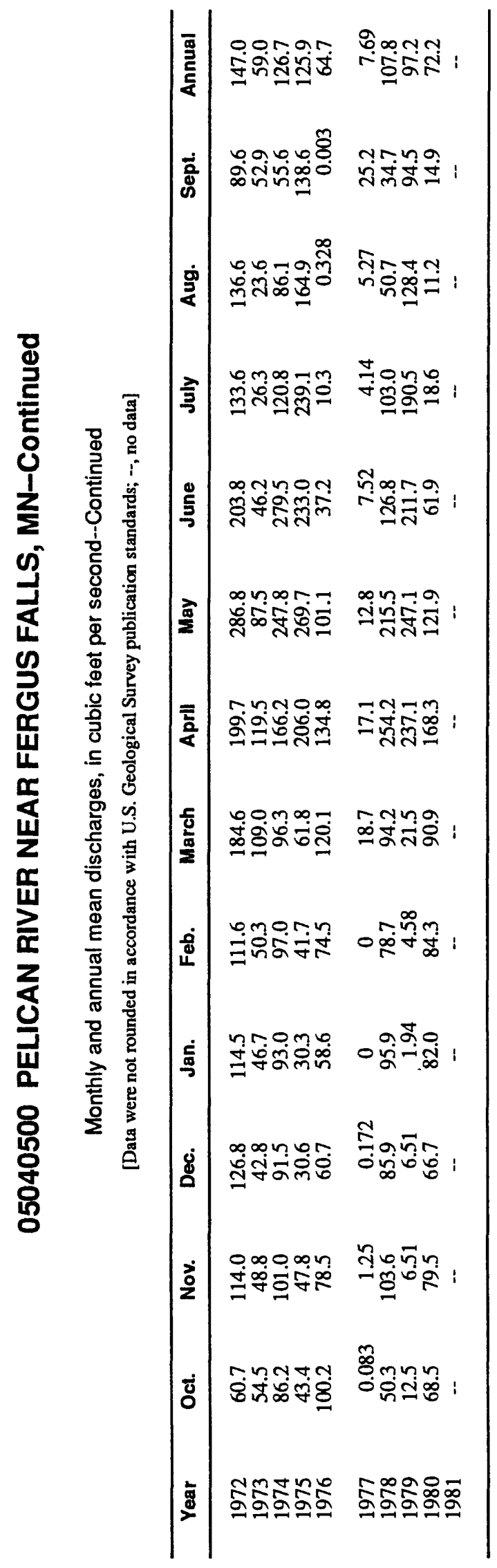




\title{
05046000 OTTER TAIL RIVER BELOW ORWELL DAM NEAR FERGUS FALLS, MN
}

\author{
Station Description
}

LOCATION.--Lat $46^{\circ} 12^{\prime} 35^{\prime \prime}$, long $96^{\circ} 11^{\prime} 05^{\prime \prime}$, in NE $1 / 4$ sec.34, T.132 N., R.44 W., Otter Tail County, Hydrologic Unit 09020103 , on left bank $0.7 \mathrm{mi}$ downstream from Orwell Dam, $6.1 \mathrm{mi}$ downstream from Dayton Hollow Dam, $8 \mathrm{mi}$ southwest of Fergus Falls, and $11.1 \mathrm{mi}$ downstream from Pelican River.

DRAINAGE AREA.--1,830 $\mathrm{mi}^{2}$, approximately.

PERIOD OF RECORD.--October 1930 to current year. Prior to October 1952, published as Otter Tail River below Pelican River near Fergus Falls. Monthly discharges only for some periods, published in Water-Supply Paper 1308.

GAGE.--Water-stage recorder. Datum of gage is $1,029.65 \mathrm{ft}$ above sea level, adjustment of 1912 . Oct. 11 , 1930, to Nov. 17,1933 , at same site at datum $2.00 \mathrm{ft}$ higher, Nov. 18, 1933, to Mar. 21, 1953, at site $6.1 \mathrm{mi}$ upstream at datum $40.30 \mathrm{ft}$ higher.

EXTREMES FOR PERIOD OF RECORD.--Maximum discharge, 1,710 $\mathrm{ft}^{3} / \mathrm{s}$, June 17, 1953; maximum gage height, $5.60 \mathrm{ft}$, June 17, 1953; minimum discharge, $0.70 \mathrm{ft}^{3} / \mathrm{s}$, Aug. 5, 1970 (minimum gage height $1.28 \mathrm{ft}$ ), caused by regulation.

Annual mean discharge

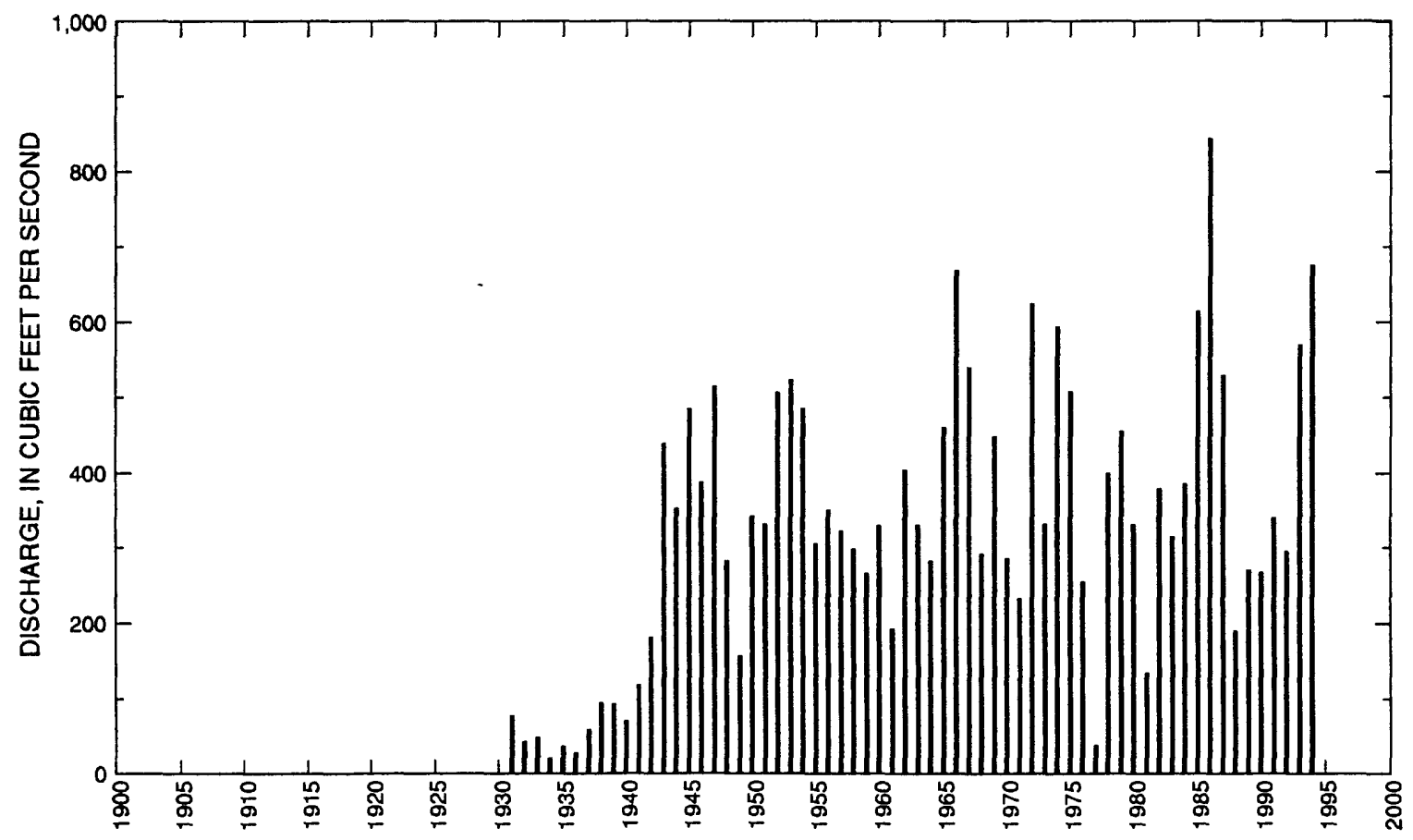




\section{OTTER TAIL RIVER BELOW ORWELL DAM NEAR FERGUS FALLS, MN-Continued}

Pre-regulation period, 1931-52

Statistics of monthly and annual mean discharges, pre-regulation period

\begin{tabular}{|c|c|c|c|c|c|c|c|c|}
\hline \multirow[b]{2}{*}{ Month } & \multicolumn{2}{|c|}{ Maximum } & \multicolumn{2}{|c|}{ Minimum } & \multicolumn{4}{|c|}{ Mean } \\
\hline & $\begin{array}{c}\text { Discharge } \\
\left(\mathrm{ft}^{3} / \mathbf{s}\right)\end{array}$ & $\begin{array}{c}\text { Water year } \\
\text { of } \\
\text { occurrence }\end{array}$ & $\begin{array}{c}\text { Discharge } \\
\left(\mathrm{ft}^{3} / \mathbf{s}\right)\end{array}$ & $\begin{array}{c}\text { Water year } \\
\text { of } \\
\text { occurrence }\end{array}$ & $\begin{array}{c}\text { Discharge } \\
\left(\mathrm{ft}^{3} / \mathbf{s}\right)\end{array}$ & $\begin{array}{c}\text { Standard } \\
\text { deviation } \\
\left(\mathrm{ft}^{3} / \mathrm{s}\right)\end{array}$ & $\begin{array}{l}\text { Coeffl- } \\
\text { cient of } \\
\text { variation }\end{array}$ & $\begin{array}{l}\text { Percentage } \\
\text { of annual } \\
\text { dlscharge }\end{array}$ \\
\hline October & 695 & 1945 & 10.5 & 1936 & 160 & 191 & 1.20 & 6.29 \\
\hline November & 667 & 1945 & 13.7 & 1935 & 158 & 187 & 1.19 & 6.20 \\
\hline December & 503 & 1945 & 14.1 & 1935 & 146 & 158 & 1.08 & 5.75 \\
\hline January & 479 & 1952 & 15.1 & 1937 & 137 & 143 & 1.04 & 5.40 \\
\hline February & 484 & 1952 & 10.8 & 1935 & 133 & 127 & 0.96 & 5.23 \\
\hline March & 528 & 1945 & 23.5 & 1937 & 197 & 143 & 0.73 & 7.74 \\
\hline April & 787 & 1952 & 39.5 & 1934 & 305 & 246 & 0.81 & 12.0 \\
\hline May & 900 & 1947 & 18.0 & 1934 & 377 & 301 & 0.80 & 14.8 \\
\hline June & 884 & 1950 & 14.2 & 1934 & 348 & 291 & 0.84 & 13.7 \\
\hline July & 667 & 1950 & 12.8 & 1936 & 243 & 224 & 0.92 & 9.54 \\
\hline August & 611 & 1946 & 11.5 & 1934 & 171 & 177 & 1.04 & 6.71 \\
\hline September & 581 & 1944 & 7.99 & 1934 & 169 & 178 & 1.05 & 6.63 \\
\hline Annual & 515 & 1947 & 20.4 & 1934 & 212 & 176 & 0.83 & 100 \\
\hline
\end{tabular}

Annual flow duration, pre-regulation period

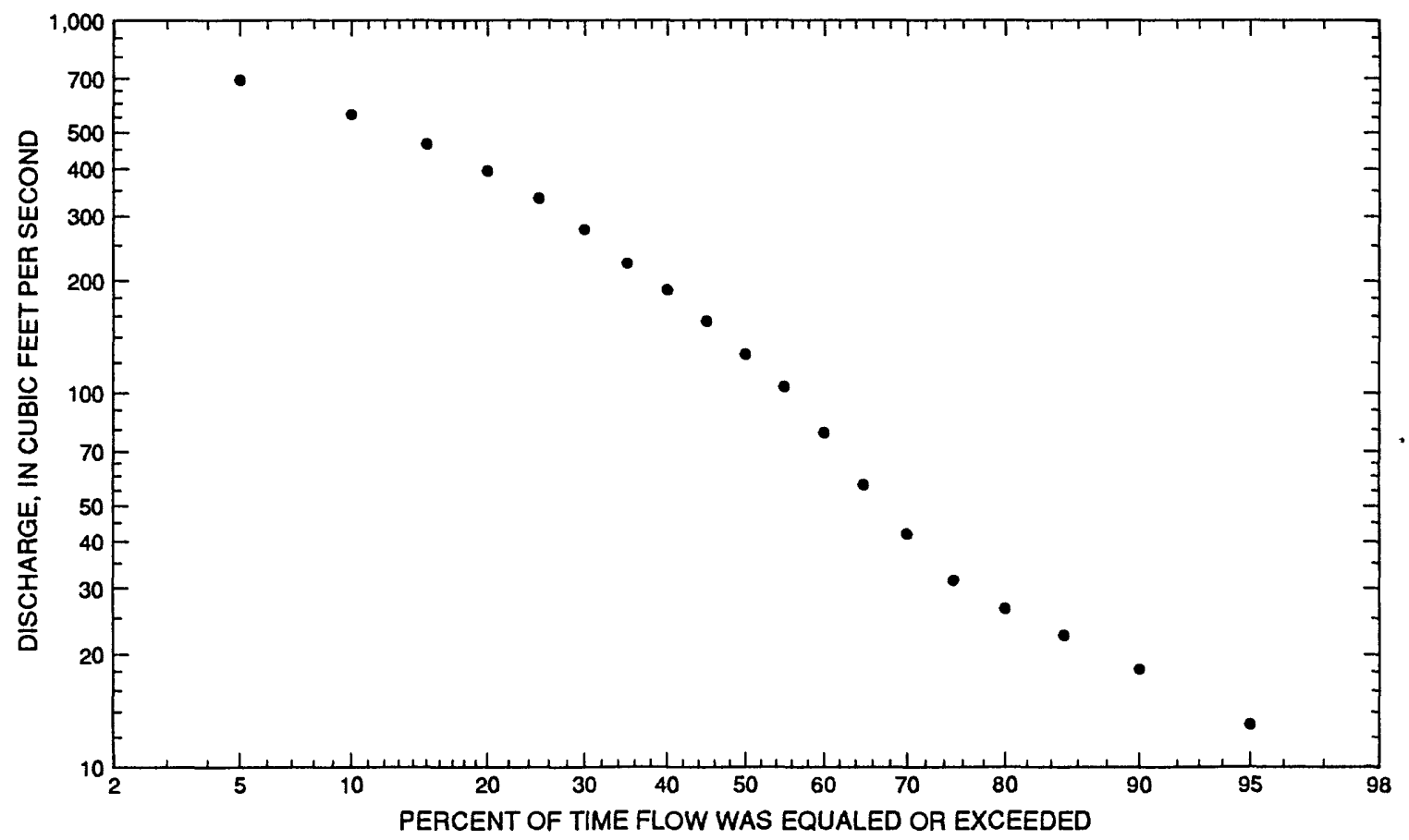




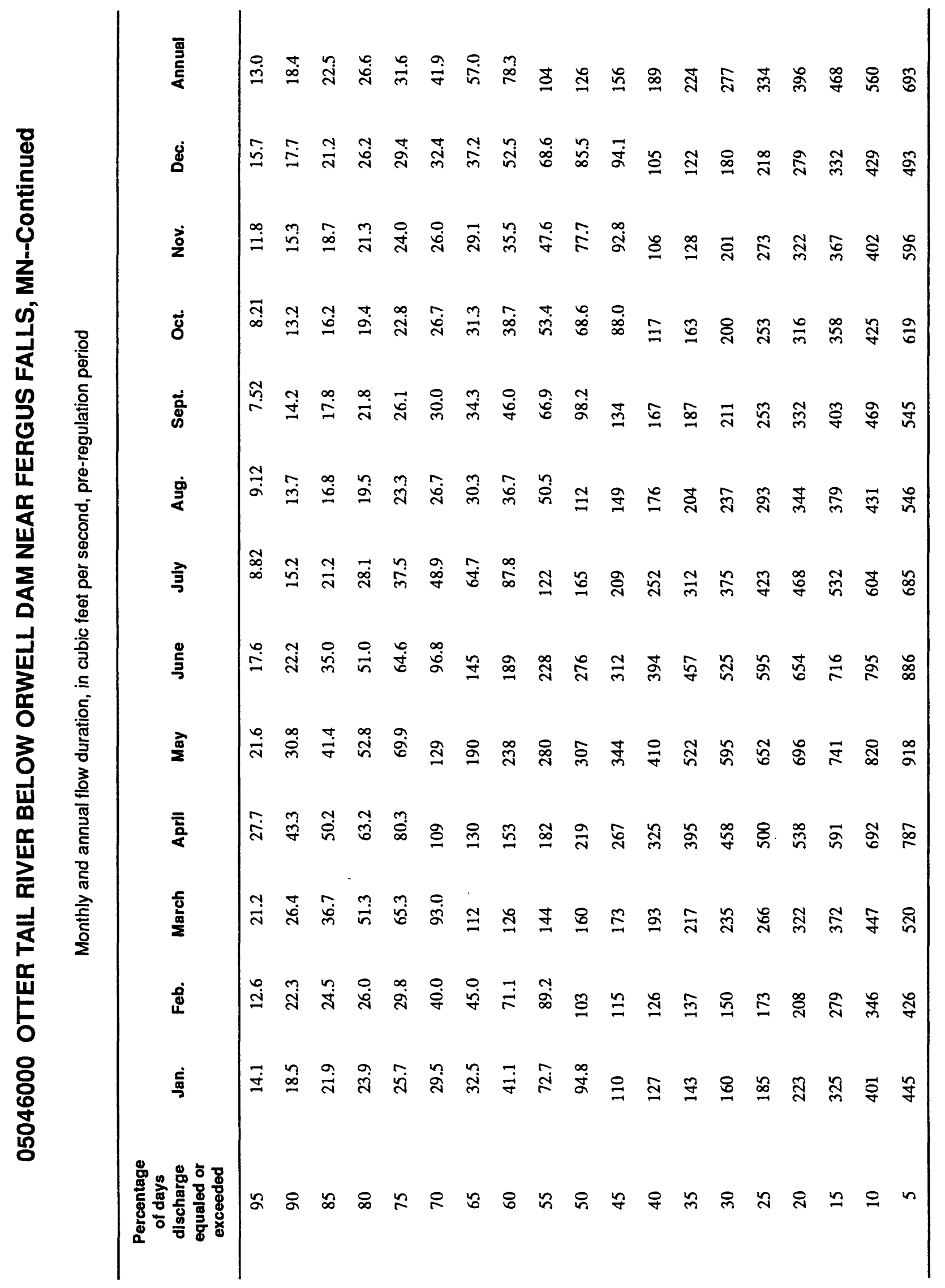




\section{OTTER TAIL RIVER BELOW ORWELL DAM NEAR FERGUS FALLS, MN-Continued}

Probability of annual high discharges, pre-regulation period

[ng, statistic not given]

\begin{tabular}{|c|c|c|c|c|c|c|}
\hline \multirow[b]{2}{*}{$\begin{array}{l}\text { Exceedance } \\
\text { probability }\end{array}$} & \multirow[b]{2}{*}{$\begin{array}{l}\text { Recurrence } \\
\text { Interval } \\
\text { (years) }\end{array}$} & \multirow[b]{2}{*}{$\begin{array}{c}\text { Maximum } \\
\text { Instantaneous } \\
\left(\mathrm{ft}^{3} / \mathbf{s}\right)\end{array}$} & \multicolumn{4}{|c|}{$\begin{array}{l}\text { Maximum average discharge } \\
\qquad\left(\mathrm{ft}^{3} / \mathrm{s}\right)\end{array}$} \\
\hline & & & 3-day period & 7-day period & 15-day period & 30-day period \\
\hline 0.99 & 1.01 & 317 & 56.4 & 40.7 & 32.8 & 24.4 \\
\hline 0.95 & 1.05 & 406 & 117 & 90.8 & 76.4 & 61.2 \\
\hline 0.90 & 1.11 & 462 & 167 & 134 & 115 & 95.3 \\
\hline 0.80 & 1.25 & 542 & 248 & 208 & 182 & 156 \\
\hline 0.50 & 2 & 734 & 482 & 433 & 392 & 357 \\
\hline 0.20 & 5 & 994 & 835 & 493 & 738 & 701 \\
\hline 0.10 & 10 & 1,160 & 1,070 & 1,040 & 976 & 943 \\
\hline 0.04 & 25 & 1,380 & 1,340 & 1,340 & 1,270 & 1,240 \\
\hline 0.02 & 50 & 1,540 & 1,530 & 1,550 & 1,480 & 1,460 \\
\hline 0.01 & 100 & 1,700 & 1,700 & 1,750 & 1,670 & 1,660 \\
\hline 0.005 & 200 & 1,860 & 1,870 & 1,940 & 1,860 & 1,850 \\
\hline 0.002 & 500 & 2,070 & ng & ng & $\mathrm{ng}$ & $\mathrm{ng}$ \\
\hline
\end{tabular}

Probability of annual low discharges, pre-regulation period

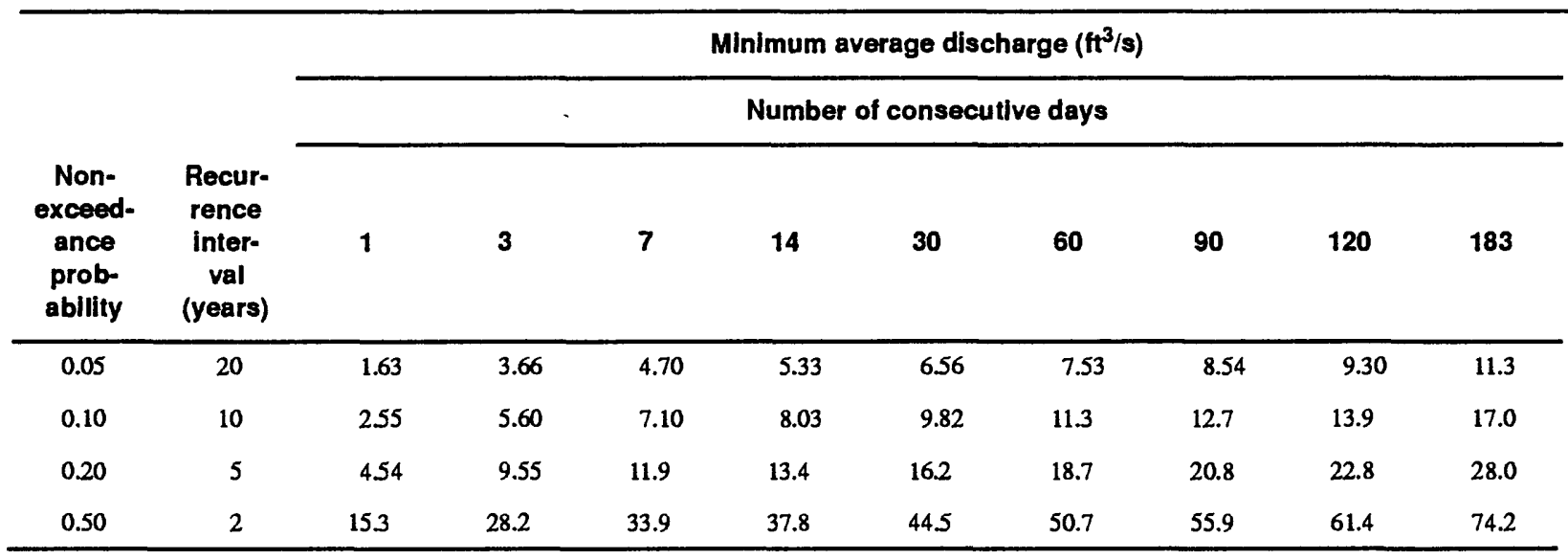




\section{OTTER TAIL RIVER BELOW ORWELL DAM NEAR FERGUS FALLS, MN-Continued}

Probability of seasonal low discharges, pre-regulation period

\begin{tabular}{|c|c|c|c|c|c|c|c|c|c|}
\hline \multirow[b]{3}{*}{$\begin{array}{c}\text { Non- } \\
\text { exceedance } \\
\text { probability }\end{array}$} & \multirow[b]{3}{*}{$\begin{array}{c}\text { Recurrence } \\
\text { interval } \\
\text { (years) }\end{array}$} & \multicolumn{8}{|c|}{ Minimum average discharge $\left(\mathrm{ft}^{3} / \mathrm{s}\right)$} \\
\hline & & \multicolumn{8}{|c|}{ Number of consecutive days } \\
\hline & & 1 & 7 & 14 & 30 & 1 & 7 & 14 & 30 \\
\hline & & \multicolumn{4}{|c|}{ December-January-February } & \multicolumn{4}{|c|}{ March-April-May } \\
\hline 0.05 & 20 & 3.44 & 8.99 & 9.50 & 10.0 & 4.35 & 13.2 & 16.4 & 19.8 \\
\hline 0.10 & 10 & 5.77 & 12.8 & 13.6 & 14.6 & 7.73 & 20.5 & 25.0 & 31.1 \\
\hline 0.20 & 5 & 10.7 & 20.1 & 21.3 & 23.3 & 15.1 & 34.3 & 40.8 & 52.2 \\
\hline \multirow[t]{2}{*}{0.50} & 2 & 34.6 & 50.5 & 53.3 & 59.4 & 50.4 & 86.9 & 98.8 & 129 \\
\hline & & \multicolumn{4}{|c|}{ June-July-August } & \multicolumn{4}{|c|}{ September-October-November } \\
\hline 0.05 & 20 & 3.30 & 5.15 & 6.19 & 8.00 & 2.08 & 4.92 & 5.80 & 6.75 \\
\hline 0.10 & 10 & 5.69 & 9.02 & 10.7 & 13.4 & 3.37 & 7.70 & 9.12 & 10.6 \\
\hline 0.20 & 5 & 11.0 & 17.6 & 20.5 & 24.8 & 6.28 & 13.6 & 16.1 & 18.6 \\
\hline 0.50 & 2 & 39.6 & 61.0 & 68.2 & 78.4 & 23.4 & 43.3 & 50.5 & 57.3 \\
\hline
\end{tabular}




\section{OTTER TAIL RIVER BELOW ORWELL DAM NEAR FERGUS FALLS, MN-Continued}

Post-regulation period, 1953-94

Statistics of monthly and annual mean discharges, post-regulation period

\begin{tabular}{|c|c|c|c|c|c|c|c|c|}
\hline \multirow[b]{2}{*}{ Month } & \multicolumn{2}{|c|}{ Maximum } & \multicolumn{2}{|c|}{ Minimum } & \multicolumn{4}{|c|}{ Mean } \\
\hline & $\begin{array}{c}\text { Discharge } \\
\left(\mathrm{ft}^{3} / \mathrm{s}\right)\end{array}$ & $\begin{array}{c}\text { Water year } \\
\text { of } \\
\text { occurrence }\end{array}$ & $\begin{array}{c}\text { Discharge } \\
\left(\mathrm{ft}^{3} / \mathrm{s}\right)\end{array}$ & $\begin{array}{c}\text { Water year } \\
\text { of } \\
\text { occurrence }\end{array}$ & $\begin{array}{c}\text { Discharge } \\
\left(\mathrm{ft}^{3} / \mathrm{s}\right)\end{array}$ & $\begin{array}{c}\text { Standard } \\
\text { deviation } \\
\left(\mathrm{ft}^{3} / \mathrm{s}\right)\end{array}$ & $\begin{array}{l}\text { Coeffi- } \\
\text { cient of } \\
\text { variation }\end{array}$ & $\begin{array}{l}\text { Percentage } \\
\text { of annuai } \\
\text { discharge }\end{array}$ \\
\hline October & 973 & 1994 & 9.15 & 1977 & 274 & 207 & 0.75 & 5.87 \\
\hline November & 831 & 1986 & 8.42 & 1977 & 286 & 200 & 0.70 & 6.13 \\
\hline December & 706 & 1987 & 8.10 & 1977 & 271 & 180 & 0.66 & 5.81 \\
\hline January & 603 & 1986 & 19.8 & 1977 & 262 & 150 & 0.57 & 5.62 \\
\hline February & 647 & 1994 & 22.4 & 1977 & 270 & 137 & 0.51 & 5.79 \\
\hline March & 724 & 1994 & 30.4 & 1977 & 360 & 140 & 0.39 & 7.72 \\
\hline April & 1,050 & 1986 & 45.8 & 1977 & 539 & 204 & 0.38 & 11.6 \\
\hline May & 1,430 & 1986 & 14.1 & 1977 & 656 & 311 & 0.47 & 14.0 \\
\hline June & 1,420 & 1986 & 55.0 & 1977 & 661 & 352 & 0.53 & 14.2 \\
\hline July & 1,250 & 1953 & 59.8 & 1977 & 491 & 282 & 0.57 & 10.5 \\
\hline August & 1,080 & 1985 & 50.4 & 1977 & 329 & 256 & 0.78 & 7.05 \\
\hline September & 1,030 & 1993 & 9.17 & 1976 & 268 & 226 & 0.84 & 5.73 \\
\hline Annual & 842 & 1986 & 37.5 & 1977 & 389 & 162 & 0.42 & 100 \\
\hline
\end{tabular}

Annual flow duration, post-regulation period

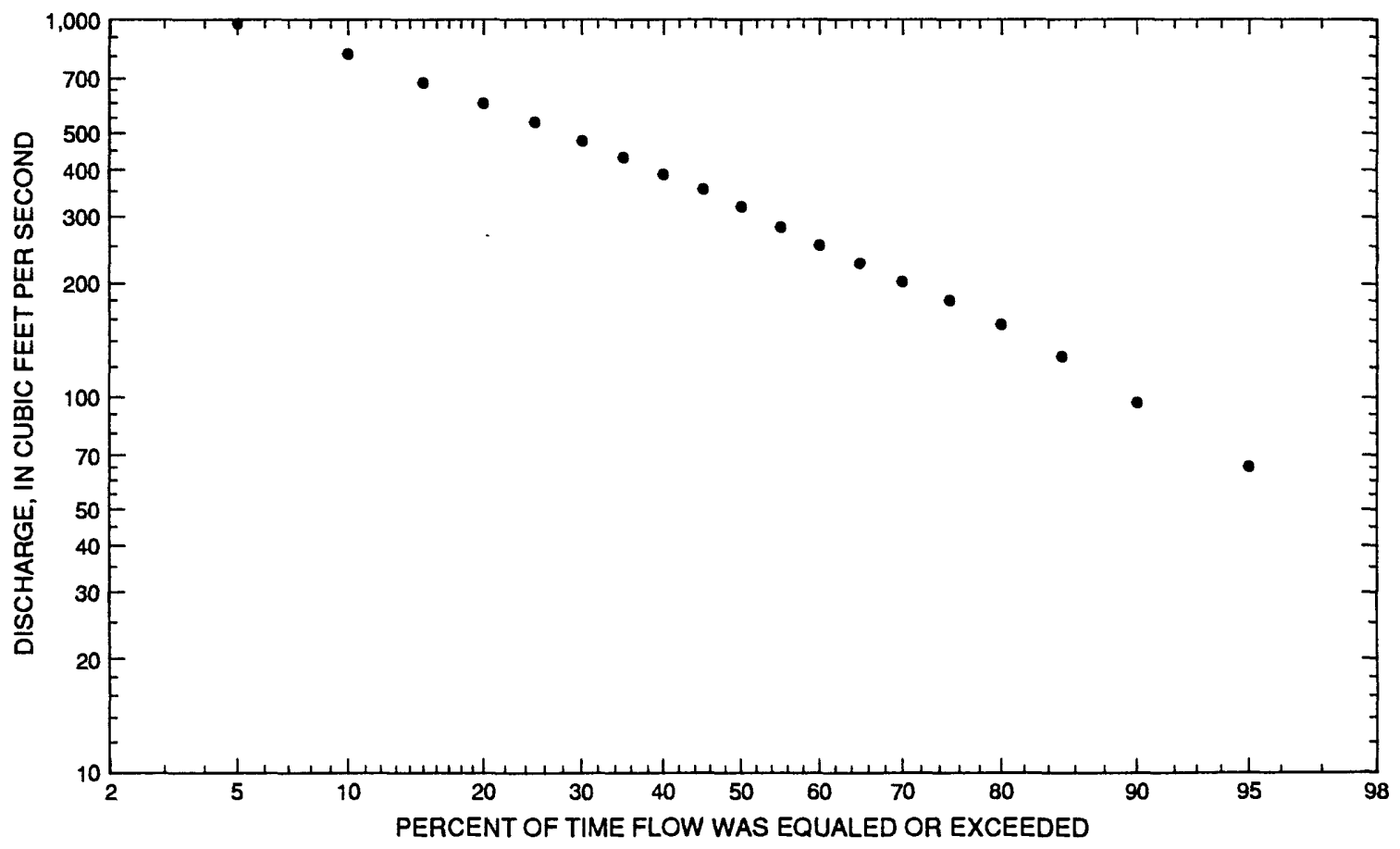




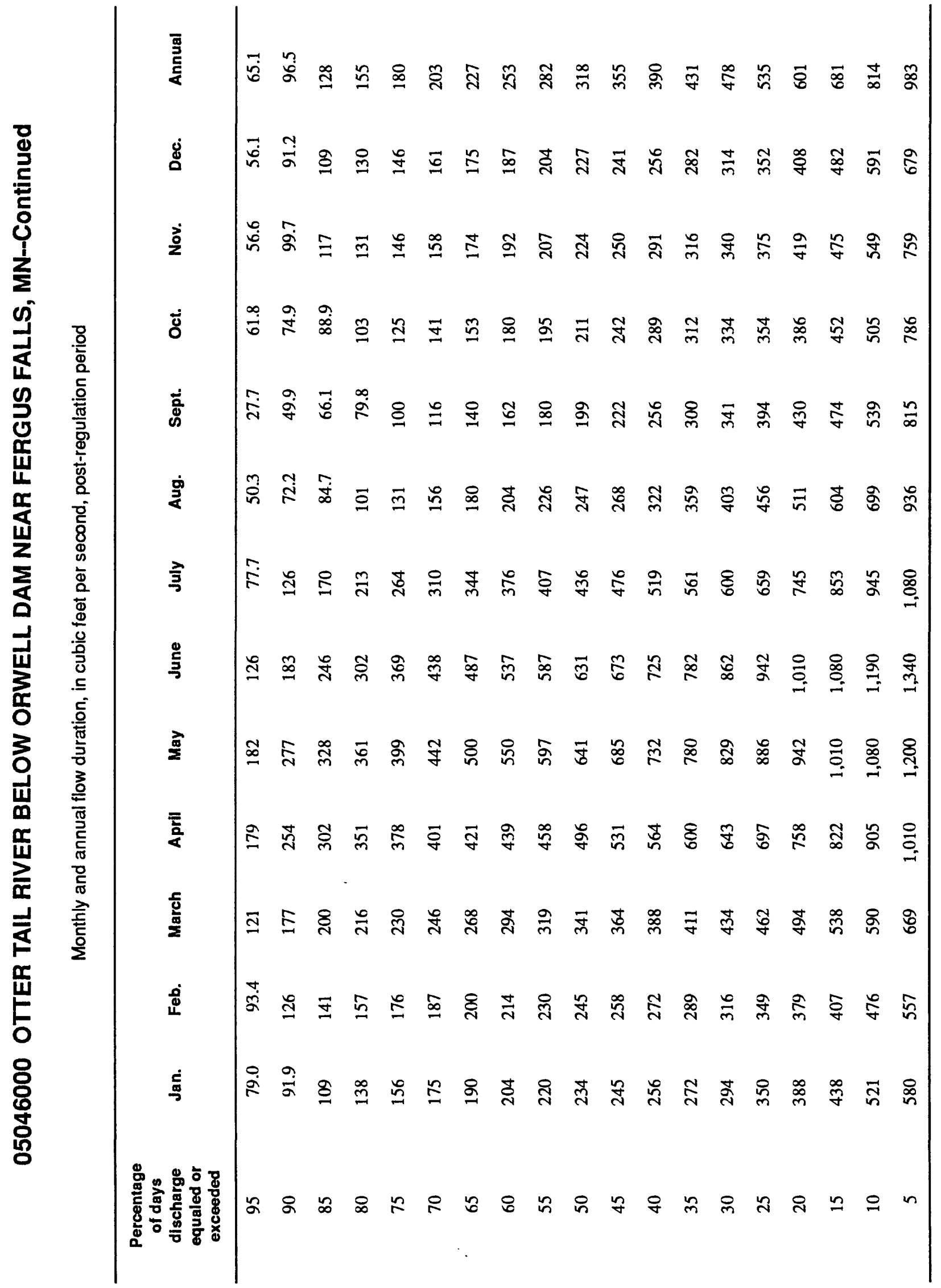




\section{OTTER TAIL RIVER BELOW ORWELL DAM NEAR FERGUS FALLS, MN-Continued}

Probability of annual high discharges, post-regulation period

[ng, statistic not given]

\begin{tabular}{lcccccc}
\hline & & & \multicolumn{5}{c}{$\begin{array}{c}\text { Maximum average discharge } \\
\left(\mathrm{ft}^{3} / \mathbf{s}\right)\end{array}$} \\
\cline { 5 - 7 } $\begin{array}{c}\text { Exceedance } \\
\text { probability }\end{array}$ & $\begin{array}{c}\text { Recurrence } \\
\text { intervai } \\
\text { (years) }\end{array}$ & $\begin{array}{c}\text { Maximum } \\
\text { instantaneous } \\
\left(\mathbf{f t}^{3} / \mathbf{s}\right)\end{array}$ & 3-day period & 7-day period & 15-day period & 30-day period \\
\hline 0.99 & 1.01 & $\mathrm{ng}$ & 206 & 196 & 172 & 147 \\
0.95 & 1.05 & 446 & 350 & 332 & 301 & 271 \\
0.90 & 1.11 & 535 & 448 & 425 & 391 & 359 \\
0.80 & 1.25 & 657 & 585 & 556 & 519 & 486 \\
0.50 & 2 & 930 & 884 & 849 & 808 & 771 \\
0.20 & 5 & 1,250 & 1,200 & 1,160 & 1,120 & 1,070 \\
0.10 & 10 & 1,420 & 1,340 & 1,320 & 1,270 & 1,210 \\
0.04 & 25 & 1,620 & 1,480 & 1,480 & 1,420 & 1,340 \\
0.02 & 50 & 1,740 & 1,560 & 1,560 & 1,500 & 1,400 \\
0.01 & 100 & 1,850 & 1,620 & 1,630 & 1,570 & 1,460 \\
0.005 & 200 & 1,950 & 1,670 & 1,690 & 1,620 & 1,500 \\
0.002 & 500 & 2,070 & ng & ng & ng & ng \\
\hline
\end{tabular}

Probability of annual low discharges, post-regulation period

\begin{tabular}{|c|c|c|c|c|c|c|c|c|c|c|}
\hline \multirow[b]{3}{*}{$\begin{array}{l}\text { Non- } \\
\text { exceed- } \\
\text { ance } \\
\text { prob- } \\
\text { ability }\end{array}$} & \multirow[b]{3}{*}{$\begin{array}{c}\text { Recur- } \\
\text { rence } \\
\text { inter- } \\
\text { val } \\
\text { (years) }\end{array}$} & \multicolumn{9}{|c|}{ Minimum average discharge $\left(\mathrm{ft}^{3} / \mathrm{s}\right)$} \\
\hline & & \multicolumn{9}{|c|}{ Number of consecutive days } \\
\hline & & 1 & 3 & 7 & 14 & 30 & 60 & 90 & 120 & 183 \\
\hline 0.05 & 20 & 11.7 & 13.9 & 14.9 & 16.1 & 19.5 & 29.7 & 34.2 & 36.8 & 45.7 \\
\hline 0.10 & 10 & 18.1 & 20.5 & 23.0 & 25.5 & 30.8 & 45.8 & 53.4 & 58.0 & 69.2 \\
\hline 0.20 & 5 & 29.7 & 32.4 & 38.0 & 43.0 & 51.6 & 73.7 & 86.3 & 94.4 & 108 \\
\hline 0.50 & 2 & 70.9 & 74.4 & 91.8 & 106 & 124 & 159 & 183 & 198 & 218 \\
\hline
\end{tabular}




\section{OTTER TAIL RIVER BELOW ORWELL DAM NEAR FERGUS FALLS, MN-Continued}

Probability of seasonal low discharges, post-regulation period

[ng, statistic not given]

\begin{tabular}{|c|c|c|c|c|c|c|c|c|c|}
\hline \multirow[b]{3}{*}{$\begin{array}{c}\text { Non- } \\
\text { exceedance } \\
\text { probabillty }\end{array}$} & \multirow[b]{3}{*}{$\begin{array}{c}\text { Recurrence } \\
\text { Interval } \\
\text { (yeara) }\end{array}$} & \multicolumn{8}{|c|}{ Minimum average discharge $\left(\mathrm{ft}^{3} / \mathrm{s}\right)$} \\
\hline & & \multicolumn{8}{|c|}{ Number of consecutive days } \\
\hline & & 1 & 7 & 14 & 30 & 1 & 7 & 14 & 30 \\
\hline & & \multicolumn{4}{|c|}{ December-January-February } & \multicolumn{4}{|c|}{ March-April-May } \\
\hline 0.05 & 20 & 34.8 & 37.2 & 39.1 & 41.3 & 32.0 & 53.1 & 66.7 & ng \\
\hline 0.10 & 10 & 54.8 & 59.5 & 63.3 & 67.7 & 49.3 & 88.1 & 116 & ng \\
\hline 0.20 & 5 & 88.6 & 97.3 & 104 & 112 & 78.3 & 143 & 192 & ng \\
\hline \multirow[t]{2}{*}{0.50} & 2 & 183 & 201 & 215 & 230 & 159 & 258 & 324 & ng \\
\hline & & \multicolumn{4}{|c|}{ June-July-August } & \multicolumn{4}{|c|}{ September-October-November } \\
\hline 0.05 & 20 & 15.9 & 26.5 & 33.9 & 61.2 & 16.4 & 17.8 & 20.2 & 28.7 \\
\hline 0.10 & 10 & 26.2 & 39.7 & 49.5 & 81.5 & 25.5 & 27.7 & 31.5 & 44.0 \\
\hline 0.20 & 5 & 46.2 & 63.7 & 77.2 & 115 & 42.7 & 46.2 & 52.7 & 71.6 \\
\hline 0.50 & 2 & 122 & 151 & 175 & 224 & 108 & 116 & 130 & 164 \\
\hline
\end{tabular}




\section{OTTER TAIL RIVER BELOW ORWELL DAM NEAR FERGUS FALLS, MN-Continued}

Annual peak discharge and corresponding gage height, period of record

$$
[-, \text { no data] }
$$

\begin{tabular}{|c|c|c|c|c|c|c|c|}
\hline $\begin{array}{l}\text { Water } \\
\text { year }\end{array}$ & Date & $\begin{array}{c}\text { Gage } \\
\text { height } \\
\text { (feet) }\end{array}$ & $\begin{array}{c}\text { Peak } \\
\text { discharge } \\
\left(\mathrm{ft}^{3} / \mathrm{s}\right)\end{array}$ & $\begin{array}{l}\text { Water } \\
\text { year }\end{array}$ & Date & $\begin{array}{l}\text { Gage } \\
\text { height } \\
\text { (feet) }\end{array}$ & $\begin{array}{c}\text { Peak } \\
\text { discharge } \\
\left(\mathrm{ft}^{3} / \mathrm{s}\right)\end{array}$ \\
\hline \multicolumn{8}{|c|}{ Annual peak discharge, by year, and corresponding gage height } \\
\hline 1931 & May 18 & 2.03 & 686 & 1963 & June 17 & 3.65 & 745 \\
\hline 1932 & June 14 & - & 551 & 1964 & May 11 & 3.73 & 861 \\
\hline 1933 & June 18 & - & 577 & 1965 & June 14 & 4.74 & 1,330 \\
\hline 1934 & May 29 & 2.92 & 448 & 1966 & June 1 & 4.59 & 1,490 \\
\hline 1935 & June 26 & - & 472 & 1967 & May 18 & 4.14 & 1,130 \\
\hline 1936 & April 14 & 2.97 & 468 & 1968 & May 14 & 3.37 & 714 \\
\hline 1937 & July 30 & 3.27 & 518 & 1969 & May 31 & 4.43 & 1,260 \\
\hline 1938 & June 16 & - & 544 & 1970 & June 22 & 3.88 & 935 \\
\hline 1939 & -- & 3.20 & 603 & 1971 & April 9 & 3.53 & 755 \\
\hline 1940 & September 2 & 3.28 & 600 & 1972 & June 3 & 4.44 & 1,360 \\
\hline 1941 & July 2 & - & 611 & 1973 & March 15 & 3.48 & 714 \\
\hline 1942 & June 6 & 3.52 & 747 & 1974 & June 3 & 4.31 & 1,310 \\
\hline 1943 & April 2 & 4.31 & 1,150 & 1975 & June 21 & 4.09 & 1,090 \\
\hline 1944 & June 4 & 4.31 & 1,200 & 1976 & March 29 & 3.27 & 663 \\
\hline 1945 & November 14 & 4.13 & 1,120 & 1977 & September 19 & 2.88 & 300 \\
\hline 1946 & July 19 & - & 777 & 1978 & April 9 & 4.07 & 1,040 \\
\hline 1947 & June 10 & 4.81 & 1,370 & 1979 & April 17 & 4.11 & 1,110 \\
\hline 1948 & May 18 & - & 900 & 1980 & April 9 & 3.84 & 903 \\
\hline 1949 & July 8 & 3.61 & 564 & 1981 & August 4 & 2.75 & 267 \\
\hline 1950 & May 23 & - & 1,100 & 1982 & May 29 & 3.71 & 849 \\
\hline 1951 & April 5 & - & 1,160 & 1983 & July 20 & 3.11 & 524 \\
\hline 1952 & April 10 & 3.91 & 1,040 & 1984 & June 15 & 3.64 & 808 \\
\hline 1953 & June 17 & 5.60 & 1,710 & 1985 & June 27 & 4.29 & 1,270 \\
\hline 1954 & June 20 & 4.26 & 1,210 & 1986 & May 27 & 4.75 & 1,600 \\
\hline 1955 & August 6 & 3.53 & 730 & 1987 & June 30 & 3.97 & 1,050 \\
\hline 1956 & May 29 & 4.05 & 1,080 & 1988 & March 25 & 3.05 & 408 \\
\hline 1957 & May 23 & 3.70 & 794 & 1989 & April 10 & 4.17 & 1,180 \\
\hline 1958 & March 4 & 3.19 & 534 & 1990 & June 14 & 3.37 & 650 \\
\hline 1959 & June 8 & 3.32 & 612 & 1991 & July 1 & 4.00 & 1,050 \\
\hline 1960 & May 26 & 3.66 & 810 & 1992 & July 25 & - & 589 \\
\hline 1961 & May 23 & 3.44 & 664 & 1993 & July 24 & 4.34 & 1,290 \\
\hline 1962 & June 26 & 4.40 & 1,260 & 1994 & May 6 & 4.30 & 1,280 \\
\hline \multicolumn{8}{|c|}{ Annual peak discharge, from highest to lowest, and corresponding gage height } \\
\hline 1953 & June 17 & 5.60 & 1,710 & 1962 & June 26 & 4.40 & 1,260 \\
\hline 1986 & May 27 & 4.75 & 1,600 & 1969 & May 31 & 4.43 & 1,260 \\
\hline 1966 & June 1 & 4.59 & 1,490 & 1954 & June 20 & 4.26 & 1,210 \\
\hline 1947 & June 10 & 4.81 & 1,370 & 1944 & June 4 & 4.31 & 1,200 \\
\hline 1972 & June 3 & 4.44 & 1,360 & 1989 & April 10 & 4.17 & 1,180 \\
\hline 1965 & June 14 & 4.74 & 1,330 & 1951 & April 5 & - & 1,160 \\
\hline 1974 & June 3 & 4.31 & 1,310 & 1943 & April 2 & 4.31 & 1,150 \\
\hline 1993 & July 24 & 4.34 & 1,290 & 1967 & May 18 & 4.14 & 1,130 \\
\hline 1994 & May 6 & 4.30 & 1,280 & 1945 & November 14 & 4.13 & 1,120 \\
\hline 1985 & June 27 & 4.29 & 1,270 & 1979 & April 17 & 4.11 & 1,110 \\
\hline
\end{tabular}


05046000 OTTER TAIL RIVER BELOW ORWELL DAM NEAR FERGUS FALLS, MN-Continued

Annual peak discharge and corresponding gage height, period of record--Continued

$$
[-, \text { no data }]
$$

\begin{tabular}{|c|c|c|c|c|c|c|c|}
\hline $\begin{array}{l}\text { Water } \\
\text { year }\end{array}$ & Date & $\begin{array}{c}\text { Gage } \\
\text { height } \\
\text { (feet) }\end{array}$ & $\begin{array}{c}\text { Peak } \\
\text { discharge } \\
\left(\mathrm{ft}^{3} / \mathrm{s}\right)\end{array}$ & $\begin{array}{l}\text { Water } \\
\text { year }\end{array}$ & Date & $\begin{array}{c}\text { Gage } \\
\text { helght } \\
\text { (feet) }\end{array}$ & $\begin{array}{c}\text { Peak } \\
\text { discharge } \\
\left(\mathrm{ft}^{3} / \mathrm{s}\right)\end{array}$ \\
\hline \multicolumn{8}{|c|}{ Annual peak discharge, from highest to lowest, and corresponding gage height--Continued } \\
\hline 1950 & May 23 & - & 1,100 & 1931 & May 18 & 2.03 & 686 \\
\hline 1975 & June 21 & 4.09 & 1,090 & 1961 & May 23 & 3.44 & 664 \\
\hline 1956 & May 29 & 4.05 & 1,080 & 1976 & March 29 & 3.27 & 663 \\
\hline 1987 & June 30 & 3.97 & 1,050 & 1990 & June 14 & 3.37 & 650 \\
\hline 1991 & July 1 & 4.00 & 1,050 & 1959 & June 8 & 3.32 & 612 \\
\hline 1952 & April 10 & 3.91 & 1,040 & 1941 & July 2 & - & 611 \\
\hline 1978 & April 9 & 4.07 & 1,040 & 1939 & -- & 3.20 & 603 \\
\hline 1970 & June 22 & 3.88 & 935 & 1940 & September 2 & 3.28 & 600 \\
\hline 1980 & April 9 & 3.84 & 903 & 1992 & July 25 & - & 589 \\
\hline 1948 & May 18 & - & 900 & 1933 & June 18 & - & 577 \\
\hline 1964 & May 11 & 3.73 & 861 & 1949 & July 8 & 3.61 & 564 \\
\hline 1982 & May 29 & 3.71 & 849 & 1932 & June 14 & - & 551 \\
\hline 1960 & May 26 & 3.66 & 810 & 1938 & June 16 & -- & 544 \\
\hline 1984 & June 15 & 3.64 & 808 & 1958 & March 4 & 3.19 & 534 \\
\hline 1957 & May 23 & 3.70 & 794 & 1983 & July 20 & 3.11 & 524 \\
\hline 1946 & July 19 & - & 777 & 1937 & July 30 & 3.27 & 518 \\
\hline 1971 & April 9 & 3.53 & 755 & 1935 & June 26 & -- & 472 \\
\hline 1942 & June 6 & 3.52 & 747 & 1936 & April 14 & 2.97 & 468 \\
\hline 1963 & June 17 & 3.65 & 745 & 1934 & May 29 & 2.92 & 448 \\
\hline 1955 & August 6 & 3.53 & 730 & 1988 & March 25 & 3.05 & 408 \\
\hline 1968 & May 14 & 3.37 & 714 & 1977 & September 19 & 2.88 & 300 \\
\hline 1973 & March 15 & 3.48 & 714 & 1981 & August 4 & 2.75 & 267 \\
\hline
\end{tabular}




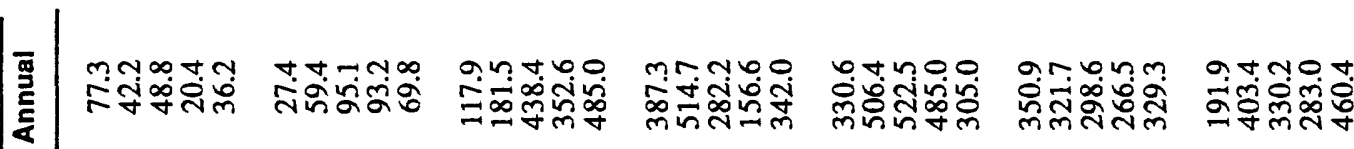

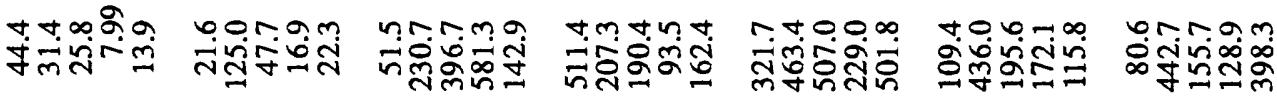

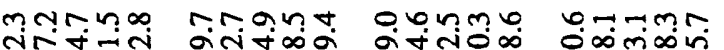

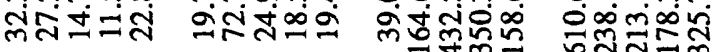

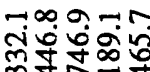

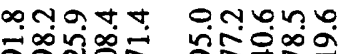

or-mo ma0tn

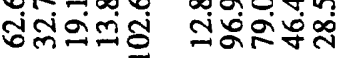

$0 \infty-1-m$

$m+N-0$

no rn

0.7090

$\forall m 000$

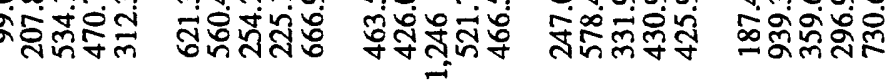

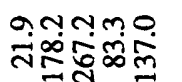

nึmog

$\forall \infty \infty$

nฺंत̄a

웜ำ

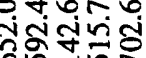

$\infty \quad \forall$ लभกजठ nळ त्रूm

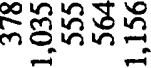

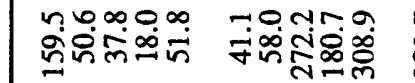

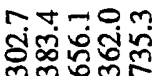

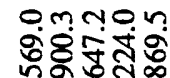

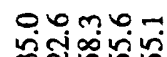

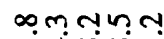

กุกกุดา

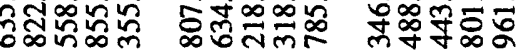

ormnn ogoon

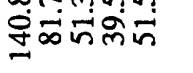

กิำㅇำ

F⿻mm

robon

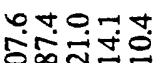

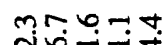

monnn

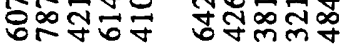

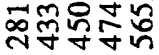

- $00 \infty \infty$

mี่ํํำ

nnmosin

$n-7-\infty$

กัต่ํํำ

-กฺฺด0

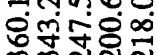

nro- -0

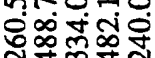

$0 \infty 0 \infty-$

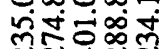

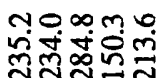

ที่งิษี่์

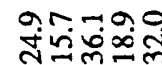

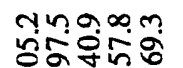

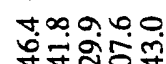

가우눈

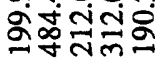

웅ำ

montin

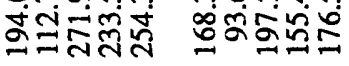

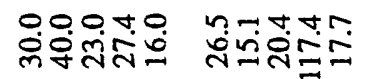

ำกำ:움

กตรต

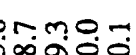

nongo.

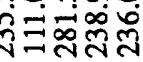

namo

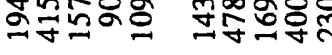

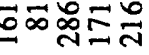

mㅇํㅇํํㅇ

i

Onm+l -

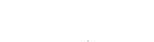

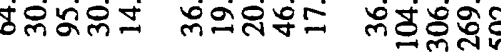

สิรัซจำ

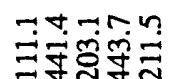

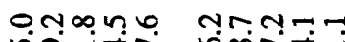

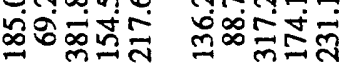

monn ชึ্ฺ่

जู่กิส

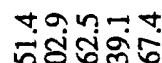

magno

NMNOO

$0,0.0-4$

$0.70 \infty n$

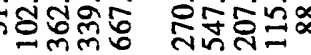

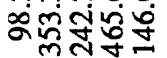

तुंगु

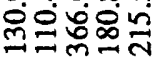

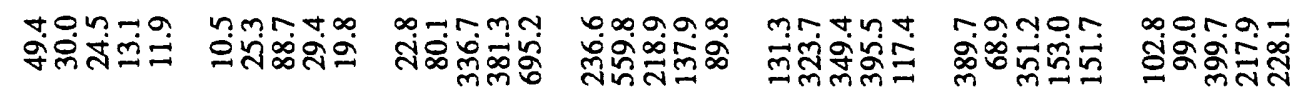

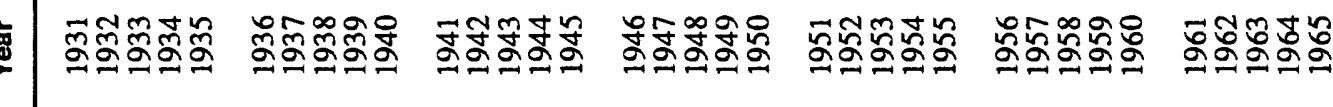




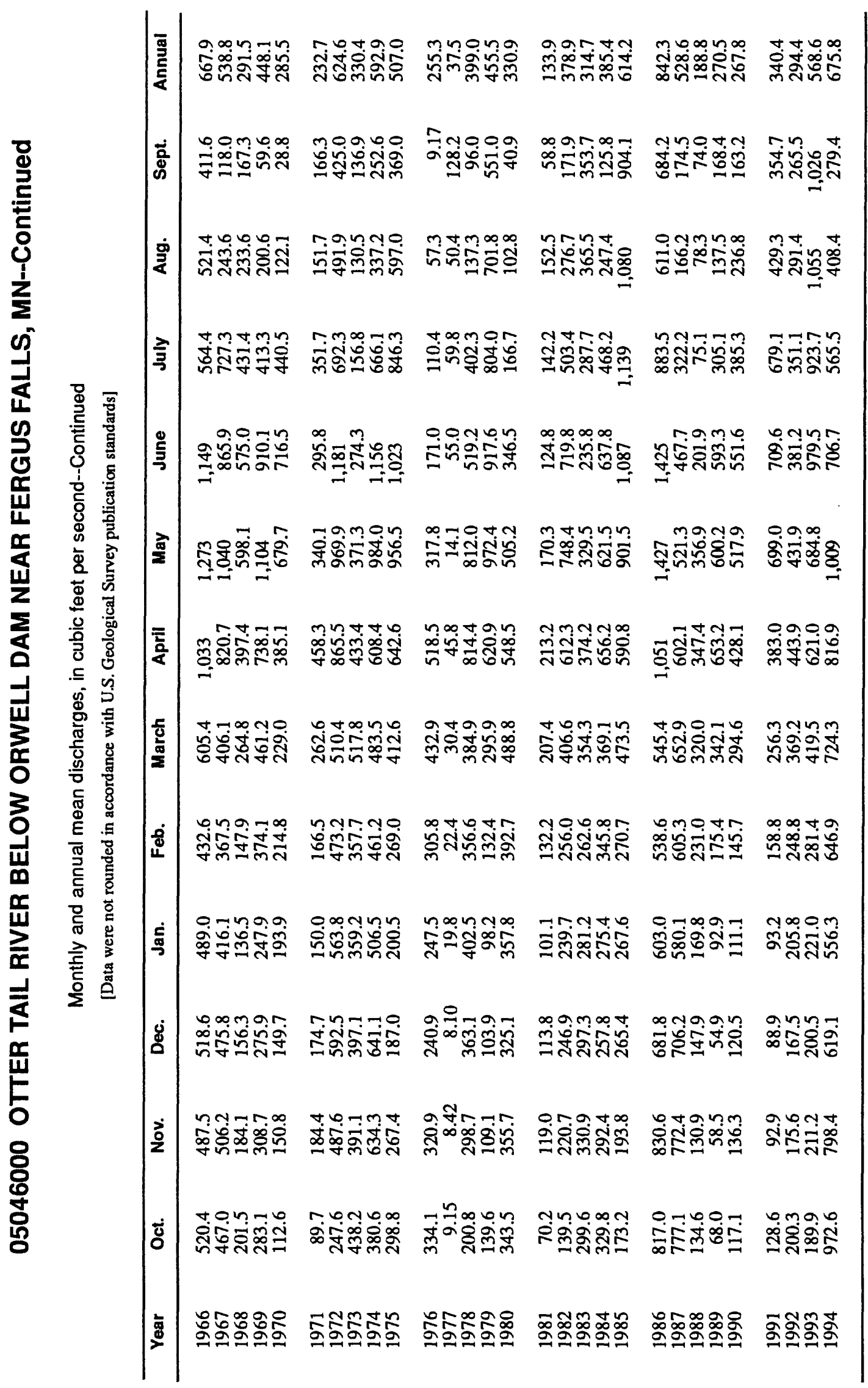




\section{MUSTINKA DITCH ABOVE WEST BRANCH MUSTINKA RIVER NEAR CHARLESVILLE, MN}

\section{Station Description}

LOCATION.--Lat 45'53'25", long 96²1'30", in SW $1 / 4 \mathrm{SW}^{1} / 4$ sec.17, T.128 N., R.45 W., Traverse County, Hydrologic Unit 09020102, near right bank on upstream side of highway bridge, $0.2 \mathrm{mi}$ upstream from West Branch Mustinka River, $6 \mathrm{mi}$ southwest of Charlesville, and $7.8 \mathrm{mi}$ northeast of Wheaton.

DRAINAGE AREA.--Not determined.

PERIOD OF RECORD.--April 1943 to September 1955. Monthly discharge only for some periods, published in Water-Supply Paper 1308. Prior to October 1949, published as "above Twelve Mile Creek near Charlesville".

GAGE.--Staff gage. Datum of gage is $990.00 \mathrm{ft}$ above sea level, adjustment of 1912 . Prior to May 11, 1948, reference mark at same site referred to same datum.

EXTREMES FOR PERIOD OF RECORD.--Maximum discharge, 1,550 $\mathrm{ft}^{3} / \mathrm{s}$, Apr. 9, 1952; maximum gage height, $14.19 \mathrm{ft}$, Apr. 2, 1952; no flow for several months most years.

Annual mean discharge

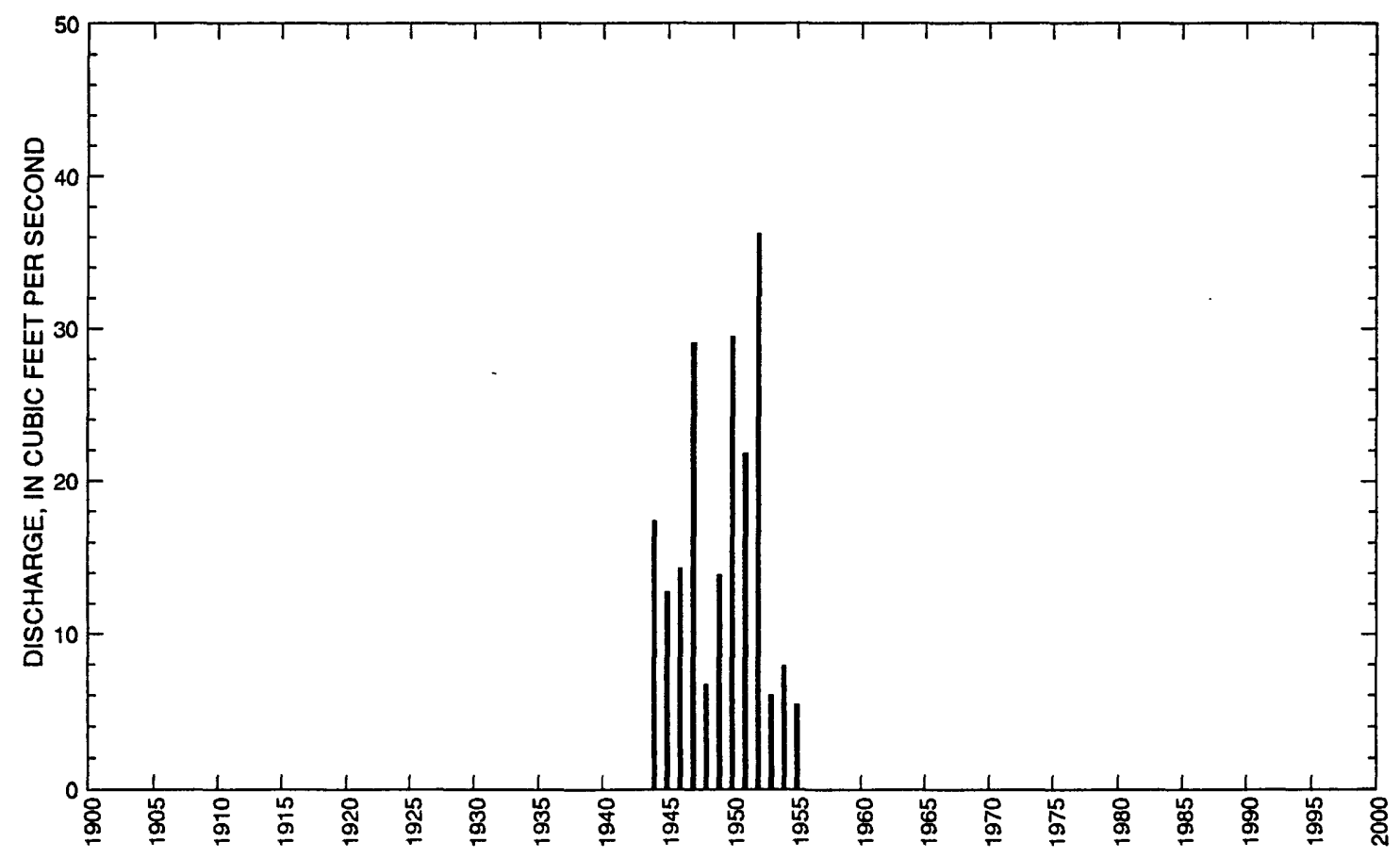




\section{MUSTINKA DITCH ABOVE WEST BRANCH MUSTINKA RIVER NEAR CHARLESVILLE, MN--Continued}

Statistics of monthly and annual mean discharges

[m, more than 1 year of occurrence]

\begin{tabular}{|c|c|c|c|c|c|c|c|c|}
\hline \multirow[b]{2}{*}{ Month } & \multicolumn{2}{|c|}{ Maximum } & \multicolumn{2}{|c|}{ Minimum } & \multicolumn{4}{|c|}{ Mean } \\
\hline & $\begin{array}{c}\text { Discharge } \\
\left(\mathrm{ft}^{3} / \mathrm{s}\right)\end{array}$ & $\begin{array}{c}\text { Water year } \\
\text { of } \\
\text { occurrence }\end{array}$ & $\begin{array}{c}\text { Discharge } \\
\left(\mathrm{ft}^{3} / \mathrm{s}\right)\end{array}$ & $\begin{array}{c}\text { Water year } \\
\text { of } \\
\text { occurrence }\end{array}$ & $\begin{array}{c}\text { Discharge } \\
\left(\mathrm{ft}^{3 / 8}\right)\end{array}$ & $\begin{array}{c}\text { Standard } \\
\text { deviation } \\
\left(\mathrm{ft}^{3} / \mathrm{s}\right)\end{array}$ & $\begin{array}{l}\text { Coeffl- } \\
\text { cient of } \\
\text { variation }\end{array}$ & $\begin{array}{l}\text { Percentage } \\
\text { of annual } \\
\text { discharge }\end{array}$ \\
\hline October & 7.77 & 1952 & 0.068 & 1953 & 2.55 & 2.66 & 1.04 & 1.21 \\
\hline November & 5.48 & 1947 & 0.440 & 1953 & 2.23 & 1.79 & 0.80 & 1.06 \\
\hline December & 2.24 & 1952 & 0.032 & 1951 & 0.580 & 0.73 & 1.26 & 0.28 \\
\hline January & 0.813 & 1952 & 0 & $\mathrm{~m}$ & 0.130 & 0.25 & 2.01 & 0.06 \\
\hline February & 0.304 & 1953 & 0 & $\mathrm{~m}$ & 0.050 & 0.10 & 2.03 & 0.02 \\
\hline March & 78.0 & 1946 & 0 & $\mathrm{~m}$ & 18.7 & 26.1 & 1.40 & 8.88 \\
\hline April & 311 & 1952 & 12.6 & 1949 & 86.6 & 93.2 & 1.08 & 41.2 \\
\hline May & 153 & 1950 & 1.64 & 1949 & 39.2 & 42.8 & 1.09 & 18.7 \\
\hline June & 89.2 & 1944 & 3.19 & 1946 & 29.1 & 27.9 & 0.96 & 13.9 \\
\hline July & 111 & 1949 & 2.47 & 1948 & 24.4 & 29.6 & 1.21 & 11.6 \\
\hline August & 25.4 & 1943 & 0.342 & 1945 & 4.60 & 6.75 & 1.47 & 2.19 \\
\hline September & 8.63 & 1943 & 0 & 1953 & 2.00 & 2.40 & 1.20 & 0.95 \\
\hline Annual & 36.2 & 1952 & 5.40 & 1955 & 16.8 & 10.3 & 0.62 & 100 \\
\hline
\end{tabular}

Annual flow duration

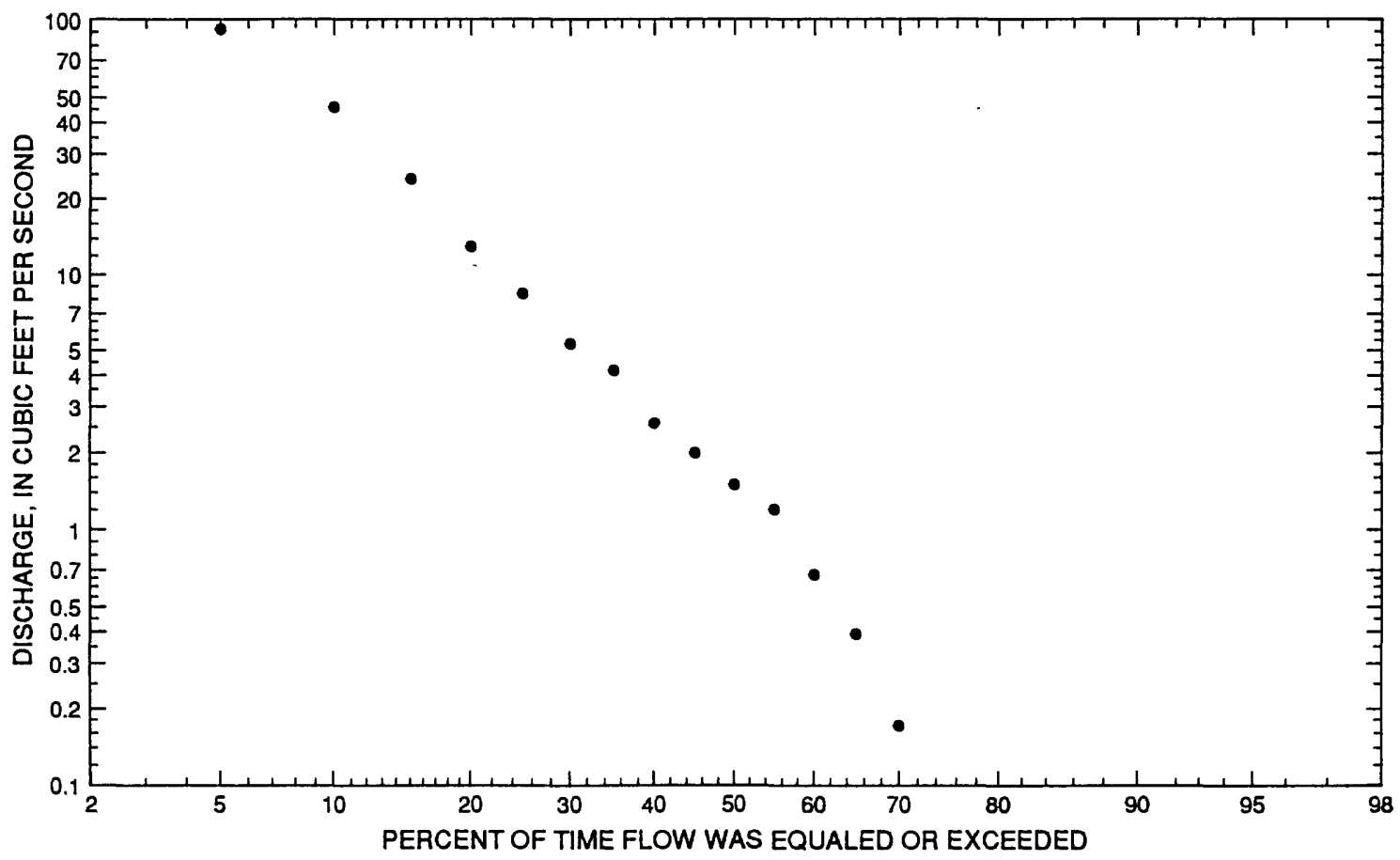




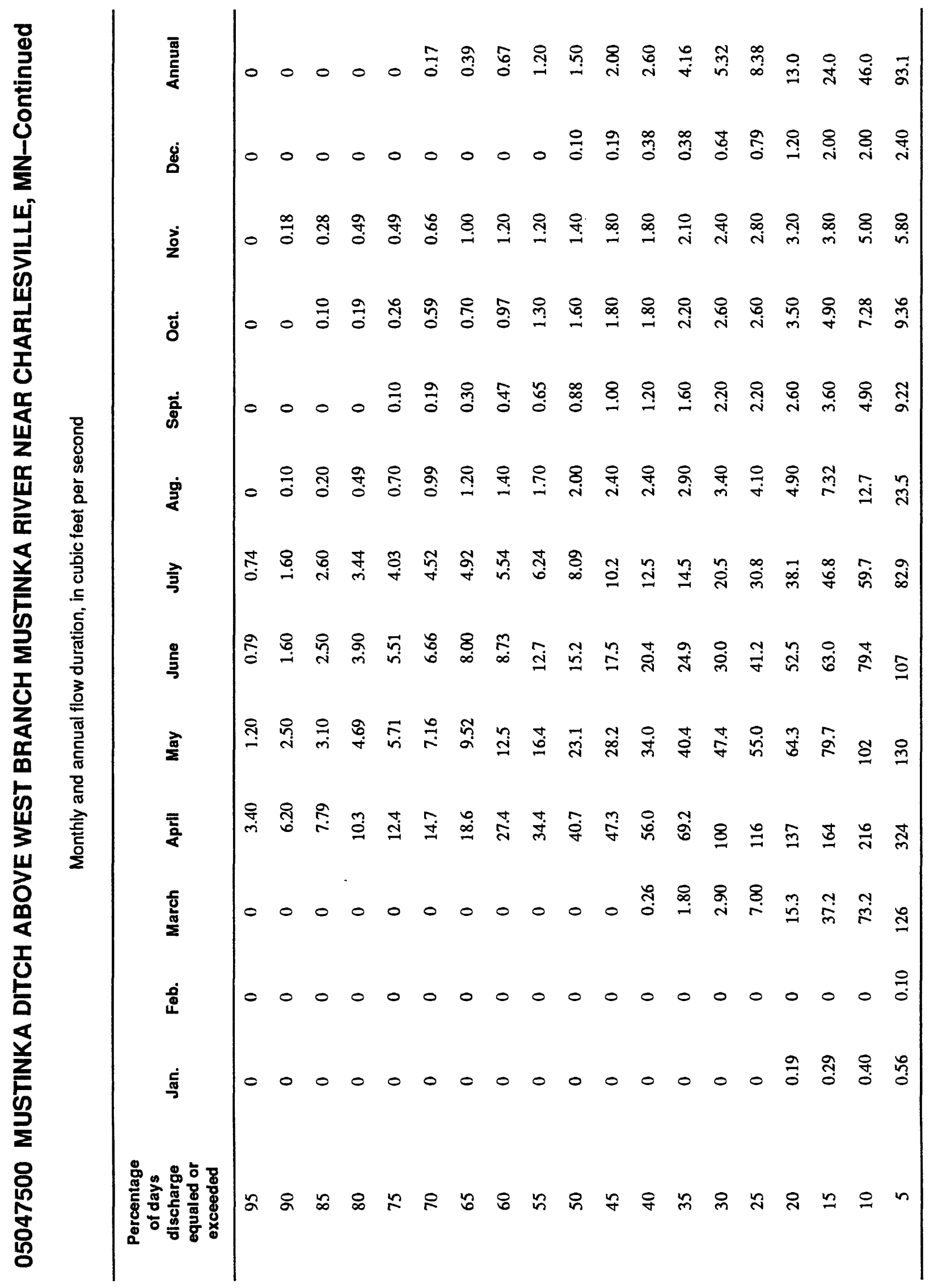




\section{MUSTINKA DITCH ABOVE WEST BRANCH MUSTINKA RIVER NEAR CHARLESVILLE, MN--Continued}

Probability of annual high discharges

[ng, statistic not given]

\begin{tabular}{lcccccc}
\hline & & & \multicolumn{5}{c}{$\begin{array}{c}\text { Maximum average discharge } \\
\left(\mathbf{f t}^{3} / \mathbf{s}\right)\end{array}$} \\
\cline { 5 - 7 } $\begin{array}{c}\text { Exceedance } \\
\text { probability }\end{array}$ & $\begin{array}{c}\text { Recurrence } \\
\text { interval } \\
\text { (years) }\end{array}$ & $\begin{array}{c}\text { Maximum } \\
\text { instantaneous } \\
\left(\mathbf{f t}^{3} / \mathbf{s}\right)\end{array}$ & 3-day period & 7-day period & 15-day period & 30-day period \\
\hline 0.99 & 1.01 & 45.2 & 38.6 & 30.9 & 21.4 & 16.1 \\
0.95 & 1.05 & 78.5 & 66.2 & 52.2 & 37.0 & 27.8 \\
0.90 & 1.11 & 105 & 87.9 & 68.9 & 49.4 & 37.0 \\
0.80 & 1.25 & 149 & 124 & 96.4 & 69.8 & 52.1 \\
0.50 & 2 & 290 & 235 & 183 & 133 & 98.6 \\
0.20 & 5 & 559 & 440 & 346 & 250 & 183 \\
0.10 & 10 & 784 & 607 & 481 & 345 & 251 \\
0.04 & 25 & 1,120 & 854 & 685 & 485 & 350 \\
0.02 & 50 & 1,420 & 1,060 & 859 & 602 & 432 \\
0.01 & 100 & 1,740 & 1,290 & 1,050 & 730 & 522 \\
0.005 & 200 & 2,100 & 1,540 & 1,270 & 869 & 619 \\
0.002 & 500 & 2,640 & ng & ng & ng & ng \\
\hline
\end{tabular}

Probability of annual low discharges

[ng, statistic not given]

\begin{tabular}{|c|c|c|c|c|c|c|c|c|c|c|}
\hline \multirow{3}{*}{$\begin{array}{l}\text { Non- } \\
\text { exceed- } \\
\text { ance } \\
\text { prob- } \\
\text { ability }\end{array}$} & \multirow{3}{*}{$\begin{array}{c}\text { Recur- } \\
\text { rence } \\
\text { inter- } \\
\text { val } \\
\text { (years) }\end{array}$} & \multicolumn{9}{|c|}{ Mlnimum average discharge $\left(\mathrm{ft}^{3} / \mathrm{s}\right)$} \\
\hline & & \multicolumn{9}{|c|}{ Number of consecutive days } \\
\hline & & 1 & 3 & 7 & 14 & 30 & 60 & 90 & 120 & 183 \\
\hline 0.05 & 20 & ng & ng & $\mathrm{ng}$ & ng & ng & ng & 0 & 0 & 0.163 \\
\hline 0.10 & 10 & ng & ng & $\mathrm{ng}$ & ng & ng & ng & 0 & 0.030 & 0.224 \\
\hline 0.20 & 5 & ng & ng & ng & $\mathbf{n g}$ & ng & ng & 0 & 0.093 & 0.330 \\
\hline 0.50 & 2 & ng & ng & $\mathrm{ng}$ & ng & $\mathrm{ng}$ & $\mathrm{ng}$ & 0 & 0.268 & 0.705 \\
\hline
\end{tabular}




\section{MUSTINKA DITCH ABOVE WEST BRANCH MUSTINKA RIVER NEAR CHARLESVILLE, MN--Continued}

Probability of seasonal low discharges

[ng, statistic not given]

\begin{tabular}{|c|c|c|c|c|c|c|c|c|c|}
\hline \multirow[b]{3}{*}{$\begin{array}{c}\text { Non- } \\
\text { exceedance } \\
\text { probability }\end{array}$} & \multirow[b]{3}{*}{$\begin{array}{l}\text { Recurrence } \\
\text { interval } \\
\text { (years) }\end{array}$} & \multicolumn{8}{|c|}{ Minimum average discharge $\left(\mathrm{ft}^{3} / \mathrm{s}\right)$} \\
\hline & & \multicolumn{8}{|c|}{ Number of consecutive days } \\
\hline & & 1 & 7 & 14 & 30 & 1 & 7 & 14 & 30 \\
\hline & & \multicolumn{4}{|c|}{ December-January-February } & \multicolumn{4}{|c|}{ March-April-May } \\
\hline 0.05 & 20 & $\mathrm{ng}$ & $\mathrm{ng}$ & ng & ng & ng & 0 & 0 & 0 \\
\hline 0.10 & 10 & ng & ng & ng & ng & $\mathrm{ng}$ & 0 & 0 & 0 \\
\hline 0.20 & 5 & ng & $\mathrm{ng}$ & $\mathrm{ng}$ & $\mathrm{ng}$ & ng & 0 & 0 & 0 \\
\hline \multirow[t]{2}{*}{0.50} & 2 & ng & $\mathrm{ng}$ & $\mathrm{ng}$ & ng & ng & 0 & 0 & 3.84 \\
\hline & & \multicolumn{4}{|c|}{ June-July-August } & \multicolumn{4}{|c|}{ September-October-November } \\
\hline 0.05 & 20 & 0 & 0 & 0.015 & 0.370 & 0 & 0 & 0 & 0 \\
\hline 0.10 & 10 & 0 & 0 & 0.040 & 0.522 & 0 & 0 & 0 & 0 \\
\hline 0.20 & 5 & 0 & 0.086 & 0.123 & 0.809 & 0 & 0 & 0 & 0.040 \\
\hline 0.50 & 2 & 0.392 & 0.644 & 0.820 & 1.99 & 0 & 0.089 & 0.245 & 0.759 \\
\hline
\end{tabular}




\section{MUSTINKA DITCH ABOVE WEST BRANCH MUSTINKA RIVER NEAR CHARLESVILLE, MN--Continued}

Annual peak discharge and corresponding gage height, period of record

$$
[--, \text { no data }]
$$

\begin{tabular}{|c|c|c|c|c|c|c|c|}
\hline $\begin{array}{l}\text { Water } \\
\text { year }\end{array}$ & Date & $\begin{array}{c}\text { Gage } \\
\text { height } \\
\text { (foet) }\end{array}$ & $\begin{array}{c}\text { Peak } \\
\text { discharge } \\
\left(\mathrm{ft}^{3} / \mathrm{s}\right)\end{array}$ & $\begin{array}{l}\text { Water } \\
\text { year }\end{array}$ & Date & $\begin{array}{c}\text { Gage } \\
\text { height } \\
\text { (foet) }\end{array}$ & $\begin{array}{c}\text { Peak } \\
\text { discharge } \\
\left(\mathrm{ft}^{3} / \mathrm{s}\right)\end{array}$ \\
\hline \multicolumn{8}{|c|}{ Annual peak discharge, by year, and corresponding gage height } \\
\hline 1944 & June 5 & 9.50 & 186 & 1950 & May 9 & - & 440 \\
\hline 1945 & March 16 & 8.32 & 186 & 1951 & April 7 & .- & 534 \\
\hline 1946 & $\operatorname{March} 20$ & - & 310 & 1952 & April 9 & 14.19 & 1,550 \\
\hline 1947 & April 11 & 12.35 & 431 & 1953 & June 27 & - & 77.0 \\
\hline 1948 & April 3 & - & 132 & 1954 & June 8 & 8.84 & 280 \\
\hline 1949 & July 10 & 10.75 & 422 & 1955 & July 13 & 2.17 & 162 \\
\hline \multicolumn{8}{|c|}{ Annual peak discharge, from highest to lowest, and corresponding gage height } \\
\hline 1952 & April 9 & 14.19 & 1,550 & 1954 & June 8 & 8.84 & 280 \\
\hline 1951 & April 7 & - & 534 & 1944 & June 5 & 9.50 & 186 \\
\hline 1950 & May 9 & - & 440 & 1945 & March 16 & 8.32 & 186 \\
\hline 1947 & April 11 & 12.35 & 431 & 1955 & July 13 & 2.17 & 162 \\
\hline 1949 & July 10 & 10.75 & 422 & 1948 & April 3 & - & 132 \\
\hline 1946 & March 20 & - & 310 & 1953 & June 27 & - & 77.0 \\
\hline
\end{tabular}




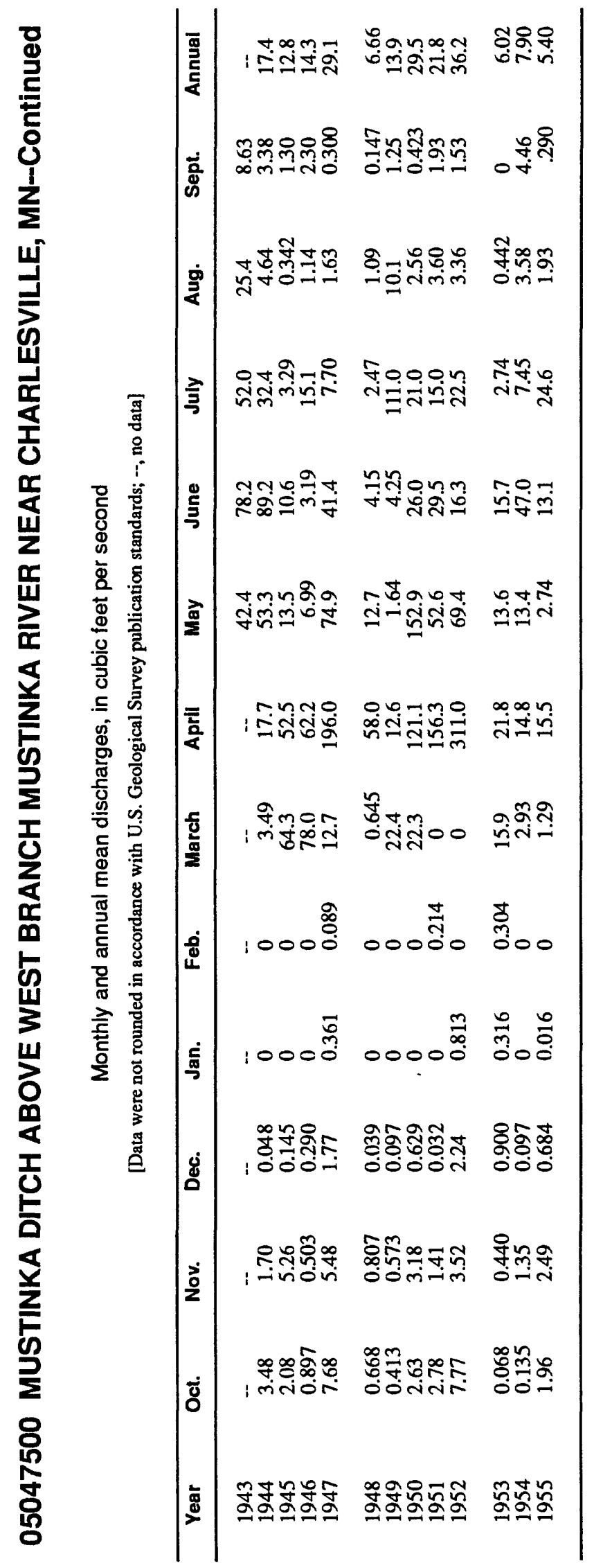




\title{
05048000 MUSTINKA DITCH BELOW WEST BRANCH MUSTINKA RIVER NEAR CHARLESVILLE, MN
}

\author{
Station Description
}

LOCATION.--Lat 45 53'20", long 96 $21^{\prime} 45^{\prime \prime}$, in SE $1 / 4 \mathrm{SE}^{1} / 4$ sec.18, T.128 N., R.45 W., Traverse County, Hydrologic Unit 09020102, on right bank $100 \mathrm{ft}$ downstream from inlet structure to West Branch Mustinka River, $6 \mathrm{mi}$ southwest of Charlesville, and $7.7 \mathrm{mi}$ northeast of Wheaton.

DRAINAGE AREA.--Not determined.

PERIOD OF RECORD.--April 1943 to September 1955. Prior to October 1949, published as "below Twelve Mile Creek near Charlesville".

GAGE.--Staff gage. Datum of gage is $987.00 \mathrm{ft}$ above sea level, adjustment of 1912. Prior to May 11, 1948, reference mark and May 11, 1948, to July 10, 1955, staff gage, at site on West Branch Mustinka River $40 \mathrm{ft}$ downstream from inlet to Mustinka ditch, at datum $3.00 \mathrm{ft}$ higher.

EXTREMES FOR PERIOD OF RECORD.--Maximum discharge, $997 \mathrm{ft}^{3} / \mathrm{s}$, Apr. 9, 1952; maximum gage height, $13.73 \mathrm{ft}$, Apr. 2, 1952 (from floodmark); no flow for several months each year.

Annual mean discharge

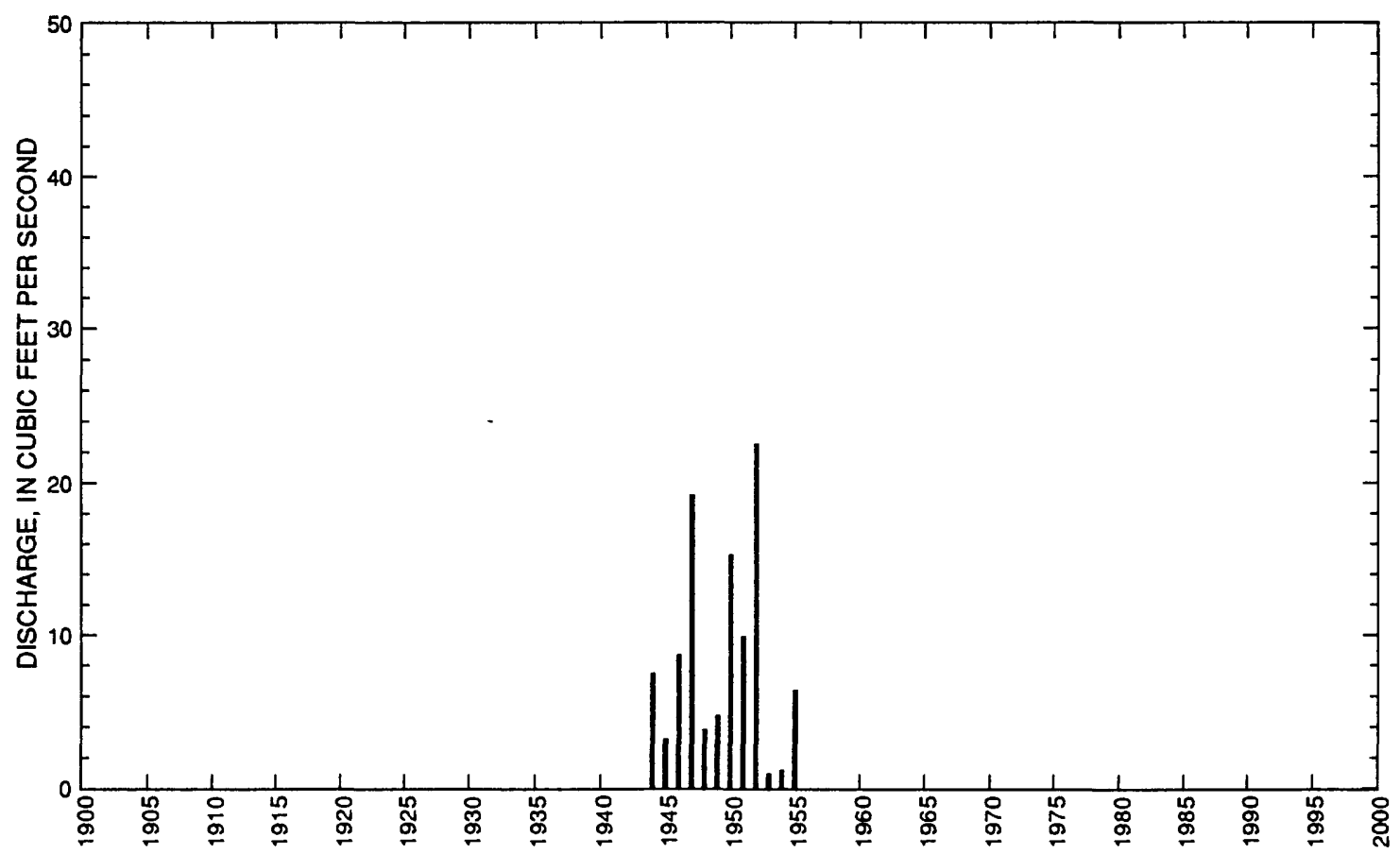




\section{MUSTINKA DITCH BELOW WEST BRANCH MUSTINKA RIVER NEAR CHARLESVILLE, MN-Continued}

Statistics of monthly and annual mean discharges

[m, more than 1 year of occurrence; ng, statistic not given]

\begin{tabular}{|c|c|c|c|c|c|c|c|c|}
\hline \multirow[b]{2}{*}{ Month } & \multicolumn{2}{|c|}{ Maximum } & \multicolumn{2}{|c|}{ Minimum } & \multicolumn{4}{|c|}{ Mean } \\
\hline & $\begin{array}{c}\text { Discharge } \\
\left(\mathrm{ft}^{3} / \mathrm{s}\right)\end{array}$ & $\begin{array}{l}\text { Water year } \\
\text { of } \\
\text { occurrence }\end{array}$ & $\begin{array}{c}\text { Discharge } \\
\left(\mathrm{ft}^{3} / \mathbf{s}\right)\end{array}$ & $\begin{array}{c}\text { Water year } \\
\text { of } \\
\text { occurrence }\end{array}$ & $\begin{array}{c}\text { Discharge } \\
\left(\mathrm{ft}^{3} / \mathrm{s}\right)\end{array}$ & $\begin{array}{c}\text { Standard } \\
\text { deviation } \\
\left(\mathrm{ft}^{3} / \mathrm{s}\right)\end{array}$ & $\begin{array}{l}\text { Coeffi- } \\
\text { clent of } \\
\text { variation }\end{array}$ & $\begin{array}{l}\text { Percentage } \\
\text { of annual } \\
\text { discharge }\end{array}$ \\
\hline October & 0.032 & 1947 & 0 & $\mathrm{~m}$ & 0 & 0.01 & 3.46 & 0 \\
\hline November & 0.007 & 1944 & 0 & $\mathrm{~m}$ & 0 & 0 & 3.46 & 0 \\
\hline December & 0 & $\mathrm{~m}$ & 0 & $\mathrm{~m}$ & 0 & 0 & $\mathrm{ng}$ & 0 \\
\hline January & 0 & $\mathrm{~m}$ & 0 & $\mathrm{~m}$ & 0 & 0 & $\mathrm{ng}$ & 0 \\
\hline February & 0 & $\mathrm{~m}$ & 0 & $\mathrm{~m}$ & 0 & 0 & $\mathrm{ng}$ & 0 \\
\hline March & 82.6 & 1946 & 0 & $\mathrm{~m}$ & 13.8 & 23.7 & 1.72 & 12.9 \\
\hline April & 252 & 1952 & 0.103 & 1953 & 55.9 & 82.8 & 1.48 & 52.4 \\
\hline May & 74.4 & 1950 & 0 & 1949 & 11.9 & 20.5 & 1.73 & 11.2 \\
\hline June & 66.7 & 1944 & 0 & $\mathrm{~m}$ & 13.6 & 20.9 & 1.53 & 12.8 \\
\hline July & 49.1 & 1955 & 0 & $\mathrm{~m}$ & 11.0 & 17.5 & 1.59 & 10.3 \\
\hline August & 3.88 & 1955 & 0 & $\mathrm{~m}$ & 0.420 & 1.12 & 2.70 & 0.39 \\
\hline September & 0.367 & 1955 & 0 & $\mathrm{~m}$ & 0.030 & 0.10 & 3.35 & 0.03 \\
\hline Annual & 22.5 & 1952 & 0.918 & 1953 & 8.60 & 7.01 & 0.81 & 100 \\
\hline
\end{tabular}

Annual flow duration

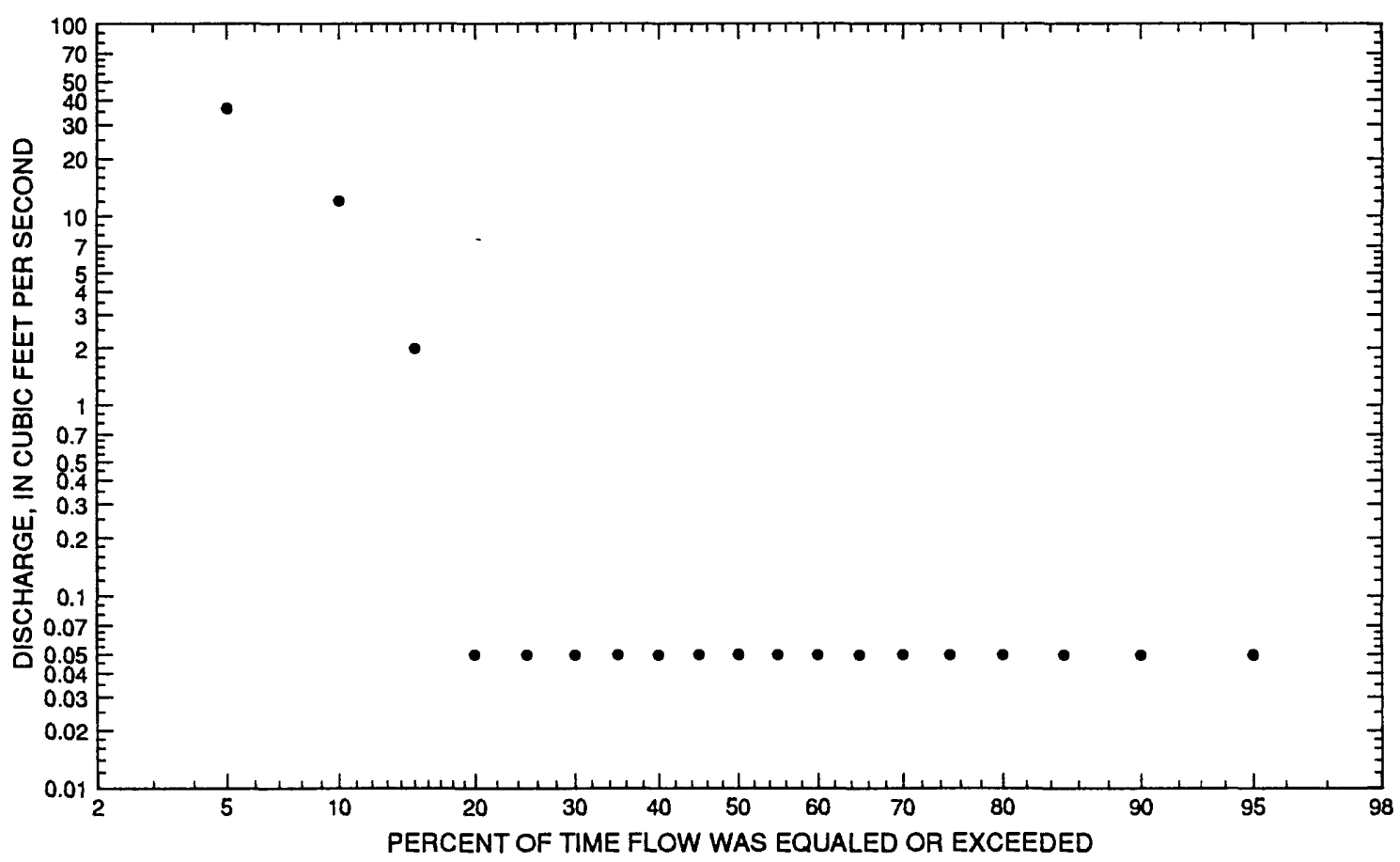




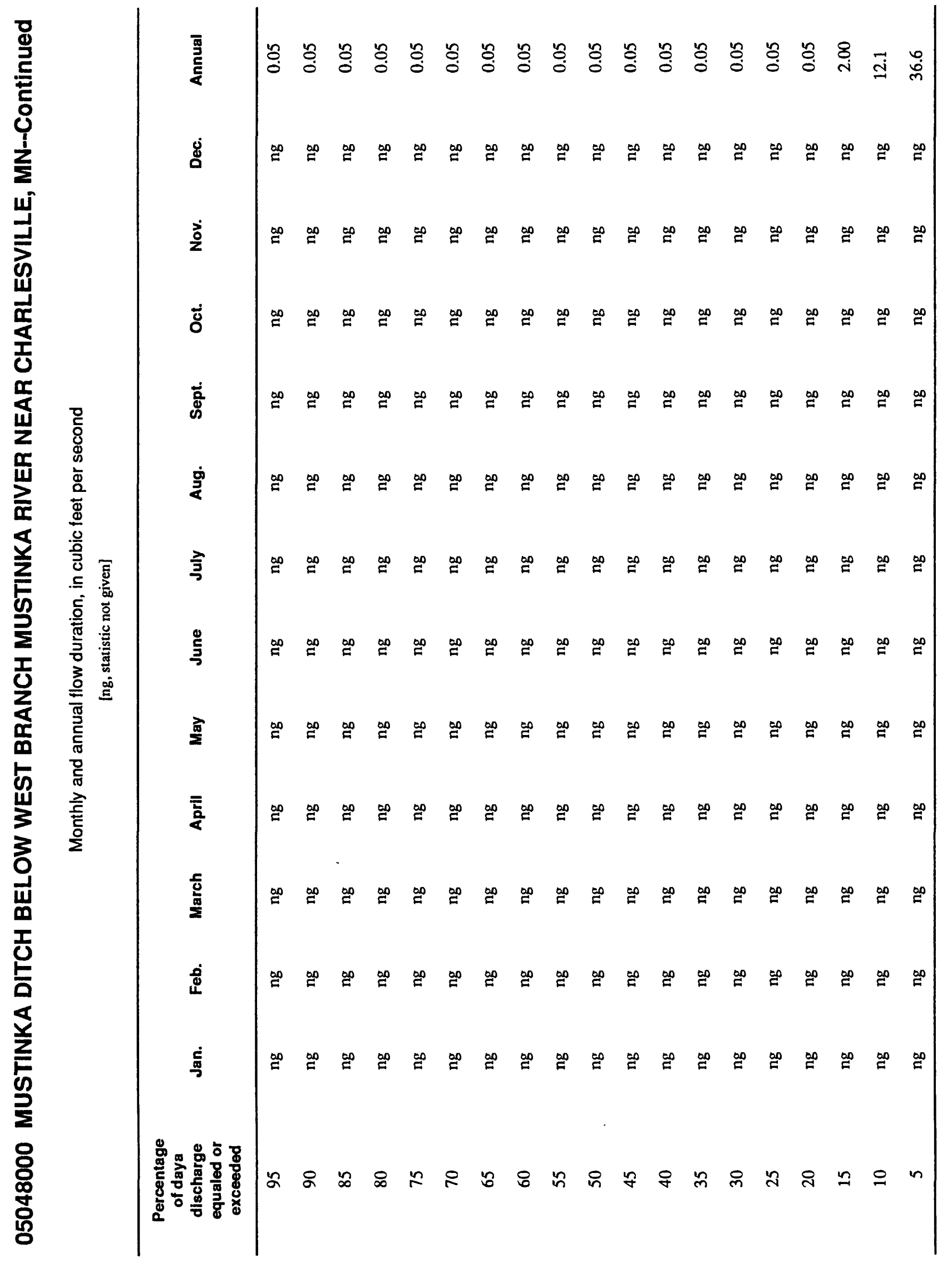




\section{MUSTINKA DITCH BELOW WEST BRANCH MUSTINKA RIVER NEAR CHARLESVILLE, MN--Continued}

Probability of annual high discharges

[ng, statistic not given]

\begin{tabular}{|c|c|c|c|c|c|c|}
\hline \multirow[b]{2}{*}{$\begin{array}{l}\text { Excoedance } \\
\text { probability }\end{array}$} & \multirow[b]{2}{*}{$\begin{array}{l}\text { Recurrence } \\
\text { interval } \\
\text { (years) }\end{array}$} & \multirow[b]{2}{*}{$\begin{array}{c}\text { Maximum } \\
\text { instantaneous } \\
\left(\mathrm{ft}^{3} / \mathbf{s}\right)\end{array}$} & \multicolumn{4}{|c|}{$\begin{array}{l}\text { Maximum average discharge } \\
\qquad\left(\mathrm{ft}^{3} / \mathrm{s}\right)\end{array}$} \\
\hline & & & 3-day period & 7-day period & 15-day period & 30-day period \\
\hline 0.99 & 1.01 & ng & 19.9 & 11.9 & 7.49 & 3.18 \\
\hline 0.95 & 1.05 & ng & 48.6 & 29.9 & 17.7 & 8.90 \\
\hline 0.90 & 1.11 & 140 & 74.6 & 46.7 & 27.1 & 14.7 \\
\hline 0.80 & 1.25 & 189 & 120 & 77.1 & 44.2 & 25.6 \\
\hline 0.50 & 2 & 326 & 259 & 179 & 103 & 65.1 \\
\hline 0.20 & 5 & 543 & 478 & 358 & 217 & 140 \\
\hline 0.10 & 10 & 700 & 620 & 487 & 306 & 197 \\
\hline 0.04 & 25 & 908 & 786 & 651 & 430 & 270 \\
\hline 0.02 & 50 & 1,070 & 898 & 770 & 527 & 324 \\
\hline 0.01 & 100 & 1,230 & 998 & 884 & 627 & 376 \\
\hline 0.005 & 200 & 1,400 & 1,090 & 993 & 728 & 426 \\
\hline 0.002 & 500 & 1,630 & $\mathrm{ng}$ & $\mathrm{ng}$ & $\mathrm{ng}$ & $\mathrm{ng}$ \\
\hline
\end{tabular}

Probability of annual low discharges

[ng, statistic not given]

\begin{tabular}{|c|c|c|c|c|c|c|c|c|c|c|}
\hline \multirow{3}{*}{$\begin{array}{l}\text { Non- } \\
\text { excoed- } \\
\text { ance } \\
\text { prob- } \\
\text { ability }\end{array}$} & \multirow{3}{*}{$\begin{array}{c}\text { Recur- } \\
\text { rence } \\
\text { inter- } \\
\text { val } \\
\text { (years) }\end{array}$} & \multicolumn{9}{|c|}{ Minimum average discharge $\left(\mathrm{ft}^{3} / \mathrm{s}\right)$} \\
\hline & & \multicolumn{9}{|c|}{ Number of consecutive days } \\
\hline & & 1 & 3 & 7 & 14 & 30 & 60 & 90 & 120 & 183 \\
\hline 0.05 & 20 & ng & $\mathrm{ng}$ & ng & ng & ng & ng & ng & ng & ng \\
\hline 0.10 & 10 & ng & ng & ng & ng & ng & ng & ng & ng & ng \\
\hline 0.20 & 5 & ng & ng & ng & ng & ng & ng & ng & ng & ng \\
\hline 0.50 & 2 & ng & $\mathrm{ng}$ & ng & ng & ng & ng & ng & ng & ng \\
\hline
\end{tabular}




\section{MUSTINKA DITCH BELOW WEST BRANCH MUSTINKA RIVER NEAR CHARLESVILLE, MN--Continued}

Probability of seasonal low discharges

[ng, statistic not given]

\begin{tabular}{|c|c|c|c|c|c|c|c|c|c|}
\hline \multirow[b]{3}{*}{$\begin{array}{c}\text { Non- } \\
\text { exceedance } \\
\text { probability }\end{array}$} & \multirow[b]{3}{*}{$\begin{array}{c}\text { Recurrence } \\
\text { interval } \\
\text { (years) }\end{array}$} & \multicolumn{8}{|c|}{ Minimum average discharge $\left(\mathrm{ft}^{3} / \mathrm{s}\right)$} \\
\hline & & \multicolumn{8}{|c|}{ Number of consecutive days } \\
\hline & & 1 & 7 & 14 & 30 & 1 & 7 & 14 & 30 \\
\hline & & \multicolumn{4}{|c|}{ December-January-February } & \multicolumn{4}{|c|}{ March-April-May } \\
\hline 0.05 & 20 & ng & ng & ng & ng & ng & ng & $\mathrm{ng}$ & 0 \\
\hline 0.10 & 10 & ng & ng & $\mathrm{ng}$ & ng & ng & ng & $\mathrm{ng}$ & 0 \\
\hline 0.20 & 5 & ng & ng & ng & ng & ng & ng & ng & 0 \\
\hline \multirow[t]{2}{*}{0.50} & 2 & ng & ng & ng & ng & ng & $\mathrm{ng}$ & ng & 0 \\
\hline & & \multicolumn{4}{|c|}{ June-July-August } & \multicolumn{4}{|c|}{ September-October-November } \\
\hline 0.05 & 20 & ng & $\mathrm{ng}$ & ng & ng & ng & $\mathrm{ng}$ & $\mathrm{ng}$ & ng \\
\hline 0.10 & 10 & $\mathrm{ng}$ & ng & ng & ng & $\mathrm{ng}$ & $\mathrm{ng}$ & ng & $\mathrm{ng}$ \\
\hline 0.20 & 5 & ng & ng & ng & $\mathrm{ng}$ & $\mathrm{ng}$ & $\mathrm{ng}$ & $\mathrm{ng}$ & ng \\
\hline 0.50 & 2 & $\mathrm{ng}$ & ng & $\mathrm{ng}$ & $\mathrm{ng}$ & $\mathrm{ng}$ & $\mathrm{ng}$ & ng & $\mathrm{ng}$ \\
\hline
\end{tabular}




\section{MUSTINKA DITCH BELOW WEST BRANCH MUSTINKA RIVER NEAR CHARLESVILLE, MN--Continued}

Annual peak discharge and corresponding gage height, period of record

$$
[\cdots, \text { no data }]
$$

\begin{tabular}{|c|c|c|c|c|c|c|c|}
\hline $\begin{array}{l}\text { Water } \\
\text { year }\end{array}$ & Date & $\begin{array}{c}\text { Gage } \\
\text { height } \\
\text { (feet) }\end{array}$ & $\begin{array}{c}\text { Peak } \\
\text { discharge } \\
\left(\mathrm{ft}^{3} / \mathrm{s}\right)\end{array}$ & $\begin{array}{l}\text { Water } \\
\text { year }\end{array}$ & Date & $\begin{array}{c}\text { Gage } \\
\text { height } \\
\text { (feet) }\end{array}$ & $\begin{array}{c}\text { Peak } \\
\text { discharge } \\
\left(\mathrm{ft}^{3} / \mathrm{s}\right)\end{array}$ \\
\hline \multicolumn{8}{|c|}{ Annual peak discharge, by year, and corresponding gage height } \\
\hline 1944 & June 4 & 8.92 & 318 & 1950 & May 9 & 9.31 & 374 \\
\hline 1945 & March 16 & 6.68 & 165 & 1951 & April 7 & - & 500 \\
\hline 1946 & March 20 & 11.00 & 512 & 1952 & April 9 & 13.73 & 997 \\
\hline 1947 & April 12 & - & 600 & 1953 & June 27 & 4.86 & 47.0 \\
\hline 1948 & April 7 & 6.62 & 132 & 1954 & June 8 & 7.91 & 207 \\
\hline 1949 & July 10 & 9.87 & 292 & 1955 & July 13 & 5.12 & 318 \\
\hline \multicolumn{8}{|c|}{ Annual peak discharge, from highest to lowest, and corresponding gage height } \\
\hline 1952 & April 9 & 13.73 & 997 & 1955 & July 13 & 5.12 & 318 \\
\hline 1947 & April 12 & -- & 600 & 1949 & July 10 & 9.87 & 292 \\
\hline 1946 & March 20 & 11.00 & 512 & 1954 & June 8 & 7.91 & 207 \\
\hline 1951 & April 7 & - & 500 & 1945 & March 16 & 6.68 & 165 \\
\hline 1950 & May 9 & 9.31 & 374 & 1948 & April 7 & 6.62 & 132 \\
\hline 1944 & June 4 & 8.92 & 318 & 1953 & June 27 & 4.86 & 47.0 \\
\hline
\end{tabular}




\title{
05048500 WEST BRANCH MUSTINKA RIVER BELOW MUSTINKA DITCH NEAR CHARLESVILLE, MN
}

\author{
Station Description
}

LOCATION.--Lat $45^{\circ} 53^{\prime} 20^{\prime \prime}$, long $96^{\circ} 21^{\prime} 45^{\prime \prime}$, in SE $1 / 4 \mathrm{SE}^{1 / 4}$ sec.18, T.128 N., R.45 W., Traverse County, Hydrologic Unit 09020102, on left bank $150 \mathrm{ft}$ downstream from inlet from Mustinka ditch, $1.75 \mathrm{mi}$ upstream from mouth, $6 \mathrm{mi}$ southwest of Charlesville, and $7.8 \mathrm{mi}$ northeast of Wheaton.

DRAINAGE AREA.--Not determined.

PERIOD OF RECORD.--April 1943 to September 1955. Monthly discharges only for some periods, published in WSP 1308. Prior to October 1949, published as Twelve Mile Creek below Mustinka ditch, near Charlesville.

GAGE.--Staff gage. Datum of gage is $990.00 \mathrm{ft}$ above mean sea level, adjustment of 1912 (levels by Corps of Enigeers). Prior to May 11, 1948, reference mark and May 11, 1948, to July 10, 1955, staff gage, at site $110 \mathrm{ft}$ upstream at same datum.

EXTREMES FOR PERIOD OF RECORD.--Maximum discharge, 13,700 $\mathrm{ft}^{3} / \mathrm{s}$, Apr. 9, 1952 (gage height, $13.73 \mathrm{ft}$, from floodmarks), from rating curve extended above $1,200 \mathrm{ft}^{3} / \mathrm{s}$ on basis of slope-area measurement of peak flow; no flow most winters.

Annual mean discharge

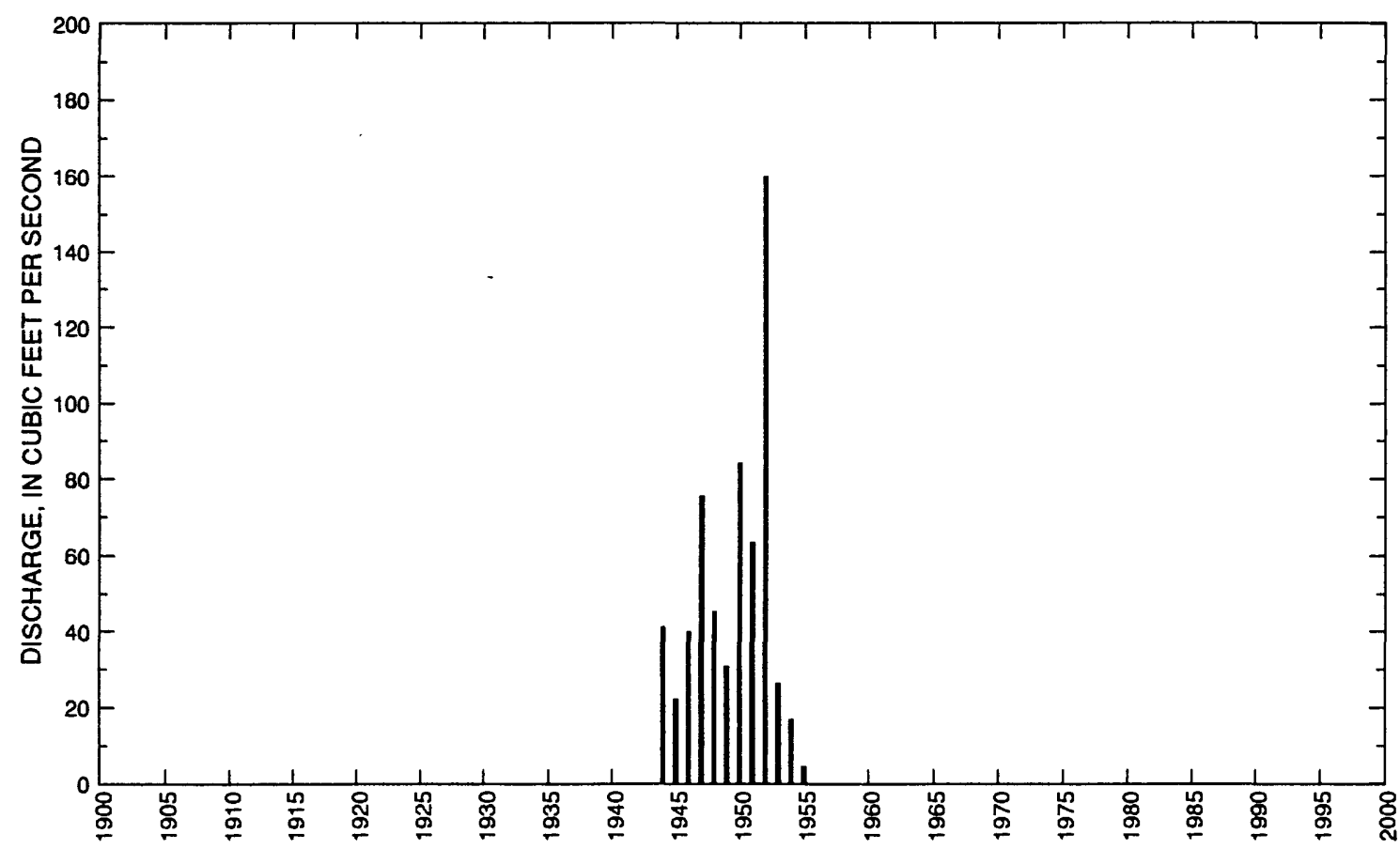




\section{WEST BRANCH MUSTINKA RIVER BELOW MUSTINKA DITCH NEAR CHARLESVILLE, MN--Continued}

Statistics of monthly and annual mean discharges

[m, more than 1 year of occurrence]

\begin{tabular}{|c|c|c|c|c|c|c|c|c|}
\hline \multirow[b]{2}{*}{ Month } & \multicolumn{2}{|c|}{ Maximum } & \multicolumn{2}{|c|}{ Minimum } & \multicolumn{4}{|c|}{ Mean } \\
\hline & $\begin{array}{c}\text { DIscharge } \\
\left(\mathrm{ft}^{3} / \mathbf{s}\right)\end{array}$ & $\begin{array}{c}\text { Water year } \\
\text { of } \\
\text { occurrence }\end{array}$ & $\begin{array}{c}\text { Dlscharge } \\
\left(\mathrm{ft}^{3} / \mathrm{s}\right)\end{array}$ & $\begin{array}{c}\text { Water year } \\
\text { of } \\
\text { occurrence }\end{array}$ & $\begin{array}{c}\text { Discharge } \\
\left(\mathrm{ft}^{3} / \mathrm{s}\right)\end{array}$ & $\begin{array}{c}\text { Standard } \\
\text { deviatlon } \\
\left(\mathrm{ft}^{3} / \mathrm{s}\right)\end{array}$ & $\begin{array}{l}\text { Coeffi- } \\
\text { cient of } \\
\text { variation }\end{array}$ & $\begin{array}{l}\text { Percentage } \\
\text { of annual } \\
\text { discharge }\end{array}$ \\
\hline October & 12.8 & 1947 & 0.200 & 1954 & 3.60 & 3.69 & 1.02 & 0.57 \\
\hline November & 9.00 & 1947 & 0.487 & 1946 & 2.91 & 2.56 & 0.88 & 0.46 \\
\hline December & 3.71 & 1952 & 0.035 & 1951 & 0.92 & 1.12 & 1.22 & 0.15 \\
\hline January & 1.52 & 1947 & 0 & $\mathrm{~m}$ & 0.29 & 0.53 & 1.84 & 0.05 \\
\hline February & 0.821 & 1953 & 0 & $\mathrm{~m}$ & 0.13 & 0.31 & 2.34 & 0.02 \\
\hline March & 284 & 1946 & 0 & 1952 & 80.0 & 89.5 & 1.12 & 12.7 \\
\hline April & 1,580 & 1952 & 27.8 & 1949 & 307 & 447 & 1.46 & 48.9 \\
\hline May & 320 & 1950 & 1.99 & 1949 & 86.0 & 91.9 & 1.07 & 13.7 \\
\hline June & 277 & 1944 & 0 & 1955 & 77.5 & 83.3 & 1.07 & 12.3 \\
\hline July & 272 & 1949 & 5.46 & 1955 & 59.2 & 74.2 & 1.25 & 9.42 \\
\hline August & 39.2 & 1943 & 0 & 1955 & 7.87 & 10.6 & 1.35 & 1.25 \\
\hline September & 16.1 & 1943 & 0 & $\mathrm{~m}$ & 3.05 & 4.40 & 1.44 & 0.49 \\
\hline Annual & 160 & 1952 & 4.39 & 1955 & 50.8 & 41.7 & 0.82 & 100 \\
\hline
\end{tabular}

Annual flow duration

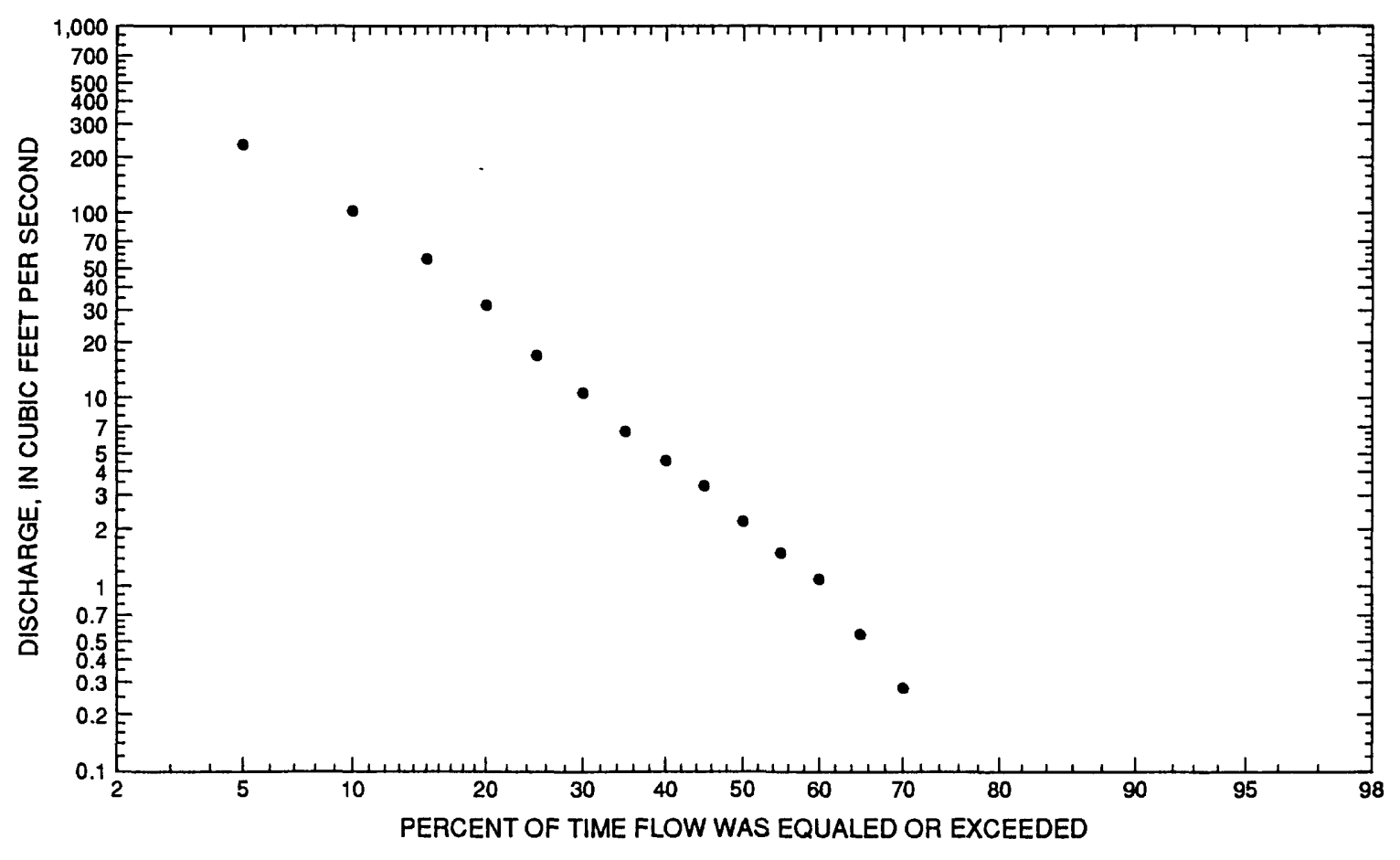




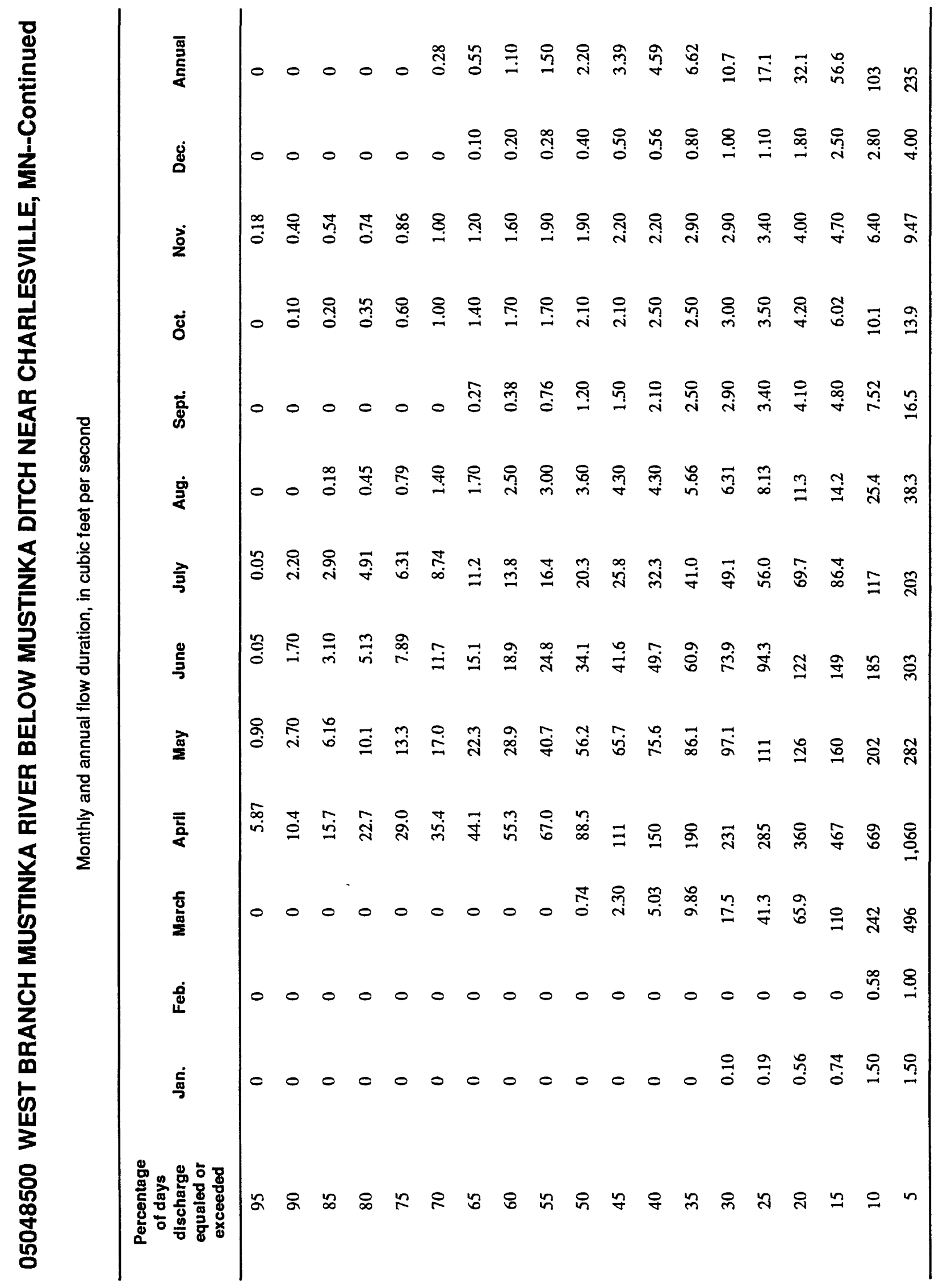




\section{WEST BRANCH MUSTINKA RIVER BELOW MUSTINKA DITCH NEAR CHARLESVILLE, MN--Continued}

Probability of annual high discharges

[ng, statistic not given]

\begin{tabular}{|c|c|c|c|c|c|c|}
\hline \multirow[b]{2}{*}{$\begin{array}{l}\text { Exceedance } \\
\text { probability }\end{array}$} & \multirow[b]{2}{*}{$\begin{array}{c}\text { Recurrence } \\
\text { Interval } \\
\text { (years) }\end{array}$} & \multirow[b]{2}{*}{$\begin{array}{c}\text { Maximum } \\
\text { instantaneous } \\
\left(\mathrm{ft}^{3} / \mathrm{s}\right)\end{array}$} & \multicolumn{4}{|c|}{$\begin{array}{l}\text { Maximum average discharge } \\
\left(\mathrm{ft}^{3} / \mathrm{s}\right)\end{array}$} \\
\hline & & & 3-day period & 7-day period & 15-day period & 30-day period \\
\hline 0.99 & 1.01 & 104 & 123 & 68.0 & 35.8 & 19.8 \\
\hline 0.95 & 1.05 & 213 & 205 & 136 & 80.4 & 49.5 \\
\hline 0.90 & 1.11 & 312 & 276 & 196 & 121 & 77.8 \\
\hline 0.80 & 1.25 & 496 & 403 & 304 & 195 & 130 \\
\hline 0.50 & 2 & 1,210 & 882 & 695 & 458 & 312 \\
\hline 0.20 & 5 & 2,940 & 2,100 & 1,560 & 998 & 661 \\
\hline 0.10 & 10 & 4,680 & 3,420 & 2,370 & 1.460 & 933 \\
\hline 0.04 & 25 & 7,690 & 5,920 & 3,690 & 2,130 & 1,300 \\
\hline 0.02 & 50 & 10,600 & 8,550 & 4,880 & 2,700 & 1,590 \\
\hline 0.01 & 100 & 14,200 & 12,000 & 6,280 & 3,320 & 1,870 \\
\hline 0.005 & 200 & 18,400 & 16,600 & 7,900 & 3,970 & 2,160 \\
\hline 0.002 & 500 & 25,400 & $\mathrm{ng}$ & $\mathrm{ng}$ & $\mathrm{ng}$ & $\mathrm{ng}$ \\
\hline
\end{tabular}

Probability of annual low discharges

[ng, statistic not given]

\begin{tabular}{|c|c|c|c|c|c|c|c|c|c|c|}
\hline \multirow{3}{*}{$\begin{array}{l}\text { Non- } \\
\text { exceed- } \\
\text { ance } \\
\text { prob- } \\
\text { abllity }\end{array}$} & \multirow{3}{*}{$\begin{array}{c}\text { Recur- } \\
\text { rence } \\
\text { Inter- } \\
\text { val } \\
\text { (years) }\end{array}$} & \multicolumn{9}{|c|}{ Minimum average discharge $\left(\mathrm{ft}^{3} / \mathrm{s}\right)$} \\
\hline & & \multicolumn{9}{|c|}{ Number of consecutive days } \\
\hline & & 1 & 3 & 7 & 14 & 30 & 60 & 90 & 120 & 183 \\
\hline 0.05 & 20 & $\mathrm{ng}$ & $\mathrm{ng}$ & $\mathrm{ng}$ & ng & ng & ng & 0 & 0.043 & 0.204 \\
\hline 0.10 & 10 & $\mathrm{ng}$ & ng & ng & ng & $\mathrm{ng}$ & ng & 0 & 0.077 & 0.296 \\
\hline 0.20 & 5 & ng & ng & $\mathrm{ng}$ & ng & ng & $\mathrm{ng}$ & 0 & 0.148 & 0.459 \\
\hline 0.50 & 2 & $\mathrm{ng}$ & $\mathrm{ng}$ & $\mathrm{ng}$ & ng & $\mathrm{ng}$ & ng & 0.019 & 0.445 & 1.05 \\
\hline
\end{tabular}




\section{WEST BRANCH MUSTINKA RIVER BELOW MUSTINKA DITCH NEAR CHARLESVILLE, MN--Continued}

Probability of seasonal low discharges

[ng, statistic not given]

\begin{tabular}{|c|c|c|c|c|c|c|c|c|c|}
\hline \multirow[b]{3}{*}{$\begin{array}{l}\text { Non- } \\
\text { exceedance } \\
\text { probability }\end{array}$} & \multirow[b]{3}{*}{$\begin{array}{c}\text { Recurrence } \\
\text { Interval } \\
\text { (years) }\end{array}$} & \multicolumn{8}{|c|}{ Minimum average discharge $\left(\mathrm{ft}^{3} / \mathrm{s}\right)$} \\
\hline & & \multicolumn{8}{|c|}{ Number of consecutive days } \\
\hline & & 1 & 7 & 14 & 30 & 1 & 7 & 14 & 30 \\
\hline & & \multicolumn{4}{|c|}{ December-January-February } & \multicolumn{4}{|c|}{ March-April-May } \\
\hline 0.05 & 20 & ng & $\mathrm{ng}$ & ng & $\mathrm{ng}$ & ng & 0 & 0 & 0 \\
\hline 0.10 & 10 & ng & $\mathrm{ng}$ & $\mathrm{ng}$ & ng & ng & 0 & 0 & 0.108 \\
\hline 0.20 & 5 & ng & $\mathrm{ng}$ & $\mathrm{ng}$ & $\mathrm{ng}$ & $\mathrm{ng}$ & 0 & 0 & 1.45 \\
\hline \multirow[t]{2}{*}{0.50} & 2 & ng & ng & $\mathrm{ng}$ & ng & ng & 0 & 0 & 12.9 \\
\hline & & \multicolumn{4}{|c|}{ June-July-August } & \multicolumn{4}{|c|}{ September-October-November } \\
\hline 0.05 & 20 & 0 & 0 & 0 & 0 & 0 & 0 & 0 & 0 \\
\hline 0.10 & 10 & 0 & 0 & 0 & 0.422 & 0 & 0 & 0 & 0.009 \\
\hline 0.20 & 5 & 0 & 0 & 0.165 & 1.08 & 0 & 0 & 0 & 0.115 \\
\hline 0.50 & 2 & 0.947 & 1.31 & 1.74 & 3.56 & 0 & 0.142 & 0.318 & 0.860 \\
\hline
\end{tabular}




\section{WEST BRANCH MUSTINKA RIVER BELOW MUSTINKA DITCH NEAR CHARLESVILLE, MN--Continued}

Annual peak discharge and corresponding gage height, period of record

$$
[--, \text { no data }]
$$

\begin{tabular}{|c|c|c|c|c|c|c|c|}
\hline $\begin{array}{l}\text { Water } \\
\text { year }\end{array}$ & Date & $\begin{array}{c}\text { Gage } \\
\text { helght } \\
\text { (feet) }\end{array}$ & $\begin{array}{c}\text { Peak } \\
\text { discharge } \\
\left(\mathrm{ft}^{3} / \mathrm{s}\right)\end{array}$ & $\begin{array}{l}\text { Water } \\
\text { year }\end{array}$ & Date & $\begin{array}{c}\text { Gage } \\
\text { height } \\
\text { (feet) }\end{array}$ & $\begin{array}{c}\text { Peak } \\
\text { discharge } \\
\left(\mathrm{ft}^{3} / \mathrm{s}\right)\end{array}$ \\
\hline \multicolumn{8}{|c|}{ Annual peak discharge, by year, and corresponding gage height } \\
\hline 1944 & June 4 & 8.92 & 1,030 & 1950 & March 28 & 10.51 & 1,140 \\
\hline 1945 & March 15 & - & 490 & 1951 & April 7 & - & 2,800 \\
\hline 1946 & March 20 & - & 1,580 & 1952 & April 9 & 13.73 & 13,700 \\
\hline 1947 & April 12 & 10.65 & 1,720 & 1953 & March 23 & 7.10 & 532 \\
\hline 1948 & March 30 & 10.95 & 1,670 & 1954 & June 8 & 7.91 & 794 \\
\hline 1949 & July 10 & 9.87 & 1,280 & 1955 & April 1 & - & 178 \\
\hline \multicolumn{8}{|c|}{ Annual peak discharge, from highest to lowest, and corresponding gage height } \\
\hline 1952 & April 9 & 13.73 & 13,700 & 1950 & March 28 & 10.51 & 1,140 \\
\hline 1951 & April 7 & - & 2,800 & 1944 & June 4 & 8.92 & 1,030 \\
\hline 1947 & April 12 & 10.65 & 1,720 & 1954 & June 8 & 7.91 & 794 \\
\hline 1948 & March 30 & 10.95 & 1,670 & 1953 & March 23 & 7.10 & 532 \\
\hline 1946 & March 20 & - & 1,580 & 1945 & March 15 & - & 490 \\
\hline 1949 & July 10 & 9.87 & 1,280 & 1955 & April 1 & - & 178 \\
\hline
\end{tabular}




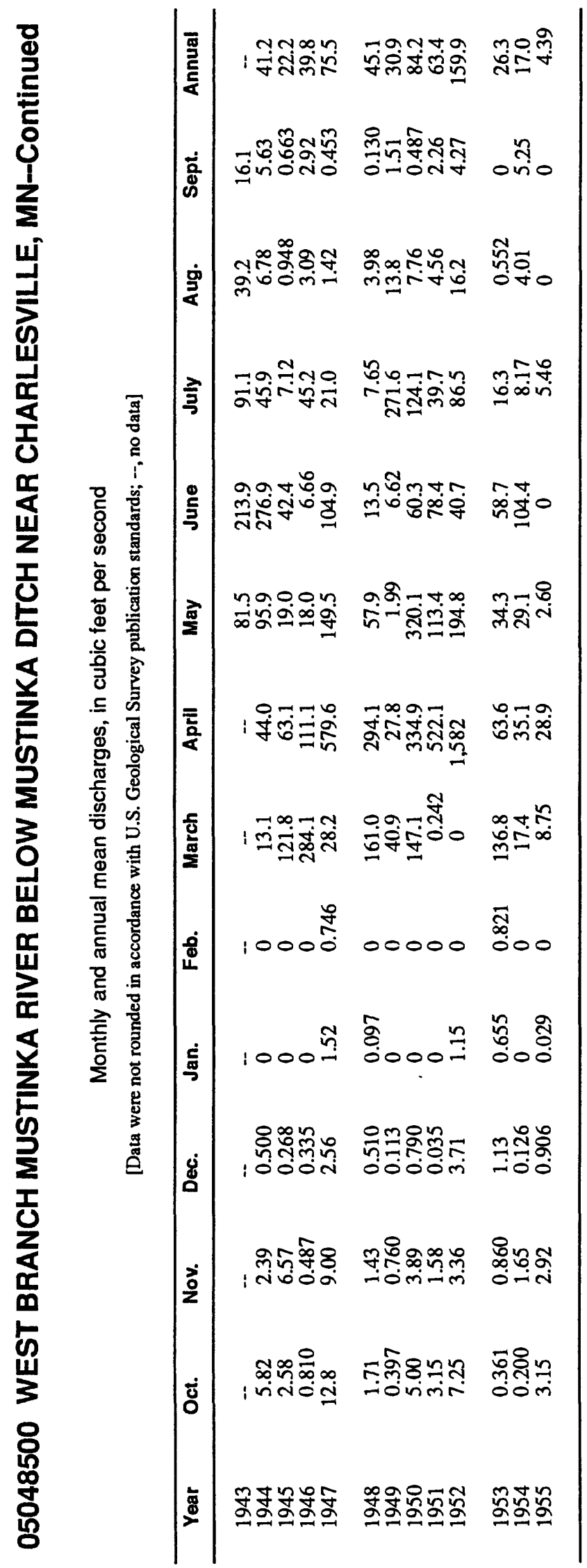




\section{MUSTINKA RIVER ABOVE WHEATON, MN}

\section{Station Description}

LOCATION.--Lat 45'49'15", long 96'29'25', in SW $1 / 4$ sec.8, T.127 N., R.46 W., Traverse County, Hydrologic Unit 09020102, at bridge on U.S. Highway 75, 1 mi upstream from Chicago, Milwaukee and St. Paul railroad bridge, $0.5 \mathrm{mi}$ north of Wheaton, about $8 \mathrm{mi}$ above Lake Traverse.

DRAINAGE AREA.--834 $\mathrm{mi}^{2}$.

PERIOD OF RECORD.--October 1915 to September 1924, October 1930 to September 1958. Monthly discharge only for some periods, published in Water-Supply Paper 1308. Peaks only for 1985 to current year.

GAGE.--History of gage published in Water-Supply Paper 1728.

EXTREMES FOR PERIOD OF RECORD.--Maximum discharge, 7,320 $\mathrm{ft}^{3} / \mathrm{s}$, Apr. 10, 1952 (gage height, $16.56 \mathrm{ft}$ ); maximum gage height, $20.01 \mathrm{ft}$, Mar. 29, 1989; no flow at times in most years.

Annual mean discharge

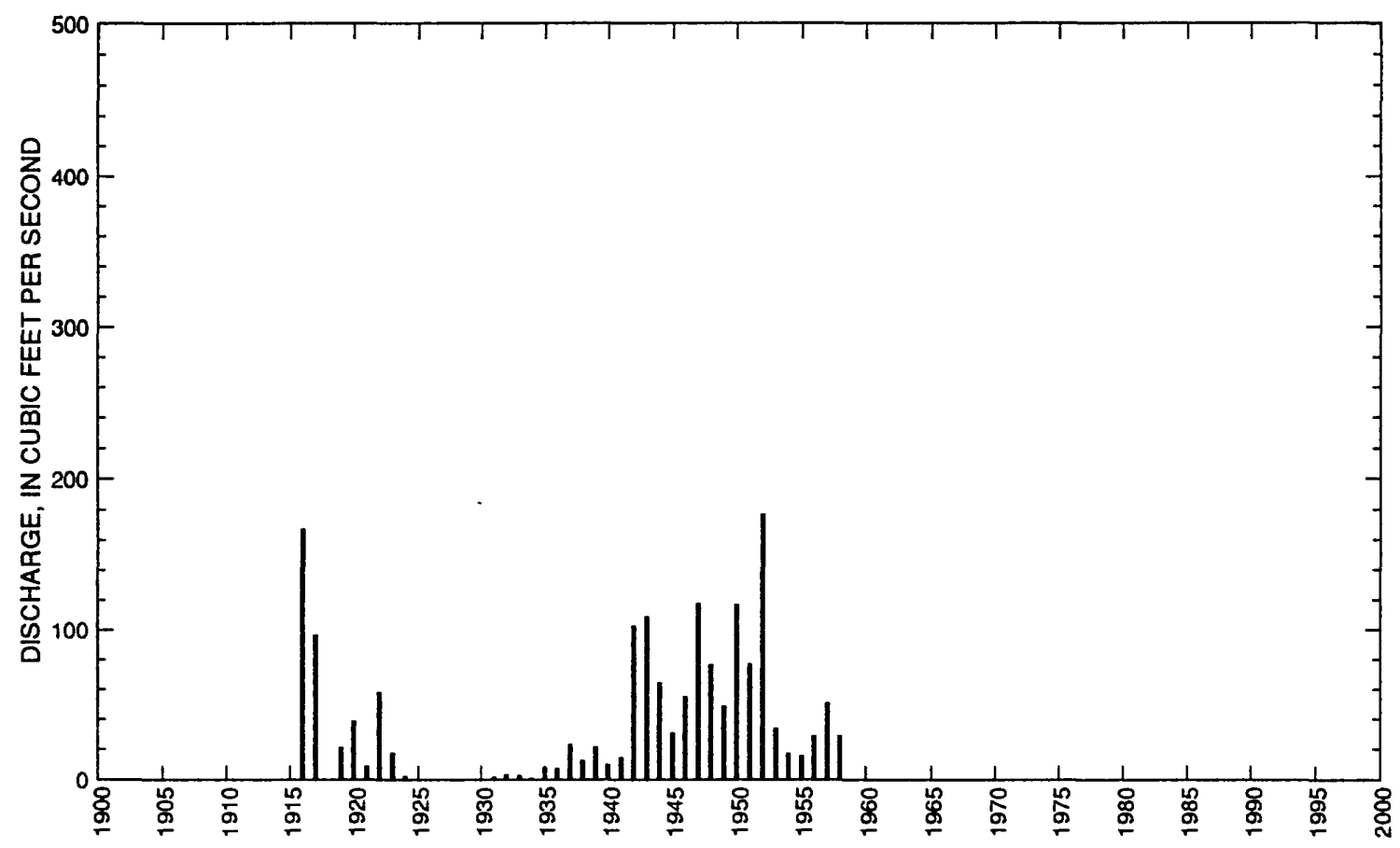




\section{MUSTINKA RIVER ABOVE WHEATON, MN-Continued}

Statistics of monthly and annual mean discharges

[ $\mathrm{m}$, more than 1 year of occurrence]

\begin{tabular}{|c|c|c|c|c|c|c|c|c|}
\hline \multirow[b]{2}{*}{ Month } & \multicolumn{2}{|c|}{ Maximum } & \multicolumn{2}{|c|}{ Minimum } & \multicolumn{4}{|c|}{ Mean } \\
\hline & $\begin{array}{c}\text { Discharge } \\
\left(\mathrm{ft}^{3} / \mathrm{s}\right)\end{array}$ & $\begin{array}{c}\text { Water year } \\
\text { of } \\
\text { occurrence }\end{array}$ & $\begin{array}{c}\text { Dlscharge } \\
\left(\mathrm{ft}^{3} / \mathbf{s}\right)\end{array}$ & $\begin{array}{c}\text { Water year } \\
\text { of } \\
\text { occurrence }\end{array}$ & $\begin{array}{c}\text { Discharge } \\
\left(\mathrm{ft}^{3} / \mathrm{s}\right)\end{array}$ & $\begin{array}{c}\text { Standard } \\
\text { devlatlon } \\
\left(\mathrm{ft}^{3} / \mathrm{s}\right)\end{array}$ & $\begin{array}{l}\text { Coeffi- } \\
\text { clent of } \\
\text { variation }\end{array}$ & $\begin{array}{l}\text { Percentage } \\
\text { of annual } \\
\text { discharge }\end{array}$ \\
\hline October & 30.9 & 1917 & 0 & $\mathrm{~m}$ & 3.29 & 6.58 & 2.00 & 0.60 \\
\hline November & 41.3 & 1958 & 0 & $\mathrm{~m}$ & 3.82 & 8.15 & 2.13 & 0.69 \\
\hline December & 4.47 & 1958 & 0 & $\mathrm{~m}$ & 0.94 & 1.26 & 1.34 & 0.17 \\
\hline January & 3.80 & 1917 & 0 & $\mathrm{~m}$ & 0.39 & 0.83 & 2.14 & 0.07 \\
\hline February & 11.0 & 1958 & 0 & $\mathrm{~m}$ & 0.63 & 1.87 & 2.94 & 0.12 \\
\hline March & 435 & 1946 & 0.039 & 1934 & 76.6 & 110 & 1.43 & 13.9 \\
\hline April & 1,770 & 1952 & 1.33 & 1931 & 237 & 369 & 1.56 & 43.0 \\
\hline May & 571 & 1942 & 0.129 & 1934 & 74.6 & 124 & 1.67 & 13.5 \\
\hline June & 524 & 1942 & 0.090 & 1934 & 74.7 & 127 & 1.69 & 13.6 \\
\hline July & 821 & 1916 & 0 & $\mathrm{~m}$ & 63.3 & 153 & 2.42 & 11.5 \\
\hline August & 108 & 1916 & 0 & $\mathrm{~m}$ & 10.1 & 22.2 & 2.19 & 1.84 \\
\hline September & 44.1 & 1916 & 0 & $\mathrm{~m}$ & 5.83 & 11.2 & 1.92 & 1.06 \\
\hline Annual & 176 & 1952 & 0.192 & 1934 & 45.9 & 46.9 & 1.02 & 100 \\
\hline
\end{tabular}

Annual flow duration

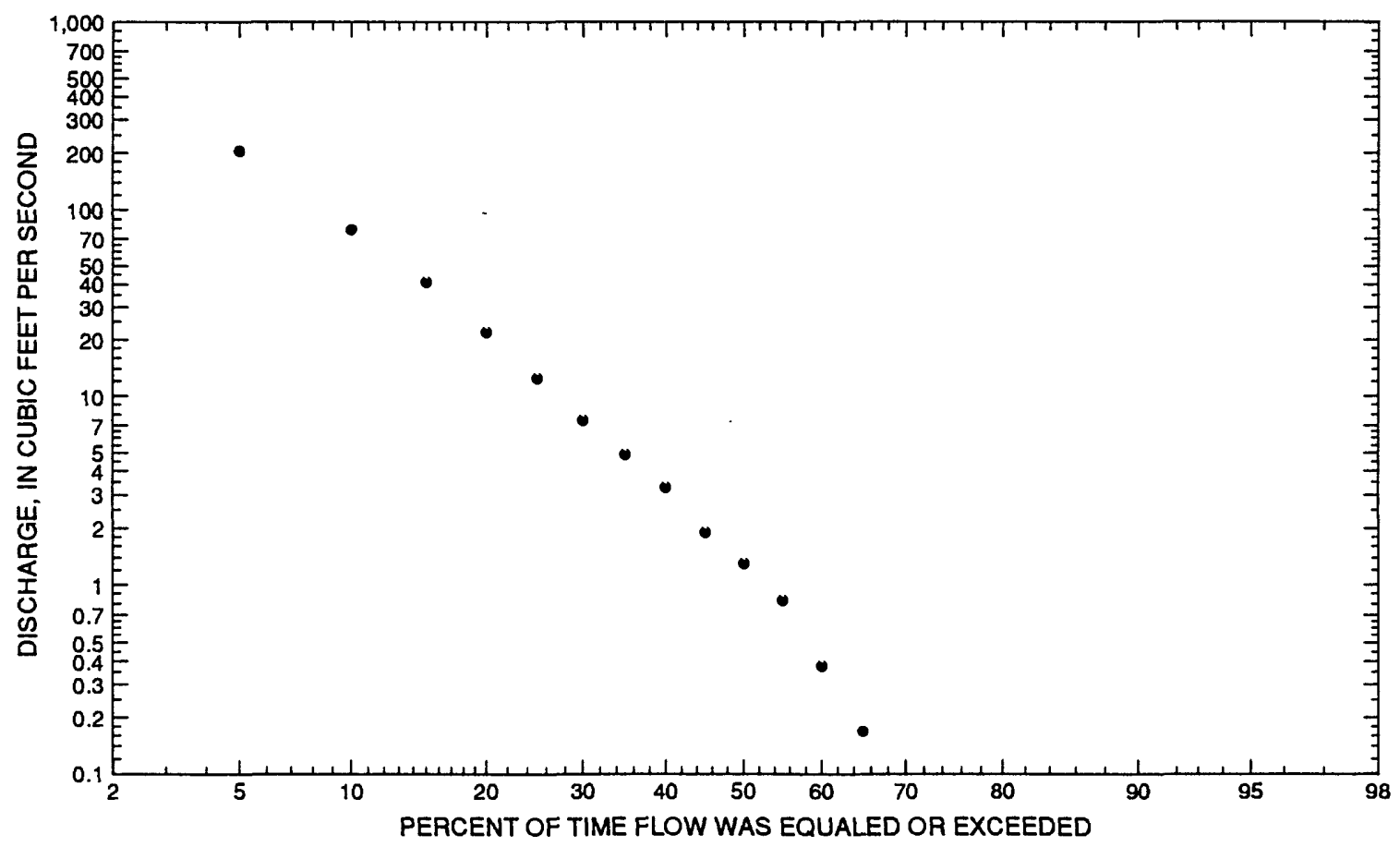




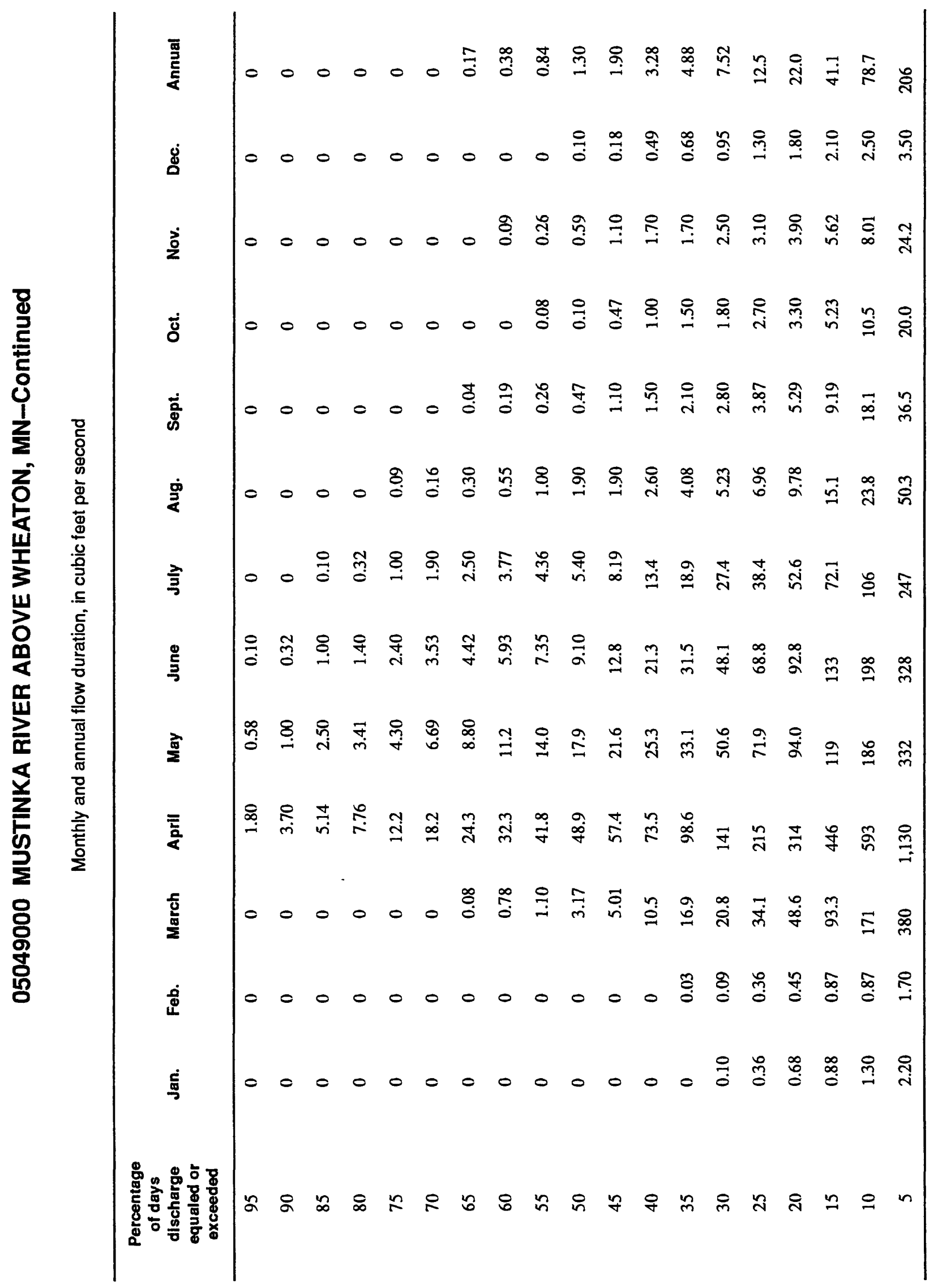


05049000 MUSTINKA RIVER ABOVE WHEATON, MN-Continued

Probability of annual high discharges

[ng, statistic not given]

\begin{tabular}{ccccccc}
\hline & & & \multicolumn{5}{c}{$\begin{array}{c}\text { Maximum average discharge } \\
\text { (ft/s) }\end{array}$} \\
\cline { 5 - 7 } $\begin{array}{c}\text { Exceedance } \\
\text { probability }\end{array}$ & $\begin{array}{c}\text { Recurrence } \\
\text { interval } \\
\text { (years) }\end{array}$ & $\begin{array}{c}\text { Maximum } \\
\text { instantaneous } \\
\left(\mathrm{ft}^{3} / \mathbf{s}\right)\end{array}$ & 3-day period & 7-day period & 15-day period & 30-day period \\
\hline 0.99 & 1.01 & $\mathrm{ng}$ & 7.17 & 4.43 & 2.62 & 1.70 \\
0.95 & 1.05 & 77.4 & 35.8 & 23.6 & 14.5 & 9.19 \\
0.90 & 1.11 & 136 & 76.2 & 52.0 & 32.4 & 20.4 \\
0.80 & 1.25 & 260 & 174 & 123 & 78.2 & 49.1 \\
0.50 & 2 & 817 & 649 & 493 & 320 & 202 \\
0.20 & 5 & 2,270 & 1,760 & 1,420 & 935 & 606 \\
0.10 & 10 & 3,700 & 2,640 & 2,200 & 1,450 & 957 \\
0.04 & 25 & 6,010 & 3,780 & 3,230 & 2,140 & 1,440 \\
0.02 & 50 & 8,080 & 4,570 & 3,980 & 2,640 & 1,800 \\
0.01 & 100 & 10,400 & 5,310 & 4,690 & 3,100 & 2,140 \\
0.005 & 200 & 13,000 & 5,970 & 5,340 & 3,530 & 2,470 \\
0.002 & 500 & 16,800 & $\mathrm{ng}$ & $\mathrm{ng}$ & $\mathrm{ng}$ & $\mathrm{ng}$ \\
\hline
\end{tabular}

Probability of annual low discharges

\begin{tabular}{|c|c|c|c|c|c|c|c|c|c|c|}
\hline \multirow[b]{3}{*}{$\begin{array}{l}\text { Non- } \\
\text { exceed- } \\
\text { ance } \\
\text { prob- } \\
\text { ability }\end{array}$} & \multirow[b]{3}{*}{$\begin{array}{c}\text { Recur- } \\
\text { rence } \\
\text { inter- } \\
\text { val } \\
\text { (years) }\end{array}$} & \multicolumn{9}{|c|}{ Minimum average discharge $\left(\mathrm{ft}^{3} / \mathrm{s}\right)$} \\
\hline & & \multicolumn{9}{|c|}{ Number of consecutive days } \\
\hline & & 1 & 3 & 7 & 14 & 30 & 60 & 90 & 120 & 183 \\
\hline 0.05 & 20 & 0 & 0 & 0 & 0 & 0 & 0 & 0 & 0 & 0 \\
\hline 0.10 & 10 & 0 & 0 & 0 & 0 & 0 & 0 & 0 & 0 & 0 \\
\hline 0.20 & 5 & 0 & 0 & 0 & 0 & 0 & 0 & 0 & 0 & 0 \\
\hline 0.50 & 2 & 0 & 0 & 0 & 0 & 0 & 0 & 0 & 0.196 & 0.361 \\
\hline
\end{tabular}


05049000 MUSTINKA RIVER ABOVE WHEATON, MN-Continued

Probability of seasonal low discharges

\begin{tabular}{|c|c|c|c|c|c|c|c|c|c|}
\hline \multirow[b]{3}{*}{$\begin{array}{l}\text { Non- } \\
\text { exceedance } \\
\text { probability }\end{array}$} & \multirow[b]{3}{*}{$\begin{array}{c}\text { Recurrence } \\
\text { intervai } \\
\text { (years) }\end{array}$} & \multicolumn{8}{|c|}{ Minimum average discharge $\left(\mathrm{ft}^{3} / \mathrm{s}\right)$} \\
\hline & & \multicolumn{8}{|c|}{ Number of consecutive days } \\
\hline & & 1 & 7 & 14 & 30 & 1 & 7 & 14 & 30 \\
\hline & & \multicolumn{4}{|c|}{ December-January-February } & \multicolumn{4}{|c|}{ March-April-May } \\
\hline 0.05 & 20 & 0 & 0 & 0 & 0 & 0 & 0 & 0 & 0 \\
\hline 0.10 & 10 & 0 & 0 & 0 & 0 & 0 & 0 & 0 & 0.120 \\
\hline 0.20 & 5 & 0 & 0 & 0 & 0 & 0 & 0 & 0 & 0.762 \\
\hline \multirow[t]{2}{*}{0.50} & 2 & 0 & 0 & 0 & 0 & 0 & 0 & 0.355 & 7.43 \\
\hline & & \multicolumn{4}{|c|}{ June-July-August } & \multicolumn{4}{|c|}{ September-October-November } \\
\hline 0.05 & 20 & 0 & 0 & 0 & 0 & 0 & 0 & 0 & 0 \\
\hline 0.10 & 10 & 0 & 0 & 0 & 0 & 0 & 0 & 0 & 0 \\
\hline 0.20 & 5 & 0 & 0 & 0 & 0.139 & 0 & 0 & 0 & 0 \\
\hline 0.50 & 2 & 0.314 & 0.422 & 0.468 & 1.43 & 0 & 0 & 0 & 0.062 \\
\hline
\end{tabular}




\section{MUSTINKA RIVER ABOVE WHEATON, MN-Continued}

Annual peak discharge and corresponding gage height, period of record

$$
[-, \text { no data }]
$$

\begin{tabular}{|c|c|c|c|c|c|c|c|}
\hline $\begin{array}{l}\text { Water } \\
\text { Year }\end{array}$ & Date & $\begin{array}{c}\text { Gage } \\
\text { height } \\
\text { (feet) }\end{array}$ & $\begin{array}{c}\text { Peak } \\
\text { discharge } \\
\left(\mathrm{ft}^{3} / \mathrm{s}\right)\end{array}$ & $\begin{array}{l}\text { Water } \\
\text { year }\end{array}$ & Date & $\begin{array}{c}\text { Gage } \\
\text { height } \\
\text { (feet) }\end{array}$ & $\begin{array}{c}\text { Peak } \\
\text { discharge } \\
\left(\mathrm{ft}^{3} / \mathbf{s}\right)\end{array}$ \\
\hline \multicolumn{8}{|c|}{ Annual peak discharge, by year, and corresponding gage height } \\
\hline 1916 & -- & 17.40 & 2,980 & 1946 & March 21 & 14.56 & 2,460 \\
\hline 1917 & April 1 & 14.73 & 2,250 & 1947 & April 13 & 14.68 & 2,710 \\
\hline 1918 & -- & -. & -- & 1948 & April 1 & - & 2,080 \\
\hline 1919 & June 5 & 7.37 & 559 & 1949 & July 12 & 12.98 & 1,770 \\
\hline 1920 & May 11 & 9.50 & 970 & 1950 & May 11 & -- & 1,690 \\
\hline 1921 & April 2 & 5.50 & 287 & 1951 & April 8 & - & 3,180 \\
\hline 1922 & $\operatorname{March} 22$ & 13.08 & 1,330 & 1952 & April 10 & 16.56 & 7,320 \\
\hline 1923 & April 15 & - & 746 & 1953 & March 26 & -- & 572 \\
\hline 1924 & April 5 & 2.65 & 45.0 & 1954 & June 9 & 6.61 & 393 \\
\hline 1931 & June 10 & 1.80 & 41.0 & 1955 & July 13 & 5.58 & 484 \\
\hline 1932 & April 8 & 4.50 & 300 & 1956 & May 29 & - & 655 \\
\hline 1933 & March 2 & 4.84 & 78.0 & 1957 & April 21 & 10.68 & 1,610 \\
\hline 1934 & April 10 & 1.36 & 9.10 & 1958 & April 8 & 6.84 & 690 \\
\hline 1935 & March 17 & - & 120 & 1985 & May 31 & 9.37 & 1,190 \\
\hline 1936 & March 23 & 10.51 & 354 & 1986 & March 29 & 18.91 & 5,500 \\
\hline 1937 & May 1 & -- & 400 & 1987 & June 1 & 4.78 & 275 \\
\hline 1938 & July 4 & 6.42 & 267 & 1988 & May ${ }^{1}$ & - & 250 \\
\hline 1939 & March 25 & 13.62 & 1,420 & 1989 & March 29 & 20.01 & 5,400 \\
\hline 1940 & April 2 & 8.08 & 349 & 1990 & April 4 & 3.83 & 130 \\
\hline 1941 & June 23 & - & 240 & 1991 & July 2 & 11.22 & 1,420 \\
\hline 1942 & May 17 & 14.08 & 1,480 & 1992 & March 7 & 5.45 & 220 \\
\hline 1943 & April 4 & - & 1,940 & 1993 & March 30 & 18.07 & 4,400 \\
\hline 1944 & June 6 & 13.29 & 1,520 & 1994 & March 25 & 16.95 & 4,310 \\
\hline 1945 & March 16 & - & 892 & & & & \\
\hline \multicolumn{8}{|c|}{ Annual peak discharge, from highest to lowest, and corresponding gage height } \\
\hline 1952 & April 10 & 16.56 & 7,320 & 1922 & March 22 & 13.08 & 1,330 \\
\hline 1986 & March 29 & 18.91 & $.5,500$ & 1985 & May 31 & 9.37 & 1,190 \\
\hline 1989 & March 29 & 20.01 & 5,400 & 1920 & May 11 & 9.50 & 970 \\
\hline 1993 & March 30 & 18.07 & 4,400 & 1945 & March 16 & - & 892 \\
\hline 1994 & March 25 & 16.95 & 4,310 & 1923 & April 15 & - & 746 \\
\hline 1951 & April 8 & - & 3,180 & 1958 & April 8 & 6.84 & 690 \\
\hline 1916 & -- & 17.40 & 2,980 & 1956 & May 29 & - & 655 \\
\hline 1947 & April 13 & 14.68 & 2,710 & 1953 & March 26 & -- & 572 \\
\hline 1946 & March 21 & 14.56 & 2,460 & 1919 & June 5 & 7.37 & 559 \\
\hline 1917 & April 1 & 14.73 & 2,250 & 1955 & July 13 & 5.58 & 484 \\
\hline 1948 & April 1 & - & 2,080 & 1937 & May 1 & -- & 400 \\
\hline 1943 & April 4 & - & 1,940 & 1954 & June 9 & 6.61 & 393 \\
\hline 1949 & July 12 & 12.98 & 1,770 & 1936 & March 23 & 10.51 & 354 \\
\hline 1950 & May 11 & -- & 1,690 & 1940 & April 2 & 8.08 & 349 \\
\hline 1957 & April 21 & 10.68 & 1,610 & 1932 & April 8 & 4.50 & 300 \\
\hline 1944 & June 6 & 13.29 & 1,520 & 1921 & April 2 & 5.50 & 287 \\
\hline 1942 & May 17 & 14.08 & 1,480 & 1987 & June 1 & 4.78 & 275 \\
\hline 1939 & March 25 & 13.62 & 1,420 & 1938 & July 4 & 6.42 & 267 \\
\hline 1991 & July 2 & 11.22 & 1,420 & 1988 & May ${ }^{1}$ & - & 250 \\
\hline
\end{tabular}




\section{MUSTINKA RIVER ABOVE WHEATON, MN-Continued}

Annual peak discharge and corresponding gage height, period of record--Continued

$$
[--, \text { no data }]
$$

\begin{tabular}{cccccccc}
\hline $\begin{array}{c}\text { Water } \\
\text { Year }\end{array}$ & \multicolumn{1}{c}{ Date } & $\begin{array}{c}\text { Gage } \\
\text { helght } \\
(\text { feet })\end{array}$ & $\begin{array}{c}\text { Peak } \\
\text { discharge } \\
\left(\mathrm{ft}^{3} / \mathbf{s}\right)\end{array}$ & $\begin{array}{c}\text { Water } \\
\text { year }\end{array}$ & Date & $\begin{array}{c}\text { Gage } \\
\text { helght } \\
(\text { feet })\end{array}$ & $\begin{array}{c}\text { Peak } \\
\text { discharge } \\
\left(\mathbf{f t}^{3} / \mathbf{s}\right)\end{array}$ \\
\hline \multicolumn{7}{c}{ Annual peak discharge, from highest to lowest, and corresponding gage height-.Continued } \\
1941 & June 23 & - & 240 & 1933 & March 2 & 4.84 & 78.0 \\
1992 & March 7 & 5.45 & 220 & 1924 & April 5 & 2.65 & 45.0 \\
1990 & April 4 & 3.83 & 130 & 1931 & June 10 & 1.80 & 41.0 \\
1935 & March 17 & -- & 120 & 1934 & April 10 & 1.36 & 9.10 \\
\hline
\end{tabular}

${ }^{1}$ Day of month unknown. 


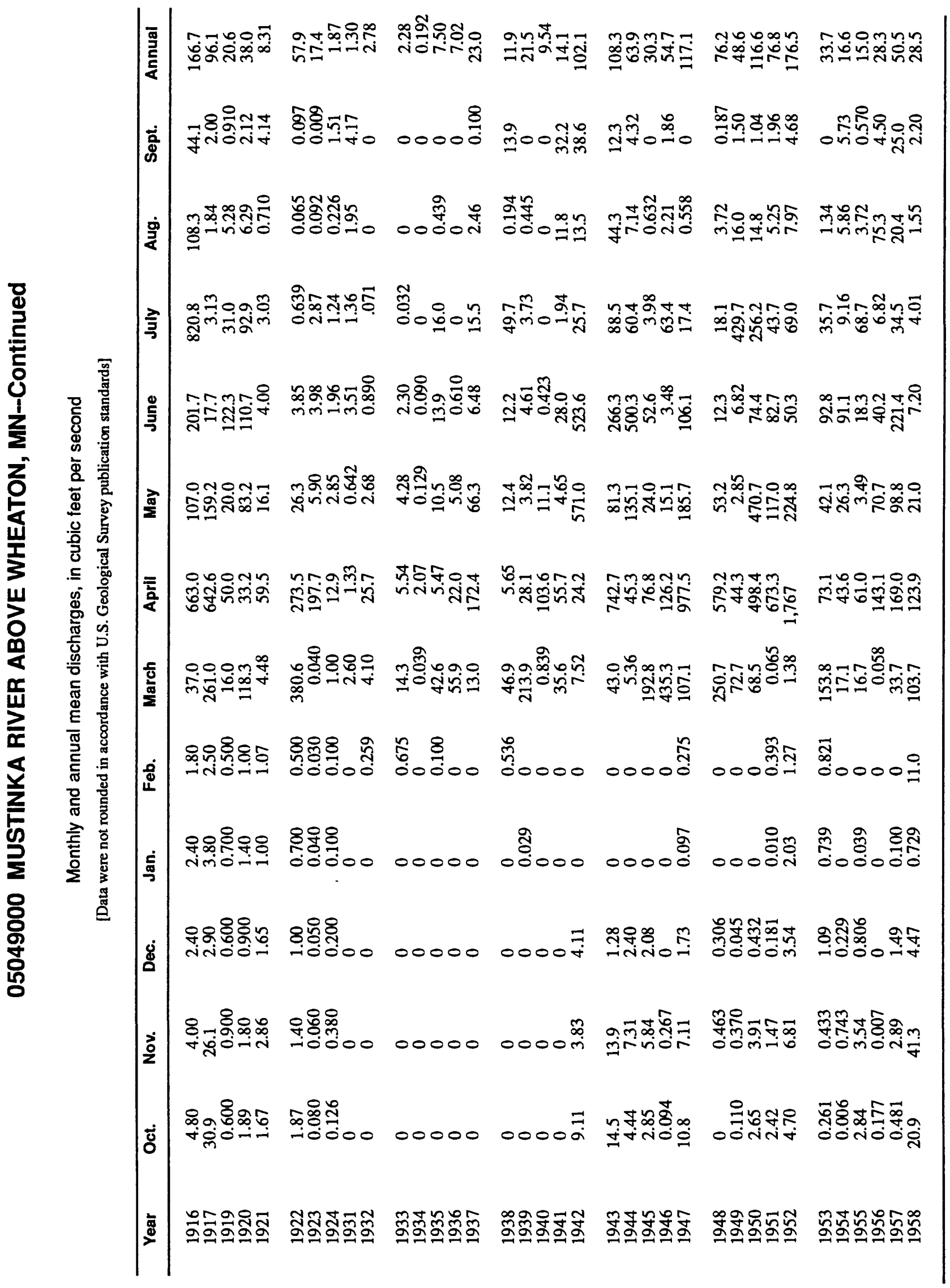




\section{BOIS DE SIOUX RIVER NEAR WHITE ROCK, SD}

\section{Station Description}

LOCATION.--Lat $45^{\circ} 51^{\prime} 45^{\prime \prime}$, long $96^{\circ} 34^{\prime} 25^{\prime \prime}$, in SW $1 / 4 \mathrm{SW}^{1} / 4$ sec.27, T.128 N., R.47 W., Roberts County, Hydrologic Unit 09020101, on Sisseton Indian Reservation, on left bank just downstream from Big Slough Outlet, $300 \mathrm{ft}$ downstream from White Rock Dam, $4 \mathrm{mi}$ south of White Rock, SD and $5 \mathrm{mi}$ northwest of Wheaton.

DRAINAGE AREA.--1,160 $\mathrm{mi}^{2}$, approximately.

PERIOD OF RECORD.--October 1941 to current year.

GAGE.--.Water-stage recorder. Datum of gage is $960.00 \mathrm{ft}$, adjustment of 1912 (levels by U.S. Army Corps of Engineers). Prior to Jan. 14, 1943, nonrecording gage at same site at datum $0.11 \mathrm{ft}$ lower. Jan. 15,1943 , to Sept. 30,1963 , water-stage recorder at same site at datum $0.11 \mathrm{ft}$ lower.

EXTREMES FOR PERIOD OF RECORD.--Maximum discharge, 3,770 $\mathrm{ft}^{3} / \mathrm{s}$, Apr. 19-21, 1969; maximum gage height, $15.07 \mathrm{ft}$, Apr. 19-21, 1969 (from floodmark); no flow at times in most years.

Annual mean discharge

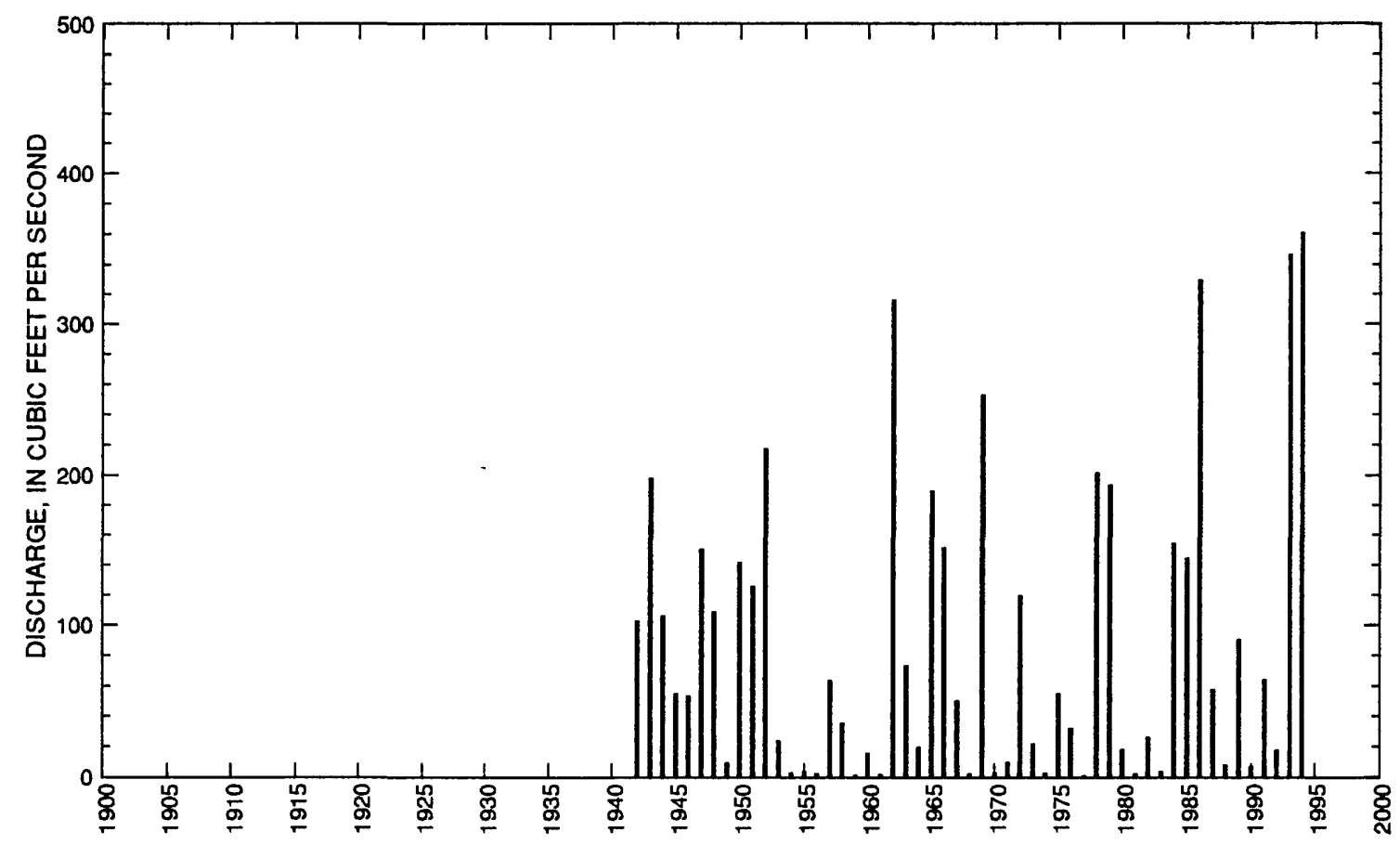




\section{BOIS DE SIOUX RIVER NEAR WHITE ROCK, SD-Continued}

Statistics of monthly and annual mean discharges

[m, more than 1 year of occurrence]

\begin{tabular}{|c|c|c|c|c|c|c|c|c|}
\hline \multirow[b]{2}{*}{ Month } & \multicolumn{2}{|c|}{ Maximum } & \multicolumn{2}{|c|}{ Minimum } & \multicolumn{4}{|c|}{ Mean } \\
\hline & $\begin{array}{c}\text { Discharge } \\
\left(\mathrm{ft}^{3} / \mathrm{s}\right)\end{array}$ & $\begin{array}{c}\text { Water year } \\
\text { of } \\
\text { occurrence }\end{array}$ & $\begin{array}{c}\text { Discharge } \\
\left(\mathrm{ft}^{3} / \mathrm{s}\right)\end{array}$ & $\begin{array}{c}\text { Water year } \\
\text { of } \\
\text { occurrence }\end{array}$ & $\begin{array}{c}\text { Discharge } \\
\left(\mathrm{ft}^{3} / \mathrm{s}\right)\end{array}$ & $\begin{array}{c}\text { Standard } \\
\text { devlation } \\
\left(\mathrm{ft}^{3} / \mathrm{s}\right)\end{array}$ & $\begin{array}{l}\text { Coeffi- } \\
\text { clent of } \\
\text { variation }\end{array}$ & $\begin{array}{l}\text { Percentage } \\
\text { of annual } \\
\text { dlscharge }\end{array}$ \\
\hline October & 535 & 1994 & 0 & $\mathrm{~m}$ & 25.8 & 88.4 & 3.43 & 2.42 \\
\hline November & 258 & 1985 & 0 & $\mathrm{~m}$ & 11.8 & 38.4 & 3.26 & 1.11 \\
\hline December & 57.5 & 1985 & 0 & $\mathrm{~m}$ & 4.87 & 11.3 & 2.32 & 0.46 \\
\hline January & 36.0 & 1987 & 0 & $\mathrm{~m}$ & 2.59 & 6.51 & 2.51 & 0.24 \\
\hline February & 53.0 & 1966 & 0 & $\mathrm{~m}$ & 3.31 & 8.74 & 2.64 & 0.31 \\
\hline March & 227 & 1985 & 0 & $\mathrm{~m}$ & 28.8 & 53.8 & 1.87 & 2.70 \\
\hline April & 1,320 & 1969 & 0 & 1942 & 216 & 289 & 1.34 & 20.2 \\
\hline May & 1,310 & 1969 & 0.228 & 1977 & 260 & 367 & 1.41 & 24.4 \\
\hline June & 1,100 & 1986 & 0.010 & 1977 & 235 & 304 & 1.29 & 22.0 \\
\hline July & 1,040 & 1962 & 0 & $\mathrm{~m}$ & 167 & 253 & 1.51 & 15.6 \\
\hline August & 1,180 & 1993 & 0 & $\mathrm{~m}$ & 74.9 & 225 & 3.00 & 7.02 \\
\hline September & 1,060 & 1993 & 0 & $\mathrm{~m}$ & 37.0 & 152 & 4.10 & 3.47 \\
\hline Annual & 360 & 1994 & 0.377 & 1977 & 89.2 & 99.4 & 1.11 & 100 \\
\hline
\end{tabular}

Annual flow duration

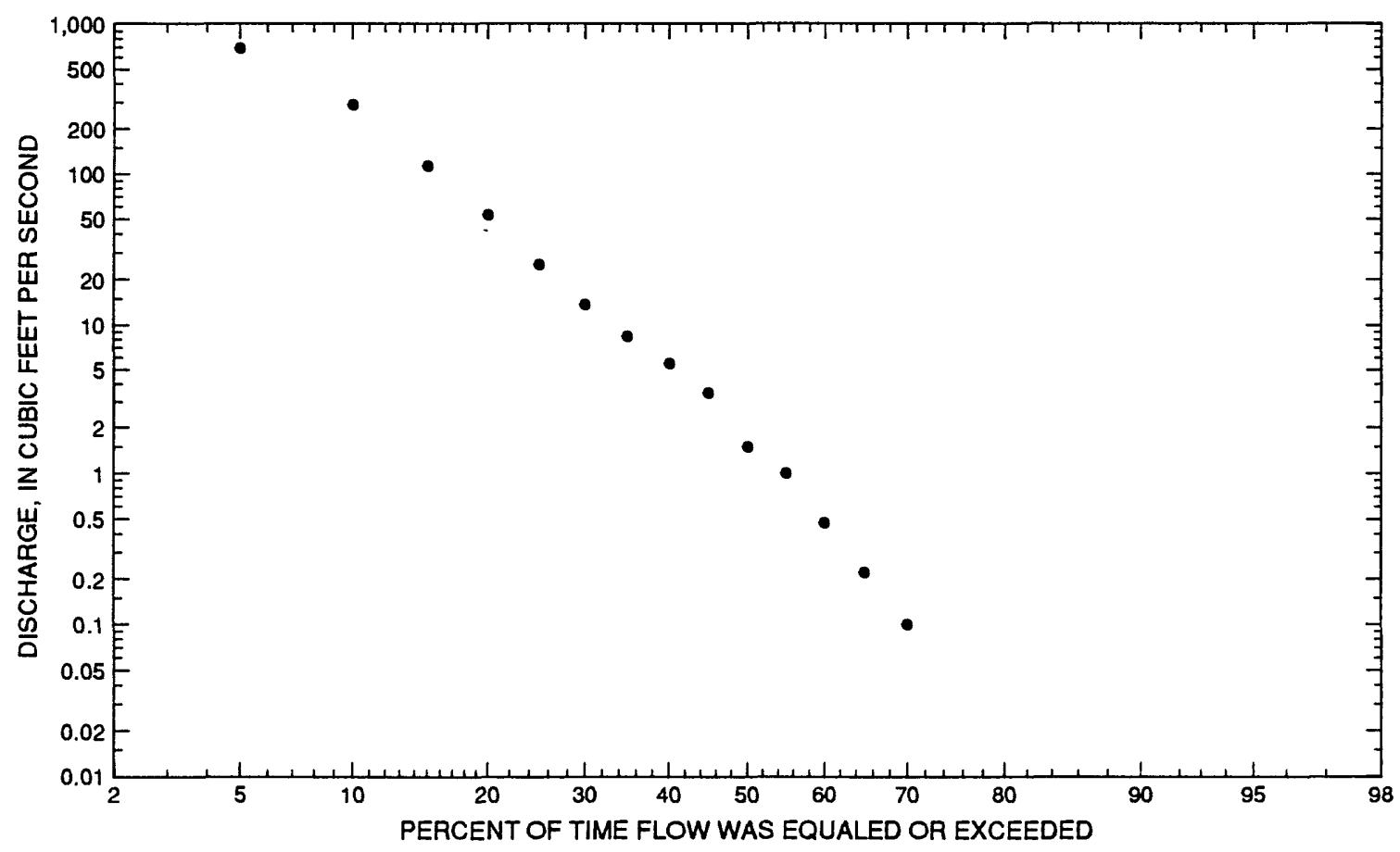




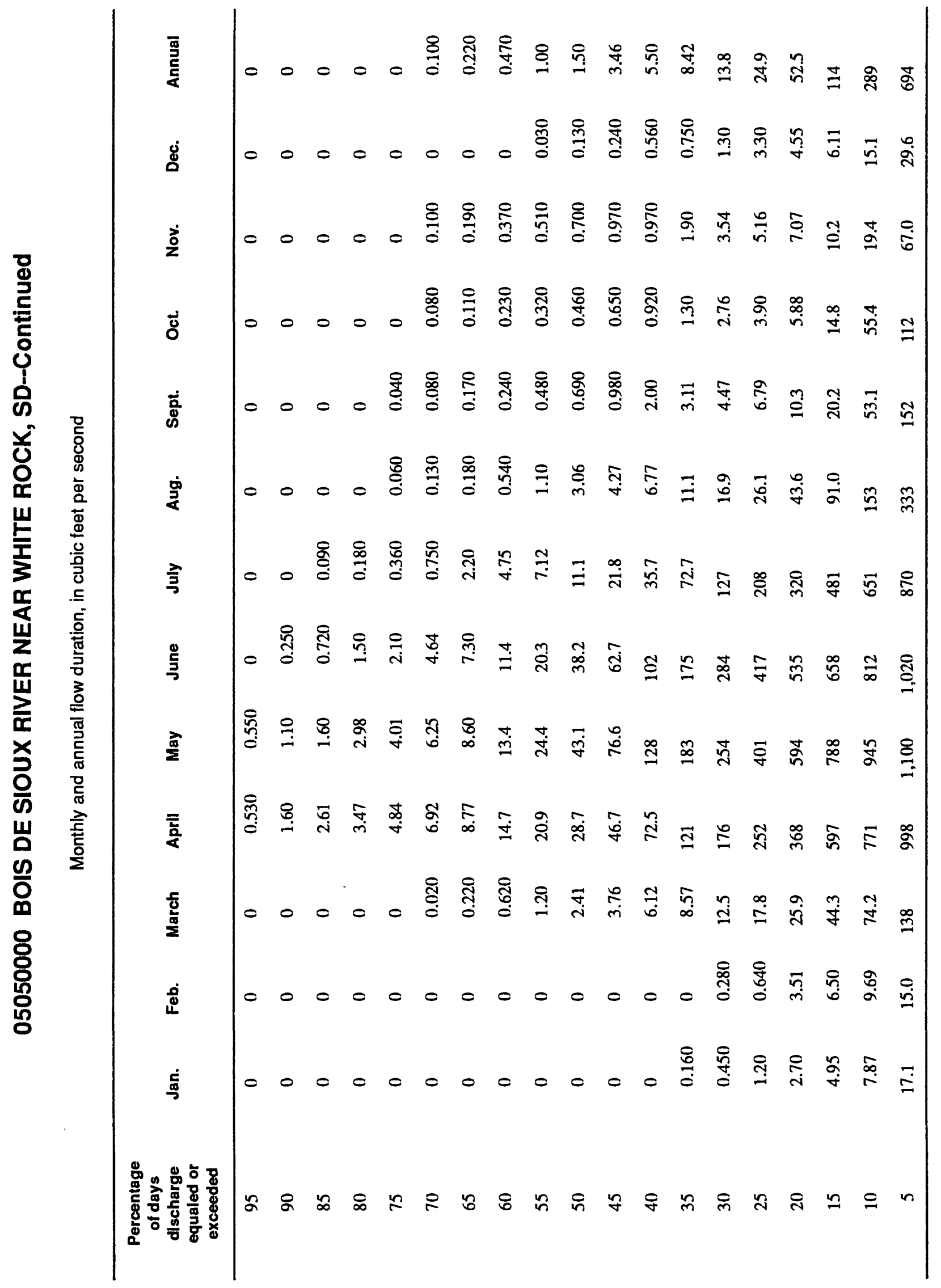


05050000 BOIS DE SIOUX RIVER NEAR WHITE ROCK, SD--Continued

Probability of annual high discharges

[ng, statistic not given]

\begin{tabular}{lcccccc}
\hline & & & \multicolumn{5}{c}{$\begin{array}{c}\text { Maximum average discharge } \\
\left(\mathrm{ft}^{3} / \mathbf{s}\right)\end{array}$} \\
\cline { 6 - 7 } $\begin{array}{c}\text { Exceedance } \\
\text { probability }\end{array}$ & $\begin{array}{c}\text { Recurrence } \\
\text { interval } \\
\text { (years) }\end{array}$ & $\begin{array}{c}\text { Maximum } \\
\text { instantaneous } \\
\left(\mathrm{ft}^{3} / \mathbf{s}\right)\end{array}$ & 3-day period & 7-day period & 15-day period & 30-day period \\
\hline 0.99 & 1.01 & 17.2 & 4.29 & 2.88 & 1.87 & 1.14 \\
0.95 & 1.05 & 51.0 & 20.2 & 15.1 & 10.9 & 7.13 \\
0.90 & 1.11 & 87.1 & 42.3 & 33.5 & 25.2 & 17.2 \\
0.80 & 1.25 & 160 & 96.2 & 80.5 & 64.1 & 45.7 \\
0.50 & 2 & 454 & 370 & 339 & 395 & 229 \\
0.20 & 5 & 1,110 & 1,080 & 1,060 & 988 & 841 \\
0.10 & 10 & 1,670 & 1,710 & 1,730 & 1,660 & 1,480 \\
0.04 & 25 & 2,470 & 2,600 & 2,700 & 2,660 & 2,480 \\
0.02 & 50 & 3,120 & 3,290 & 3,460 & 3,460 & 3,330 \\
0.01 & 100 & 3,800 & 3,970 & 4,230 & 4,270 & 4,220 \\
0.005 & 200 & 4,490 & 4,640 & 4,980 & 5,080 & 5,140 \\
0.002 & 500 & 5,430 & ng & ng & ng & ng \\
\hline
\end{tabular}

Probability of annual low discharges

\begin{tabular}{|c|c|c|c|c|c|c|c|c|c|c|}
\hline \multirow[b]{3}{*}{$\begin{array}{l}\text { Non- } \\
\text { exceed- } \\
\text { ance } \\
\text { prob- } \\
\text { ability }\end{array}$} & \multirow[b]{3}{*}{$\begin{array}{c}\text { Recur- } \\
\text { rence } \\
\text { Inter- } \\
\text { val } \\
\text { (years) }\end{array}$} & \multicolumn{9}{|c|}{ Minimum average discharge $\left(\mathrm{ft}^{3} / \mathrm{s}\right)$} \\
\hline & & \multicolumn{9}{|c|}{ Number of consecutive days } \\
\hline & & 1 & 3 & 7 & 14 & 30 & 60 & 90 & 120 & 183 \\
\hline 0.05 & 20 & 0 & 0 & 0 & 0 & 0 & 0 & 0 & 0 & 0 \\
\hline 0.10 & 10 & 0 & 0 & 0 & 0 & 0 & 0 & 0 & 0 & 0 \\
\hline 0.20 & 5 & 0 & 0 & 0 & 0 & 0 & 0 & 0 & 0 & 0.065 \\
\hline 0.50 & 2 & 0 & 0 & 0 & 0 & 0 & 0 & 0.016 & 0.253 & 1.18 \\
\hline
\end{tabular}


05050000 BOIS DE SIOUX RIVER NEAR WHITE ROCK, SD--Continued

Probability of seasonal low discharges

\begin{tabular}{|c|c|c|c|c|c|c|c|c|c|}
\hline \multirow[b]{3}{*}{$\begin{array}{l}\text { Non- } \\
\text { exceedance } \\
\text { probability }\end{array}$} & \multirow[b]{3}{*}{$\begin{array}{l}\text { Recurrence } \\
\text { Interval } \\
\text { (years) }\end{array}$} & \multicolumn{8}{|c|}{ Minimum average discharge $\left(\mathrm{ft}^{3} / \mathrm{s}\right)$} \\
\hline & & \multicolumn{8}{|c|}{ Number of consecutive days } \\
\hline & & 1 & 7 & 14 & 30 & 1 & 7 & 14 & 30 \\
\hline & & \multicolumn{4}{|c|}{ December-January-February } & \multicolumn{4}{|c|}{ March-April-May } \\
\hline 0.05 & 20 & 0 & 0 & 0 & 0 & 0 & 0 & 0 & 0 \\
\hline 0.10 & 10 & 0 & 0 & 0 & 0 & 0 & 0 & 0 & 0.015 \\
\hline 0.20 & 5 & 0 & 0 & 0 & 0 & 0 & 0 & 0 & 0.381 \\
\hline \multirow[t]{2}{*}{0.50} & 2 & 0 & 0 & 0 & 0 & 0 & 0.073 & 0.521 & 3.74 \\
\hline & & \multicolumn{4}{|c|}{ June-July-August } & \multicolumn{4}{|c|}{ September-October-November } \\
\hline 0.05 & 20 & 0 & 0 & 0 & 0 & 0 & 0 & 0 & 0 \\
\hline 0.10 & 10 & 0 & 0 & 0 & 0 & 0 & 0 & 0 & 0 \\
\hline 0.20 & 5 & 0 & 0 & 0 & 0.094 & 0 & 0 & 0 & 0 \\
\hline 0.50 & 2 & 0.111 & 0.292 & 0.773 & 2.42 & 0 & 0.045 & 0.094 & 0.274 \\
\hline
\end{tabular}


05050000 BOIS DE SIOUX RIVER NEAR WHITE ROCK, SD--Continued

Annual peak discharge and corresponding gage height, period of record

$$
[--, \text { no data }]
$$

\begin{tabular}{|c|c|c|c|c|c|c|c|}
\hline $\begin{array}{c}\text { Water } \\
\text { year }\end{array}$ & Date & $\begin{array}{c}\text { Gage } \\
\text { height } \\
\text { (feet) }\end{array}$ & $\begin{array}{c}\text { Peak } \\
\text { discharge } \\
\left(\mathrm{ft}^{3} / \mathrm{s}\right)\end{array}$ & $\begin{array}{l}\text { Water } \\
\text { year }\end{array}$ & Date & $\begin{array}{c}\text { Gage } \\
\text { height } \\
\text { (feet) }\end{array}$ & $\begin{array}{c}\text { Peak } \\
\text { discharge } \\
\left(\mathrm{ft}^{3} / \mathrm{s}\right)\end{array}$ \\
\hline \multicolumn{8}{|c|}{ Annual peak discharge, by year, and corresponding gage height } \\
\hline 1942 & July 11 & - & 845 & 1969 & April $^{1}$ & 15.07 & 3,770 \\
\hline 1943 & May 24 & 8.95 & 1,120 & 1970 & October 14 & 4.68 & 102 \\
\hline 1944 & June 23 & 9.28 & 1,080 & 1971 & June 26 & 5.93 & 188 \\
\hline 1945 & April 4 & 8.10 & 900 & 1972 & April 10 & 9.39 & 776 \\
\hline 1946 & April 8 & 7.89 & 850 & 1973 & March 27 & 7.00 & 381 \\
\hline 1947 & May 1 & 8.67 & 975 & 1974 & May 1 & 4.22 & 54.0 \\
\hline 1948 & April 27 & 8.20 & 1,020 & 1975 & July 16 & 8.95 & 552 \\
\hline 1949 & July 14 & - & 210 & 1976 & March 21 & 8.60 & 522 \\
\hline 1950 & July 8 & 9.16 & 1,060 & 1977 & March 13 & 5.52 & 27.0 \\
\hline 1951 & May 16 & -- & 959 & 1978 & April 19 & 11.59 & 929 \\
\hline 1952 & June 3 & 10.36 & 1,410 & 1979 & May 4 & 10.15 & 1,030 \\
\hline 1953 & May 27 & - & 187 & 1980 & June 14 & 6.19 & 169 \\
\hline 1954 & September 17 & 4.67 & 124 & 1981 & July 19 & 4.16 & 27.0 \\
\hline 1955 & July 11 & 6.01 & 152 & 1982 & April 16 & 8.25 & 414 \\
\hline 1956 & August 8 & 6.94 & 303 & 1983 & November 29 & 6.63 & 150 \\
\hline 1957 & June 22 & 9.09 & 610 & 1984 & April 14 & 11.00 & 978 \\
\hline 1958 & April 16 & 7.05 & 418 & 1985 & March 31 & 9.19 & 860 \\
\hline 1959 & July 5 & - & 43.0 & 1986 & May 7 & 12.12 & 1,820 \\
\hline 1960 & June 11 & 7.31 & 131 & 1987 & October 9 & 7.71 & 530 \\
\hline 1961 & September 14 & 5.16 & 125 & 1988 & April 12 & 5.84 & 180 \\
\hline 1962 & August 6 & 11.52 & 1,620 & 1989 & April 14 & 10.72 & 693 \\
\hline 1963 & June 18 & 8.65 & 945 & 1990 & March 13 & 4.85 & 89.0 \\
\hline 1964 & April 22 & 5.44 & 209 & 1991 & July 7 & 11.14 & 685 \\
\hline 1965 & June 9 & 11.05 & 1,320 & 1992 & August 1 & 7.17 & 139 \\
\hline 1966 & April 29 & 8.77 & 921 & 1993 & August 4 & 11.52 & 1,300 \\
\hline 1967 & April 17 & 7.33 & 530 & 1994 & April 8 & 11.88 & 1,550 \\
\hline 1968 & April 23 & 3.94 & 58.0 & & & & \\
\hline \multicolumn{8}{|c|}{ Annual peak discharge, from highest to lowest, and corresponding gage height } \\
\hline 1969 & April $^{1}$ & 15.07 & 3,770 & 1978 & April 19 & 11.59 & 929 \\
\hline 1986 & May 7 & 12.12 & 1,820 & 1966 & April 29 & 8.77 & 921 \\
\hline 1962 & August 6 & 11.52 & 1,620 & 1945 & April 4 & 8.10 & 900 \\
\hline 1994 & April 8 & 11.88 & 1,550 & 1985 & March 31 & 9.19 & 860 \\
\hline 1952 & June 3 & 10.36 & 1,410 & 1946 & April 8 & 7.89 & 850 \\
\hline 1965 & June 9 & 11.05 & 1,320 & 1942 & July 11 & - & 845 \\
\hline 1993 & August 4 & 11.52 & 1,300 & 1972 & April 10 & 9.39 & 776 \\
\hline 1943 & May 24 & 8.95 & 1,120 & 1989 & April 14 & 10.72 & 693 \\
\hline 1944 & June 23 & 9.28 & 1,080 & 1991 & July 7 & 11.14 & 685 \\
\hline 1950 & July 8 & 9.16 & 1,060 & 1957 & June 22 & 9.09 & 610 \\
\hline 1979 & May 4 & 10.15 & 1,030 & 1975 & July 16 & 8.95 & 552 \\
\hline 1948 & April 27 & 8.20 & 1,020 & 1967 & April 17 & 7.33 & 530 \\
\hline 1984 & April 14 & 11.00 & 978 & 1986 & October 9 & 7.71 & 530 \\
\hline 1947 & May 1 & 8.67 & 975 & 1976 & March 21 & 8.60 & 522 \\
\hline 1951 & May 16 & -- & 959 & 1958 & April 16 & 7.05 & 418 \\
\hline 1963 & June 18 & 8.65 & 945 & 1982 & April 16 & 8.25 & 414 \\
\hline
\end{tabular}




\section{BOIS DE SIOUX RIVER NEAR WHITE ROCK, SD--Continued}

Annual peak discharge and corresponding gage height, period of record--Continued

$$
[-, \text { no data }]
$$

\begin{tabular}{|c|c|c|c|c|c|c|c|}
\hline $\begin{array}{l}\text { Water } \\
\text { year }\end{array}$ & Date & $\begin{array}{c}\text { Gage } \\
\text { height } \\
\text { (feet) }\end{array}$ & $\begin{array}{c}\text { Peak } \\
\text { discharge } \\
\left(\mathrm{ft}^{3} / \mathrm{s}\right)\end{array}$ & $\begin{array}{c}\text { Water } \\
\text { year }\end{array}$ & Date & $\begin{array}{c}\text { Gage } \\
\text { helght } \\
\text { (feet) }\end{array}$ & $\begin{array}{c}\text { Peak } \\
\text { discharge } \\
\left(\mathrm{ft}^{3} / \mathrm{s}\right)\end{array}$ \\
\hline \multicolumn{8}{|c|}{ Annual peak discharge, from highest to lowest, and corresponding gage height.-Continued } \\
\hline 1973 & March 27 & 7.00 & 381 & 1960 & June 11 & 7.31 & 131 \\
\hline 1956 & August 8 & 6.94 & 303 & 1961 & September 14 & 5.16 & 125 \\
\hline 1949 & July 14 & -- & 210 & 1954 & September 17 & 4.67 & 124 \\
\hline 1964 & April 22 & 5.44 & 209 & 1969 & October 14 & 4.68 & 102 \\
\hline 1971 & June 26 & 5.93 & 188 & 1990 & March 13 & 4.85 & 89.0 \\
\hline 1953 & May 27 & - & 187 & 1968 & April 23 & 3.94 & 58.0 \\
\hline 1988 & April 12 & 5.84 & 180 & 1974 & May 1 & 4.22 & 54.0 \\
\hline 1980 & June 14 & 6.19 & 169 & 1959 & July 5 & - & 43.0 \\
\hline 1955 & July 11 & 6.01 & 152 & 1977 & March 13 & 5.52 & 270 \\
\hline 1982 & November 29 & 6.63 & 150 & 1981 & July 19 & 4.16 & 27.0 \\
\hline 1992 & August 1 & 7.17 & 139 & & & & \\
\hline
\end{tabular}

${ }^{1}$ Day of month unknown. 


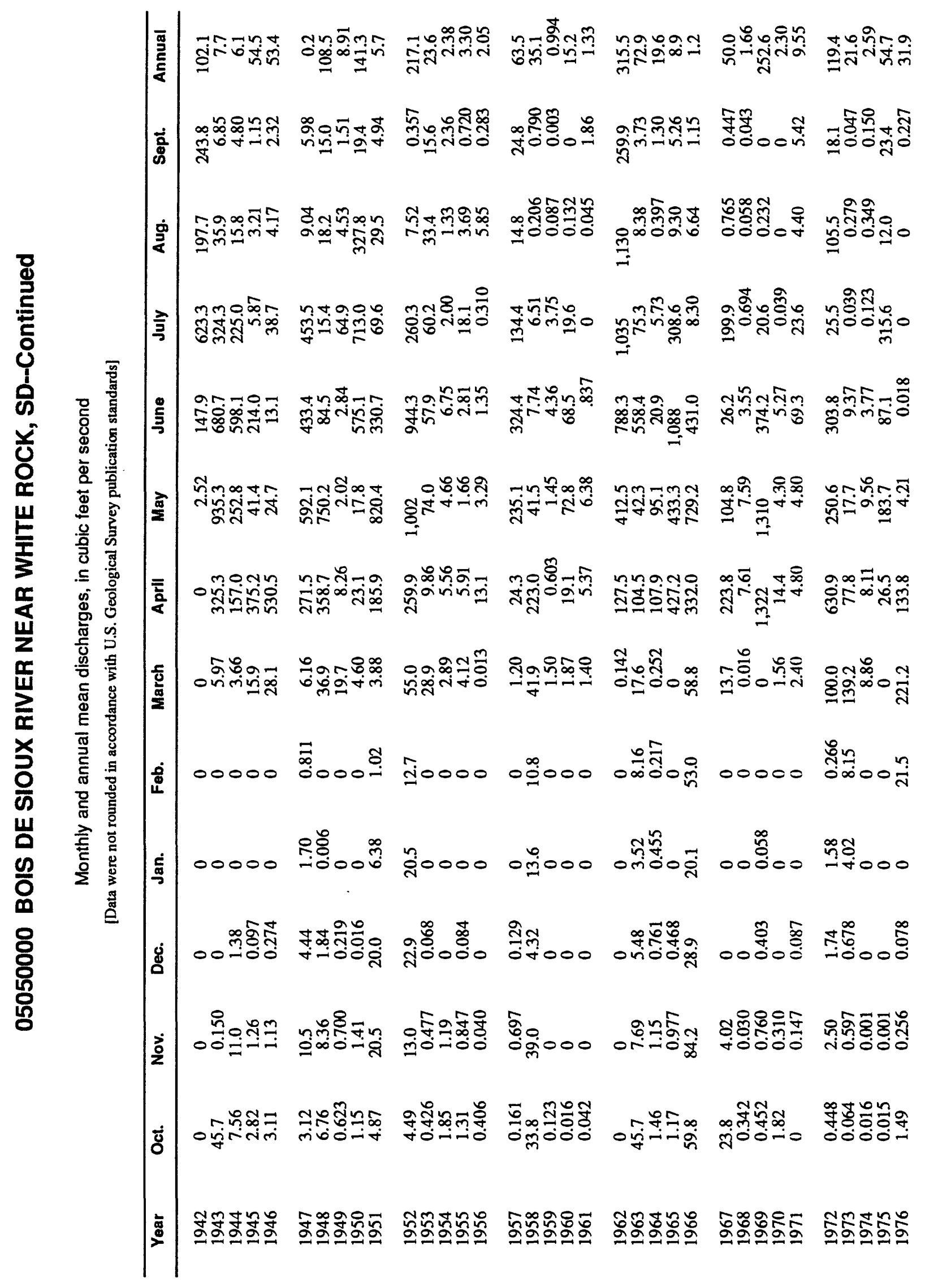




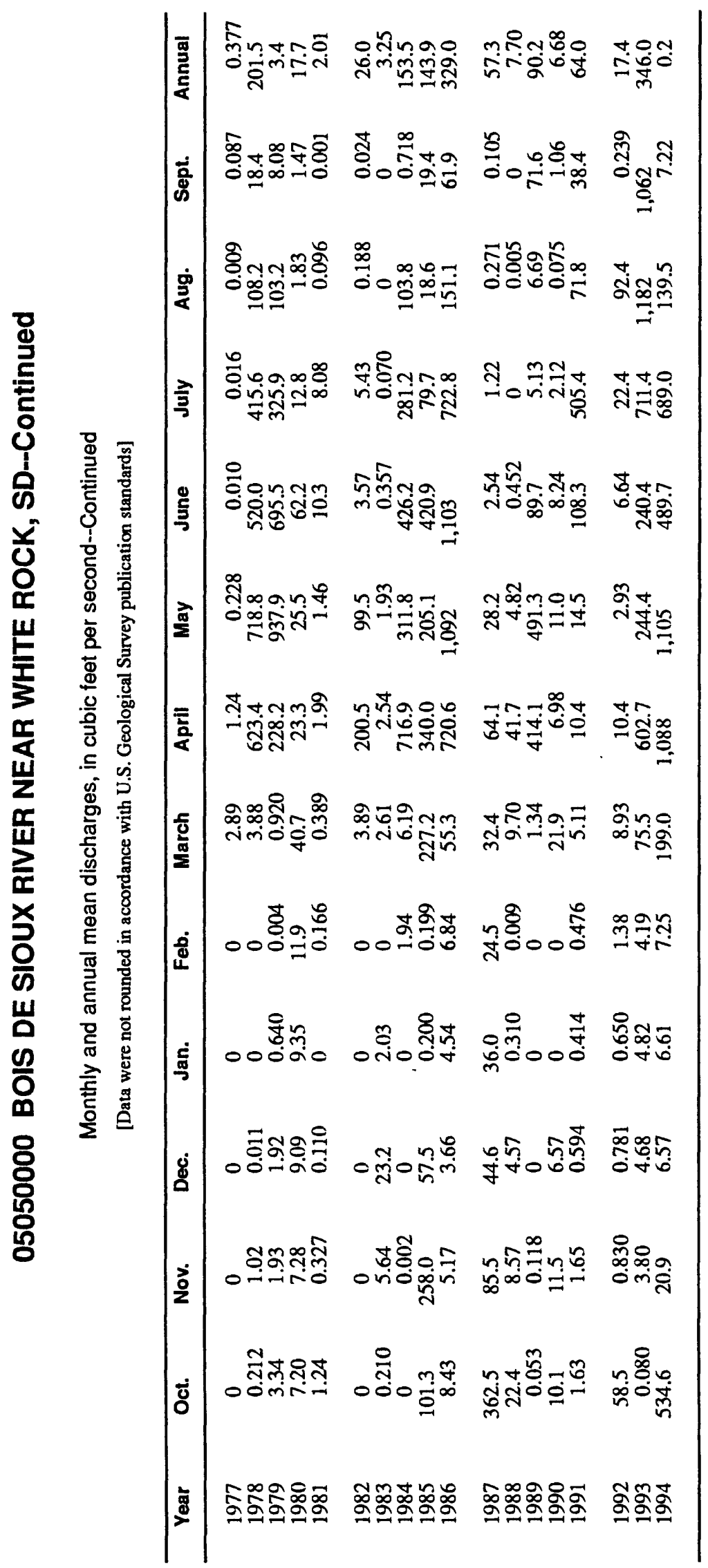




\section{BOIS DE SIOUX RIVER NEAR FAIRMOUNT, ND}

\section{Station Description}

LOCATION.--Lat 460.'00', long 96 33'25', on line between secs.22 and 27, T.130 N., R.47 W., Richland County, Hydrologic Unit 09020101, at bridge on Minnesota Highway 55 and North Dakota Highway 11, $0.75 \mathrm{mi}$ upstream from Minneapolis, St. Paul \& Sault Ste. Marie Railway bridge, $2 \mathrm{mi}$ east of Fairmount, $5 \mathrm{mi}$ west of Tenny, Minn., and $15 \mathrm{mi}$ downstream from Lake Traverse.

DRAINAGE AREA.--1,540 $\mathrm{mi}^{2}$, approximately.

PERIOD OF RECORD.--April 1919 to September 1944.

GAGE.--Chain gage. Datum of gage is $952.13 \mathrm{ft}$ above sea level, adjustment of 1912. Prior to October 1919, staff gage at same site at datum $10.00 \mathrm{ft}$ higher. Apr. 1, 1920, to Sept. 30, 1939, staff gage at site $0.75 \mathrm{mi}$ downstream at datum $8.95 \mathrm{ft}$ higher. Oct. 1, 1939, to Dec. 5, 1940, staff gage at present site at datum $10.00 \mathrm{ft}$ higher.

EXTREMES FOR PERIOD OF RECORD.--Maximum discharge, 1,430 $\mathrm{ft}^{3} / \mathrm{s}$, Apr. 4,1943; maximum gage height, $16.40 \mathrm{ft}$, Apr. 4, 1943; no flow at times in most years.

Annual mean discharge

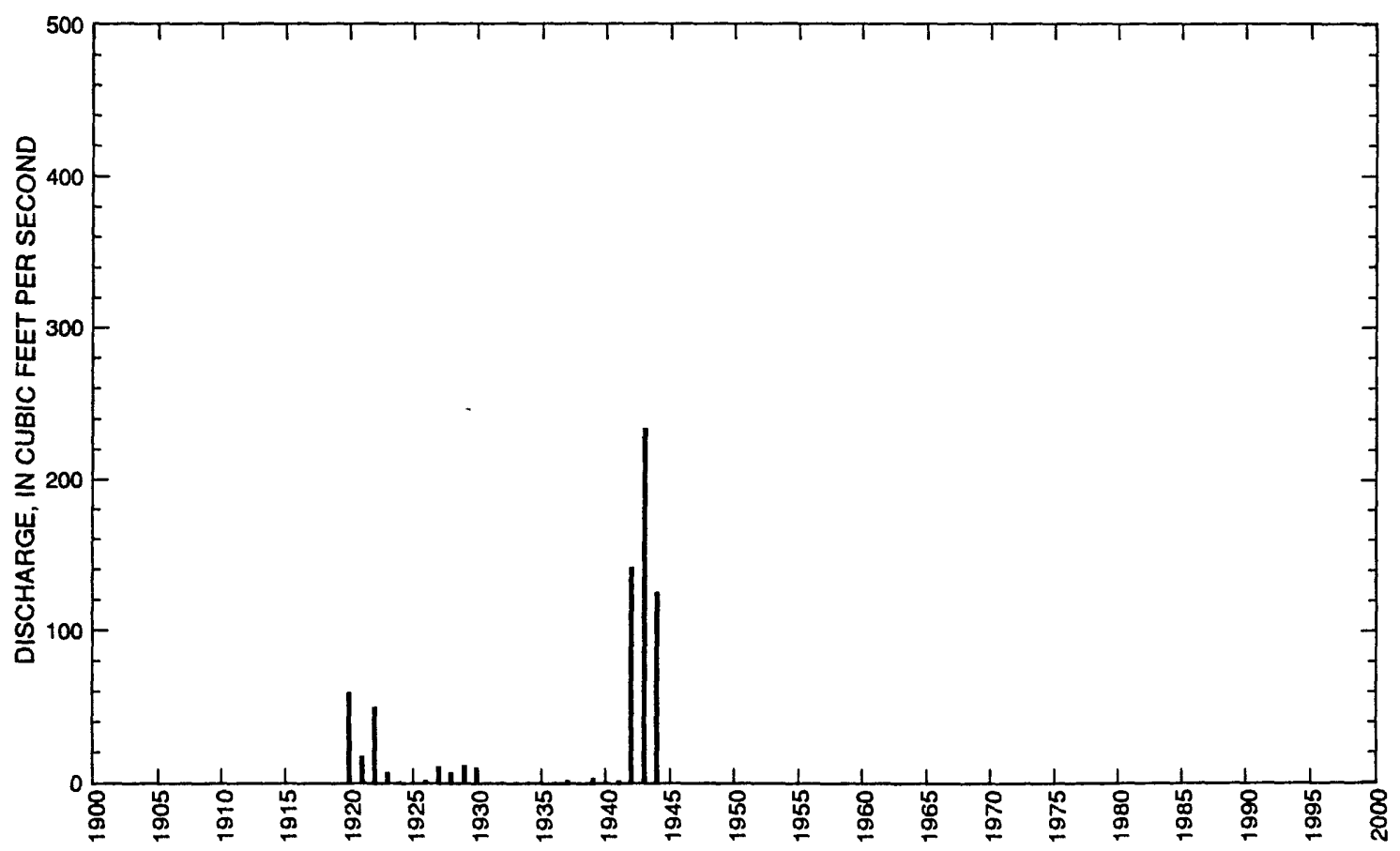


05050500 BOIS DE SIOUX RIVER NEAR FAIRMOUNT, ND-Continued

Statistics of monthly and annual mean discharges

[ $\mathrm{m}$, more than 1 year of occurrence]

\begin{tabular}{|c|c|c|c|c|c|c|c|c|}
\hline \multirow[b]{2}{*}{ Month } & \multicolumn{2}{|c|}{ Maximum } & \multicolumn{2}{|c|}{ Minimum } & \multicolumn{4}{|c|}{ Mean } \\
\hline & $\begin{array}{c}\text { Discharge } \\
\left(\mathrm{ft}^{3} / \mathrm{s}\right)\end{array}$ & $\begin{array}{c}\text { Water year } \\
\text { of } \\
\text { occurrence }\end{array}$ & $\begin{array}{c}\text { Discharge } \\
\left(\mathrm{ft}^{3} / \mathrm{s}\right)\end{array}$ & $\begin{array}{c}\text { Water year } \\
\text { of } \\
\text { occurrence }\end{array}$ & $\begin{array}{c}\text { Discharge } \\
\left(\mathrm{ft}^{3} / \mathrm{s}\right)\end{array}$ & $\begin{array}{l}\text { Standard } \\
\text { deviation } \\
\left(\mathrm{ft}^{3} / \mathrm{s}\right)\end{array}$ & $\begin{array}{l}\text { Coeffi- } \\
\text { cient of } \\
\text { variation }\end{array}$ & $\begin{array}{l}\text { Percentage } \\
\text { of annual } \\
\text { discharge }\end{array}$ \\
\hline October & 57.4 & 1943 & 0 & $\mathrm{~m}$ & 5.08 & 13.5 & 2.66 & 1.61 \\
\hline November & 23.5 & 1921 & 0 & $\mathrm{~m}$ & 1.83 & 5.01 & 2.73 & 0.58 \\
\hline December & 14.8 & 1921 & 0 & $\mathrm{~m}$ & 0.770 & 2.99 & 3.89 & 0.24 \\
\hline January & 6.74 & 1921 & 0 & $\mathrm{~m}$ & 0.270 & 1.35 & 5.00 & 0.09 \\
\hline February & 8.64 & 1930 & 0 & $\mathrm{~m}$ & 0.490 & 1.81 & 3.70 & 0.16 \\
\hline March & 72.3 & 1943 & 0 & $\mathrm{~m}$ & 11.7 & 19.6 & 1.67 & 3.72 \\
\hline April & 625 & 1943 & 0 & $\mathrm{~m}$ & 55.8 & 133 & 2.38 & 17.7 \\
\hline May & 928 & 1943 & 0 & $\mathrm{~m}$ & 73.4 & 191 & 2.60 & 23.3 \\
\hline June & 726 & 1943 & 0 & $\mathrm{~m}$ & 81.8 & 203 & 2.48 & 25.9 \\
\hline July & 662 & 1942 & 0 & $\mathrm{~m}$ & 56.1 & 149 & 2.66 & 17.8 \\
\hline August & 208 & 1942 & 0 & $\mathrm{~m}$ & 14.6 & 42.2 & 2.89 & 4.63 \\
\hline September & 250 & 1942 & 0 & $\mathrm{~m}$ & 13.5 & 49.3 & 3.66 & 4.27 \\
\hline Annual & 233 & 1943 & 0 & $\mathrm{~m}$ & 26.8 & 57.1 & 2.13 & 100 \\
\hline
\end{tabular}

Annual flow duration

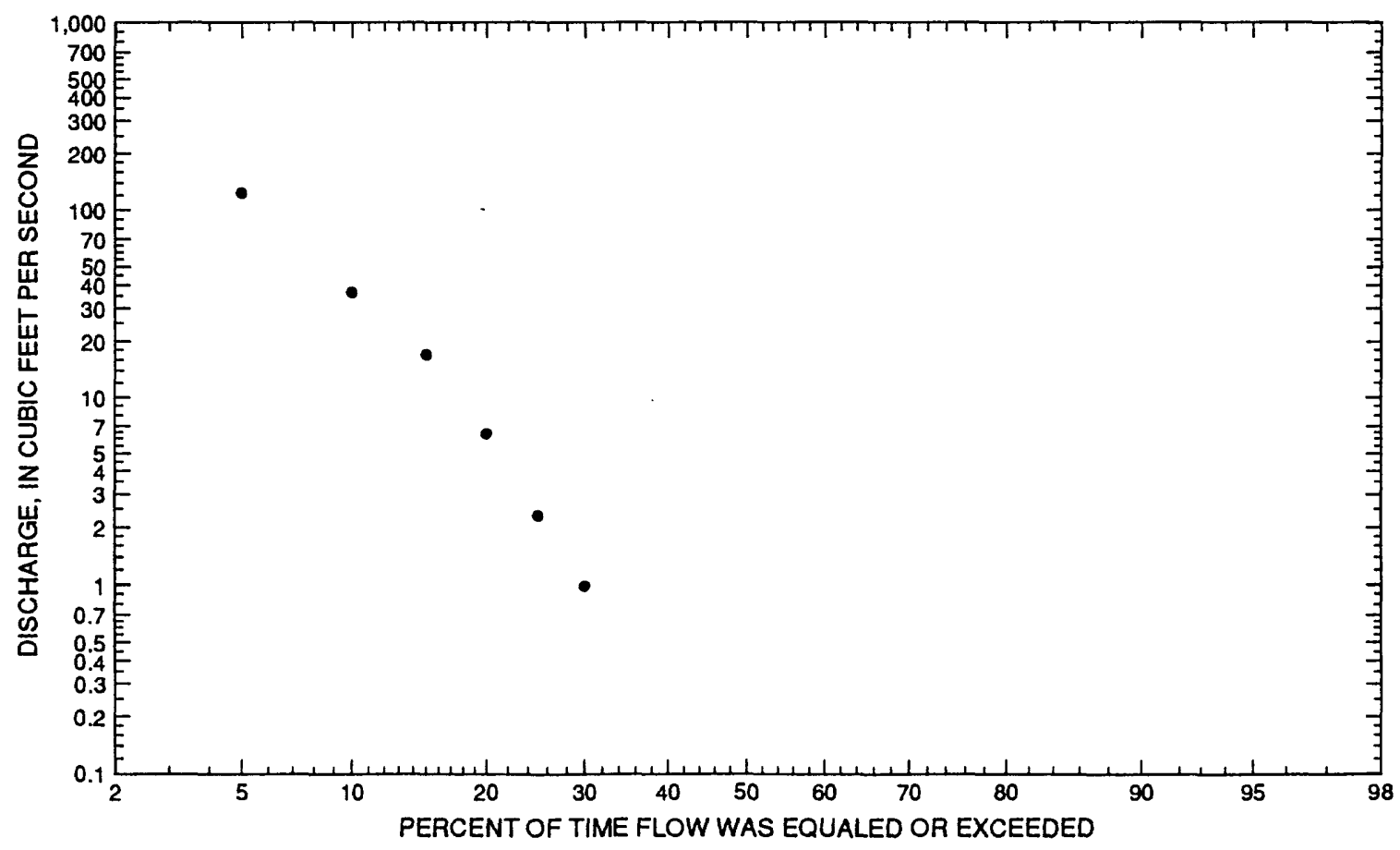




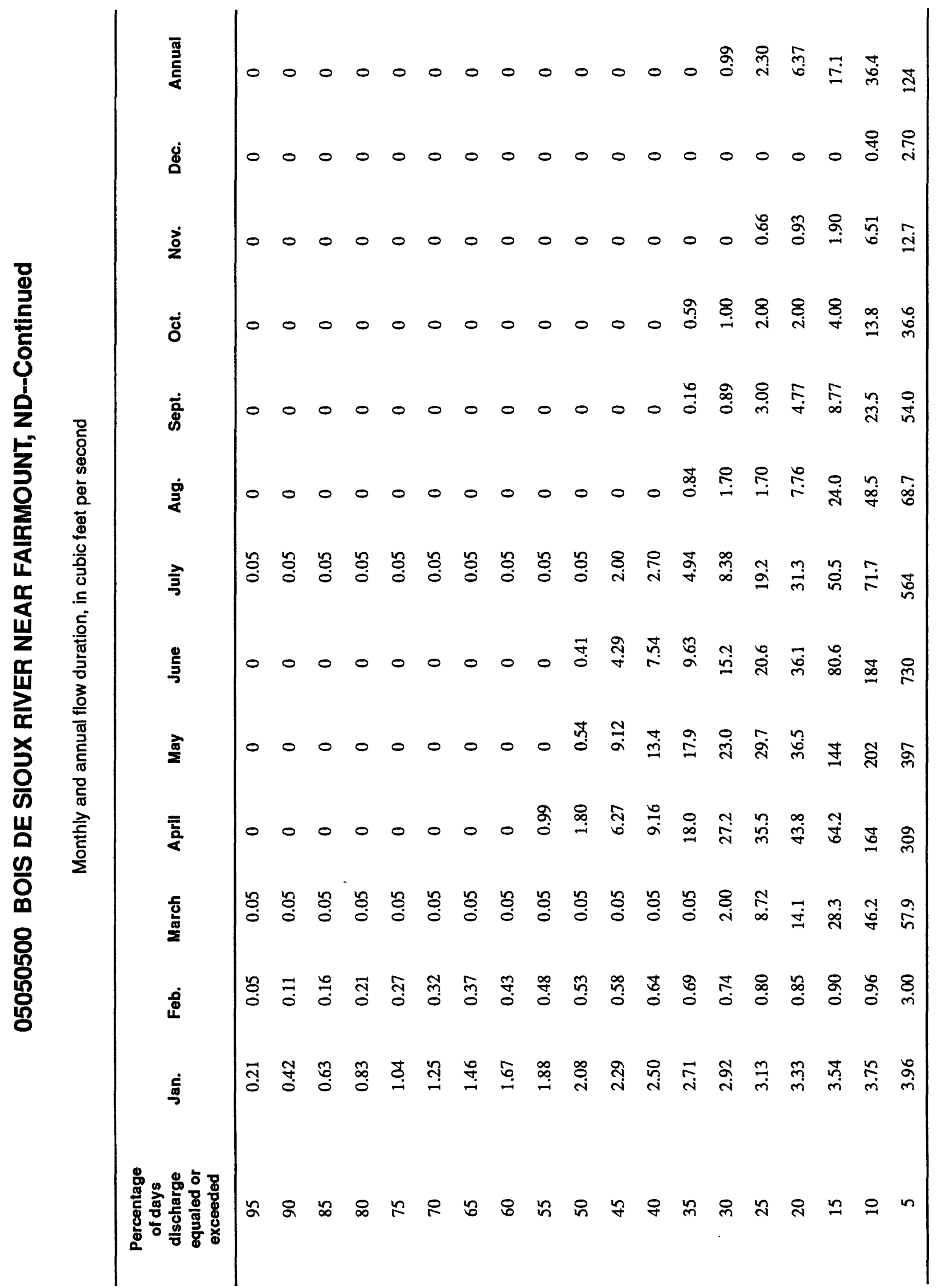


Probability of annual high discharges

[ng, statistic not given]

\begin{tabular}{lcccccc}
\hline & & & \multicolumn{5}{c}{$\begin{array}{c}\text { Maximum average discharge } \\
\text { (ft } \mathbf{3}^{3 / \mathbf{s})}\end{array}$} \\
\cline { 5 - 7 } $\begin{array}{c}\text { Exceedance } \\
\text { probability }\end{array}$ & $\begin{array}{c}\text { Recurrence } \\
\text { interval } \\
\text { (years) }\end{array}$ & $\begin{array}{c}\text { Maximum } \\
\text { Instantaneous } \\
\left(\mathrm{ft}^{3} / \mathbf{s}\right)\end{array}$ & 3-day period & 7-day period & 15-day period & 30-day period \\
\hline 0.99 & 1.01 & $\mathrm{ng}$ & 0 & 0 & 0 & 0 \\
0.95 & 1.05 & $\mathrm{ng}$ & 0 & 0 & 0 & 0 \\
0.90 & 1.11 & $\mathrm{ng}$ & 0 & 0 & 0 & 0 \\
0.80 & 1.25 & $\mathrm{ng}$ & 0 & 0 & 0 & 0 \\
0.50 & 2 & 27.8 & 24.1 & 20.4 & 15.5 & 11.1 \\
0.20 & 5 & 226 & 213 & 199 & 180 & 155 \\
0.10 & 10 & 590 & 525 & 485 & 436 & 385 \\
0.04 & 25 & 1,490 & 1,230 & 1,100 & 939 & 832 \\
0.02 & 50 & 2,570 & 2,060 & 1,780 & 1,450 & 1,270 \\
0.01 & 100 & 4,070 & 3,330 & 2,750 & 2,110 & 1,820 \\
0.005 & 200 & 6,040 & 5,050 & 3,990 & 2,880 & 2,430 \\
0.002 & 500 & 9,420 & $\mathrm{ng}$ & $\mathrm{ng}$ & $\mathrm{ng}$ & $\mathrm{ng}$ \\
\hline
\end{tabular}

Probability of annual low discharges

[ng, statistic not given]

\begin{tabular}{|c|c|c|c|c|c|c|c|c|c|c|}
\hline \multirow{3}{*}{$\begin{array}{l}\text { Non- } \\
\text { exceed- } \\
\text { ance } \\
\text { prob- } \\
\text { ability }\end{array}$} & \multirow{3}{*}{$\begin{array}{l}\text { Recur- } \\
\text { rence } \\
\text { inter- } \\
\text { val } \\
\text { (years) }\end{array}$} & \multicolumn{9}{|c|}{ MInimum average dlscharge $\left(\mathrm{ft}^{3} / \mathrm{s}\right)$} \\
\hline & & \multicolumn{9}{|c|}{ Number of consecutlve days } \\
\hline & & 1 & 3 & 7 & 14 & 30 & 60 & 90 & 120 & 183 \\
\hline 0.05 & 20 & ng & $\mathrm{ng}$ & ng & ng & ng & ng & $\mathrm{ng}$ & 0 & 0 \\
\hline 0.10 & 10 & ng & $\mathrm{ng}$ & ng & ng & $\mathrm{ng}$ & ng & ng & 0 & 0 \\
\hline 0.20 & 5 & ng & $\mathrm{ng}$ & $\mathbf{n g}$ & ng & ng & ng & ng & 0 & 0 \\
\hline 0.50 & 2 & $\mathrm{ng}$ & ng & ng & ng & ng & ng & ng & 0 & 0 \\
\hline
\end{tabular}




\section{BOIS DE SIOUX RIVER NEAR FAIRMOUNT, ND--Continued}

Probability of seasonal low discharges

[ng, statistic not given]

\begin{tabular}{|c|c|c|c|c|c|c|c|c|c|}
\hline \multirow[b]{3}{*}{$\begin{array}{c}\text { Non- } \\
\text { exceedance } \\
\text { probability }\end{array}$} & \multirow[b]{3}{*}{$\begin{array}{l}\text { Recurrence } \\
\text { interval } \\
\text { (years) }\end{array}$} & \multicolumn{8}{|c|}{ Minimum average discharge $\left(\mathrm{ft}^{3} / \mathrm{s}\right)$} \\
\hline & & \multicolumn{8}{|c|}{ Number of consecutive days } \\
\hline & & 1 & 7 & 14 & 30 & 1 & 7 & 14 & 30 \\
\hline & & \multicolumn{4}{|c|}{ December-January-February } & \multicolumn{4}{|c|}{ March-April-May } \\
\hline 0.05 & 20 & ng & $\mathrm{ng}$ & ng & ng & 0 & 0 & 0 & 0 \\
\hline 0.10 & 10 & ng & $\mathrm{ng}$ & ng & ng & 0 & 0 & 0 & 0 \\
\hline 0.20 & 5 & $\mathrm{ng}$ & $\mathrm{ng}$ & ng & ng & 0 & 0 & 0 & 0 \\
\hline \multirow[t]{2}{*}{0.50} & 2 & ng & ng & ng & ng & 0 & 0 & 0 & 0.062 \\
\hline & & \multicolumn{4}{|c|}{ June-July-August } & \multicolumn{4}{|c|}{ September-October-November } \\
\hline 0.05 & 20 & 0 & 0 & 0 & 0 & 0 & 0 & 0 & 0 \\
\hline 0.10 & 10 & 0 & 0 & 0 & 0 & 0 & 0 & 0 & 0 \\
\hline 0.20 & 5 & 0 & 0 & 0 & 0 & 0 & 0 & 0 & 0 \\
\hline 0.50 & 2 & 0 & 0 & 0 & 0 & 0 & 0 & 0 & 0 \\
\hline
\end{tabular}




\section{BOIS DE SIOUX RIVER NEAR FAIRMOUNT, ND--Continued}

Annual peak discharge and corresponding gage height, period of record

$$
[--, \text { no data }]
$$

\begin{tabular}{|c|c|c|c|c|c|c|c|}
\hline $\begin{array}{l}\text { Water } \\
\text { year }\end{array}$ & Date & $\begin{array}{c}\text { Gage } \\
\text { height } \\
\text { (feet) }\end{array}$ & $\begin{array}{c}\text { Peak } \\
\text { discharge } \\
\left(\mathrm{ft}^{3} / \mathrm{s}\right) \\
\end{array}$ & $\begin{array}{l}\text { Water } \\
\text { year }\end{array}$ & Date & $\begin{array}{c}\text { Gage } \\
\text { height } \\
\text { (feet) }\end{array}$ & $\begin{array}{c}\text { Peak } \\
\text { discharge } \\
\left(\mathrm{ft}^{3} / \mathbf{s}\right)\end{array}$ \\
\hline \multicolumn{8}{|c|}{ Annual peak discharge, by year, and corresponding gage height } \\
\hline 1920 & April 2 & 5.00 & 250 & 1933 & -- & 5.70 & 0 \\
\hline 1921 & April 2 & -- & 67.0 & 1934 & - & 5.70 & 0 \\
\hline 1922 & April 21 & 5.70 & 390 & 1935 & -- & 5.70 & $\mathbf{0}$ \\
\hline 1923 & April 14 & 3.65 & 80.0 & 1936 & April 13 & 5.70 & 2.50 \\
\hline 1924 & -- & -- & 0 & 1937 & May 1 & 3.15 & 20.0 \\
\hline 1925 & -- & - & 0 & 1938 & -- & 5.70 & 0 \\
\hline 1926 & March 18 & -- & 32.0 & 1939 & March 29 & 5.40 & 105 \\
\hline 1927 & April 2 & 3.50 & 70.0 & 1940 & - & - & 0 \\
\hline 1928 & - & 3.50 & 40.0 & 1941 & June 21 & -- & 130 \\
\hline 1929 & - & 3.90 & 53.0 & 1942 & June 7 & 11.72 & 1,180 \\
\hline 1930 & -- & 3.90 & 64.0 & 1943 & April 4 & 16.40 & 1,430 \\
\hline 1931 & -- & 5.70 & 0 & 1944 & June 23 & - & 1,180 \\
\hline 1932 & - & 5.70 & 0 & & & & \\
\hline \multicolumn{8}{|c|}{ Annual peak discharge, from highest to lowest, and corresponding gage height } \\
\hline 1943 & April 4 & 16.40 & 1,430 & 1926 & March 18 & - & 32.0 \\
\hline 1944 & June 23 & -- & 1,180 & 1937 & May 1 & 3.15 & 20.0 \\
\hline 1942 & June 7 & 11.72 & 1,180 & 1936 & April 13 & 5.70 & 2.50 \\
\hline 1922 & April 21 & 5.70 & 390 & 1924 & - & - & 0 \\
\hline 1920 & April 2 & 5.00 & 250 & 1925 & -- & - & 0 \\
\hline 1941 & June 21 & -- & 130 & 1931 & -- & 5.70 & 0 \\
\hline 1939 & March 29 & 5.40 & 105 & 1932 & -- & 5.70 & 0 \\
\hline 1923 & April 14 & 3.65 & 80.0 & 1933 & -- & 5.70 & 0 \\
\hline 1927 & April 2 & 3.50 & 70.0 & 1934 & - & 5.70 & 0 \\
\hline 1921 & April 2 & -- & 67.0 & 1935 & -- & 5.70 & 0 \\
\hline 1930 & -- & 3.90 & 64.0 & 1938 & -- & 5.70 & 0 \\
\hline 1929 & -- & 3.90 & 53.0 & 1940 & -- & - & 0 \\
\hline 1928 & -- & 3.50 & 40.0 & & & & \\
\hline
\end{tabular}




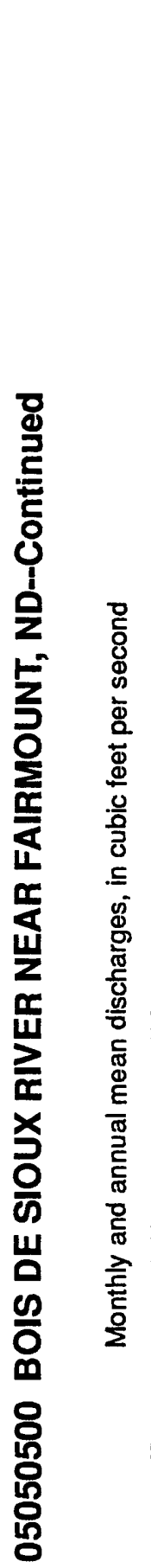

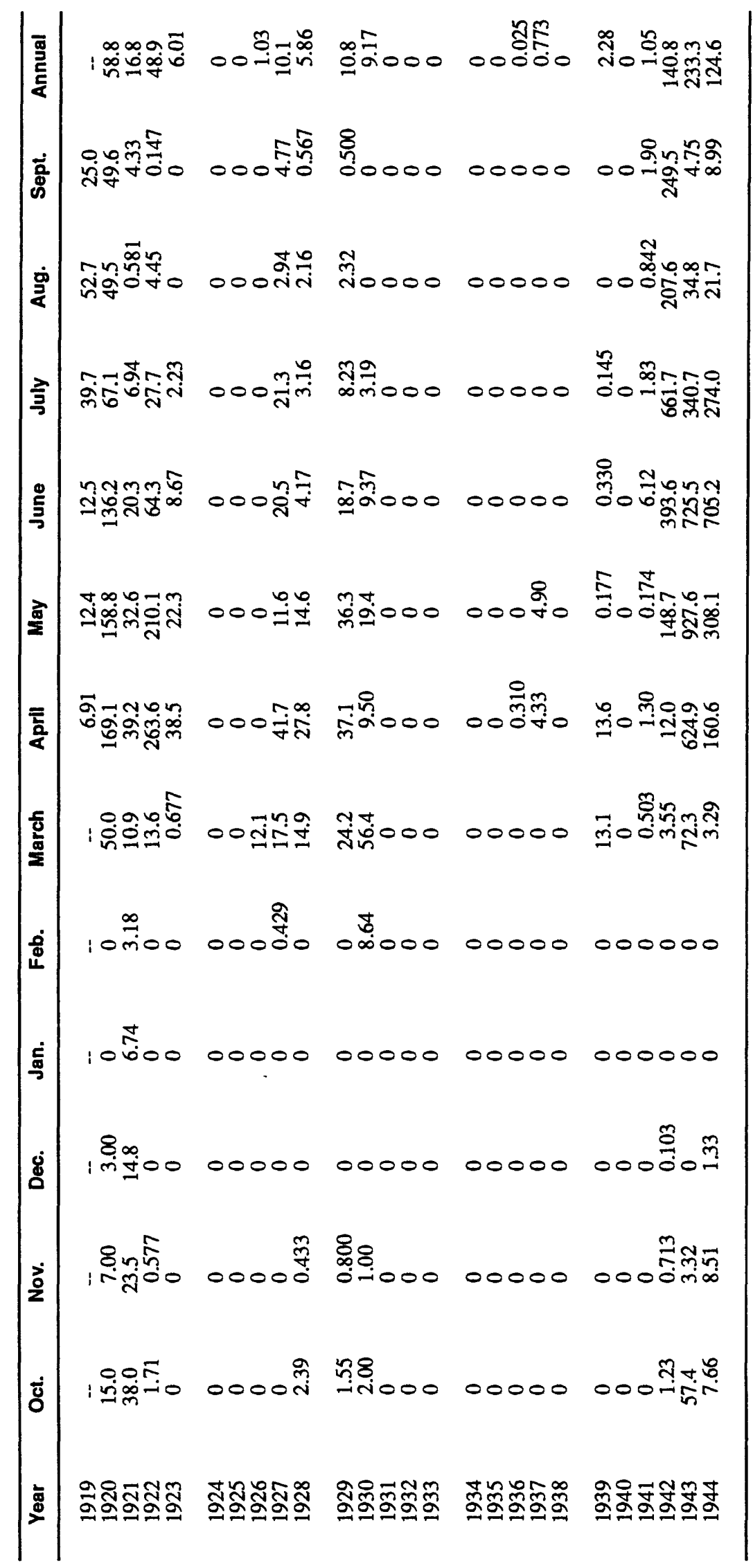




\title{
05051000 RABBIT CREEK AT CAMPBELL, MN
}

\author{
Station Description
}

LOCATION.--Lat $46^{\circ} 05^{\prime} 40^{\prime \prime}$, long $96^{\circ} 24^{\prime} 40^{\prime \prime}$, in $\mathrm{SE}^{1} / 4^{\mathrm{SE}}{ }^{1} / 4 \mathrm{sec} .2$, T.130 N., R.46 W., Wilken County, Hydrologic Unit 09020101, near center of span on upstream side of highway bridge in Campbell, $1 \mathrm{mi}$ downstream from South Fork and 10 mi upstream from mouth.

DRAINAGE AREA.--266 $\mathrm{mi}^{2}$.

PERIOD OF RECORD.--June 1942 to November 1951.

GAGE.--Wire-weight gage. Datum of gage is $970 \mathrm{ft}$ (from topographic map). Prior to Mar. 29, 1943, staff gage and Apr. 8, 1943, to Aug. 10, 1948, chain gage, at same site and datum.

EXTREMES FOR PERIOD OF RECORD.--Maximum discharge, 1,940 ft 3 s, Apr. 7, 1951 (gage height, $12.10 \mathrm{ft}$ ); maximum gage height, $15.07 \mathrm{ft}$, Apr. 2, 1943, from floodmark (backwater from ice); no flow during large part of each year.

EXTREMES OUTSIDE PERIOD OF RECORD.--Maximum discharge 7,000 ft³/s, April 1952 (gage height, $16.4 \mathrm{ft}$, backwater from ice).

Annual mean discharge

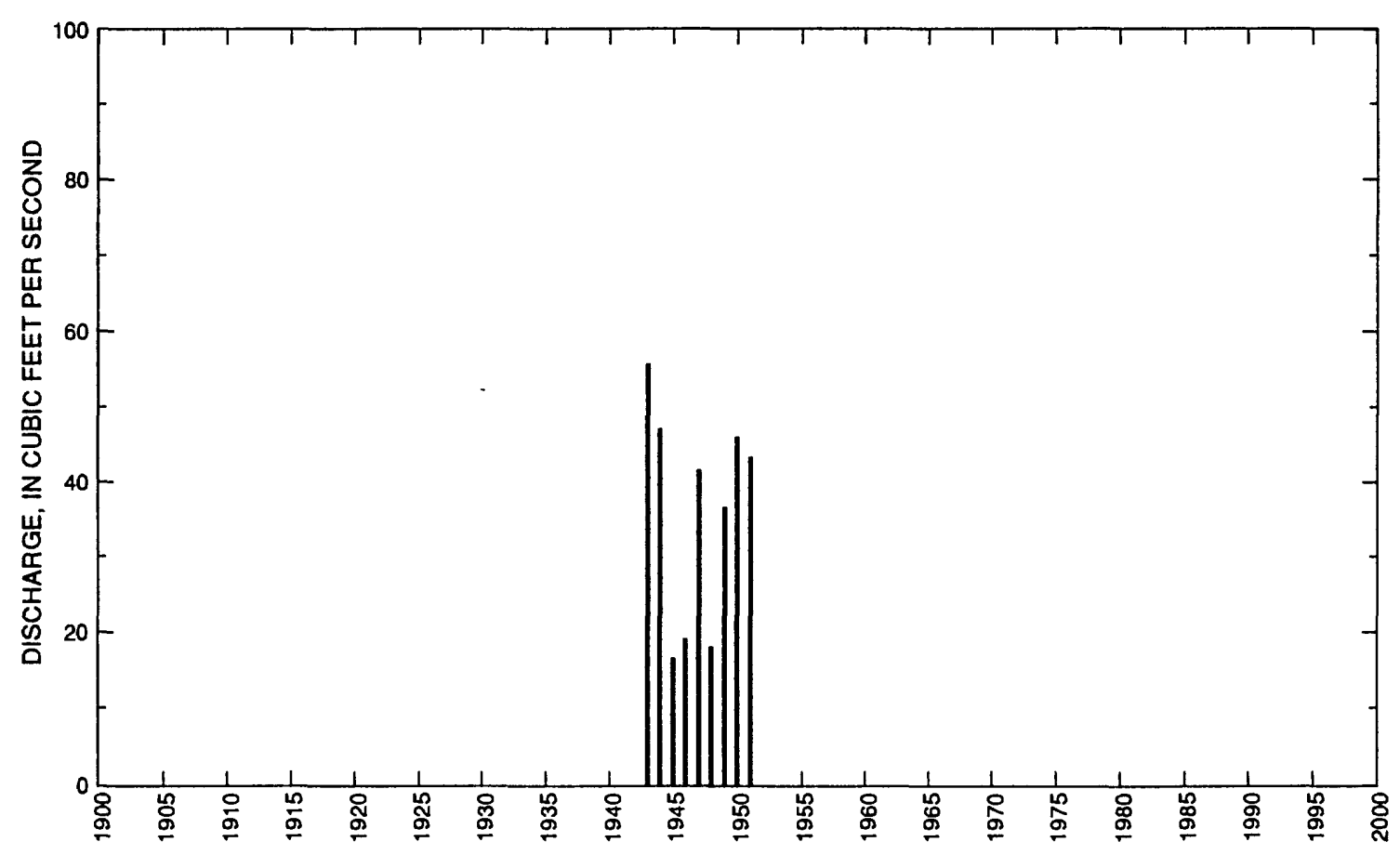




\section{RABBIT CREEK AT CAMPBELL, MN-Continued}

Statistics of monthly and annual mean discharges

[m, more than 1 year of occurrence; ng, statistic not given]

\begin{tabular}{|c|c|c|c|c|c|c|c|c|}
\hline \multirow[b]{2}{*}{ Month } & \multicolumn{2}{|c|}{ Maximum } & \multicolumn{2}{|c|}{ Minimum } & \multicolumn{4}{|c|}{ Mean } \\
\hline & $\begin{array}{c}\text { Discharge } \\
\left(\mathrm{ft}^{3} / \mathbf{s}\right)\end{array}$ & $\begin{array}{c}\text { Water year } \\
\text { of } \\
\text { occurrence }\end{array}$ & $\begin{array}{c}\text { Discharge } \\
\left(\mathrm{ft}^{3} / \mathrm{s}\right)\end{array}$ & $\begin{array}{c}\text { Water year } \\
\text { of } \\
\text { occurrence }\end{array}$ & $\begin{array}{c}\text { Discharge } \\
\left(\mathrm{ft}^{3} / \mathrm{s}\right)\end{array}$ & $\begin{array}{c}\text { Standard } \\
\text { devlatton } \\
\left(\mathrm{ft}^{3} / \mathrm{s}\right)\end{array}$ & $\begin{array}{l}\text { Coeffl- } \\
\text { clent of } \\
\text { variation }\end{array}$ & $\begin{array}{l}\text { Percentage } \\
\text { of annual } \\
\text { dlscharge }\end{array}$ \\
\hline October & 1.56 & 1944 & 0 & $\mathrm{~m}$ & 0.590 & 0.61 & 1.03 & 0.14 \\
\hline November & 2.20 & 1944 & 0 & $\mathrm{~m}$ & 0.530 & 0.68 & 1.29 & 0.12 \\
\hline December & 0.226 & 1944 & 0 & $\mathrm{~m}$ & 0.040 & 0.08 & 1.87 & 0.01 \\
\hline January & 0 & $\mathrm{~m}$ & 0 & $\mathrm{~m}$ & 0 & 0 & $\mathrm{ng}$ & 0 \\
\hline February & 0 & $\mathrm{~m}$ & 0 & $\mathrm{~m}$ & 0 & 0 & $\mathrm{ng}$ & 0 \\
\hline March & 188 & 1946 & 4.55 & 1951 & 60.2 & 70.1 & 1.16 & 13.9 \\
\hline April & 488 & 1951 & 14.3 & 1945 & 202 & 196 & 0.97 & 46.9 \\
\hline May & 279 & 1951 & 0.190 & 1946 & 55.2 & 101 & 1.83 & 12.8 \\
\hline June & 324 & 1944 & 0 & 1946 & 56.8 & 107 & 1.89 & 13.1 \\
\hline July & 335 & 1949 & 0.168 & 1945 & 51.5 & 108 & 2.10 & 11.9 \\
\hline August & 24.1 & 1943 & 0 & $\mathrm{~m}$ & 4.11 & 8.33 & 2.03 & 0.95 \\
\hline September & 4.67 & 1943 & 0 & $\mathrm{~m}$ & 0.670 & 1.51 & 2.27 & 0.15 \\
\hline Annual & 55.7 & 1943 & 16.5 & 1945 & 36.0 & 14.5 & 0.40 & 100 \\
\hline
\end{tabular}

Annual flow duration

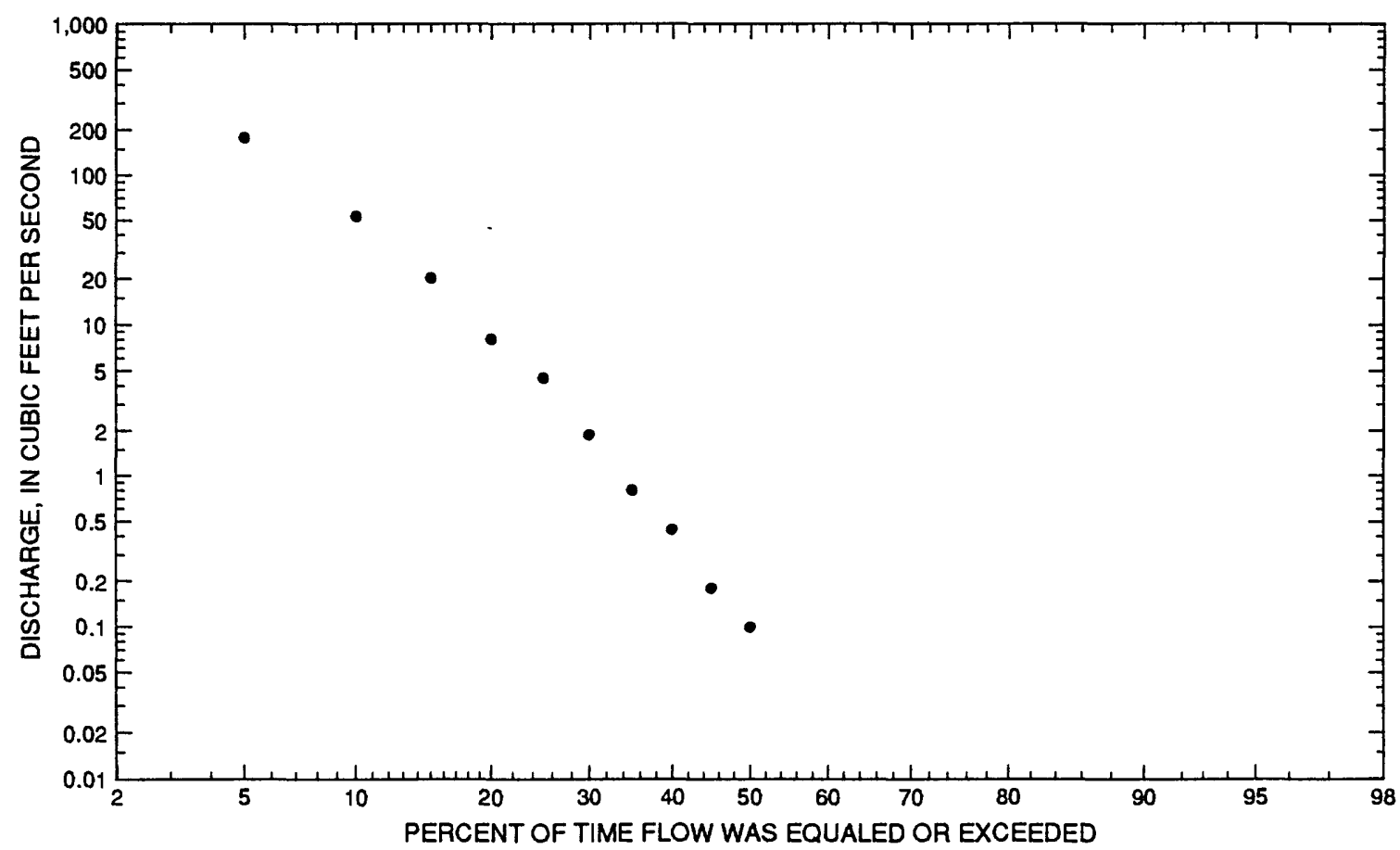




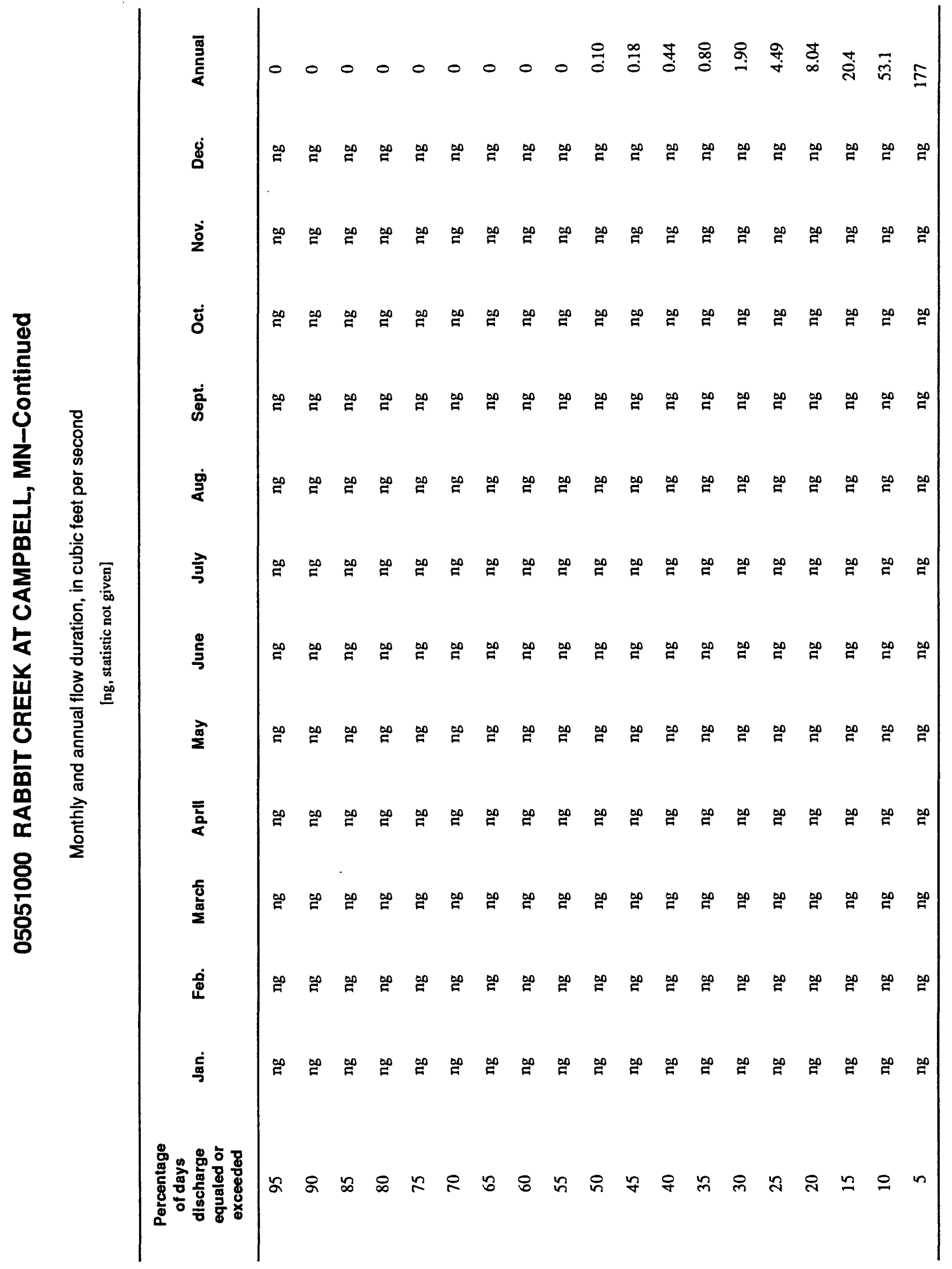




\section{RABBIT CREEK AT CAMPBELL, MN-Continued}

Probability of annual high discharges

[ng, statistic not given]

\begin{tabular}{|c|c|c|c|c|c|c|}
\hline \multirow[b]{2}{*}{$\begin{array}{l}\text { Exceedance } \\
\text { probability }\end{array}$} & \multirow[b]{2}{*}{$\begin{array}{l}\text { Recurrence } \\
\text { interval } \\
\text { (years) }\end{array}$} & \multirow[b]{2}{*}{$\begin{array}{c}\text { Maximum } \\
\text { Instantaneous } \\
\left(\mathrm{f}^{3} / \mathrm{s}\right)\end{array}$} & \multicolumn{4}{|c|}{$\begin{array}{c}\text { Maximum average discharge } \\
\left(\mathrm{ft}^{3} / \mathrm{s}\right)\end{array}$} \\
\hline & & & 3-day perlod & 7-day perlod & 15-day perlod & 30-day perlod \\
\hline 0.99 & 1.01 & 258 & 281 & 312 & 229 & 119 \\
\hline 0.95 & 1.05 & 421 & 433 & 420 & 301 & 166 \\
\hline 0.90 & 1.11 & 548 & 536 & 490 & 347 & 197 \\
\hline 0.80 & 1.25 & 754 & 684 & 591 & 411 & 238 \\
\hline 0.50 & 2 & 1,400 & 1,040 & 839 & 561 & 333 \\
\hline 0.20 & 5 & 2,610 & 1,500 & 1,180 & 755 & 448 \\
\hline 0.10 & 10 & 3,630 & 1,770 & 1,410 & 877 & 516 \\
\hline 0.04 & 25 & 5,160 & 2,090 & 1,700 & 1,020 & 592 \\
\hline 0.02 & 50 & 6,490 & 2,300 & 1,910 & 1,130 & 643 \\
\hline 0.01 & 100 & 7,980 & 2,500 & 2,120 & 1,230 & 691 \\
\hline 0.005 & 200 & 9,660 & 2,690 & 2,340 & 1,330 & 735 \\
\hline 0.002 & 500 & 12,200 & $\mathrm{ng}$ & $\mathrm{ng}$ & $\mathrm{ng}$ & ng \\
\hline
\end{tabular}

Probability of annual low discharges

[ng, statistic not given]

\begin{tabular}{|c|c|c|c|c|c|c|c|c|c|c|}
\hline \multirow[b]{3}{*}{$\begin{array}{l}\text { Non- } \\
\text { exceed- } \\
\text { ance } \\
\text { prob- } \\
\text { abllity }\end{array}$} & \multirow[b]{3}{*}{$\begin{array}{l}\text { Recur- } \\
\text { rence } \\
\text { inter- } \\
\text { val } \\
\text { (years) }\end{array}$} & \multicolumn{9}{|c|}{ Minimum average dlscharge $\left(\mathrm{ft}^{3} / \mathrm{s}\right)$} \\
\hline & & \multicolumn{9}{|c|}{ Number of consecutlve days } \\
\hline & & 1 & 3 & 7 & 14 & 30 & 60 & 90 & 120 & 183 \\
\hline 0.05 & 20 & ng & ng & ng & ng & ng & ng & ng & 0 & 0 \\
\hline 0.10 & 10 & ng & ng & ng & ng & ng & ng & ng & 0 & 0 \\
\hline 0.20 & 5 & ng & ng & ng & ng & ng & ng & ng & 0 & 0 \\
\hline 0.50 & 2 & ng & ng & ng & ng & ng & ng & ng & 0 & 0.061 \\
\hline
\end{tabular}




\section{RABBIT CREEK AT CAMPBELL, MN-Continued}

Probability of seasonal low discharges

[ng, statistic not given]

\begin{tabular}{|c|c|c|c|c|c|c|c|c|c|}
\hline \multirow[b]{3}{*}{$\begin{array}{c}\text { Non- } \\
\text { exceedance } \\
\text { probability }\end{array}$} & \multirow[b]{3}{*}{$\begin{array}{l}\text { Recurrence } \\
\text { Interval } \\
\text { (years) }\end{array}$} & \multicolumn{8}{|c|}{ Minimum average discharge $\left(\mathrm{ft}^{3} / \mathrm{s}\right)$} \\
\hline & & \multicolumn{8}{|c|}{ Number of consecutive days } \\
\hline & & 1 & 7 & 14 & 30 & 1 & 7 & 14 & 30 \\
\hline & & \multicolumn{4}{|c|}{ December-January-February } & \multicolumn{4}{|c|}{ March-April-May } \\
\hline 0.05 & 20 & $\mathrm{ng}$ & ng & ng & ng & ng & ng & 0 & 0.199 \\
\hline 0.10 & 10 & ng & ng & $\mathrm{ng}$ & ng & ng & ng & 0 & 0.444 \\
\hline 0.20 & 5 & $\mathrm{ng}$ & ng & ng & ng & ng & ng & 0 & 1.06 \\
\hline \multirow[t]{2}{*}{0.50} & 2 & ng & ng & ng & $\mathrm{ng}$ & ng & ng & 0 & 4.09 \\
\hline & & \multicolumn{4}{|c|}{ June-July-August } & \multicolumn{4}{|c|}{ September-October-November } \\
\hline 0.05 & 20 & 0 & 0 & 0 & 0 & ng & $\mathrm{ng}$ & $\mathrm{ng}$ & 0 \\
\hline 0.10 & 10 & 0 & 0 & 0 & 0 & $\mathrm{ng}$ & $\mathrm{ng}$ & $\mathrm{ng}$ & 0 \\
\hline 0.20 & 5 & 0 & 0 & 0 & 0 & $\mathrm{ng}$ & $\mathrm{ng}$ & $\mathrm{ng}$ & 0 \\
\hline 0.50 & 2 & 0 & 0 & 0 & 0.233 & $\mathrm{ng}$ & $\mathrm{ng}$ & $\mathrm{ng}$ & 0 \\
\hline
\end{tabular}




\section{RABBIT CREEK AT CAMPBELL, MN-Continued}

Annual peak discharge and corresponding gage height, period of record

$$
[--, \text { no data }]
$$

\begin{tabular}{|c|c|c|c|c|c|c|c|}
\hline $\begin{array}{l}\text { Water } \\
\text { year }\end{array}$ & Date & $\begin{array}{c}\text { Gage } \\
\text { height } \\
\text { (feet) }\end{array}$ & $\begin{array}{c}\text { Peak } \\
\text { discharge } \\
\left(\mathrm{ft}^{3} / \mathrm{s}\right)\end{array}$ & $\begin{array}{l}\text { Water } \\
\text { year }\end{array}$ & Date & $\begin{array}{c}\text { Gage } \\
\text { height } \\
\text { (feet) }\end{array}$ & $\begin{array}{c}\text { Peak } \\
\text { discharge } \\
\left(\mathrm{ft}^{3} / \mathrm{s}\right)\end{array}$ \\
\hline \multicolumn{8}{|c|}{ Annual peak discharge, by year, and corresponding gage height } \\
\hline 1943 & April 3 & - & 1,850 & 1948 & April 3 & 10.82 & 734 \\
\hline 1944 & June 4 & 11.88 & 1,860 & 1949 & July 9 & 9.53 & 1,300 \\
\hline 1945 & March 15 & - & 495 & 1950 & May 9 & - & 1,430 \\
\hline 1946 & March 21 & - & 674 & 1951 & April 7 & 12.10 & 1,940 \\
\hline 1947 & April 11 & 10.05 & 1,410 & 1952 & April $^{1}$ & 16.40 & 7,000 \\
\hline \multicolumn{8}{|c|}{ Annual peak discharge, from highest to lowest, and corresponding gage height } \\
\hline 1952 & April $^{1}$ & 16.40 & 7,000 & 1947 & April 11 & 10.05 & 1,410 \\
\hline 1951 & April 7 & 12.10 & 1,940 & 1949 & July 9 & 9.53 & 1,300 \\
\hline 1944 & June 4 & 11.88 & 1,860 & 1948 & April 3 & 10.82 & 734 \\
\hline 1943 & April 3 & - & 1,850 & 1946 & March 21 & - & 674 \\
\hline 1950 & May 9 & - & 1,430 & 1945 & March 15 & - & 495 \\
\hline
\end{tabular}

${ }^{1}$ Day of month unknown. 


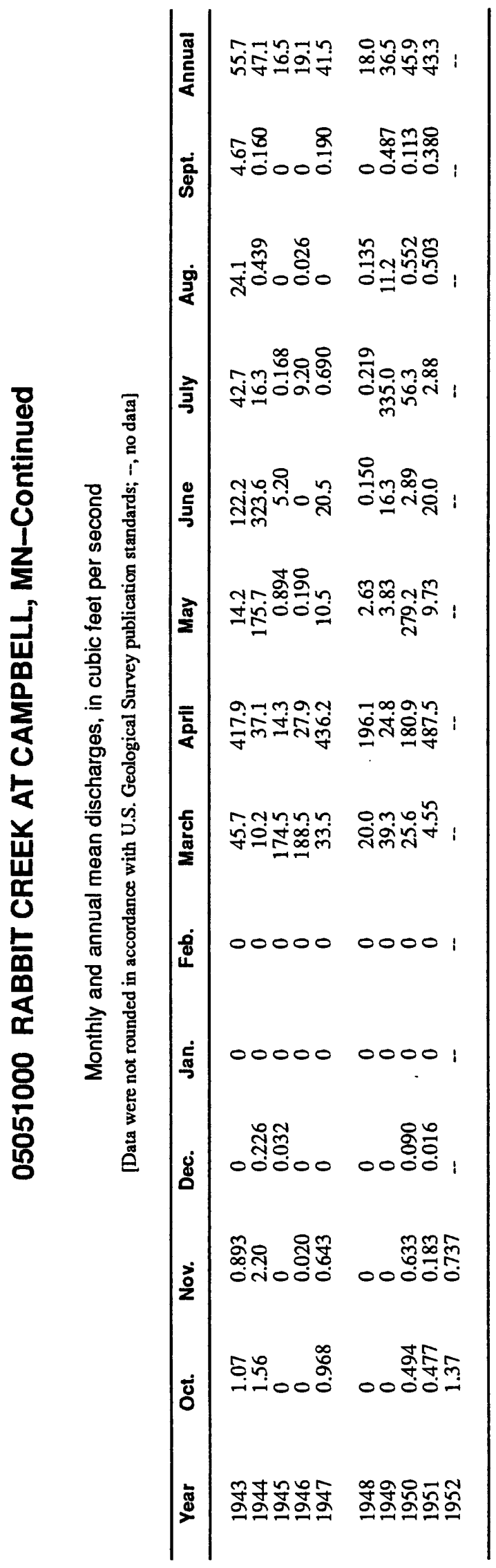




\title{
05051500 RED RIVER OF THE NORTH AT WAHPETON, ND
}

\author{
Station Description
}

LOCATION.--Lat $46^{\circ} 15^{\prime} 55^{\prime \prime}$, long $96^{\circ} 35^{\prime} 40^{\prime \prime}$, in NE ${ }^{1 / 4}$ sec.8, T.132 N., R.47 W., Richland County, Hydrologic Unit 09020104, on left bank in Wahpeton, $800 \mathrm{ft}$ downstream from confluence of Bois de Sioux and Otter Tail Rivers, and at mile 548.6.

DRAINAGE AREA.--4,010 $\mathrm{mi}^{2}$, approximately.

PERIOD OF RECORD.--April 1942 to October 1942, March 1943 to current year. Gage-height records collected in this vicinity since 1917 are contained in reports of the National Weather Service.

GAGE.--Water-stage recorder and concrete and wooden dam. Datum of gage is $942.97 \mathrm{ft}$ above sea level. Prior to Aug. 6, 1943, National Weather Service nonrecording gage $800 \mathrm{ft}$ downstream, converted to present datum. Aug. 6, 1943, to Oct. 27, 1950, nonrecording gage at present site and datum.

EXTREMES FOR PERIOD OF RECORD.--Maximum discharge, 9,200 $\mathrm{ft}^{3} / \mathrm{s}$, Apr. 10, 1969 (gage height, $16.34 \mathrm{ft}$ ); maximum gage height, $17.95 \mathrm{ft}$, Apr. 5, 1989; minimum daily discharge, $1.7 \mathrm{ft}^{3} / \mathrm{s}$, Aug. 28 to Sept. 5, 1976, and Sept. 9-10, 1976; minimum gage height 0.63 feet, Aug. 29, 1976.

EXTREMES OUTSIDE PERIOD OF RECORD.--A stage of $17.0 \mathrm{ft}$, discharge, $10,500 \mathrm{ft}^{3} / \mathrm{s}$, occurred in the spring of 1897 and has not been exceeded since.

Annual mean discharge

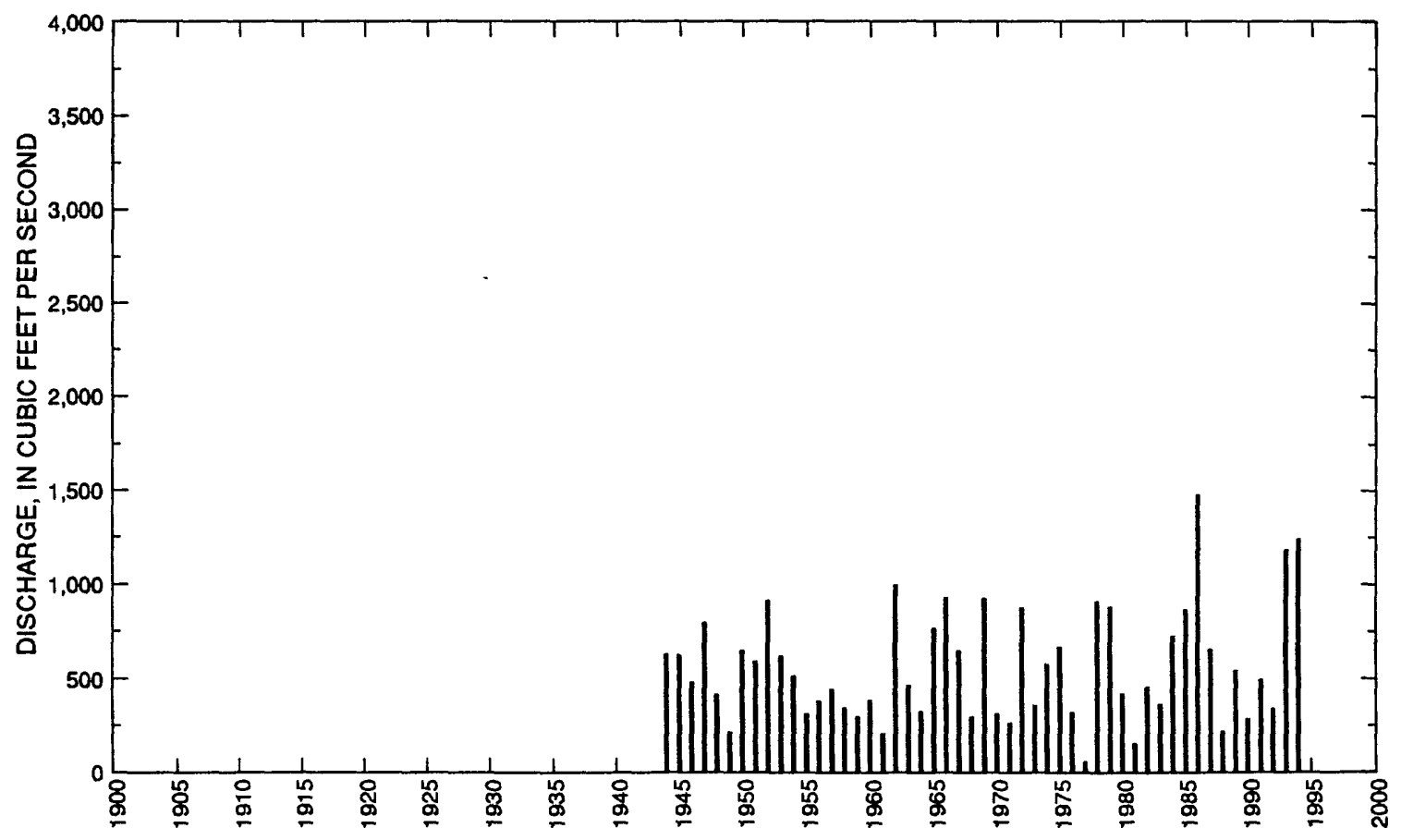




\section{RED RIVER OF THE NORTH AT WAHPETON, ND-Continued}

Statistics of monthly and annual mean discharges

[m, more than 1 year of occurrence]

\begin{tabular}{|c|c|c|c|c|c|c|c|c|}
\hline \multirow[b]{2}{*}{ Month } & \multicolumn{2}{|c|}{ Maximum } & \multicolumn{2}{|c|}{ Minimum } & \multicolumn{4}{|c|}{ Mean } \\
\hline & $\begin{array}{c}\text { Discharge } \\
\left(\mathrm{ft}^{3} / \mathrm{s}\right)\end{array}$ & $\begin{array}{c}\text { Water year } \\
\text { of } \\
\text { occurrence }\end{array}$ & $\begin{array}{c}\text { Discharge } \\
\left(\mathrm{ft}^{3} / \mathbf{s}\right)\end{array}$ & $\begin{array}{c}\text { Water year } \\
\text { of } \\
\text { occurrence }\end{array}$ & $\begin{array}{c}\text { Discharge } \\
\left(\mathrm{ft}^{3} / \mathrm{s}\right)\end{array}$ & $\begin{array}{l}\text { Standard } \\
\text { devlation } \\
\left(\mathrm{ft}^{3 / 3}\right)\end{array}$ & $\begin{array}{l}\text { Coeffi- } \\
\text { clent of } \\
\text { variation }\end{array}$ & $\begin{array}{l}\text { Percentage } \\
\text { of annual } \\
\text { discharge }\end{array}$ \\
\hline October & 1,600 & 1994 & 5.72 & 1977 & 320 & 287 & 0.90 & 4.69 \\
\hline November & 952 & 1987 & 7.40 & 1977 & 296 & 211 & 0.71 & 4.34 \\
\hline December & 820 & 1987 & 6.60 & 1977 & 266 & 180 & 0.68 & 3.90 \\
\hline January & 678 & 1986 & 8.81 & 1977 & 258 & 159 & 0.61 & 3.78 \\
\hline February & 687 & 1987 & 18.0 & 1977 & 268 & 151 & 0.57 & 3.92 \\
\hline March & 1,970 & 1994 & 84.3 & 1977 & 604 & 426 & 0.70 & 8.85 \\
\hline April & 4,440 & 1969 & 138 & 1977 & 1,230 & 963 & 0.78 & 18.1 \\
\hline May & 3,080 & 1986 & 22.5 & 1977 & 1,020 & 669 & 0.65 & 15.0 \\
\hline June & 2,680 & 1962 & 90.0 & 1977 & 1,030 & 665 & 0.65 & 15.0 \\
\hline July & 2,790 & 1993 & 65.6 & 1977 & 777 & 610 & 0.79 & 11.4 \\
\hline August & 2,500 & 1993 & 53.5 & $\mathrm{~m}$ & 419 & 434 & 1.03 & 6.14 \\
\hline September & 2,150 & 1993 & 2.18 & 1976 & 332 & 365 & 1.10 & 4.87 \\
\hline Annual & 1,480 & 1986 & 54.0 & 1977 & 564 & 300 & 0.53 & 100 \\
\hline
\end{tabular}

Annual flow duration

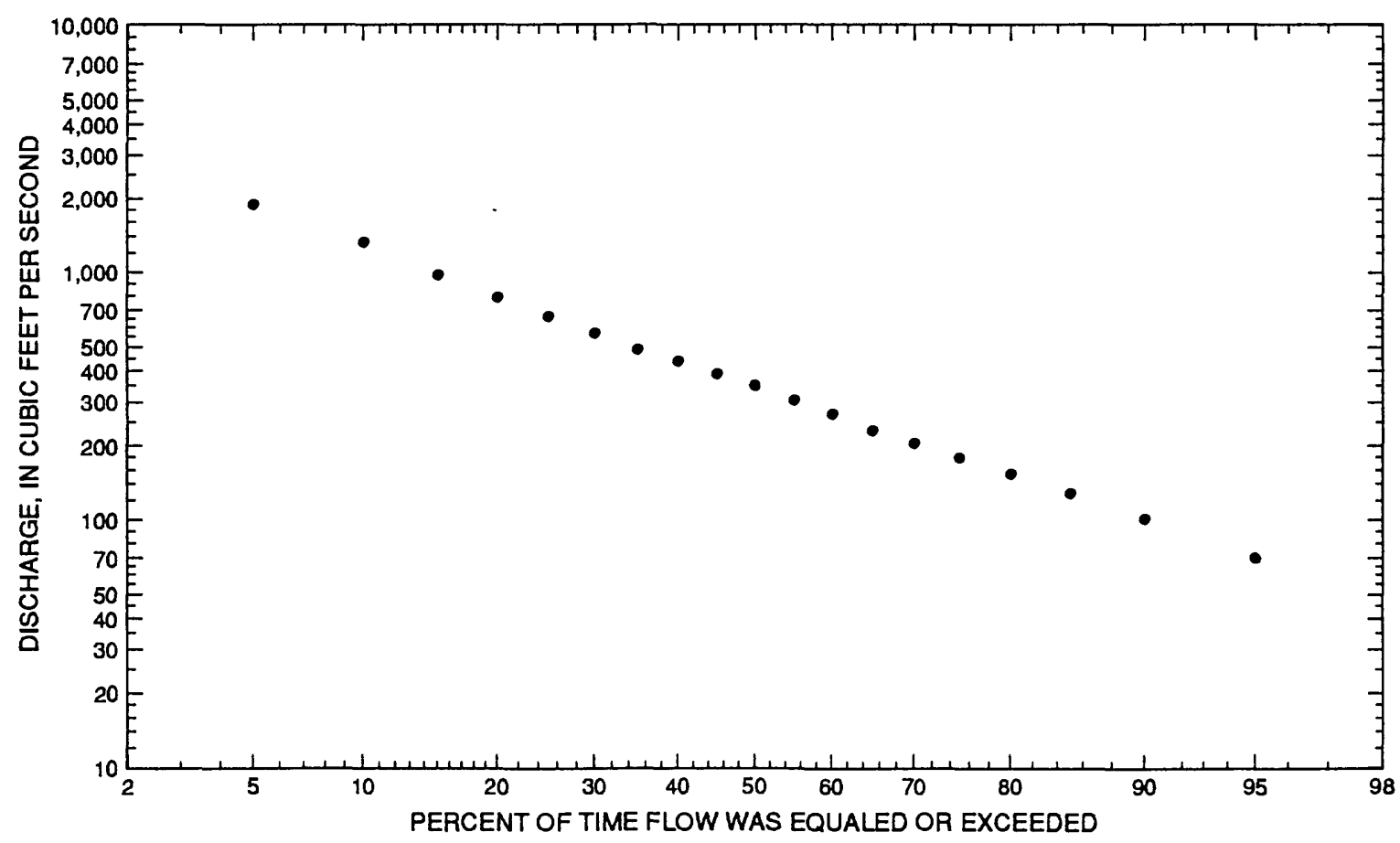




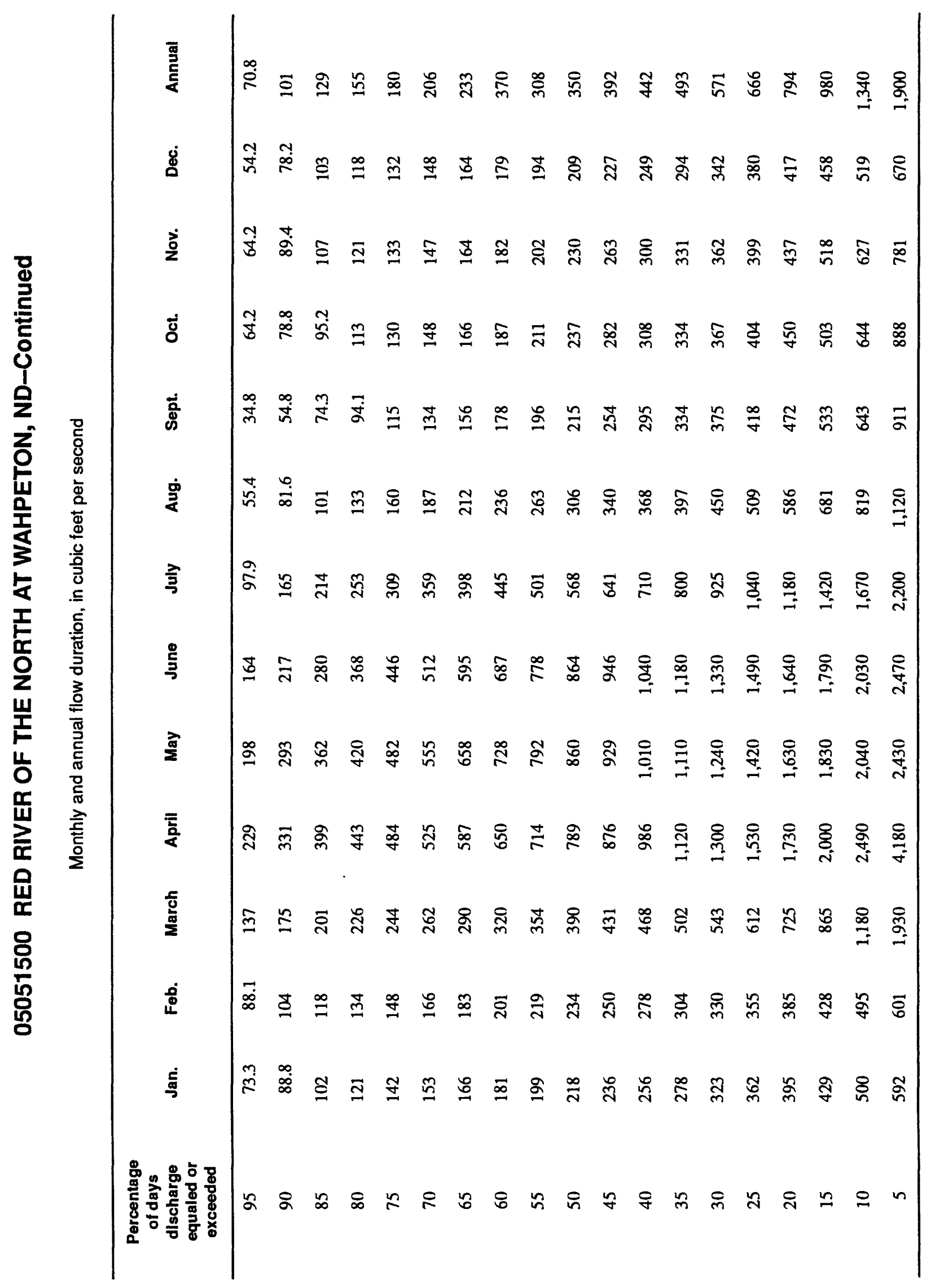




\section{RED RIVER OF THE NORTH AT WAHPETON, ND-Continued}

Probability of annual high discharges

[ng, statistic not given]

\begin{tabular}{lcccccc}
\hline & & & \multicolumn{5}{c}{$\begin{array}{c}\text { Maximum average discharge } \\
\left(\mathbf{f t}^{\mathbf{3}} / \mathbf{s}\right)\end{array}$} \\
\cline { 5 - 7 } $\begin{array}{c}\text { Exceedance } \\
\text { probability }\end{array}$ & $\begin{array}{c}\text { Recurrence } \\
\text { interval } \\
\text { (years) }\end{array}$ & $\begin{array}{c}\text { Maximum } \\
\text { instantaneous } \\
\left(\mathbf{f t}^{3} \mathbf{s}\right)\end{array}$ & 3-day perlod & 7-day perlod & 15-day period & 30-day period \\
\hline 0.99 & 1.01 & 333 & 293 & 257 & 223 & 181 \\
0.95 & 1.05 & 657 & 569 & 486 & 415 & 354 \\
0.90 & 1.11 & 923 & 797 & 673 & 570 & 493 \\
0.80 & 1.25 & 1,360 & 1,180 & 986 & 824 & 719 \\
0.50 & 2 & 2,700 & 2,360 & 1,970 & 1,600 & 1,380 \\
0.20 & 5 & 4,940 & 4,460 & 3,760 & 2,950 & 2,410 \\
0.10 & 10 & 6,580 & 6,060 & 5,170 & 3,970 & 3,130 \\
0.04 & 25 & 8,720 & 8,260 & 7,170 & 5,370 & 4,020 \\
0.02 & 50 & 10,300 & 9,990 & 8,790 & 6,470 & 4,670 \\
0.01 & 100 & 12,000 & 11,800 & 10,500 & 7,620 & 5,300 \\
0.005 & 200 & 13,600 & 13,600 & 12,300 & 8,800 & 5,910 \\
0.002 & 500 & 15,700 & ng & ng & ng & ng \\
\hline
\end{tabular}

Probability of annual low discharges

\begin{tabular}{|c|c|c|c|c|c|c|c|c|c|c|}
\hline \multirow[b]{3}{*}{$\begin{array}{l}\text { Non- } \\
\text { exceed- } \\
\text { ance } \\
\text { prob- } \\
\text { ability }\end{array}$} & \multirow[b]{3}{*}{$\begin{array}{c}\text { Recur- } \\
\text { rence } \\
\text { Inter- } \\
\text { val } \\
\text { (years) }\end{array}$} & \multicolumn{9}{|c|}{ Minimum average dlscharge $\left(\mathrm{ft}^{3} / \mathrm{s}\right)$} \\
\hline & & \multicolumn{9}{|c|}{ Number of consecutive days } \\
\hline & & 1 & 3 & 7 & 14 & 30 & 60 & 90 & 120 & 183 \\
\hline 0.05 & 20 & 7.50 & 8.87 & 10.7 & 13.3 & 16.5 & 25.4 & 32.8 & 35.8 & 44.6 \\
\hline 0.10 & 10 & 13.0 & 15.7 & 19.5 & 24.0 & 30.2 & 44.8 & 55.0 & 59.7 & 71.1 \\
\hline 0.20 & 5 & 24.2 & 29.7 & 37.3 & 45.3 & 57.0 & 80.2 & 94.1 & 102 & 117 \\
\hline 0.50 & 2 & 69.5 & 84.6 & 104 & 121 & 146 & 183 & 203 & 218 & 244 \\
\hline
\end{tabular}




\section{RED RIVER OF THE NORTH AT WAHPETON, ND-Continued}

Probability of seasonal low discharges

\begin{tabular}{|c|c|c|c|c|c|c|c|c|c|}
\hline \multirow[b]{3}{*}{$\begin{array}{l}\text { Non- } \\
\text { exceedance } \\
\text { probability }\end{array}$} & \multirow[b]{3}{*}{$\begin{array}{l}\text { Recurrence } \\
\text { interval } \\
\text { (years) }\end{array}$} & \multicolumn{8}{|c|}{ Minimum average discharge $\left(\mathrm{ft}^{3} / \mathrm{s}\right)$} \\
\hline & & \multicolumn{8}{|c|}{ Number of consecutive days } \\
\hline & & 1 & 7 & 14 & 30 & 1 & 7 & 14 & 30 \\
\hline & & \multicolumn{4}{|c|}{ December-January-February } & \multicolumn{4}{|c|}{ March-April-May } \\
\hline 0.05 & 20 & 26.3 & 31.5 & 33.4 & 39.0 & 50.3 & 64.4 & 73.3 & 108 \\
\hline 0.10 & 10 & 42.3 & 52.1 & 55.7 & 62.6 & 76.9 & 101 & 115 & 156 \\
\hline 0.20 & 5 & 70.3 & 88.1 & 94.5 & 103 & 120 & 158 & 180 & 234 \\
\hline \multirow[t]{2}{*}{0.50} & 2 & 153 & 189 & 201 & 212 & 232 & 286 & 323 & 448 \\
\hline & & \multicolumn{4}{|c|}{ June-July-August } & \multicolumn{4}{|c|}{ September-October-November } \\
\hline 0.05 & 20 & 12.2 & 16.6 & 30.9 & 71.3 & 9.51 & 14.1 & 16.7 & 22.1 \\
\hline 0.10 & 10 & 24.2 & 33.0 & 50.7 & 95.6 & 16.7 & 25.4 & 30.3 & 40.5 \\
\hline 0.20 & 5 & 51.0 & 68.8 & 88.4 & 137 & 31.5 & 48.2 & 57.3 & 76.5 \\
\hline 0.50 & 2 & 165 & 210 & 224 & 272 & 91.6 & 133 & 155 & 196 \\
\hline
\end{tabular}




\section{RED RIVER OF THE NORTH AT WAHPETON, ND-Continued}

Annual peak discharge and corresponding gage height, period of record

\begin{tabular}{|c|c|c|c|c|c|c|c|}
\hline $\begin{array}{l}\text { Water } \\
\text { year }\end{array}$ & Date & $\begin{array}{c}\text { Gage } \\
\text { height } \\
\text { (feet) }\end{array}$ & $\begin{array}{c}\text { Peak } \\
\text { discharge } \\
\left(\mathrm{ft}^{3} / \mathrm{s}\right)\end{array}$ & $\begin{array}{l}\text { Water } \\
\text { year }\end{array}$ & Date & $\begin{array}{c}\text { Gage } \\
\text { height } \\
\text { (feet) }\end{array}$ & $\begin{array}{c}\text { Peak } \\
\text { discharge } \\
\left(f t^{3 / s}\right)\end{array}$ \\
\hline \multicolumn{8}{|c|}{ Annual peak discharge, by year, and corresponding gage height } \\
\hline 1942 & June 7 & 10.49 & 3,280 & 1969 & April 10 & 16.34 & 9,200 \\
\hline 1943 & April 2 & 14.75 & 5,000 & 1970 & April 8 & 6.93 & 1,450 \\
\hline 1944 & June 6 & 12.11 & 4,360 & 1971 & March 18 & 6.74 & 927 \\
\hline 1945 & March 17 & 11.44 & 3,910 & 1972 & March 19 & 11.67 & 3,380 \\
\hline 1946 & March 22 & 9.74 & 3,110 & 1973 & March 15 & 6.08 & 1,220 \\
\hline 1947 & April 12 & 11.90 & 4,610 & 1974 & May 24 & 6.14 & 1,250 \\
\hline 1948 & April 6 & 8.58 & 2,300 & 1975 & July 3 & 10.84 & 3,850 \\
\hline 1949 & July 10 & 9.24 & 2,290 & 1976 & March 26 & 9.00 & 2,700 \\
\hline 1950 & April 2 & 11.62 & 4,190 & 1977 & June 25 & 4.49 & 526 \\
\hline 1951 & April 7 & 14.01 & 6,090 & 1978 & March 31 & 14.04 & 6,250 \\
\hline 1952 & April 12 & 14.99 & 7,130 & 1979 & April 14 & 15.44 & 7,050 \\
\hline 1953 & June 21 & 9.87 & 3,150 & 1980 & April 1 & 10.71 & 3,100 \\
\hline 1954 & June 9 & 7.59 & 1,860 & 1981 & August 2 & 4.54 & 512 \\
\hline 1955 & April 2 & 6.99 & 1,150 & 1982 & April 1 & 12.26 & 3,120 \\
\hline 1956 & April 14 & 7.88 & 1,980 & 1983 & March 13 & 5.70 & 880 \\
\hline 1957 & April 22 & 8.82 & 2,290 & 1984 & March 28 & 13.43 & 4,710 \\
\hline 1958 & April 15 & 5.59 & 866 & 1985 & June 1 & 10.71 & 3,690 \\
\hline 1959 & May 27 & 6.17 & 1,050 & 1986 & March 30 & 14.31 & 6,140 \\
\hline 1960 & April 7 & 8.89 & 2,370 & 1987 & October 1 & 7.46 & 1,770 \\
\hline 1961 & June 8 & 4.72 & 548 & 1988 & March 27 & 5.58 & 911 \\
\hline 1962 & June 11 & 13.98 & 5,650 & 1989 & April 5 & 17.95 & 8,370 \\
\hline 1963 & June 11 & 11.38 & 3,830 & 1990 & March 18 & 5.72 & 900 \\
\hline 1964 & May 6 & 7.39 & 1,700 & 1991 & July 3 & 9.52 & 2,980 \\
\hline 1965 & April 11 & 14.34 & 5,690 & 1992 & March 8 & 8.46 & 2,000 \\
\hline 1966 & March 18 & 12.91 & 4,760 & 1993 & March 31 & 14.33 & 6,080 \\
\hline 1967 & June 15 & 8.81 & 2,500 & 1994 & March 23 & 13.59 & 5,000 \\
\hline 1968 & May 18 & 4.95 & 708 & & & & \\
\hline \multicolumn{8}{|c|}{ Annual peak discharge, from highest to lowest, and corresponding gage height } \\
\hline 1969 & April 10 & 16.34 & 9,200 & 1950 & April 2 & 11.62 & 4,190 \\
\hline 1989 & April 5 & 17.95 & 8,370 & 1945 & March 17 & 11.44 & 3,910 \\
\hline 1952 & April 12 & 14.99 & 7,130 & 1975 & July 3 & 10.84 & 3,850 \\
\hline 1979 & April 14 & 15.44 & 7,050 & 1963 & June 11 & 11.38 & 3,830 \\
\hline 1978 & March 31 & 14.04 & 6,250 & 1985 & June 1 & 10.71 & 3,690 \\
\hline 1986 & March 30 & 14.31 & 6,140 & 1972 & March 19 & 11.67 & 3,380 \\
\hline 1951 & April 7 & 14.01 & 6,090 & 1942 & June 7 & 10.49 & 3,280 \\
\hline 1993 & March 31 & 14.33 & 6,080 & 1953 & June 21 & 9.87 & 3,150 \\
\hline 1965 & April 11 & 14.34 & 5,690 & 1982 & April 1 & 12.26 & 3,120 \\
\hline 1962 & June 11 & 13.98 & 5,650 & 1946 & March 22 & 9.74 & 3,110 \\
\hline 1943 & April 2 & 14.75 & 5,000 & 1980 & April 1 & 10.71 & 3,100 \\
\hline 1994 & March 23 & 13.59 & 5,000 & 1991 & July 3 & 9.52 & 2,980 \\
\hline 1966 & March 18 & 12.91 & 4,760 & 1976 & March 26 & 9.00 & 2,700 \\
\hline 1984 & March 28 & 13.43 & 4,710 & 1967 & June 15 & 8.81 & 2,500 \\
\hline 1947 & April 12 & 11.90 & 4,610 & 1960 & April 7 & 8.89 & 2,370 \\
\hline 1944 & June 6 & 12.11 & 4,360 & 1948 & April 6 & 8.58 & 2,300 \\
\hline
\end{tabular}




\section{RED RIVER OF THE NORTH AT WAHPETON, ND-Continued}

Annual peak discharge and corresponding gage height, period of record--Continued

\begin{tabular}{|c|c|c|c|c|c|c|c|}
\hline $\begin{array}{l}\text { Water } \\
\text { year }\end{array}$ & Date & $\begin{array}{c}\text { Gage } \\
\text { height } \\
\text { (feet) }\end{array}$ & $\begin{array}{c}\text { Peak } \\
\text { discharge } \\
\left(\mathrm{ft}^{3} / \mathrm{s}\right)\end{array}$ & $\begin{array}{l}\text { Water } \\
\text { year }\end{array}$ & Date & $\begin{array}{c}\text { Gage } \\
\text { height } \\
\text { (feet) }\end{array}$ & $\begin{array}{c}\text { Peak } \\
\text { discharge } \\
\left(\mathrm{ft}^{3} / \mathrm{s}\right)\end{array}$ \\
\hline \multicolumn{8}{|c|}{ Annual peak discharge, from highest to lowest, and corresponding gage height--Continued } \\
\hline 1949 & July 10 & 9.24 & 2,290 & 1959 & May 27 & 6.17 & 1,050 \\
\hline 1957 & April 22 & 8.82 & 2,290 & 1971 & March 18 & 6.74 & 927 \\
\hline 1992 & March 8 & 8.46 & 2,000 & 1988 & March 27 & 5.58 & 911 \\
\hline 1956 & April 14 & 7.88 & 1,980 & 1990 & March 18 & 5.72 & 900 \\
\hline 1954 & June 9 & 7.59 & 1,860 & 1983 & March 13 & 5.70 & 880 \\
\hline 1987 & October 1 & 7.46 & 1,770 & 1958 & April 15 & 5.59 & 866 \\
\hline 1964 & May 6 & 7.39 & 1,700 & 1968 & May 18 & 4.95 & 708 \\
\hline 1970 & April 8 & 6.93 & 1,450 & 1961 & June 8 & 4.72 & 548 \\
\hline 1974 & May 24 & 6.14 & 1,250 & 1977 & June 25 & 4.49 & 526 \\
\hline 1973 & March 15 & 6.08 & 1,220 & 1981 & August 2 & 4.54 & 512 \\
\hline 1955 & April 2 & 6.99 & 1,150 & & & & \\
\hline
\end{tabular}


กำ.

nom-r.

구응

४तनฺ

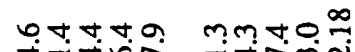

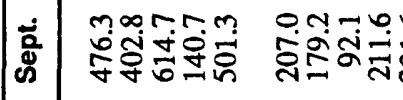

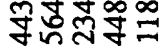

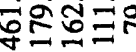
ริำสำ

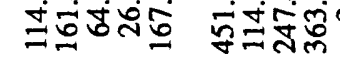

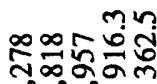

mr

क⿺辶่

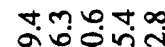

눙ㅇㅇ

ㅍำำ

누웅

o.mmn

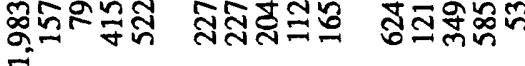

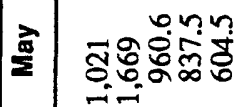

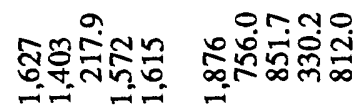

9r-⿻口卄.

ทั่าำ-

ํิสี

ทีत-

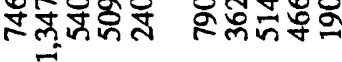
ते तु

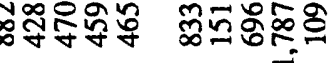

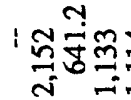

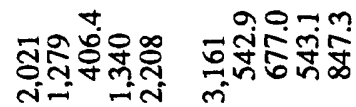

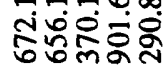

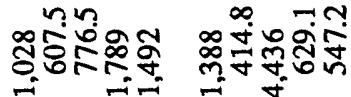

我

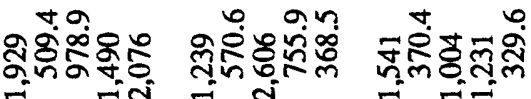

Nanno

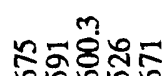

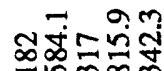

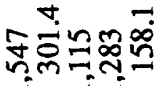

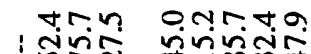

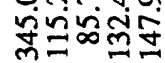

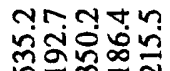

9.700.n

mis

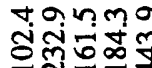

पำสำ

$m 040$

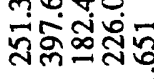

ทดตษับ

ติ่ง

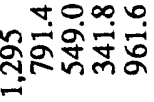

\section{.}

: : ถูติ

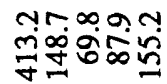
nทक幺?

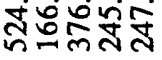

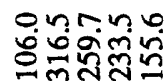

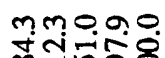

:mnno ranom

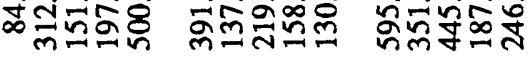

: :

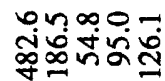

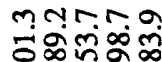
กู่ inn:0.0. gogon troor คำ

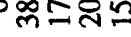

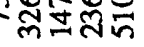

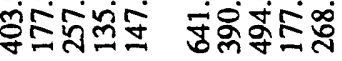

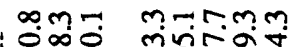

9)0nm r.900

ํํㅇ요웜

orom.

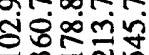

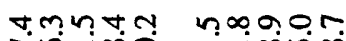

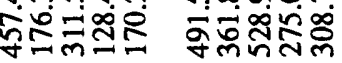

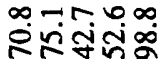

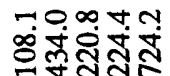

antino

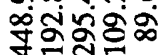

ल⿻一𠃋十 更

.

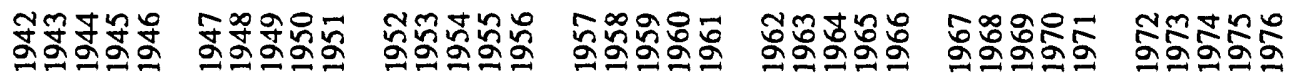




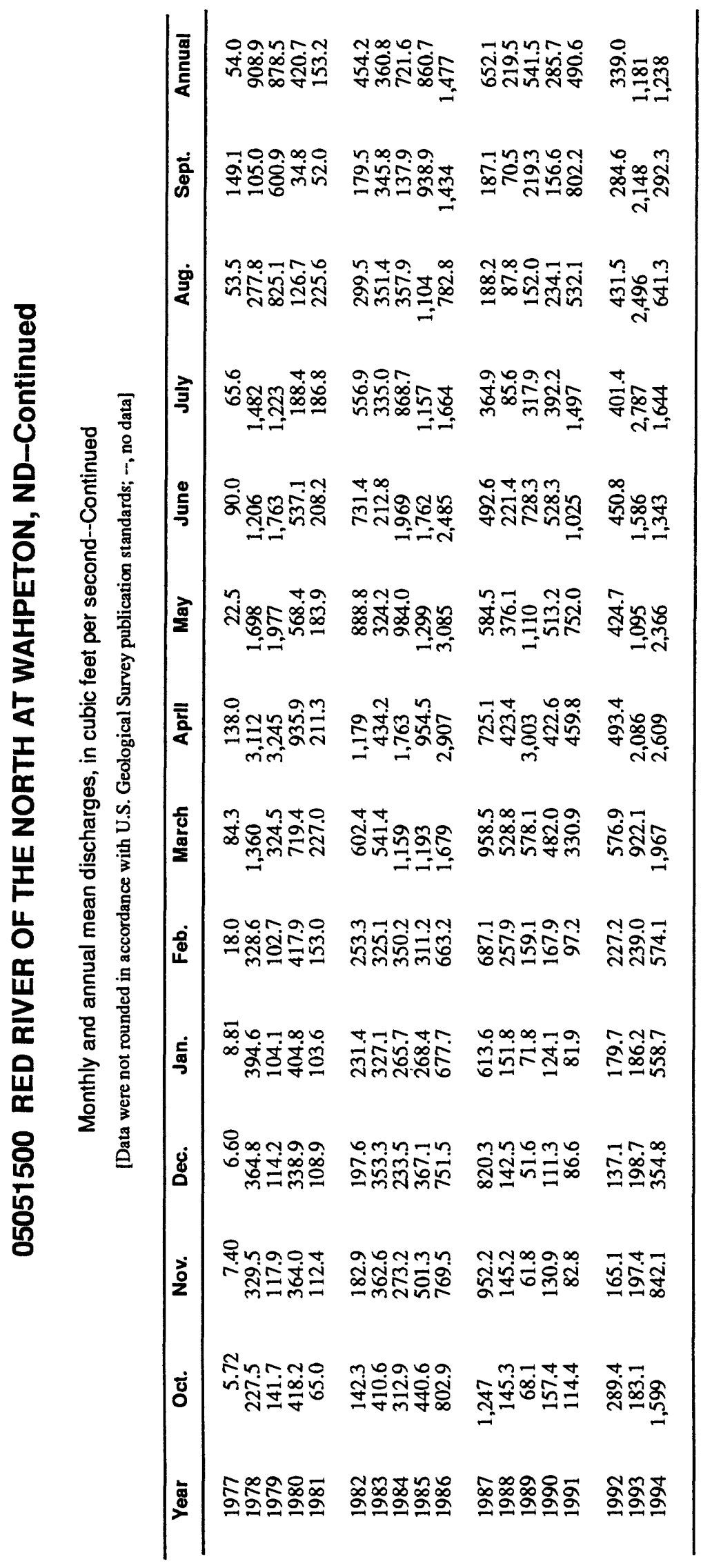




\section{RED RIVER OF THE NORTH AT HICKSON, ND}

\section{Station Description}

LOCATION.--Lat 46³9'35', long 9647'44”, in SW1/4 sec.19, T.137 N., R.48 W., Clay County, MN, Hydrologic Unit 09020104 , on right bank $60 \mathrm{ft}$ downstream from bridge on township road, and $1 \mathrm{mi}$ southeast of Hickson.

DRAINAGE AREA.--4,300 $\mathrm{mi}^{2}$, approximately.

PERIOD OF RECORD.--October 1975 to current year.

GAGE.--Water-stage recorder and concrete control. Datum of gage is $877.06 \mathrm{ft}$ above sea level.

EXTREMES FOR PERIOD OF RECORD.--Maximum discharge, 12,900 $\mathrm{ft}^{3} / \mathrm{s}$, Apr. 7, 1989; maximum gage height, $35.81 \mathrm{ft}$, Apr. 7, 1989; no flow from Oct. 26, 1977, to Jan. 9, 1978.

Annual mean discharge

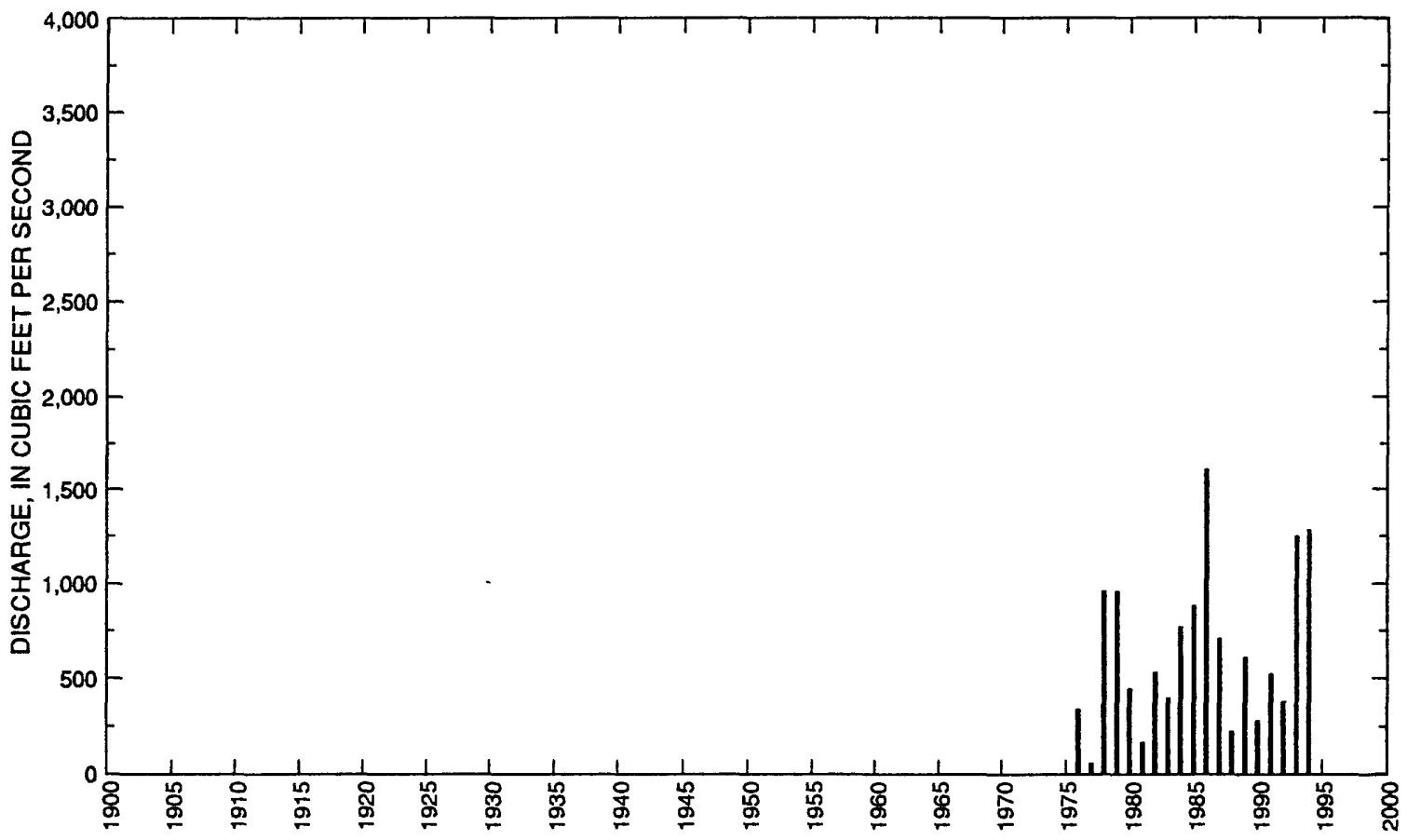


05051522 RED RIVER OF THE NORTH AT HICKSON, ND--Continued

Statistics of monthly and annual mean discharges

\begin{tabular}{|c|c|c|c|c|c|c|c|c|}
\hline \multirow[b]{2}{*}{ Month } & \multicolumn{2}{|c|}{ Maximum } & \multicolumn{2}{|c|}{ Minimum } & \multicolumn{4}{|c|}{ Mean } \\
\hline & $\begin{array}{c}\text { Discharge } \\
\left(\mathrm{ft}^{3} / \mathrm{s}\right)\end{array}$ & $\begin{array}{l}\text { Water year } \\
\text { of } \\
\text { occurrence }\end{array}$ & $\begin{array}{c}\text { Discharge } \\
\left(\mathrm{ft}^{3} / \mathrm{s}\right)\end{array}$ & $\begin{array}{l}\text { Water year } \\
\text { of } \\
\text { occurrence }\end{array}$ & $\begin{array}{c}\text { Discharge } \\
\left(\mathrm{ft}^{3} / \mathrm{s}\right)\end{array}$ & $\begin{array}{c}\text { Standard } \\
\text { deviation } \\
\left(\mathrm{ft}^{3} / \mathbf{s}\right)\end{array}$ & $\begin{array}{l}\text { Coeffl- } \\
\text { cient of } \\
\text { variation }\end{array}$ & $\begin{array}{l}\text { Percentage } \\
\text { of annual } \\
\text { dlscharge }\end{array}$ \\
\hline October & 1,560 & 1994 & 2.02 & 1977 & 388 & 418 & 1.08 & 4.99 \\
\hline November & 900 & 1987 & 0 & 1977 & 310 & 265 & 0.86 & 3.98 \\
\hline December & 817 & 1986 & 0 & 1977 & 270 & 226 & 0.84 & 3.47 \\
\hline January & 747 & 1986 & 4.95 & 1977 & 261 & 198 & 0.76 & 3.35 \\
\hline February & 745 & 1987 & 14.0 & 1977 & 305 & 202 & 0.66 & 3.92 \\
\hline March & 2,080 & 1994 & 75.9 & 1977 & 783 & 503 & 0.64 & 10.1 \\
\hline April & 4,160 & 1978 & 165 & 1977 & 1,710 & 1,430 & 0.84 & 21.9 \\
\hline May & 3,390 & 1986 & 22.0 & 1977 & 1,010 & 837 & 0.83 & 13.0 \\
\hline June & 2,480 & 1986 & 86.4 & 1977 & 951 & 707 & 0.74 & 12.2 \\
\hline July & 2,670 & 1993 & 73.4 & 1977 & 834 & 735 & 0.88 & 10.7 \\
\hline August & 2,670 & 1993 & 35.6 & 1977 & 515 & 600 & 1.16 & 6.63 \\
\hline September & 2,140 & 1993 & 12.6 & 1976 & 445 & 558 & 1.25 & 5.71 \\
\hline Annual & 1,600 & 1986 & 53.1 & 1977 & 649 & 418 & 0.64 & 100 \\
\hline
\end{tabular}

Annual flow duration

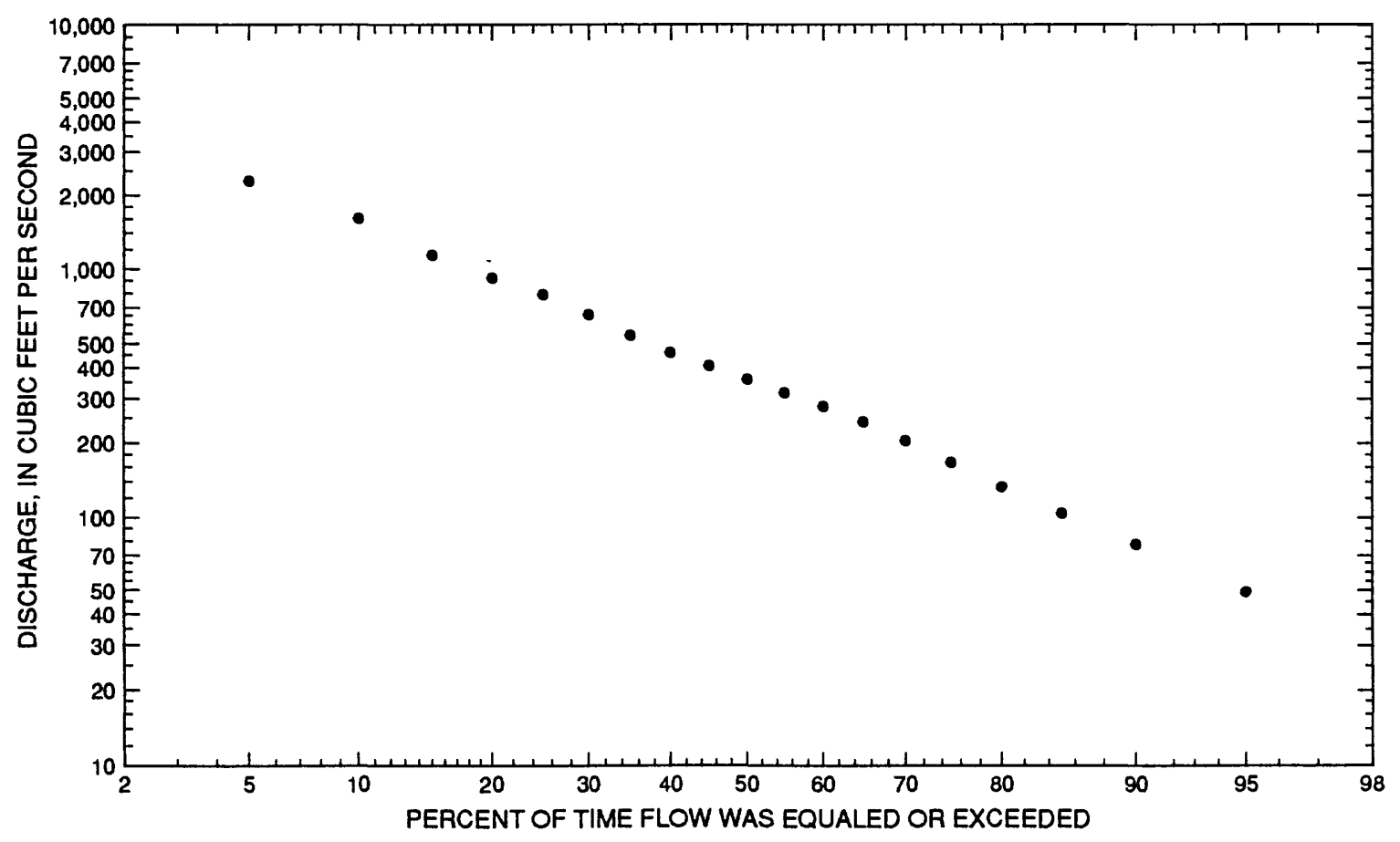




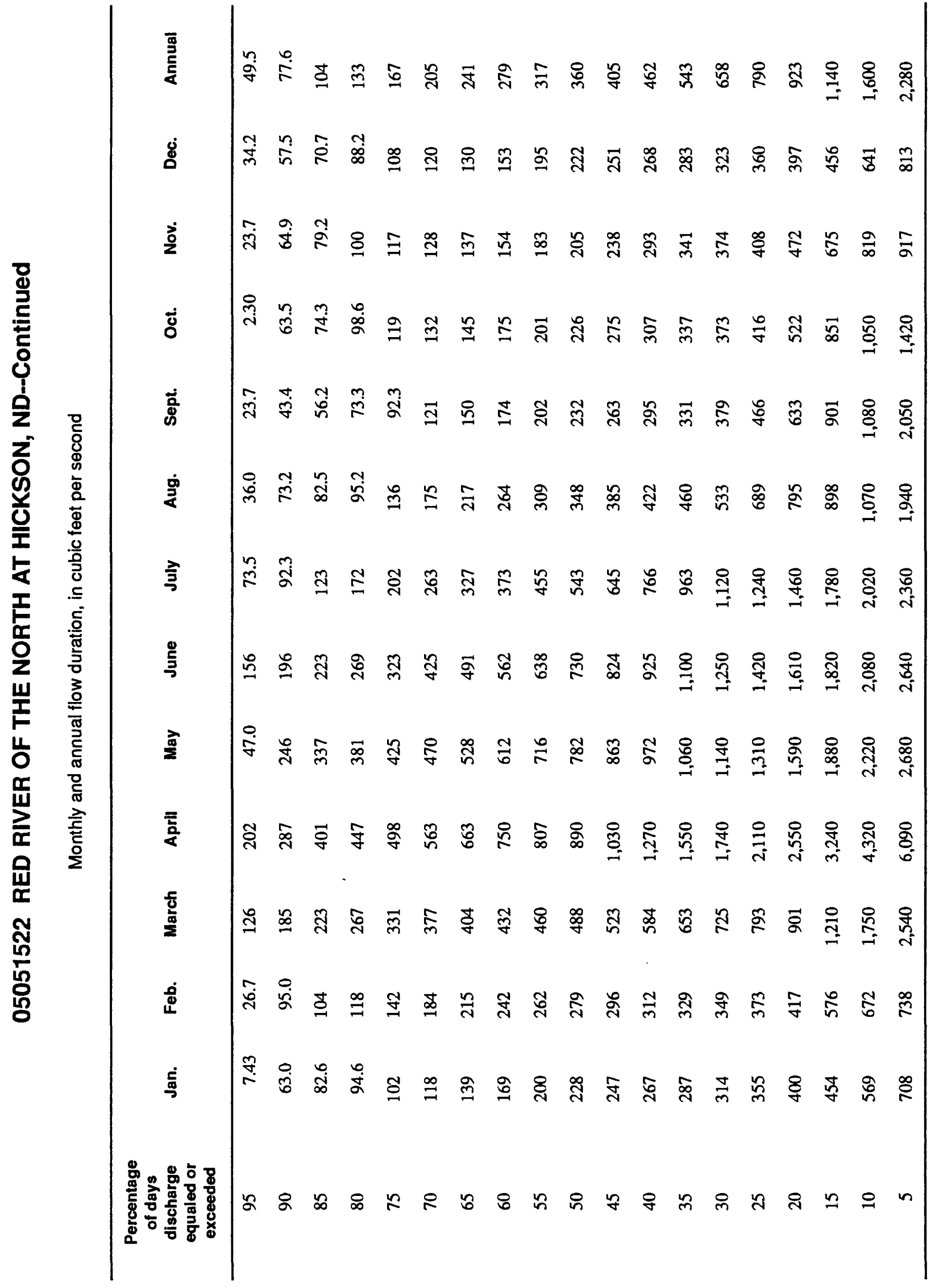




\section{RED RIVER OF THE NORTH AT HICKSON, ND--Continued}

Probability of annual high discharges

[ng, statistic not given]

\begin{tabular}{|c|c|c|c|c|c|c|}
\hline \multirow[b]{2}{*}{$\begin{array}{l}\text { Exceedance } \\
\text { probability }\end{array}$} & \multirow[b]{2}{*}{$\begin{array}{c}\text { Recurrence } \\
\text { interval } \\
\text { (years) }\end{array}$} & \multirow[b]{2}{*}{$\begin{array}{c}\text { Maximum } \\
\text { Instantaneous } \\
\left(\mathrm{ft}^{3} / \mathrm{s}\right)\end{array}$} & \multicolumn{4}{|c|}{$\begin{array}{l}\text { Maximum average discharge } \\
\left(\mathrm{ft}^{3} / \mathrm{s}\right)\end{array}$} \\
\hline & & & 3-day period & 7-day period & 15-day period & 30-day perloc \\
\hline 0.99 & 1.01 & 187 & 166 & 150 & 114 & 99.4 \\
\hline 0.95 & 1.05 & 460 & 423 & 380 & 297 & 258 \\
\hline 0.90 & 1.11 & 722 & 673 & 602 & 477 & 408 \\
\hline 0.80 & 1.25 & 1,210 & 1,140 & 1,020 & 813 & 683 \\
\hline 0.50 & 2 & 3,010 & 2,870 & 2,550 & 2,030 & 1,610 \\
\hline 0.20 & 5 & 6,750 & 6,390 & 5,680 & 4,400 & 3,270 \\
\hline 0.10 & 10 & 9,900 & 9,260 & 8,270 & 6,260 & 4,460 \\
\hline 0.04 & 25 & 14,500 & 13,300 & 11,900 & 8,800 & 5,980 \\
\hline 0.02 & 50 & 18,200 & 16,600 & 14,900 & 10,700 & 7,060 \\
\hline 0.01 & 100 & 22,200 & 19,900 & 18,000 & 12,700 & 8,100 \\
\hline 0.005 & 200 & 26,400 & 23,400 & 21,200 & 14,700 & 9,090 \\
\hline 0.002 & 500 & 32,200 & $\mathrm{ng}$ & $\mathrm{ng}$ & $\mathrm{ng}$ & $\mathrm{ng}$ \\
\hline
\end{tabular}

Probability of annual low discharges

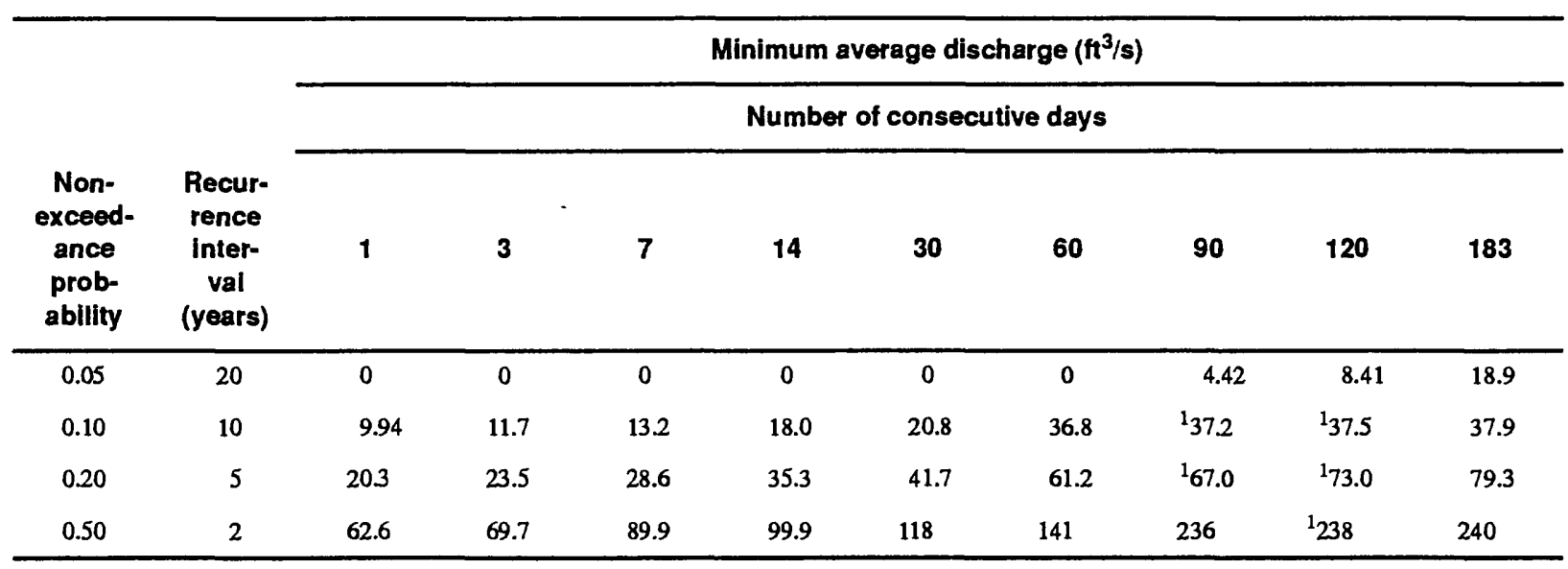

${ }^{1}$ Graphical interpretation. 
05051522 RED RIVER OF THE NORTH AT HICKSON, ND--Continued

Probability of seasonal low discharges

\begin{tabular}{|c|c|c|c|c|c|c|c|c|c|}
\hline \multirow[b]{3}{*}{$\begin{array}{l}\text { Non- } \\
\text { exceedance } \\
\text { probability }\end{array}$} & \multirow[b]{3}{*}{$\begin{array}{c}\text { Recurrence } \\
\text { interval } \\
\text { (years) }\end{array}$} & \multicolumn{8}{|c|}{ Minimum average discharge $\left(\mathrm{ft}^{3} / \mathrm{s}\right)$} \\
\hline & & \multicolumn{8}{|c|}{ Number of consecutive days } \\
\hline & & 1 & 7 & 14 & 30 & 1 & 7 & 14 & 30 \\
\hline & & \multicolumn{4}{|c|}{ December-January-February } & \multicolumn{4}{|c|}{ March-April-May } \\
\hline 0.05 & 20 & 0 & 0 & 0 & 0 & 39.7 & 48.9 & 52.7 & 71.1 \\
\hline 0.10 & 10 & 42.0 & 48.3 & 51.7 & 55.8 & 74.8 & 90.6 & 100 & 133 \\
\hline 0.20 & 5 & 65.2 & 76.6 & 82.4 & 89.9 & 140 & 165 & 186 & 252 \\
\hline \multirow[t]{2}{*}{0.50} & 2 & 134 & 160 & 171 & 188 & 312 & 349 & 392 & 600 \\
\hline & & \multicolumn{4}{|c|}{ June-July-August } & \multicolumn{4}{|c|}{ September-October-November } \\
\hline 0.05 & 20 & 12.5 & 16.7 & 25.4 & 47.9 & 0 & 0 & 0 & 0 \\
\hline 0.10 & 10 & 23.1 & 30.7 & 44.0 & 73.1 & 21.7 & 28.0 & 31.7 & 38.1 \\
\hline 0.20 & 5 & 46.6 & 61.3 & 82.1 & 120 & 34.3 & 45.5 & 51.7 & 63.2 \\
\hline 0.50 & 2 & 159 & 199 & 237 & 293 & 79.1 & 107 & 123 & 150 \\
\hline
\end{tabular}




\section{RED RIVER OF THE NORTH AT HICKSON, ND--Continued}

Annual peak discharge and corresponding gage height, period of record

\begin{tabular}{|c|c|c|c|c|c|c|c|}
\hline $\begin{array}{l}\text { Water } \\
\text { year }\end{array}$ & Date & $\begin{array}{c}\text { Gage } \\
\text { height } \\
\text { (feet) }\end{array}$ & $\begin{array}{c}\text { Peak } \\
\text { discharge } \\
\left(\mathrm{ft}^{3} / \mathrm{s}\right)\end{array}$ & $\begin{array}{l}\text { Water } \\
\text { year }\end{array}$ & Date & $\begin{array}{c}\text { Gage } \\
\text { height } \\
\text { (feet) }\end{array}$ & $\begin{array}{c}\text { Peak } \\
\text { discharge } \\
\left(\mathrm{ft}^{3} / \mathrm{s}\right)\end{array}$ \\
\hline \multicolumn{8}{|c|}{ Annual peak discharge, by year, and corresponding gage height } \\
\hline 1976 & March 31 & 16.94 & 2,500 & 1986 & April 1 & 27.27 & 6,720 \\
\hline 1977 & June 27 & 10.30 & 408 & 1987 & March 26 & 15.34 & 2,460 \\
\hline 1978 & April 2 & 33.54 & 9,200 & 1988 & March 30 & 10.97 & 826 \\
\hline 1979 & April 18 & 33.03 & 9,600 & 1989 & April 7 & 35.81 & 12,900 \\
\hline 1980 & April 4 & 19.13 & 3,250 & 1990 & April 2 & 11.26 & 857 \\
\hline 1981 & August 4 & 10.41 & 544 & 1991 & July 5 & 16.15 & 2,820 \\
\hline 1982 & April 4 & 23.07 & 4,200 & 1992 & March 10 & 13.62 & 1,750 \\
\hline 1983 & March 19 & 11.08 & 824 & 1993 & April 3 & 28.30 & 6,400 \\
\hline 1984 & March 31 & 25.58 & 5,100 & 1994 & April 1 & 26.43 & 6,320 \\
\hline 1985 & June 3 & 18.71 & 3,680 & & & & \\
\hline \multicolumn{8}{|c|}{ Annual peak discharge, from highest to lowest, and corresponding gage height } \\
\hline 1989 & April 7 & 35.81 & 12,900 & 1991 & July 5 & 16.15 & 2,820 \\
\hline 1979 & April 18 & 33.03 & 9,600 & 1976 & March 31 & 16.94 & 2,500 \\
\hline 1978 & April 2 & 33.54 & 9,200 & 1987 & March 26 & 15.34 & 2,460 \\
\hline 1986 & April 1 & 27.27 & 6,720 & 1992 & March 10 & 13.62 & 1,750 \\
\hline 1993 & April 3 & 28.30 & 6,400 & 1990 & April 2 & 11.26 & 857 \\
\hline 1994 & April 1 & 26.43 & 6,320 & 1988 & March 30 & 10.97 & 826 \\
\hline 1984 & March 31 & 25.58 & 5,100 & 1983 & March 19 & 11.08 & 824 \\
\hline 1982 & April 4 & 23.07 & 4,200 & 1981 & August 4 & 10.41 & 544 \\
\hline 1985 & June 3 & 18.71 & 3,680 & 1977 & June 27 & 10.30 & 408 \\
\hline 1980 & April 4 & 19.13 & 3,250 & & & & \\
\hline
\end{tabular}




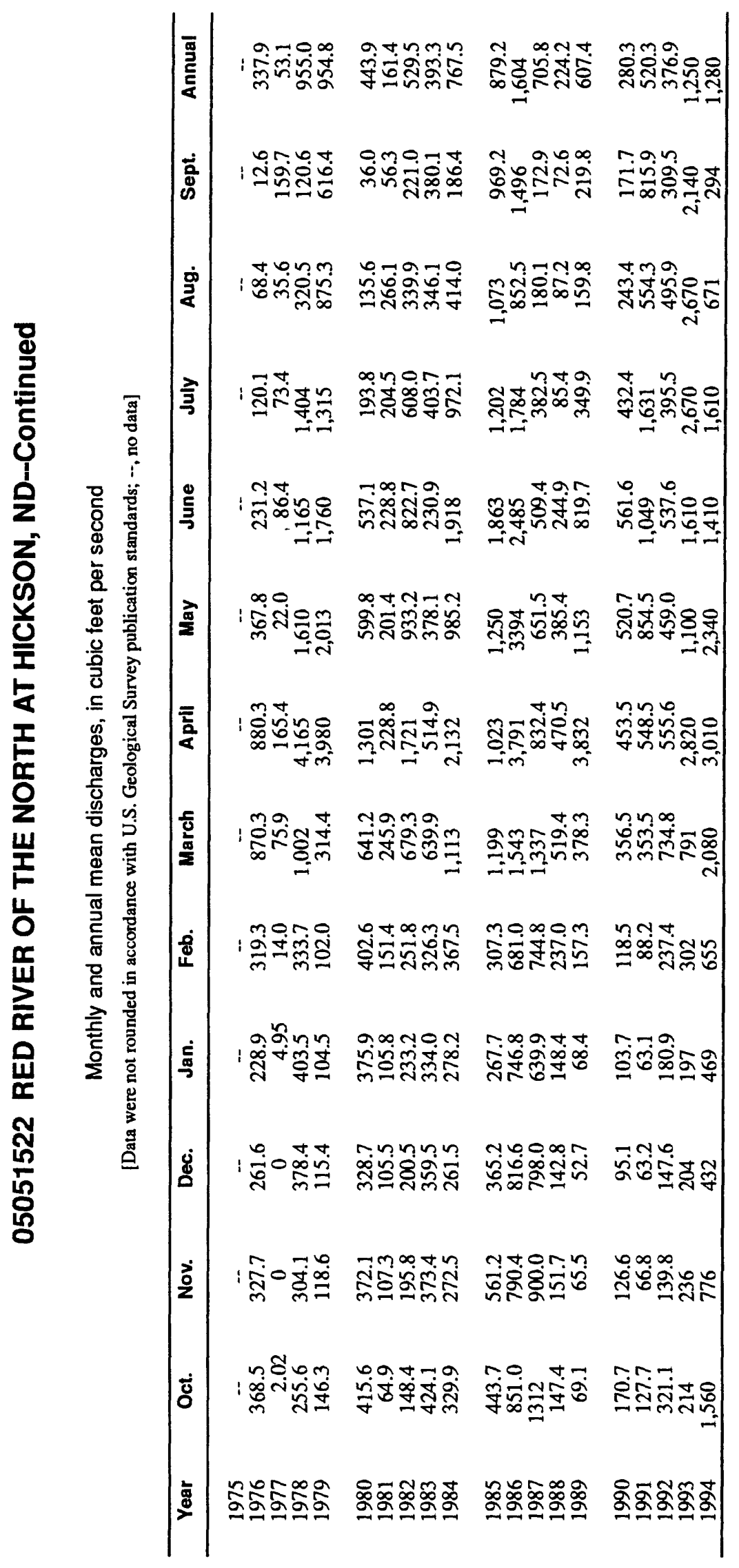




\section{WILD RICE RIVER NEAR RUTLAND, ND}

\section{Station Description}

LOCATION.--Lat 4601 '20', long 97³0'40”, in SE $1 / 4 \mathrm{SE}^{1} / 4$ sec.36, T.130 N., R.55 W., Sargent County, Hydrologic Unit 09020105 , on right bank $1,000 \mathrm{ft}$ upstream from bridge on county highway, $2 \mathrm{mi}$ south of Rutland, and 10 mi upstream from Lake Tewaukon.

DRAINAGE AREA.--546 $\mathrm{mi}^{2}$, of which about $250 \mathrm{mi}^{2}$ is probably noncontributing.

PERIOD OF RECORD.--October 1959 to current year (seasonal records only since 1982).

GAGE.--Water-stage recorder. Datum of gage is $1,197.73 \mathrm{ft}$ above sea level. Prior to Dec. 11, 1960, nonrecording gage at same site and datum.

EXTREMES FOR PERIOD OF RECORD.--Maximum discharge, 1,270 $\mathrm{ft}^{3} / \mathrm{s}$, Apr. 8, 1969; maximum gage height, $8.78 \mathrm{ft}$, Apr. 8, 1969; no flow at times each year.

Annual mean discharge

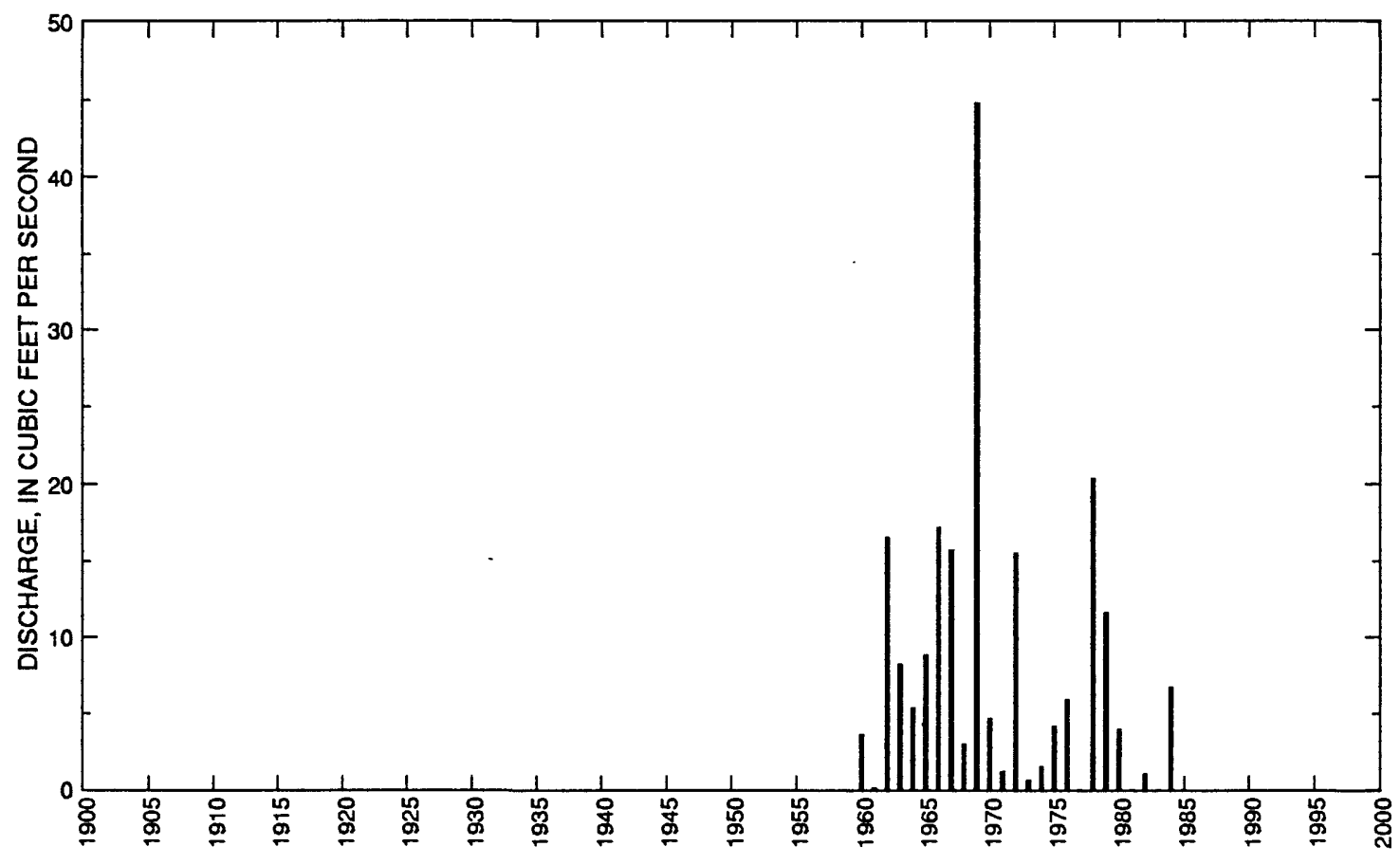




\section{WILD RICE RIVER NEAR RUTLAND, ND--Continued}

Statistics of monthly and annual mean discharges

[m, more than 1 year of occurrence]

\begin{tabular}{|c|c|c|c|c|c|c|c|c|}
\hline \multirow[b]{2}{*}{ Month } & \multicolumn{2}{|c|}{ Maximum } & \multicolumn{2}{|c|}{ Minimum } & \multicolumn{4}{|c|}{ Mean } \\
\hline & $\begin{array}{c}\text { Discharge } \\
\left(\mathrm{ft}^{3} / \mathrm{s}\right)\end{array}$ & $\begin{array}{c}\text { Water year } \\
\text { of } \\
\text { occurrence }\end{array}$ & $\begin{array}{c}\text { Discharge } \\
\left(\mathrm{ft}^{3} / \mathrm{s}\right)\end{array}$ & $\begin{array}{c}\text { Water year } \\
\text { of } \\
\text { occurrence }\end{array}$ & $\begin{array}{c}\text { Discharge } \\
\left(\mathrm{ft}^{3} / \mathrm{s}\right)\end{array}$ & $\begin{array}{l}\text { Standard } \\
\text { deviation } \\
\left(\mathrm{ft}^{3} / \mathrm{s}\right)\end{array}$ & $\begin{array}{l}\text { Coeffi- } \\
\text { clent of } \\
\text { varlation }\end{array}$ & $\begin{array}{l}\text { Percentage } \\
\text { of annual } \\
\text { discharge }\end{array}$ \\
\hline October & 4.81 & 1963 & 0 & $\mathrm{~m}$ & 0.540 & 1.18 & 2.19 & 0.54 \\
\hline November & 5.87 & 1963 & 0 & $\mathrm{~m}$ & 0.360 & 1.23 & 3.44 & 0.36 \\
\hline December & 2.90 & 1963 & 0 & $\mathrm{~m}$ & 0.140 & 0.59 & 4.19 & 0.14 \\
\hline January & 0.103 & 1963 & 0 & $\mathrm{~m}$ & 0 & 0.02 & 4.90 & 0 \\
\hline February & 1.00 & 1976 & 0 & $\mathbf{m}$ & 0.070 & 0.22 & 3.40 & 0.07 \\
\hline March & 138 & 1966 & 0 & $\mathbf{m}$ & 17.7 & 30.6 & 1.73 & 17.6 \\
\hline April & 348 & 1969 & 0 & $\mathbf{m}$ & 40.6 & 65.8 & 1.62 & 40.5 \\
\hline May & 69.3 & 1986 & 0 & $\mathrm{~m}$ & 13.4 & 19.0 & 1.41 & 13.4 \\
\hline June & 61.4 & 1967 & 0 & $\mathrm{~m}$ & 9.38 & 15.2 & 1.62 & 9.35 \\
\hline July & 103 & 1993 & 0 & $\mathrm{~m}$ & 13.6 & 24.7 & 1.82 & 13.5 \\
\hline August & 47.0 & 1993 & 0 & $\mathrm{~m}$ & 3.41 & 9.01 & 2.64 & 3.39 \\
\hline September & 9.07 & 1969 & 0 & $\mathrm{~m}$ & 1.20 & 2.43 & 2.03 & 1.19 \\
\hline Annual & 44.8 & 1969 & 0 & m & 8.36 & 9.94 & 1.19 & 100 \\
\hline
\end{tabular}

Annual flow duration

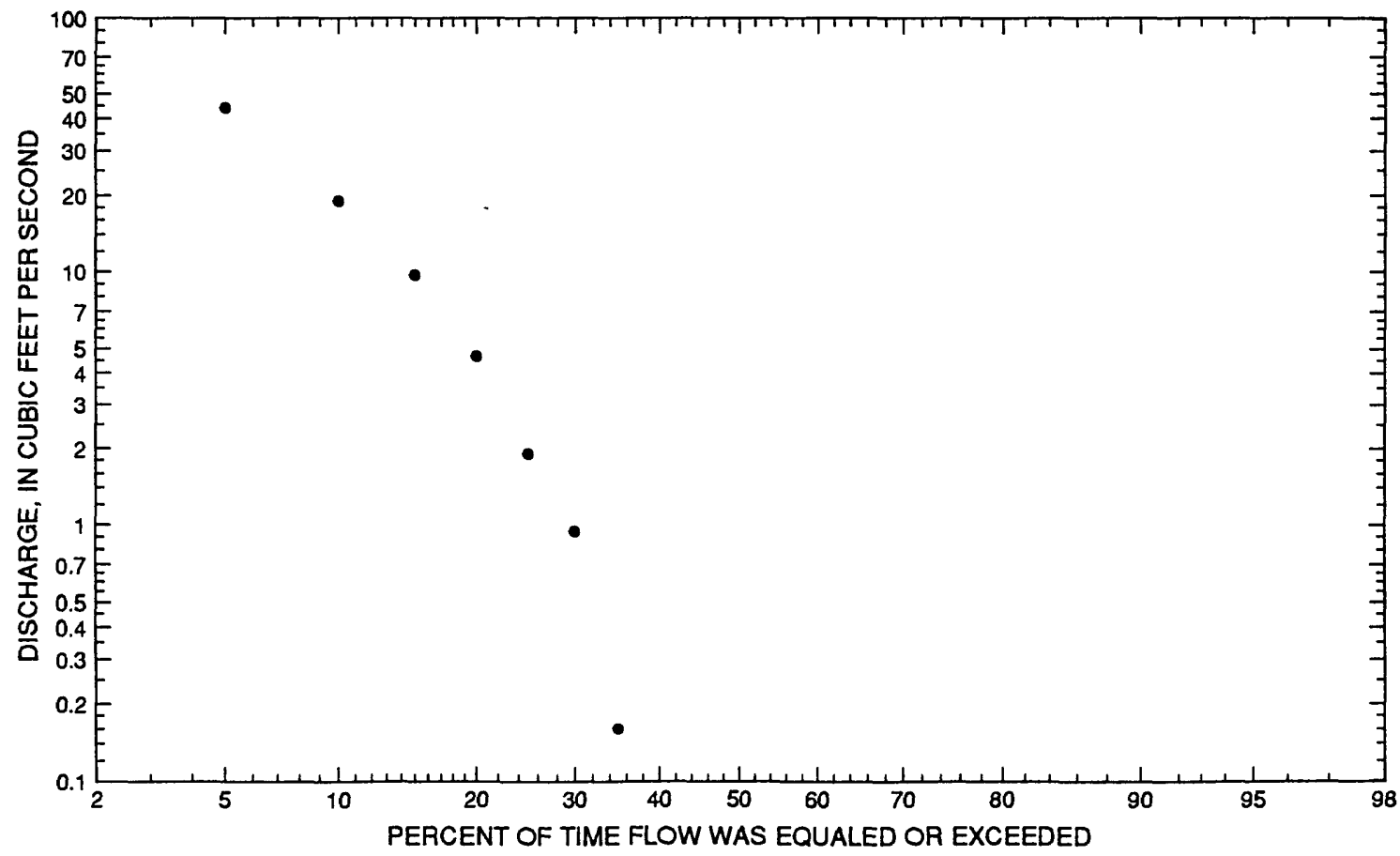




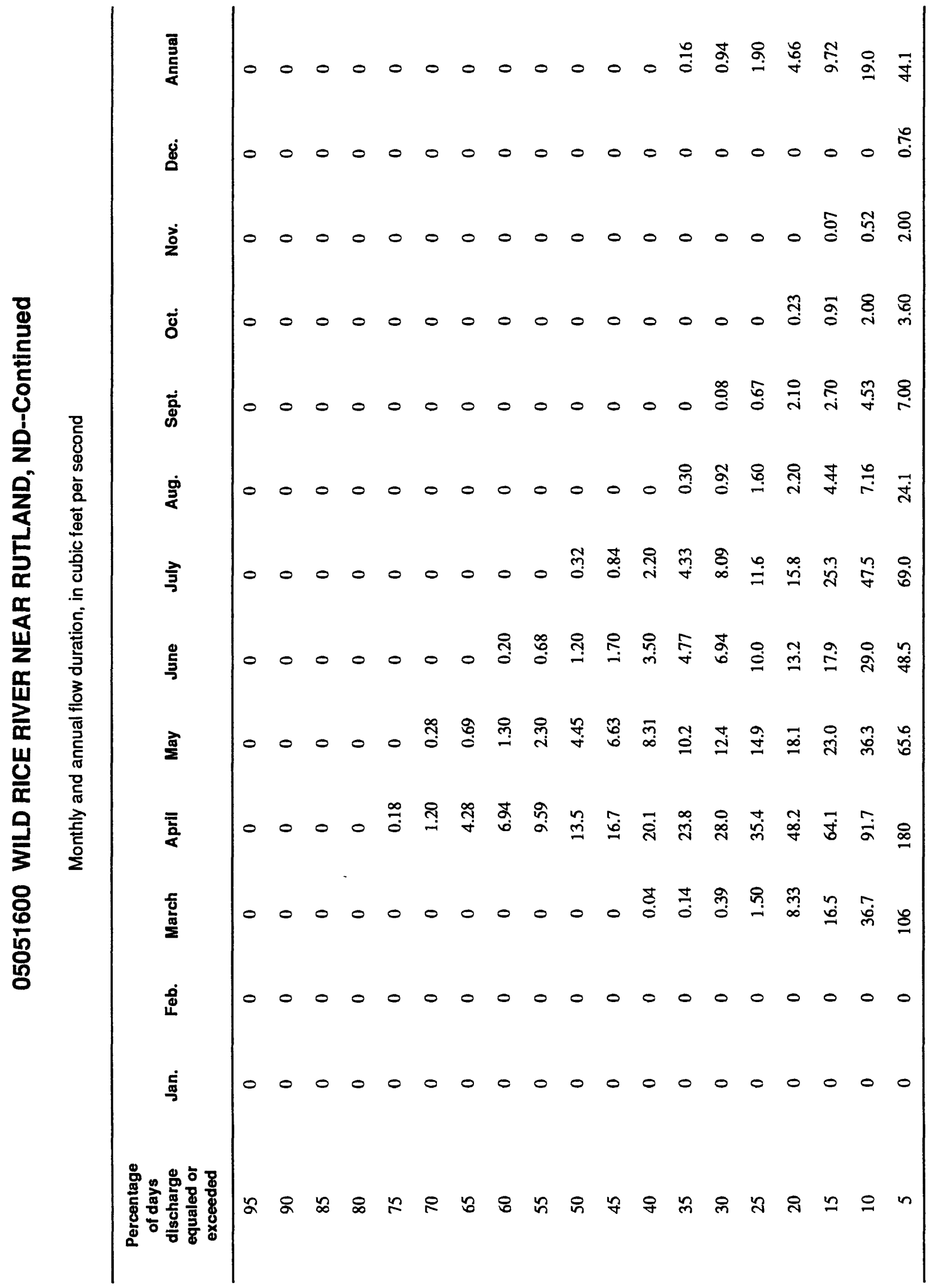




\section{WILD RICE RIVER NEAR RUTLAND, ND--Continued}

Probability of annual high discharges

[ng, statistic not given]

\begin{tabular}{|c|c|c|c|c|c|c|}
\hline \multirow[b]{2}{*}{$\begin{array}{l}\text { Exceedance } \\
\text { probability }\end{array}$} & \multirow[b]{2}{*}{$\begin{array}{l}\text { Recurrence } \\
\text { Interval } \\
\text { (years) }\end{array}$} & \multirow[b]{2}{*}{$\begin{array}{c}\text { Maximum } \\
\text { Instantaneous } \\
\left(\mathrm{ft}^{3} / \mathrm{s}\right)\end{array}$} & \multicolumn{4}{|c|}{$\begin{array}{l}\text { Maximum average discharge } \\
\qquad\left(\mathrm{ft}^{3} / \mathrm{s}\right)\end{array}$} \\
\hline & & & 3-day period & 7-day period & 15-day perlod & 30-day period \\
\hline 0.99 & 1.01 & ng & 0 & 0 & 0 & 0 \\
\hline 0.95 & 1.05 & $\mathrm{ng}$ & 0 & 0 & 0 & 0 \\
\hline 0.90 & 1.11 & ng & 2.34 & 1.91 & 1.62 & 1.19 \\
\hline 0.80 & 1.25 & 21.3 & 14.1 & 12.0 & 9.93 & 7.48 \\
\hline 0.50 & 2 & 91.0 & 76.0 & 65.7 & 51.9 & 37.1 \\
\hline 0.20 & 5 & 316 & 249 & 214 & 160 & 104 \\
\hline 0.10 & 10 & 560 & 419 & 357 & 257 & 156 \\
\hline 0.04 & 25 & 974 & 677 & 569 & 395 & 223 \\
\hline 0.02 & 50 & 1,350 & 898 & 748 & 506 & 272 \\
\hline 0.01 & 100 & 1,780 & 1,140 & 940 & 621 & 317 \\
\hline 0.005 & 200 & 2,260 & 1,390 & 1,130 & 734 & 359 \\
\hline 0.002 & 500 & 2,950 & ng & $\mathrm{ng}$ & $\mathrm{ng}$ & $\mathrm{ng}$ \\
\hline
\end{tabular}

Probability of annual low discharges

[ng, statistic not given]

\begin{tabular}{|c|c|c|c|c|c|c|c|c|c|c|}
\hline \multirow[b]{3}{*}{$\begin{array}{l}\text { Non- } \\
\text { excoed- } \\
\text { ance } \\
\text { prob- } \\
\text { ability }\end{array}$} & \multirow[b]{3}{*}{$\begin{array}{c}\text { Recur- } \\
\text { rence } \\
\text { inter- } \\
\text { val } \\
\text { (years) }\end{array}$} & \multicolumn{9}{|c|}{ Minimum average discharge $\left(\mathrm{ft}^{3} / \mathrm{s}\right)$} \\
\hline & & \multicolumn{9}{|c|}{ Number of consecutive days } \\
\hline & & 1 & $\mathbf{3}$ & 7 & 14 & 30 & 60 & 90 & 120 & 183 \\
\hline 0.05 & 20 & ng & ng & ng & ng & ng & ng & ng & 0 & 0 \\
\hline 0.10 & 10 & ng & ng & ng & ng & ng & ng & $\mathrm{ng}$ & 0 & 0 \\
\hline 0.20 & 5 & ng & ng & ng & ng & ng & ng & ng & 0 & 0 \\
\hline 0.50 & 2 & ng & ng & ng & ng & ng & ng & ng & 0 & 0 \\
\hline
\end{tabular}




\section{WILD RICE RIVER NEAR RUTLAND, ND--Continued}

Probability of seasonal low discharges

[ng, statistic not given]

\begin{tabular}{|c|c|c|c|c|c|c|c|c|c|}
\hline \multirow[b]{3}{*}{$\begin{array}{l}\text { Non- } \\
\text { exceedance } \\
\text { probabillty }\end{array}$} & \multirow[b]{3}{*}{$\begin{array}{c}\text { Recurrence } \\
\text { interval } \\
\text { (years) }\end{array}$} & \multicolumn{8}{|c|}{ Minimum average discharge $\left(\mathrm{ft}^{3} / \mathrm{s}\right)$} \\
\hline & & \multicolumn{8}{|c|}{ Number of consecutive days } \\
\hline & & 1 & 7 & 14 & 30 & 1 & 7 & 14 & 30 \\
\hline & & \multicolumn{4}{|c|}{ December-January-February } & \multicolumn{4}{|c|}{ March-April-May } \\
\hline 0.05 & 20 & ng & ng & ng & $\mathrm{ng}$ & $\mathrm{ng}$ & 0 & 0 & 0 \\
\hline 0.10 & 10 & ng & ng & ng & ng & ng & 0 & 0 & 0 \\
\hline 0.20 & 5 & ng & ng & $\mathrm{ng}$ & ng & $\mathrm{ng}$ & 0 & 0 & 0 \\
\hline \multirow[t]{2}{*}{0.50} & 2 & ng & ng & ng & ng & ng & 0 & 0 & 0.518 \\
\hline & & \multicolumn{4}{|c|}{ June-July-August } & \multicolumn{4}{|c|}{ September-October-November } \\
\hline 0.05 & 20 & 0 & 0 & 0 & 0 & 0 & 0 & 0 & 0 \\
\hline 0.10 & 10 & 0 & 0 & 0 & 0 & 0 & 0 & 0 & 0 \\
\hline 0.20 & 5 & 0 & 0 & 0 & 0 & 0 & 0 & 0 & 0 \\
\hline 0.50 & 2 & 0 & 0 & 0 & 0 & 0 & 0 & 0 & 0 \\
\hline
\end{tabular}




\section{WILD RICE RIVER NEAR RUTLAND, ND--Continued}

Annual peak discharge and corresponding gage height, period of record

$$
[-, \text { no data }]
$$

\begin{tabular}{|c|c|c|c|c|c|c|c|}
\hline $\begin{array}{l}\text { Water } \\
\text { year }\end{array}$ & Date & $\begin{array}{c}\text { Gage } \\
\text { helght } \\
\text { (feet) }\end{array}$ & $\begin{array}{c}\text { Peak } \\
\text { discharge } \\
\left(\mathrm{ft}^{3} / \mathrm{s}\right)\end{array}$ & $\begin{array}{l}\text { Water } \\
\text { year }\end{array}$ & Date & $\begin{array}{c}\text { Gage } \\
\text { height } \\
\text { (feet) }\end{array}$ & $\begin{array}{c}\text { Peak } \\
\text { discharge } \\
\left(\mathrm{ft}^{3} / \mathrm{s}\right)\end{array}$ \\
\hline \multicolumn{8}{|c|}{ Annual peak discharge, by year, and corresponding gage height } \\
\hline 1960 & April 15 & 4.80 & 102 & 1978 & March 29 & 7.64 & 600 \\
\hline 1961 & $\operatorname{March} 27$ & 2.25 & 2.50 & 1979 & April 21 & 5.39 & 365 \\
\hline 1962 & July 6 & 5.69 & 135 & 1980 & March 23 & 4.50 & 130 \\
\hline 1963 & April 4 & 4.19 & 68.0 & 1981 & -- & -- & 0 \\
\hline 1964 & April 25 & 3.52 & 46.0 & 1982 & April 17 & 2.88 & 20.0 \\
\hline 1965 & April 13 & 4.80 & 173 & 1983 & October 9 & 2.45 & 7.60 \\
\hline 1966 & March 15 & 7.12 & 660 & 1984 & March 28 & 4.62 & 150 \\
\hline 1967 & June 15 & 5.39 & 257 & 1985 & March 18 & 3.20 & 38.0 \\
\hline 1968 & April 7 & 3.86 & 91.0 & 1986 & April $20^{\circ}$ & 5.51 & 361 \\
\hline 1969 & April 8 & 8.77 & 1,270 & 1987 & March 30 & 4.06 & 138 \\
\hline 1970 & June 16 & 3.21 & 39.0 & 1988 & February 27 & - & 1.00 \\
\hline 1971 & March 16 & 4.04 & 70.0 & 1989 & March 31 & 8.47 & 700 \\
\hline 1972 & March 18 & 6.64 & 435 & 1990 & -. & - & 0 \\
\hline 1973 & March 26 & 2.47 & 13.0 & 1991 & July 3 & 3.29 & 25.0 \\
\hline 1974 & March 20 & 2.93 & 30.0 & 1992 & June 22 & 3.50 & 25.0 \\
\hline 1975 & July 1 & 4.52 & 133 & 1993 & March 28 & 6.12 & 300 \\
\hline 1976 & March 23 & 5.37 & 200 & 1994 & $\operatorname{March} 21$ & 7.08 & 400 \\
\hline 1977 & -- & - & 0 & & & & \\
\hline \multicolumn{8}{|c|}{ Annual peak discharge, from highest to lowest, and corresponding gage height } \\
\hline 1969 & April 8 & 8.77 & 1,270 & 1968 & April 7 & 3.86 & 91.0 \\
\hline 1989 & March 31 & 8.47 & 700 & 1971 & March 16 & 4.04 & 70.0 \\
\hline 1966 & March 15 & 7.12 & 660 & 1963 & April 4 & 4.19 & 68.0 \\
\hline 1978 & March 29 & 7.64 & 600 & 1964 & April 25 & 3.52 & 46.0 \\
\hline 1972 & March 18 & 6.64 & 435 & 1970 & June 16 & 3.21 & 39.0 \\
\hline 1994 & March 21 & 7.08 & 400 & 1985 & March 18 & 3.20 & 38.0 \\
\hline 1979 & April 21 & 5.39 & 365 & 1974 & March 20 & 2.93 & 30.0 \\
\hline 1986 & April 20 & 5.51 & 361 & 1991 & July 3 & 3.29 & 25.0 \\
\hline 1993 & March 28 & 6.12 & 300 & 1992 & June 22 & 3.50 & 25.0 \\
\hline 1967 & June 15 & 5.39 & 257 & 1982 & April 17 & 2.88 & 20.0 \\
\hline 1976 & March 23 & 5.37 & 200 & 1973 & March 26 & 2.47 & 13.0 \\
\hline 1965 & April 13 & 4.80 & 173 & 1983 & October 9 & 2.45 & 7.60 \\
\hline 1984 & March 28 & 4.62 & 150 & 1961 & March 27 & 2.25 & 2.50 \\
\hline 1987 & March 30 & 4.06 & 138 & 1988 & February 27 & - & 1.00 \\
\hline 1962 & July 6 & 5.69 & 135 & 1977 & -- & - & 0 \\
\hline 1975 & July 1 & 4.52 & 133 & 1981 & -- & -- & 0 \\
\hline 1980 & March 23 & 4.50 & 130 & 1990 & -- & - & 0 \\
\hline 1960 & April 15 & 4.80 & 102 & & & & \\
\hline
\end{tabular}




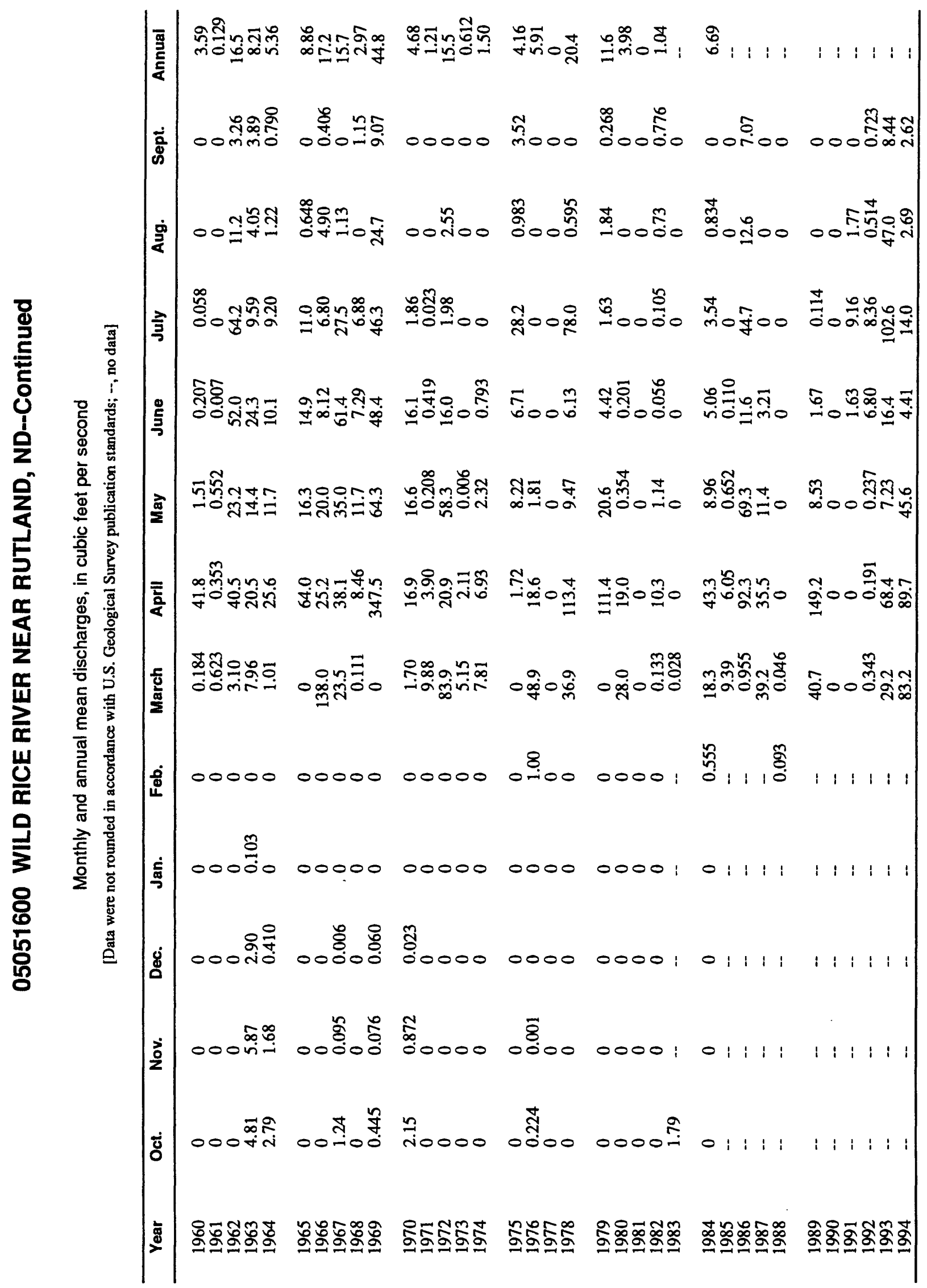




\section{WILD RICE RIVER NEAR CAYUGA, ND}

\section{Station Description}

LOCATION.--Lat $46^{\circ} 07^{\prime} 30^{\prime \prime}$, long 97 $21^{\prime} 40^{\prime \prime}$, on line between secs.29 and 30, T.131 N., R.53 W., Sargent County, Hydrologic Unit 09020105 , on left bank $20 \mathrm{ft}$ downstream from county highway bridge, $1.2 \mathrm{mi}$ downstream from Shortfoot Creek, $2.5 \mathrm{mi}$ downstream from Crooked Creek, and $3.5 \mathrm{mi}$ northeast of Cayuga.

DRAINAGE AREA.--955 $\mathrm{mi}^{2}$, of which about $390 \mathrm{mi}^{2}$ is probably noncontributing.

PERIOD OF RECORD.--May 1956 to September 1979.

GAGE.--Water-stage recorder. Datum of gage is 1,095.64 ft National Geodetic Vertical Datum of 1929. Prior to Oct. 9, 1957 , nonrecording gage $0.8 \mathrm{mi}$ upstream at different datum.

EXTREMES FOR PERIOD OF RECORD.--Maximum discharge, 1,710 ft 3 /s, Apr. 12, 1969 (gage height, $9.32 \mathrm{ft}$ ); maximum gage height, $10.90 \mathrm{ft}$, Apr. 7, 1969, backwater from ice; no flow at times each year.

Annual mean discharge

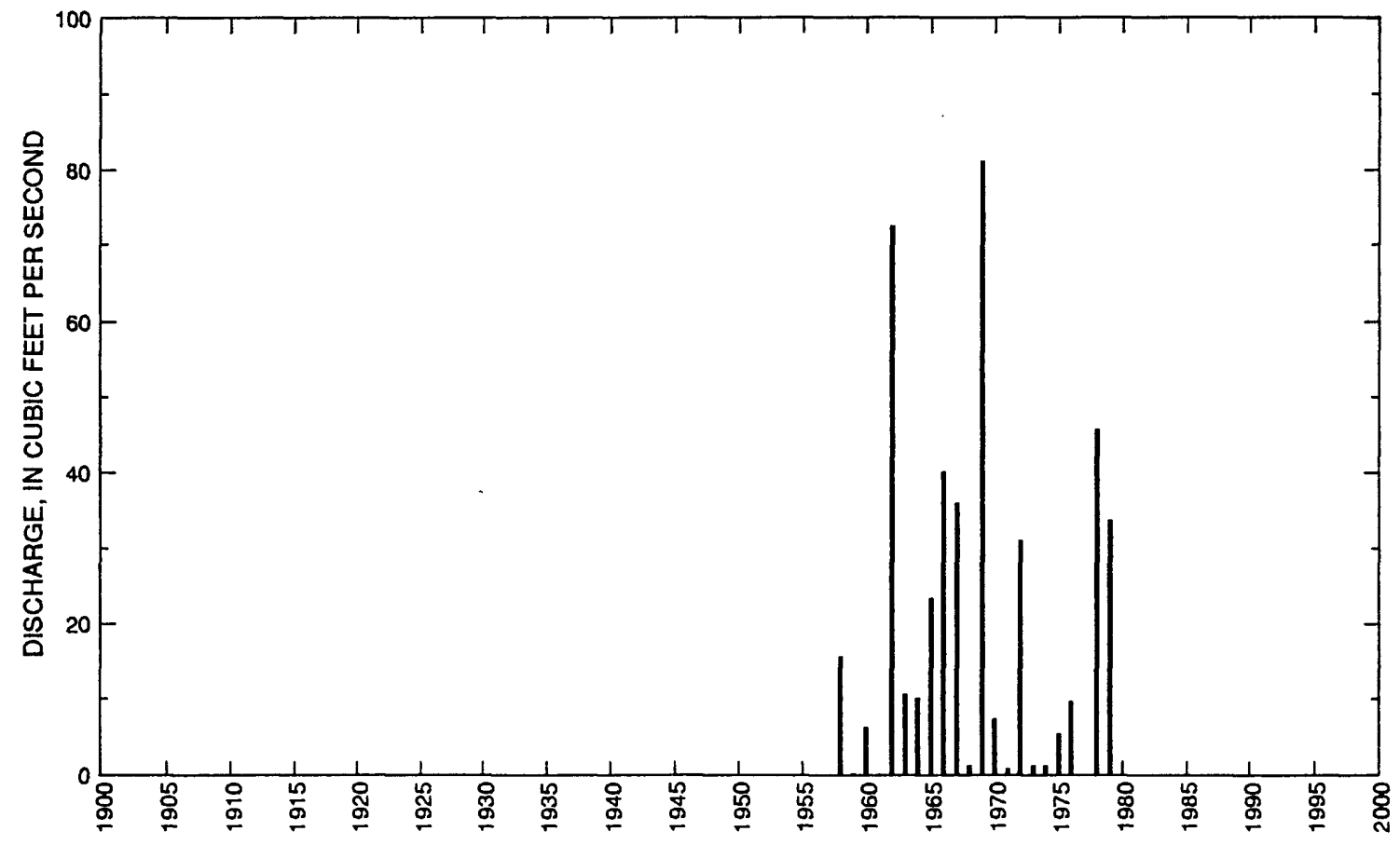




\section{WILD RICE RIVER NEAR CAYUGA, ND-Continued}

Statistics of monthly and annual mean discharges

[ $m$, more than 1 year of occurrence]

\begin{tabular}{|c|c|c|c|c|c|c|c|c|}
\hline \multirow[b]{2}{*}{ Month } & \multicolumn{2}{|c|}{ Maximum } & \multicolumn{2}{|c|}{ Minimum } & \multicolumn{4}{|c|}{ Mean } \\
\hline & $\begin{array}{c}\text { Discharge } \\
\left(\mathrm{ft}^{3} / \mathrm{s}\right)\end{array}$ & $\begin{array}{c}\text { Water year } \\
\text { of } \\
\text { occurrence }\end{array}$ & $\begin{array}{c}\text { Discharge } \\
\left(\mathrm{ft}^{3} / \mathrm{s}\right)\end{array}$ & $\begin{array}{c}\text { Water year } \\
\text { of } \\
\text { occurrence }\end{array}$ & $\begin{array}{c}\text { Discharge } \\
\left(\mathrm{ft}^{3} / \mathrm{s}\right)\end{array}$ & $\begin{array}{c}\text { Standard } \\
\text { deviation } \\
\left(\mathrm{f}^{3} / \mathrm{s}\right)\end{array}$ & $\begin{array}{l}\text { Coeffl- } \\
\text { cient of } \\
\text { variation }\end{array}$ & $\begin{array}{l}\text { Percentage } \\
\text { of annual } \\
\text { discharge }\end{array}$ \\
\hline October & 11.2 & 1958 & 0 & $\mathrm{~m}$ & 0.990 & 2.94 & 2.97 & 0.42 \\
\hline November & 16.2 & 1958 & 0 & $\mathrm{~m}$ & 1.23 & 3.92 & 3.18 & 0.52 \\
\hline December & 5.35 & 1958 & 0 & $\mathrm{~m}$ & 0.390 & 1.22 & 3.10 & 0.17 \\
\hline January & 0.719 & 1958 & 0 & $\mathrm{~m}$ & 0.040 & 0.15 & 4.23 & 0.02 \\
\hline February & 3.31 & 1976 & 0 & $\mathrm{~m}$ & 0.210 & 0.74 & 3.54 & 0.09 \\
\hline March & 273 & 1966 & 0 & $\mathrm{~m}$ & 26.8 & 62.9 & 2.34 & 11.4 \\
\hline April & 715 & 1969 & 0 & $\mathrm{~m}$ & 99.0 & 159 & 1.60 & 42.0 \\
\hline May & 153 & 1969 & 0 & $\mathrm{~m}$ & 38.4 & 46.5 & 1.21 & 16.3 \\
\hline June & 161 & 1962 & 0 & $\mathrm{~m}$ & 28.1 & 42.6 & 1.52 & 11.9 \\
\hline July & 351 & 1962 & 0 & $\mathrm{~m}$ & 35.1 & 78.4 & 2.24 & 14.9 \\
\hline August & 58.7 & 1962 & 0 & $\mathrm{~m}$ & 5.05 & 12.8 & 2.53 & 2.14 \\
\hline September & 2.28 & 1969 & 0 & $\mathrm{~m}$ & 0.390 & 0.66 & 1.72 & 0.16 \\
\hline Annual & 81.1 & 1969 & 0.009 & 1977 & 19.6 & 23.5 & 1.20 & 100 \\
\hline
\end{tabular}

Annual flow duration

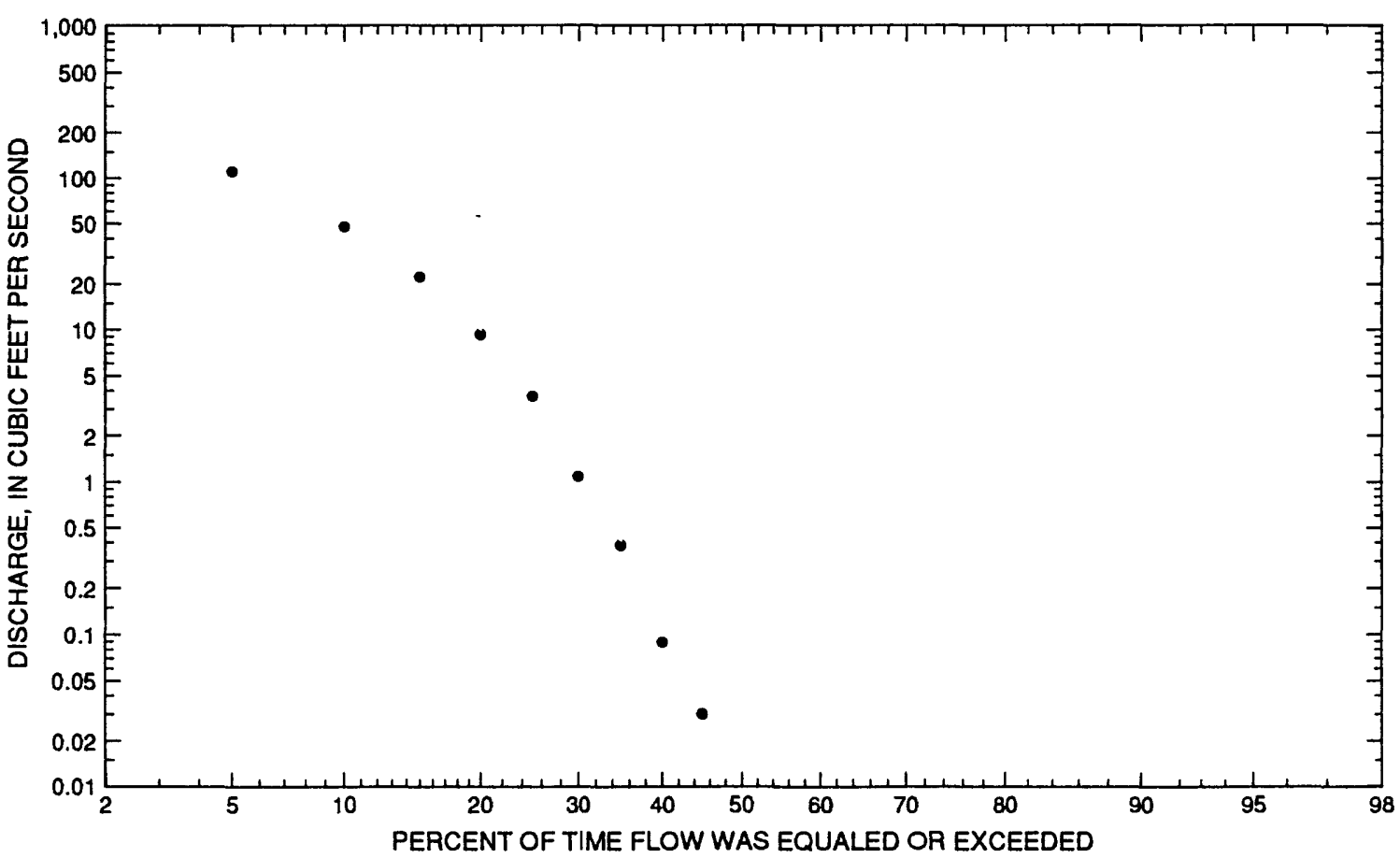




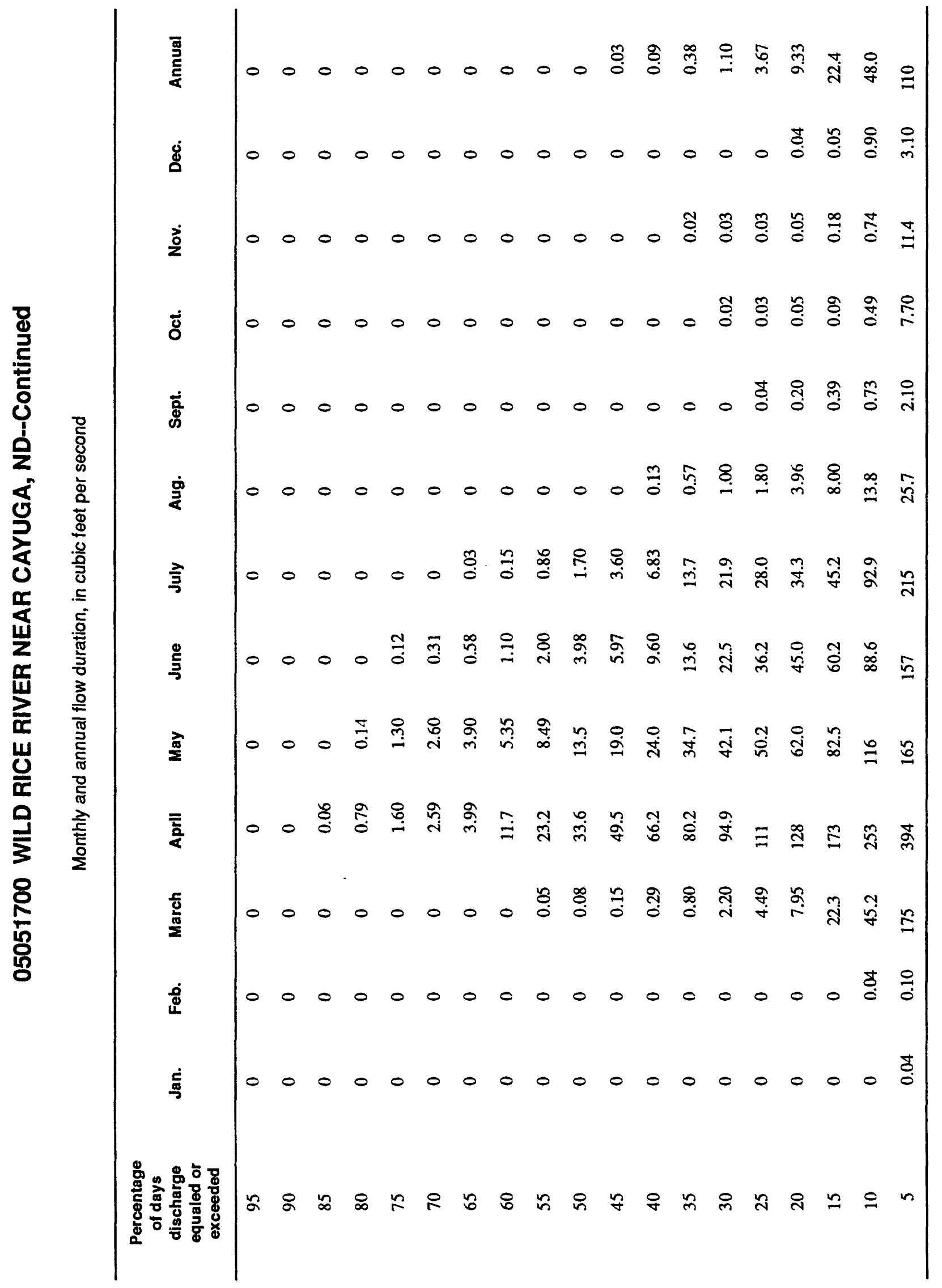




\section{WILD RICE RIVER NEAR CAYUGA, ND--Continued}

Probability of annual high discharges

[ng, statistic not given]

\begin{tabular}{lcccccc}
\hline & & & \multicolumn{5}{c}{$\begin{array}{c}\text { Maximum average discharge } \\
\left(\mathrm{ft}^{3} / \mathbf{s}\right)\end{array}$} \\
\cline { 5 - 7 } $\begin{array}{c}\text { Exceedance } \\
\text { probability }\end{array}$ & $\begin{array}{c}\text { Recurrence } \\
\text { intervai } \\
\text { (years) }\end{array}$ & $\begin{array}{c}\text { Maximum } \\
\text { instantaneous } \\
\left(\mathbf{f t}^{\mathbf{3}} / \mathbf{s}\right)\end{array}$ & 3-day period & 7-day period & 15-day period & 30-day period \\
\hline 0.99 & 1.01 & $\mathrm{ng}$ & 0.101 & 0.066 & 0.036 & 0.020 \\
0.95 & 1.05 & 12.1 & 1.48 & 0.986 & 0.580 & 0.358 \\
0.90 & 1.11 & 24.3 & 5.08 & 3.44 & 2.12 & 1.37 \\
0.80 & 1.25 & 53.1 & 18.8 & 13.2 & 8.58 & 5.80 \\
0.50 & 2 & 198 & 138 & 107 & 76.9 & 54.8 \\
0.20 & 5 & 586 & 548 & 481 & 385 & 279 \\
0.10 & 10 & 951 & 913 & 861 & 728 & 526 \\
0.04 & 25 & 1,500 & 1,370 & 1,400 & 1,250 & 895 \\
0.02 & 50 & 1,950 & 1,680 & 1,790 & 1,660 & 1,180 \\
0.01 & 100 & 2,420 & 1,940 & 2,160 & 2,050 & 1,440 \\
0.005 & 200 & 2,910 & 2,150 & 2,480 & 2,420 & 1,690 \\
0.002 & 500 & 3,550 & $n g$ & $n g$ & $n g$ & ng \\
\hline
\end{tabular}

Probability of annual low discharges

[ng, statistic not given]

\begin{tabular}{|c|c|c|c|c|c|c|c|c|c|c|}
\hline \multirow{3}{*}{$\begin{array}{c}\text { Non- } \\
\text { exceed- } \\
\text { ance } \\
\text { prob- } \\
\text { ability }\end{array}$} & \multirow{3}{*}{$\begin{array}{l}\text { Recur- } \\
\text { rence } \\
\text { inter- } \\
\text { val } \\
\text { (years) }\end{array}$} & \multicolumn{9}{|c|}{ Minimum average discharge $\left(\mathrm{ft}^{3} / \mathrm{s}\right)$} \\
\hline & & \multicolumn{9}{|c|}{ Number of consecutive days } \\
\hline & & 1 & 3 & 7 & 14 & 30 & 60 & 90 & 120 & 183 \\
\hline 0.05 & 20 & ng & ng & ng & ng & ng & ng & 0 & 0 & 0 \\
\hline 0.10 & 10 & ng & ng & ng & ng & $\mathrm{ng}$ & ng & 0 & 0 & 0 \\
\hline 0.20 & 5 & ng & ng & ng & ng & ng & ng & 0 & 0 & 0 \\
\hline 0.50 & 2 & ng & ng & ng & ng & ng & ng & 0 & 0 & 0 \\
\hline
\end{tabular}




\section{WILD RICE RIVER NEAR CAYUGA, ND--Continued}

Probability of seasonal low discharges

[ng, statistic not given]

\begin{tabular}{|c|c|c|c|c|c|c|c|c|c|}
\hline \multirow[b]{3}{*}{$\begin{array}{l}\text { Non- } \\
\text { exceedance } \\
\text { probability }\end{array}$} & \multirow[b]{3}{*}{$\begin{array}{l}\text { Recurrence } \\
\text { interval } \\
\text { (years) }\end{array}$} & \multicolumn{8}{|c|}{ Minimum average discharge $\left(\mathrm{ft}^{3} / \mathrm{s}\right)$} \\
\hline & & \multicolumn{8}{|c|}{ Number of consecutive days } \\
\hline & & 1 & 7 & 14 & 30 & 1 & 7 & 14 & 30 \\
\hline & & \multicolumn{4}{|c|}{ December-January-February } & \multicolumn{4}{|c|}{ March-April-May } \\
\hline 0.05 & 20 & ng & ng & ng & ng & 0 & 0 & 0 & 0 \\
\hline 0.10 & 10 & ng & ng & $\mathrm{ng}$ & ng & 0 & 0 & 0 & 0 \\
\hline 0.20 & 5 & ng & ng & ng & $\mathrm{ng}$ & 0 & 0 & 0 & 0 \\
\hline \multirow[t]{2}{*}{0.50} & 2 & ng & ng & ng & ng & 0 & 0 & 0.007 & 0.878 \\
\hline & & 1 & \multicolumn{3}{|c|}{ June-July-August } & \multicolumn{4}{|c|}{ September-October-November } \\
\hline 0.05 & 20 & 0 & 0 & 0 & 0 & $\mathrm{ng}$ & $\mathrm{ng}$ & $\mathrm{ng}$ & 0 \\
\hline 0.10 & 10 & 0 & 0 & 0 & 0 & $\mathrm{ng}$ & $\mathrm{ng}$ & $\mathrm{ng}$ & 0 \\
\hline 0.20 & 5 & 0 & 0 & 0 & 0 & ng & $\mathrm{ng}$ & $\mathrm{ng}$ & 0 \\
\hline 0.50 & 2 & 0 & 0 & 0 & 0 & $\mathrm{ng}$ & $\mathrm{ng}$ & $\mathrm{ng}$ & 0 \\
\hline
\end{tabular}


05051700 WILD RICE RIVER NEAR CAYUGA, ND--Continued

Annual peak discharge and corresponding gage height

\begin{tabular}{|c|c|c|c|c|c|c|c|}
\hline $\begin{array}{l}\text { Water } \\
\text { years }\end{array}$ & Date & $\begin{array}{c}\text { Gage } \\
\text { height } \\
\text { (feet) }\end{array}$ & $\begin{array}{c}\text { Peak } \\
\text { discharge } \\
\left(\mathrm{ft}^{3} / \mathrm{s}\right)\end{array}$ & $\begin{array}{l}\text { Water } \\
\text { year }\end{array}$ & Date & $\begin{array}{c}\text { Gage } \\
\text { height } \\
\text { (feet) }\end{array}$ & $\begin{array}{c}\text { Peak } \\
\text { dlscharge } \\
\left(\mathrm{ft}^{3} / \mathbf{s}\right)\end{array}$ \\
\hline \multicolumn{8}{|c|}{ Annual peak discharge, by year, and corresponding gage height } \\
\hline 1957 & April 19 & 6.07 & 150 & 1969 & April 12 & 9.32 & 1,710 \\
\hline 1958 & April 15 & 5.08 & 131 & 1970 & June 17 & 4.24 & 177 \\
\hline 1959 & June 12 & 3.51 & 54.0 & 1971 & March 14 & 4.02 & 55.0 \\
\hline 1960 & April 19 & 4.40 & 123 & 1972 & March 27 & 6.05 & 488 \\
\hline 1961 & March 12 & 2.77 & 1.20 & 1973 & July 1 & 3.88 & 114 \\
\hline 1962 & July 6 & 8.95 & 1,080 & 1974 & March 7 & 3.62 & 53.0 \\
\hline 1963 & June 10 & 3.95 & 107 & 1975 & July 1 & 7.66 & 636 \\
\hline 1964 & April 27 & 4.30 & 153 & 1976 & March 28 & 4.30 & 185 \\
\hline 1965 & April 14 & 6.08 & 375 & 1977 & March 26 & 2.98 & 3.60 \\
\hline 1966 & March 19 & 7.28 & 757 & 1978 & April 4 & 6.24 & 499 \\
\hline 1967 & July 8 & 5.72 & 309 & 1979 & April 12 & 7.29 & 600 \\
\hline 1968 & July 17 & 3.63 & 55.0 & & & & \\
\hline \multicolumn{8}{|c|}{ Annual peak discharge, from highest to lowest, and corresponding gage height } \\
\hline 1969 & April 12 & 9.32 & 1,710 & 1957 & April 19 & 6.07 & 150 \\
\hline 1962 & July 6 & 8.95 & 1,080 & 1958 & April 15 & 5.08 & 131 \\
\hline 1966 & March 19 & 7.28 & 757 & 1960 & April 19 & 4.40 & 123 \\
\hline 1975 & July 1 & 7.66 & 636 & 1973 & July 1 & 3.88 & 114 \\
\hline 1979 & April 12 & 7.29 & 600 & 1963 & June 10 & 3.95 & 107 \\
\hline 1978 & April 4 & 6.24 & 499 & 1968 & July 17 & 3.63 & 55.0 \\
\hline 1972 & March 27 & 6.05 & 488 & 1971 & March 14 & 4.02 & 55.0 \\
\hline 1965 & April 14 & 6.08 & 375 & 1959 & June 12 & 3.51 & 54.0 \\
\hline 1967 & July 8 & 5.72 & 309 & 1974 & March 7 & 3.62 & 53.0 \\
\hline 1976 & March 28 & 4.30 & 185 & 1977 & March 26 & 2.98 & 3.60 \\
\hline 1970 & June 17 & 4.24 & 177 & 1961 & March 12 & 2.77 & 1.20 \\
\hline 1964 & April 27 & 4.30 & 153 & & & & \\
\hline
\end{tabular}




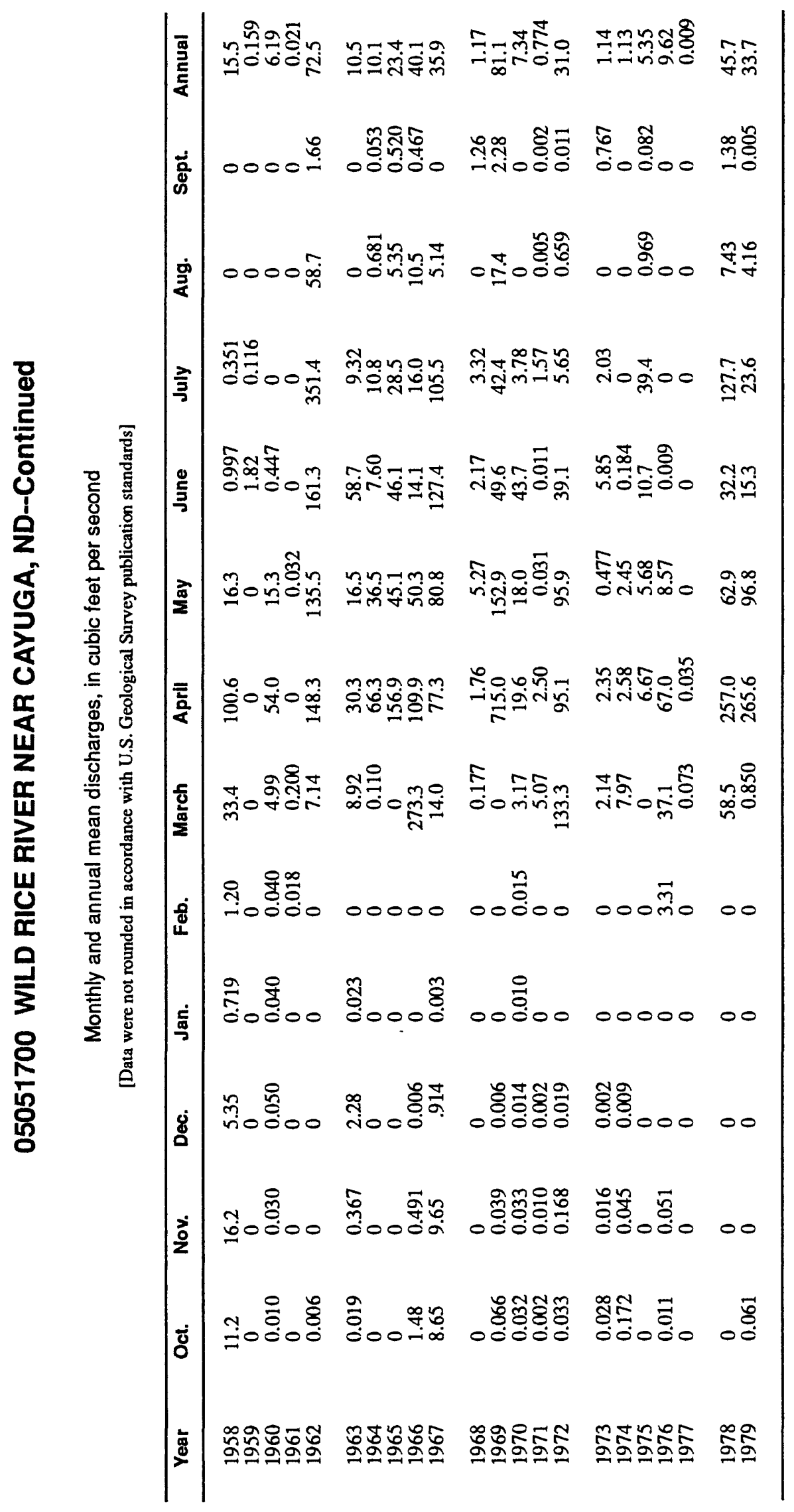




\section{WILD RICE RIVER NEAR ABERCROMBIE, ND}

\section{Station Description}

LOCATION.--Lat $46^{\circ} 28^{\prime} 05^{\prime \prime}$, long $96^{\circ} 47^{\prime} 00^{\prime \prime}$, in NE $1 / 4 \mathrm{NE}^{1 / 4} \mathrm{sec} .36, \mathrm{~T} .135 \mathrm{~N}$., R.49 W., Richland County, Hydrologic Unit 09020105 , on right bank $420 \mathrm{ft}$ upstream from bridge on county highway, $0.75 \mathrm{mi}$ upstream from rubble masonry dam which serves as control, $3.2 \mathrm{mi}$ northwest of Abercrombie, and $7 \mathrm{mi}$ downstream from Antelope Creek.

DRAINAGE AREA.--2,080 $\mathrm{mi}^{2}$, of which about $590 \mathrm{mi}^{2}$ is probably noncontributing.

PERIOD OF RECORD.--April 1932 to current year. Monthly discharge only for some periods, published in Water-Supply Paper 1308.

GAGE.--Water-stage recorder and masonry control. Datum of gage is $907.94 \mathrm{ft}$ above sea level. Prior to Dec. 7, 1939, nonrecording gage at site $420 \mathrm{ft}$ downstream at datum $5.0 \mathrm{ft}$ lower. Dec. 7, 1939, to Nov. 24,1952 , nonrecording gage at site $0.75 \mathrm{mi}$ downstream at present datum.

EXTREMES FOR PERIOD OF RECORD.--Maximum discharge, 9,540 $\mathrm{ft}^{3} / \mathrm{s}$, Apr. 11, 1969; maximum gage height, $24.58 \mathrm{ft}$, Apr. 11, 1969; no flow at times.

EXTREMES OUTSIDE PERIOD OF RECORD.--Flood in spring of 1897 reached a stage of $27.5 \mathrm{ft}$, present site and datum, from floodmarks pointed out by local residents.

Annual mean discharge

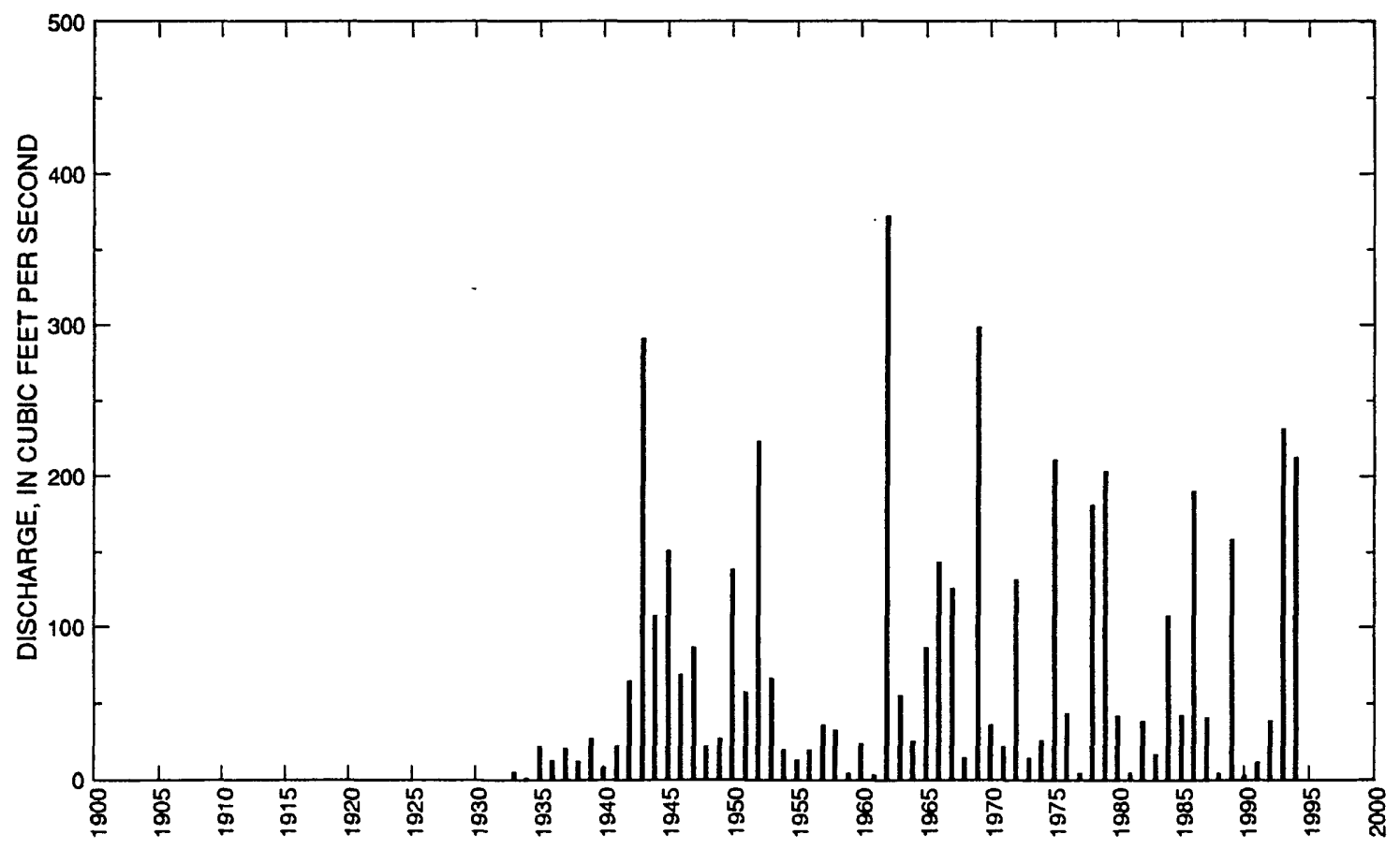




\section{WILD RICE RIVER NEAR ABERCROMBIE, ND--Continued}

Pre-regulation period, 1933-57

Statistics of monthly and annual mean discharges, pre-regulation period

[m, more than 1 year of occurrence]

\begin{tabular}{|c|c|c|c|c|c|c|c|c|}
\hline \multirow[b]{2}{*}{ Month } & \multicolumn{2}{|c|}{ Maximum } & \multicolumn{2}{|c|}{ Minimum } & \multicolumn{4}{|c|}{ Mean } \\
\hline & $\begin{array}{c}\text { Discharge } \\
\left(\mathrm{ft}^{3} / \mathrm{s}\right)\end{array}$ & $\begin{array}{c}\text { Water year } \\
\text { of } \\
\text { occurrence }\end{array}$ & $\begin{array}{c}\text { Discharge } \\
\left(\mathrm{ft}^{3} / \mathrm{s}\right)\end{array}$ & $\begin{array}{c}\text { Water year } \\
\text { of } \\
\text { occurrence }\end{array}$ & $\begin{array}{c}\text { Discharge } \\
\left(\mathrm{ft}^{3} / \mathbf{s}\right)\end{array}$ & $\begin{array}{c}\text { Standard } \\
\text { deviation } \\
\left(\mathrm{ft}^{3} / \mathrm{s}\right)\end{array}$ & $\begin{array}{l}\text { Coeffi- } \\
\text { cient of } \\
\text { variation }\end{array}$ & $\begin{array}{l}\text { Percentage } \\
\text { of annual } \\
\text { dlscharge }\end{array}$ \\
\hline October & 111 & 1943 & 0 & $\mathrm{~m}$ & 6.17 & 22.2 & 3.60 & 0.86 \\
\hline November & 60.4 & 1943 & 0 & $\mathrm{~m}$ & 4.98 & 13.4 & 2.69 & 0.69 \\
\hline December & 19.2 & 1943 & 0 & $\mathrm{~m}$ & 2.21 & 4.77 & 2.16 & 0.31 \\
\hline January & 6.69 & 1943 & 0 & $\mathrm{~m}$ & 0.450 & 1.35 & 2.99 & 0.06 \\
\hline February & 1.83 & 1943 & 0 & $\mathrm{~m}$ & 0.240 & 0.47 & 1.93 & 0.03 \\
\hline March & 1,010 & 1945 & 0 & $\mathrm{~m}$ & 118 & 219 & 1.85 & 16.4 \\
\hline April & 2,350 & 1952 & 3.71 & 1934 & 332 & 570 & 1.71 & 46.2 \\
\hline May & 594 & 1950 & 0.110 & 1934 & 91.1 & 131 & 1.44 & 12.7 \\
\hline June & 707 & 1943 & 0.357 & 1936 & 87.2 & 156 & 1.79 & 12.1 \\
\hline July & 464 & 1944 & 0 & $\mathrm{~m}$ & 51.5 & 109 & 2.12 & 7.16 \\
\hline August & 134 & 1944 & 0 & $m$ & 12.7 & 28.1 & 2.21 & 1.76 \\
\hline September & 100 & 1942 & 0 & $\mathrm{~m}$ & 11.8 & 28.8 & 2.44 & 1.64 \\
\hline Annual & 291 & 1943 & 0.482 & 1934 & 60.6 & 72.2 & 1.19 & 100 \\
\hline
\end{tabular}

Annual flow duration, pre-regulation period

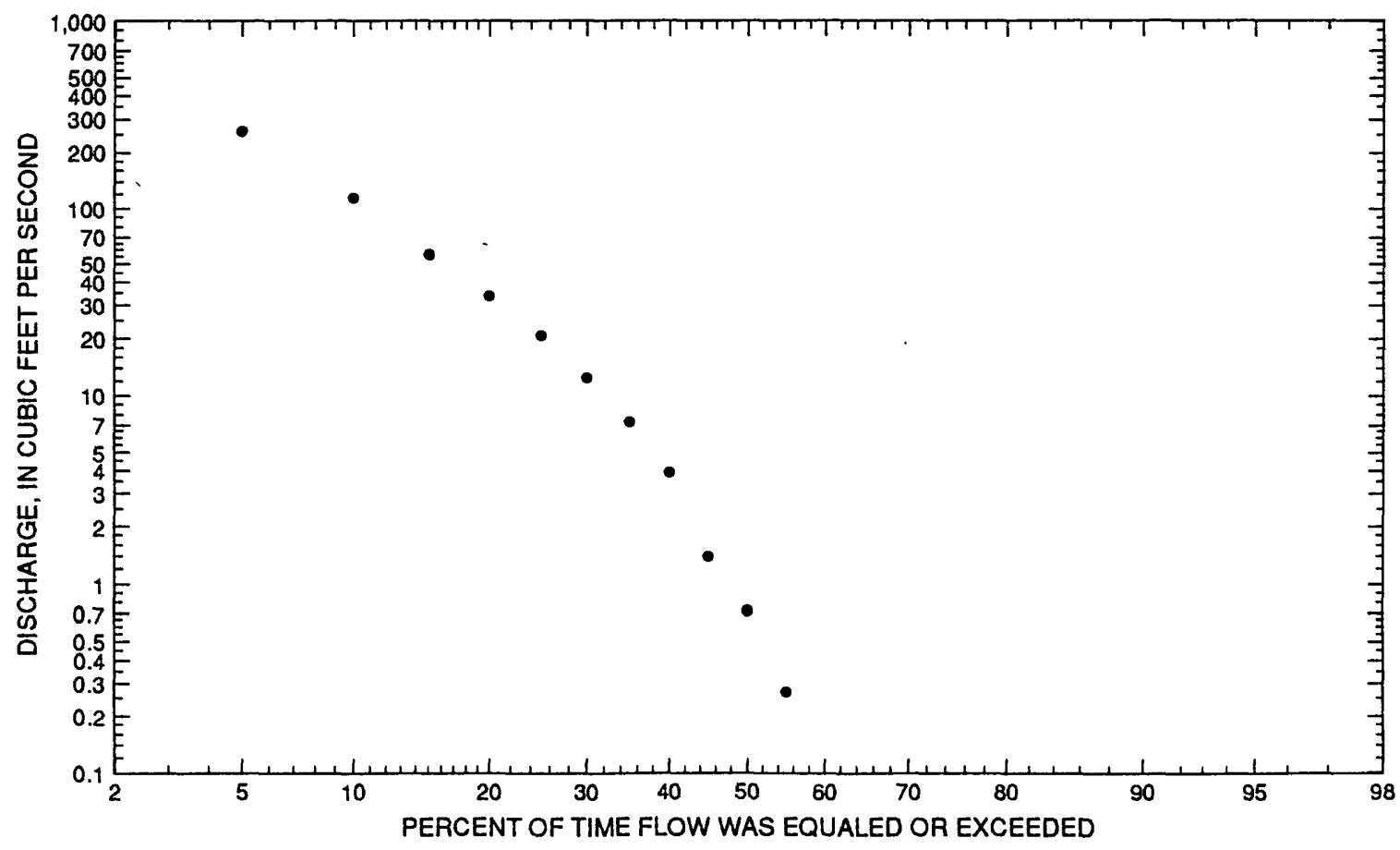




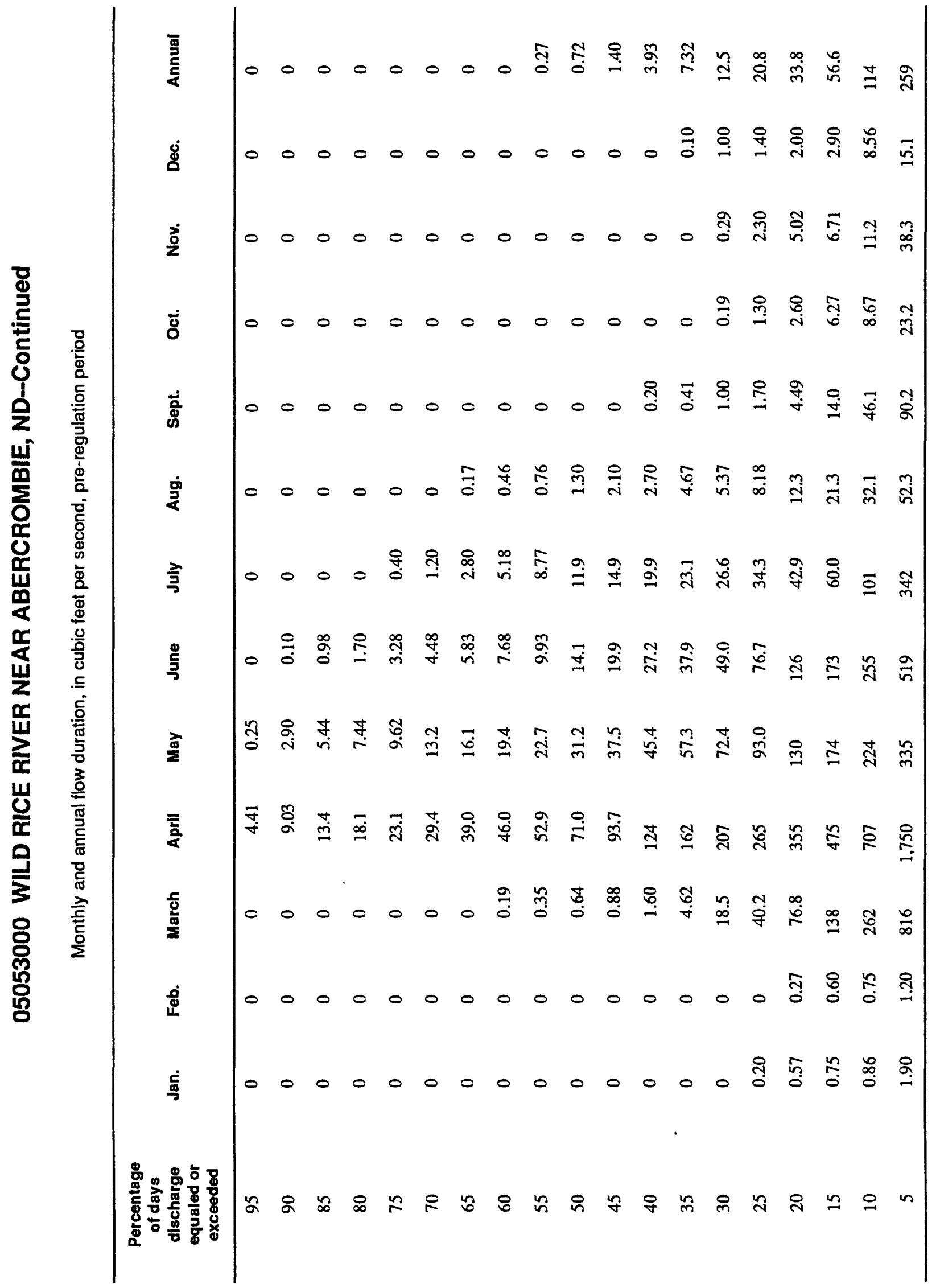




\section{WILD RICE RIVER NEAR ABERCROMBIE, ND--Continued}

Probability of annual high discharges, pre-regulation period

[ng, statistic not given]

\begin{tabular}{lcccccc}
\hline & & & \multicolumn{5}{c}{$\begin{array}{c}\text { Maximum average discharge } \\
\left(\mathbf{f t}^{3} / \mathbf{s}\right)\end{array}$} \\
\cline { 5 - 7 } $\begin{array}{c}\text { Exceedance } \\
\text { probability }\end{array}$ & $\begin{array}{c}\text { Recurrence } \\
\text { Interval } \\
\text { (years) }\end{array}$ & $\begin{array}{c}\text { Maximum } \\
\text { instantaneous } \\
\left(\mathbf{f t}^{3} / \mathbf{s}\right)\end{array}$ & 3-day period & 7-day period & 15-day period & 30-day period \\
\hline 0.99 & 1.01 & $\mathrm{ng}$ & 13.1 & 11.2 & 7.61 & 4.56 \\
0.95 & 1.05 & 145 & 55.9 & 45.4 & 30.0 & 19.0 \\
0.90 & 1.11 & 223 & 111 & 88.7 & 58.1 & 37.5 \\
0.80 & 1.25 & 367 & 237 & 186 & 122 & 79.9 \\
0.50 & 2 & 906 & 799 & 631 & 417 & 277 \\
0.20 & 5 & 2,090 & 2,040 & 1,670 & 1,140 & 748 \\
0.10 & 10 & 3,160 & 3,020 & 2,530 & 1,770 & 1,150 \\
0.04 & 25 & 4,810 & 4,280 & 3,710 & 2,670 & 1,690 \\
0.02 & 50 & 6,240 & 5,180 & 4,590 & 3,380 & 2,110 \\
0.01 & 100 & 7,840 & 6,020 & 5,460 & 4,100 & 2,520 \\
0.005 & 200 & 9,610 & 6,790 & 6,290 & 4,810 & 2,920 \\
0.002 & 500 & 12,200 & ng & ng & ng & ng \\
\hline
\end{tabular}

Probability of annual low discharges, pre-regulation period

[ng, statistic not given]

\begin{tabular}{|c|c|c|c|c|c|c|c|c|c|c|}
\hline \multirow{3}{*}{$\begin{array}{l}\text { Non- } \\
\text { exceed- } \\
\text { ance } \\
\text { prob- } \\
\text { ablility }\end{array}$} & \multirow{3}{*}{$\begin{array}{c}\text { Recur- } \\
\text { rence } \\
\text { Inter- } \\
\text { val } \\
\text { (years) }\end{array}$} & \multicolumn{9}{|c|}{ Minimum average discharge $\left(\mathrm{ft}^{3} / \mathrm{s}\right)$} \\
\hline & & \multicolumn{9}{|c|}{ Number of consecutive days } \\
\hline & & 1 & 3 & 7 & 14 & 30 & 60 & 90 & 120 & 183 \\
\hline 0.05 & 20 & $\mathrm{ng}$ & $\mathrm{ng}$ & $\mathrm{ng}$ & 0 & 0 & 0 & 0 & 0 & 0 \\
\hline 0.10 & 10 & $\mathrm{ng}$ & ng & $\mathrm{ng}$ & 0 & 0 & 0 & 0 & 0 & 0 \\
\hline 0.20 & 5 & ng & ng & $\mathrm{ng}$ & 0 & 0 & 0 & 0 & 0 & 0 \\
\hline 0.50 & 2 & $\mathrm{ng}$ & $\mathrm{ng}$ & $\mathrm{ng}$ & 0 & 0 & 0 & 0 & 0 & 0 \\
\hline
\end{tabular}




\section{WILD RICE RIVER NEAR ABERCROMBIE, ND--Continued}

Probability of seasonal low discharges, pre-regulation period

[ng, statistic not given]

\begin{tabular}{|c|c|c|c|c|c|c|c|c|c|}
\hline \multirow[b]{3}{*}{$\begin{array}{c}\text { Non- } \\
\text { exceedance } \\
\text { probability }\end{array}$} & \multirow[b]{3}{*}{$\begin{array}{c}\text { Recurrence } \\
\text { Interval } \\
\text { (years) }\end{array}$} & \multicolumn{8}{|c|}{ Minimum average dlscharge $\left(\mathrm{ft}^{3} / \mathrm{s}\right)$} \\
\hline & & \multicolumn{8}{|c|}{ Number of consecutive days } \\
\hline & & 1 & 7 & 14 & 30 & 1 & 7 & 14 & 30 \\
\hline & & \multicolumn{4}{|c|}{ December-January-February } & \multicolumn{4}{|c|}{ March-April-May } \\
\hline 0.05 & 20 & ng & 0 & 0 & 0 & 0 & 0 & 0 & 0 \\
\hline 0.10 & 10 & ng & 0 & 0 & 0 & 0 & 0 & 0 & 0 \\
\hline 0.20 & 5 & $\mathrm{ng}$ & 0 & 0 & 0 & 0 & 0 & 0 & 0.992 \\
\hline \multirow[t]{2}{*}{0.50} & 2 & ng & 0 & 0 & 0 & 0 & 0 & 0.256 & 12.2 \\
\hline & & \multicolumn{4}{|c|}{ June-July-August } & \multicolumn{4}{|c|}{ September-October-November } \\
\hline 0.05 & 20 & 0 & 0 & 0 & 0 & 0 & 0 & 0 & 0 \\
\hline 0.10 & 10 & 0 & 0 & 0 & 0 & 0 & 0 & 0 & 0 \\
\hline 0.20 & 5 & 0 & 0 & 0 & 0 & 0 & 0 & 0 & 0 \\
\hline 0.50 & 2 & 0 & 0.085 & 0.518 & 1.71 & 0 & 0 & 0 & 0 \\
\hline
\end{tabular}




\section{WILD RICE RIVER NEAR ABERCROMBIE, ND--Continued}

Post-regulation period, 1958-94

Statistics of monthly and annual mean discharges, post-regulation period

[m, more than 1 year of occurrence]

\begin{tabular}{|c|c|c|c|c|c|c|c|c|}
\hline \multirow[b]{2}{*}{ Month } & \multicolumn{2}{|c|}{ Maximum } & \multicolumn{2}{|c|}{ MInImum } & \multicolumn{4}{|c|}{ Moan } \\
\hline & $\begin{array}{c}\text { Dlscharge } \\
\left(\mathrm{ft}^{3} / \mathrm{s}\right)\end{array}$ & $\begin{array}{c}\text { Water year } \\
\text { of } \\
\text { occurrence }\end{array}$ & $\begin{array}{c}\text { Discharge } \\
\left(\mathrm{ft}^{3} / \mathrm{s}\right)\end{array}$ & $\begin{array}{c}\text { Water year } \\
\text { of } \\
\text { occurrence }\end{array}$ & $\begin{array}{c}\text { Discharge } \\
\left(\mathrm{ft}^{3} / \mathrm{s}\right)\end{array}$ & $\begin{array}{c}\text { Standard } \\
\text { deviation } \\
\left(\mathrm{ft}^{3} / \mathrm{s}\right)\end{array}$ & $\begin{array}{l}\text { Coeffl- } \\
\text { clent of } \\
\text { varlation }\end{array}$ & $\begin{array}{l}\text { Percentage } \\
\text { of annual } \\
\text { discharge }\end{array}$ \\
\hline October & 71.6 & 1987 & 0 & $\overline{\mathrm{m}}$ & 6.59 & 13.8 & 2.10 & 0.64 \\
\hline November & 37.5 & 1987 & 0 & $\mathrm{~m}$ & 5.40 & 8.70 & 1.61 & 0.52 \\
\hline December & 18.8 & 1987 & 0 & $\mathrm{~m}$ & 3.15 & 4.71 & 1.50 & 0.30 \\
\hline January & 14.5 & 1994 & 0 & $\mathrm{~m}$ & 1.34 & 2.65 & 1.98 & 0.13 \\
\hline February & 37.6 & 1984 & 0 & $\mathrm{~m}$ & 2.65 & 7.33 & 2.77 & 0.26 \\
\hline March & 902 & 1966 & 0 & $\mathrm{~m}$ & 152 & 227 & 1.50 & 14.6 \\
\hline April & 3,080 & 1969 & 2.81 & 1991 & 432 & 647 & 1.50 & 41.7 \\
\hline May & 728 & 1962 & 1.43 & 1988 & 121 & 181 & 1.49 & 11.7 \\
\hline June & 929 & 1962 & 0.085 & 1988 & 108 & 185 & 1.72 & 10.4 \\
\hline July & 1,790 & 1962 & 0 & 1961 & 158 & 376 & 2.39 & 15.2 \\
\hline August & 462 & 1993 & 0 & $\mathrm{~m}$ & 35.4 & 94.2 & 2.67 & 3.42 \\
\hline September & 159 & 1986 & 0 & $\mathrm{~m}$ & 10.7 & 30.3 & 2.84 & 1.03 \\
\hline Annual & 372 & 1962 & 2.70 & 1990 & 86.3 & 94.4 & 1.09 & 100 \\
\hline
\end{tabular}

Annual flow duration, post-regulation period

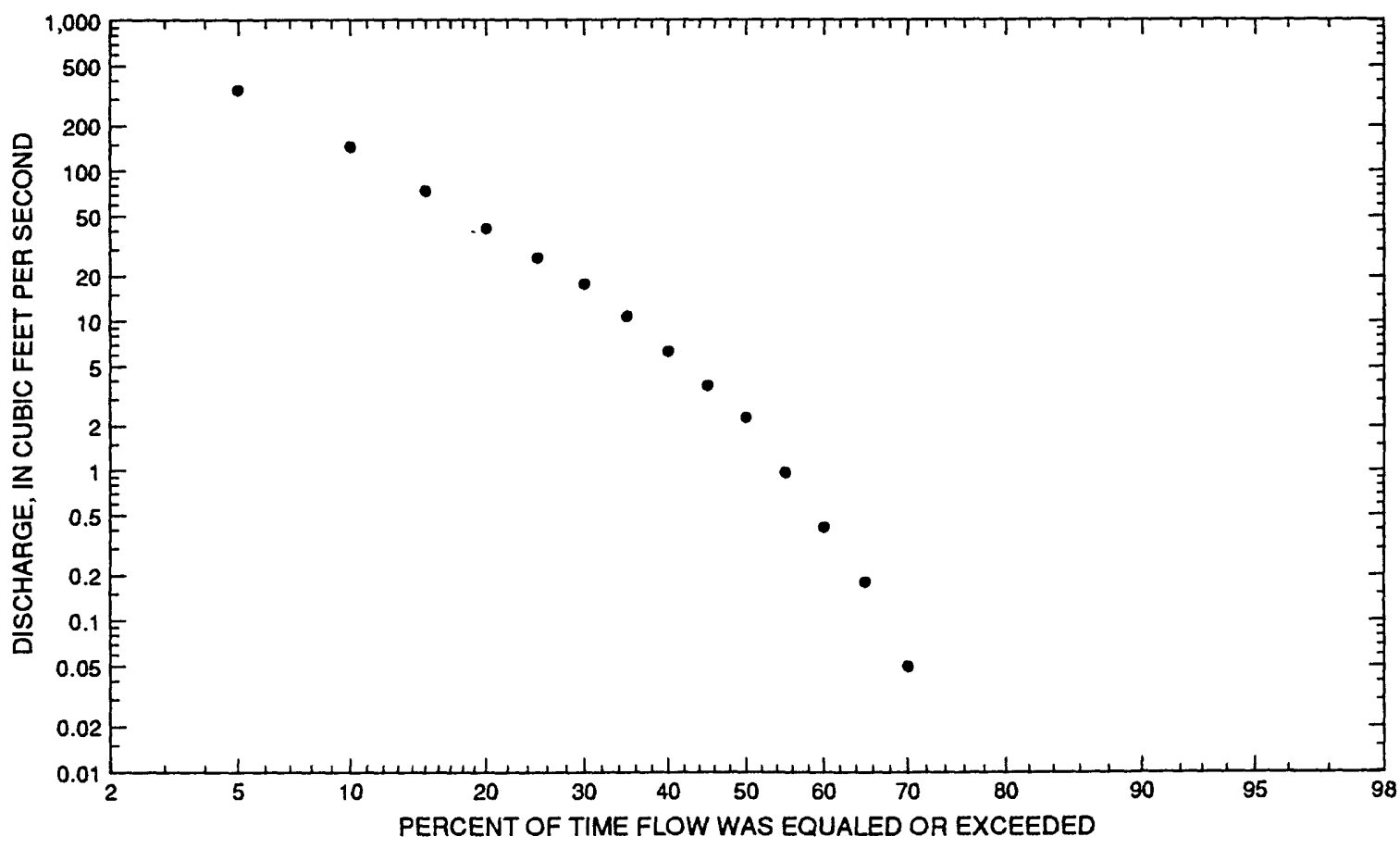




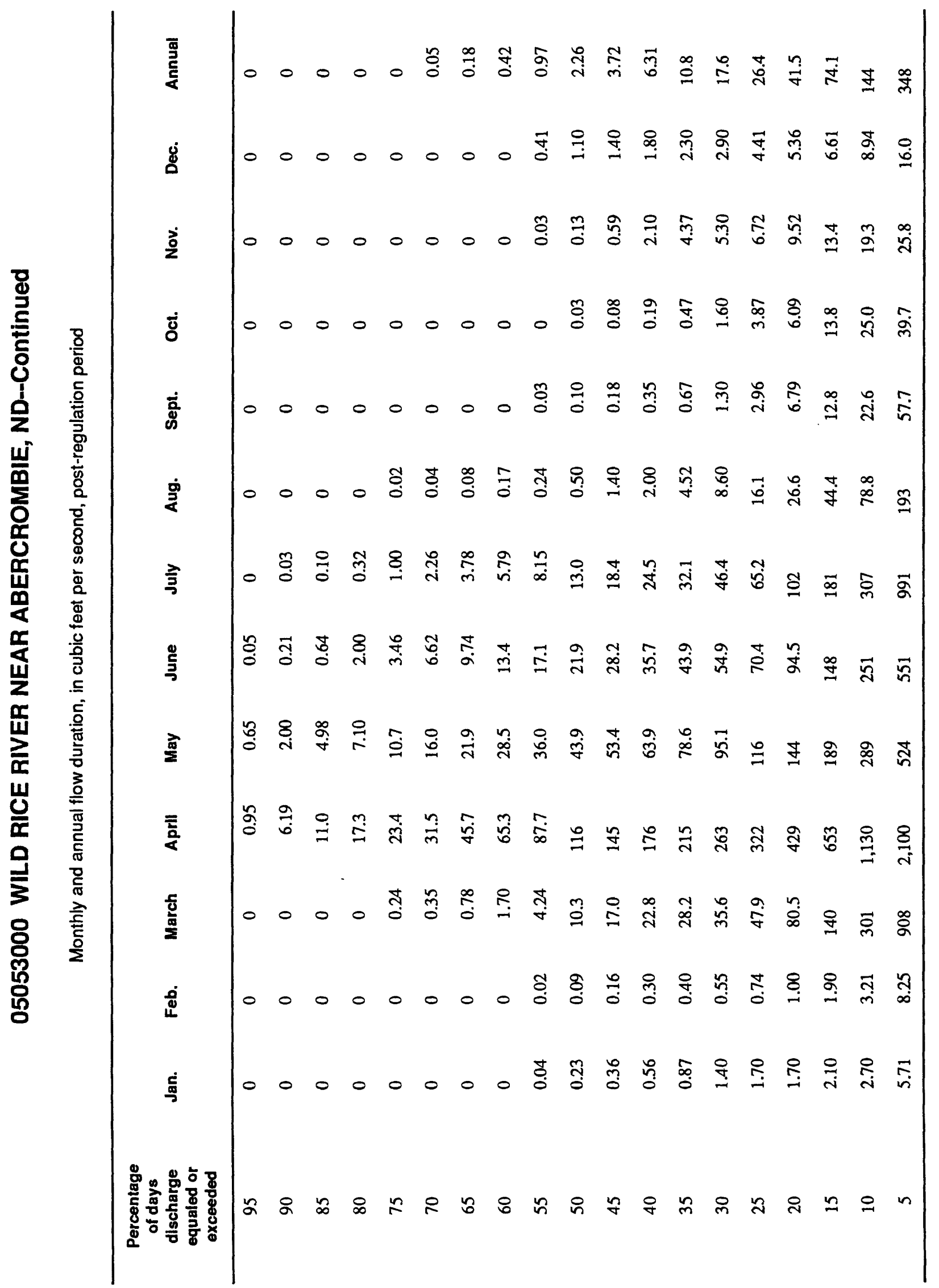




\section{WILD RICE RIVER NEAR ABERCROMBIE, ND--Continued}

Probability of annual high discharges, post-regulation period

[ng, statistic not given]

\begin{tabular}{lcccccc}
\hline & & & \multicolumn{5}{c}{$\begin{array}{c}\text { Maximum average discharge } \\
\left(\mathbf{f t}^{3} \mathbf{s}\right)\end{array}$} \\
\cline { 6 - 7 } $\begin{array}{c}\text { Exceedance } \\
\text { probability }\end{array}$ & $\begin{array}{c}\text { Recurrence } \\
\text { interval } \\
\text { (years) }\end{array}$ & $\begin{array}{c}\text { Maximum } \\
\text { instantaneous } \\
\left(\mathbf{f t}^{3} / \mathbf{s}\right)\end{array}$ & 3-day period & 7-day period & 15-day period & 30-day period \\
\hline 0.99 & 1.01 & 15.0 & 10.9 & 8.68 & 7.05 & 6.04 \\
0.95 & 1.05 & 58.8 & 45.5 & 35.5 & 27.0 & 21.2 \\
0.90 & 1.11 & 116 & 92.2 & 71.6 & 52.9 & 39.9 \\
0.80 & 1.25 & 251 & 206 & 160 & 115 & 83.2 \\
0.50 & 2 & 953 & 833 & 657 & 454 & 307 \\
0.20 & 5 & 3,030 & 2,800 & 2,280 & 1,550 & 1,000 \\
0.10 & 10 & 5,190 & 4,910 & 4,100 & 2,790 & 1,770 \\
0.04 & 25 & 8,770 & 8,500 & 7,330 & 5,020 & 3,140 \\
0.02 & 50 & 12,000 & 11,800 & 10,400 & 7,170 & 4,460 \\
0.01 & 100 & 15,600 & 15,600 & 14,000 & 9,750 & 6,050 \\
0.005 & 200 & 19,700 & 19,800 & 18,200 & 12,800 & 7,900 \\
0.002 & 500 & 25,500 & ng & ng & ng & ng \\
\hline
\end{tabular}

Probability of annual low discharges, post-regulation period

\begin{tabular}{|c|c|c|c|c|c|c|c|c|c|c|}
\hline \multirow[b]{3}{*}{$\begin{array}{l}\text { Non- } \\
\text { exceed- } \\
\text { ance } \\
\text { prob- } \\
\text { abillty }\end{array}$} & \multirow[b]{3}{*}{$\begin{array}{c}\text { Recur- } \\
\text { rence } \\
\text { inter- } \\
\text { val } \\
\text { (years) }\end{array}$} & \multicolumn{9}{|c|}{ Minimum average discharge $\left(f^{3} / s\right)$} \\
\hline & & \multicolumn{9}{|c|}{ Number of consecutlve days } \\
\hline & & 1 & 3 & 7 & 14 & 30 & 60 & 90 & 120 & 183 \\
\hline 0.05 & 20 & 0 & 0 & 0 & 0 & 0 & 0 & 0 & 0 & 0 \\
\hline 0.10 & 10 & 0 & 0 & 0 & 0 & 0 & 0 & 0 & 0 & 0 \\
\hline 0.20 & 5 & 0 & 0 & 0 & 0 & 0 & 0 & 0 & 0 & 0 \\
\hline 0.50 & 2 & 0 & 0 & 0 & 0 & 0 & 0.023 & 0.179 & 0.434 & 0.870 \\
\hline
\end{tabular}




\section{WILD RICE RIVER NEAR ABERCROMBIE, ND--Continued}

Probability of seasonal low discharges, post-regulation period

\begin{tabular}{|c|c|c|c|c|c|c|c|c|c|}
\hline \multirow[b]{3}{*}{$\begin{array}{c}\text { Non- } \\
\text { exceedance } \\
\text { probability }\end{array}$} & \multirow[b]{3}{*}{$\begin{array}{c}\text { Recurrence } \\
\text { intervai } \\
\text { (years) }\end{array}$} & \multicolumn{8}{|c|}{ Minimum average discharge $\left(\mathrm{ft}^{3} / \mathrm{s}\right)$} \\
\hline & & \multicolumn{8}{|c|}{ Number of consecutive days } \\
\hline & & 1 & 7 & 14 & 30 & 1 & 7 & 14 & 30 \\
\hline & & \multicolumn{4}{|c|}{ December-January-February } & \multicolumn{4}{|c|}{ March-April-May } \\
\hline 0.05 & 20 & 0 & 0 & 0 & 0 & 0 & 0 & 0 & 0 \\
\hline 0.10 & 10 & 0 & 0 & 0 & 0 & 0 & 0 & 0 & 0.364 \\
\hline 0.20 & 5 & 0 & 0 & 0 & 0 & 0 & 0 & 0 & 1.70 \\
\hline \multirow[t]{2}{*}{0.50} & 2 & 0 & 0 & 0 & 0.088 & 0.090 & 0.440 & 1.54 & 14.2 \\
\hline & & \multicolumn{4}{|c|}{ June-July-August } & \multicolumn{4}{|c|}{ September-October-November } \\
\hline 0.05 & 20 & 0 & 0 & 0 & 0 & 0 & 0 & 0 & 0 \\
\hline 0.10 & 10 & 0 & 0 & 0 & 0 & 0 & 0 & 0 & 0 \\
\hline 0.20 & 5 & 0 & 0 & 0 & 0.031 & 0 & 0 & 0 & 0 \\
\hline 0.50 & 2 & 0.037 & 0.142 & 0.279 & 1.20 & 0 & 0 & 0 & 0 \\
\hline
\end{tabular}


05053000 WILD RICE RIVER NEAR ABERCROMBIE, ND--Continued

Annual peak discharge and corresponding gage height, period of record

$$
[--, \text { no data] }
$$

\begin{tabular}{|c|c|c|c|c|c|c|c|}
\hline $\begin{array}{l}\text { Water } \\
\text { year }\end{array}$ & Date & $\begin{array}{c}\text { Gage } \\
\text { helght } \\
\text { (feet) }\end{array}$ & $\begin{array}{c}\text { Peak } \\
\text { dlscharge } \\
\left(\mathrm{ft}^{3} / \mathrm{s}\right)\end{array}$ & $\begin{array}{l}\text { Water } \\
\text { year }\end{array}$ & Date & $\begin{array}{c}\text { Gage } \\
\text { height } \\
\text { (feet) }\end{array}$ & $\begin{array}{c}\text { Peak } \\
\text { dlscharge } \\
\left(\mathrm{ft}^{3} / \mathbf{s}\right)\end{array}$ \\
\hline \multicolumn{8}{|c|}{ Annual peak discharge, by year, and corresponding gage height } \\
\hline 1933 & March 13 & 4.10 & 75.0 & 1964 & April 17 & 3.65 & 415 \\
\hline 1934 & April 7 & 2.10 & 15.0 & 1965 & April 12 & 16.50 & 2,820 \\
\hline 1935 & March 16 & 10.21 & 513 & 1966 & March 19 & 18.43 & 2,850 \\
\hline 1936 & March 22 & 8.00 & 415 & 1967 & June 17 & 14.17 & 2,050 \\
\hline 1937 & April 9 & 8.71 & 540 & 1968 & April 28 & 2.53 & 127 \\
\hline 1938 & March 17 & 7.28 & 318 & 1969 & April 11 & 24.58 & 9,540 \\
\hline 1939 & March 25 & 15.17 & 1,350 & 1970 & June 17 & 4.18 & 556 \\
\hline 1940 & April 8 & 3.20 & 300 & 1971 & July 4 & 4.18 & 508 \\
\hline 1941 & June 11 & 3.90 & 608 & 1972 & March 18 & 14.69 & 2,100 \\
\hline 1942 & June 10 & 3.77 & 579 & 1973 & March 17 & 3.67 & 426 \\
\hline 1943 & April 2 & 21.02 & 5,500 & 1974 & April 12 & 4.73 & 630 \\
\hline 1944 & July 7 & 6.58 & 956 & 1975 & July 4 & 19.16 & 3,500 \\
\hline 1945 & March 19 & 15.00 & 2,840 & 1976 & March 24 & 6.90 & 870 \\
\hline 1946 & March 24 & 13.07 & 2,320 & 1977 & September 27 & 2.41 & 91.0 \\
\hline 1947 & April 12 & - & 2,450 & 1978 & March 30 & 20.03 & 4,900 \\
\hline 1948 & April 11 & 4.66 & 729 & 1979 & April 15 & 22.39 & 6,000 \\
\hline 1949 & April 3 & 5.60 & 650 & 1980 & April 1 & 12.22 & 1,800 \\
\hline 1950 & April 3 & 16.28 & 2,300 & 1981 & April 2 & 1.69 & 25.8 \\
\hline 1951 & April 6 & 11.95 & 1,890 & 1982 & April 1 & 11.62 & 1,550 \\
\hline 1952 & April 12 & 20.62 & 5,400 & 1983 & March 19 & 2.79 & 265 \\
\hline 1953 & May 29 & 14.45 & 2,500 & 1984 & March 29 & 16.74 & 2,970 \\
\hline 1954 & July 2 & 5.93 & 800 & 1985 & June 3 & 9.01 & 1,210 \\
\hline 1955 & April 1 & 5.76 & 550 & 1986 & March 28 & 14.07 & 2,210 \\
\hline 1956 & April 12 & 6.17 & 750 & 1987 & March 27 & 5.34 & 701 \\
\hline 1957 & April 19 & 3.47 & 408 & 1988 & March 10 & 2.33 & 105 \\
\hline 1958 & April 9 & 3.03 & 262 & 1989 & April 7 & 23.27 & 7,150 \\
\hline 1959 & June 17 & 2.84 & 222 & 1990 & April 2 & 2.12 & 74.0 \\
\hline 1960 & April 6 & 5.00 & 640 & 1991 & July 5 & 3.72 & 410 \\
\hline 1961 & March 3 & 2.20 & 36.0 & 1992 & June 18 & 8.32 & 1,000 \\
\hline 1962 & July 9 & 18.38 & 3,610 & 1993 & April 1 & 17.52 & 3,630 \\
\hline 1963 & June 13 & 10.21 & 1,460 & 1994 & July 23 & 12.83 & 2,430 \\
\hline \multicolumn{8}{|c|}{ Annual peak discharge, from highest to lowest, and corresponding gage height } \\
\hline 1969 & April 11 & 24.58 & 9,540 & 1945 & March 19 & 15.00 & 2,840 \\
\hline 1989 & April 7 & 23.27 & 7,150 & 1965 & April 12 & 16.50 & 2,820 \\
\hline 1979 & April 15 & 22.39 & 6,000 & 1953 & May 29 & 14.45 & 2,500 \\
\hline 1943 & April 2 & 21.02 & 5,500 & 1947 & April 12 & - & 2,450 \\
\hline 1952 & April 12 & 20.62 & 5,400 & 1994 & July 23 & 12.83 & 2,430 \\
\hline 1978 & March 30 & 20.03 & 4,900 & 1946 & March 24 & 13.07 & 2,320 \\
\hline 1993 & April 1 & 17.52 & 3,630 & 1950 & April 3 & 16.28 & 2,300 \\
\hline 1962 & July 9 & 18.38 & 3,610 & 1986 & March 28 & 14.07 & 2,210 \\
\hline 1975 & July 4 & 19.16 & 3,500 & 1972 & March 18 & 14.69 & 2,100 \\
\hline 1984 & March 29 & 16.74 & 2,970 & 1967 & June 17 & 14.17 & 2,050 \\
\hline 1966 & March 19 & 18.43 & 2,850 & 1951 & April 6 & 11.95 & 1,890 \\
\hline
\end{tabular}




\section{WILD RICE RIVER NEAR ABERCROMBIE, ND--Continued}

Annual peak discharge and corresponding gage height, period of record--Continued

$$
[--, \text { no data }]
$$

\begin{tabular}{cccccccc}
\hline $\begin{array}{c}\text { Water } \\
\text { year }\end{array}$ & Date & $\begin{array}{c}\text { Gage } \\
\text { height } \\
\text { (foet) }\end{array}$ & $\begin{array}{c}\text { Peak } \\
\text { discharge } \\
\left(\mathrm{ft}^{3} / \mathbf{s}\right)\end{array}$ & $\begin{array}{c}\text { Water } \\
\text { year }\end{array}$ & Date & $\begin{array}{c}\text { Gage } \\
\text { height } \\
\text { (feet) }\end{array}$ & $\begin{array}{c}\text { Peak } \\
\text { discharge } \\
\left(\mathrm{ft}^{3} / \mathbf{s}\right)\end{array}$ \\
\hline \multicolumn{7}{c}{ Annual peak discharge, from hlghest to lowest, and corresponding gage helght.-Continued } \\
1980 & April 1 & 12.22 & 1,800 & 1935 & March 16 & 10.21 & 513 \\
1982 & April 1 & 11.62 & 1,550 & 1971 & July 4 & 4.18 & 508 \\
1963 & June 13 & 10.21 & 1,460 & 1973 & March 17 & 3.67 & 426 \\
1939 & March 25 & 15.17 & 1,350 & 1936 & March 22 & 8.00 & 415 \\
1985 & June 3 & 9.01 & 1,210 & 1964 & April 17 & 3.65 & 415 \\
1992 & June 18 & 8.32 & 1,000 & 1991 & July 5 & 3.72 & 410 \\
1944 & July 7 & 6.58 & 956 & 1957 & April 19 & 3.47 & 408 \\
1976 & March 24 & 6.90 & 870 & 1938 & March 17 & 7.28 & 318 \\
1954 & July 2 & 5.93 & 800 & 1940 & April 8 & 3.20 & 300 \\
1956 & April 12 & 6.17 & 750 & 1983 & March 19 & 2.79 & 265 \\
1948 & April 11 & 4.66 & 729 & 1958 & April 9 & 3.03 & 262 \\
1987 & March 27 & 5.34 & 701 & 1959 & June 17 & 2.84 & 222 \\
1949 & April 3 & 5.60 & 650 & 1968 & April 28 & 2.53 & 127 \\
1960 & April 6 & 5.00 & 640 & 1988 & March 10 & 2.33 & 105 \\
1974 & April 12 & 4.73 & 630 & 1977 & September 27 & 2.41 & 91.0 \\
1941 & June 11 & 3.90 & 608 & 1933 & March 13 & 4.10 & 75.0 \\
1942 & June 10 & 3.77 & 579 & 1990 & April 2 & 2.12 & 74.0 \\
1970 & June 17 & 4.18 & 556 & 1961 & March 3 & 2.20 & 36.0 \\
1955 & April 1 & 5.76 & 550 & 1981 & April 2 & 1.69 & 25.8 \\
1937 & April 9 & 8.71 & 540 & 1934 & April 7 & 2.10 & 15.0 \\
\hline
\end{tabular}




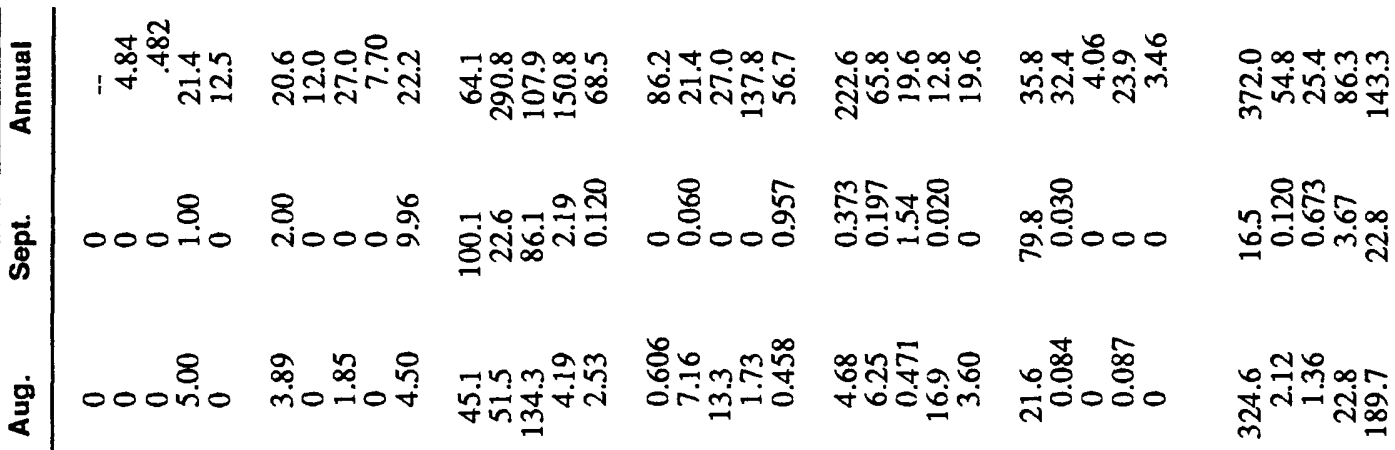

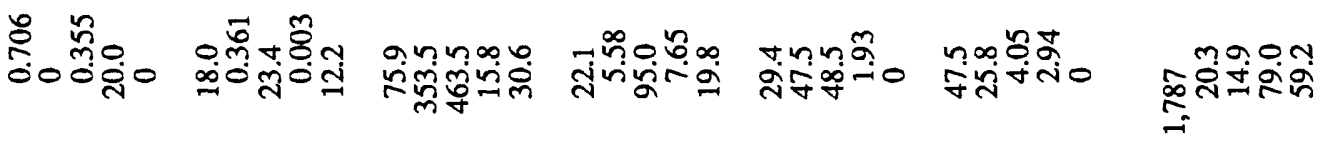

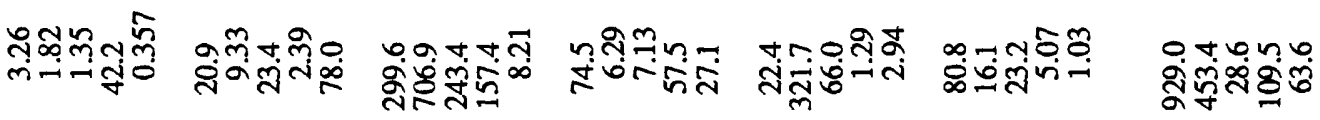

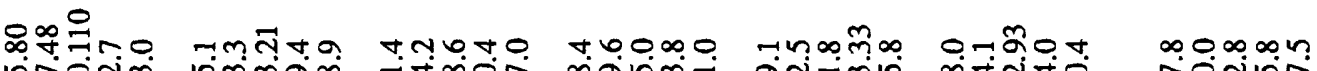

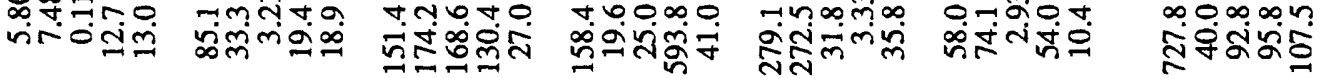

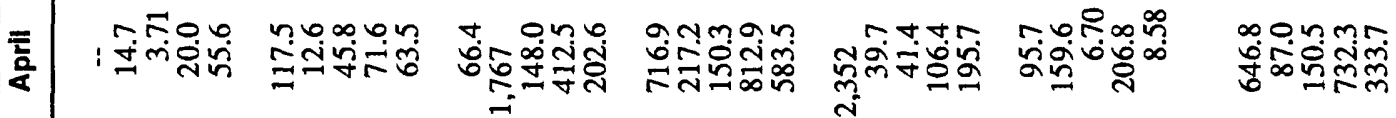

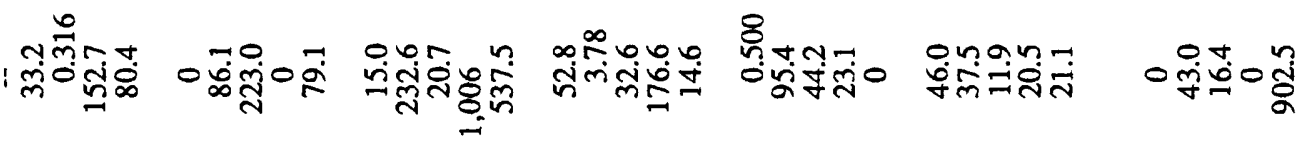
iั

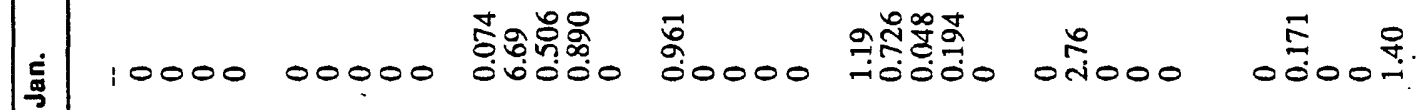

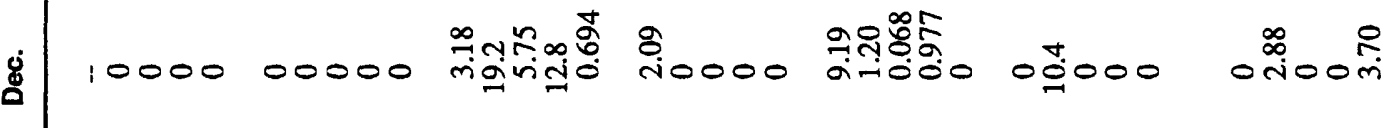

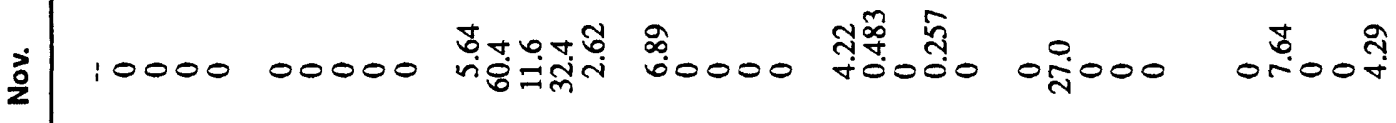

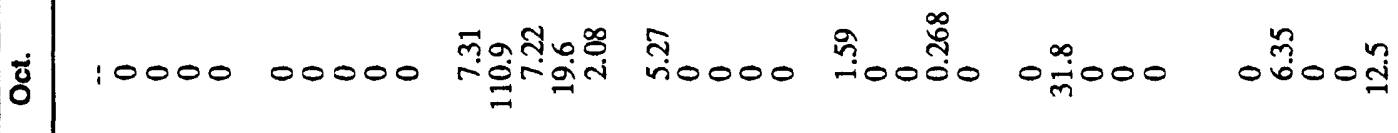

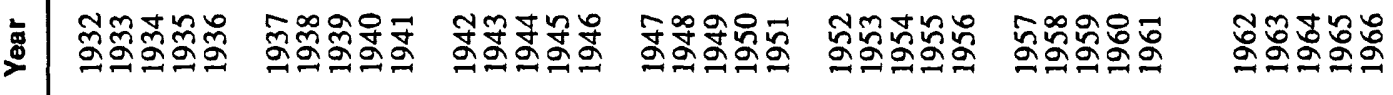




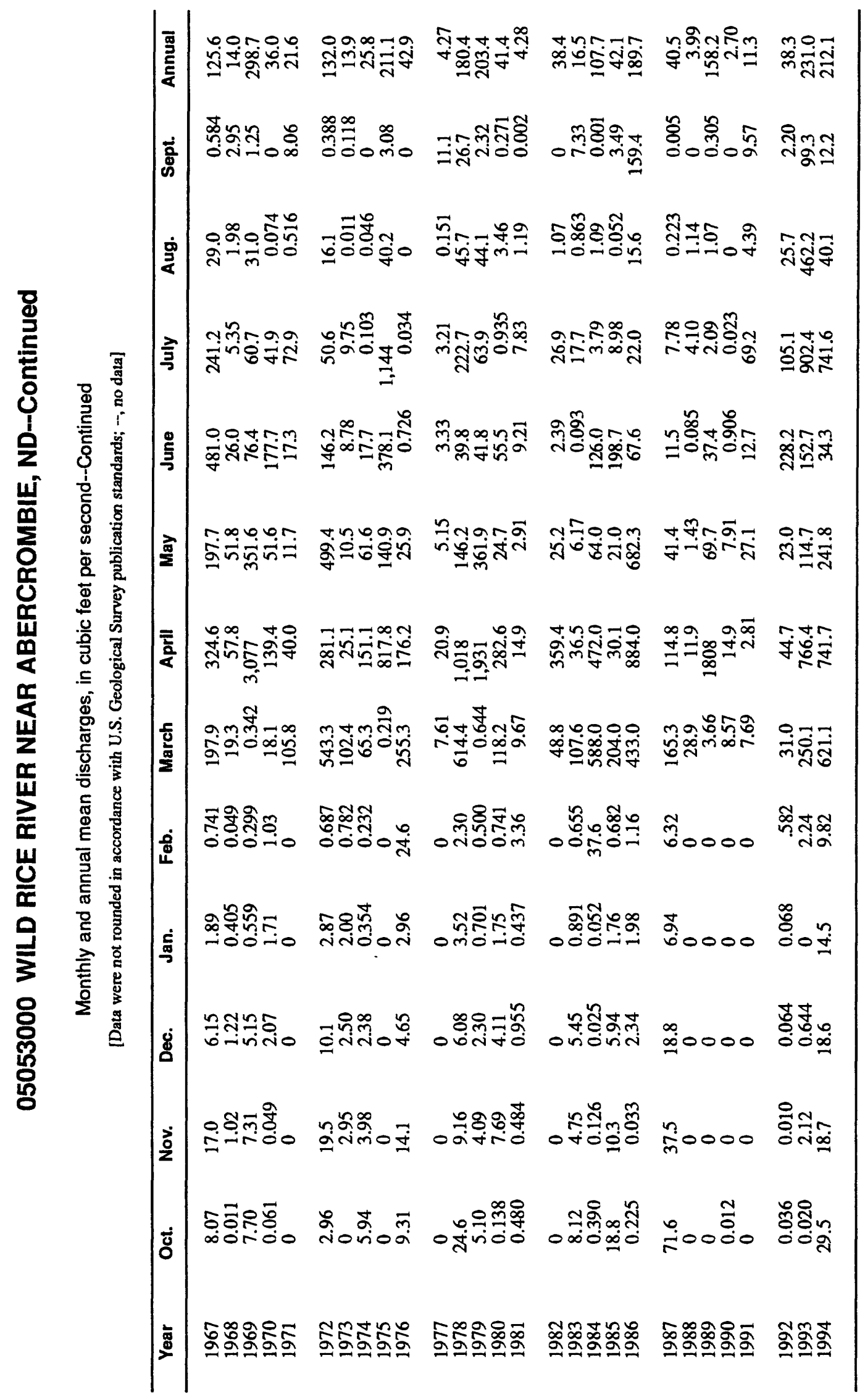




\section{RED RIVER OF THE NORTH AT FARGO, ND}

\section{Station Description}

LOCATION.--Lat $46^{\circ} 51^{\prime} 40^{\prime \prime}$, long $96^{\circ} 47^{\prime} 00^{\prime \prime}$, in NW $1 / 4 \mathrm{NE}^{1} / 4 \mathrm{sec} .18, \mathrm{~T} .139$ N., R.48 W., Cass County, Hydrologic Unit 09020104, at waterplant on 4th St. S. in Fargo, 25 mi upstream from mouth of Sheyenne River, and at mile 453.

DRAINAGE AREA.--6,800 $\mathrm{mi}^{2}$, approximately.

PERIOD OF RECORD.--May 1901 to current year. Published as “at Moorhead, Minn.”, 1901. Monthly discharge only for some periods, published in Water-Supply Paper 1308.

GAGE.--Water-stage recorder and concrete control. Datum of gage is $861.8 \mathrm{ft}$ above sea level. Oct. 1, 1960, to Sept. 30, 1962, water-stage recorder at present site at datum $5.6 \mathrm{ft}$ higher. See Water-Supply Paper 1728 or 1913 for history of changes prior to Oct. 1, 1960.

EXTREMES FOR PERIOD OF RECORD.--Maximum discharge, 25,300 $\mathrm{ft}^{3} / \mathrm{s}$, Apr. 15, 1969; maximum gage height, $37.34 \mathrm{ft}$, Apr. 15, 1969; no flow for many days in each year during 1932-41, Sept. 30 and Oct. 1-2, 1970, and Oct. 10-19, 1976.

EXTREMES OUTSIDE PERIOD OF RECORD.--Flood of Apr. 7, 1897, reached a stage of $40.1 \mathrm{ft}$ present datum, discharge, $25,000 \mathrm{ft}^{3} / \mathrm{s}$ at site $1.5 \mathrm{mi}$ downstream.

Annual mean discharge

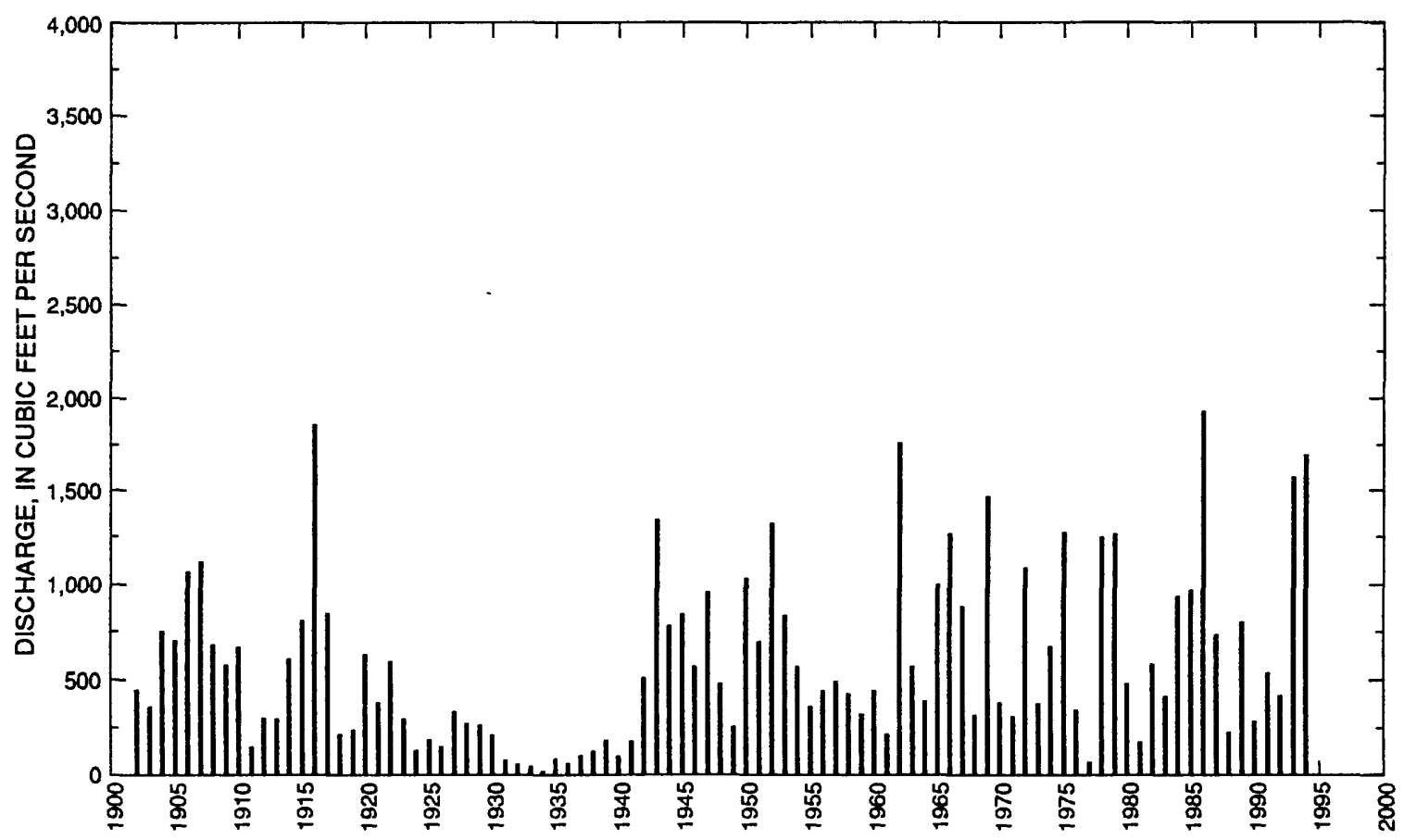




\section{RED RIVER OF THE NORTH AT FARGO, ND-Continued}

Period of record, 1902-94

Statistics of monthly and annual mean discharges, period of record

[m, more than 1 year of occurrence]

\begin{tabular}{|c|c|c|c|c|c|c|c|c|}
\hline \multirow[b]{2}{*}{ Month } & \multicolumn{2}{|c|}{ Maximum } & \multicolumn{2}{|c|}{ Minimum } & \multicolumn{4}{|c|}{ Mean } \\
\hline & $\begin{array}{c}\text { Discharge } \\
\left(\mathrm{ft}^{3} / \mathrm{s}\right)\end{array}$ & $\begin{array}{c}\text { Water year } \\
\text { of } \\
\text { occurrence }\end{array}$ & $\begin{array}{c}\text { Discharge } \\
\left(\mathrm{ft}^{3} / \mathrm{s}\right)\end{array}$ & $\begin{array}{c}\text { Water year } \\
\text { of } \\
\text { occurrence }\end{array}$ & $\begin{array}{c}\text { Discharge } \\
\left(\mathrm{ft}^{3} / \mathrm{s}\right)\end{array}$ & $\begin{array}{c}\text { Standard } \\
\text { deviation } \\
\left(\mathrm{ft}^{3} / \mathrm{s}\right)\end{array}$ & $\begin{array}{l}\text { Coeffi- } \\
\text { cient of } \\
\text { variation }\end{array}$ & $\begin{array}{l}\text { Percentage } \\
\text { of annual } \\
\text { discharge }\end{array}$ \\
\hline October & 1,740 & 1994 & 0 & $\mathrm{~m}$ & 306 & 306 & 1.00 & 4.29 \\
\hline November & 942 & 1907 & 0 & 1937 & 266 & 221 & 0.83 & 3.73 \\
\hline December & 800 & 1987 & 0 & 1938 & 220 & 185 & 0.84 & 3.08 \\
\hline January & 740 & 1986 & 0 & $\mathrm{~m}$ & 202 & 167 & 0.83 & 2.84 \\
\hline February & 778 & 1987 & 0.179 & 1933 & 202 & 160 & 0.79 & 2.84 \\
\hline March & 3,760 & 1966 & 26.8 & 1937 & 661 & 680 & 1.03 & 9.27 \\
\hline April & 9,920 & 1969 & 102 & 1934 & 1,700 & 1,940 & 1.14 & 23.8 \\
\hline May & 4,590 & 1986 & 8.12 & 1934 & 995 & 860 & 0.86 & 14.0 \\
\hline June & 5,120 & 1962 & 2.87 & 1936 & 1,010 & 883 & 0.88 & 14.1 \\
\hline July & 5,690 & 1962 & 0 & $\mathrm{~m}$ & 847 & 1,080 & 1.27 & 11.9 \\
\hline August & 3,290 & 1993 & 0 & $\mathrm{~m}$ & 411 & 516 & 1.25 & 5.77 \\
\hline September & 2,280 & 1993 & 0 & $\mathrm{~m}$ & 316 & 371 & 1.17 & 4.43 \\
\hline Annual & 1,930 & 1986 & 17.5 & 1934 & 596 & 451 & 0.76 & 100 \\
\hline
\end{tabular}

Annual flow duration, period of record

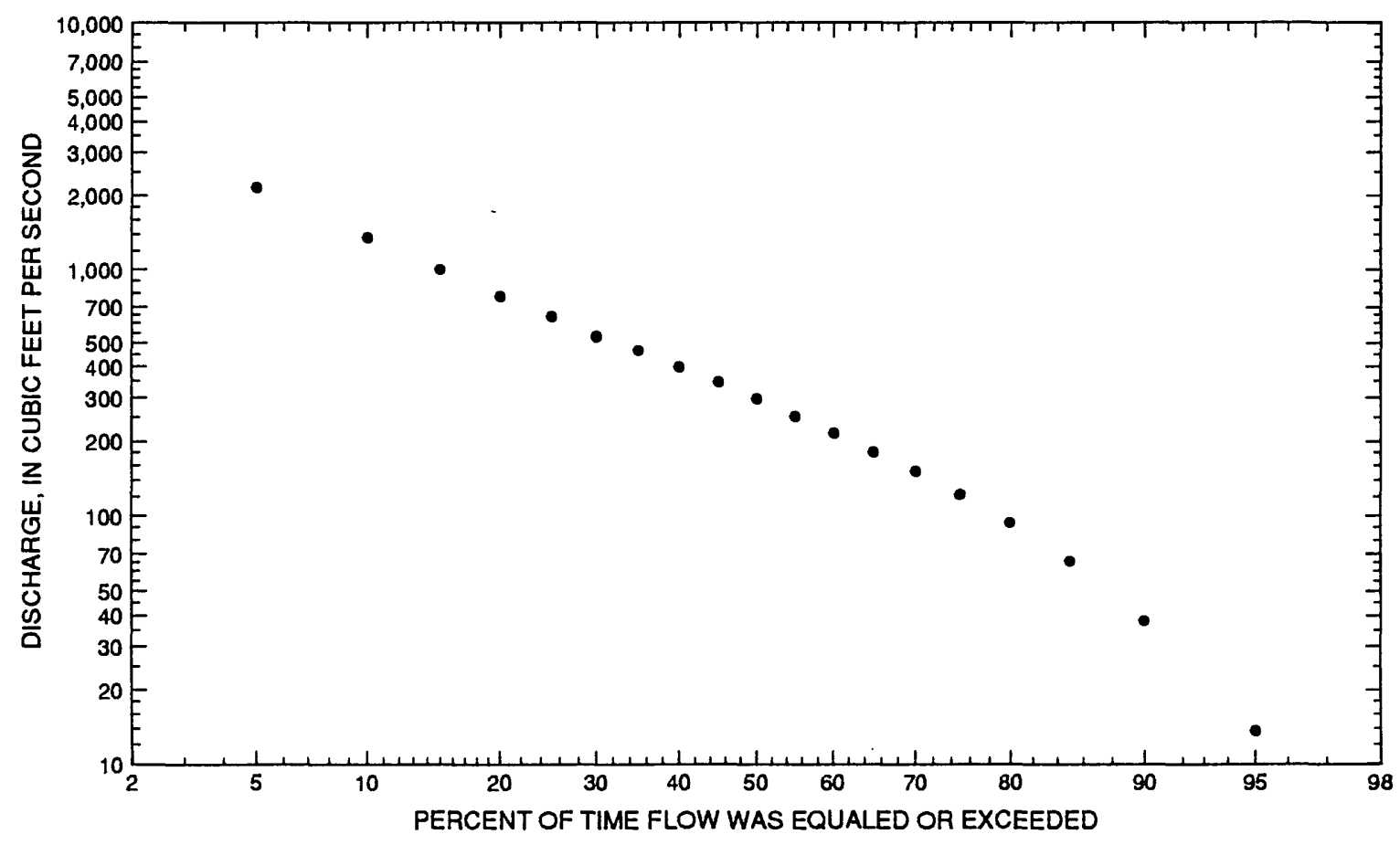




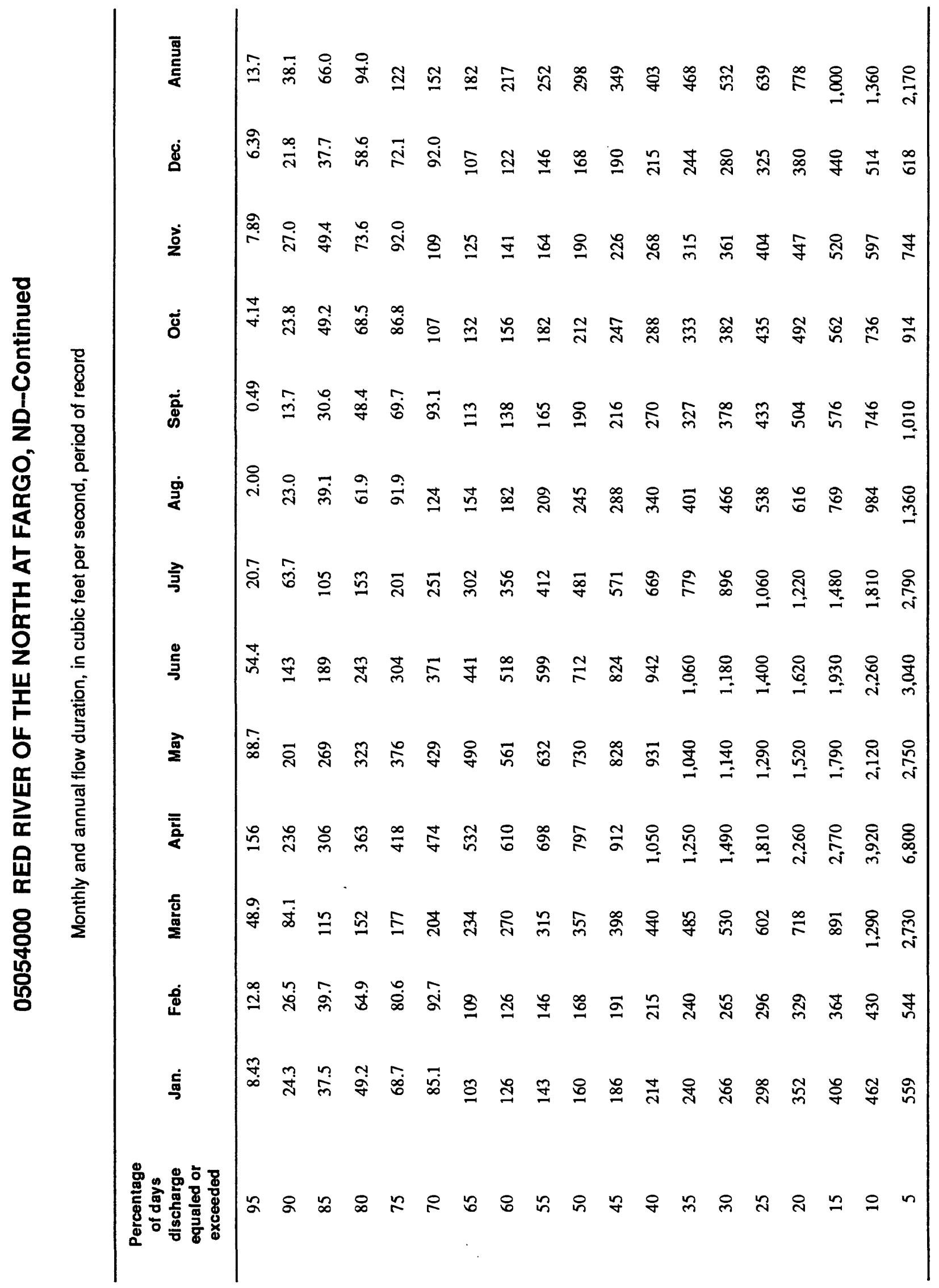




\section{RED RIVER OF THE NORTH AT FARGO, ND-Continued}

Probability of annual high discharges, period of record

[ng, statistic not given]

\begin{tabular}{|c|c|c|c|c|c|c|}
\hline \multirow[b]{2}{*}{$\begin{array}{l}\text { Exceedance } \\
\text { probability }\end{array}$} & \multirow[b]{2}{*}{$\begin{array}{l}\text { Recurrence } \\
\text { interval } \\
\text { (years) }\end{array}$} & \multirow[b]{2}{*}{$\begin{array}{c}\text { Maximum } \\
\text { instantaneous } \\
\left(\mathrm{ft}^{3} / \mathrm{s}\right)^{1}\end{array}$} & \multicolumn{4}{|c|}{$\begin{array}{l}\text { Maximum average discharge } \\
\left(\left(\mathrm{ft}^{3} / \mathrm{s}\right)\right.\end{array}$} \\
\hline & & & 3-day period & 7-day period & 15-day period & 30-day period \\
\hline 0.99 & 1.01 & 301 & 264 & 220 & 176 & 138 \\
\hline 0.95 & 1.05 & 609 & 531 & 449 & 356 & 285 \\
\hline 0.90 & 1.11 & 884 & 770 & 655 & 517 & 416 \\
\hline 0.80 & 1.25 & 1,380 & 1,200 & 1,030 & 810 & 652 \\
\hline 0.50 & 2 & 3,210 & 2,810 & 2,450 & 1,900 & 1,500 \\
\hline 0.20 & 5 & 7,330 & 6,500 & 5,780 & 4,410 & 3,320 \\
\hline 0.10 & 10 & 11,200 & 10,000 & 9,010 & 6,830 & 4,970 \\
\hline 0.04 & 25 & 17,600 & 15,900 & 14,500 & 10,900 & 7,560 \\
\hline 0.02 & 50 & 23,400 & 21,400 & 19,600 & 14,600 & 9,860 \\
\hline 0.01 & 100 & 30,300 & 27,900 & 25,700 & 19,100 & 12,500 \\
\hline 0.005 & 200 & 38,300 & 35,600 & 33,000 & 24,300 & 15,400 \\
\hline 0.002 & 500 & 50,800 & $\mathrm{ng}$ & $\mathrm{ng}$ & $\mathrm{ng}$ & ng \\
\hline
\end{tabular}

${ }^{1}$ Historic adjustment made to Log Pearson Type III peak frequency.

Probability of annual low discharges, period of record

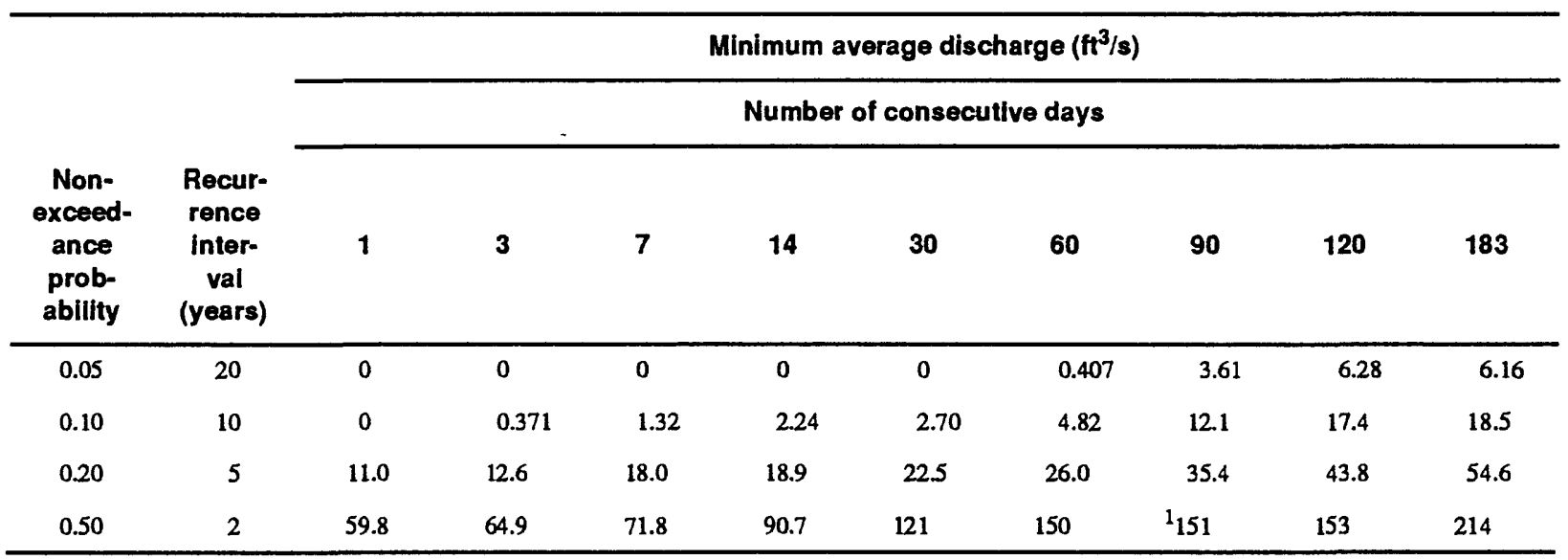

${ }^{1}$ Graphical interpretation. 


\section{RED RIVER OF THE NORTH AT FARGO, ND-Continued}

Probability of seasonal low discharges, period of record

\begin{tabular}{|c|c|c|c|c|c|c|c|c|c|}
\hline \multirow[b]{3}{*}{$\begin{array}{l}\text { Non- } \\
\text { exceedance } \\
\text { probability }\end{array}$} & \multirow[b]{3}{*}{$\begin{array}{l}\text { Recurrence } \\
\text { interval } \\
\text { (years) }\end{array}$} & \multicolumn{8}{|c|}{ Minimum average discharge $\left(\mathrm{ft}^{3} / \mathrm{s}\right)$} \\
\hline & & \multicolumn{8}{|c|}{ Number of consecutive days } \\
\hline & & 1 & 7 & 14 & 30 & 1 & 7 & 14 & 30 \\
\hline & & \multicolumn{4}{|c|}{ December-January-February } & \multicolumn{4}{|c|}{ March-April-May } \\
\hline 0.05 & 20 & 0 & 0 & 0 & 1.84 & 7.15 & 18.2 & 18.5 & 56.5 \\
\hline 0.10 & 10 & 9.66 & 14.4 & 17.3 & $1_{21.0}$ & 22.8 & 37.7 & 45.0 & 91.8 \\
\hline 0.20 & 5 & 28.7 & 36.4 & 40.7 & ${ }^{1} 45.0$ & 62.2 & 79.0 & 105 & 156 \\
\hline \multirow[t]{2}{*}{0.50} & 2 & 102 & 113 & 119 & 153 & 211 & 220 & 290 & 370 \\
\hline & & \multicolumn{4}{|c|}{ June-July-August } & \multicolumn{4}{|c|}{ September-October-November } \\
\hline 0.05 & 20 & 0 & 0 & 0.331 & 6.27 & 0 & 0 & 0 & 0.390 \\
\hline 0.10 & 10 & 7.24 & 13.0 & ${ }^{1} 17.8$ & 22.5 & 0.255 & 5.01 & ${ }^{1} 6.80$ & 8.58 \\
\hline 0.20 & 5 & 27.0 & 38.9 & 42.3 & 61.4 & 14.2 & 22.8 & 24.8 & 37.1 \\
\hline 0.50 & 2 & 135 & 158 & 242 & 245 & 91.5 & 108 & 152 & 188 \\
\hline
\end{tabular}

${ }^{1}$ Graphical interpretation. 


\section{RED RIVER OF THE NORTH AT FARGO, ND-Continued}

Pre-regulation period, $1902-41$

Statistics of monthly and annual mean discharges, pre-regulation period

[m, more than 1 year of occurrence]

\begin{tabular}{|c|c|c|c|c|c|c|c|c|}
\hline \multirow[b]{2}{*}{ Month } & \multicolumn{2}{|c|}{ Maximum } & \multicolumn{2}{|c|}{ Minimum } & \multicolumn{4}{|c|}{ Mean } \\
\hline & $\begin{array}{c}\text { Discharge } \\
\left(\mathrm{ft}^{3} / \mathrm{s}\right)\end{array}$ & $\begin{array}{c}\text { Water year } \\
\text { of } \\
\text { occurrence }\end{array}$ & $\begin{array}{c}\text { Discharge } \\
\left(\mathrm{ft}^{3} / \mathrm{s}\right)\end{array}$ & $\begin{array}{l}\text { Water year } \\
\text { of } \\
\text { occurrence }\end{array}$ & $\begin{array}{c}\text { Discharge } \\
\left(\mathrm{ft}^{3} / \mathbf{s}\right)\end{array}$ & $\begin{array}{c}\text { Standard } \\
\text { deviation } \\
\left(\mathrm{ft}^{3} / \mathrm{s}\right)\end{array}$ & $\begin{array}{l}\text { Coeffi- } \\
\text { clent of } \\
\text { variation }\end{array}$ & $\begin{array}{l}\text { Percentage } \\
\text { of annual } \\
\text { discharge }\end{array}$ \\
\hline October & 1,030 & 1917 & 0 & $\mathrm{~m}$ & 266 & 288 & 1.08 & 5.51 \\
\hline November & 942 & 1907 & 0 & 1937 & 230 & 236 & 1.02 & 4.77 \\
\hline December & 600 & 1907 & 0 & 1938 & 166 & 175 & 1.05 & 3.44 \\
\hline January & 504 & 1907 & 0 & $\mathrm{~m}$ & 133 & 138 & 1.04 & 2.75 \\
\hline February & 367 & 1907 & 0.179 & 1933 & 119 & 110 & 0.92 & 2.47 \\
\hline March & 2,130 & 1910 & 26.8 & 1937 & 522 & 528 & 1.01 & 10.8 \\
\hline April & 6,080 & 1916 & 102 & 1934 & 971 & 1,140 & 1.17 & 20.1 \\
\hline May & 2,540 & 1916 & 8.12 & 1934 & 642 & 547 & 0.85 & 13.3 \\
\hline June & 2,200 & 1907 & 2.87 & 1936 & 647 & 590 & 0.91 & 13.4 \\
\hline July & 5,460 & 1916 & 0 & $\mathrm{~m}$ & 562 & 907 & 1.62 & 11.6 \\
\hline August & 1,850 & 1916 & 0 & $\mathrm{~m}$ & 308 & 410 & 1.33 & 6.38 \\
\hline September & 1,250 & 1916 & 0 & $\mathrm{~m}$ & 261 & 318 & 1.22 & 5.42 \\
\hline Annual & 1,850 & 1916 & 17.5 & 1934 & 403 & 374 & 0.93 & 100 \\
\hline
\end{tabular}

Annual flow duration, pre-regulation period

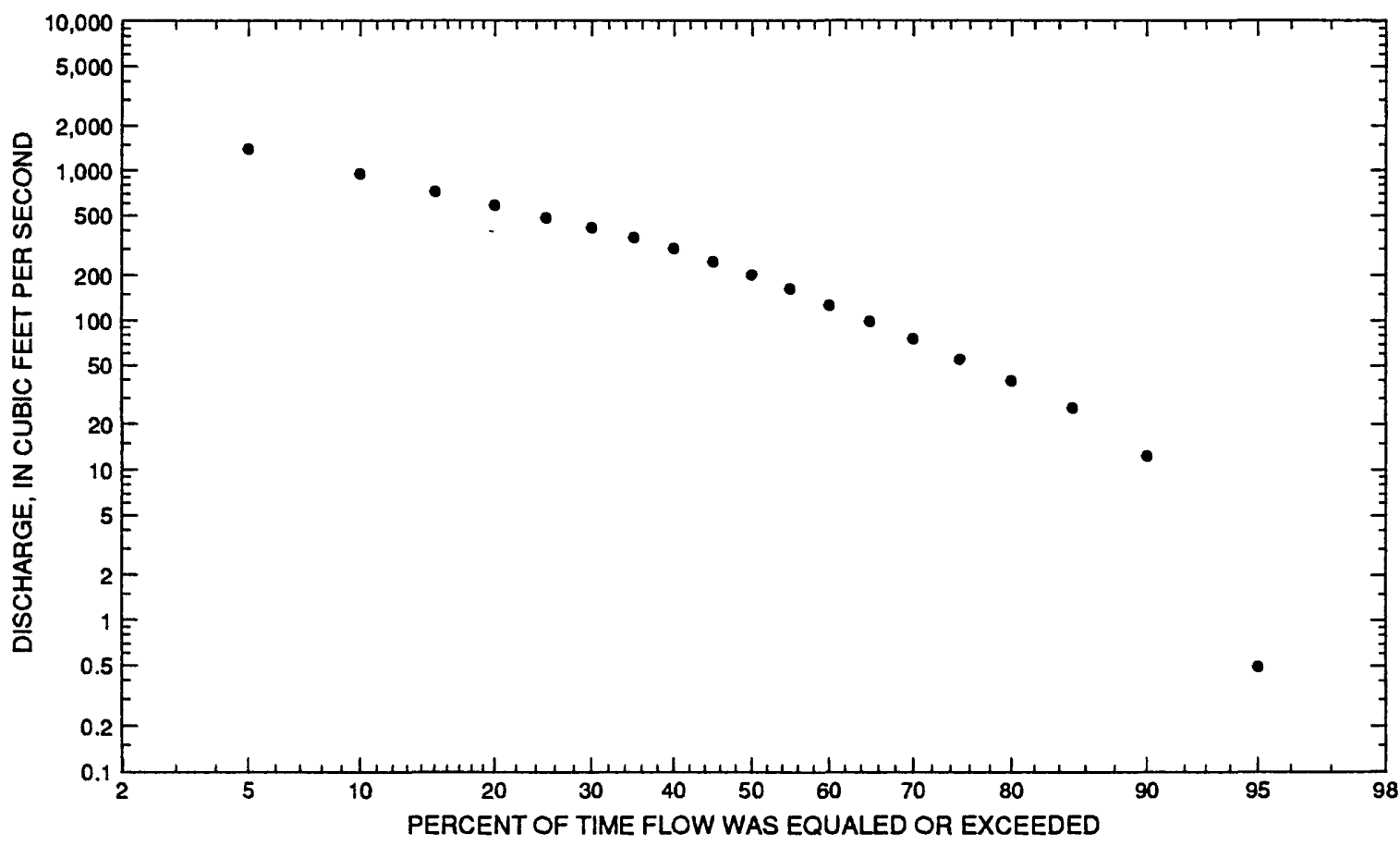




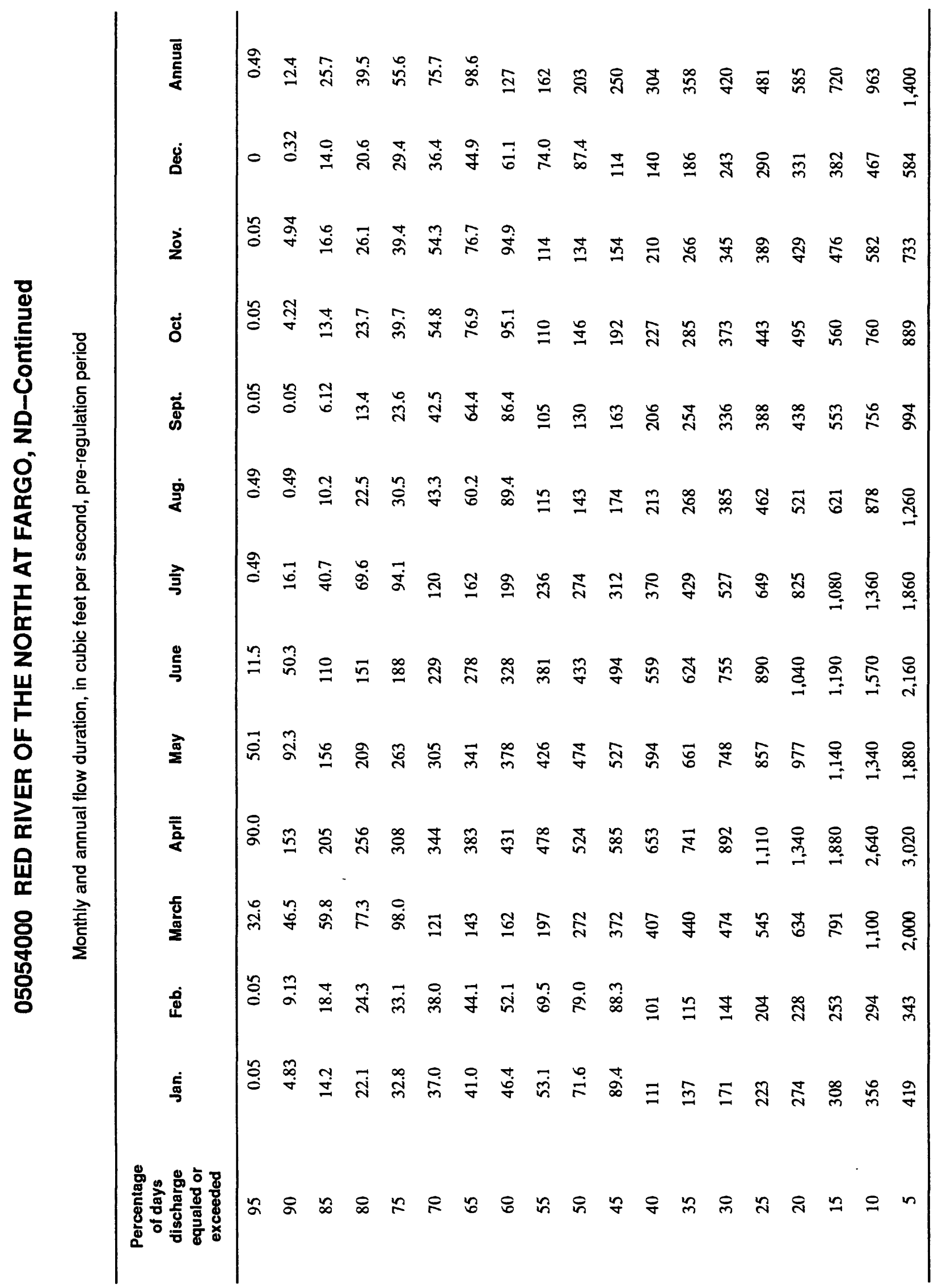




\section{RED RIVER OF THE NORTH AT FARGO, ND-Continued}

Probability of annual high discharges, pre-regulation period

[ng, statistic not given]

\begin{tabular}{|c|c|c|c|c|c|c|}
\hline \multirow[b]{2}{*}{$\begin{array}{l}\text { Exceedance } \\
\text { probabillity }\end{array}$} & \multirow[b]{2}{*}{$\begin{array}{l}\text { Recurrence } \\
\text { interval } \\
\text { (years) }\end{array}$} & \multirow[b]{2}{*}{$\begin{array}{c}\text { Maximum } \\
\text { instantaneous } \\
\left(\mathrm{ft}^{3} / \mathrm{s}\right)\end{array}$} & \multicolumn{4}{|c|}{$\begin{array}{l}\text { Maximum average discharge } \\
\qquad\left(\mathrm{ft}^{3} / \mathrm{s}\right)\end{array}$} \\
\hline & & & 3-day period & 7-day perlod & 15-day perlod & 30-day period \\
\hline 0.99 & 1.01 & 224 & 213 & 174 & 138 & 106 \\
\hline 0.95 & 1.05 & 436 & 404 & 340 & 270 & 208 \\
\hline 0.90 & 1.11 & 615 & 563 & 482 & 382 & 295 \\
\hline 0.80 & 1.25 & 922 & 834 & 727 & 576 & 446 \\
\hline 0.50 & 2 & 1,920 & 1,730 & 1,550 & 1,220 & 956 \\
\hline 0.20 & 5 & 3,830 & 3,460 & 3,160 & 2,470 & 1,970 \\
\hline 0.10 & 10 & 5,390 & 4,910 & 4,510 & 3,520 & 2,840 \\
\hline 0.04 & 25 & 7,660 & 7,070 & 6,530 & 5,060 & 4,140 \\
\hline 0.02 & 50 & 9,530 & 8,900 & 8,240 & 6,360 & 5,250 \\
\hline 0.01 & 100 & 11,600 & 10,900 & 10,100 & 7,770 & 6,470 \\
\hline 0.005 & 200 & 13,700 & 13,100 & 12,200 & 9,310 & 7,820 \\
\hline 0.002 & 500 & 16,800 & $\mathrm{ng}$ & $\mathrm{ng}$ & $\mathrm{ng}$ & $\mathrm{ng}$ \\
\hline
\end{tabular}

Probability of annual low discharges, pre-regulation period

\begin{tabular}{|c|c|c|c|c|c|c|c|c|c|c|}
\hline \multirow[b]{3}{*}{$\begin{array}{l}\text { Non- } \\
\text { exceed- } \\
\text { ance } \\
\text { prob- } \\
\text { ability }\end{array}$} & \multirow[b]{3}{*}{$\begin{array}{c}\text { Recur- } \\
\text { rence } \\
\text { inter- } \\
\text { val } \\
\text { (years) }\end{array}$} & \multicolumn{9}{|c|}{ Minimum average discharge $\left(\mathrm{ft}^{3} / \mathrm{s}\right)$} \\
\hline & & \multicolumn{9}{|c|}{ Number of consecutlve days } \\
\hline & & 1 & 3 & 7 & 14 & 30 & 60 & 90 & 120 & 183 \\
\hline 0.05 & 20 & 0 & 0 & 0 & 0 & 0 & 0 & 0 & 0 & 1.42 \\
\hline 0.10 & 10 & 0 & 0 & 0 & 0 & 0 & 0.119 & 2.30 & 4.37 & 4.87 \\
\hline 0.20 & 5 & 0 & 0 & 0 & 0 & 0.712 & 3.64 & 9.93 & 15.0 & 17.5 \\
\hline 0.50 & 2 & 35.4 & 40.6 & 47.2 & 51.8 & 74.0 & 63.6 & 66.7 & 79.0 & 111 \\
\hline
\end{tabular}




\section{RED RIVER OF THE NORTH AT FARGO, ND-Continued}

Probability of seasonal low discharges, pre-regulation period

\begin{tabular}{|c|c|c|c|c|c|c|c|c|c|}
\hline \multirow[b]{3}{*}{$\begin{array}{l}\text { Non- } \\
\text { exceedance } \\
\text { probability }\end{array}$} & \multirow[b]{3}{*}{$\begin{array}{c}\text { Recurrence } \\
\text { interval } \\
\text { (years) }\end{array}$} & \multicolumn{8}{|c|}{ Minimum average discharge $\left(\mathrm{ft}^{3} / \mathrm{s}\right)$} \\
\hline & & \multicolumn{8}{|c|}{ Number of consecutive days } \\
\hline & & 1 & 7 & 14 & 30 & 1 & 7 & 14 & 30 \\
\hline & & \multicolumn{4}{|c|}{ December-January-February } & \multicolumn{4}{|c|}{ March-April-May } \\
\hline 0.05 & 20 & 0 & 0 & 0 & 0 & 0 & 5.71 & 6.08 & 29.2 \\
\hline 0.10 & 10 & 0 & 0 & 0 & 0 & 8.92 & 14.9 & 16.9 & 51.2 \\
\hline 0.20 & 5 & 6.21 & 10.7 & 13.8 & 15.7 & 26.8 & 36.4 & 47.6 & 95.0 \\
\hline \multirow[t]{2}{*}{0.50} & 2 & 48.7 & 55.8 & 59.4 & 64.0 & 120 & 137 & 195 & 259 \\
\hline & & \multicolumn{4}{|c|}{ June-July-August } & \multicolumn{4}{|c|}{ September-October-November } \\
\hline 0.05 & 20 & 0 & 0 & 0 & 0 & 0 & 0 & 0 & 0 \\
\hline 0.10 & 10 & 0 & 0 & 0.004 & 3.63 & 0 & 0 & 0 & 0 \\
\hline 0.20 & 5 & 8.61 & 12.6 & ${ }^{1} 16.4$ & 20.2 & 0.896 & 5.19 & 17.34 & 9.49 \\
\hline 0.50 & 2 & 80.9 & 98.3 & 139 & ${ }^{1} 160$ & 60.2 & 80.4 & 111 & 133 \\
\hline
\end{tabular}

${ }^{1}$ Graphical interpretation. 


\section{RED RIVER OF THE NORTH AT FARGO, ND-Continued}

Post-regulation period, 1942-94

Statistics of monthly and annual mean discharges, post-regulation period

[m, more than 1 year of occurrence]

\begin{tabular}{|c|c|c|c|c|c|c|c|c|}
\hline \multirow[b]{2}{*}{ Month } & \multicolumn{2}{|c|}{ Maximum } & \multicolumn{2}{|c|}{ Minimum } & \multicolumn{4}{|c|}{ Mean } \\
\hline & $\begin{array}{c}\text { Discharge } \\
\left(\mathrm{ft}^{3} / \mathrm{s}\right)\end{array}$ & $\begin{array}{l}\text { Water year } \\
\text { of } \\
\text { occurrence }\end{array}$ & $\begin{array}{c}\text { Discharge } \\
\left(\mathrm{ft}^{3} / \mathrm{s}\right)\end{array}$ & $\begin{array}{c}\text { Water year } \\
\text { of } \\
\text { occurrence }\end{array}$ & $\begin{array}{c}\text { Discharge } \\
\left(\mathrm{ft}^{3} / \mathrm{s}\right)\end{array}$ & $\begin{array}{c}\text { Standard } \\
\text { devlation } \\
\left(\mathrm{ft}^{3} / \mathrm{s}\right)\end{array}$ & $\begin{array}{l}\text { Coeffi- } \\
\text { cient of } \\
\text { variation }\end{array}$ & $\begin{array}{c}\text { Percentage } \\
\text { of annual } \\
\text { discharge }\end{array}$ \\
\hline October & 1,740 & 1994 & 3.77 & 1977 & 336 & 318 & 0.95 & 3.78 \\
\hline November & 893 & 1987 & 12.0 & 1977 & 293 & 207 & 0.70 & 3.30 \\
\hline December & 800 & 1987 & 11.7 & 1977 & 261 & 184 & 0.71 & 2.93 \\
\hline January & 740 & 1986 & 14.8 & 1977 & 255 & 169 & 0.66 & 2.87 \\
\hline February & 778 & 1987 & 19.0 & 1977 & 265 & 163 & 0.62 & 2.98 \\
\hline March & 3,760 & 1966 & 85.2 & 1977 & 766 & 763 & 1.00 & 8.62 \\
\hline April & 9,920 & 1969 & 194 & 1977 & 2,250 & 2,230 & 0.99 & 25.3 \\
\hline May & 4,590 & 1986 & 77.1 & 1977 & 1,260 & 957 & 0.76 & 14.2 \\
\hline June & 5,120 & 1962 & 91.1 & 1977 & 1,280 & 973 & 0.76 & 14.4 \\
\hline July & 5,690 & 1962 & 63.0 & 1988 & 1,070 & 1,150 & 1.08 & 12.0 \\
\hline August & 3,290 & 1993 & 18.5 & 1977 & 491 & 576 & 1.17 & 5.53 \\
\hline September & 2,280 & 1993 & 12.3 & 1976 & 359 & 406 & 1.13 & 4.03 \\
\hline Annual & 1,930 & 1986 & 64.7 & 1977 & 741 & 454 & 0.61 & 100 \\
\hline
\end{tabular}

Annual flow duration, post-regulation period

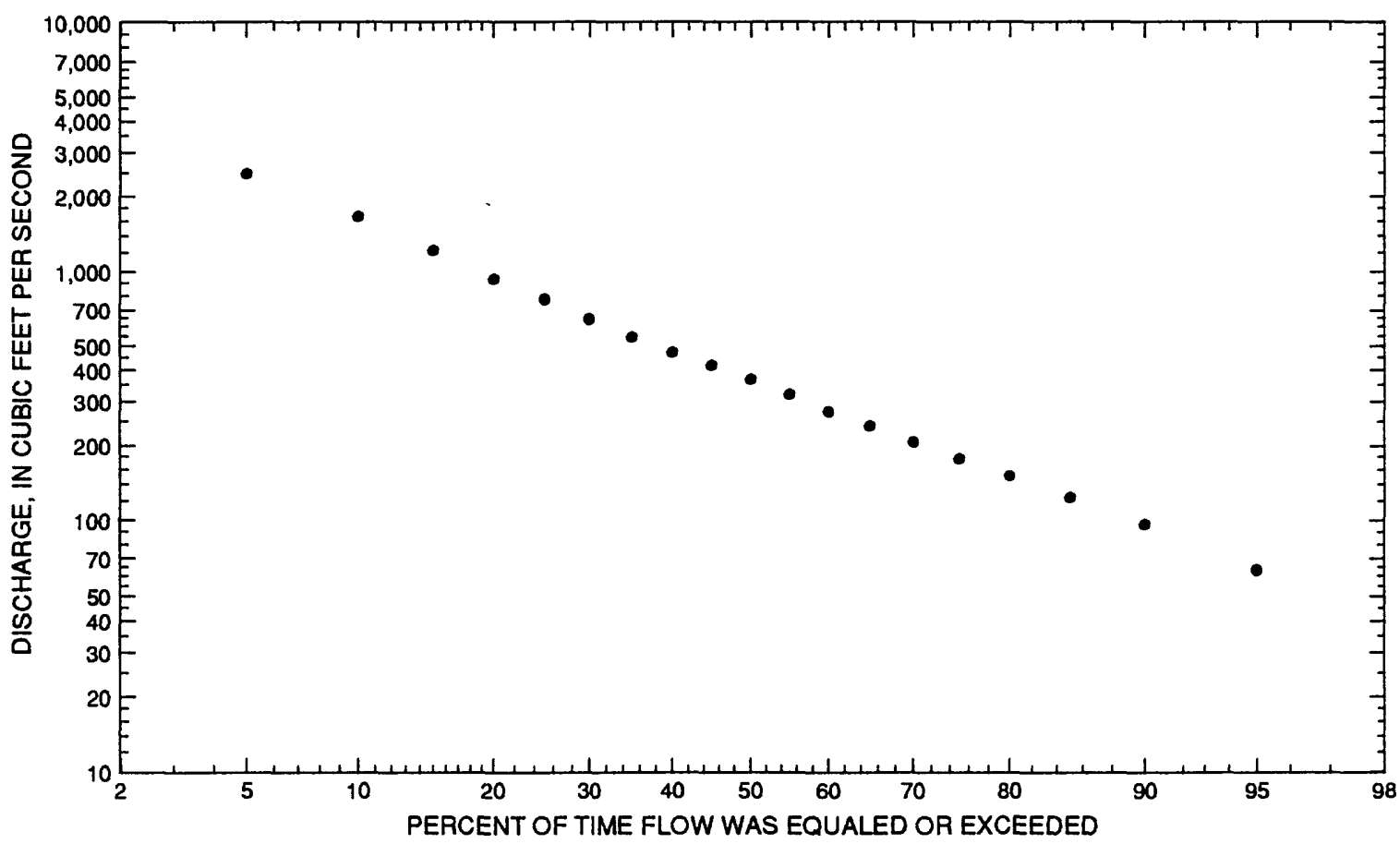




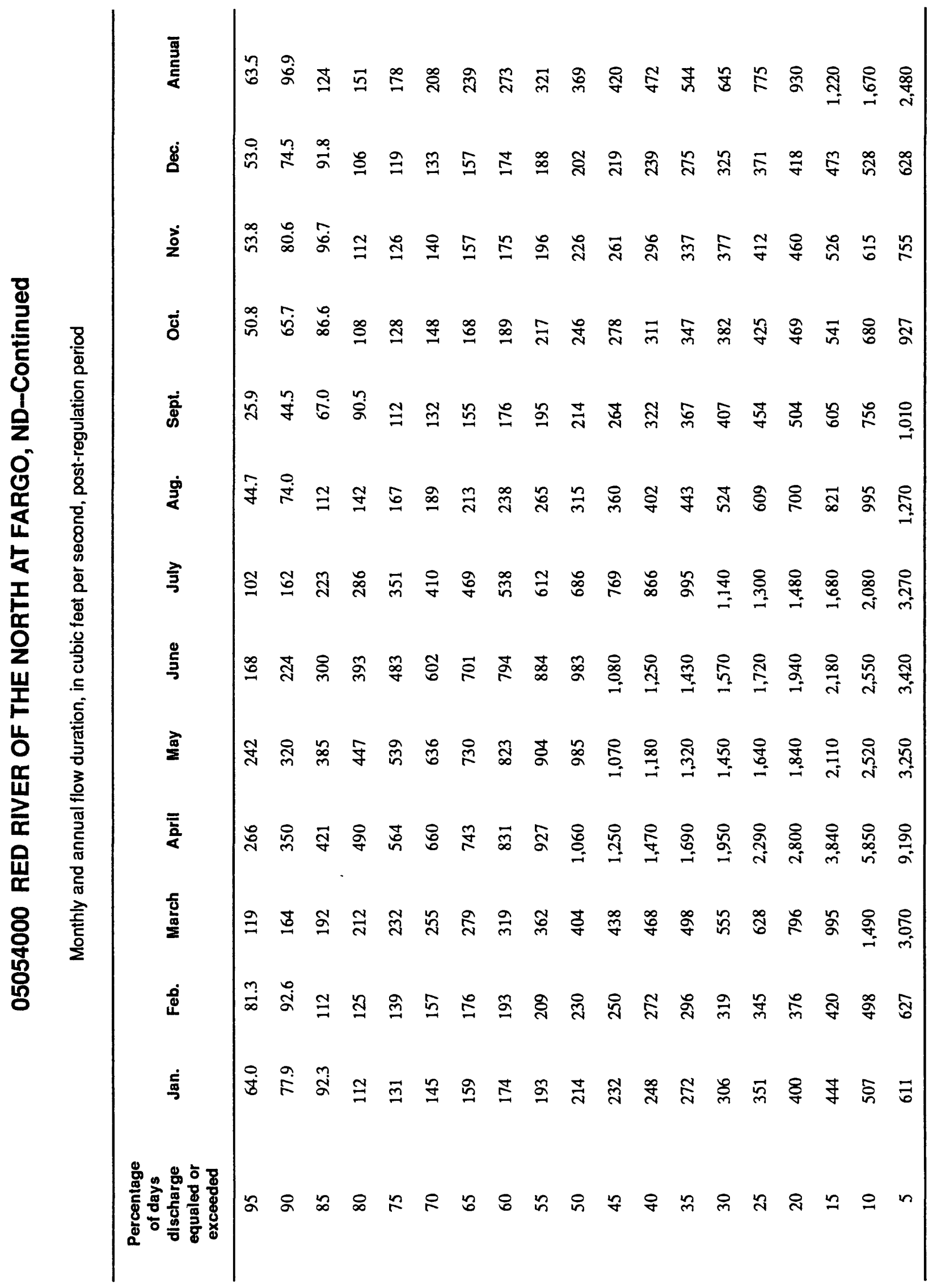




\section{RED RIVER OF THE NORTH AT FARGO, ND-Continued}

Probability of annual high discharges, post-regulation period

[ng, statistic not given]

\begin{tabular}{lcccccc}
\hline & & & \multicolumn{5}{c}{$\begin{array}{c}\text { Maximum average discharge } \\
\left(\mathrm{ft}^{3} / \mathbf{s}\right)\end{array}$} \\
\cline { 5 - 7 } $\begin{array}{c}\text { Exceedance } \\
\text { probabillty }\end{array}$ & $\begin{array}{c}\text { Recurrence } \\
\text { interval } \\
\text { (years) }\end{array}$ & $\begin{array}{c}\text { Maximum } \\
\text { instantaneous } \\
\left(\mathbf{f t}^{\mathbf{3}} / \mathbf{s}\right)\end{array}$ & 3-day period & 7-day period & 15-day period & 30-day period \\
\hline 0.99 & 1.01 & 522 & 391 & 319 & 252 & 222 \\
0.95 & 1.05 & 1,010 & 814 & 670 & 524 & 447 \\
0.90 & 1.11 & 1,430 & 1,190 & 985 & 765 & 640 \\
0.80 & 1.25 & 2,150 & 1,860 & 1,550 & 1,200 & 977 \\
0.50 & 2 & 4,590 & 4,210 & 3,610 & 2,750 & 2,120 \\
0.20 & 5 & 9,500 & 9,130 & 8,080 & 6,100 & 4,390 \\
0.10 & 10 & 13,700 & 13,400 & 12,100 & 9,110 & 6,320 \\
0.04 & 25 & 20,100 & 20,000 & 18,500 & 13,800 & 9,190 \\
0.02 & 50 & 25,600 & 25,700 & 24,100 & 18,000 & 11,600 \\
0.01 & 100 & 31,700 & 32,100 & 30,500 & 22,700 & 14,300 \\
0.005 & 200 & 38,500 & 39,100 & 37,700 & 28,100 & 17,200 \\
0.002 & 500 & 48,500 & ng & ng & ng & ng \\
\hline
\end{tabular}

Probability of annual low discharges, post-regulation period

\begin{tabular}{|c|c|c|c|c|c|c|c|c|c|c|}
\hline \multirow[b]{3}{*}{$\begin{array}{l}\text { Non- } \\
\text { exceed- } \\
\text { ance } \\
\text { prob- } \\
\text { ability }\end{array}$} & \multirow[b]{3}{*}{$\begin{array}{c}\text { Recur- } \\
\text { rence } \\
\text { Inter- } \\
\text { val } \\
\text { (years) }\end{array}$} & \multicolumn{9}{|c|}{ Minimum average discharge $\left(\mathrm{ft}^{3} / \mathrm{s}\right)$} \\
\hline & & \multicolumn{9}{|c|}{ Number of consecutive days } \\
\hline & & 1 & 3 & 7 & 14 & 30 & 60 & 90 & 120 & 183 \\
\hline 0.05 & 20 & 1.83 & 4.06 & 9.63 & ${ }^{1} 13.6$ & 17.6 & 29.0 & 36.3 & 40.5 & 49.9 \\
\hline 0.10 & 10 & 8.85 & 10.6 & 17.9 & ${ }^{1} 23.7$ & 29.5 & 44.8 & 54.9 & 61.0 & 73.6 \\
\hline 0.20 & 5 & 23.9 & 25.9 & 33.7 & 40.7 & 52.4 & 72.5 & 86.8 & 95.8 & 113 \\
\hline 0.50 & 2 & 85.3 & 89.5 & 92.9 & 130 & 133 & 161 & 184 & 200 & 232 \\
\hline
\end{tabular}

${ }^{1}$ Graphical interpretation. 


\section{RED RIVER OF THE NORTH AT FARGO, ND-Continued}

Probability of seasonal low discharges, post-regulation period

[ng, statistic not given]

\begin{tabular}{|c|c|c|c|c|c|c|c|c|c|}
\hline \multirow[b]{3}{*}{$\begin{array}{c}\text { Non- } \\
\text { exceedance } \\
\text { probability }\end{array}$} & \multirow[b]{3}{*}{$\begin{array}{l}\text { Recurrence } \\
\text { interval } \\
\text { (years) }\end{array}$} & \multicolumn{8}{|c|}{ Minimum average discharge $\left(\mathrm{ft}^{3} / \mathrm{s}\right)$} \\
\hline & & \multicolumn{8}{|c|}{ Number of consecutive days } \\
\hline & & 1 & 7 & 14 & 30 & 1 & 7 & 14 & 30 \\
\hline & & \multicolumn{4}{|c|}{ December-January-February } & \multicolumn{4}{|c|}{ March-April-May } \\
\hline 0.05 & 20 & 30.4 & 34.8 & 36.7 & 40.9 & ng & 59.9 & 75.8 & 154 \\
\hline 0.10 & 10 & 44.0 & 51.2 & 54.5 & 60.6 & ng & 93.7 & 112 & 186 \\
\hline 0.20 & 5 & 67.0 & 78.9 & 84.7 & 93.5 & ng & 148 & 169 & 237 \\
\hline \multirow[t]{2}{*}{0.50} & 2 & 138 & 163 & 175 & 191 & ng & 278 & 304 & 403 \\
\hline & & \multicolumn{4}{|c|}{ June-July-August } & \multicolumn{4}{|c|}{ September-October-November } \\
\hline 0.05 & 20 & 11.2 & 21.8 & 33.1 & 55.2 & 8.54 & 11.9 & ${ }^{1} 16.8$ & 21.7 \\
\hline 0.10 & 10 & 23.7 & 38.3 & 53.9 & 82.9 & 19.5 & 22.7 & $1_{30.4}$ & 38.2 \\
\hline 0.20 & 5 & 53.2 & 72.2 & 93.7 & 133 & 35.7 & 43.3 & 52.2 & 70.6 \\
\hline 0.50 & 2 & 187 & 209 & 242 & 308 & 93.0 & 121 & 173 & 186 \\
\hline
\end{tabular}

${ }^{1}$ Graphical interpretation. 


\section{RED RIVER OF THE NORTH AT FARGO, ND-Continued}

Annual peak discharge and corresponding gage height, period of record

$[--$, no data $]$

\begin{tabular}{|c|c|c|c|c|c|c|c|}
\hline $\begin{array}{l}\text { Water } \\
\text { year }\end{array}$ & Date & $\begin{array}{c}\text { Gage } \\
\text { height } \\
\text { (feet) }\end{array}$ & $\begin{array}{c}\text { Peak } \\
\text { discharge } \\
\left(\mathrm{ft}^{3} / \mathrm{s}\right)\end{array}$ & $\begin{array}{l}\text { Water } \\
\text { year }\end{array}$ & Date & $\begin{array}{c}\text { Gage } \\
\text { height } \\
\text { (feet) }\end{array}$ & $\begin{array}{c}\text { Peak } \\
\text { discharge } \\
\left(\mathrm{ft}^{3} / \mathrm{s}\right)\end{array}$ \\
\hline \multicolumn{8}{|c|}{ Annual peak discharge, by year, and corresponding gage height } \\
\hline${ }^{1} 1882$ & April 11 & 36.80 & 20,000 & 1945 & March 22 & 20.70 & 7,700 \\
\hline${ }^{1} 1897$ & April 7 & 39.10 & 25,000 & 1946 & March 27 & 17.13 & 5,970 \\
\hline 1902 & May 23 & 9.50 & 1,180 & 1947 & April 15 & 22.93 & 9,300 \\
\hline 1903 & April 6 & 12.90 & 2,450 & 1948 & April 10 & 12.45 & 3,390 \\
\hline 1904 & April 20 & 20.30 & 5,220 & 1949 & July 12 & 11.27 & 2,660 \\
\hline 1905 & May 17 & 17.40 & 4,250 & 1950 & April 7 & 20.88 & 7,800 \\
\hline 1906 & April 9 & 14.50 & 3,050 & 1951 & April 11 & 20.73 & 8,010 \\
\hline 1907 & March 31 & 28.80 & 7,000 & 1952 & April 16 & 28.79 & 16,300 \\
\hline 1908 & June 13 & 13.70 & 2,600 & 1953 & June 1 & 18.05 & 6,720 \\
\hline 1909 & May 30 & - & 1,780 & 1954 & July 4 & 10.53 & 1,920 \\
\hline 1910 & March 19 & 22.20 & 5,000 & 1955 & April 4 & 11.12 & 2,760 \\
\hline 1911 & April 11 & 7.70 & 608 & 1956 & April 16 & 12.54 & 3,870 \\
\hline 1912 & May 14 & 9.60 & 1,100 & 1957 & April 24 & 11.10 & 2,540 \\
\hline 1913 & July 8 & 10.90 & 1,560 & 1958 & July 6 & 10.90 & 2,280 \\
\hline 1914 & June 12 & 16.10 & 3,140 & 1959 & July 8 & 10.42 & 1,250 \\
\hline 1915 & July 3 & 9.73 & 3,130 & 1960 & April 8 & 12.48 & 3,900 \\
\hline 1916 & July 11 & - & 7,740 & 1961 & June 9 & 9.24 & 1,020 \\
\hline 1917 & April 3 & 17.80 & 5,240 & 1962 & June 14 & 22.83 & 9,580 \\
\hline 1918 & March 31 & 6.87 & 874 & 1963 & June 14 & 19.97 & 4,930 \\
\hline 1919 & April 6 & 6.50 & 680 & 1964 & April 18 & 16.22 & 2,400 \\
\hline 1920 & March 28 & 17.20 & 6,200 & 1965 & April 15 & 30.50 & 11,400 \\
\hline 1921 & April 6 & 8.40 & 1,970 & 1966 & March 22 & 30.16 & 10,700 \\
\hline 1922 & April 11 & 14.70 & 5,200 & 1967 & June 19 & 22.34 & 5,900 \\
\hline 1923 & June 29 & 11.60 & 3,960 & 1968 & April 30 & 14.71 & 788 \\
\hline 1924 & April 30 & 6.20 & 530 & 1969 & April 15 & 37.34 & 25,300 \\
\hline 1925 & June 21 & 7.00 & 940 & 1970 & June 18 & 16.27 & 2,480 \\
\hline 1926 & March 24 & 8.00 & 1,600 & 1971 & July 7 & 15.87 & 1,910 \\
\hline 1927 & March 19 & 9.10 & 2,650 & 1972 & March 24 & 25.36 & 7,250 \\
\hline 1928 & March 28 & 13.30 & 3,840 & 1973 & March 15 & 16.41 & 1,950 \\
\hline 1929 & March 20 & 12.80 & 4,440 & 1974 & April 14 & 20.25 & 4,150 \\
\hline 1930 & March 17 & 10.00 & 1,340 & 1975 & July 4 & 33.26 & 13,200 \\
\hline 1931 & April 3 & 8.55 & 365 & 1976 & March 30 & 18.70 & 3,200 \\
\hline 1932 & April 11 & 9.45 & 875 & 1977 & July 4 & 14.99 & 878 \\
\hline 1933 & April 5 & 9.04 & 605 & 1978 & April 2 & 34.41 & 17,500 \\
\hline 1934 & April 10 & 8.55 & 323 & 1979 & April 19 & 34.93 & 17,300 \\
\hline 1935 & March 20 & 9.72 & 942 & 1980 & April 5 & 20.74 & 5,470 \\
\hline 1936 & April 14 & 9.90 & 1,050 & 1981 & May 24 & 15.84 & 1,710 \\
\hline 1937 & April 12 & 10.17 & 1,390 & 1982 & April 4 & 25.07 & 5,920 \\
\hline 1938 & May 2 & 10.02 & 1,350 & 1983 & July 4 & 15.99 & 1,750 \\
\hline 1939 & March 31 & 13.00 & 3,870 & 1984 & April 1 & 28.27 & 9,550 \\
\hline 1940 & April 8 & 9.63 & 1,030 & 1985 & June 5 & 20.08 & 4,690 \\
\hline 1941 & April 3 & 10.10 & 1,390 & 1986 & April 2 & 27.19 & 8,640 \\
\hline 1942 & June 11 & 12.27 & 3,380 & 1987 & March 27 & 17.75 & 3,300 \\
\hline 1943 & April 7 & 28.40 & 16,000 & 1988 & March 11 & 15.10 & 981 \\
\hline 1944 & June 10 & 14.26 & 4,150 & 1989 & April 9 & 35.39 & 18,900 \\
\hline
\end{tabular}




\section{RED RIVER OF THE NORTH AT FARGO, ND-Continued}

Annual peak discharge and corresponding gage height, period of record--Continued

$$
[--, \text { no data }]
$$

\begin{tabular}{|c|c|c|c|c|c|c|c|}
\hline $\begin{array}{l}\text { Water } \\
\text { year }\end{array}$ & Date & $\begin{array}{c}\text { Gage } \\
\text { height } \\
\text { (feet) }\end{array}$ & $\begin{array}{c}\text { Peak } \\
\text { discharge } \\
\left(\mathrm{ft}^{3} / \mathrm{s}\right) \\
\end{array}$ & $\begin{array}{l}\text { Water } \\
\text { year }\end{array}$ & Date & $\begin{array}{c}\text { Gage } \\
\text { height } \\
\text { (feet) }\end{array}$ & $\begin{array}{c}\text { Peak } \\
\text { discharge } \\
\left(\mathrm{ft}^{3} / \mathbf{s}\right)\end{array}$ \\
\hline \multicolumn{8}{|c|}{ Annual peak discharge, by year, and corresponding gage height.-Continued } \\
\hline 1990 & June 2 & 15.40 & 1,220 & 1993 & April 5 & 28.27 & 10,100 \\
\hline 1991 & July 6 & 16.99 & 2,630 & 1994 & April 3 & 26.69 & 11,200 \\
\hline 1992 & June 19 & 16.93 & 2,590 & & & & \\
\hline \multicolumn{8}{|c|}{ Annual peak discharge, from highest to lowest, and corresponding gage height } \\
\hline 1969 & April 15 & 37.34 & 25,300 & 1960 & April 8 & 12.48 & 3,900 \\
\hline${ }^{1} 1897$ & April 7 & 39.10 & 25,000 & 1939 & March 31 & 13.00 & 3,870 \\
\hline${ }^{1} 1882$ & April 11 & 36.80 & 20,000 & 1956 & April 16 & 12.54 & 3,870 \\
\hline 1989 & April 9 & 35.39 & 18,900 & 1928 & March 28 & 13.30 & 3,840 \\
\hline 1978 & April 2 & 34.41 & 17,500 & 1948 & April 10 & 12.45 & 3,390 \\
\hline 1979 & April 19 & 34.93 & 17,300 & 1942 & June 11 & 12.27 & 3,380 \\
\hline 1952 & April 16 & 28.79 & 16,300 & 1987 & March 27 & 17.75 & 3,300 \\
\hline 1943 & April 7 & 28.40 & 16,000 & 1976 & March 30 & 18.70 & 3,200 \\
\hline 1975 & July 4 & 33.26 & 13,200 & 1914 & June 12 & 16.10 & 3,140 \\
\hline 1965 & April 15 & 30.50 & 11,400 & 1915 & July 3 & 9.73 & 3,130 \\
\hline 1994 & April 3 & 26.69 & 11,200 & 1906 & April 9 & 14.50 & 3,050 \\
\hline 1966 & $\operatorname{March} 22$ & 30.16 & 10,700 & 1955 & April 4 & 11.12 & 2,760 \\
\hline 1993 & April 5 & 28.27 & 10,100 & 1949 & July 12 & 11.27 & 2,660 \\
\hline 1962 & June 14 & 22.83 & 9,580 & 1927 & March 19 & 9.10 & 2,650 \\
\hline 1984 & April 1 & 28.27 & 9,550 & 1991 & July 6 & 16.99 & 2,630 \\
\hline 1947 & April 15 & 22.93 & 9,300 & 1908 & June 13 & 13.70 & 2,600 \\
\hline 1986 & April 2 & 27.19 & 8,640 & 1992 & June 19 & 16.93 & 2,590 \\
\hline 1951 & April 11 & 20.73 & 8,010 & 1957 & April 24 & 11.10 & 2,540 \\
\hline 1950 & April 7 & 20.88 & 7,800 & 1970 & June 18 & 16.27 & 2,480 \\
\hline 1916 & July 11 & - & 7,740 & 1903 & April 6 & 12.90 & 2,450 \\
\hline 1945 & March 22 & 20.70 & 7,700 & 1964 & April 18 & 16.22 & 2,400 \\
\hline 1972 & March 24 & 25.36 & 7,250 & 1958 & July 6 & 10.90 & 2,280 \\
\hline 1907 & May 30 & 28.80 & 7,000 & 1921 & April 6 & 8.40 & 1,970 \\
\hline 1953 & June 1 & 18.05 & 6,720 & 1973 & March 15 & 16.41 & 1,950 \\
\hline 1920 & March 28 & 17.20 & 6,200 & 1954 & July 4 & 10.53 & 1,920 \\
\hline 1946 & March 27 & 17.13 & 5,970 & 1971 & July 7 & 15.87 & 1,910 \\
\hline 1982 & April 4 & 25.07 & 5,920 & 1909 & May 30 & - & 1,780 \\
\hline 1967 & June 19 & 22.34 & 5,900 & 1983 & July & 15.99 & 1,750 \\
\hline 1980 & April 5 & 20.74 & 5,470 & 1981 & May 24 & 15.84 & 1,710 \\
\hline 1917 & April 3 & 17.80 & 5,240 & 1926 & $\operatorname{March} 24$ & 8.00 & 1,600 \\
\hline 1904 & April 20 & 20.30 & 5,220 & 1913 & July 8 & 10.90 & 1,560 \\
\hline 1922 & April 11 & 14.70 & 5,200 & 1937 & April 12 & 10.17 & 1,390 \\
\hline 1910 & March 19 & 22.20 & 5,000 & 1941 & April 3 & 10.10 & 1,390 \\
\hline 1963 & June 14 & 19.97 & 4,930 & 1938 & May 2 & 10.02 & 1,350 \\
\hline 1985 & June 5 & 20.08 & 4,690 & 1930 & March 17 & 10.00 & 1,340 \\
\hline 1929 & March 20 & 12.80 & 4,440 & 1959 & July 8 & 10.42 & 1,250 \\
\hline 1905 & May 17 & 17.40 & 4,250 & 1990 & June 2 & 15.40 & 1,220 \\
\hline 1944 & June 10 & 14.26 & 4,150 & 1902 & May 23 & 9.50 & 1,180 \\
\hline 1974 & April 14 & 20.25 & 4,150 & 1912 & May 14 & 9.60 & 1,100 \\
\hline 1923 & June 29 & 11.60 & 3,960 & 1936 & April 14 & 9.90 & 1,050 \\
\hline
\end{tabular}




\section{RED RIVER OF THE NORTH AT FARGO, ND-Continued}

Annual peak discharge and corresponding gage height, period of record--Continued

$$
[--, \text { no data] }
$$

\begin{tabular}{|c|c|c|c|c|c|c|c|}
\hline $\begin{array}{l}\text { Water } \\
\text { year }\end{array}$ & Date & $\begin{array}{c}\text { Gage } \\
\text { helght } \\
\text { (feet) }\end{array}$ & $\begin{array}{c}\text { Peak } \\
\text { dlscharge } \\
\left(\mathrm{ft}^{3} / \mathrm{s}\right)\end{array}$ & $\begin{array}{l}\text { Water } \\
\text { year }\end{array}$ & Date & $\begin{array}{c}\text { Gage } \\
\text { helght } \\
\text { (feet) }\end{array}$ & $\begin{array}{c}\text { Peak } \\
\text { dlscharge } \\
\left(\mathrm{ft}^{3} / \mathrm{s}\right)\end{array}$ \\
\hline \multicolumn{8}{|c|}{ Annual peak discharge, from highest to lowest, and corresponding gage height--Continued } \\
\hline 1940 & April 8 & 9.63 & 1,030 & 1968 & April 30 & 14.71 & 788 \\
\hline 1961 & June 9 & 9.24 & 1,020 & 1919 & April 6 & 6.50 & 680 \\
\hline 1988 & March 11 & 15.10 & 981 & 1911 & April 11 & 7.70 & 608 \\
\hline 1935 & March 20 & 9.72 & 942 & 1933 & April 5 & 9.04 & 605 \\
\hline 1925 & June 21 & 7.00 & 940 & 1924 & April 30 & 6.20 & 530 \\
\hline 1977 & July 4 & 14.99 & 878 & 1931 & April 3 & 8.55 & 365 \\
\hline 1932 & April 11 & 9.45 & 875 & 1934 & April 10 & 8.55 & 323 \\
\hline 1918 & March 31 & 6.87 & 874 & & & & \\
\hline
\end{tabular}

${ }^{1}$ Historic data, not used in statistics. 


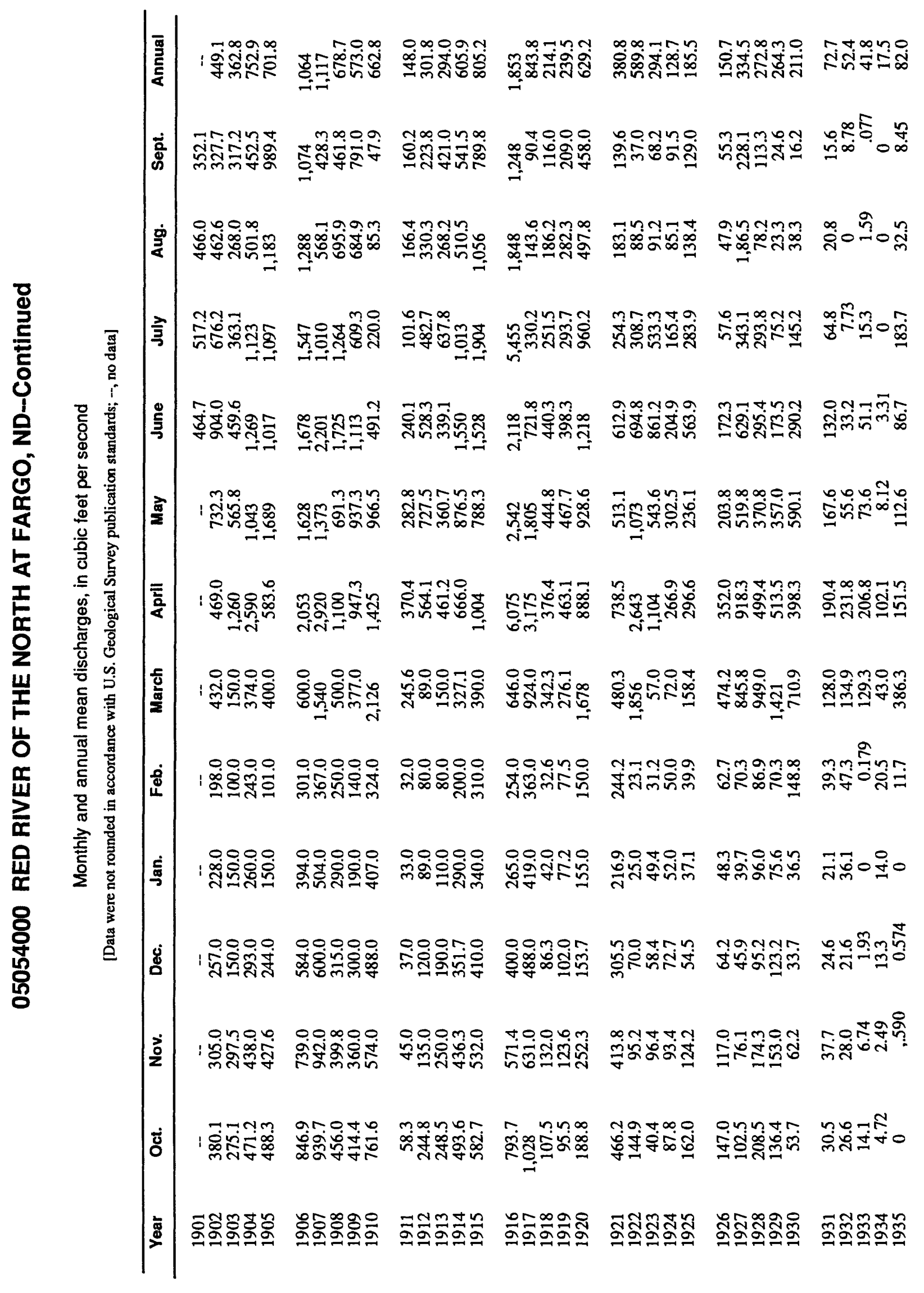




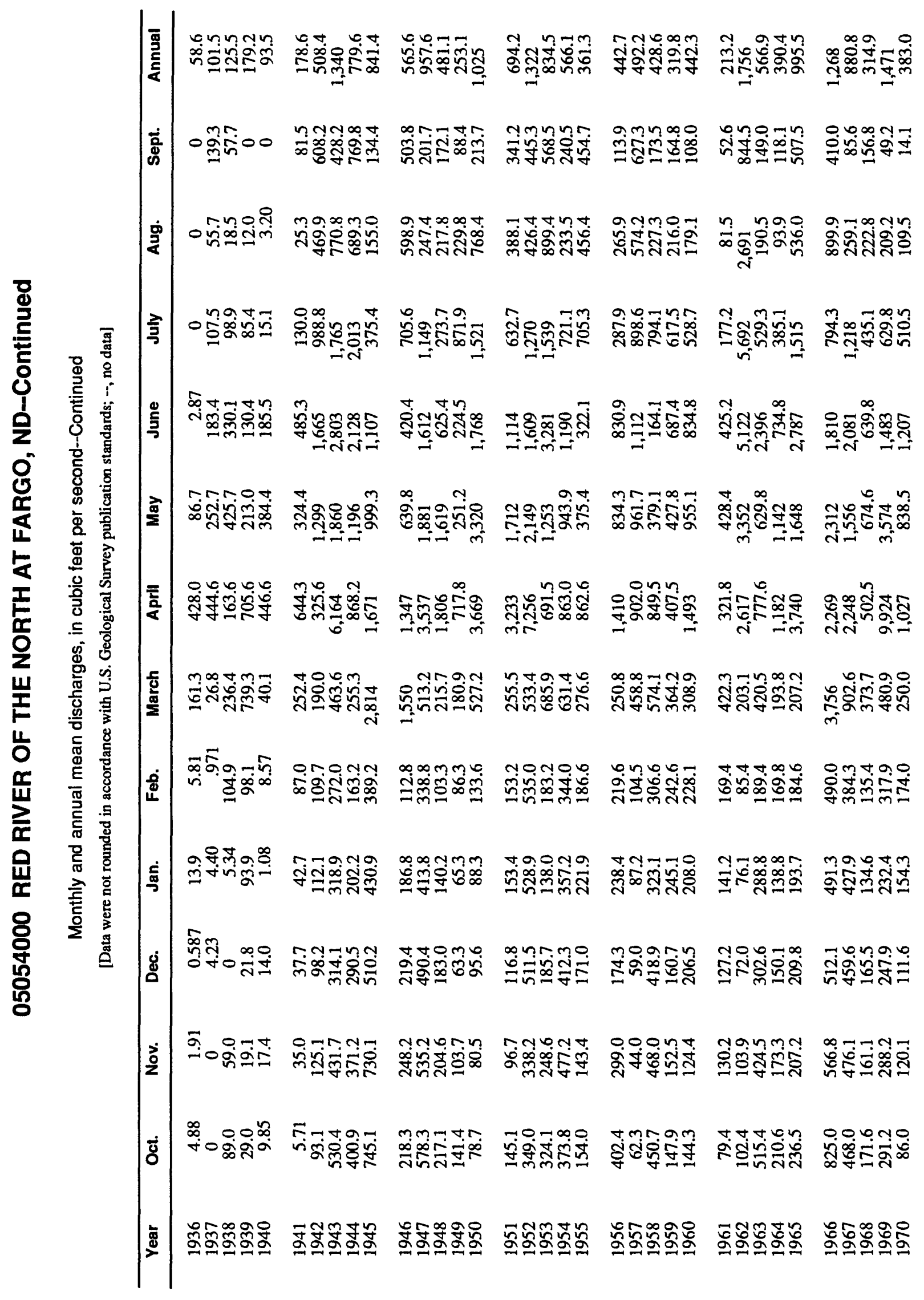




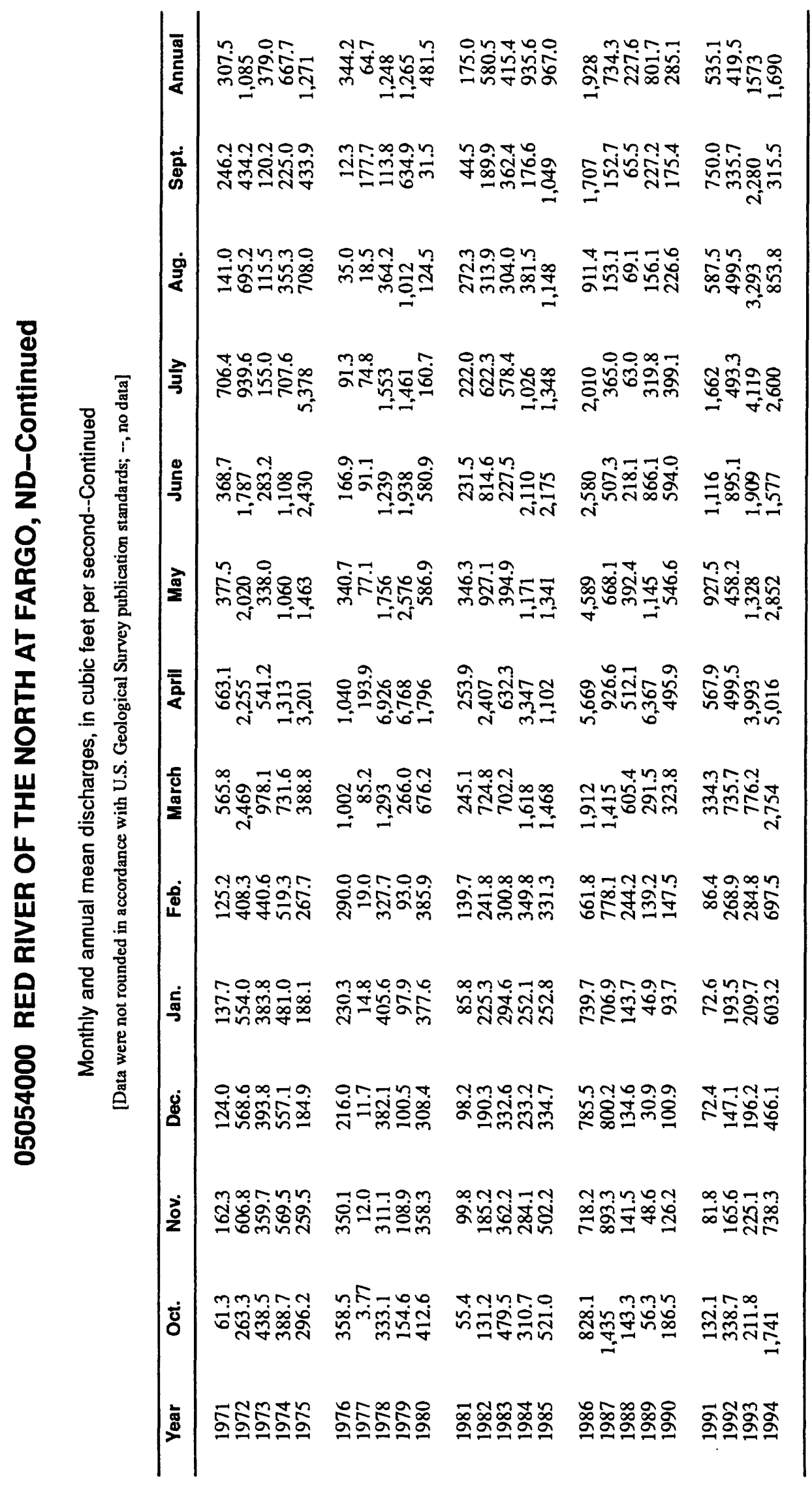




\section{SHEYENNE RIVER ABOVE HARVEY, ND}

\section{Station Description}

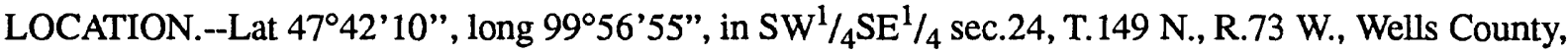
Hydrologic Unit 09020202, on right bank just downstream from county road, and 4.5 mi south of Harvey.

DRAINAGE AREA.- $-424 \mathrm{mi}^{2}$, of which about $270 \mathrm{mi}^{2}$ is probably noncontributing.

PERIOD OF RECORD.--September 1955 to current year.

GAGE.--Water-stage recorder. Datum of gage is $1,547.30 \mathrm{ft}$ above sea level.

EXTREMES FOR PERIOD OF RECORD.--Maximum discharge, 1,000 $\mathrm{ft}^{3} / \mathrm{s}$, Apr. 20, 1979 (gage height, $9.45 \mathrm{ft}$ ); maximum gage height, 10.30, Apr. 1, 1971, backwater from ice; no flow at times most years.

Annual mean discharge

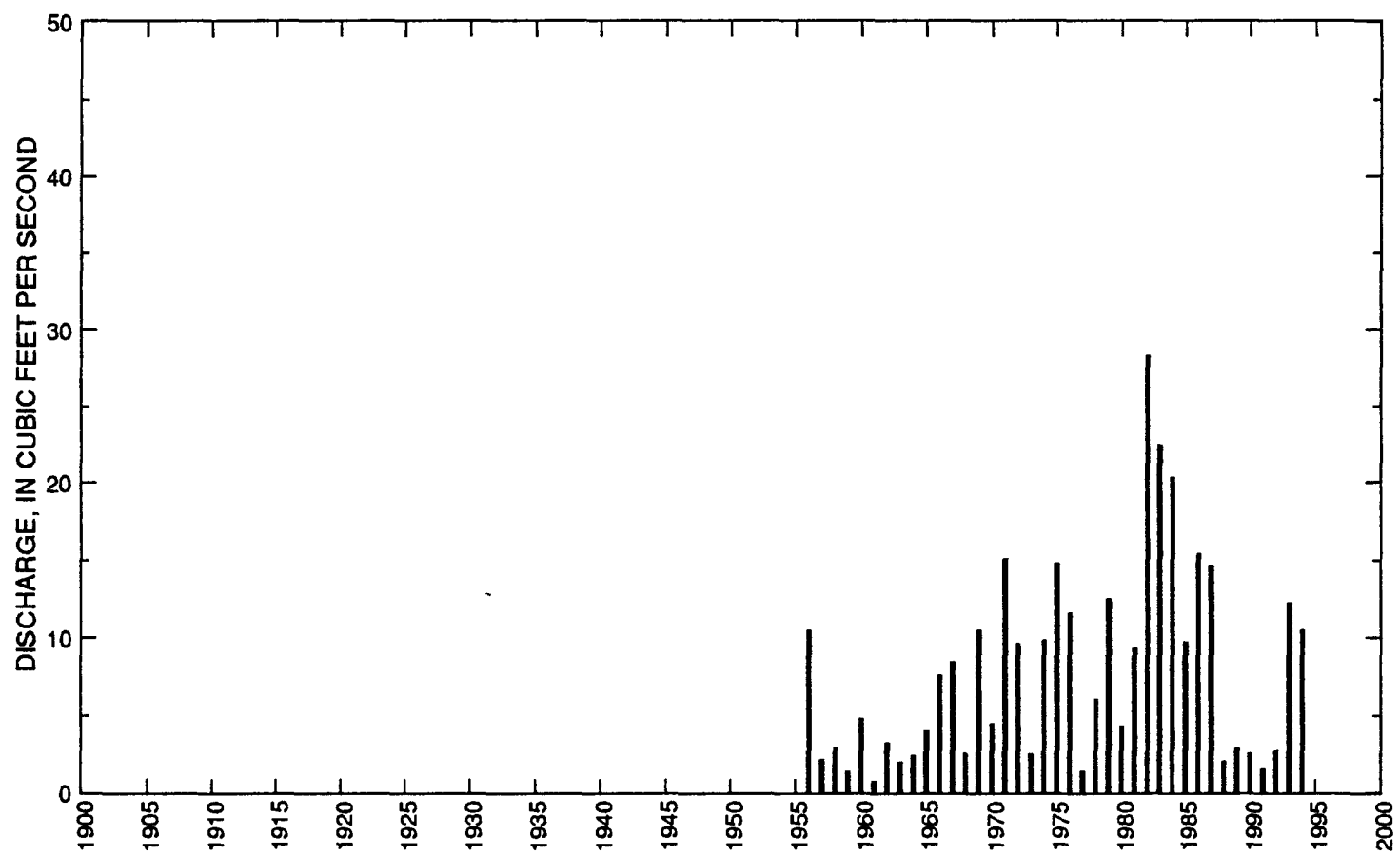




\section{SHEYENNE RIVER ABOVE HARVEY, ND-Continued}

Statistics of monthly and annual mean discharges

[ $m$, more than 1 year of occurrence]

\begin{tabular}{|c|c|c|c|c|c|c|c|c|}
\hline \multirow[b]{2}{*}{ Month } & \multicolumn{2}{|c|}{ Maximum } & \multicolumn{2}{|c|}{ Minimum } & \multicolumn{4}{|c|}{ Mean } \\
\hline & $\begin{array}{c}\text { Discharge } \\
\left(\mathrm{ft}^{3} / \mathrm{s}\right)\end{array}$ & $\begin{array}{c}\text { Water year } \\
\text { of } \\
\text { occurrence }\end{array}$ & $\begin{array}{c}\text { Discharge } \\
\left(\mathrm{ft}^{3} / \mathrm{s}\right)\end{array}$ & $\begin{array}{c}\text { Water year } \\
\text { of } \\
\text { occurrence }\end{array}$ & $\begin{array}{c}\text { Discharge } \\
\left(\mathrm{ft}^{3} / \mathrm{s}\right)\end{array}$ & $\begin{array}{l}\text { Standard } \\
\text { devlation } \\
\left(\mathrm{ft}^{3} / \mathrm{s}\right)\end{array}$ & $\begin{array}{l}\text { Coeffi- } \\
\text { cient of } \\
\text { varlation }\end{array}$ & $\begin{array}{l}\text { Percentage } \\
\text { of annual } \\
\text { discharge }\end{array}$ \\
\hline October & 11.8 & 1983 & 0.432 & 1991 & 2.47 & 2.48 & 1.00 & 2.60 \\
\hline November & 10.4 & 1983 & 0.265 & 1977 & 2.15 & 1.69 & 0.78 & 2.26 \\
\hline December & 6.27 & 1983 & 0.090 & 1962 & 0.910 & 1.03 & 1.12 & 0.96 \\
\hline January & 4.10 & 1983 & $\mathrm{~m}$ & 1959 & 0.480 & 0.89 & 1.86 & 0.50 \\
\hline February & 26.7 & 1983 & $\mathrm{~m}$ & 1956 & 2.61 & 5.56 & 2.13 & 2.75 \\
\hline March & 123 & 1982 & 0 & 1969 & 25.8 & 30.7 & 1.19 & 27.1 \\
\hline April & 100 & 1969 & 2.13 & 1991 & 28.4 & 31.0 & 1.09 & 29.9 \\
\hline May & 62.1 & 1984 & 1.59 & 1977 & 13.3 & 15.5 & 1.16 & 14.0 \\
\hline June & 54.0 & 1971 & 0.303 & 1961 & 7.52 & 9.53 & 1.27 & 7.91 \\
\hline July & 58.9 & 1993 & 0.071 & 1961 & 7.00 & 12.7 & 1.82 & 7.36 \\
\hline August & 25.6 & 1985 & $\mathrm{~m}$ & 1959 & 2.68 & 5.28 & 1.97 & 2.82 \\
\hline September & 4.79 & 1977 & 0.061 & 1976 & 1.72 & 1.32 & 0.77 & 1.81 \\
\hline Annual & 28.3 & 1982 & 0.760 & 1961 & 7.94 & 6.52 & 0.82 & 100 \\
\hline
\end{tabular}

Annual flow duration

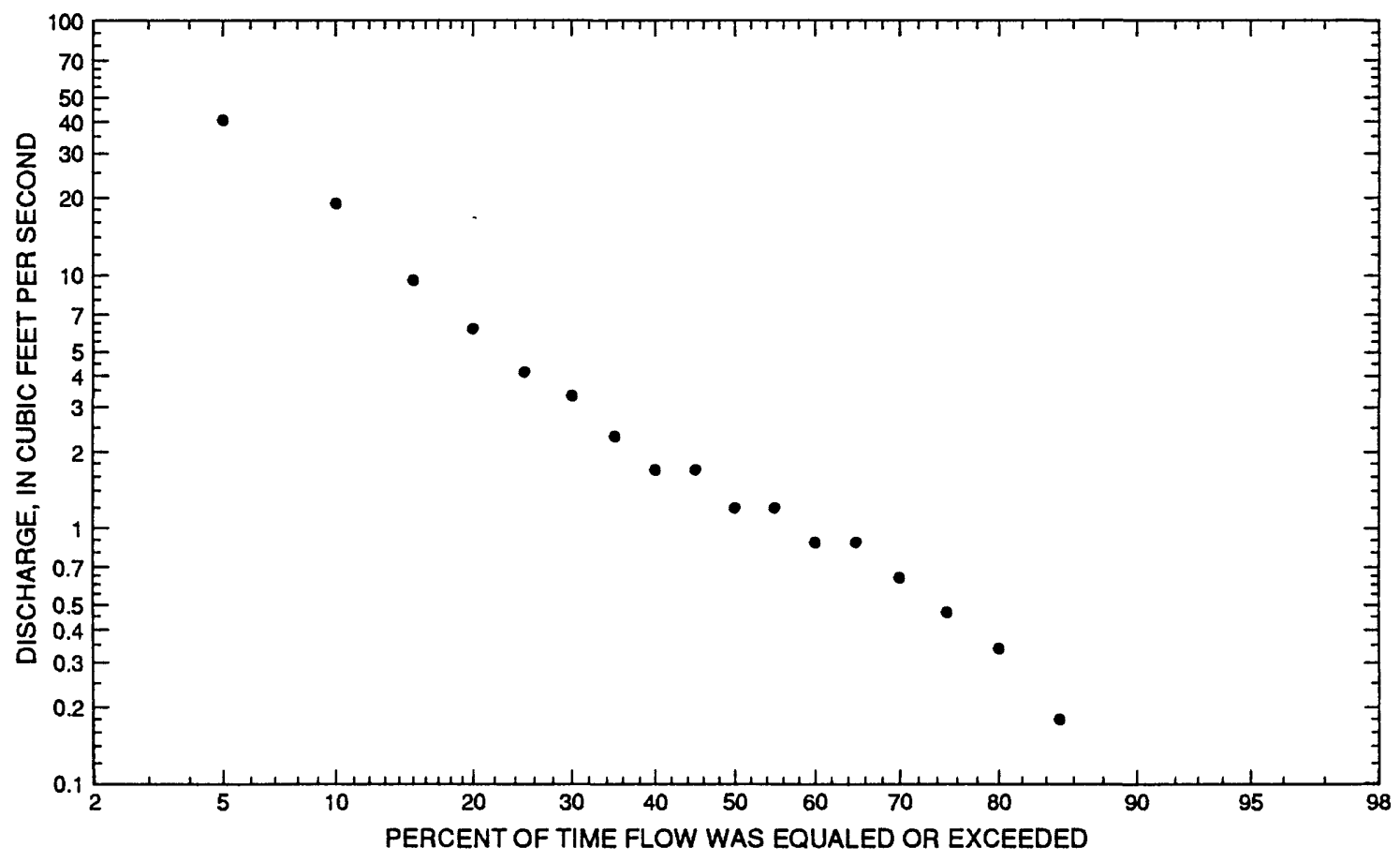




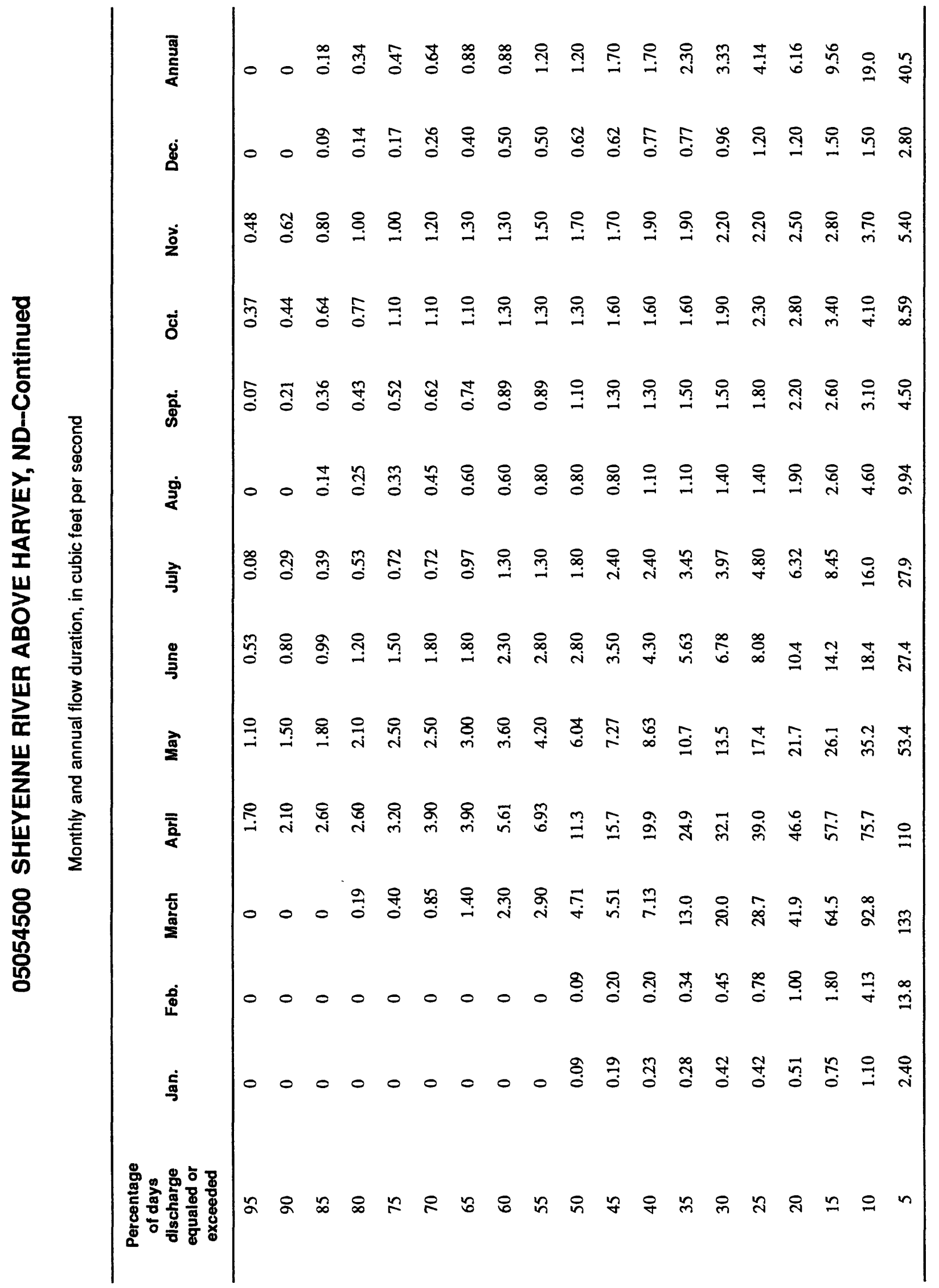




\section{SHEYENNE RIVER ABOVE HARVEY, ND--Continued}

Probability of annual high discharges

[ng, statistic not given]

\begin{tabular}{|c|c|c|c|c|c|c|}
\hline \multirow[b]{2}{*}{$\begin{array}{l}\text { Exceedance } \\
\text { probability }\end{array}$} & \multirow[b]{2}{*}{$\begin{array}{l}\text { Recurrence } \\
\text { Interval } \\
\text { (years) }\end{array}$} & \multirow[b]{2}{*}{$\begin{array}{c}\text { Maximum } \\
\text { Instantaneous } \\
\left(\mathrm{ft}^{3} / \mathbf{s}\right)\end{array}$} & \multicolumn{4}{|c|}{$\begin{array}{l}\text { Maximum average discharge } \\
\qquad\left(\mathrm{ft}^{3} / \mathrm{s}\right)\end{array}$} \\
\hline & & & 3-day period & 7-day period & 15-day period & 30-day period \\
\hline 0.99 & 1.01 & 5.50 & 3.44 & 2.71 & 2.02 & 1.64 \\
\hline 0.95 & 1.05 & 14.3 & 9.76 & 7.70 & 5.51 & 4.15 \\
\hline 0.90 & 1.11 & 23.2 & 16.3 & 12.9 & 9.09 & 6.65 \\
\hline 0.80 & 1.25 & 40.4 & 29.3 & 23.0 & 16.1 & 11.5 \\
\hline 0.50 & 2 & 108 & 79.9 & 62.3 & 44.0 & 30.7 \\
\hline 0.20 & 5 & 264 & 189 & 145 & 106 & 75.5 \\
\hline 0.10 & 10 & 406 & 282 & 214 & 161 & 117 \\
\hline 0.04 & 25 & 625 & 414 & 311 & 243 & 182 \\
\hline 0.02 & 50 & 813 & 520 & 387 & 312 & 239 \\
\hline 0.01 & 100 & 1,020 & 631 & 466 & 386 & 302 \\
\hline 0.005 & 200 & 1,250 & 744 & 545 & 464 & 373 \\
\hline 0.002 & 500 & 1,580 & $\mathrm{ng}$ & $\mathrm{ng}$ & $\mathrm{ng}$ & $\mathrm{ng}$ \\
\hline
\end{tabular}

Probability of annual low discharges

\begin{tabular}{|c|c|c|c|c|c|c|c|c|c|c|}
\hline \multirow[b]{3}{*}{$\begin{array}{l}\text { Non- } \\
\text { exceed- } \\
\text { ance } \\
\text { prob- } \\
\text { abillty }\end{array}$} & \multirow[b]{3}{*}{$\begin{array}{c}\text { Recur- } \\
\text { rence } \\
\text { inter- } \\
\text { val } \\
\text { (years) }\end{array}$} & \multicolumn{9}{|c|}{ Minimum average discharge $\left(\mathrm{ft}^{3} / \mathrm{s}\right)$} \\
\hline & & \multicolumn{9}{|c|}{ Number of consecutive days } \\
\hline & & 1 & 3 & 7 & 14 & 30 & 60 & 90 & 120 & 183 \\
\hline 0.05 & 20 & 0 & 0 & 0 & 0 & 0 & 0 & 0 & 0.117 & 0.348 \\
\hline 0.10 & 10 & 0 & 0 & 0 & 0 & 0 & 0 & 0.020 & 0.175 & 0.440 \\
\hline 0.20 & 5 & 0 & 0 & 0 & 0 & 0 & 0 & 0.074 & 0.279 & 0.588 \\
\hline 0.50 & 2 & 0 & 0 & 0 & 0 & 0 & 0.103 & 0.307 & 0.642 & 1.04 \\
\hline
\end{tabular}




\section{SHEYENNE RIVER ABOVE HARVEY, ND--Continued}

Probability of seasonal low discharges

\begin{tabular}{|c|c|c|c|c|c|c|c|c|c|}
\hline \multirow[b]{3}{*}{$\begin{array}{l}\text { Non- } \\
\text { exceedance } \\
\text { probability }\end{array}$} & \multirow[b]{3}{*}{$\begin{array}{l}\text { Recurrence } \\
\text { interval } \\
\text { (years) }\end{array}$} & \multicolumn{8}{|c|}{ Minimum average discharge $\left(\mathrm{ft}^{3} / \mathrm{s}\right)$} \\
\hline & & \multicolumn{8}{|c|}{ Number of consecutive days } \\
\hline & & 1 & 7 & 14 & 30 & 1 & 7 & 14 & 30 \\
\hline & & \multicolumn{4}{|c|}{ December-January-February } & \multicolumn{4}{|c|}{ March-April-May } \\
\hline 0.05 & 20 & 0 & 0 & 0 & 0 & 0 & 0 & 0 & 0.279 \\
\hline 0.10 & 10 & 0 & 0 & 0 & 0 & 0 & 0 & 0 & 0.629 \\
\hline 0.20 & 5 & 0 & 0 & 0 & 0 & 0 & 0 & 0.132 & 1.36 \\
\hline \multirow[t]{2}{*}{0.50} & 2 & 0 & 0 & 0 & 0 & 0.279 & 0.521 & 1.52 & 4.53 \\
\hline & & \multicolumn{4}{|c|}{ June-July-August } & \multicolumn{4}{|c|}{ September-October-November } \\
\hline 0.05 & 20 & 0 & 0 & 0 & 0 & 0 & 0 & 0.048 & 0.179 \\
\hline 0.10 & 10 & 0 & 0 & 0 & 0.067 & 0.068 & 0.086 & 0.125 & 0.286 \\
\hline 0.20 & 5 & 0 & 0.082 & 0.175 & 0.217 & 0.171 & 0.239 & 0.282 & 0.475 \\
\hline 0.50 & 2 & 0.324 & 0.411 & 0.546 & 0.962 & 0.441 & 0.618 & 0.794 & 1.05 \\
\hline
\end{tabular}




\section{SHEYENNE RIVER ABOVE HARVEY, ND--Continued}

Annual peak discharge and corresponding gage height, period of record

$$
[-, \text { no data }]
$$

\begin{tabular}{|c|c|c|c|c|c|c|c|}
\hline $\begin{array}{l}\text { Water } \\
\text { year }\end{array}$ & Date & $\begin{array}{c}\text { Gage } \\
\text { height } \\
\text { (feet) }\end{array}$ & $\begin{array}{c}\text { Peak } \\
\text { discharge } \\
\left(\mathrm{ft}^{3} / \mathrm{s}\right)\end{array}$ & $\begin{array}{l}\text { Water } \\
\text { year }\end{array}$ & Date & $\begin{array}{c}\text { Gage } \\
\text { height } \\
\text { (feet) }\end{array}$ & $\begin{array}{c}\text { Peak } \\
\text { discharge } \\
\left(\mathrm{ft}^{3 / s}\right)\end{array}$ \\
\hline \multicolumn{8}{|c|}{ Annual peak discharge, by year, and corresponding gage height } \\
\hline 1956 & April 11 & 8.92 & 224 & 1976 & March 20 & - & 220 \\
\hline 1957 & March 23 & 8.14 & 26.0 & 1977 & September 24 & 5.51 & 20.0 \\
\hline 1958 & June 30 & 7.26 & 58.0 & 1978 & March 27 & 8.78 & 148 \\
\hline 1959 & March 28 & 5.70 & 15.0 & 1979 & April 20 & 9.45 & 1,000 \\
\hline 1960 & March 27 & 8.94 & 209 & 1980 & August 20 & 6.33 & 34.0 \\
\hline 1961 & April 5 & 5.19 & 14.0 & 1981 & June 16 & 7.15 & 53.0 \\
\hline 1962 & March 24 & 8.51 & 110 & 1982 & April 3 & - & 410 \\
\hline 1963 & June 8 & 7.32 & 65.0 & 1983 & March 13 & 9.57 & 240 \\
\hline 1964 & April 1 & 8.18 & 54.0 & 1984 & April 14 & 8.38 & 145 \\
\hline 1965 & April 9 & 8.43 & 95.0 & 1985 & August 19 & 8.80 & 224 \\
\hline 1966 & March 15 & 9.21 & 410 & 1986 & March 4 & 9.16 & 180 \\
\hline 1967 & March 26 & 9.94 & 300 & 1987 & July 24 & 9.08 & 434 \\
\hline 1968 & March 7 & 7.59 & 62.0 & 1988 & March 26 & - & 80.0 \\
\hline 1969 & April 11 & 9.45 & 370 & 1989 & March 29 & 7.95 & 70.0 \\
\hline 1970 & April 6 & 7.74 & 58.0 & 1990 & September 29 & 5.81 & 18.0 \\
\hline 1971 & April 2 & 10.25 & 300 & 1991 & September 23 & - & 10.0 \\
\hline 1972 & March 15 & 9.09 & 177 & 1992 & March 2 & 8.27 & 55.0 \\
\hline 1973 & February 27 & 9.41 & 18.0 & 1993 & July 28 & 9.67 & 224 \\
\hline 1974 & May 20 & 8.36 & 160 & 1994 & March 24 & 9.04 & 210 \\
\hline 1975 & April 29 & - & 160 & & & & \\
\hline \multicolumn{8}{|c|}{ Annual peak discharge, from highest to lowest, and corresponding gage height } \\
\hline 1979 & April 20 & 9.45 & 1,000 & 1962 & March 24 & 8.51 & 110 \\
\hline 1987 & July 24 & 9.08 & 434 & 1965 & April 9 & 8.43 & 95.0 \\
\hline 1966 & March 15 & 9.21 & 410 & 1988 & March 26 & - & 80.0 \\
\hline 1982 & April 3 & - & 410 & 1989 & March 29 & 7.95 & 70.0 \\
\hline 1969 & April 11 & 9.45 & 370 & 1963 & June 8 & 7.32 & 65.0 \\
\hline 1967 & March 26 & 9.94 & 300 & 1968 & March 7 & 7.59 & 62.0 \\
\hline 1971 & April 2 & 10.25 & 300 & 1958 & June 30 & 7.26 & 58.0 \\
\hline 1983 & March 13 & 9.57 & 240 & 1970 & April 6 & 7.74 & 58.0 \\
\hline 1956 & April 11 & 8.92 & 224 & 1992 & March 2 & 8.27 & 55.0 \\
\hline 1985 & August 19 & 8.80 & 224 & 1964 & April 1 & 8.18 & 54.0 \\
\hline 1993 & July 28 & 9.67 & 224 & 1981 & June 16 & 7.15 & 53.0 \\
\hline 1976 & March 20 & - & 220 & 1980 & August 20 & 6.33 & 34.0 \\
\hline 1994 & March 24 & 9.04 & 210 & 1957 & March 23 & 8.14 & 26.0 \\
\hline 1960 & March 27 & 8.94 & 209 & 1977 & September 24 & 5.51 & 20.0 \\
\hline 1986 & March 4 & 9.16 & 180 & 1973 & February 27 & 9.41 & 18.0 \\
\hline 1972 & March 15 & 9.09 & 177 & 1990 & September 29 & 5.81 & 18.0 \\
\hline 1974 & May 20 & 8.36 & 160 & 1959 & March 28 & 5.70 & 15.0 \\
\hline 1975 & April 29 & - & 160 & 1961 & April 5 & 5.19 & 14.0 \\
\hline 1978 & March 27 & 8.78 & 148 & 1991 & September 23 & -- & 10.0 \\
\hline 1984 & April 14 & 8.38 & 145 & & & & \\
\hline
\end{tabular}




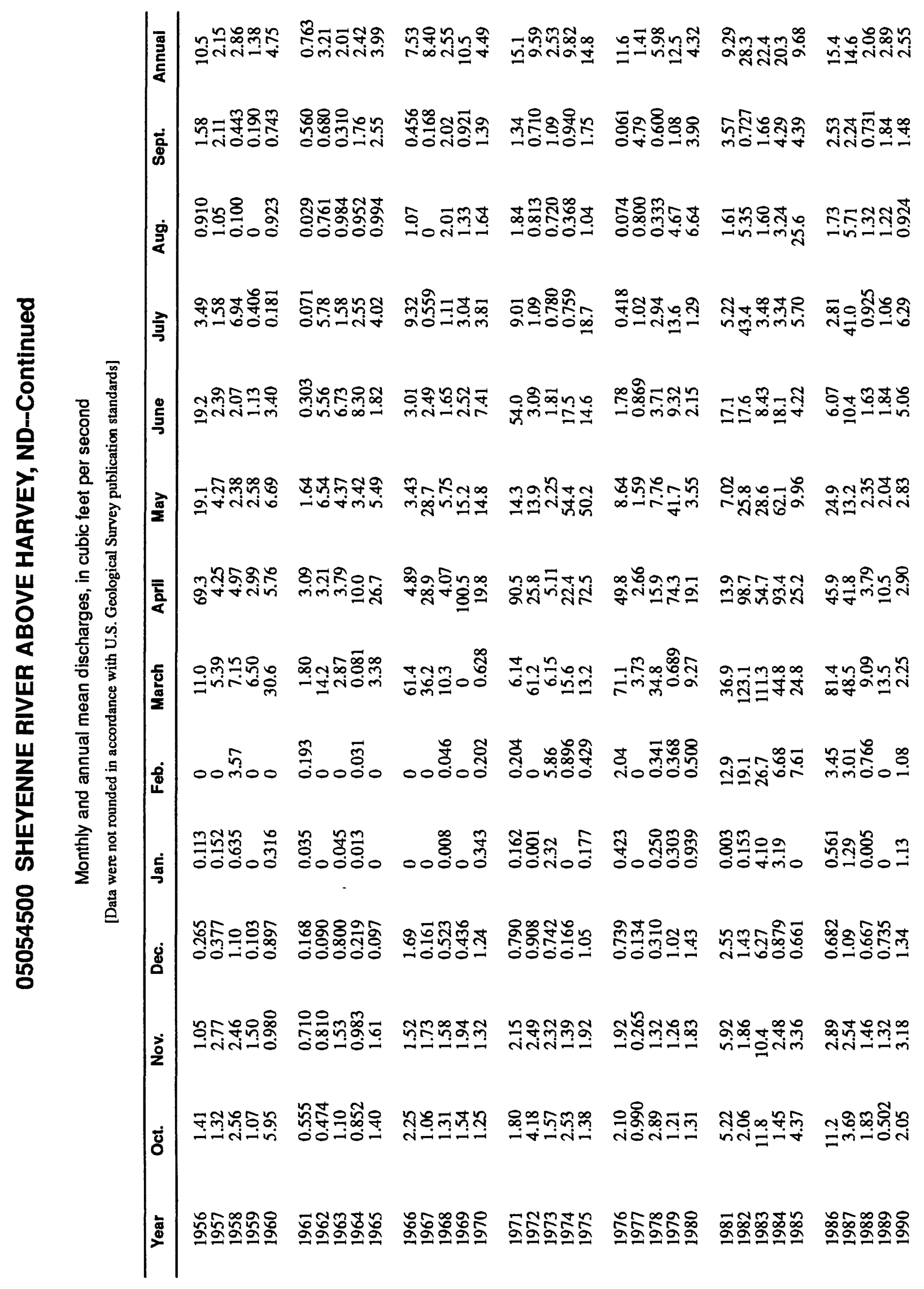




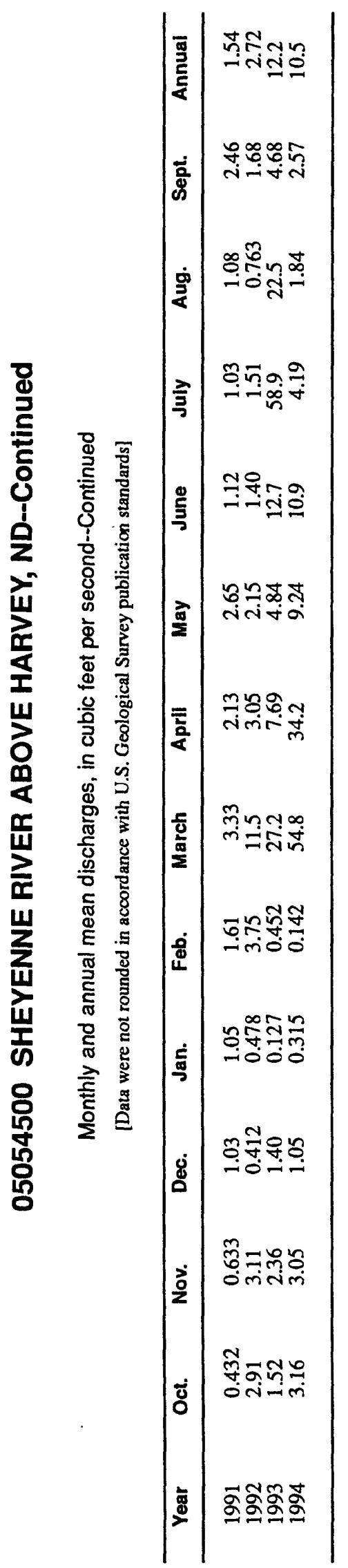




\section{SHEYENNE RIVER NEAR HARVEY, ND}

\section{Station Description}

LOCATION.--Lat $47^{\circ} 47^{\prime} 25^{\prime \prime}$, long $99^{\circ} 53^{\prime} 25^{\prime \prime}$, in SE $1 / 4 \mathrm{SW}^{1} / 4$ sec. 21, T.150 N., R.72 W., Wells County, Hydrologic Unit 09020202, $90 \mathrm{ft}$ north of Harvey Water Works, $0.4 \mathrm{mi}$ upstream from small tributary, and $2.25 \mathrm{mi}$ northeast of Harvey.

DRAINAGE AREA.--534 $\mathrm{mi}^{2}$ (revised), of which about $360 \mathrm{mi}^{2}$ is probably noncontributing.

PERIOD OF RECORD.--October 1945 to September 1956.

GAGE.--Staff gage. Datum of gage is $1,520.10 \mathrm{ft}$ above mean sea level, datum of 1929 . Prior to June 11 , 1946, staff gage at site $3 \mathrm{mi}$ upstream at different datum.

EXTREMES FOR PERIOD OF RECORD.--Maximum discharge, 1,430 $\mathrm{ft}^{3} / \mathrm{s}$, Apr. 18, 1950 (gage height, $6.62 \mathrm{ft}$ ); maximum gage height, 6.95, Apr. 17, 1950, affected by ice; no flow during several months in most years.

Annual mean discharge

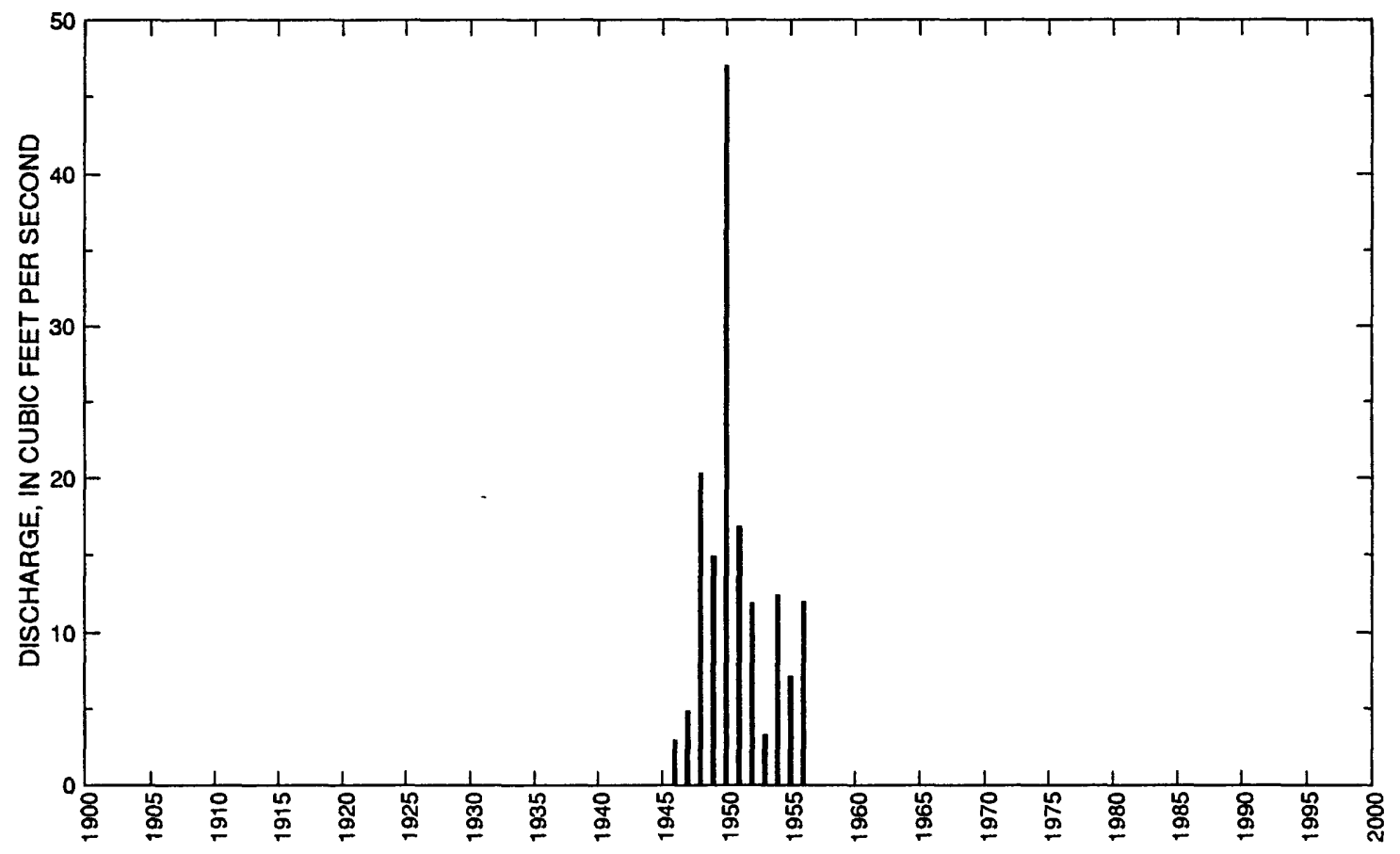




\section{SHEYENNE RIVER NEAR HARVEY, ND--Continued}

Statistics of monthly and annual mean discharges

[ $\mathrm{m}$, more than 1 year of occurrence]

\begin{tabular}{|c|c|c|c|c|c|c|c|c|}
\hline \multirow[b]{2}{*}{ Month } & \multicolumn{2}{|c|}{ Maximum } & \multicolumn{2}{|c|}{ Minimum } & \multicolumn{4}{|c|}{ Mean } \\
\hline & $\begin{array}{c}\text { Discharge } \\
\left(\mathrm{ft}^{3} / \mathrm{s}\right)\end{array}$ & $\begin{array}{c}\text { Water year } \\
\text { of } \\
\text { occurrence }\end{array}$ & $\begin{array}{c}\text { Discharge } \\
\left(\mathrm{ft}^{3} / \mathrm{s}\right)\end{array}$ & $\begin{array}{l}\text { Water year } \\
\text { of } \\
\text { occurrence }\end{array}$ & $\begin{array}{c}\text { Discharge } \\
\left(\mathrm{ft}^{3} / \mathrm{s}\right)\end{array}$ & $\begin{array}{c}\text { Standard } \\
\text { deviation } \\
\left(\mathrm{ft}^{3} / \mathrm{s}\right)\end{array}$ & $\begin{array}{l}\text { Coetfl- } \\
\text { cient of } \\
\text { variation }\end{array}$ & $\begin{array}{l}\text { Percentage } \\
\text { of annual } \\
\text { discharge }\end{array}$ \\
\hline October & 3.33 & 1955 & 0 & $\mathrm{~m}$ & 0.530 & 1.01 & 1.90 & 0.32 \\
\hline November & 2.66 & 1955 & 0 & $\mathrm{~m}$ & 0.580 & 0.87 & 1.50 & 0.35 \\
\hline December & 1.77 & 1955 & 0 & $\mathrm{~m}$ & 0.540 & 0.71 & 1.32 & 0.32 \\
\hline January & 1.77 & 1951 & 0 & $\mathrm{~m}$ & 0.200 & 0.52 & 2.58 & 0.12 \\
\hline February & 8.93 & 1954 & 0 & $\mathrm{~m}$ & 0.880 & 2.68 & 3.03 & 0.53 \\
\hline March & 23.9 & 1947 & 0.161 & 1948 & 9.25 & 8.83 & 0.950 & 5.50 \\
\hline April & 324 & 1950 & 7.16 & 1946 & 99.5 & 102 & 1.03 & 59.1 \\
\hline May & 201 & 1950 & 1.15 & 1946 & 29.3 & 57.5 & 1.97 & 17.4 \\
\hline June & 86.2 & 1954 & 0.193 & 1946 & 20.2 & 25.2 & 1.25 & 12.0 \\
\hline July & 27.0 & 1954 & 1.15 & 1949 & 5.72 & 7.32 & 1.28 & 3.40 \\
\hline August & 5.42 & 1954 & 0 & $\mathrm{~m}$ & 0.720 & 1.59 & 2.20 & 0.43 \\
\hline September & 5.72 & 1954 & 0 & $\mathrm{~m}$ & 0.860 & 1.82 & 2.12 & 0.51 \\
\hline Annual & 47.0 & 1950 & 2.93 & 1946 & 14.0 & 12.3 & 0.880 & 100 \\
\hline
\end{tabular}

Annual flow duration

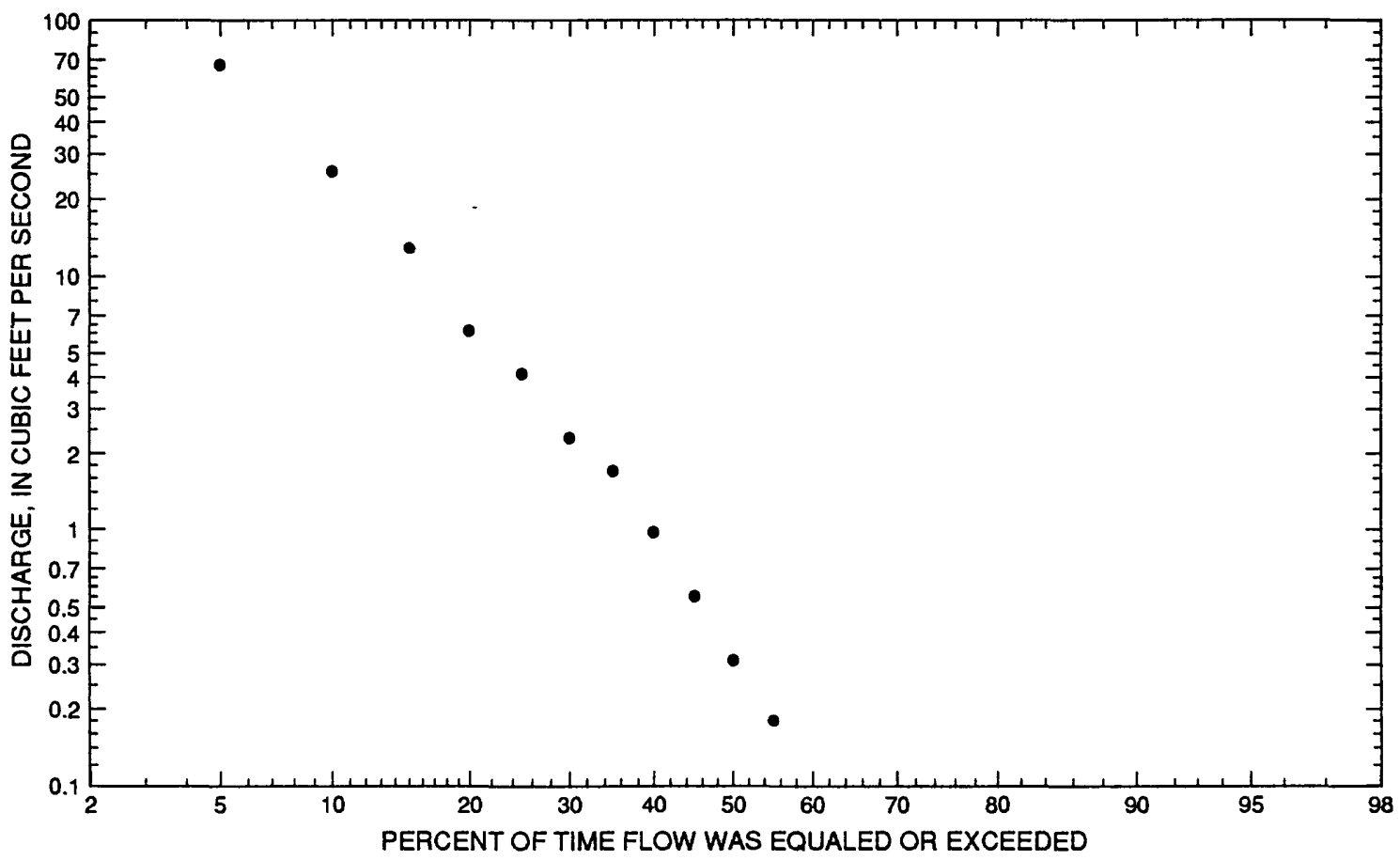




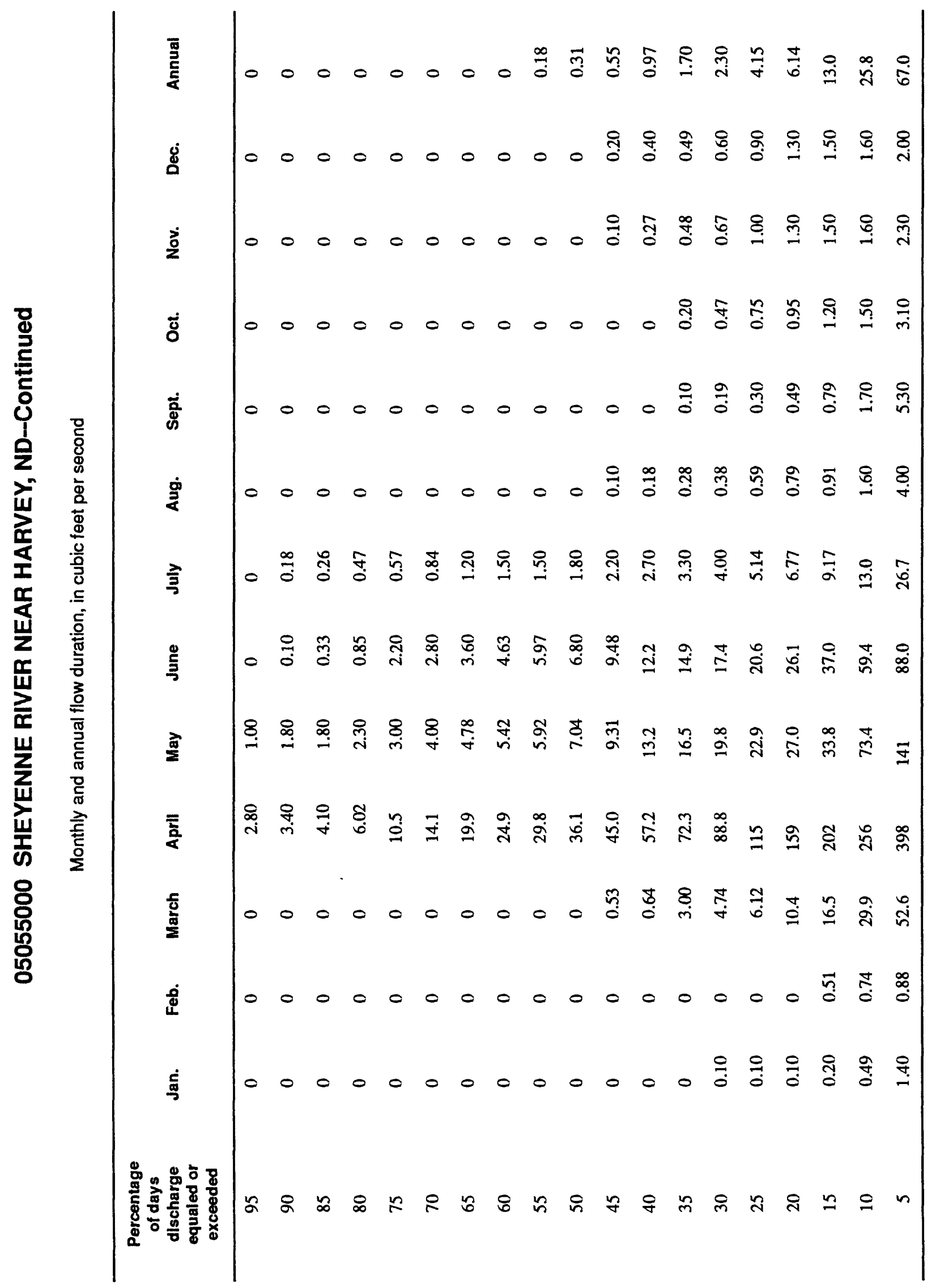


05055000 SHEYENNE RIVER NEAR HARVEY, ND--Continued

Probability of annual high discharges

[ng, statistic not given]

\begin{tabular}{lcccccc}
\hline & & & \multicolumn{5}{c}{$\begin{array}{c}\text { Maximum average discharge } \\
\left(\mathbf{f t}^{\mathbf{3}} / \mathbf{s}\right)\end{array}$} \\
\cline { 6 - 7 } $\begin{array}{c}\text { Exceedance } \\
\text { probability }\end{array}$ & $\begin{array}{c}\text { Recurrence } \\
\text { Interval } \\
\text { (years) }\end{array}$ & $\begin{array}{c}\text { Maximum } \\
\text { instantaneous } \\
\left(\mathbf{f t}^{3} / \mathbf{s}\right)\end{array}$ & 3-day period & 7-day period & 15-day period & 30-day period \\
\hline 0.99 & 1.01 & 29.6 & 13.4 & 11.9 & 9.08 & 6.34 \\
0.95 & 1.05 & 63.4 & 33.2 & 28.4 & 21.4 & 14.6 \\
0.90 & 1.11 & 93.8 & 52.5 & 44.1 & 32.8 & 22.1 \\
0.80 & 1.25 & 148 & 89.3 & 73.6 & 53.9 & 36.0 \\
0.50 & 2 & 342 & 231 & 184 & 130 & 85.9 \\
0.20 & 5 & 744 & 545 & 423 & 287 & 189 \\
0.10 & 10 & 1,090 & 826 & 634 & 419 & 276 \\
0.04 & 25 & 1,620 & 1,250 & 955 & 612 & 405 \\
0.02 & 50 & 2,080 & 1,620 & 1,230 & 772 & 513 \\
0.01 & 100 & 2,580 & 2,020 & 1,530 & 942 & 628 \\
0.005 & 200 & 3,120 & 2,460 & 1,850 & 1,120 & 752 \\
0.002 & 500 & 3,920 & $\mathrm{ng}$ & $\mathrm{ng}$ & $\mathrm{ng}$ & $\mathrm{ng}$ \\
\hline
\end{tabular}

Probability of annual low discharges

[ng, statistic not given]

\begin{tabular}{|c|c|c|c|c|c|c|c|c|c|c|}
\hline \multirow{3}{*}{$\begin{array}{l}\text { Non- } \\
\text { exceed- } \\
\text { ance } \\
\text { prob- } \\
\text { ablilty }\end{array}$} & \multirow{3}{*}{$\begin{array}{c}\text { Recur- } \\
\text { rence } \\
\text { inter- } \\
\text { val } \\
\text { (years) }\end{array}$} & \multicolumn{9}{|c|}{ MinImum average dlscharge $\left(\mathrm{tt}^{3} / \mathrm{s}\right)$} \\
\hline & & \multicolumn{9}{|c|}{ Number of consecutive days } \\
\hline & & 1 & 3 & 7 & 14 & 30 & 60 & 90 & 120 & 183 \\
\hline 0.05 & 20 & ng & ng & ng & $\mathrm{ng}$ & $\mathrm{ng}$ & ng & 0 & 0 & 0 \\
\hline 0.10 & 10 & ng & ng & ng & ng & ng & ng & 0 & 0 & 0 \\
\hline 0.20 & 5 & ng & ng & ng & $\mathrm{ng}$ & ng & ng & 0 & 0 & 0 \\
\hline 0.50 & 2 & ng & ng & ng & ng & $\mathrm{ng}$ & ng & 0 & 0 & 0.025 \\
\hline
\end{tabular}




\section{SHEYENNE RIVER NEAR HARVEY, ND--Continued}

Probability of seasonal low discharges

[ng, statistic not given]

\begin{tabular}{|c|c|c|c|c|c|c|c|c|c|}
\hline \multirow[b]{3}{*}{$\begin{array}{c}\text { Non- } \\
\text { exceedance } \\
\text { probability }\end{array}$} & \multirow[b]{3}{*}{$\begin{array}{c}\text { Recurrence } \\
\text { interval } \\
\text { (years) }\end{array}$} & \multicolumn{8}{|c|}{ Minimum average discharge $\left(\mathrm{ft}^{3} / \mathrm{s}\right)$} \\
\hline & & \multicolumn{8}{|c|}{ Number of consecutive days } \\
\hline & & 1 & 7 & 14 & 30 & 1 & 7 & 14 & 30 \\
\hline & & \multicolumn{4}{|c|}{ December-January-February } & \multicolumn{4}{|c|}{ March-April-May } \\
\hline 0.05 & 20 & $\mathrm{ng}$ & ng & $\mathrm{ng}$ & $\mathrm{ng}$ & ng & $\mathrm{ng}$ & 0 & 0.100 \\
\hline 0.10 & 10 & ng & $\mathrm{ng}$ & $\mathrm{ng}$ & ng & $\mathrm{ng}$ & $\mathrm{ng}$ & 0 & 0.240 \\
\hline 0.20 & 5 & $\mathrm{ng}$ & $\mathrm{ng}$ & $\mathrm{ng}$ & $\mathrm{ng}$ & $\mathrm{ng}$ & $\mathrm{ng}$ & 0 & 0.600 \\
\hline \multirow[t]{2}{*}{0.50} & 2 & ng & ng & $\mathrm{ng}$ & ng & ng & ng & 0 & 2.58 \\
\hline & & \multicolumn{4}{|c|}{ June-July-August } & \multicolumn{4}{|c|}{ September-October-November } \\
\hline 0.05 & 20 & $\mathrm{ng}$ & 0 & 0 & 0 & ng & ng & 0 & 0 \\
\hline 0.10 & 10 & $\mathrm{ng}$ & 0 & 0 & 0 & $\mathrm{ng}$ & $\mathrm{ng}$ & 0 & 0 \\
\hline 0.20 & 5 & $\mathrm{ng}$ & 0 & 0 & 0 & $\mathrm{ng}$ & $\mathrm{ng}$ & 0 & 0 \\
\hline 0.50 & 2 & $\mathrm{ng}$ & 0 & 0 & 0.120 & $\mathrm{ng}$ & $\mathrm{ng}$ & 0 & 0 \\
\hline
\end{tabular}




\section{SHEYENNE RIVER NEAR HARVEY, ND--Continued}

Annual peak discharge and corresponding gage height, period of record

\begin{tabular}{|c|c|c|c|c|c|c|c|}
\hline $\begin{array}{l}\text { Water } \\
\text { year }\end{array}$ & Date & $\begin{array}{l}\text { Gage } \\
\text { height } \\
\text { (feet) }\end{array}$ & $\begin{array}{c}\text { Peak } \\
\text { discharge } \\
\left(\mathrm{ft}^{3} / \mathrm{s}\right)\end{array}$ & $\begin{array}{l}\text { Water } \\
\text { year }\end{array}$ & Date & $\begin{array}{l}\text { Gage } \\
\text { height } \\
\text { (feet) }\end{array}$ & $\begin{array}{c}\text { Peak } \\
\text { dlscharge } \\
\left(\mathrm{ft}^{3} / \mathrm{s}\right)\end{array}$ \\
\hline \multicolumn{8}{|c|}{ Annual peak discharge, by year, and corresponding gage height } \\
\hline 1946 & March 21 & 2.26 & 132 & 1952 & April 7 & 5.23 & 258 \\
\hline 1947 & March 23 & 5.44 & 200 & 1953 & June 30 & 3.60 & 60.0 \\
\hline 1948 & April 18 & 6.45 & 1,220 & 1954 & June 17 & 5.57 & 351 \\
\hline 1949 & April 7 & 6.20 & 846 & 1955 & March 31 & 4.76 & 120 \\
\hline 1950 & April 18 & 6.62 & 1,430 & 1956 & April 10 & 5.65 & 316 \\
\hline 1951 & April 7 & 5.85 & 340 & & & & \\
\hline \multicolumn{8}{|c|}{ Annual peak discharge, from highest to lowest, and corresponding gage height } \\
\hline 1950 & April 18 & 6.62 & 1,430 & 1952 & April 7 & 5.23 & 258 \\
\hline 1948 & April 18 & 6.45 & 1,220 & 1947 & March 23 & 5.44 & 200 \\
\hline 1949 & April 7 & 6.20 & 846 & 1946 & March 21 & 2.26 & 132 \\
\hline 1954 & June 17 & 5.57 & 351 & 1955 & March 31 & 4.76 & 120 \\
\hline 1951 & April 7 & 5.85 & 340 & 1953 & June 30 & 3.60 & 60.0 \\
\hline 1956 & April 10 & 5.65 & 316 & & & & \\
\hline
\end{tabular}




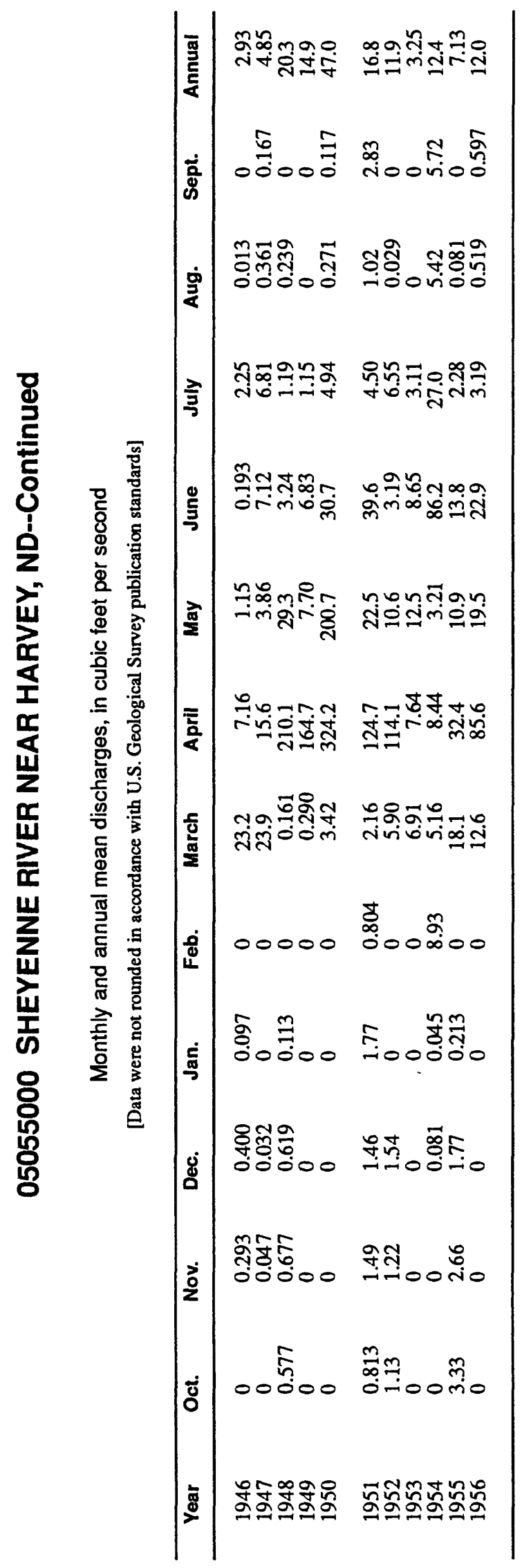




\section{NORTH FORK SHEYENNE RIVER NEAR WELLSBURG, ND}

\section{Station Description}

LOCATION.--Lat 4752'34", long 9943'05”, at north line of sec.26, T.151 N., R.71 W., Benson County, Hydrologic Unit 09020202, on right bank $7 \mathrm{ft}$ downstream from bridge on county highway, $1 \mathrm{mi}$ upstream from mouth, and $3.5 \mathrm{mi}$ northeast of Wellsburg.

DRAINAGE AREA.--693 $\mathrm{mi}^{2}$, of which about $490 \mathrm{mi}^{2}$ is probably noncontributing (includes $227 \mathrm{mi}^{2}$ in closed basins).

PERIOD OF RECORD.--September 1957 to September 1967.

GAGE.--Water-stage recorder. Altitude of gage is $1,455 \mathrm{ft}$ (from topographic map).

EXTREMES FOR PERIOD OF RECORD.--Maximum discharge, about $240 \mathrm{ft}^{3} / \mathrm{s}$, Mar. 16, 1966; maximum gage height, $6.02 \mathrm{ft}$, Mar. 16, 1966 (backwater from ice); no flow for several months each year.

Annual mean discharge

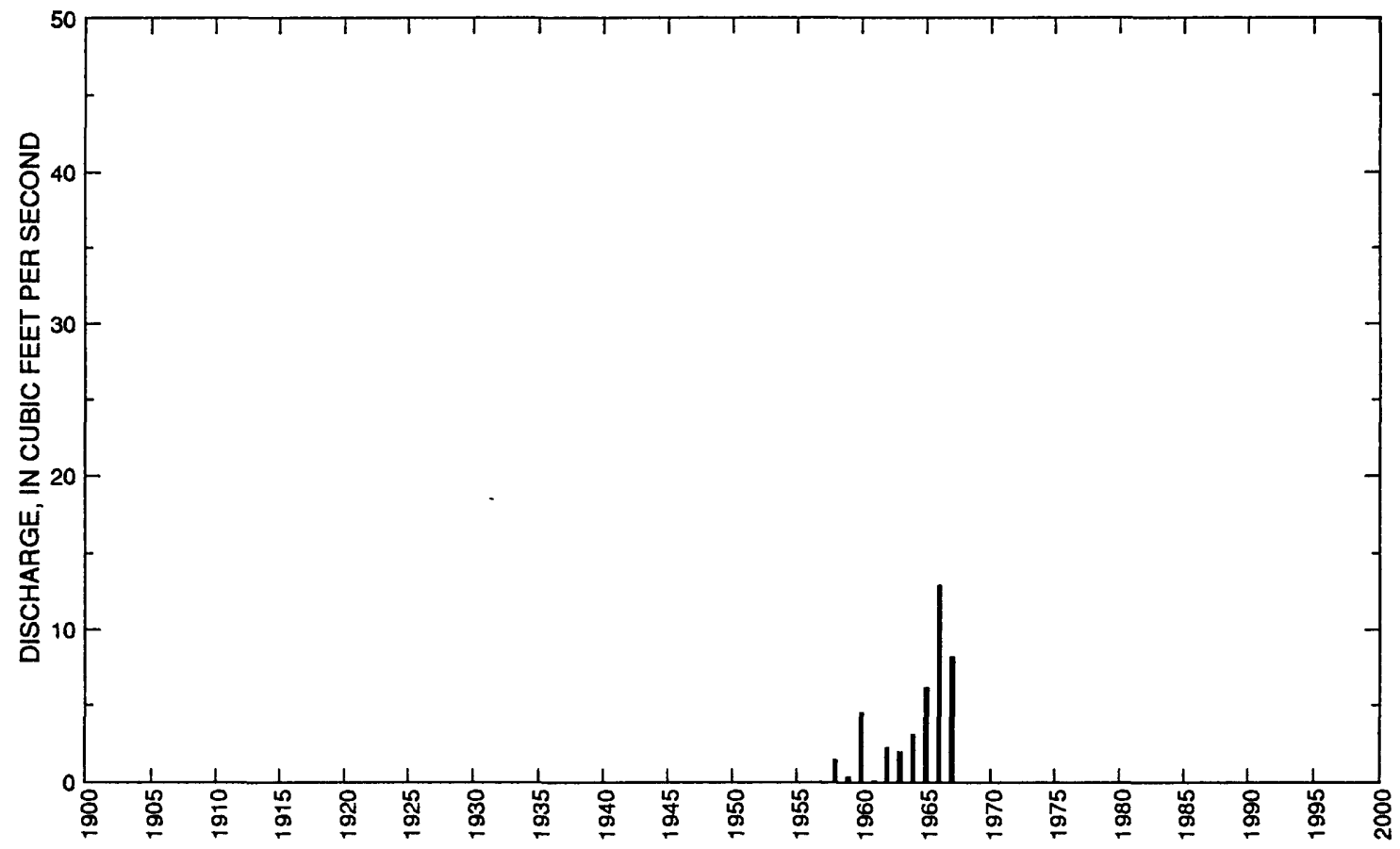


05055100 NORTH FORK SHEYENNE RIVER NEAR WELLSBURG, ND--Continued

Statistics of monthly and annual mean discharges

[m, more than 1 year of occurrence; ng, statistic not given]

\begin{tabular}{|c|c|c|c|c|c|c|c|c|}
\hline \multirow[b]{2}{*}{ Month } & \multicolumn{2}{|c|}{ Maximum } & \multicolumn{2}{|c|}{ Minimum } & \multicolumn{4}{|c|}{ Mean } \\
\hline & $\begin{array}{c}\text { Discharge } \\
\left(\mathrm{ft}^{3} / \mathrm{s}\right)\end{array}$ & $\begin{array}{l}\text { Water year } \\
\text { of } \\
\text { occurrence }\end{array}$ & $\begin{array}{c}\text { Discharge } \\
\left(\mathrm{ft}^{3} / \mathrm{s}\right)\end{array}$ & $\begin{array}{l}\text { Water year } \\
\text { of } \\
\text { occurrence }\end{array}$ & $\begin{array}{c}\text { Discharge } \\
\left(\mathrm{ft}^{3 / s}\right)\end{array}$ & $\begin{array}{c}\text { Standard } \\
\text { deviation } \\
\left(\mathrm{ft}^{3} / \mathbf{s}\right)\end{array}$ & $\begin{array}{l}\text { Coeffi- } \\
\text { cient of } \\
\text { variation }\end{array}$ & $\begin{array}{l}\text { Percentage } \\
\text { of annual } \\
\text { discharge }\end{array}$ \\
\hline October & 11.0 & 1966 & 0 & $\mathrm{~m}$ & 1.17 & 3.46 & 2.96 & 2.39 \\
\hline November & 4.00 & 1966 & 0 & $\mathbf{m}$ & 0.530 & 1.28 & 2.42 & 1.08 \\
\hline December & 0.303 & 1966 & 0 & $\mathbf{m}$ & 0.040 & 0.10 & 2.72 & 0.07 \\
\hline January & ng & ng & 0 & $\mathrm{~m}$ & 0 & 0 & $\mathrm{ng}$ & 0 \\
\hline February & 0.368 & 1958 & 0 & $\mathrm{~m}$ & 0.040 & 0.12 & 3.10 & 0.08 \\
\hline March & 79.5 & 1966 & 0 & 1961 & 12.5 & 25.7 & 2.06 & 25.6 \\
\hline April & 42.5 & 1960 & 0.803 & 1961 & 15.6 & 14.2 & 0.91 & 31.9 \\
\hline May & 25.6 & 1967 & 0.245 & 1959 & 6.96 & 7.70 & 1.11 & 14.2 \\
\hline June & 14.7 & 1964 & 0 & 1961 & 5.05 & 5.09 & 1.01 & 10.3 \\
\hline July & 22.3 & 1965 & 0 & $\mathrm{~m}$ & 4.18 & 6.77 & 1.62 & 8.54 \\
\hline August & 11.9 & 1963 & 0 & $\mathrm{~m}$ & 2.04 & 4.30 & 2.11 & 4.16 \\
\hline September & 8.39 & 1965 & 0 & $\mathrm{~m}$ & 0.850 & 2.65 & 3.13 & 1.73 \\
\hline Annual & 12.9 & 1966 & 0.095 & 1961 & 4.10 & 4.01 & 0.98 & 100 \\
\hline
\end{tabular}

Annual flow duration

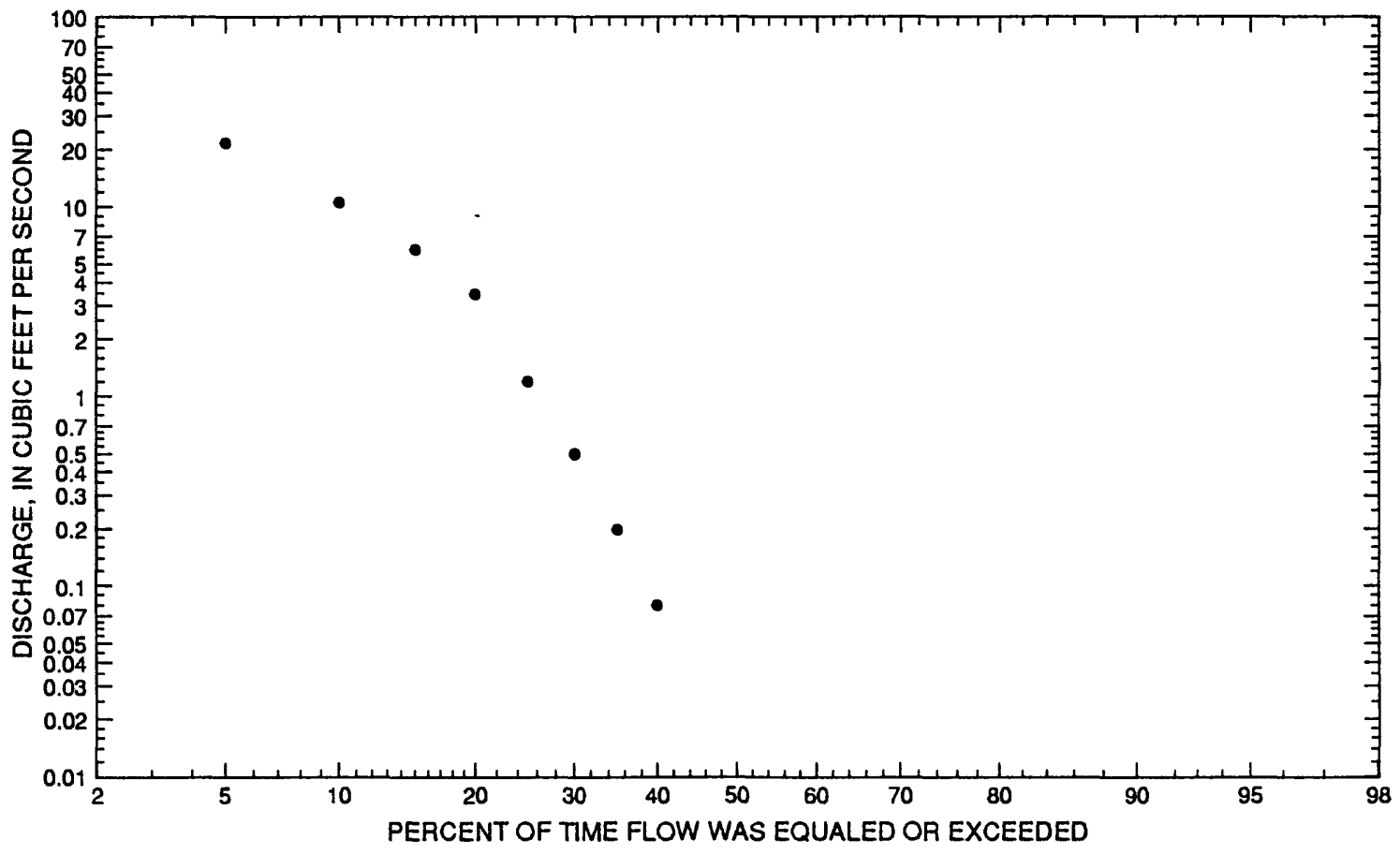




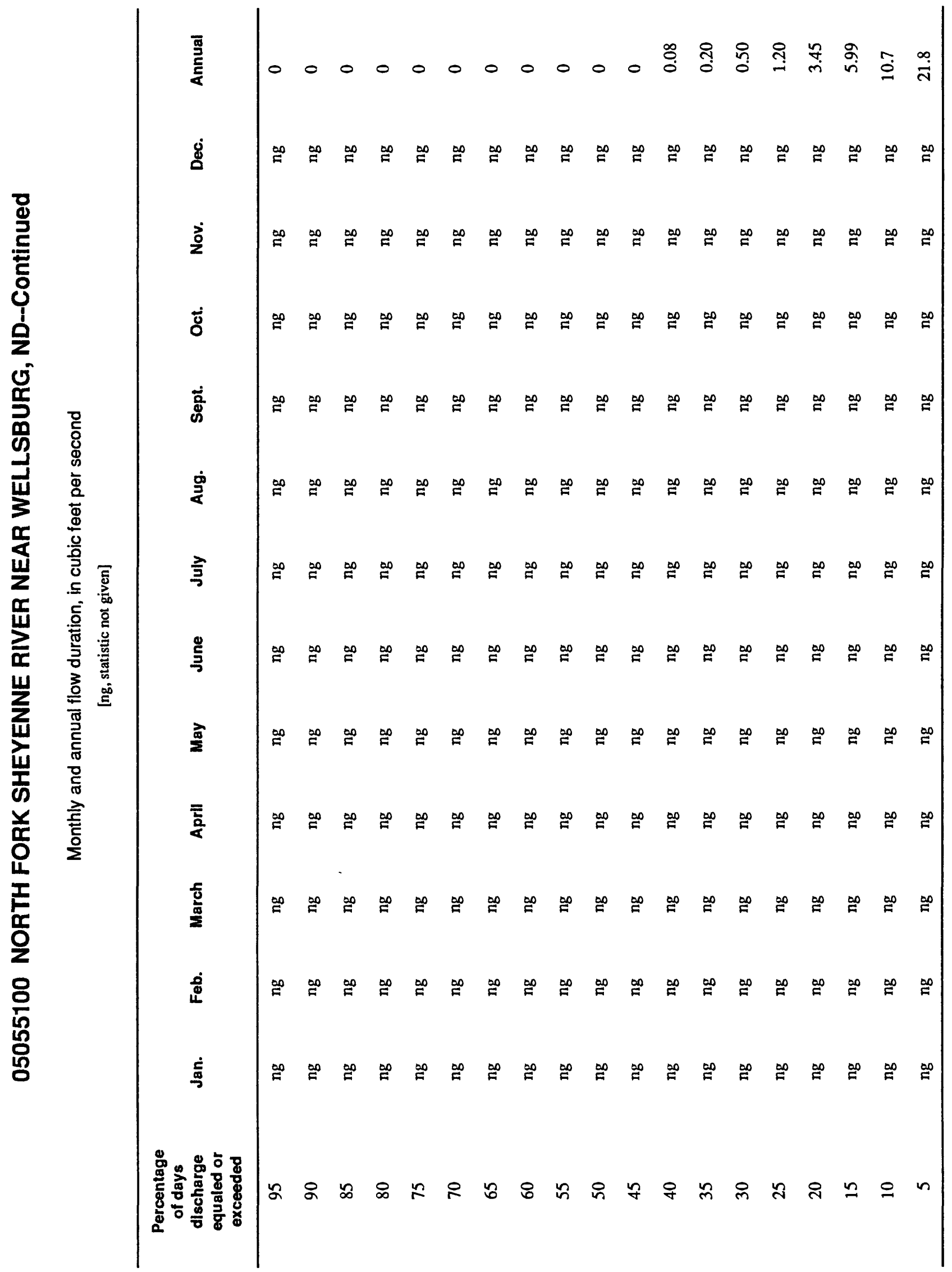


05055100 NORTH FORK SHEYENNE RIVER NEAR WELLSBURG, ND--Continued

Probability of annual high discharges

[ng, statistic not given]

\begin{tabular}{|c|c|c|c|c|c|c|}
\hline \multirow[b]{2}{*}{$\begin{array}{l}\text { Exceedance } \\
\text { probability }\end{array}$} & \multirow[b]{2}{*}{$\begin{array}{l}\text { Recurrence } \\
\text { interval } \\
\text { (years) }\end{array}$} & \multirow[b]{2}{*}{$\begin{array}{c}\text { Maximum } \\
\text { instantaneous } \\
\left(\mathrm{ft}^{3} / \mathrm{s}\right)\end{array}$} & \multicolumn{4}{|c|}{$\begin{array}{l}\text { Maximum average discharge } \\
\qquad\left(\mathrm{ft}^{3} / \mathrm{s}\right)\end{array}$} \\
\hline & & & 3-day period & 7-day period & 15-day period & 30-day period \\
\hline 0.99 & 1.01 & 0.500 & 0.296 & 0.322 & 0.350 & 0.299 \\
\hline 0.95 & 1.05 & 2.20 & 1.87 & 1.86 & 1.75 & 1.29 \\
\hline 0.90 & 1.11 & 4.80 & 4.42 & 4.23 & 3.71 & 2.57 \\
\hline 0.80 & 1.25 & 11.5 & 11.2 & 10.3 & 8.41 & 5.51 \\
\hline 0.50 & 2 & 50.3 & 48.7 & 41.9 & 30.8 & 19.0 \\
\hline 0.20 & 5 & 175 & 145 & 119 & 81.2 & 49.8 \\
\hline 0.10 & 10 & 307 & 223 & 180 & 120 & 74.6 \\
\hline 0.04 & 25 & 527 & 323 & 257 & 168 & 107 \\
\hline 0.02 & 50 & 723 & 393 & 310 & 202 & 131 \\
\hline 0.01 & 100 & 940 & 455 & 358 & 231 & 154 \\
\hline 0.005 & 200 & 1,180 & 511 & 400 & 258 & 175 \\
\hline 0.002 & 500 & 1,510 & $\mathrm{ng}$ & $\mathrm{ng}$ & $\mathrm{ng}$ & $\mathrm{ng}$ \\
\hline
\end{tabular}

Probability of annual low discharges

[ng, statistic not given]

\begin{tabular}{|c|c|c|c|c|c|c|c|c|c|c|}
\hline \multirow[b]{3}{*}{$\begin{array}{c}\text { Non- } \\
\text { exceed- } \\
\text { ance } \\
\text { prob- } \\
\text { ability }\end{array}$} & \multirow[b]{3}{*}{$\begin{array}{l}\text { Recur- } \\
\text { rence } \\
\text { Inter- } \\
\text { val } \\
\text { (years) }\end{array}$} & \multicolumn{9}{|c|}{ MInimum average discharge $\left(\mathrm{ft}^{3} / \mathrm{s}\right)$} \\
\hline & & \multicolumn{9}{|c|}{ Number of consecutive days } \\
\hline & & 1 & 3 & 7 & 14 & 30 & 60 & 90 & 120 & 183 \\
\hline 0.05 & 20 & ng & ng & ng & ng & ng & ng & ng & ng & ng \\
\hline 0.10 & 10 & ng & ng & ng & ng & ng & ng & ng & ng & ng \\
\hline 0.20 & 5 & ng & $\mathrm{ng}$ & ng & ng & ng & ng & ng & ng & ng \\
\hline 0.50 & 2 & ng & ng & ng & $\mathrm{ng}$ & $\mathrm{ng}$ & ng & $\mathrm{ng}$ & ng & ng \\
\hline
\end{tabular}


05055100 NORTH FORK SHEYENNE RIVER NEAR WELLSBURG, ND--Continued

Probability of seasonal low discharges

[ng, statistic not given]

\begin{tabular}{|c|c|c|c|c|c|c|c|c|c|}
\hline \multirow[b]{3}{*}{$\begin{array}{c}\text { Non- } \\
\text { exceedance } \\
\text { probability }\end{array}$} & \multirow[b]{3}{*}{$\begin{array}{c}\text { Recurrence } \\
\text { Interval } \\
\text { (years) }\end{array}$} & \multicolumn{8}{|c|}{ Minimum average discharge $\left(\mathrm{ft}^{3} / \mathrm{s}\right)$} \\
\hline & & \multicolumn{8}{|c|}{ Number of consecutive days } \\
\hline & & 1 & 7 & 14 & 30 & 1 & 7 & 14 & 30 \\
\hline & & \multicolumn{4}{|c|}{ December-January-February } & \multicolumn{4}{|c|}{ March-April-May } \\
\hline 0.05 & 20 & $\mathrm{ng}$ & ng & ng & ng & ng & ng & 0 & 0 \\
\hline 0.10 & 10 & ng & ng & ng & ng & ng & ng & 0 & 0 \\
\hline 0.20 & 5 & ng & ng & ng & $\mathrm{ng}$ & $\mathrm{ng}$ & ng & $\mathbf{0}$ & 0.035 \\
\hline \multirow[t]{2}{*}{0.50} & 2 & ng & $\mathrm{ng}$ & ng & $\mathrm{ng}$ & ng & ng & 0 & 0.300 \\
\hline & & \multicolumn{4}{|c|}{ June-July-August } & \multicolumn{4}{|c|}{ September-October-November } \\
\hline 0.05 & 20 & ng & ng & ng & $\mathbf{0}$ & ng & $\mathrm{ng}$ & ng & ng \\
\hline 0.10 & 10 & ng & $\mathrm{ng}$ & ng & $\mathbf{0}$ & ng & $\mathrm{ng}$ & ng & ng \\
\hline 0.20 & 5 & $\mathrm{ng}$ & ng & $\mathrm{ng}$ & 0 & ng & $\mathrm{ng}$ & $\mathrm{ng}$ & $\mathrm{ng}$ \\
\hline 0.50 & 2 & $\mathrm{ng}$ & ng & $\mathrm{ng}$ & 0 & ng & $\mathrm{ng}$ & ng & $\mathrm{ng}$ \\
\hline
\end{tabular}




\section{NORTH FORK SHEYENNE RIVER NEAR WELLSBURG, ND-Continued}

Annual peak discharge and corresponding gage height, period of record

\begin{tabular}{|c|c|c|c|c|c|c|c|}
\hline $\begin{array}{l}\text { Water } \\
\text { year }\end{array}$ & Date & $\begin{array}{c}\text { Gage } \\
\text { height } \\
\text { (feet) }\end{array}$ & $\begin{array}{c}\text { Peak } \\
\text { discharge } \\
\left(\mathrm{ft}^{3} / \mathrm{s}\right)\end{array}$ & $\begin{array}{c}\text { Water } \\
\text { year }\end{array}$ & Date & $\begin{array}{c}\text { Gage } \\
\text { height } \\
\text { (feet) }\end{array}$ & $\begin{array}{c}\text { Peak } \\
\text { discharge } \\
\left(\mathrm{ft}^{3} / \mathrm{s}\right)\end{array}$ \\
\hline \multicolumn{8}{|c|}{ Annual peak discharge, by year, and corresponding gage height } \\
\hline 1958 & April 2 & 2.94 & 29.0 & 1963 & August 3 & 3.52 & 32.0 \\
\hline 1959 & $\operatorname{March}^{1}$ & 2.10 & 4.70 & 1964 & June 20 & 3.64 & 51.0 \\
\hline 1960 & April 2 & 3.39 & 216 & 1965 & July 23 & 4.78 & 115 \\
\hline 1961 & April 21 & 1.92 & 1.70 & 1966 & March 16 & 6.02 & 240 \\
\hline 1962 & March 29 & 2.75 & 51.0 & 1967 & March 26 & 4.00 & 180 \\
\hline \multicolumn{8}{|c|}{ Annual peak discharge, from highest to lowest, and corresponding gage height } \\
\hline 1966 & March 16 & 6.02 & 240 & 1964 & June 20 & 3.64 & 51.0 \\
\hline 1960 & April 2 & 3.39 & 216 & 1963 & August 3 & 3.52 & 32.0 \\
\hline 1967 & March 26 & 4.00 & 180 & 1958 & April 2 & 2.94 & 29.0 \\
\hline 1965 & July 23 & 4.78 & 115 & 1959 & March $^{1}$ & 2.10 & 4.70 \\
\hline 1962 & March 29 & 2.75 & 51.0 & 1961 & April 21 & 1.92 & 1.70 \\
\hline
\end{tabular}

${ }^{1}$ Day of month unknown. 


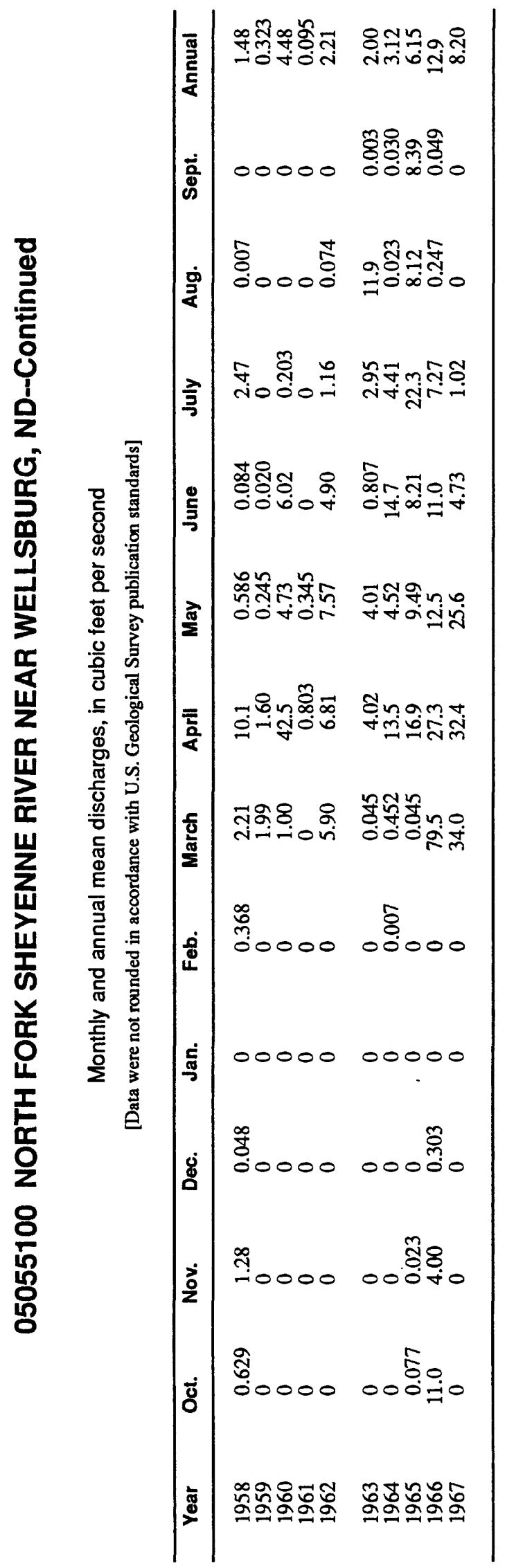




\section{BIG COULEE NEAR MADDOCK, ND}

\section{Station Description}

LOCATION.--Lat $47^{\circ} 55^{\prime} 10^{\prime \prime}$, long $99^{\circ} 34^{\prime} 47^{\prime \prime}$, on north line of sec.11, T.151 N., R.70 W., Benson County, Hydrologic Unit 09020202, at culvert on county highway, $3.5 \mathrm{mi}$ southwest of Maddock.

DRAINAGE AREA.--146 mi², of which about $49 \mathrm{mi}^{2}$ is probably noncontributing.

PERIOD OF RECORD.--October 1956 to September 1967. Annual maximums, water years 1969-73.

GAGE.--Nonrecording gage. Prior to Oct. 20, 1964, water-stage recorder at site $3 \mathrm{mi}$ upstream at different datum. June 22, 1965, to June 17, 1966, water-stage recorder and June 18, 1966, to Sept. 30, 1967, nonrecording gage, at present site and datum.

EXTREMES FOR PERIOD OF RECORD.--Maximum discharge, $810 \mathrm{ft}^{3} / \mathrm{s}$, Apr. 8, 1971; maximum gage height, $12.00 \mathrm{ft}$, Apr. 8, 1971; no flow during part of each year.

Annual mean discharge

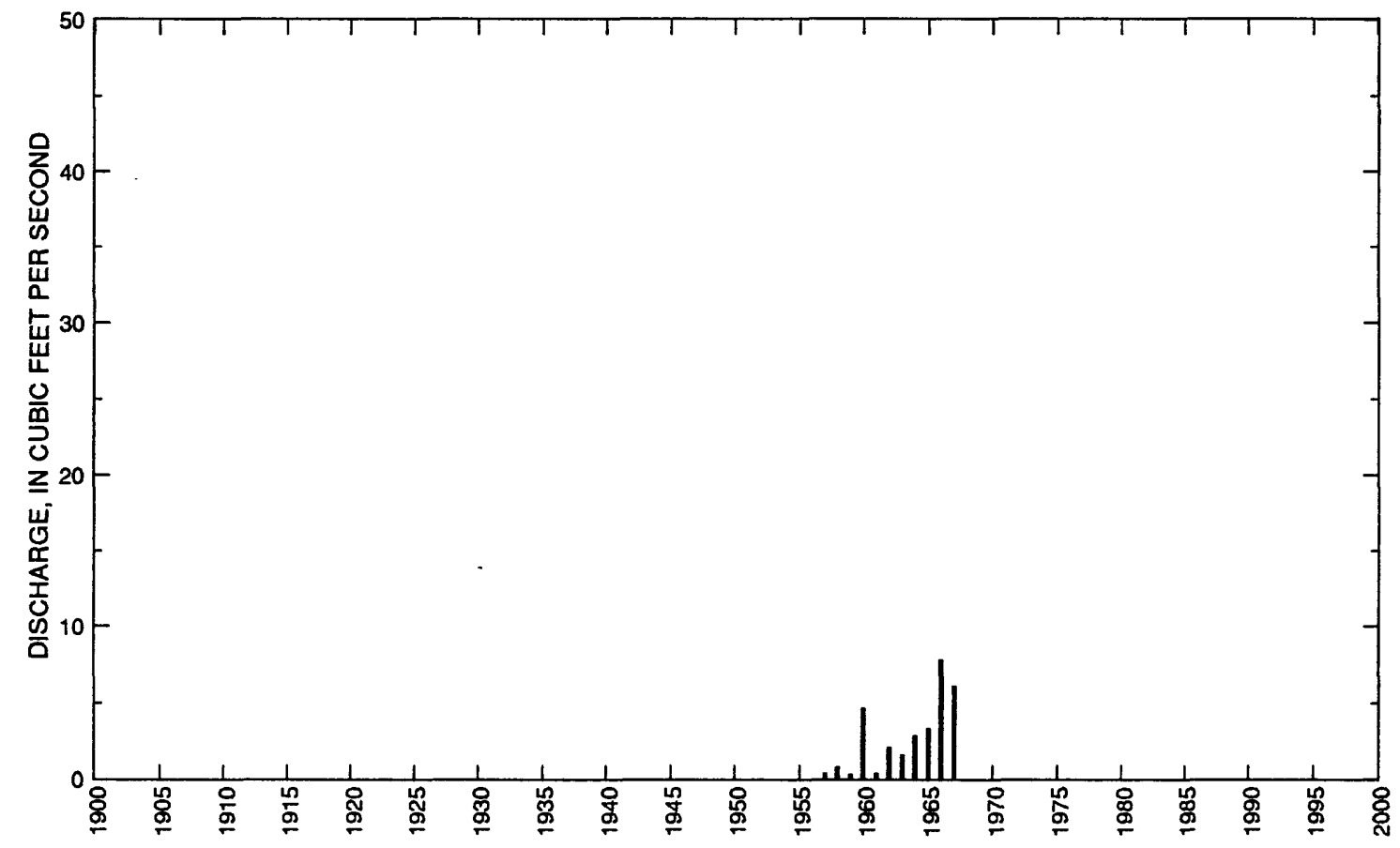




\section{BIG COULEE NEAR MADDOCK, ND-Continued}

Statistics of monthly and annual mean discharges

[m, more than 1 year of occurrence]

\begin{tabular}{|c|c|c|c|c|c|c|c|c|}
\hline \multirow[b]{2}{*}{ Month } & \multicolumn{2}{|c|}{ Maximum } & \multicolumn{2}{|c|}{ Minimum } & \multicolumn{4}{|c|}{ Mean } \\
\hline & $\begin{array}{c}\text { Discharge } \\
\left(\mathrm{ft}^{3} / \mathbf{s}\right)\end{array}$ & $\begin{array}{c}\text { Water year } \\
\text { of } \\
\text { occurrence }\end{array}$ & $\begin{array}{c}\text { Dlscharge } \\
\left(\mathrm{ft}^{3} / \mathrm{s}\right)\end{array}$ & $\begin{array}{c}\text { Water year } \\
\text { of } \\
\text { occurrence }\end{array}$ & $\begin{array}{c}\text { Discharge } \\
\left(\mathrm{ft}^{3} / \mathrm{s}\right)\end{array}$ & $\begin{array}{c}\text { Standard } \\
\text { deviation } \\
\left(\mathrm{ft}^{3} / \mathrm{s}\right)\end{array}$ & $\begin{array}{l}\text { Coeffl- } \\
\text { clent of } \\
\text { varlation }\end{array}$ & $\begin{array}{l}\text { Percentage } \\
\text { of annual } \\
\text { dlscharge }\end{array}$ \\
\hline October & 4.10 & 1966 & 0 & $\mathrm{~m}$ & 0.380 & 1.23 & 3.26 & 1.16 \\
\hline November & 0.914 & 1966 & 0 & $\mathrm{~m}$ & 0.090 & 0.27 & 2.92 & 0.29 \\
\hline December & 0.419 & 1966 & 0 & $\mathrm{~m}$ & 0.040 & 0.13 & 3.32 & 0.12 \\
\hline January & 0.085 & 1966 & 0 & $\mathrm{~m}$ & 0.010 & 0.03 & 3.32 & 0.02 \\
\hline February & 2.29 & 1958 & 0 & $\mathrm{~m}$ & 0.210 & 0.69 & 3.32 & 0.64 \\
\hline March & 69.6 & 1966 & 0.065 & 1964 & 14.7 & 22.2 & 1.52 & 45.1 \\
\hline April & 28.0 & 1960 & 0.010 & 1957 & 7.73 & 9.42 & 1.22 & 23.8 \\
\hline May & 12.3 & 1967 & 0 & 1959 & 2.96 & 3.72 & 1.26 & 9.09 \\
\hline June & 14.1 & 1964 & 0 & $\mathrm{~m}$ & 2.58 & 4.26 & 1.65 & 7.93 \\
\hline July & 12.0 & 1963 & 0 & $\mathrm{~m}$ & 2.67 & 4.03 & 1.51 & 8.22 \\
\hline August & 4.21 & 1965 & 0 & $\mathrm{~m}$ & 0.810 & 1.66 & 2.04 & 2.50 \\
\hline September & 3.65 & 1965 & 0 & $\mathrm{~m}$ & 0.390 & 1.09 & 2.82 & 1.19 \\
\hline Annual & 7.74 & 1966 & 0.331 & 1959 & 2.73 & 2.50 & 0.92 & 100 \\
\hline
\end{tabular}

Annual flow duration

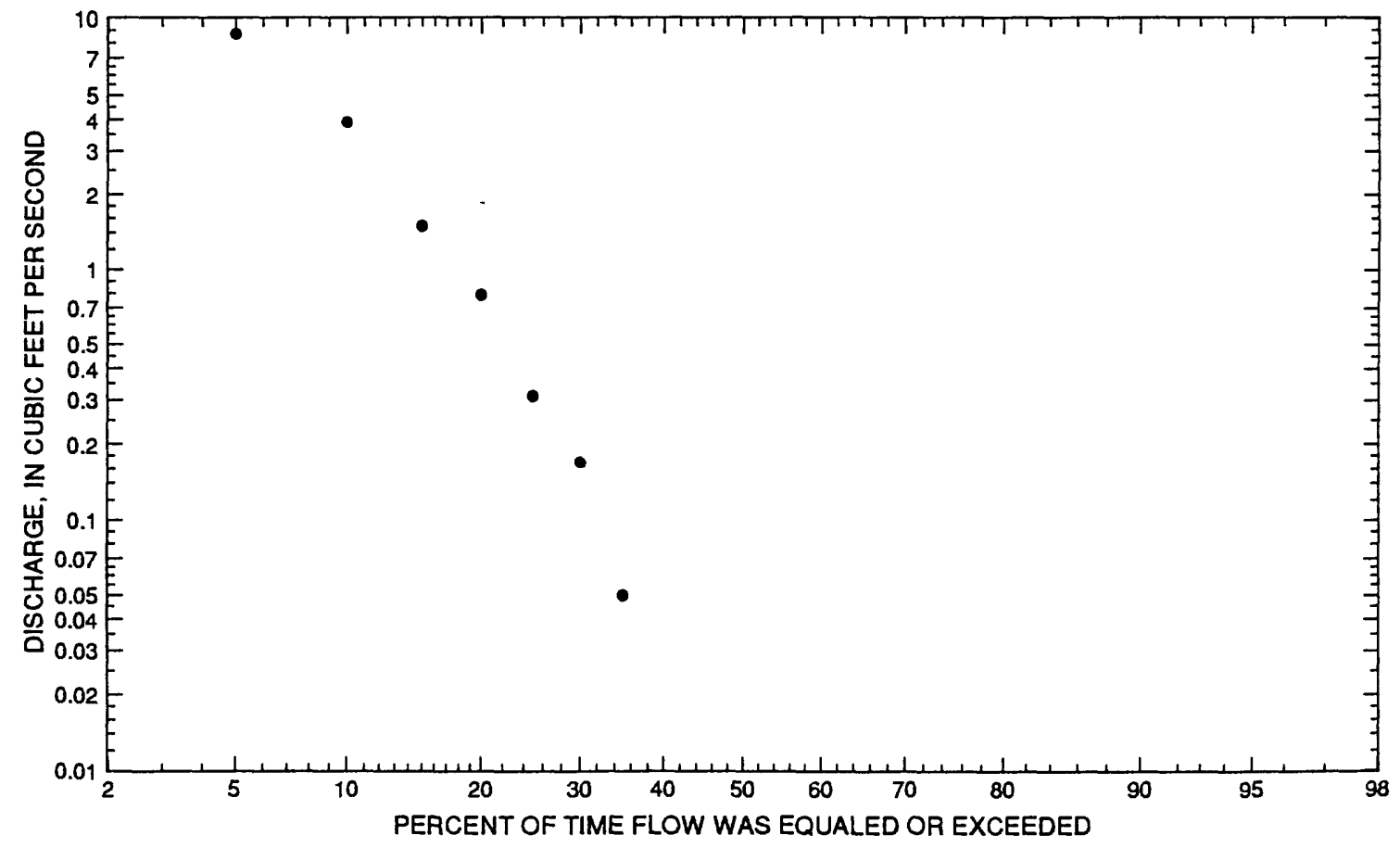




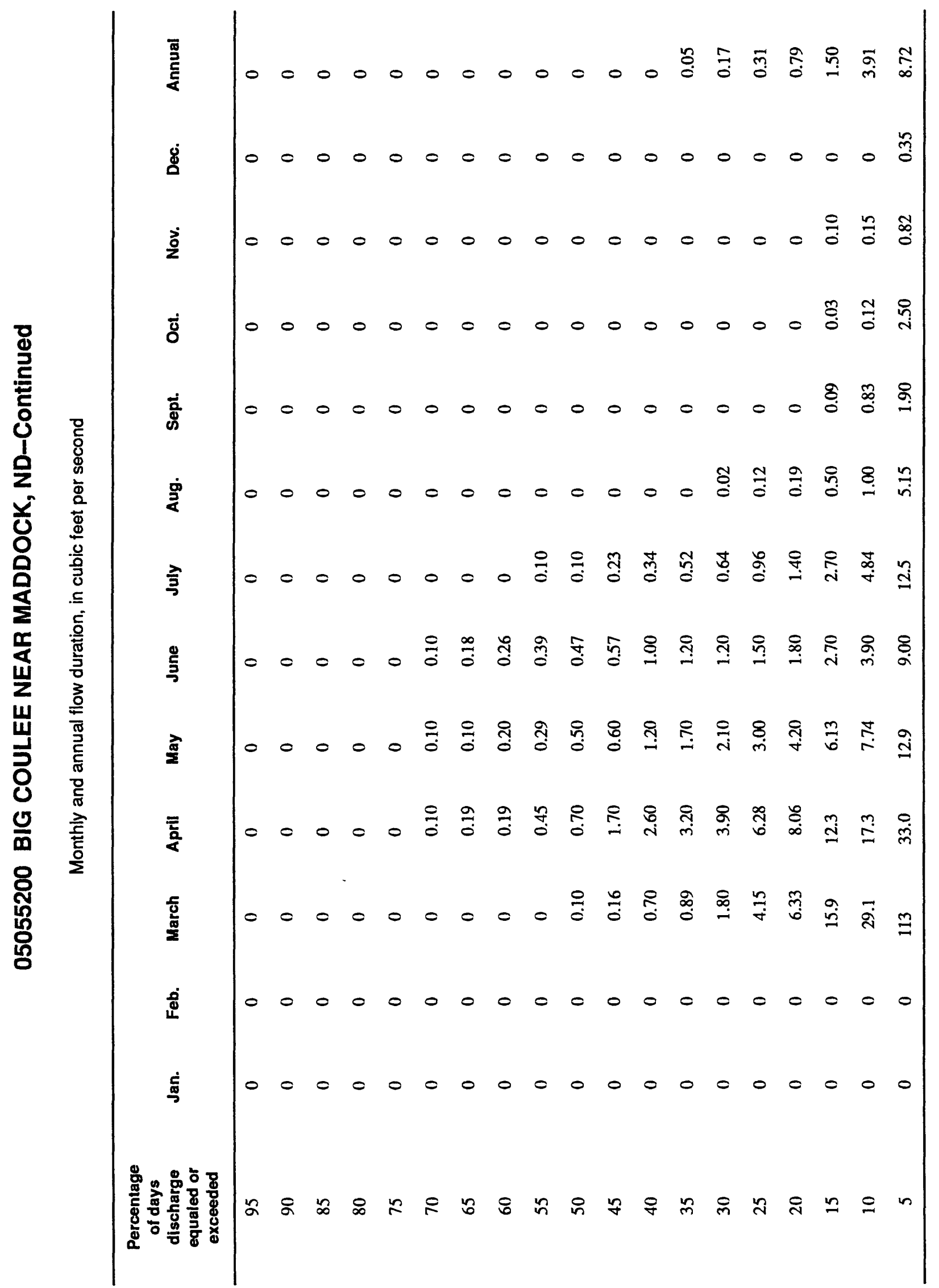




\section{BIG COULEE NEAR MADDOCK, ND-Continued}

Probability of annual high discharges

[ng, statistic not given]

\begin{tabular}{|c|c|c|c|c|c|c|}
\hline \multirow[b]{2}{*}{$\begin{array}{l}\text { Exceedance } \\
\text { probability }\end{array}$} & \multirow[b]{2}{*}{$\begin{array}{l}\text { Recurrence } \\
\text { Interval } \\
\text { (years) }\end{array}$} & \multirow[b]{2}{*}{$\begin{array}{c}\text { Maximum } \\
\text { Instantaneous } \\
\left(\mathrm{ft}^{3} / \mathbf{s}\right)\end{array}$} & \multicolumn{4}{|c|}{$\begin{array}{l}\text { Maximum average discharge } \\
\qquad\left(\mathrm{ft}^{3} / \mathrm{s}\right)\end{array}$} \\
\hline & & & 3-day period & 7-day period & 15-day period & 30-day period \\
\hline 0.99 & 1.01 & 4.80 & 5.03 & 3.07 & 1.70 & 0.862 \\
\hline 0.95 & 1.05 & 15.1 & 11.1 & 6.86 & 3.84 & 1.96 \\
\hline 0.90 & 1.11 & 26.5 & 16.8 & 10.5 & 5.90 & 3.03 \\
\hline 0.80 & 1.25 & 50.5 & 27.4 & 17.3 & 9.89 & 5.10 \\
\hline 0.50 & 2 & 154 & 67.2 & 44.8 & 26.2 & 13.7 \\
\hline 0.20 & 5 & 404 & 158 & 113 & 67.9 & 36.3 \\
\hline 0.10 & 10 & 631 & 242 & 182 & 111 & 60.0 \\
\hline 0.04 & 25 & 976 & 378 & 300 & 186 & 102 \\
\hline 0.02 & 50 & 1,270 & 500 & 413 & 260 & 144 \\
\hline 0.01 & 100 & 1,580 & 640 & 549 & 350 & 195 \\
\hline 0.005 & 200 & 1,910 & 799 & 711 & 458 & 257 \\
\hline 0.002 & 500 & 2,360 & ng & ng & $\mathrm{ng}$ & ng \\
\hline
\end{tabular}

Probability of annual low discharges

[ng, statistic not given]

\begin{tabular}{|c|c|c|c|c|c|c|c|c|c|c|}
\hline \multirow{3}{*}{$\begin{array}{l}\text { Non- } \\
\text { exceed- } \\
\text { ance } \\
\text { prob- } \\
\text { ability }\end{array}$} & \multirow{3}{*}{$\begin{array}{c}\text { Recur- } \\
\text { rence } \\
\text { Inter- } \\
\text { val } \\
\text { (years) }\end{array}$} & \multicolumn{9}{|c|}{ Minimum average discharge $\left(\mathrm{ft}^{3} / \mathrm{s}\right)$} \\
\hline & & \multicolumn{9}{|c|}{ Number of consecutive days } \\
\hline & & 1 & 3 & 7 & 14 & 30 & 60 & 90 & 120 & 183 \\
\hline 0.05 & 20 & ng & ng & ng & ng & ng & ng & ng & ng & 0 \\
\hline 0.10 & 10 & $\mathrm{ng}$ & ng & $\mathrm{ng}$ & $\mathbf{n g}$ & $\mathrm{ng}$ & ng & ng & ng & 0 \\
\hline 0.20 & 5 & ng & ng & ng & ng & ng & ng & ng & ng & 0 \\
\hline 0.50 & 2 & ng & $\mathrm{ng}$ & $\mathrm{ng}$ & ng & $\mathrm{ng}$ & ng & $\mathrm{ng}$ & $\mathrm{ng}$ & 0 \\
\hline
\end{tabular}




\section{BIG COULEE NEAR MADDOCK, ND-Continued}

Probability of seasonal low discharges

[ng, statistic not given]

\begin{tabular}{|c|c|c|c|c|c|c|c|c|c|}
\hline \multirow[b]{3}{*}{$\begin{array}{c}\text { Non- } \\
\text { exceedance } \\
\text { probability }\end{array}$} & \multirow[b]{3}{*}{$\begin{array}{c}\text { Recurrence } \\
\text { Interval } \\
\text { (years) }\end{array}$} & \multicolumn{8}{|c|}{ Minimum average discharge $\left(\mathrm{ft}^{3} / \mathrm{s}\right)$} \\
\hline & & \multicolumn{8}{|c|}{ Number of consecutive days } \\
\hline & & 1 & 7 & 14 & 30 & 1 & 7 & 14 & 30 \\
\hline & & \multicolumn{4}{|c|}{ December-January-February } & \multicolumn{4}{|c|}{ March-April-May } \\
\hline 0.05 & 20 & ng & ng & ng & ng & ng & ng & 0 & 0 \\
\hline 0.10 & 10 & ng & ng & ng & $\mathrm{ng}$ & ng & ng & 0 & 0 \\
\hline 0.20 & 5 & ng & ng & ng & ng & ng & ng & 0 & 0.006 \\
\hline \multirow[t]{2}{*}{0.50} & 2 & ng & ng & $\mathrm{ng}$ & ng & $\mathrm{ng}$ & ng & 0.012 & 0.119 \\
\hline & & \multicolumn{4}{|c|}{ June-July-August } & \multicolumn{4}{|c|}{ September-October-November } \\
\hline 0.05 & 20 & ng & ng & ng & 0 & ng & ng & $\mathrm{ng}$ & ng \\
\hline 0.10 & 10 & ng & $\mathrm{ng}$ & ng & 0 & $\mathrm{ng}$ & ng & $\mathrm{ng}$ & ng \\
\hline 0.20 & 5 & $\mathrm{ng}$ & ng & $\mathrm{ng}$ & 0 & $\mathrm{ng}$ & ng & $\mathrm{ng}$ & ng \\
\hline 0.50 & 2 & $\mathrm{ng}$ & ng & $\mathrm{ng}$ & 0 & ng & ng & $\mathrm{ng}$ & $\mathrm{ng}$ \\
\hline
\end{tabular}




\section{BIG COULEE NEAR MADDOCK, ND-Continued}

Annual peak discharge and corresponding gage height, period of record

\begin{tabular}{|c|c|c|c|c|c|c|c|}
\hline $\begin{array}{l}\text { Water } \\
\text { year }\end{array}$ & Date & $\begin{array}{c}\text { Gage } \\
\text { height } \\
\text { (feet) }\end{array}$ & $\begin{array}{c}\text { Peak } \\
\text { discharge } \\
\left(\mathrm{ft}^{3} / \mathrm{s}\right) \\
\end{array}$ & $\begin{array}{l}\text { Water } \\
\text { year }\end{array}$ & Date & $\begin{array}{c}\text { Gage } \\
\text { height } \\
\text { (feet) }\end{array}$ & $\begin{array}{c}\text { Peak } \\
\text { discharge } \\
\left(\mathrm{ft}^{3} / \mathrm{s}\right)\end{array}$ \\
\hline \multicolumn{8}{|c|}{ Annual peak discharge, by year, and corresponding gage height } \\
\hline 1957 & March 21 & 1.95 & 33.0 & 1965 & April 8 & 5.95 & 99.0 \\
\hline 1958 & February 25 & 2.05 & 21.0 & 1966 & March 14 & 6.54 & 310 \\
\hline 1959 & March 13 & 2.10 & 42.0 & 1967 & March 24 & 7.00 & 440 \\
\hline 1960 & March 27 & 2.50 & 262 & 1969 & April 11 & 7.00 & 750 \\
\hline 1961 & March 15 & 2.15 & 145 & 1970 & April 6 & 10.35 & 200 \\
\hline 1962 & March 26 & 2.27 & 209 & 1971 & April 8 & 12.00 & 810 \\
\hline 1963 & July 27 & 1.94 & 104 & 1972 & March & 9.30 & 292 \\
\hline 1964 & June 18 & 2.45 & 159 & 1973 & May 9 & 6.95 & 10.0 \\
\hline \multicolumn{8}{|c|}{ Annual peak discharge, from highest to lowest, and corresponding gage height } \\
\hline 1971 & April 8 & 12.00 & 810 & 1964 & June 18 & 2.45 & 159 \\
\hline 1969 & April 11 & 7.00 & 750 & 1961 & March 15 & 2.15 & 145 \\
\hline 1967 & March 24 & 7.00 & 440 & 1963 & July 27 & 1.94 & 104 \\
\hline 1966 & March 14 & 6.54 & 310 & 1965 & April 8 & 5.95 & 99.0 \\
\hline 1972 & March & 9.30 & 292 & 1959 & March 13 & 2.10 & 42.0 \\
\hline 1960 & March 27 & 2.50 & 262 & 1957 & March 21 & 1.95 & 33.0 \\
\hline 1962 & $\operatorname{March} 26$ & 2.27 & 209 & 1958 & February 25 & 2.05 & 21.0 \\
\hline 1970 & April 6 & 10.35 & 200 & 1973 & May 9 & 6.95 & 10.0 \\
\hline
\end{tabular}




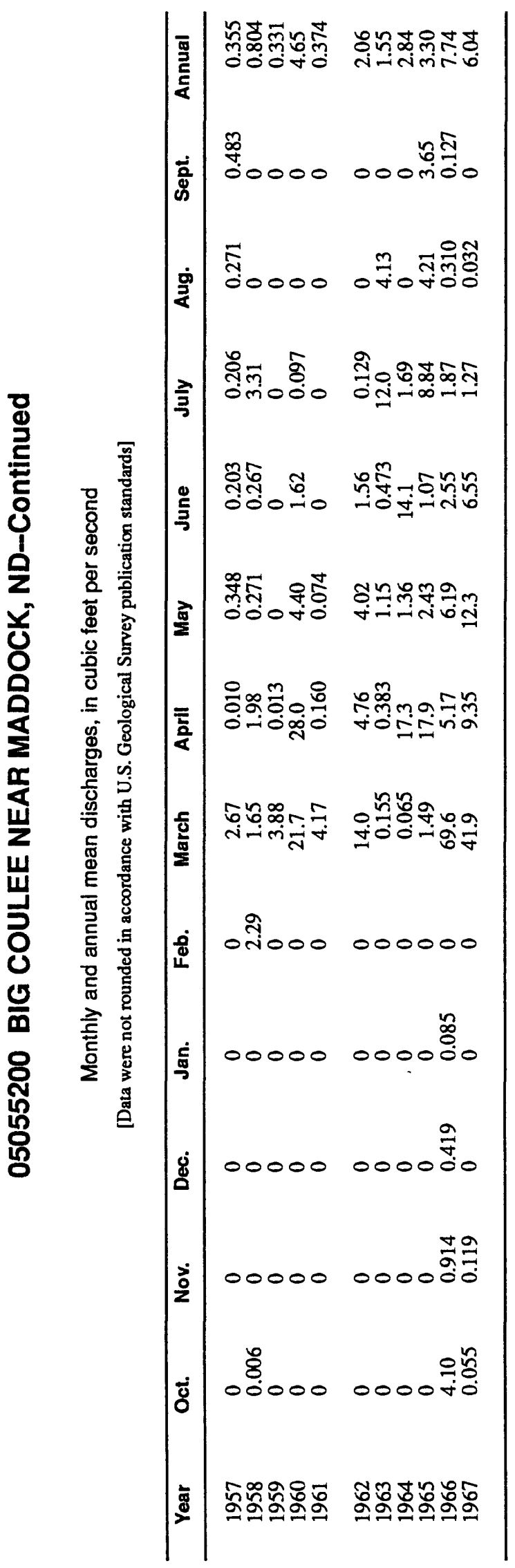




\section{SHEYENNE RIVER AT SHEYENNE, ND}

\section{Station Description}

LOCATION.--Lat 47 $50^{\prime} 20^{\prime \prime}$, long 99 07'30', in NE $1 / 4 \mathrm{SE}^{1} / 4$ sec.5, T.150 N., R.66 W., Wells County, Hydrologic Unit 09020202, at recreation-pool dam, 1 mi north of Sheyenne.

DRAINAGE AREA.--1,790 $\mathrm{mi}^{2}$ (revised), approximately, of which about $1,130 \mathrm{mi}^{2}$ is probably noncontributing (includes $227 \mathrm{mi}^{2}$ in closed basins).

PERIOD OF RECORD.--April 1929 to June 1933, October 1939 to September 1951. Monthly discharge only for some periods, published in Water-Supply Paper 1308.

GAGE.--Staff gage. Datum of gage is $1,412.54 \mathrm{ft}$ above mean sea level, datum of 1929 . Prior to Mar. 28, 1940, wire-weight and staff gages at points within $300 \mathrm{ft}$ of present site, at different datums.

EXTREMES FOR PERIOD OF RECORD.--Maximum discharge, 3,940 $\mathrm{ft}^{3} / \mathrm{s}$, Apr. 18, 1950 (gage height, $8.31 \mathrm{ft}$ ); maximum gage height, 8.51 Apr. 18-19, 1948; no flow at times in each year.

EXTREMES OUTSIDE PERIOD OF RECORD.--Flood in 1919 reached a stage about $3 \mathrm{ft}$ higher than that of April 1948.

Annual mean discharge

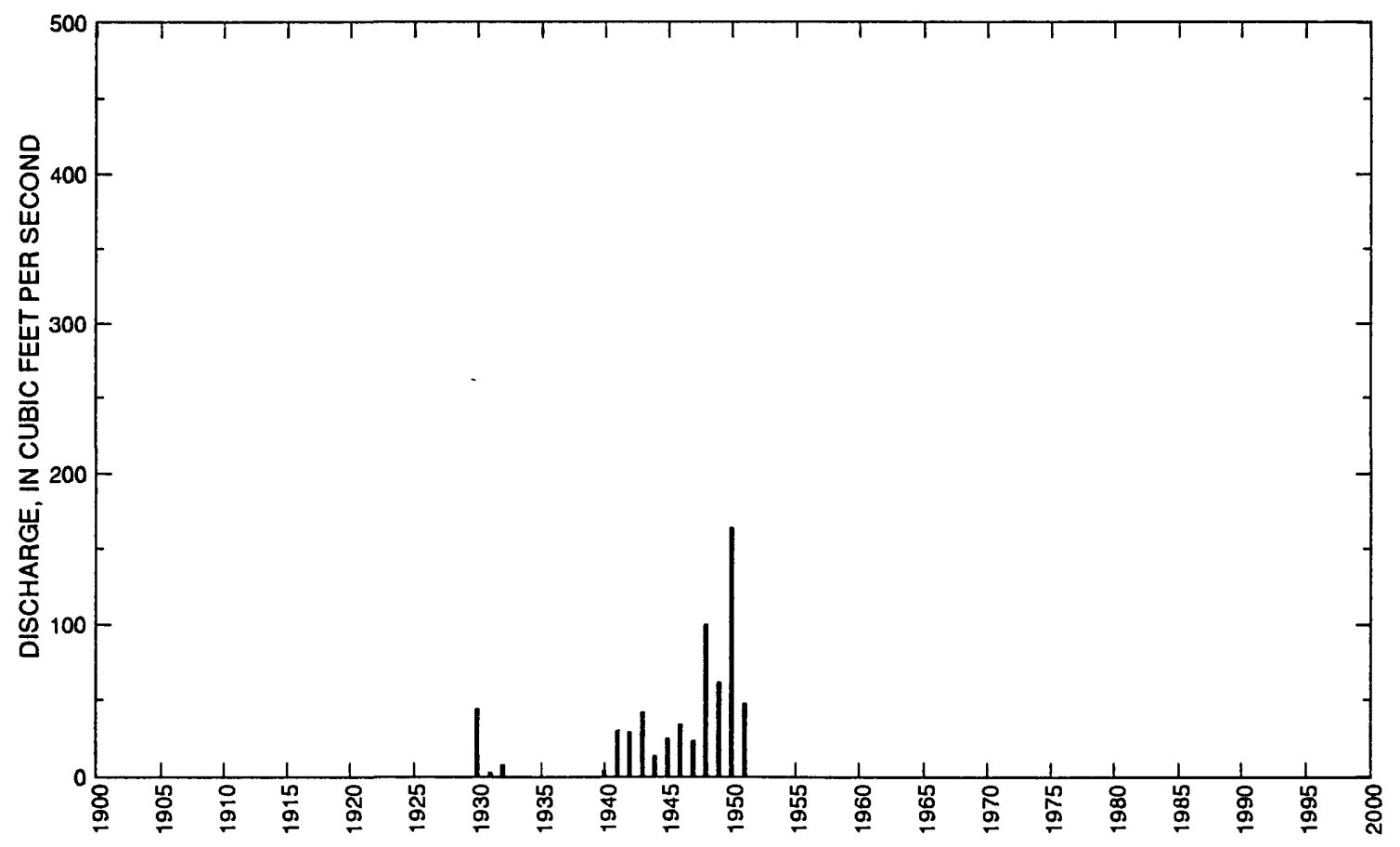




\section{SHEYENNE RIVER AT SHEYENNE, ND-Continued}

Statistics of monthly and annual mean discharges

[m, more than 1 year of occurrence]

\begin{tabular}{|c|c|c|c|c|c|c|c|c|}
\hline \multirow[b]{2}{*}{ Month } & \multicolumn{2}{|c|}{ Maximum } & \multicolumn{2}{|c|}{ Minimum } & \multicolumn{4}{|c|}{ Mean } \\
\hline & $\begin{array}{c}\text { Discharge } \\
\left(\mathrm{ft}^{3} / \mathrm{s}\right)\end{array}$ & $\begin{array}{c}\text { Water year } \\
\text { of } \\
\text { occurrence }\end{array}$ & $\begin{array}{c}\text { Discharge } \\
\left(\left(\mathrm{ft}^{3} / \mathbf{s}\right)\right.\end{array}$ & $\begin{array}{c}\text { Water year } \\
\text { of } \\
\text { occurrence }\end{array}$ & $\begin{array}{c}\text { Discharge } \\
\left(\mathrm{ft}^{3} / \mathrm{s}\right)\end{array}$ & $\begin{array}{c}\text { Standard } \\
\text { deviation } \\
\left(\mathrm{ft}^{3} / \mathrm{s}\right)\end{array}$ & $\begin{array}{l}\text { Coeffi- } \\
\text { cient of } \\
\text { variation }\end{array}$ & $\begin{array}{l}\text { Percentage } \\
\text { of annual } \\
\text { discharge }\end{array}$ \\
\hline October & 6.00 & 1942 & 0 & $\mathrm{~m}$ & 0.730 & 1.60 & 2.21 & 0.15 \\
\hline November & 10.1 & 1945 & 0 & $\mathrm{~m}$ & 1.60 & 2.75 & 1.72 & 0.33 \\
\hline December & 5.24 & 1945 & 0 & $\mathrm{~m}$ & 0.860 & 1.43 & 1.65 & 0.18 \\
\hline January & 1.34 & 1951 & 0 & $\mathrm{~m}$ & 0.210 & 0.37 & 1.76 & 0.04 \\
\hline February & 217 & 1930 & 0 & $\mathrm{~m}$ & 14.9 & 54.1 & 3.64 & 3.09 \\
\hline March & 256 & 1946 & 0 & 1940 & 81.0 & 100 & 1.24 & 16.8 \\
\hline April & 1,280 & 1950 & 8.09 & 1944 & 281 & 393 & 1.39 & 58.5 \\
\hline May & 609 & 1950 & 2.19 & 1931 & 64.3 & 144 & 2.23 & 13.4 \\
\hline June & 60.9 & 1950 & 0.350 & 1931 & 17.6 & 19.6 & 1.11 & 3.65 \\
\hline July & 73.4 & 1946 & 0 & 1940 & 10.7 & 17.7 & 1.66 & 2.22 \\
\hline August & 24.1 & 1942 & 0 & $\mathrm{~m}$ & 3.52 & 6.28 & 1.78 & 0.73 \\
\hline September & 40.1 & 1944 & 0 & $\mathrm{~m}$ & 4.44 & 10.8 & 2.43 & 0.92 \\
\hline Annual & 164 & 1950 & 2.63 & 1931 & 41.7 & 42.1 & 1.01 & 100 \\
\hline
\end{tabular}

Annual flow duration

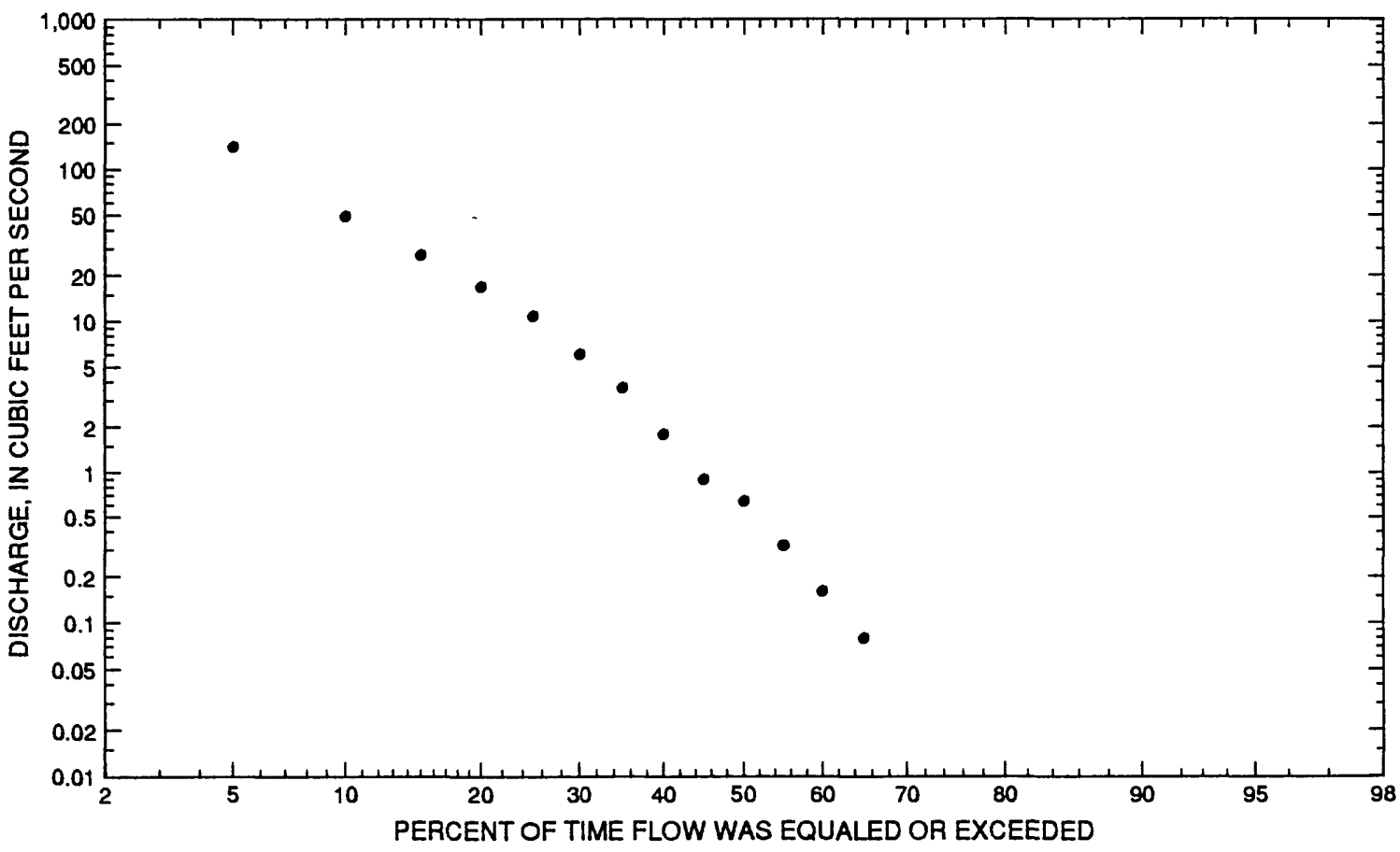




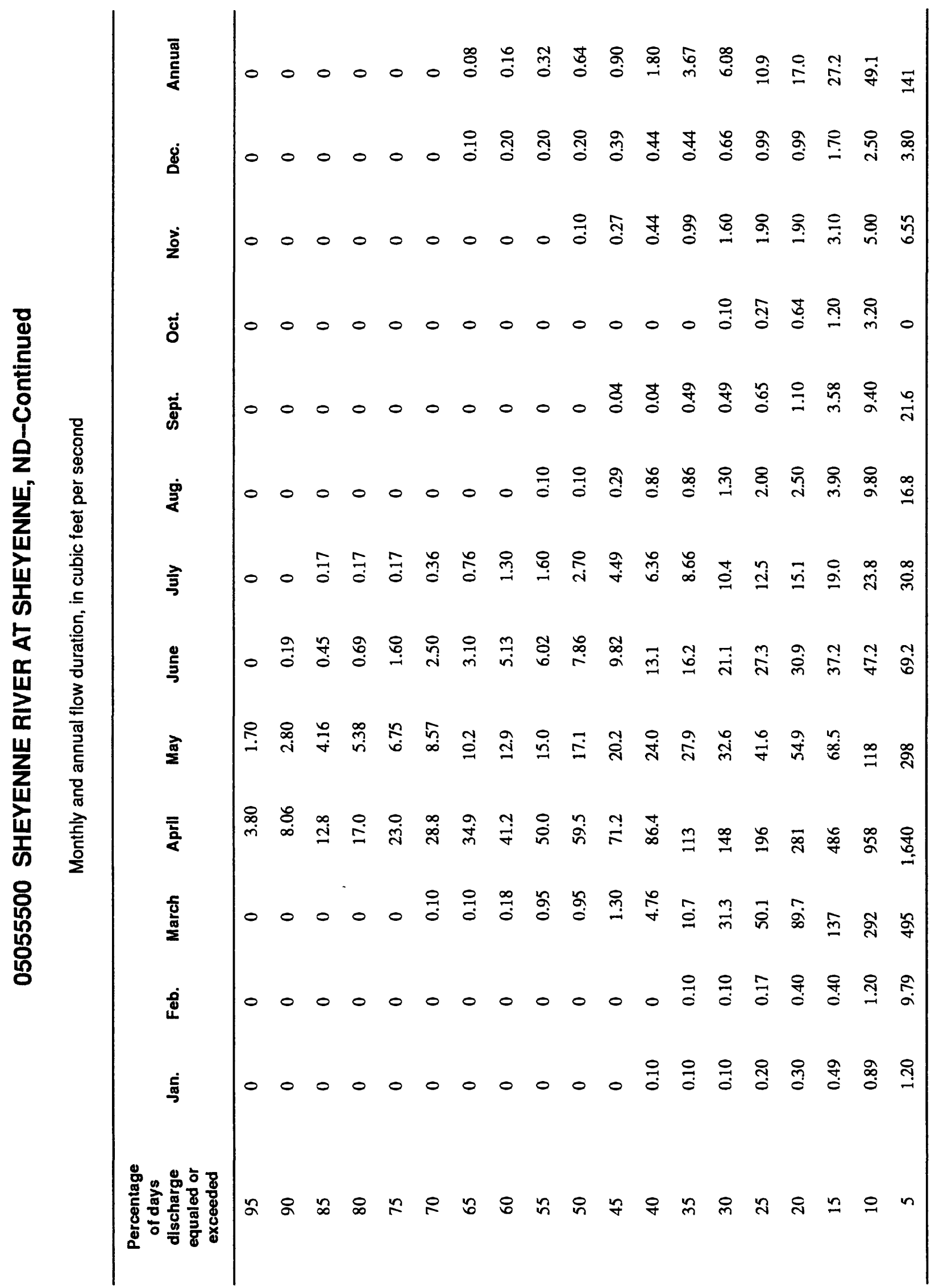




\section{SHEYENNE RIVER AT SHEYENNE, ND-Continued}

Probability of annual high discharges

[ng, statistic not given]

\begin{tabular}{lcccccc}
\hline & & & \multicolumn{5}{c}{$\begin{array}{c}\text { Maximum average discharge } \\
\left(\mathbf{f t}^{3} / \mathbf{s}\right)\end{array}$} \\
\cline { 5 - 7 } $\begin{array}{c}\text { Exceedance } \\
\text { probability }\end{array}$ & $\begin{array}{c}\text { Recurrence } \\
\text { interval } \\
\text { (years) }\end{array}$ & $\begin{array}{c}\text { Maximum } \\
\text { instantaneous } \\
\left(\mathbf{f t}^{\mathbf{3}} / \mathbf{s}\right)\end{array}$ & 3-day period & 7-day period & 15-day period & 30-day period \\
\hline 0.99 & 1.01 & 24.9 & 10.7 & 10.6 & 9.74 & 7.90 \\
0.95 & 1.05 & 83.8 & 48.7 & 43.6 & 34.2 & 24.9 \\
0.90 & 1.11 & 151 & 99.2 & 85.5 & 63.0 & 43.8 \\
0.80 & 1.25 & 292 & 216 & 180 & 125 & 83.2 \\
0.50 & 2 & 888 & 749 & 600 & 400 & 250 \\
0.20 & 5 & 2,230 & 1,930 & 1,540 & 1,050 & 640 \\
0.10 & 10 & 3,350 & 2,850 & 2,300 & 1,630 & 986 \\
0.04 & 25 & 4,930 & 4,010 & 3,290 & 2,450 & 1,500 \\
0.02 & 50 & 6,160 & 4,820 & 4,020 & 3,120 & 1,910 \\
0.01 & 100 & 7,410 & 5,570 & 4,710 & 3,800 & 2,360 \\
0.005 & 200 & 8,650 & 6,240 & 5,360 & 4,490 & 2,810 \\
0.002 & 500 & 10,300 & ng & ng & ng & ng \\
\hline
\end{tabular}

Probability of annual low discharges

[ng, statistic not given]

\begin{tabular}{|c|c|c|c|c|c|c|c|c|c|c|}
\hline \multirow[b]{3}{*}{$\begin{array}{l}\text { Non- } \\
\text { exceed- } \\
\text { ance } \\
\text { prob- } \\
\text { ablilty }\end{array}$} & \multirow[b]{3}{*}{$\begin{array}{l}\text { Recur- } \\
\text { rence } \\
\text { Inter- } \\
\text { val } \\
\text { (years) }\end{array}$} & \multicolumn{9}{|c|}{ Minimum average discharge $\left(\mathrm{ft}^{3} / \mathrm{s}\right)$} \\
\hline & & \multicolumn{9}{|c|}{ Number of consecutive days } \\
\hline & & $t$ & 3 & 7 & 14 & 30 & 60 & 90 & 120 & 183 \\
\hline 0.05 & 20 & ng & ng & ng & 0 & 0 & 0 & 0 & 0 & $\mathbf{0}$ \\
\hline 0.10 & 10 & ng & ng & ng & 0 & 0 & 0 & 0 & 0 & 0 \\
\hline 0.20 & 5 & ng & ng & ng & 0 & 0 & 0 & 0 & 0 & 0 \\
\hline 0.50 & 2 & ng & ng & ng & 0 & 0 & 0 & 0.017 & 0.140 & 0.241 \\
\hline
\end{tabular}




\section{SHEYENNE RIVER AT SHEYENNE, ND-Continued}

Probability of seasonal low discharges

[ng, statistic not given]]

\begin{tabular}{|c|c|c|c|c|c|c|c|c|c|}
\hline \multirow[b]{3}{*}{$\begin{array}{c}\text { Non- } \\
\text { exceedance } \\
\text { probability }\end{array}$} & \multirow[b]{3}{*}{$\begin{array}{c}\text { Recurrence } \\
\text { interval } \\
\text { (years) }\end{array}$} & \multicolumn{8}{|c|}{ Minimum average discharge $\left(\mathrm{ft}^{3} / \mathrm{s}\right)$} \\
\hline & & \multicolumn{8}{|c|}{ Number of consecutive days } \\
\hline & & 1 & 7 & 14 & 30 & 1 & 7 & 14 & 30 \\
\hline & & \multicolumn{4}{|c|}{ December-January-February } & \multicolumn{4}{|c|}{ March-April-May } \\
\hline 0.05 & 20 & $\mathrm{ng}$ & 0 & 0 & 0 & 0 & 0 & 0 & 0 \\
\hline 0.10 & 10 & ng & 0 & 0 & 0 & 0 & 0 & 0 & 0.118 \\
\hline 0.20 & 5 & ng & 0 & 0 & 0 & 0 & 0 & 0 & 0.709 \\
\hline \multirow[t]{2}{*}{0.50} & 2 & $\mathrm{ng}$ & 0 & 0 & 0 & 0.109 & 0.176 & 0.521 & 5.49 \\
\hline & & \multicolumn{4}{|c|}{ June-July-August } & \multicolumn{4}{|c|}{ September-October-November } \\
\hline 0.05 & 20 & $\mathrm{ng}$ & 0 & 0 & 0 & ng & $\mathrm{ng}$ & 0 & 0 \\
\hline 0.10 & 10 & $\mathrm{ng}$ & 0 & 0 & 0 & $\mathrm{ng}$ & $\mathrm{ng}$ & 0 & 0 \\
\hline 0.20 & 5 & $\mathrm{ng}$ & 0 & 0 & 0.014 & $\mathrm{ng}$ & $\mathrm{ng}$ & 0 & 0 \\
\hline 0.50 & 2 & $\mathrm{ng}$ & 0 & 0.076 & 0.560 & ng & $\mathrm{ng}$ & 0 & 0 \\
\hline
\end{tabular}




\section{SHEYENNE RIVER AT SHEYENNE, ND-Continued}

Annual peak discharge and corresponding gage height, period of record

$$
[--, \text { no data] }
$$

\begin{tabular}{|c|c|c|c|c|c|c|c|}
\hline $\begin{array}{l}\text { Water } \\
\text { year }\end{array}$ & Date & $\begin{array}{c}\text { Gage } \\
\text { height } \\
\text { (feet) }\end{array}$ & $\begin{array}{c}\text { Peak } \\
\text { discharge } \\
\left(\mathrm{ft}^{3} / \mathrm{s}\right)\end{array}$ & $\begin{array}{l}\text { Water } \\
\text { year }\end{array}$ & Date & $\begin{array}{c}\text { Gage } \\
\text { height } \\
\text { (feet) }\end{array}$ & $\begin{array}{c}\text { Peak } \\
\text { discharge } \\
\left(\mathrm{ft}^{3} / \mathrm{s}\right)\end{array}$ \\
\hline \multicolumn{8}{|c|}{ Annual peak discharge, by year, and corresponding gage height } \\
\hline 1930 & February 24 & 8.79 & 990 & 1944 & September 1 & 4.94 & 537 \\
\hline 1931 & April 9 & 2.81 & 58.0 & 1945 & March 14 & 5.70 & 932 \\
\hline 1932 & - & -- & -- & 1946 & March 21 & 5.88 & 1,120 \\
\hline 1933 & March 2 & 6.08 & 296 & 1947 & March 25 & 6.22 & 750 \\
\hline 1940 & April 16 & 3.92 & 63.0 & 1948 & April 18 & 8.51 & 3,840 \\
\hline 1941 & April 3 & 5.76 & 847 & 1949 & April 9 & 7.15 & 2,080 \\
\hline 1942 & April 5 & 6.38 & 1,140 & 1950 & April 18 & 8.31 & 3,940 \\
\hline 1943 & March 27 & 7.22 & 1,150 & 1951 & April 9 & 6.25 & 1,420 \\
\hline \multicolumn{8}{|c|}{ Annual peak discharge, from highest to lowest, and corresponding gage height } \\
\hline 1950 & April 18 & 8.31 & 3,940 & 1945 & March 14 & 5.70 & 932 \\
\hline 1948 & April 18 & 8.51 & 3,840 & 1941 & April 3 & 5.76 & 847 \\
\hline 1949 & April 9 & 7.15 & 2,080 & 1947 & March 25 & 6.22 & 750 \\
\hline 1951 & April 9 & 6.25 & 1,420 & 1944 & September 1 & 4.94 & 537 \\
\hline 1943 & March 27 & 7.22 & 1,150 & 1933 & March 2 & 6.08 & 296 \\
\hline 1942 & April 5 & 6.38 & 1,140 & 1940 & April 16 & 3.92 & 63.0 \\
\hline 1946 & March 21 & 5.88 & 1,120 & 1931 & April 9 & 2.81 & 58.0 \\
\hline 1930 & February 24 & 8.79 & 990 & & & & \\
\hline
\end{tabular}




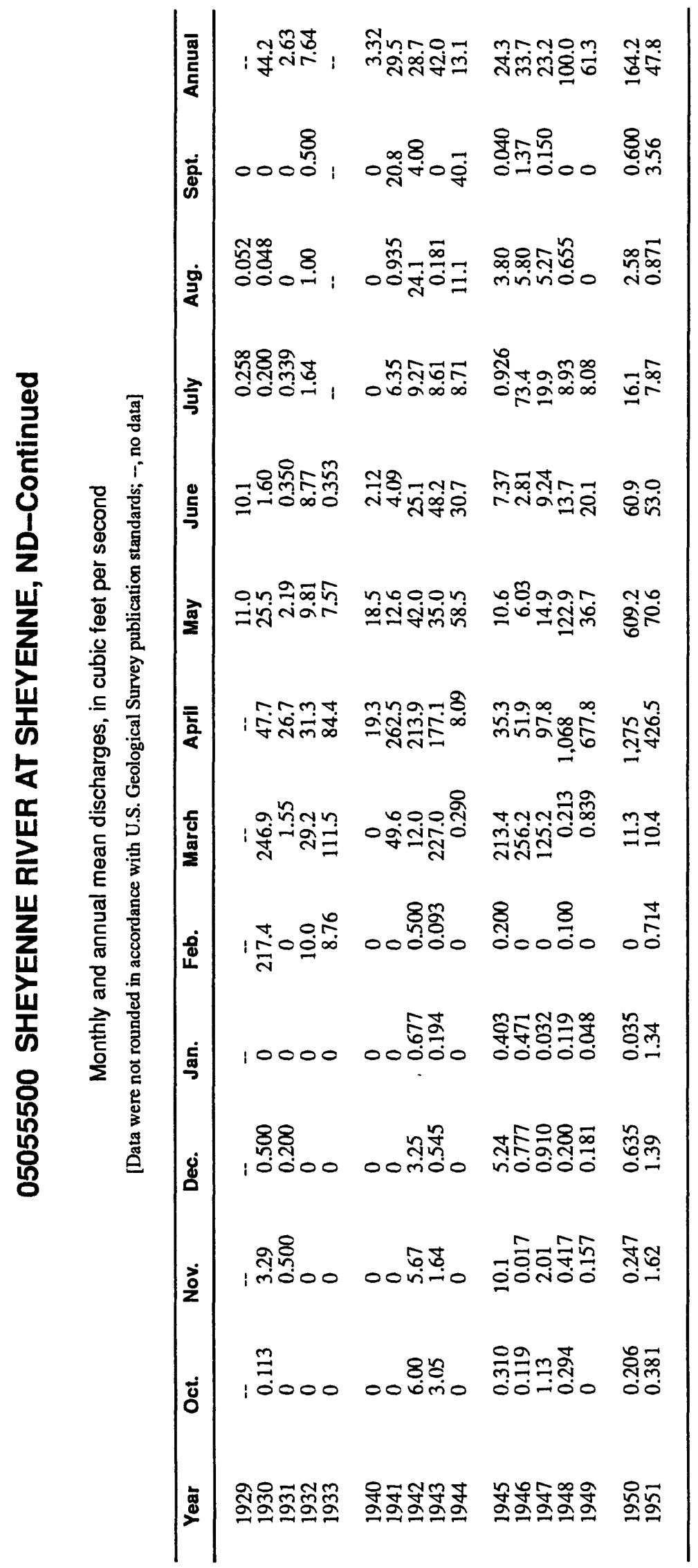




\section{SHEYENNE RIVER NEAR WARWICK, ND}

\section{Station Description}

LOCATION.--Lat $47^{\circ} 48^{\prime} 20^{\prime \prime}$, long $98^{\circ} 42^{\prime} 57^{\prime \prime}$, on south quarter of line between secs. 15 and 16, T.150 N., R.63 W., Eddy County, Hydrologic Unit 09020203, on left bank on downstream side of county highway bridge, $3.3 \mathrm{mi}$ south of Warwick.

DRAINAGE AREA.--2,070 $\mathrm{mi}^{2}$, approximately, of which about $1,310 \mathrm{mi}^{2}$ is probably noncontributing (includes $227 \mathrm{mi}^{2}$ in closed basins).

PERIOD OF RECORD.--October 1949 to current year. Monthly discharge only for some periods, published in Water-Supply Paper 1308.

GAGE.--Water-stage recorder and rubble masonry control. Elevation of gage is $1,370 \mathrm{ft}$ above sea level, by barometer.

EXTREMES FOR PERIOD OF RECORD.--Maximum discharge, 4,660 $\mathrm{ft}^{3} / \mathrm{s}$, Apr. 14, 1969 (gage height, $7.51 \mathrm{ft}$ ); maximum gage height, $7.83 \mathrm{ft}$, Apr. 18, 1956; no flow Aug. 7 to Sept. 1, and Sept. 3-9, 1961.

Annual mean discharge

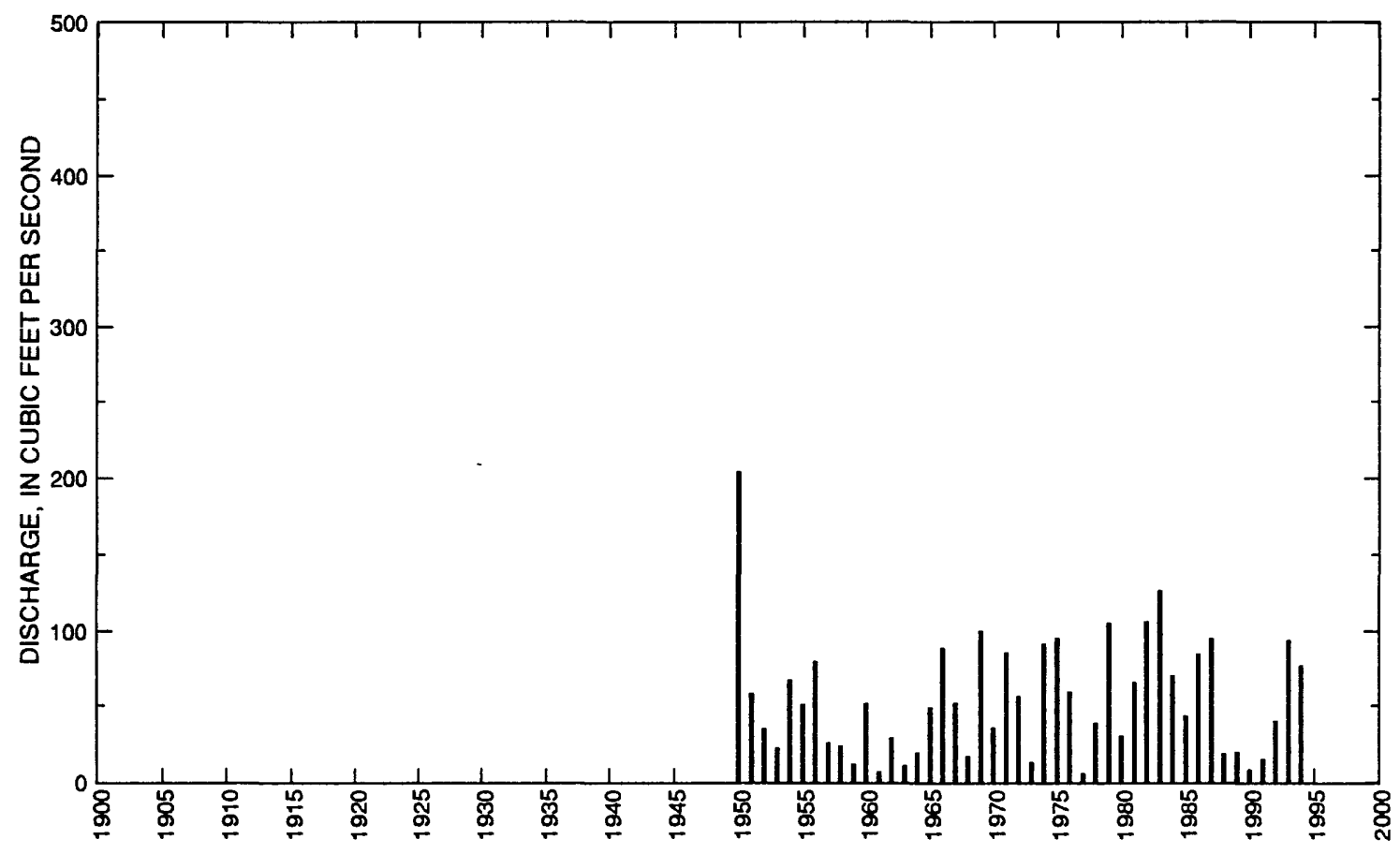


05056000 SHEYENNE RIVER NEAR WARWICK, ND--Continued

Statistics of monthly and annual mean discharges

\begin{tabular}{|c|c|c|c|c|c|c|c|c|}
\hline \multirow[b]{2}{*}{ Month } & \multicolumn{2}{|c|}{ Maximum } & \multicolumn{2}{|c|}{ Minimum } & \multicolumn{4}{|c|}{ Mean } \\
\hline & $\begin{array}{c}\text { Discharge } \\
\left(\mathrm{ft}^{3} / \mathbf{s}\right)\end{array}$ & $\begin{array}{c}\text { Water year } \\
\text { of } \\
\text { occurrence }\end{array}$ & $\begin{array}{c}\text { Discharge } \\
\left(\mathrm{ft}^{3} / \mathrm{s}\right)\end{array}$ & $\begin{array}{c}\text { Water year } \\
\text { of } \\
\text { occurrence }\end{array}$ & $\begin{array}{c}\text { Discharge } \\
\left(\mathrm{ft}^{3} / \mathbf{s}\right)\end{array}$ & $\begin{array}{l}\text { Standard } \\
\text { devlation } \\
\left(\mathrm{ft}^{3} / \mathrm{s}\right)\end{array}$ & $\begin{array}{l}\text { Coeffi- } \\
\text { cient of } \\
\text { variation }\end{array}$ & $\begin{array}{l}\text { Percentage } \\
\text { of annual } \\
\text { discharge }\end{array}$ \\
\hline October & 73.2 & 1983 & 1.16 & 1953 & 12.7 & 15.3 & 1.20 & 1.93 \\
\hline November & 50.3 & 1981 & 1.28 & 1961 & 11.4 & 9.54 & 0.84 & 1.73 \\
\hline December & 29.6 & 1983 & 0.765 & 1961 & 6.98 & 5.69 & 0.82 & 1.06 \\
\hline January & 26.3 & 1983 & 0.474 & 1990 & 4.77 & 4.47 & 0.94 & 0.72 \\
\hline February & 154 & 1981 & 0.754 & 1990 & 9.80 & 24.1 & 2.46 & 1.49 \\
\hline March & 793 & 1983 & 1.46 & 1964 & 118 & 175 & 1.48 & 17.9 \\
\hline April & 1,420 & 1950 & 15.8 & 1977 & 268 & 291 & 1.09 & 40.7 \\
\hline May & 854 & 1950 & 10.4 & 1990 & 98.2 & 148 & 1.51 & 14.9 \\
\hline June & 326 & 1954 & 1.75 & 1961 & 53.4 & 61.5 & 1.15 & 8.10 \\
\hline July & 299 & 1993 & 0.356 & 1989 & 40.7 & 58.7 & 1.44 & 6.17 \\
\hline August & 423 & 1993 & 0.090 & 1961 & 23.8 & 63.8 & 2.68 & 3.61 \\
\hline September & 63.0 & 1957 & 0.707 & 1961 & 11.5 & 14.4 & 1.25 & 1.75 \\
\hline Annual & 204 & 1950 & 5.31 & 1977 & 54.9 & 40.0 & 0.73 & 100 \\
\hline
\end{tabular}

Annual flow duration

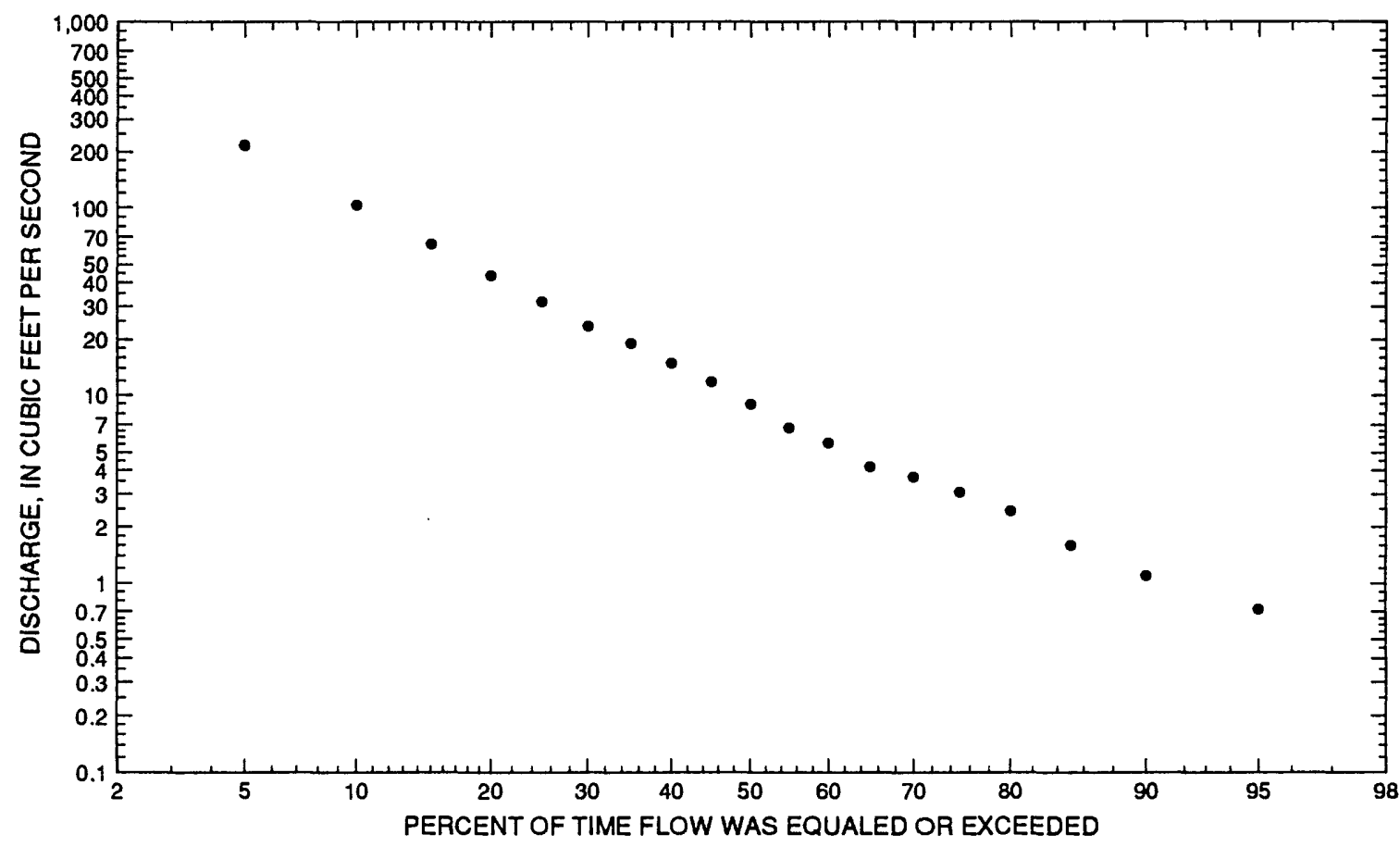




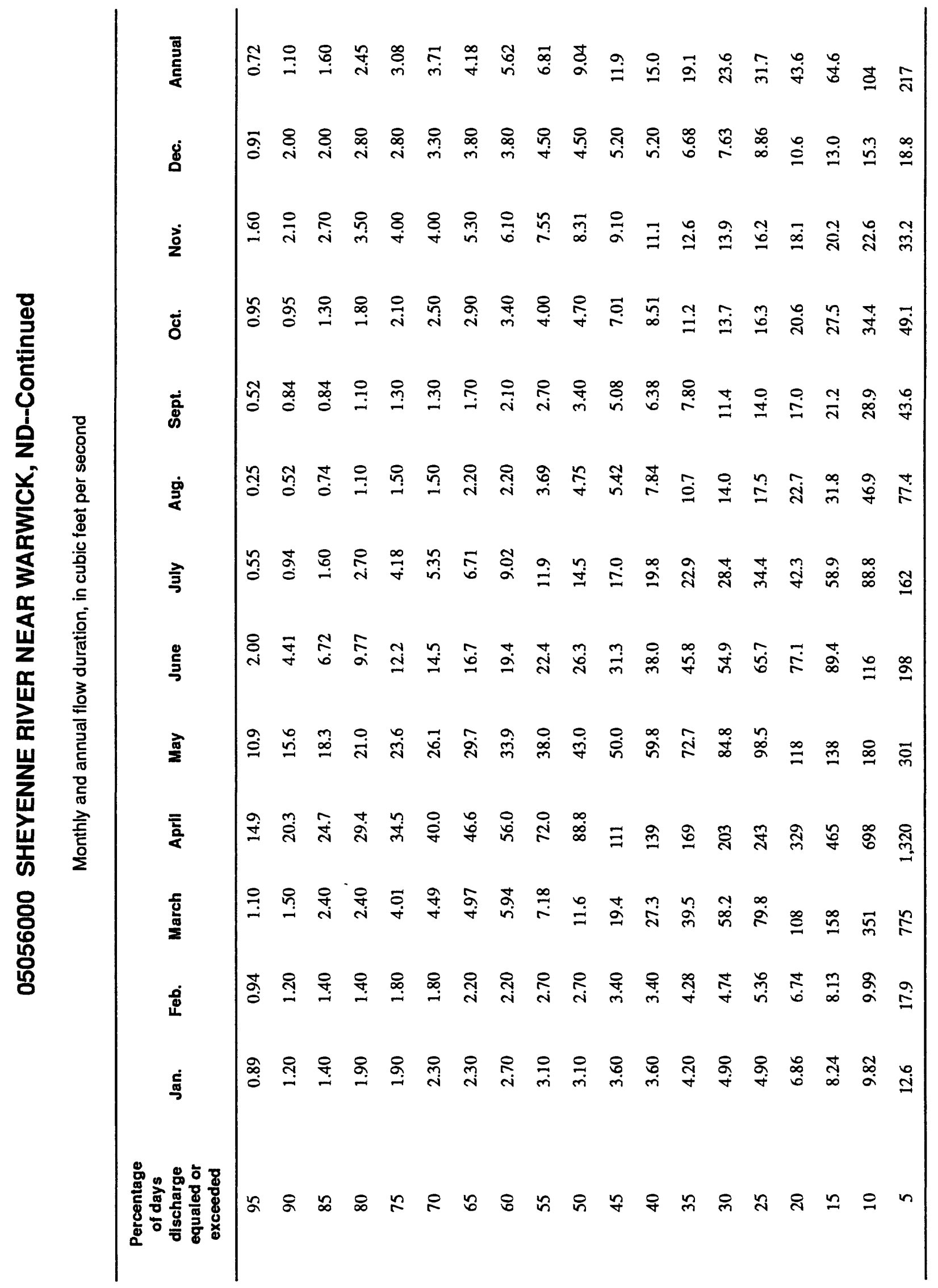




\section{SHEYENNE RIVER NEAR WARWICK, ND--Continued}

Probability of annual high discharges

[ng, statistic not given]

\begin{tabular}{lcccccc}
\hline & & & \multicolumn{5}{c}{$\begin{array}{c}\text { Maximum average discharge } \\
\left(\mathrm{ft}^{3} / \mathbf{s}\right)\end{array}$} \\
\cline { 5 - 7 } $\begin{array}{c}\text { Exceedance } \\
\text { probability }\end{array}$ & $\begin{array}{c}\text { Recurrence } \\
\text { Interval } \\
\text { (years) }\end{array}$ & $\begin{array}{c}\text { Maximum } \\
\text { instantaneous } \\
\left(\mathbf{f t}^{3} / \mathbf{s}\right)\end{array}$ & 3-day period & 7-day period & 15-day period & 30-day perlod \\
\hline 0.99 & 1.01 & 32.8 & 22.8 & 16.8 & 13.1 & 12.1 \\
0.95 & 1.05 & 92.4 & 70.5 & 55.7 & 42.8 & 34.4 \\
0.90 & 1.11 & 154 & 123 & 99.9 & 75.9 & 57.5 \\
0.80 & 1.25 & 278 & 230 & 192 & 144 & 103 \\
0.50 & 2 & 771 & 674 & 580 & 421 & 275 \\
0.20 & 5 & 1,880 & 1,680 & 1,450 & 1,020 & 631 \\
0.10 & 10 & 2,840 & 2,560 & 2,180 & 1,500 & 920 \\
0.04 & 25 & 4,260 & 3,830 & 3,210 & 2,160 & 1,320 \\
0.02 & 50 & 5,440 & 4,860 & 4,020 & 2,660 & 1,630 \\
0.01 & 100 & 6,680 & 5,930 & 4,840 & 3,160 & 1,950 \\
0.005 & 200 & 7,990 & 7,030 & 5,650 & 3,640 & 2,260 \\
0.002 & 500 & 9,800 & $n g$ & $n g$ & $n g$ & ng \\
\hline
\end{tabular}

Probability of annual low discharges

\begin{tabular}{|c|c|c|c|c|c|c|c|c|c|c|}
\hline \multirow[b]{3}{*}{$\begin{array}{l}\text { Non- } \\
\text { exceed- } \\
\text { ance } \\
\text { prob- } \\
\text { ability }\end{array}$} & \multirow[b]{3}{*}{$\begin{array}{l}\text { Recur- } \\
\text { rence } \\
\text { inter- } \\
\text { val } \\
\text { (years) }\end{array}$} & \multicolumn{9}{|c|}{ Minimum average discharge $\left(\mathrm{ft}^{3} / \mathrm{s}\right)$} \\
\hline & & \multicolumn{9}{|c|}{ Number of consecutive days } \\
\hline & & 1 & 3 & 7 & 14 & 30 & 60 & 90 & 120 & 183 \\
\hline 0.05 & 20 & 0.004 & 0.005 & 0.086 & 0.202 & 0.340 & 0.545 & 0.733 & 0.933 & 1.29 \\
\hline 0.10 & 10 & 0.076 & 0.090 & 0.207 & 0.354 & 0.526 & 0.746 & 0.987 & 1.29 & 1.75 \\
\hline 0.20 & 5 & 0.263 & 0.303 & 0.458 & 0.618 & 0.833 & 1.10 & 1.43 & 1.91 & 2.56 \\
\hline 0.50 & 2 & 1.11 & 1.24 & 1.37 & 1.52 & 1.87 & 2.35 & 3.00 & 4.06 & 5.38 \\
\hline
\end{tabular}


05056000 SHEYENNE RIVER NEAR WARWICK, ND--Continued

Probability of seasonal low discharges

\begin{tabular}{|c|c|c|c|c|c|c|c|c|c|}
\hline \multirow[b]{3}{*}{$\begin{array}{c}\text { Non- } \\
\text { exceedance } \\
\text { probability }\end{array}$} & \multirow[b]{3}{*}{$\begin{array}{c}\text { Recurrence } \\
\text { Interval } \\
\text { (years) }\end{array}$} & \multicolumn{8}{|c|}{ Minimum average dlscharge $\left(\mathrm{ft}^{3} / \mathrm{s}\right)$} \\
\hline & & \multicolumn{8}{|c|}{ Number of consecutive days } \\
\hline & & 1 & 7 & 14 & 30 & 1 & 7 & 14 & 30 \\
\hline & & \multicolumn{4}{|c|}{ December-January-February } & \multicolumn{4}{|c|}{ March-April-May } \\
\hline 0.05 & 20 & 0.594 & 0.723 & 0.794 & 0.865 & 0.865 & 0.987 & 1.24 & 2.89 \\
\hline 0.10 & 10 & 0.803 & 0.944 & 1.03 & 1.12 & 1.12 & 1.33 & 1.70 & 4.52 \\
\hline 0.20 & 5 & 1.15 & 1.30 & 1.40 & 1.54 & 1.58 & 1.95 & 2.57 & 7.70 \\
\hline \multirow[t]{2}{*}{0.50} & 2 & 2.23 & 2.40 & 2.56 & 2.87 & 3.38 & 4.42 & 6.15 & 20.5 \\
\hline & & \multicolumn{4}{|c|}{ June-July-August } & \multicolumn{4}{|c|}{ September-October-November } \\
\hline 0.05 & 20 & 0.044 & 0.097 & 0.202 & 0.328 & 0.060 & 0.276 & 0.425 & 0.581 \\
\hline 0.10 & 10 & 0.144 & 0.252 & 0.423 & 0.642 & 0.229 & 0.451 & 0.625 & 0.860 \\
\hline 0.20 & 5 & 0.429 & 0.634 & 0.896 & 1.37 & 0.487 & 0.773 & 1.01 & 1.39 \\
\hline 0.50 & 2 & 2.00 & 2.57 & 3.11 & 4.88 & 1.63 & 2.11 & 2.64 & 3.60 \\
\hline
\end{tabular}


05056000 SHEYENNE RIVER NEAR WARWICK, ND--Continued

Annual peak discharge and corresponding gage height, period of record

\begin{tabular}{|c|c|c|c|c|c|c|c|}
\hline $\begin{array}{l}\text { Water } \\
\text { year }\end{array}$ & Date & $\begin{array}{c}\text { Gage } \\
\text { height } \\
\text { (feet) }\end{array}$ & $\begin{array}{c}\text { Peak } \\
\text { discharge } \\
\left(\mathrm{ft}^{3} / \mathrm{s}\right)\end{array}$ & $\begin{array}{l}\text { Water } \\
\text { year }\end{array}$ & Date & $\begin{array}{c}\text { Gage } \\
\text { height } \\
\text { (feet) }\end{array}$ & $\begin{array}{c}\text { Peak } \\
\text { discharge } \\
\left(\mathbf{f t}^{3} / \mathbf{s}\right)\end{array}$ \\
\hline \multicolumn{8}{|c|}{ Annual peak discharge, by year, and corresponding gage height } \\
\hline 1950 & April 17 & 7.45 & 3,800 & 1973 & March 13 & 3.10 & 170 \\
\hline 1951 & April 11 & 5.01 & 1,240 & 1974 & May 24 & 6.14 & 2,030 \\
\hline 1952 & April 8 & 4.17 & 737 & 1975 & April 21 & 6.16 & 1,800 \\
\hline 1953 & July 3 & 3.08 & 204 & 1976 & March 31 & 4.70 & 1,090 \\
\hline 1954 & June 19 & 4.38 & 878 & 1977 & May 5 & 2.64 & 66.0 \\
\hline 1955 & April 3 & 5.04 & 1,330 & 1978 & April 5 & 6.10 & 1,440 \\
\hline 1956 & April 18 & 7.83 & 4,250 & 1979 & April 25 & 7.34 & 2,600 \\
\hline 1957 & September 5 & 3.17 & 249 & 1980 & April 3 & 3.81 & 560 \\
\hline 1958 & March 3 & 4.04 & 666 & 1981 & February 24 & 4.01 & 594 \\
\hline 1959 & March 21 & 2.83 & 153 & 1982 & April 4 & 5.38 & 1,420 \\
\hline 1960 & April 7 & 5.57 & 1,450 & 1983 & March 12 & 5.51 & 1,480 \\
\hline 1961 & March 26 & 2.55 & 81.0 & 1984 & March 28 & 4.78 & 1,030 \\
\hline 1962 & April 1 & 3.50 & 436 & 1985 & March 17 & 3.76 & 579 \\
\hline 1963 & June 25 & 2.68 & 116 & 1986 & March 19 & 5.16 & 1,000 \\
\hline 1964 & April 9 & 3.13 & 247 & 1987 & April 5 & 6.00 & 1,730 \\
\hline 1965 & April 12 & 4.64 & 1,000 & 1988 & March 25 & 2.88 & 151 \\
\hline 1966 & March 19 & 7.63 & 3,100 & 1989 & April 7 & 4.02 & 710 \\
\hline 1967 & March 31 & 5.24 & 1,340 & 1990 & June 5 & 2.52 & 66.0 \\
\hline 1968 & March 12 & 3.10 & 211 & 1991 & July 2 & 2.71 & 119 \\
\hline 1969 & April 14 & 7.51 & 4,660 & 1992 & March 7 & 4.53 & 960 \\
\hline 1970 & April 10 & 3.52 & 445 & 1993 & August 1 & 6.00 & 1,470 \\
\hline 1971 & April 12 & 6.55 & 2,360 & 1994 & March 27 & 5.21 & 1,170 \\
\hline 1972 & March 21 & 5.08 & 1,360 & & & & \\
\hline \multicolumn{8}{|c|}{ Annual peak discharge, from highest to lowest, and corresponding gage height } \\
\hline 1969 & April 14 & 7.51 & 4,660 & 1965 & April 12 & 4.64 & 1,000 \\
\hline 1956 & April 18 & 7.83 & 4,250 & 1986 & March 19 & 5.16 & 1,000 \\
\hline 1950 & April 17 & 7.45 & 3,800 & 1992 & March 7 & 4.53 & 960 \\
\hline 1966 & March 19 & 7.63 & 3,100 & 1954 & June 19 & 4.38 & 878 \\
\hline 1979 & April 25 & 7.34 & 2,600 & 1952 & April 8 & 4.17 & 737 \\
\hline 1971 & April 12 & 6.55 & 2,360 & 1989 & April 7 & 4.02 & 710 \\
\hline 1974 & May 24 & 6.14 & 2,030 & 1958 & March 3 & 4.04 & 666 \\
\hline 1975 & April 21 & 6.16 & 1,800 & 1981 & February 24 & 4.01 & 594 \\
\hline 1987 & April 5 & 6.00 & 1,730 & 1985 & March 17 & 3.76 & 579 \\
\hline 1983 & March 12 & 5.51 & 1,480 & 1980 & April 3 & 3.81 & 560 \\
\hline 1993 & August 1 & 6.00 & 1,470 & 1970 & April 10 & 3.52 & 445 \\
\hline 1960 & April 7 & 5.57 & 1,450 & 1962 & April 1 & 3.50 & 436 \\
\hline 1978 & April 5 & 6.10 & 1,440 & 1957 & September 5 & 3.17 & 249 \\
\hline 1982 & April 4 & 5.38 & 1,420 & 1964 & April 9 & 3.13 & 247 \\
\hline 1972 & March 21 & 5.08 & 1,360 & 1968 & March 12 & 3.10 & 211 \\
\hline 1967 & March 31 & 5.24 & 1,340 & 1953 & July 3 & 3.08 & 204 \\
\hline 1955 & April 3 & 5.04 & 1,330 & 1973 & March 13 & 3.10 & 170 \\
\hline 1951 & April 11 & 5.01 & 1,240 & 1959 & March 21 & 2.83 & 153 \\
\hline 1994 & March 27 & 5.21 & 1,170 & 1988 & March 25 & 2.88 & 151 \\
\hline 1976 & March 31 & 4.70 & 1,090 & 1991 & July 2 & 2.71 & 119 \\
\hline 1984 & March 28 & 4.78 & 1,030 & 1963 & June 25 & 2.68 & 116 \\
\hline
\end{tabular}




\section{SHEYENNE RIVER NEAR WARWICK, ND--Continued}

Annual peak discharge and corresponding gage height, period of record

\begin{tabular}{|c|c|c|c|c|c|c|c|}
\hline $\begin{array}{l}\text { Water } \\
\text { year }\end{array}$ & Date & $\begin{array}{c}\text { Gage } \\
\text { height } \\
\text { (feet) }\end{array}$ & $\begin{array}{c}\text { Peak } \\
\text { discharge } \\
\left(\mathrm{ft}^{3} / \mathrm{s}\right)\end{array}$ & $\begin{array}{l}\text { Water } \\
\text { year }\end{array}$ & Date & $\begin{array}{c}\text { Gage } \\
\text { height } \\
\text { (feet) }\end{array}$ & $\begin{array}{c}\text { Peak } \\
\text { discharge } \\
\left(\mathrm{ft}^{3} / \mathbf{s}\right)\end{array}$ \\
\hline \multicolumn{8}{|c|}{ Annual peak discharge, from highest to lowest, and corresponding gage height--Continued } \\
\hline 1961 & March 26 & 2.55 & 81.0 & 1990 & June 5 & 2.52 & 66.0 \\
\hline 1977 & May 5 & 2.64 & 66.0 & & & & \\
\hline
\end{tabular}




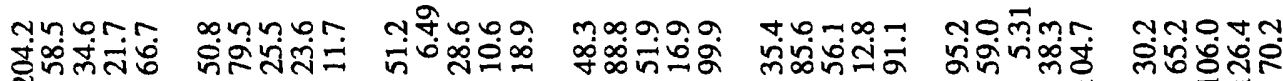
क्ष

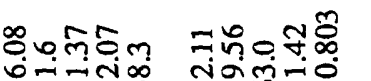
tㅎํ유.

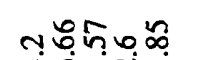

ฉทรีทั่

क no no

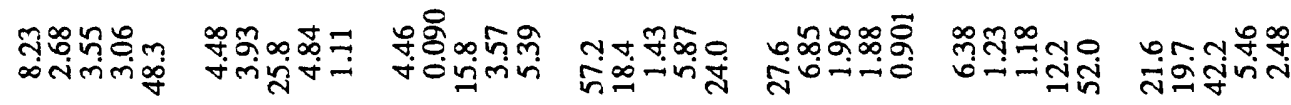

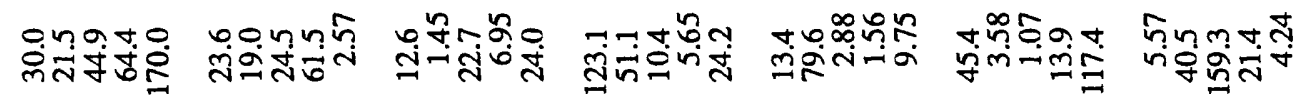

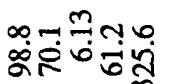

ก.า.

$\operatorname{trntan}$

ำก-7.

คํำ

ON-NM

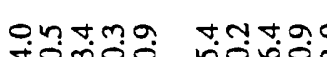
लि-क्ष

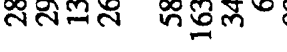

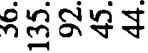

- n̊mpin

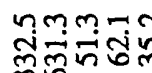

$\forall r+\Delta \infty$

ก⿻

$007=0$

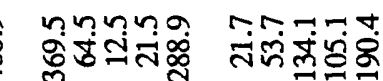

궉유

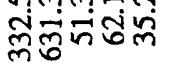

rnto

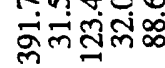

onna.

ำ่ำสำ

कृषษ⿻

궁ำกำ

$000 \mathrm{Nn}$

다융유

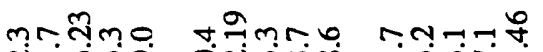

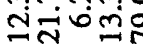

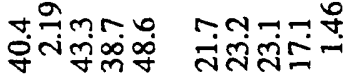

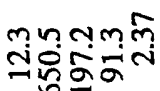

öagm

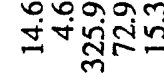

ตntu?

mmoor

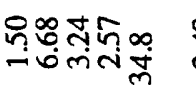

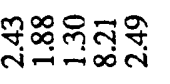

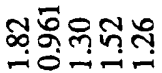

ชุำำโ

능요요

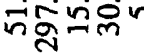

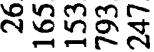

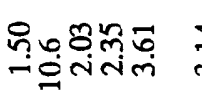

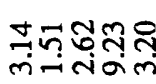

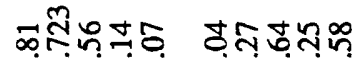

mतलिiत

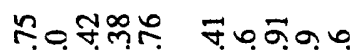
जिंทีं

\section{ชที่ง $\forall \infty$ i่m}

윰ำ

ำำำ

รำตฺ

ำริำษ

ษัำกั

लांगतंत

ลักุกตรด

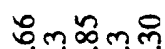
aำ 70तन मीन्तल सेंकिष

ํํํำ สุำ

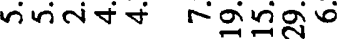

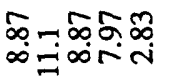

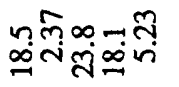

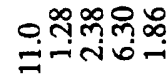

ทัดูึัฒ

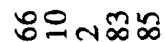

कก -

r.mos

go유눈

ทํำ- =กำำ

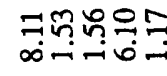

mํํำำ

ढळ

लिंतुलिio

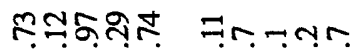

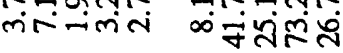




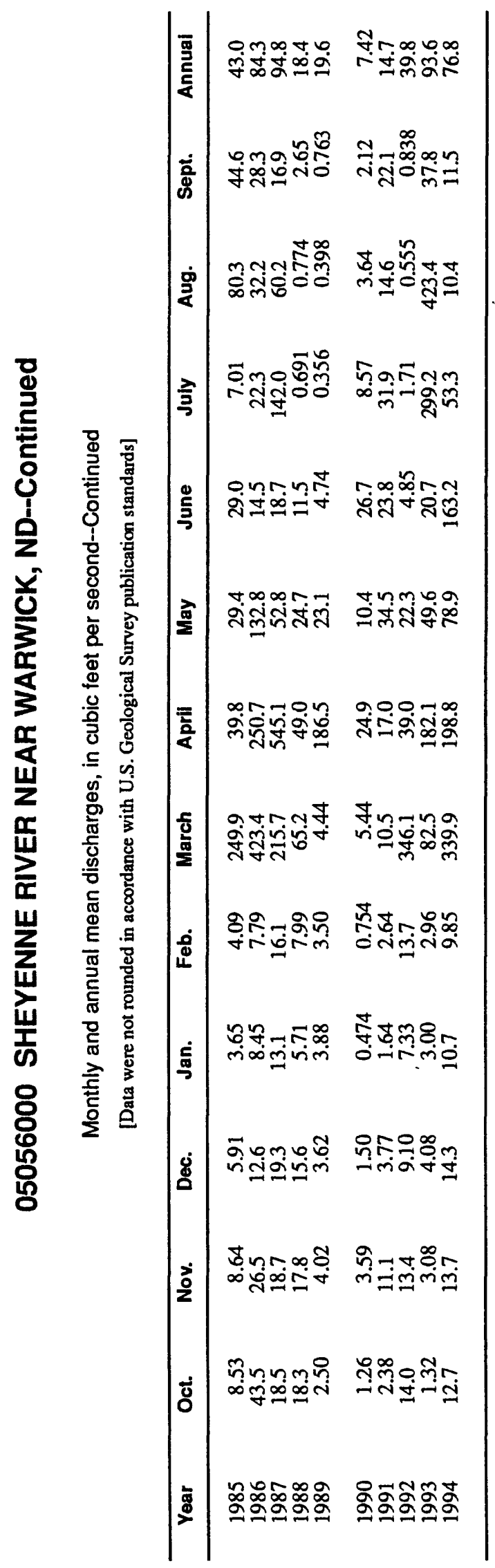




\section{SHEYENNE RIVER NEAR COOPERSTOWN, ND}

\section{Station Description}

LOCATION.--Lat $47^{\circ} 25^{\prime} 58^{\prime \prime}$, long $98^{\circ} 01^{\prime} 38^{\prime \prime}$, in NW $1 /{ }_{4} \mathrm{NW}^{1} / 4 \mathrm{SW}^{1} / 4$ sec. $26, \mathrm{~T} .146 \mathrm{~N} ., \mathrm{R} .58$ W., Griggs County, Hydrologic Unit 09020203, on right bank at Ueland Dam $0.7 \mathrm{mi}$ downstream from State Highway 200, and $5 \mathrm{mi}$ east of Cooperstown.

DRAINAGE AREA.--6,470 $\mathrm{mi}^{2}$, approximately, of which about $5,200 \mathrm{mi}^{2}$ is probably noncontributing (includes $3,800 \mathrm{mi}^{2}$ in closed basins).

PERIOD OF RECORD.--October 1944 to current year. Monthly discharge only for some periods, published in Water-Supply Paper 1308.

GAGE.--Water-stage recorder and artificial control. Datum of gage is $1,271.76 \mathrm{ft}$ above sea level (Coast and Geodetic Survey bench mark). Aug. 31, 1950, to Oct. 22, 1985, gage located on right bank $300 \mathrm{ft}$ downstream of present site and datum. Prior to Aug. 3, 1950, nonrecording gage at site $150 \mathrm{ft}$ downstream of present site at same datum.

EXTREMES FOR PERIOD OF RECORD.--Maximum discharge, 7,830 $\mathrm{ft}^{3} / \mathrm{s}$, Apr. 17, 1950; maximum gage height, $18.69 \mathrm{ft}$, Apr. 17, 1950; minimum discharge, $0 \mathrm{ft}^{3} / \mathrm{s}$, Aug. 29 to Oct. 8, 1959, Aug. 12 to Sept. 12, 1961, Jan. 25 to Mar. 6, and Aug. 24-25, 1963.

Annual mean discharge

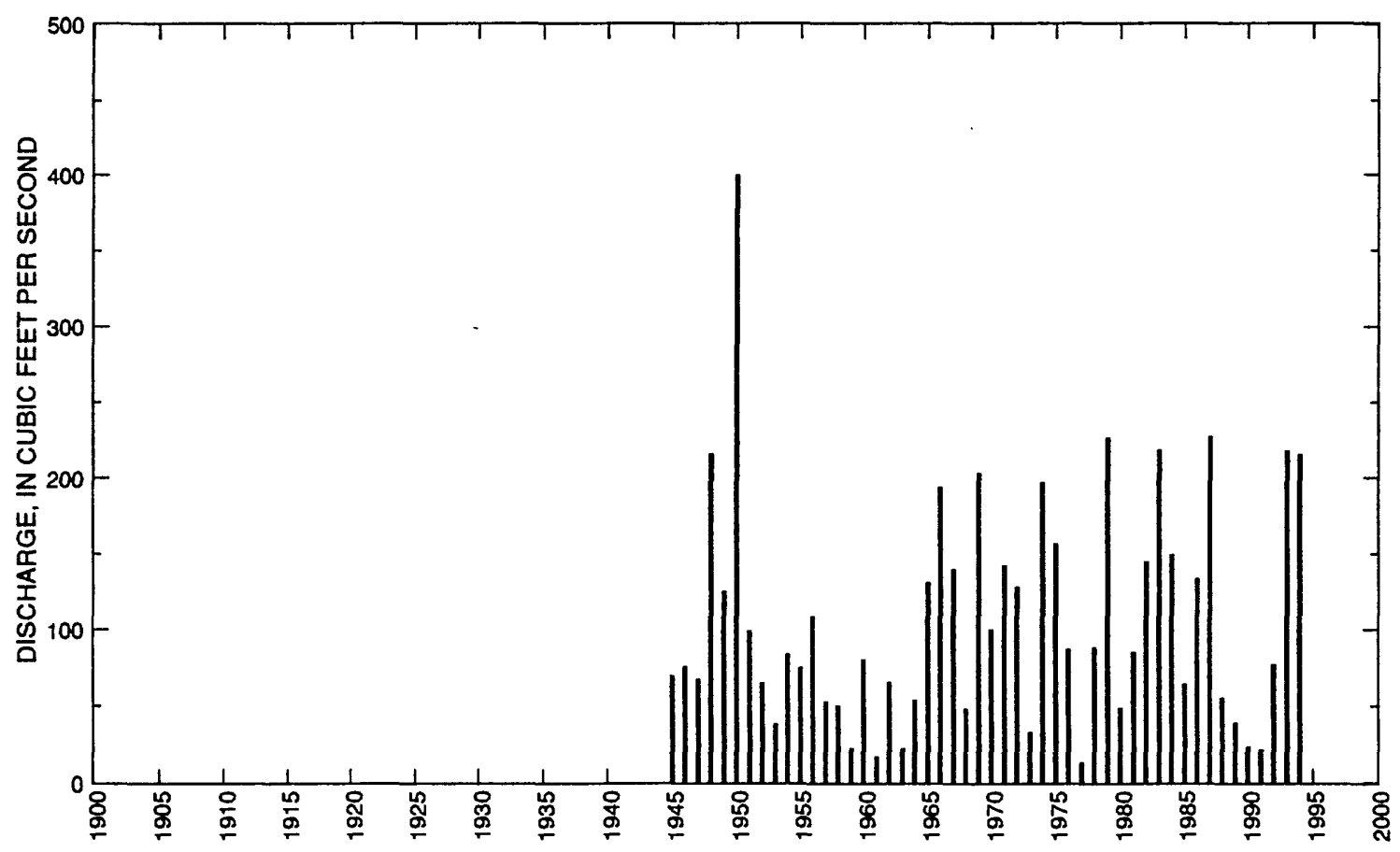




\section{SHEYENNE RIVER NEAR COOPERSTOWN, ND--Continued}

Statistics of monthly and annual mean discharges

\begin{tabular}{|c|c|c|c|c|c|c|c|c|}
\hline \multirow[b]{2}{*}{ Month } & \multicolumn{2}{|c|}{ Maximum } & \multicolumn{2}{|c|}{ Minimum } & \multicolumn{4}{|c|}{ Mean } \\
\hline & $\begin{array}{c}\text { Discharge } \\
\left(\mathrm{ft}^{3} / \mathrm{s}\right)\end{array}$ & $\begin{array}{c}\text { Water year } \\
\text { of } \\
\text { occurrence }\end{array}$ & $\begin{array}{c}\text { Discharge } \\
\left(\mathrm{ft}^{3} / \mathrm{s}\right)\end{array}$ & $\begin{array}{c}\text { Water year } \\
\text { of } \\
\text { occurrence }\end{array}$ & $\begin{array}{c}\text { Discharge } \\
\left(\mathrm{ft}^{3} / \mathrm{s}\right)\end{array}$ & $\begin{array}{c}\text { Standard } \\
\text { deviation } \\
\left(\mathrm{ft}^{3} / \mathrm{s}\right)\end{array}$ & $\begin{array}{l}\text { Coeffi- } \\
\text { clent of } \\
\text { variation }\end{array}$ & $\begin{array}{l}\text { Percentage } \\
\text { of annual } \\
\text { dlscharge }\end{array}$ \\
\hline October & 114 & 1983 & 0.829 & 1964 & 25.8 & 26.0 & 1.01 & 1.99 \\
\hline November & 71.0 & 1986 & 2.83 & 1977 & 25.2 & 17.5 & 0.70 & 1.94 \\
\hline December & 61.0 & 1983 & 3.14 & 1977 & 16.4 & 11.4 & 0.69 & 1.27 \\
\hline January & 31.4 & 1994 & 1.94 & 1964 & 10.6 & 6.66 & 0.63 & 0.82 \\
\hline February & 48.2 & 1954 & 0 & 1963 & 11.4 & 12.6 & 1.10 & 0.88 \\
\hline March & 1,090 & 1983 & 2.14 & 1964 & 166 & 235 & 1.41 & 12.9 \\
\hline April & 2,290 & 1950 & 42.4 & 1991 & 549 & 521 & 0.95 & 42.4 \\
\hline May & 1,950 & 1950 & 37.3 & 1961 & 209 & 313 & 1.50 & 16.2 \\
\hline June & 435 & 1974 & 6.66 & 1961 & 113 & 92.2 & 0.81 & 8.76 \\
\hline July & 640 & 1993 & 3.84 & 1961 & 84.0 & 105 & 1.25 & 6.49 \\
\hline August & 1,030 & 1993 & 0.681 & 1961 & 51.7 & 148 & 2.85 & 4.00 \\
\hline September & 321 & 1994 & 0 & 1959 & 31.7 & 57.3 & 1.81 & 2.45 \\
\hline Annual & 399 & 1950 & 13.2 & 1977 & 108 & 77.0 & 0.71 & 100 \\
\hline
\end{tabular}

Annual flow duration

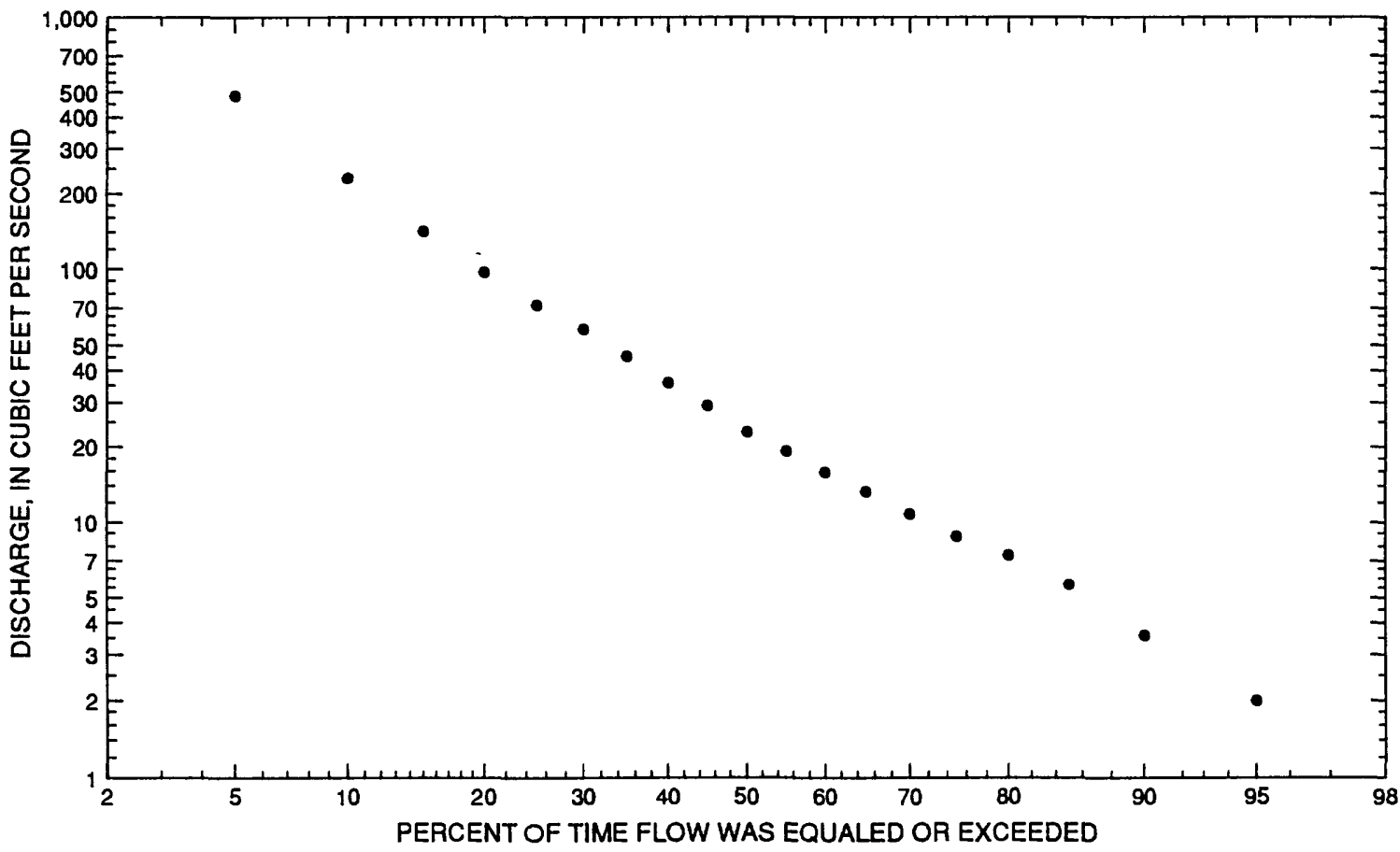




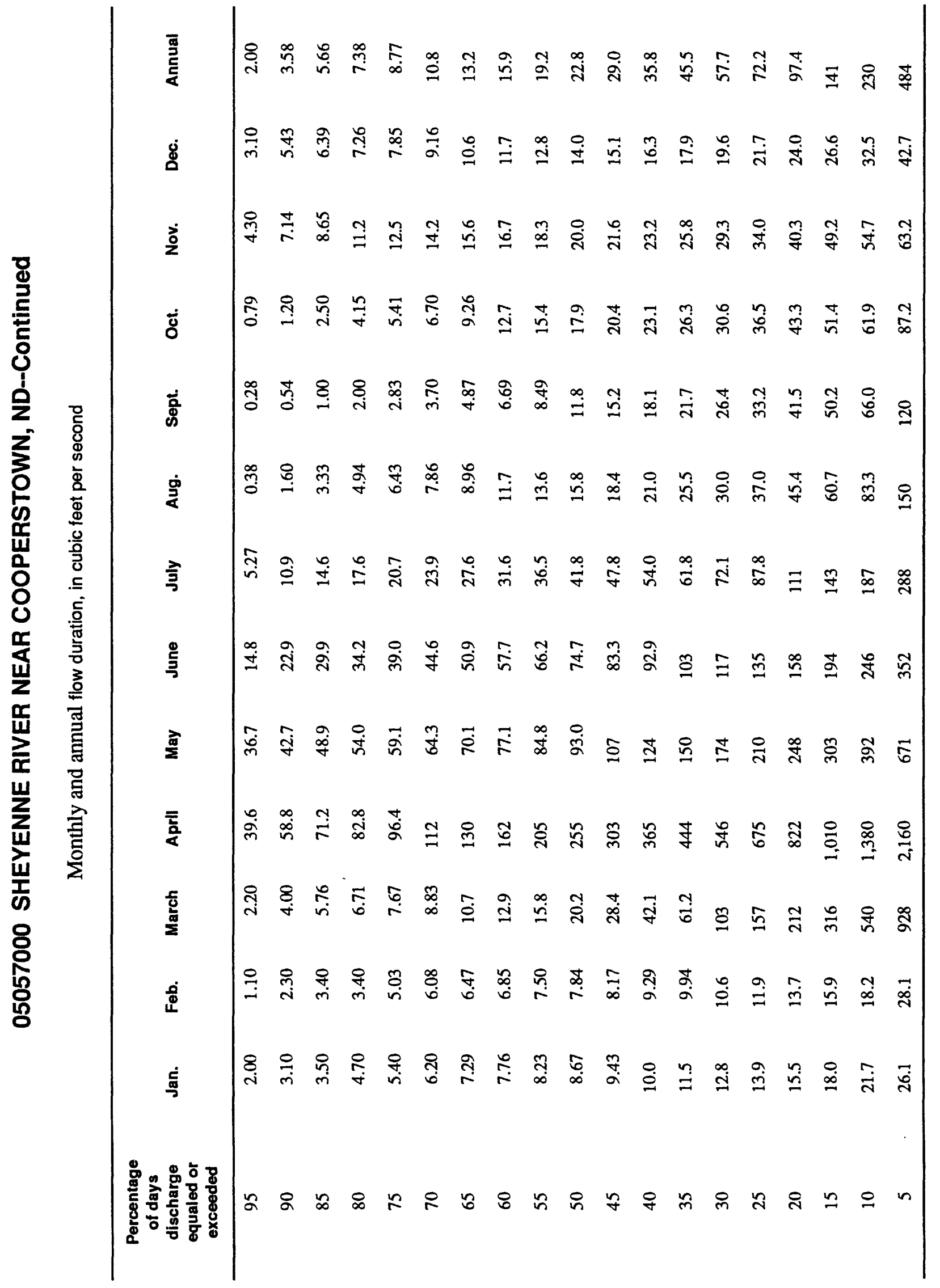




\section{SHEYENNE RIVER NEAR COOPERSTOWN, ND--Continued}

Probability of annual high discharges

[ng, statistic not given]

\begin{tabular}{|c|c|c|c|c|c|c|}
\hline \multirow[b]{2}{*}{$\begin{array}{l}\text { Exceedance } \\
\text { probability }\end{array}$} & \multirow[b]{2}{*}{$\begin{array}{l}\text { Recurrence } \\
\text { interval } \\
\text { (years) }\end{array}$} & \multirow[b]{2}{*}{$\begin{array}{c}\text { Maximum } \\
\text { instantaneous } \\
\left(\mathrm{ft}^{3} / \mathbf{s}\right)\end{array}$} & \multicolumn{4}{|c|}{$\begin{array}{l}\text { Maximum average discharge } \\
\qquad\left(\mathrm{ft}^{3} / \mathrm{s}\right)\end{array}$} \\
\hline & & & 3-day period & 7-day perlod & 15-day period & 30-day period \\
\hline 0.99 & 1.01 & 66.8 & 52.4 & 46.9 & 43.4 & 38.8 \\
\hline 0.95 & 1.05 & 165 & 140 & 126 & 111 & 90.8 \\
\hline 0.90 & 1.11 & 260 & 227 & 205 & 177 & 139 \\
\hline 0.80 & 1.25 & 438 & 394 & 356 & 299 & 226 \\
\hline 0.50 & 2 & 1,100 & 1,010 & 913 & 744 & 532 \\
\hline 0.20 & 5 & 2,490 & 2,280 & 2,030 & 1,630 & 1,130 \\
\hline 0.10 & 10 & 3,670 & 3,310 & 2,930 & 2,350 & 1,620 \\
\hline 0.04 & 25 & 5,400 & 4,760 & 4,170 & 3,340 & 2,320 \\
\hline 0.02 & 50 & 6,820 & 5,900 & 5,130 & 4,120 & 2,870 \\
\hline 0.01 & 100 & 8,340 & 7,070 & 6,110 & 4,920 & 3,450 \\
\hline 0.005 & 200 & 9,940 & 8,250 & 7,080 & 5,730 & 4,050 \\
\hline 0.002 & 500 & 12,200 & $\mathrm{ng}$ & $\mathrm{ng}$ & ng & ng \\
\hline
\end{tabular}

Probability of annual low discharges

Minimum average discharge $\left(\mathrm{ft}^{3} / \mathrm{s}\right)$

Number of consecutive days

\begin{tabular}{|c|c|c|c|c|c|c|c|c|c|c|}
\hline \multirow[b]{2}{*}{$\begin{array}{l}\text { Non- } \\
\text { exceed- } \\
\text { ance } \\
\text { prob- } \\
\text { ability }\end{array}$} & \multirow[b]{2}{*}{$\begin{array}{c}\text { Recur- } \\
\text { rence } \\
\text { inter- } \\
\text { vai } \\
\text { (years) }\end{array}$} & \multicolumn{9}{|c|}{ Number of consecutive days } \\
\hline & & 1 & 3 & 7 & 14 & 30 & 60 & 90 & 120 & 183 \\
\hline 0.05 & 20 & 0 & 0 & 0 & 0 & 0 & 0.627 & 2.06 & 2.88 & 3.16 \\
\hline 0.10 & 10 & 0 & 0.027 & 0.103 & 0.149 & 0.462 & 1.17 & 2.86 & 3.92 & 4.36 \\
\hline 0.20 & 5 & 0.254 & 0.347 & 0.493 & 0.655 & 1.24 & 2.30 & 4.22 & 5.62 & 6.37 \\
\hline 0.50 & 2 & 2.52 & 2.66 & 2.90 & 3.48 & 4.28 & 6.57 & 8.46 & 10.7 & 12.8 \\
\hline
\end{tabular}


05057000 SHEYENNE RIVER NEAR COOPERSTOWN, ND--Continued

Probability of seasonal low discharges

\begin{tabular}{|c|c|c|c|c|c|c|c|c|c|}
\hline \multirow[b]{3}{*}{$\begin{array}{c}\text { Non- } \\
\text { exceedance } \\
\text { probablity }\end{array}$} & \multirow[b]{3}{*}{$\begin{array}{c}\text { Recurrence } \\
\text { interval } \\
\text { (years) }\end{array}$} & \multicolumn{8}{|c|}{ Minimum average discharge $\left(\mathrm{ft}^{3} / \mathrm{s}\right)$} \\
\hline & & \multicolumn{8}{|c|}{ Number of consecutive days } \\
\hline & & 1 & 7 & 14 & 30 & 1 & 7 & 14 & 30 \\
\hline & & \multicolumn{4}{|c|}{ December-January-February } & \multicolumn{4}{|c|}{ March-April-May } \\
\hline 0.05 & 20 & 0.456 & 0.531 & 0.898 & 1.24 & 1.47 & ${ }^{1} 2.79$ & 14.11 & 5.44 \\
\hline 0.10 & 10 & 1.25 & $\mathbf{1}_{1.42}$ & 1.59 & 2.05 & 2.11 & ${ }^{1} 2.29$ & 2.47 & 8.92 \\
\hline 0.20 & 5 & 2.37 & 2.38 & 2.76 & 3.36 & 3.21 & 3.55 & 4.82 & 15.8 \\
\hline \multirow[t]{2}{*}{0.50} & 2 & 5.65 & 6.22 & 6.24 & 7.02 & 7.37 & 12.0 & 15.2 & 43.4 \\
\hline & & \multicolumn{4}{|c|}{ June-July-August } & \multicolumn{4}{|c|}{ September-October-November } \\
\hline 0.05 & 20 & 0 & 0.116 & 0.395 & 1.33 & 0 & 0.024 & 0.181 & 0.395 \\
\hline 0.10 & 10 & 0.094 & 0.475 & 1.05 & 2.54 & 0 & 0.196 & 0.451 & 0.896 \\
\hline 0.20 & 5 & 1.11 & 1.72 & 2.67 & 5.16 & 0.399 & 0.734 & 1.14 & 2.05 \\
\hline 0.50 & 2 & 8.08 & 9.41 & 10.5 & 16.2 & 3.68 & 4.63 & 5.15 & 7.80 \\
\hline
\end{tabular}

${ }^{1}$ Graphical interpretation. 


\section{SHEYENNE RIVER NEAR COOPERSTOWN, ND-Continued}

Annual peak discharge and corresponding gage height, period of record

\begin{tabular}{|c|c|c|c|c|c|c|c|}
\hline $\begin{array}{l}\text { Water } \\
\text { year }\end{array}$ & Date & $\begin{array}{c}\text { Gage } \\
\text { height } \\
\text { (feet) }\end{array}$ & $\begin{array}{c}\text { Peak } \\
\text { discharge } \\
\left(\mathrm{ft}^{3} / \mathrm{s}\right)\end{array}$ & $\begin{array}{l}\text { Water } \\
\text { year }\end{array}$ & Date & $\begin{array}{c}\text { Gage } \\
\text { height } \\
\text { (foet) }\end{array}$ & $\begin{array}{c}\text { Peak } \\
\text { discharge } \\
\left(\mathrm{ft}^{3} / \mathrm{s}\right)\end{array}$ \\
\hline \multicolumn{8}{|c|}{ Annual peak discharge, by year, and corresponding gage height } \\
\hline 1945 & March 20 & 10.50 & 1,000 & 1970 & April 8 & 12.80 & 1,220 \\
\hline 1946 & April 2 & 10.68 & 964 & 1971 & April 17 & 16.31 & 2,310 \\
\hline 1947 & April 8 & 13.89 & 1,150 & 1972 & March 27 & 12.26 & 1,120 \\
\hline 1948 & April 23 & 18.10 & 5,600 & 1973 & March 15 & 7.52 & 260 \\
\hline 1949 & April 17 & 15.95 & 2,290 & 1974 & April 15 & 16.40 & 2,460 \\
\hline 1950 & April 17 & 18.69 & 7,830 & 1975 & April 25 & 15.35 & 1,490 \\
\hline 1951 & April 19 & 11.05 & 989 & 1976 & April 2 & 13.45 & 1,450 \\
\hline 1952 & April 14 & 13.02 & 1,240 & 1977 & April 5 & 5.49 & 136 \\
\hline 1953 & July 3 & 6.22 & 271 & 1978 & April 12 & 14.13 & 1,460 \\
\hline 1954 & June 26 & 9.32 & 682 & 1979 & April 20 & 17.87 & 4,680 \\
\hline 1955 & April 11 & 11.41 & 1,060 & 1980 & April 9 & 10.57 & 750 \\
\hline 1956 & April 24 & 16.32 & 2,600 & 1981 & March 23 & 8.61 & 500 \\
\hline 1957 & September 4 & 6.68 & 280 & 1982 & April 12 & 15.83 & 1,900 \\
\hline 1958 & April 7 & 7.04 & 340 & 1983 & April 12 & 16.24 & 1,610 \\
\hline 1959 & April 1 & 7.95 & 360 & 1984 & April 3 & 15.13 & 1,850 \\
\hline 1960 & April 14 & 13.38 & 1,340 & 1985 & March 23 & 11.74 & 930 \\
\hline 1961 & April 3 & 5.13 & 120 & 1986 & March 23 & 15.56 & 1,760 \\
\hline 1962 & April 11 & 12.69 & 900 & 1987 & April 5 & 18.21 & 4,840 \\
\hline 1963 & April 6 & 7.25 & 300 & 1988 & April 5 & 10.96 & 389 \\
\hline 1964 & June 23 & 10.34 & 795 & 1989 & April 16 & 11.95 & 796 \\
\hline 1965 & April 15 & 16.03 & 2,320 & 1990 & June 30 & 10.30 & 159 \\
\hline 1966 & March 23 & 17.62 & 3,040 & 1991 & May 6 & 7.09 & 84.0 \\
\hline 1967 & March 31 & 15.75 & 2,160 & 1992 & March 16 & 13.72 & 1,100 \\
\hline 1968 & March 29 & 8.31 & 415 & 1993 & July 25 & 18.33 & 2,780 \\
\hline 1969 & April 17 & 18.07 & 5,050 & 1994 & April 1 & 15.31 & 1,750 \\
\hline \multicolumn{8}{|c|}{ Annual peak discharge, from highest to lowest, and corresponding gage height } \\
\hline 1950 & April 17 & 18.69 & 7,830 & 1978 & April 12 & 14.13 & 1,460 \\
\hline 1948 & April 23 & 18.10 & 5,600 & 1976 & April 2 & 13.45 & 1,450 \\
\hline 1969 & April 17 & 18.07 & 5,050 & 1960 & April 14 & 13.38 & 1,340 \\
\hline 1987 & April 5 & 18.21 & 4,840 & 1952 & April 14 & 13.02 & 1,240 \\
\hline 1979 & April 20 & 17.87 & 4,680 & 1947 & April 8 & 13.89 & 1,150 \\
\hline 1966 & March 23 & 17.62 & 3,040 & 1970 & April 8 & 12.80 & 1,220 \\
\hline 1993 & July 25 & 18.33 & 2,780 & 1972 & March 27 & 12.26 & 1,120 \\
\hline 1956 & April 24 & 16.32 & 2,600 & 1992 & March 16 & 13.72 & 1,100 \\
\hline 1974 & April 15 & 16.40 & 2,460 & 1955 & April 11 & 11.41 & 1,060 \\
\hline 1965 & April 15 & 16.03 & 2,320 & 1945 & March 20 & 10.50 & 1,000 \\
\hline 1971 & April 17 & 16.31 & 2,310 & 1951 & April 19 & 11.05 & 989 \\
\hline 1949 & April 17 & 15.95 & 2,290 & 1946 & April 2 & 10.68 & 964 \\
\hline 1967 & March 31 & 15.75 & 2,160 & 1970 & April 8 & 11.25 & 964 \\
\hline 1982 & April 12 & 15.83 & 1,900 & 1985 & March 23 & 11.74 & 930 \\
\hline 1984 & April 3 & 15.13 & 1,850 & 1962 & April 11 & 12.69 & 900 \\
\hline 1986 & March 23 & 15.56 & 1,760 & 1989 & April 16 & 11.95 & 796 \\
\hline 1994 & April 1 & 15.31 & 1,750 & 1964 & June 23 & 10.34 & 795 \\
\hline 1983 & April 12 & 16.24 & 1,610 & 1980 & April 9 & 10.57 & 750 \\
\hline 1975 & April 25 & 15.35 & 1,490 & 1954 & June 26 & 9.32 & 682 \\
\hline
\end{tabular}




\section{SHEYENNE RIVER NEAR COOPERSTOWN, ND--Continued}

Annual peak discharge and corresponding gage height, period of record--Continued

\begin{tabular}{|c|c|c|c|c|c|c|c|}
\hline $\begin{array}{l}\text { Water } \\
\text { year }\end{array}$ & Date & $\begin{array}{c}\text { Gage } \\
\text { height } \\
\text { (feet) }\end{array}$ & $\begin{array}{c}\text { Peak } \\
\text { discharge } \\
\left(\mathrm{ft}^{3} / \mathrm{s}\right)\end{array}$ & $\begin{array}{c}\text { Water } \\
\text { year }\end{array}$ & Date & $\begin{array}{c}\text { Gage } \\
\text { height } \\
\text { (feet) }\end{array}$ & $\begin{array}{c}\text { Peak } \\
\text { discharge } \\
\left(\mathrm{ft}^{3} / \mathrm{s}\right)\end{array}$ \\
\hline \multicolumn{8}{|c|}{ Annual peak discharge, from highest to lowest, and corresponding gage height-.Continued } \\
\hline 1981 & March 23 & 8.61 & 500 & 1953 & July 3 & 6.22 & 271 \\
\hline 1968 & March 29 & 8.31 & 415 & 1973 & March 15 & 7.52 & 260 \\
\hline 1988 & April 5 & 10.96 & 389 & 1990 & June 30 & 10.30 & 159 \\
\hline 1959 & April 1 & 7.95 & 360 & 1977 & April 5 & 5.49 & 136 \\
\hline 1958 & April 7 & 7.04 & 340 & 1961 & April 3 & 5.13 & 120 \\
\hline 1963 & April 6 & 7.25 & 300 & 1991 & May 6 & 7.09 & 84.0 \\
\hline 1957 & September 4 & 6.68 & 280 & & & & \\
\hline
\end{tabular}




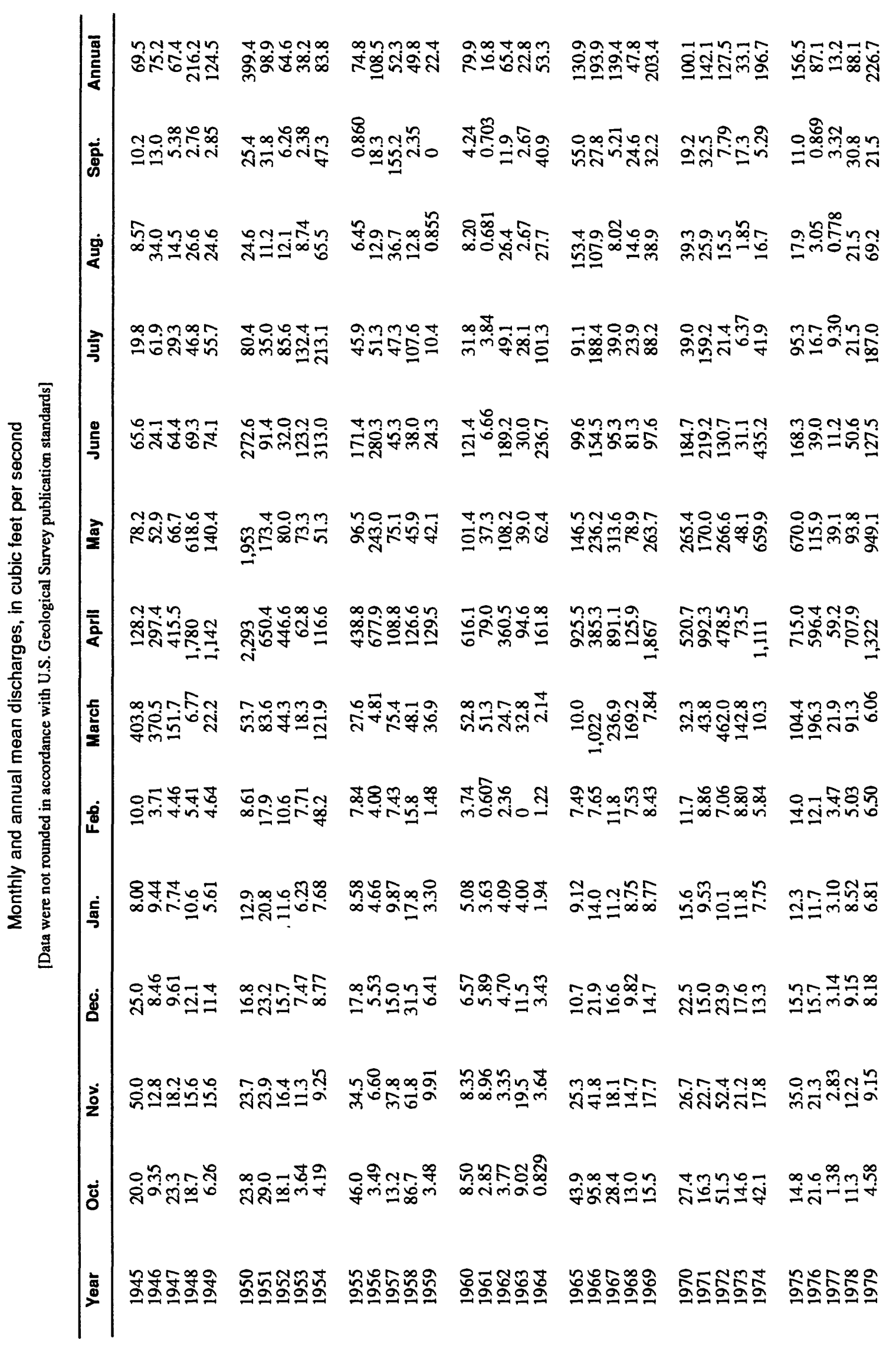




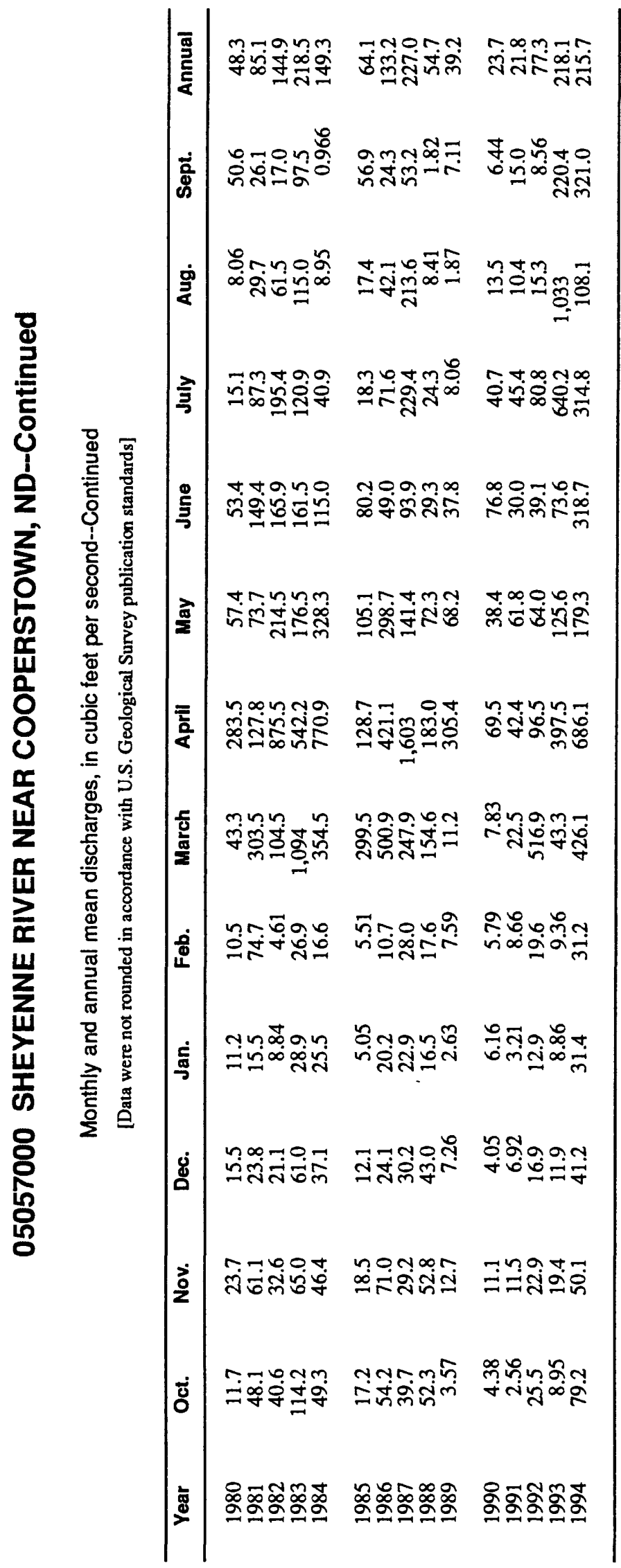




\section{BALDHILL CREEK NEAR DAZEY, ND}

\section{Station Description}

LOCATION.--Lat $47^{\circ} 13^{\prime} 45^{\prime \prime}$, long $98^{\circ} 07^{\prime} 28^{\prime \prime}$, in NW1/4 $\mathrm{SE}^{1} / \mathrm{SW}^{1} / 4$ sec. 2 , T.143 N., R.59 W., Bames County, Hydrologic Unit 09020203, on left bank $500 \mathrm{ft}$ upstream from bridge on county highway, $4.5 \mathrm{mi}$ northeast of Dazey, and $14 \mathrm{mi}$ upstream from mouth.

DRAINAGE AREA.--691 mi ${ }^{2}$, of which about $340 \mathrm{mi}^{2}$ is probably noncontributing.

PERIOD OF RECORD.--March 1956 to current year.

GAGE.--Water-stage recorder. Prior to Nov. 9, 1956, nonrecording gage $500 \mathrm{ft}$ downstream at same datum.

EXTREMES FOR PERIOD OF RECORD.--Maximum discharge, 9,000 ft ${ }^{3} / \mathrm{s}$, Apr. 19, 1979; maximum gage height, $17.78 \mathrm{ft}$, Apr. 19, 1979; minimum discharge, no flow at times.

Annual mean discharge

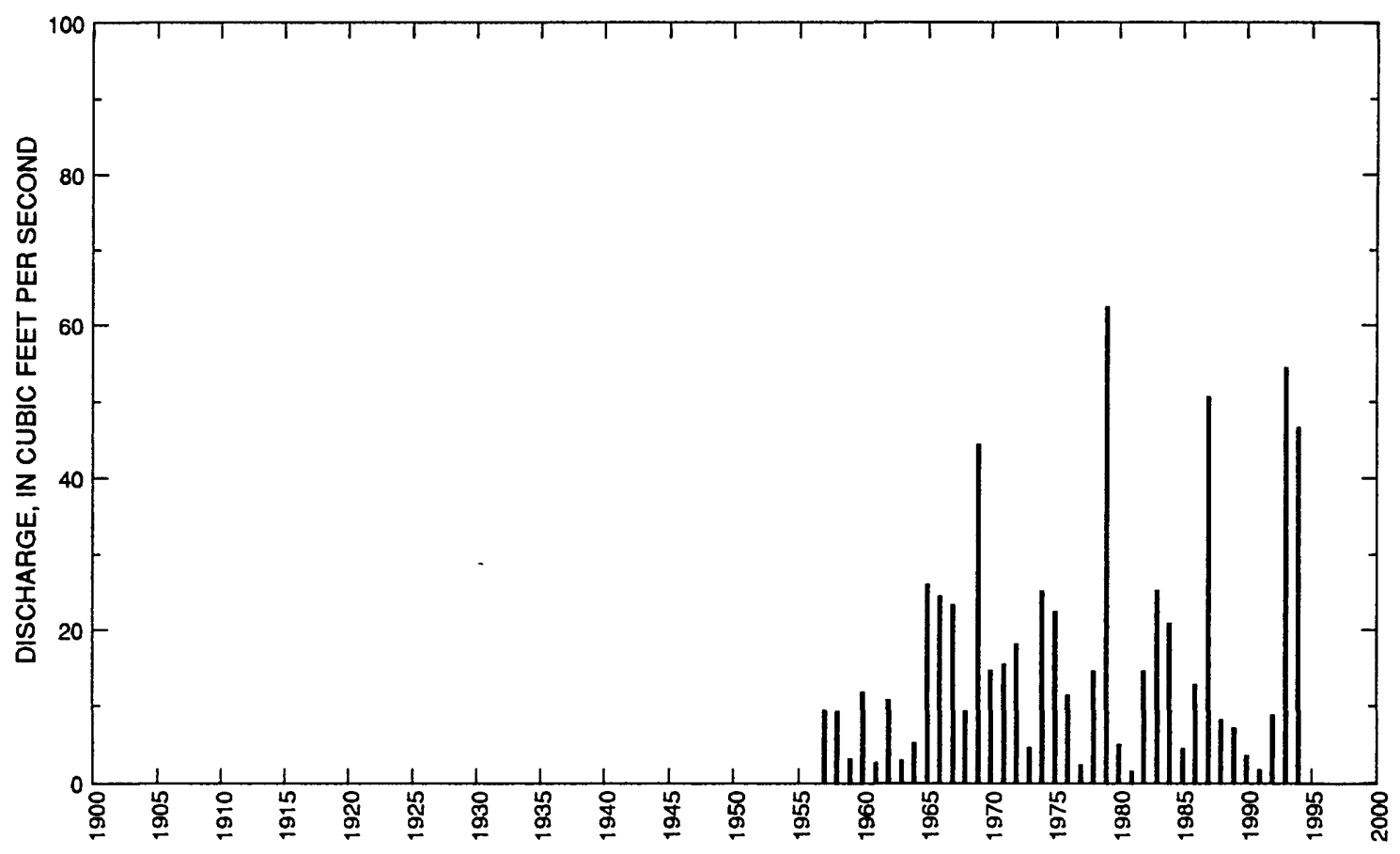


05057200 BALDHILL CREEK NEAR DAZEY, ND--Continued

Statistics of monthly and annual mean discharges

[m, more than 1 year of occurrence]

\begin{tabular}{|c|c|c|c|c|c|c|c|c|}
\hline \multirow[b]{2}{*}{ Month } & \multicolumn{2}{|c|}{ Maximum } & \multicolumn{2}{|c|}{ Minimum } & \multicolumn{4}{|c|}{ Mean } \\
\hline & $\begin{array}{c}\text { Discharge } \\
\left(\mathrm{ft}^{3} / \mathrm{s}\right)\end{array}$ & $\begin{array}{c}\text { Water year } \\
\text { of } \\
\text { occurrence }\end{array}$ & $\begin{array}{c}\text { Discharge } \\
\left(\mathrm{ft}^{3} / \mathbf{s}\right)\end{array}$ & $\begin{array}{c}\text { Water year } \\
\text { of } \\
\text { occurrence }\end{array}$ & $\begin{array}{c}\text { Discharge } \\
\left(\mathrm{ft}^{3} / \mathrm{s}\right)\end{array}$ & $\begin{array}{l}\text { Standard } \\
\text { deviation } \\
\left(\mathrm{ft}^{3} / \mathrm{s}\right)\end{array}$ & $\begin{array}{l}\text { Coeffl- } \\
\text { cient of } \\
\text { varlation }\end{array}$ & $\begin{array}{l}\text { Percentage } \\
\text { of annual } \\
\text { discharge }\end{array}$ \\
\hline October & 29.8 & 1958 & 0.473 & 1992 & 4.33 & 5.30 & 1.23 & 2.15 \\
\hline November & 20.5 & 1958 & 0.380 & 1960 & 3.86 & 3.45 & 0.90 & 1.92 \\
\hline December & 7.42 & 1958 & 0.155 & 1959 & 2.16 & 1.73 & 0.80 & 1.07 \\
\hline January & 5.38 & 1994 & 0 & $\mathrm{~m}$ & 0.930 & 1.07 & 1.15 & 0.46 \\
\hline February & 5.37 & 1983 & 0 & $\mathrm{~m}$ & 1.34 & 1.61 & 1.20 & 0.67 \\
\hline March & 270 & 1987 & 0.587 & 1964 & 47.8 & 63.9 & 1.34 & 23.7 \\
\hline April & 654 & 1979 & 2.44 & 1981 & 85.7 & 131 & 1.53 & 42.6 \\
\hline May & 82.0 & 1979 & 1.71 & 1981 & 16.9 & 16.0 & 0.94 & 8.39 \\
\hline June & 50.6 & 1970 & 0.907 & 1961 & 11.8 & 11.7 & 0.99 & 5.87 \\
\hline July & 273 & 1993 & 0.021 & 1989 & 14.5 & 43.7 & 3.01 & 7.21 \\
\hline August & 133 & 1993 & 0.076 & 1984 & 5.97 & 21.1 & 3.53 & 2.96 \\
\hline September & 58.5 & 1957 & 0.094 & 1984 & 6.07 & 13.2 & 2.18 & 3.01 \\
\hline Annual & 62.4 & 1979 & 1.52 & 1981 & 16.8 & 15.8 & 0.94 & 100 \\
\hline
\end{tabular}

Annual flow duration

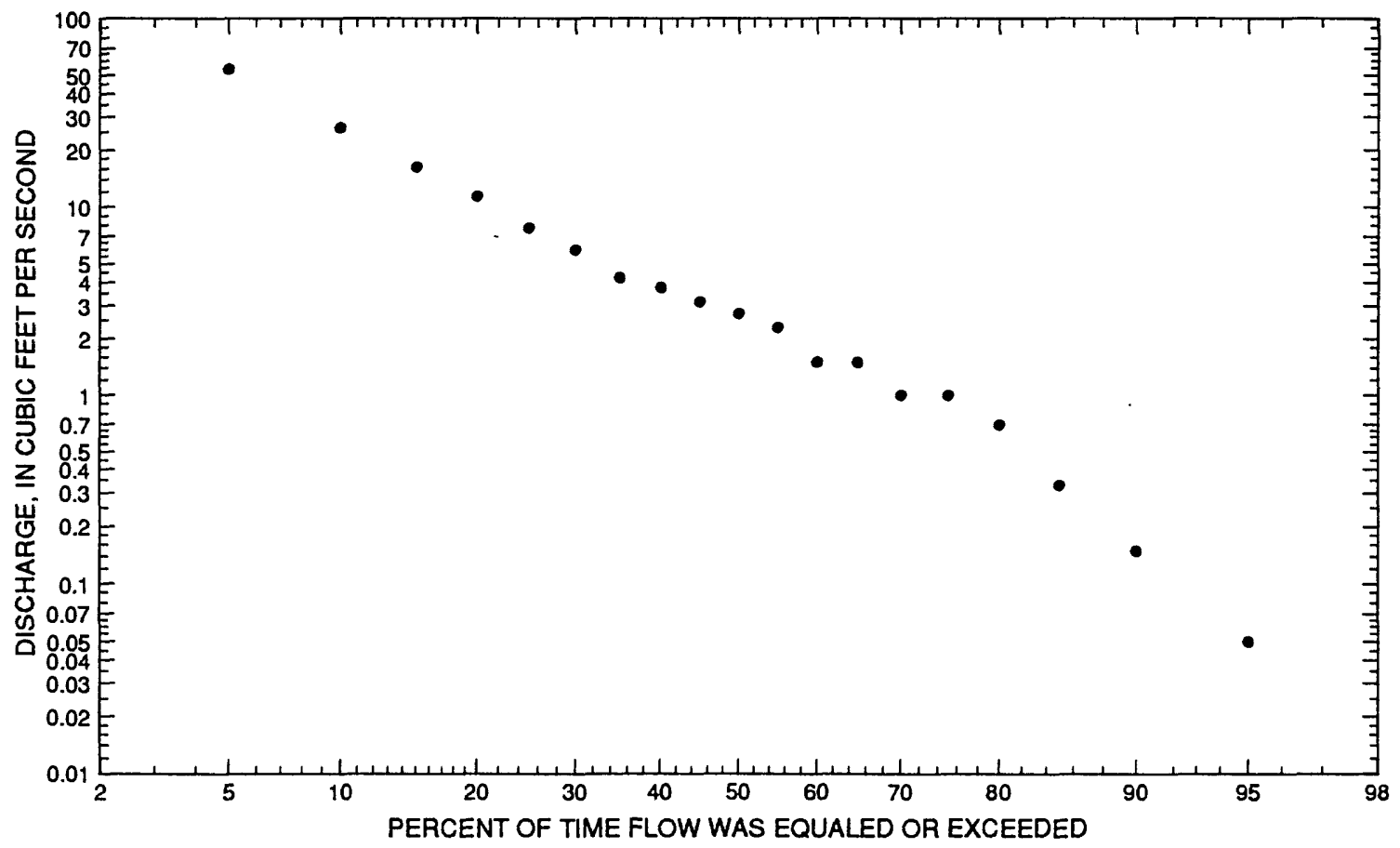




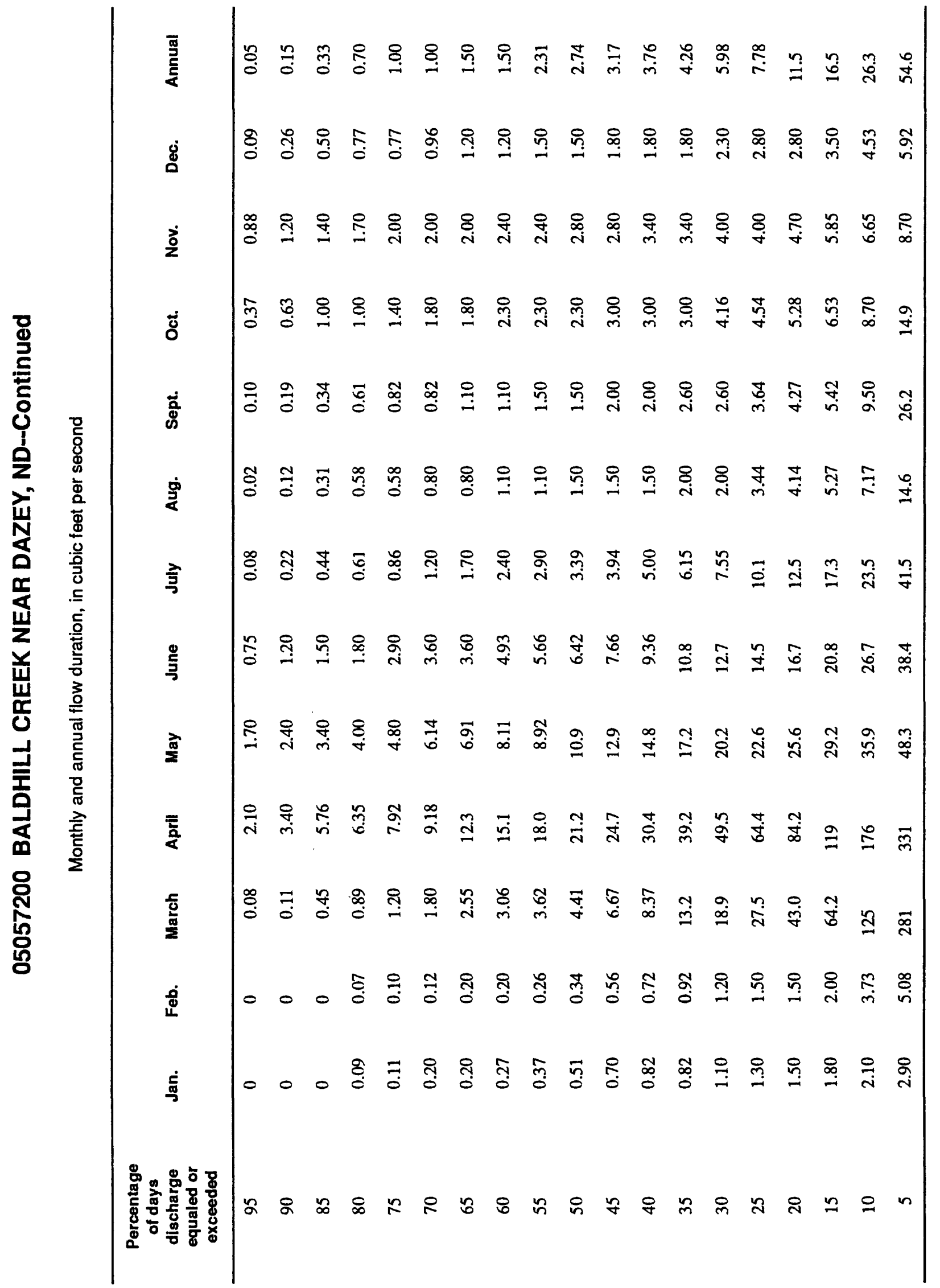


05057200 BALDHILL CREEK NEAR DAZEY, ND--Continued

Probability of annual high discharges

[ng, statistic not given]

\begin{tabular}{lcccccc}
\hline & & & \multicolumn{5}{c}{$\begin{array}{c}\text { Maximum average discharge } \\
\left.\text { (ft } \mathbf{t}^{3} / \mathbf{s}\right)\end{array}$} \\
\cline { 5 - 7 } $\begin{array}{c}\text { Exceodance } \\
\text { probability }\end{array}$ & $\begin{array}{c}\text { Recurrence } \\
\text { interval } \\
\text { (years) }\end{array}$ & $\begin{array}{c}\text { Maximum } \\
\text { instantaneous } \\
\left(\mathrm{ft}^{3} / \mathbf{s}\right)\end{array}$ & 3-day period & 7-day period & 15-day period & 30-day period \\
\hline 0.99 & 1.01 & 8.30 & 3.56 & 3.04 & 2.54 & 2.43 \\
0.95 & 1.05 & 24.1 & 12.2 & 10.4 & 8.48 & 7.10 \\
0.90 & 1.11 & 42.1 & 22.9 & 19.4 & 15.6 & 12.3 \\
0.80 & 1.25 & 81.6 & 47.9 & 40.1 & 31.4 & 23.1 \\
0.50 & 2 & 279 & 182 & 148 & 110 & 72.4 \\
0.20 & 5 & 912 & 626 & 489 & 338 & 206 \\
0.10 & 10 & 1,660 & 1,150 & 876 & 581 & 342 \\
0.04 & 25 & 3,110 & 2,150 & 1,580 & 1,000 & 573 \\
0.02 & 50 & 4,620 & 3,160 & 2,280 & 1,390 & 787 \\
0.01 & 100 & 6,570 & 4,430 & 3,130 & 1,860 & 1,040 \\
0.005 & 200 & 9,030 & 5,990 & 4,140 & 2,390 & 1,330 \\
0.002 & 500 & 13,200 & ng & ng & ng & ng \\
\hline
\end{tabular}

Probability of annual low discharges

\begin{tabular}{|c|c|c|c|c|c|c|c|c|c|c|}
\hline \multirow[b]{3}{*}{$\begin{array}{l}\text { Non- } \\
\text { exceed- } \\
\text { ance } \\
\text { prob- } \\
\text { ability }\end{array}$} & \multirow[b]{3}{*}{$\begin{array}{l}\text { Recur- } \\
\text { rence } \\
\text { Inter- } \\
\text { val } \\
\text { (years) }\end{array}$} & \multicolumn{9}{|c|}{ Minimum average discharge $\left(\mathrm{ft}^{3} / \mathrm{s}\right)$} \\
\hline & & \multicolumn{9}{|c|}{ Number of consecutive days } \\
\hline & & 1 & 3 & 7 & 14 & 30 & 60 & 90 & 120 & 183 \\
\hline 0.05 & 20 & 0 & 0 & 0 & 0 & 0 & 0 & 0.064 & 0.285 & 0.594 \\
\hline 0.10 & 10 & 0 & 0 & 0 & 0 & 0 & 0 & 0.115 & 0.395 & 0.727 \\
\hline 0.20 & 5 & 0 & 0 & 0 & 0 & 0.020 & 0.094 & 0.224 & 0.581 & 0.946 \\
\hline 0.50 & 2 & 0.072 & 0.078 & 0.097 & 0.131 & 0200 & 0.371 & 0.682 & 1.18 & 1.67 \\
\hline
\end{tabular}




\section{BALDHILL CREEK NEAR DAZEY, ND--Continued}

Probability of seasonal low discharges

\begin{tabular}{|c|c|c|c|c|c|c|c|c|c|}
\hline \multirow[b]{3}{*}{$\begin{array}{c}\text { Non- } \\
\text { exceedance } \\
\text { probability }\end{array}$} & \multirow[b]{3}{*}{$\begin{array}{l}\text { Recurrence } \\
\text { interval } \\
\text { (years) }\end{array}$} & \multicolumn{8}{|c|}{ Minimum average discharge $\left(\mathrm{ft}^{3} / \mathrm{s}\right)$} \\
\hline & & \multicolumn{8}{|c|}{ Number of consecutive days } \\
\hline & & 1 & 7 & 14 & 30 & 1 & 7 & 14 & 30 \\
\hline & & \multicolumn{4}{|c|}{ December-January-February } & \multicolumn{4}{|c|}{ March-April-May } \\
\hline 0.05 & 20 & 0 & 0 & 0 & 0 & 0 & 0 & 0 & 1.13 \\
\hline 0.10 & 10 & 0 & 0 & 0 & 0 & 0 & 0.037 & 0.228 & 1.69 \\
\hline 0.20 & 5 & 0 & 0 & 0 & 0.032 & 0.129 & 0.179 & 0.553 & 2.72 \\
\hline \multirow[t]{2}{*}{0.50} & 2 & 0.129 & 0.156 & 0.208 & 0.292 & 0.645 & 0.985 & 2.08 & 6.62 \\
\hline & & \multicolumn{4}{|c|}{ June-July-August } & \multicolumn{4}{|c|}{ September-October-November } \\
\hline 0.05 & 20 & 0 & 0 & 0 & 0.070 & 0 & 0 & 0.028 & 0.224 \\
\hline 0.10 & 10 & 0 & 0 & 0.046 & 0.164 & 0.024 & 0.052 & 0.095 & 0.359 \\
\hline 0.20 & 5 & 0.064 & 0.096 & 0.167 & 0.368 & 0.094 & 0.181 & 0.277 & 0.619 \\
\hline 0.50 & 2 & 0.568 & 0.722 & 0.926 & 1.32 & 0.594 & 0.930 & 1.23 & 1.64 \\
\hline
\end{tabular}




\section{BALDHILL CREEK NEAR DAZEY, ND--Continued}

Annual peak discharge and corresponding gage height, period of record

\begin{tabular}{|c|c|c|c|c|c|c|c|}
\hline $\begin{array}{l}\text { Water } \\
\text { year }\end{array}$ & Date & $\begin{array}{c}\text { Gage } \\
\text { height } \\
\text { (feet) }\end{array}$ & $\begin{array}{c}\text { Peak } \\
\text { discharge } \\
\left(\mathrm{ft}^{3} / \mathrm{s}\right)\end{array}$ & $\begin{array}{c}\text { Water } \\
\text { year }\end{array}$ & Date & $\begin{array}{c}\text { Gage } \\
\text { height } \\
\text { (feet) }\end{array}$ & $\begin{array}{c}\text { Peak } \\
\text { discharge } \\
\left(\mathrm{ft}^{3} / \mathbf{s}\right)\end{array}$ \\
\hline \multicolumn{8}{|c|}{ Annual peak discharge, by year, and corresponding gage height } \\
\hline 1956 & April 12 & 6.75 & 767 & 1976 & March 24 & 6.39 & 400 \\
\hline 1957 & September 3 & 5.07 & 248 & 1977 & March 15 & 3.18 & 25.0 \\
\hline 1958 & October 25 & 3.31 & 56.0 & 1978 & March 30 & 6.93 & 560 \\
\hline 1959 & March 14 & 4.42 & 30.0 & 1979 & April 19 & 17.78 & 9,000 \\
\hline 1960 & April 5 & 6.00 & 370 & 1980 & March 30 & 8.15 & 100 \\
\hline 1961 & March 2 & 3.60 & 40.0 & 1981 & February 17 & 4.65 & 28.0 \\
\hline 1962 & March 28 & 6.84 & 390 & 1982 & March 30 & 8.70 & 580 \\
\hline 1963 & April 6 & 3.47 & 24.0 & 1983 & March 7 & 9.95 & 650 \\
\hline 1964 & June 19 & 3.65 & 60.0 & 1984 & March 27 & 9.16 & 755 \\
\hline 1965 & April 12 & 9.23 & 1,780 & 1985 & March 10 & 5.50 & 88.0 \\
\hline 1966 & March 13 & 8.69 & 880 & 1986 & March 20 & 6.51 & 210 \\
\hline 1967 & March 27 & 6.90 & 650 & 1987 & April 3 & 8.99 & 960 \\
\hline 1968 & June 8 & 4.46 & 210 & 1988 & March 28 & 5.80 & 115 \\
\hline 1969 & April 11 & 10.90 & 2,510 & 1989 & April 3 & 6.64 & 303 \\
\hline 1970 & June 17 & 5.96 & 472 & 1990 & June 13 & 6.67 & 32.0 \\
\hline 1971 & March 31 & 6.20 & 305 & 1991 & May 23 & 6.74 & 50.0 \\
\hline 1972 & April 14 & 5.03 & 305 & 1992 & March 6 & 8.14 & 239 \\
\hline 1973 & March 14 & 3.84 & 100 & 1993 & July 24 & 14.50 & 1,450 \\
\hline 1974 & April 11 & 8.39 & 1,130 & 1994 & March 22 & 9.91 & 1,020 \\
\hline 1975 & April 17 & 7.51 & 680 & & & & \\
\hline \multicolumn{8}{|c|}{ Annual peak discharge, from highest to lowest, and corresponding gage height } \\
\hline 1979 & April 19 & 17.78 & 9,000 & 1972 & April 14 & 5.03 & 305 \\
\hline 1969 & April 11 & 10.90 & 2,510 & 1989 & April 3 & 6.64 & 303 \\
\hline 1965 & April 12 & 9.23 & 1,780 & 1957 & September 3 & 5.07 & 248 \\
\hline 1993 & July 24 & 14.50 & 1,450 & 1992 & March 6 & 8.14 & 239 \\
\hline 1974 & April 11 & 8.39 & 1,130 & 1968 & June 8 & 4.46 & 210 \\
\hline 1994 & March 22 & 9.91 & 1,020 & 1986 & March 20 & 6.51 & 210 \\
\hline 1987 & April 3 & 8.99 & 960 & 1988 & March 28 & 5.80 & 115 \\
\hline 1966 & March 13 & 8.69 & 880 & 1973 & March 14 & 3.84 & 100 \\
\hline 1956 & April 12 & 6.75 & 767 & 1980 & March 30 & 8.15 & 100 \\
\hline 1984 & March 27 & 9.16 & 755 & 1985 & March 10 & 5.50 & 88.0 \\
\hline 1975 & April 17 & 7.51 & 680 & 1964 & June 19 & 3.65 & 60.0 \\
\hline 1967 & March 27 & 6.90 & 650 & 1958 & October 25 & 3.31 & 56.0 \\
\hline 1983 & March 7 & 9.95 & 650 & 1991 & May 23 & 6.74 & 50.0 \\
\hline 1982 & March 30 & 8.70 & 580 & 1961 & March 2 & 3.60 & 40.0 \\
\hline 1978 & March 30 & 6.93 & 560 & 1990 & June 13 & 6.67 & 32.0 \\
\hline 1970 & June 17 & 5.96 & 472 & 1959 & March 14 & 4.42 & 30.0 \\
\hline 1976 & March 24 & 6.39 & 400 & 1981 & February 17 & 4.65 & 28.0 \\
\hline 1962 & March 28 & 6.84 & 390 & 1977 & March 15 & 3.18 & 25.0 \\
\hline 1960 & April 5 & 6.00 & 370 & 1963 & April 6 & 3.47 & 24.0 \\
\hline 1971 & March 31 & 6.20 & 305 & & & & \\
\hline
\end{tabular}




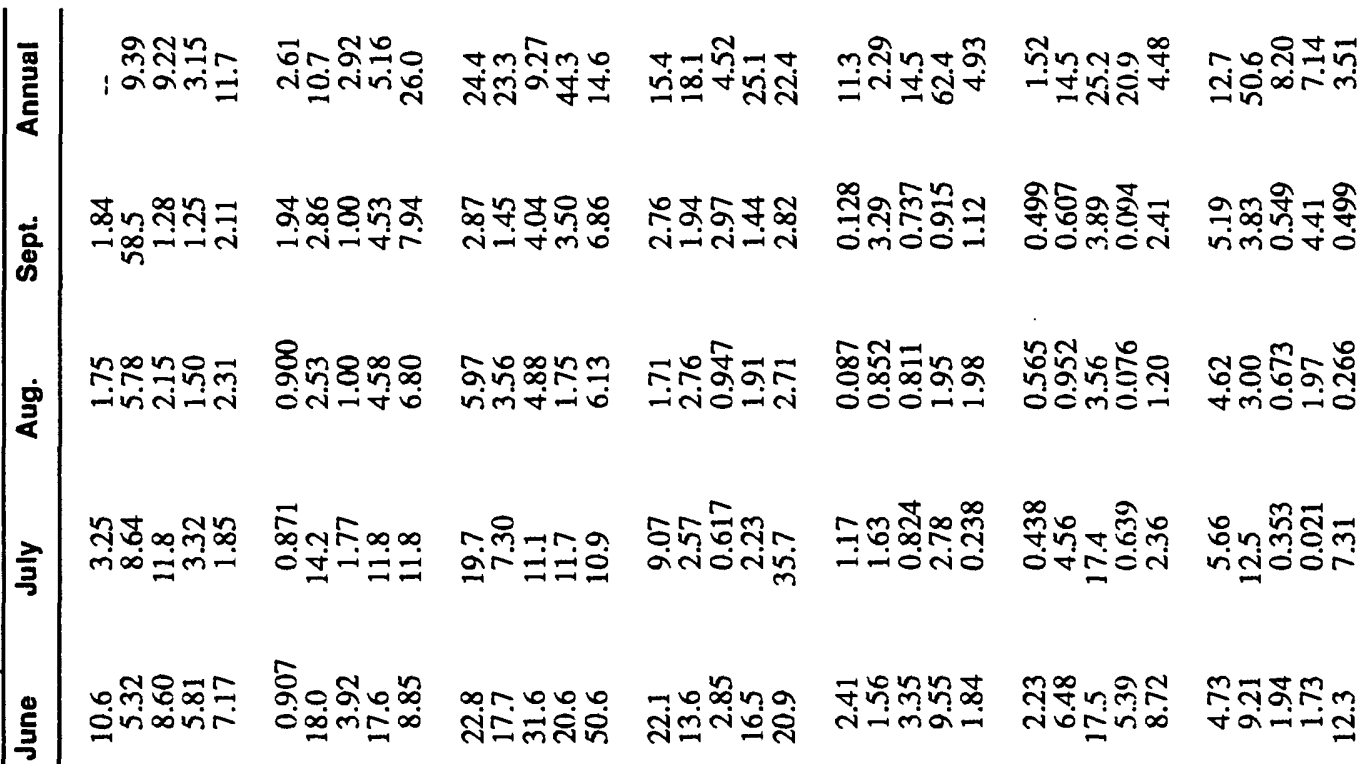

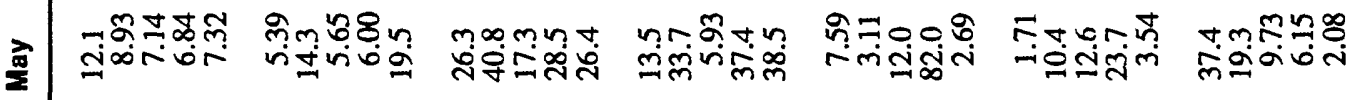

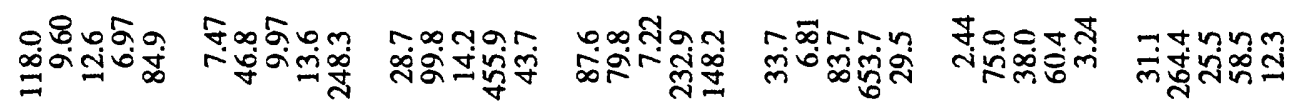

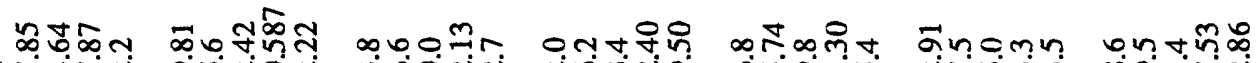

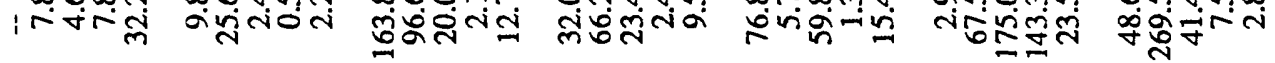
10

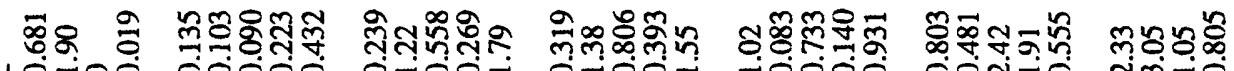

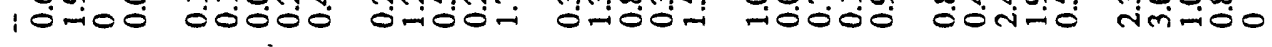

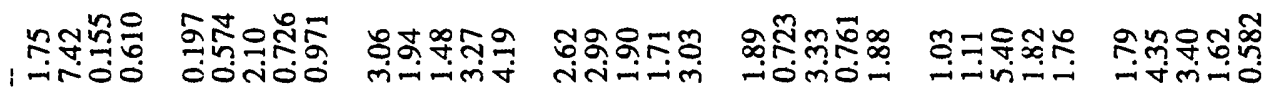

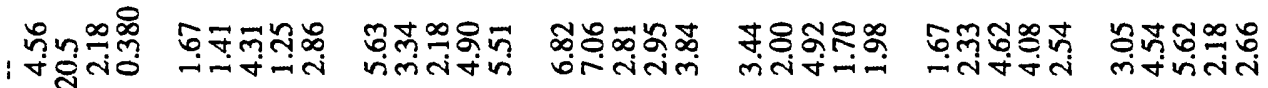

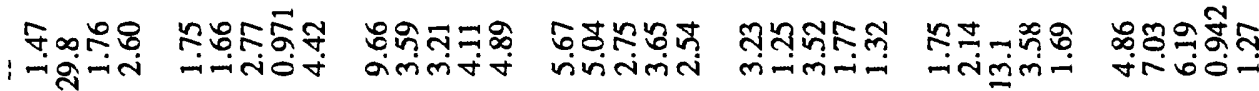

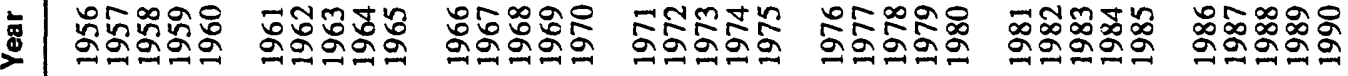




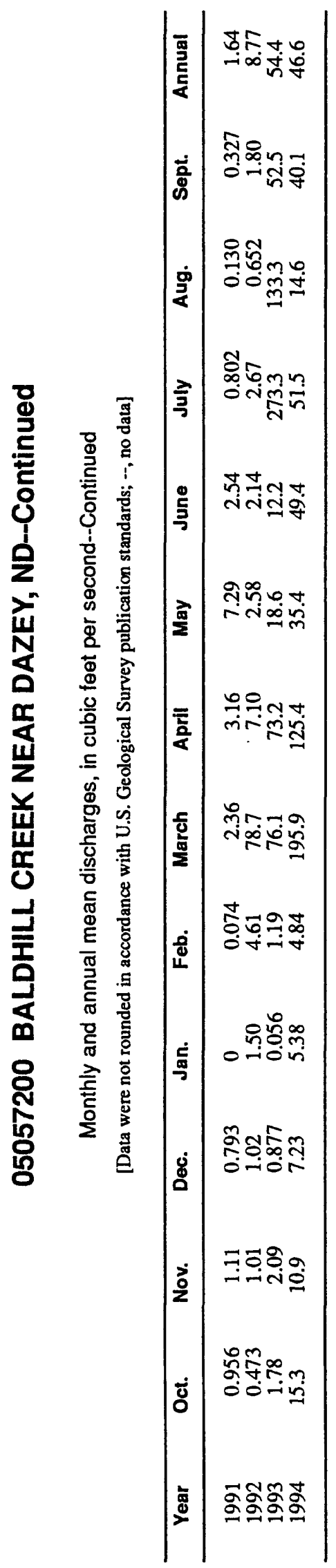




\section{SHEYENNE RIVER BELOW BALDHILL DAM, ND}

\section{Station Description}

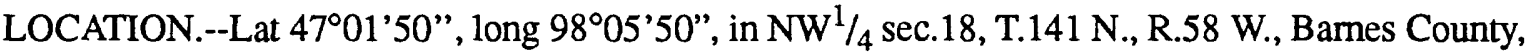
Hydrologic Unit 09020204, on right bank $600 \mathrm{ft}$ downstream from Baldhill Dam, $8 \mathrm{mi}$ northwest of Valley City, and at mile 270.5.

DRAINAGE AREA.--7,470 $\mathrm{mi}^{2}$, approximately, of which about $5,560 \mathrm{mi}^{2}$ is probably noncontributing, including $3,800 \mathrm{mi}^{2}$ in closed basin.

PERIOD OF RECORD.--October 1949 to current year. Monthly discharge only for some periods, published in WSP 1308.

GAGE.--Water-stage recorder and concrete control. Datum of gage is $1,200.00 \mathrm{ft}$ above sea level.

EXTREMES FOR PERIOD OF RECORD.--Maximum discharge, 4,740 $\mathrm{ft}^{3} / \mathrm{s}$, Apr. 24, 1979, gage height, $36.26 \mathrm{ft}$; maximum gage height, $36.26 \mathrm{ft}$, Apr. 24, 1979; minimum discharge, no flow at times in 1950 and 1952-53.

Annual mean discharge

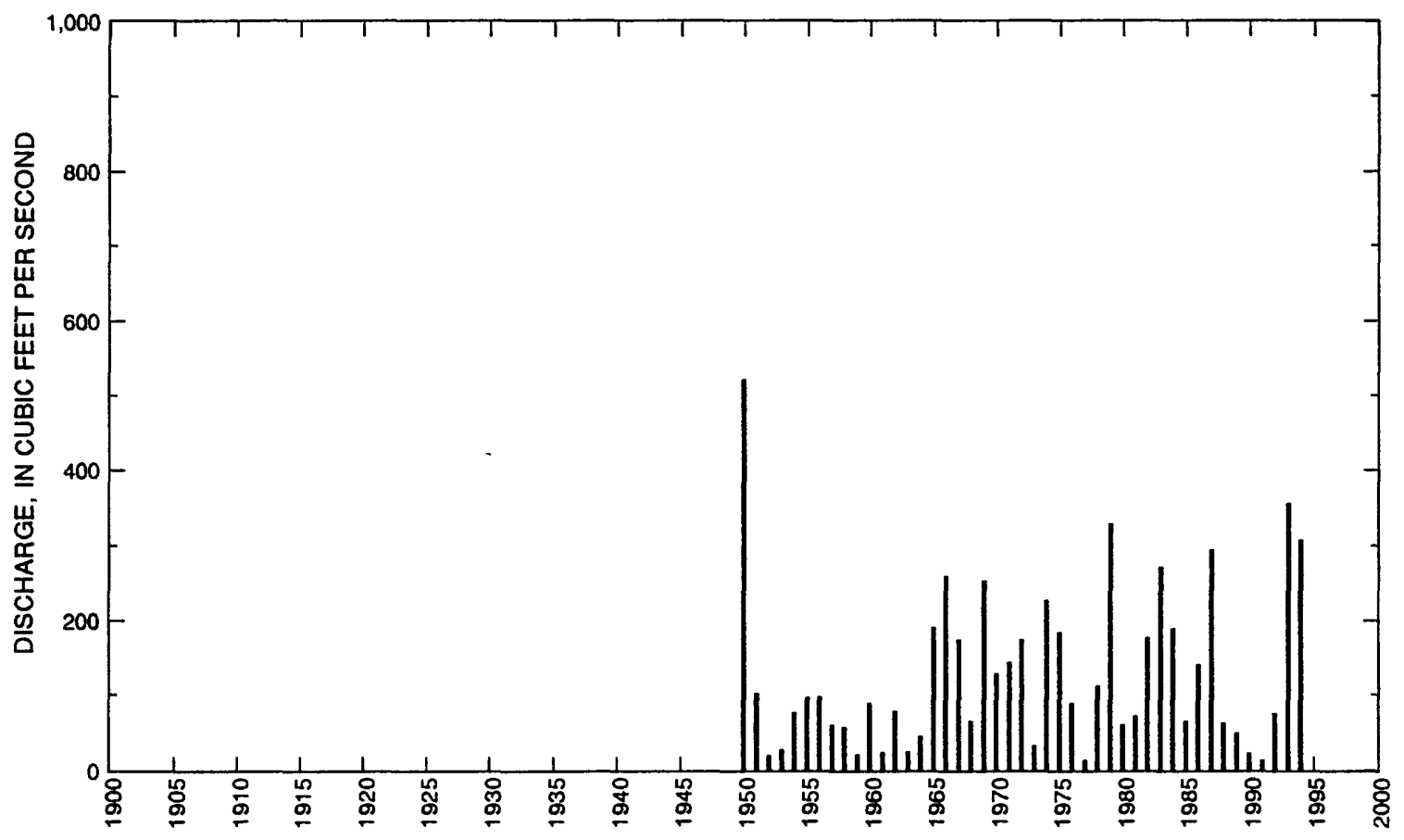




\section{SHEYENNE RIVER BELOW BALDHILL DAM, ND-Continued}

Statistics of monthly and annual mean discharges

\begin{tabular}{|c|c|c|c|c|c|c|c|c|}
\hline \multirow[b]{2}{*}{ Month } & \multicolumn{2}{|c|}{ Maximum } & \multicolumn{2}{|c|}{ Minimum } & \multicolumn{4}{|c|}{ Mean } \\
\hline & $\begin{array}{c}\text { Discharge } \\
\left(\mathrm{ft}^{3} / \mathbf{s}\right)\end{array}$ & $\begin{array}{c}\text { Water year } \\
\text { of } \\
\text { occurrence }\end{array}$ & $\begin{array}{c}\text { Discharge } \\
\left(\mathrm{ft}^{3} / \mathbf{s}\right)\end{array}$ & $\begin{array}{c}\text { Water year } \\
\text { of } \\
\text { occurrence }\end{array}$ & $\begin{array}{c}\text { Discharge } \\
\left(\mathrm{ft}^{3} / \mathrm{s}\right)\end{array}$ & $\begin{array}{c}\text { Standard } \\
\text { deviation } \\
\left(\mathrm{ft}^{3} / \mathbf{s}\right)\end{array}$ & $\begin{array}{l}\text { Coeffi- } \\
\text { cient of } \\
\text { variation }\end{array}$ & $\begin{array}{l}\text { Percentage } \\
\text { of annual } \\
\text { discharge }\end{array}$ \\
\hline October & 153 & 1982 & 1.92 & 1956 & 40.9 & 42.7 & 1.04 & 2.62 \\
\hline November & 156 & 1980 & 5.27 & 1956 & 58.7 & 51.1 & 0.87 & 3.76 \\
\hline December & 191 & 1987 & 4.32 & 1980 & 56.5 & 43.7 & 0.77 & 3.62 \\
\hline January & 140 & 1986 & 3.64 & 1956 & 53.9 & 36.3 & 0.67 & 3.45 \\
\hline February & 200 & 1969 & 7.66 & 1956 & 61.1 & 52.1 & 0.85 & 3.91 \\
\hline March & 1,240 & 1983 & 7.81 & 1955 & 173 & 269 & 1.55 & 11.1 \\
\hline April & 1,940 & 1969 & 2.07 & 1953 & 475 & 512 & 1.08 & 30.4 \\
\hline May & 2,910 & 1950 & 6.86 & 1959 & 251 & 477 & 1.90 & 16.1 \\
\hline June & 1,150 & 1950 & 5.88 & 1958 & 148 & 191 & 1.29 & 9.46 \\
\hline July & 1,270 & 1993 & 7.28 & 1959 & 115 & 203 & 1.77 & 7.36 \\
\hline August & 1,560 & 1993 & 6.72 & 1977 & 74.2 & 231 & 3.11 & 4.76 \\
\hline September & 577 & 1994 & 0.807 & 1955 & 53.3 & 103 & 1.93 & 3.42 \\
\hline Annual & 521 & 1950 & 12.8 & 1991 & 130 & 111 & 0.86 & 100 \\
\hline
\end{tabular}

Annual flow duration

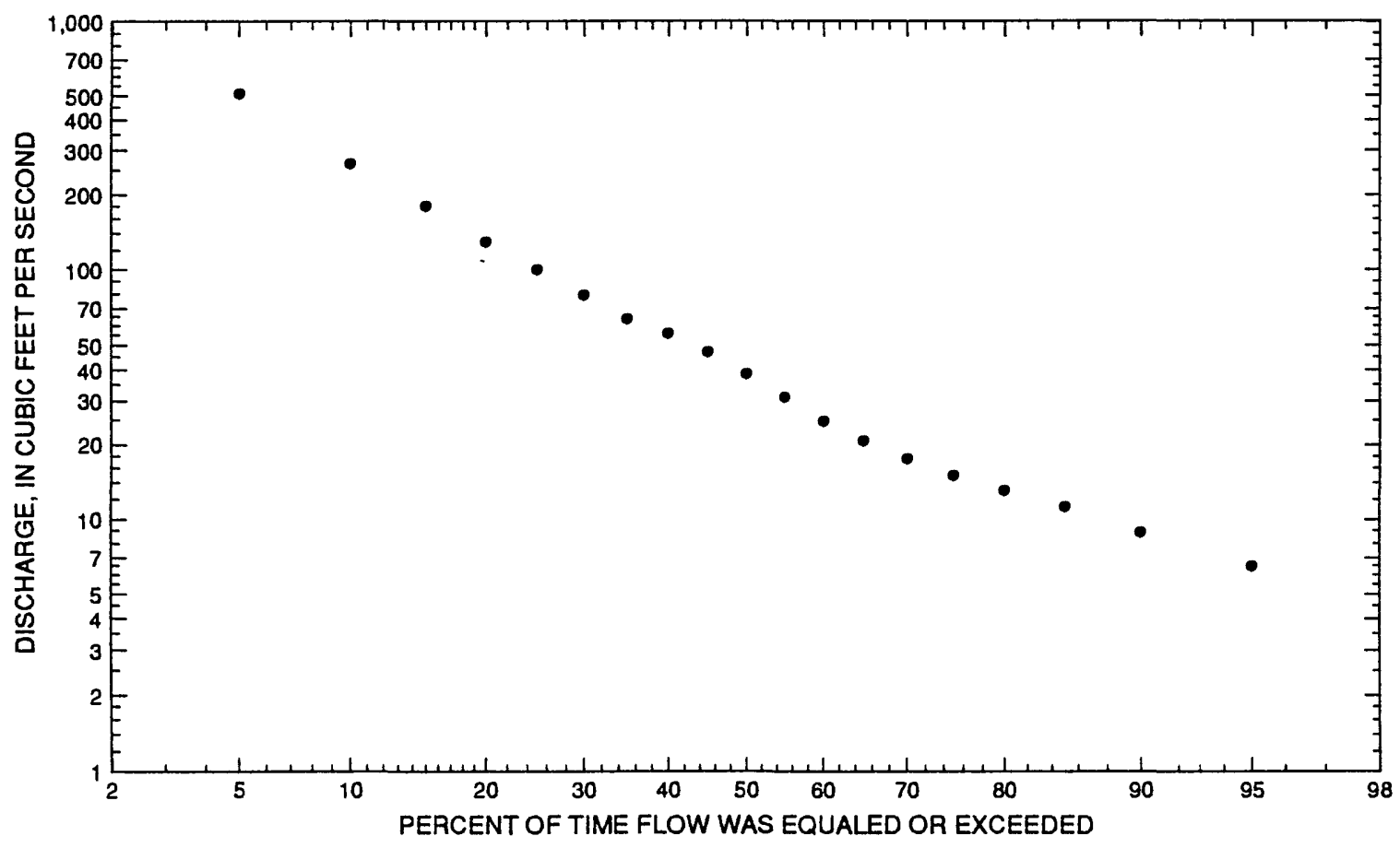




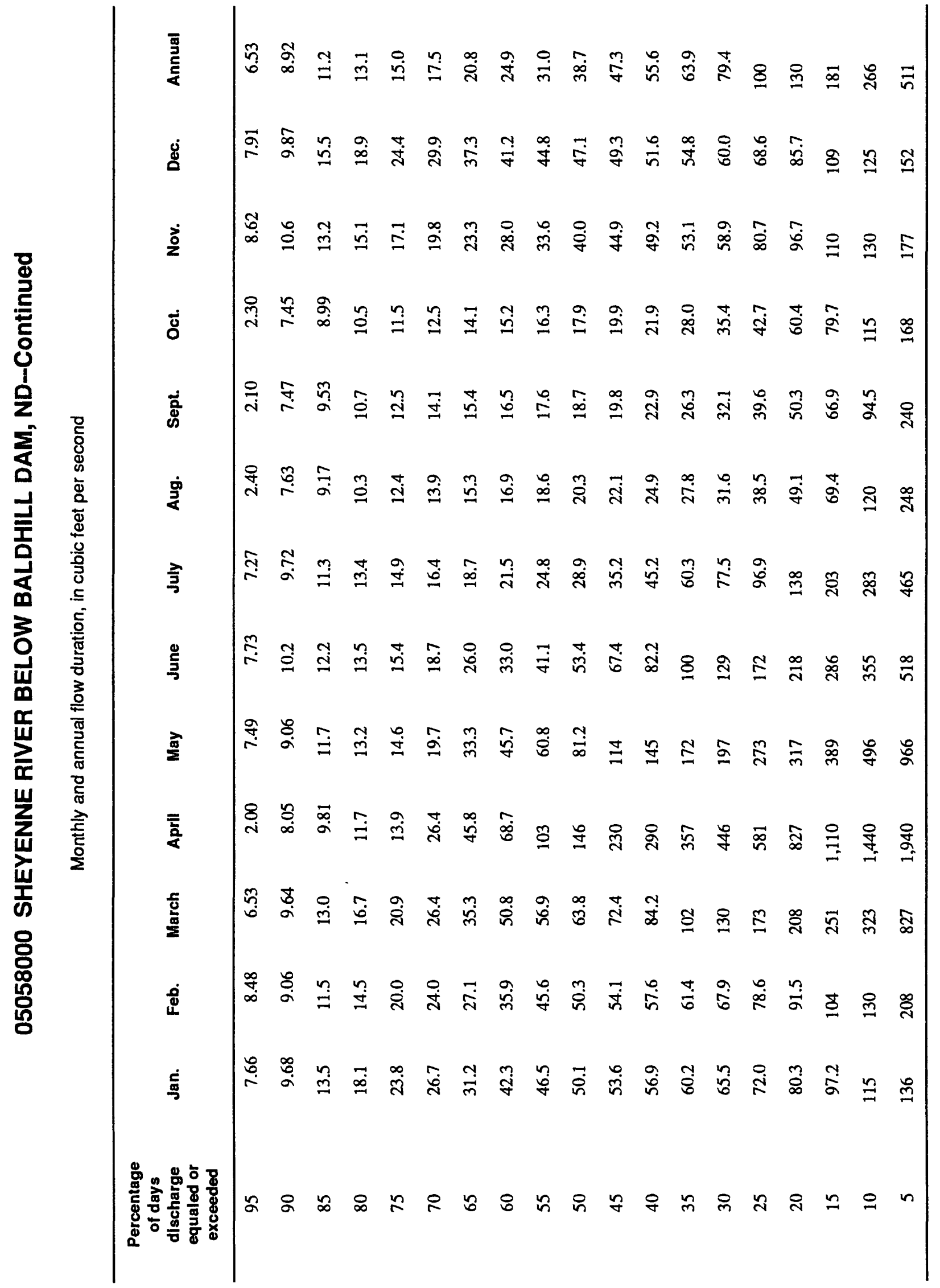




\section{SHEYENNE RIVER BELOW BALDHILL DAM, ND-Continued}

Probability of annual high discharges

[ng, statistic not given]

\begin{tabular}{lcccccc}
\hline & & & \multicolumn{5}{c}{$\begin{array}{c}\text { Maximum average discharge } \\
\left(\mathrm{ft}^{3} / \mathbf{s}\right)\end{array}$} \\
\cline { 5 - 7 } $\begin{array}{c}\text { Exceedance } \\
\text { probability }\end{array}$ & $\begin{array}{c}\text { Recurrence } \\
\text { interval } \\
\text { (years) }\end{array}$ & $\begin{array}{c}\text { Maxlmum } \\
\text { instantaneous } \\
\left(\mathrm{ft}^{3} / \mathbf{s}\right)\end{array}$ & 3-day period & 7-day period & 15-day period & 30-day period \\
\hline 0.99 & 1.01 & 29.1 & 15.3 & 14.6 & 14.4 & 14.3 \\
0.95 & 1.05 & 100 & 64.1 & 57.6 & 50.1 & 42.8 \\
0.90 & 1.11 & 181 & 126 & 111 & 92.4 & 74.2 \\
0.80 & 1.25 & 350 & 265 & 230 & 184 & 139 \\
0.50 & 2 & 1,030 & 875 & 766 & 597 & 422 \\
0.20 & 5 & 2,450 & 2,190 & 2,010 & 1,610 & 1,130 \\
0.10 & 10 & 3,560 & 3,210 & 3,050 & 2,520 & 1,790 \\
0.04 & 25 & 5,020 & 4,500 & 4,470 & 3,880 & 2,840 \\
0.02 & 50 & 6,080 & 5,400 & 5,540 & 4,990 & 3,760 \\
0.01 & 100 & 7,100 & 6,240 & 6,600 & 6,160 & 4,770 \\
0.005 & 200 & 8,070 & 7,000 & 7,620 & 7,380 & 5,880 \\
0.002 & 500 & 9,260 & ng & ng & ng & ng \\
\hline
\end{tabular}

Probability of annual low discharges

Minimum average discharge $\left(\mathrm{ft}^{3} / \mathrm{s}\right)$

Number of consecutive days

\begin{tabular}{|c|c|c|c|c|c|c|c|c|c|c|}
\hline $\begin{array}{l}\text { Non- } \\
\text { exceed- } \\
\text { ance } \\
\text { prob- } \\
\text { ability }\end{array}$ & $\begin{array}{l}\text { Recur- } \\
\text { rence } \\
\text { inter- } \\
\text { val } \\
\text { (years) }\end{array}$ & 1 & 3 & 7 & 14 & 30 & 60 & 90 & 120 & 183 \\
\hline 0.05 & 20 & 0 & 0.006 & 0.015 & 0.258 & 2.08 & 3.92 & 4.68 & 6.15 & 7.62 \\
\hline 0.10 & 10 & 0.090 & 0.201 & 0.430 & 0.870 & 3.10 & 5.02 & 6.15 & 7.91 & 10.5 \\
\hline 0.20 & 5 & 0.580 & 0.938 & 1.71 & 2.48 & 4.48 & 6.84 & 8.63 & 10.8 & 15.4 \\
\hline 0.50 & 2 & 4.01 & 5.73 & 7.95 & 8.97 & 102 & 12.9 & 16.9 & 20.5 & 30.6 \\
\hline
\end{tabular}


05058000 SHEYENNE RIVER BELOW BALDHILL DAM, ND-Continued

Probability of seasonal low discharges

\begin{tabular}{|c|c|c|c|c|c|c|c|c|c|}
\hline \multirow[b]{3}{*}{$\begin{array}{c}\text { Non- } \\
\text { exceedance } \\
\text { probability }\end{array}$} & \multirow[b]{3}{*}{$\begin{array}{l}\text { Recurrence } \\
\text { Interval } \\
\text { (years) }\end{array}$} & \multicolumn{8}{|c|}{ Minimum average discharge $\left(\mathrm{ft}^{3} / \mathrm{s}\right)$} \\
\hline & & \multicolumn{8}{|c|}{ Number of consecutive days } \\
\hline & & 1 & 7 & 14 & 30 & 1 & 7 & 14 & 30 \\
\hline & & \multicolumn{4}{|c|}{ December-January-February } & \multicolumn{4}{|c|}{ March-April-May } \\
\hline 0.05 & 20 & 2.88 & 4.67 & 5.46 & 6.14 & 1.43 & 1.56 & 2.03 & 3.05 \\
\hline 0.10 & 10 & 5.06 & 7.13 & 8.11 & 9.38 & 2.60 & 3.00 & 3.72 & 5.85 \\
\hline 0.20 & 5 & 9.34 & 11.5 & 12.7 & 15.1 & 5.10 & 6.23 & 7.45 & 12.3 \\
\hline \multirow[t]{2}{*}{0.50} & 2 & 24.7 & 26.2 & 27.9 & 33.2 & 16.1 & 21.2 & 25.1 & 43.7 \\
\hline & & \multicolumn{4}{|c|}{ June-July-August } & \multicolumn{4}{|c|}{ September-October-November } \\
\hline 0.05 & 20 & 0.204 & 1.53 & 12.78 & 4.03 & 0.077 & 0.443 & 1.26 & 3.23 \\
\hline 0.10 & 10 & 1.46 & 3.26 & ${ }^{1} 4.43$ & 5.60 & 0.330 & 1.21 & 2.41 & 4.84 \\
\hline 0.20 & 5 & 3.63 & 5.18 & 5.22 & 8.37 & 1.23 & 3.32 & 4.81 & 7.69 \\
\hline 0.50 & 2 & 10.8 & 11.0 & 16.6 & 18.3 & 7.34 & 13.1 & 13.9 & 17.3 \\
\hline
\end{tabular}

${ }^{1}$ Graphical interpretation. 


\section{SHEYENNE RIVER BELOW BALDHILL DAM, ND-Continued}

Annual peak discharge and corresponding gage height, period of record

$$
[-, \text { no data }]
$$

\begin{tabular}{|c|c|c|c|c|c|c|c|}
\hline $\begin{array}{l}\text { Water } \\
\text { year }\end{array}$ & Date & $\begin{array}{c}\text { Gage } \\
\text { height } \\
\text { (feet) }\end{array}$ & $\begin{array}{c}\text { Peak } \\
\text { discharge } \\
\left(\mathrm{ft}^{3} / \mathrm{s}\right)\end{array}$ & $\begin{array}{l}\text { Water } \\
\text { year }\end{array}$ & Date & $\begin{array}{c}\text { Gage } \\
\text { height } \\
\text { (feet) }\end{array}$ & $\begin{array}{c}\text { Peak } \\
\text { discharge } \\
\left(\mathrm{ft}^{3} / \mathrm{s}\right)\end{array}$ \\
\hline \multicolumn{8}{|c|}{ Annual peak discharge, by year, and corresponding gage height } \\
\hline 1948 & April 27 & - & 4,600 & 1972 & April 26 & 27.82 & 991 \\
\hline 1950 & May 23 & 32.62 & 3,150 & 1973 & March 29 & 26.50 & 90.0 \\
\hline 1951 & April 19 & 28.00 & 1,270 & 1974 & April 21 & 30.02 & 1,940 \\
\hline 1952 & August 29 & - & 400 & 1975 & April 29 & 29.20 & 1,640 \\
\hline 1953 & July 16 & 29.40 & 1,980 & 1976 & April 10 & 27.99 & 982 \\
\hline 1954 & March 11 & 28.28 & 1,270 & 1977 & October 1 & 26.16 & 37.0 \\
\hline 1955 & April 13 & 28.86 & 1,640 & 1978 & April 7 & 28.55 & 1,370 \\
\hline 1956 & April 26 & 30.25 & 2,070 & 1979 & April 24 & 36.26 & 4,740 \\
\hline 1957 & September 4 & 27.50 & 598 & 1980 & April 3 & 27.38 & 516 \\
\hline 1958 & July 17 & - & 240 & 1981 & April 3 & 27.37 & 493 \\
\hline 1959 & November 18 & 26.83 & 190 & 1982 & April 12 & 30.01 & 1,930 \\
\hline 1960 & April 17 & 29.24 & 1,570 & 1983 & March 9 & 29.91 & 1,910 \\
\hline 1961 & November 27 & 27.88 & 697 & 1984 & April 3 & 29.50 & 1,790 \\
\hline 1962 & April 10 & 27.67 & 778 & 1985 & May 16 & 27.73 & 680 \\
\hline 1963 & November 15 & 26.30 & 56.0 & 1986 & April 19 & 30.22 & 2,050 \\
\hline 1964 & June 28 & 27.51 & 679 & 1987 & April 14 & 30.51 & 2,200 \\
\hline 1965 & April 14 & 32.69 & 2,980 & 1988 & January 19 & 26.84 & 146 \\
\hline 1966 & March 28 & 32.72 & 3,250 & 1989 & April 4 & 27.60 & 642 \\
\hline 1967 & April 9 & 29.00 & 1,560 & 1990 & September 13 & 26.75 & 123 \\
\hline 1968 & June 11 & 27.92 & 974 & 1991 & July 25 & 26.60 & 82.0 \\
\hline 1969 & April 19 & 35.47 & 4,580 & 1992 & March 7 & 28.57 & 1,260 \\
\hline 1970 & April 19 & 28.23 & 1,230 & 1993 & July 28 & 34.98 & 3,720 \\
\hline 1971 & April 20 & 29.75 & 1,830 & 1994 & April 2 & 29.28 & 1,630 \\
\hline \multicolumn{8}{|c|}{ Annual peak discharge, from highest to lowest, and corresponding gage beight } \\
\hline 1979 & April 24 & 36.26 & 4,740 & 1954 & March 11 & 28.28 & 1,270 \\
\hline 1948 & April 27 & - & 4,600 & 1992 & March 7 & 28.57 & 1,260 \\
\hline 1969 & April 19 & 35.47 & 4,580 & 1970 & April 19 & 28.23 & 1,230 \\
\hline 1993 & July 28 & 34.98 & 3,720 & 1972 & April 26 & 27.82 & 991 \\
\hline 1966 & March 28 & 32.72 & 3,250 & 1976 & April 10 & 27.99 & 982 \\
\hline 1950 & May 23 & 32.62 & 3,150 & 1968 & June 11 & 27.92 & 974 \\
\hline 1965 & April 14 & 32.69 & 2,980 & 1962 & April 10 & 27.67 & 778 \\
\hline 1987 & April 14 & 30.51 & 2,200 & 1961 & November 27 & 27.88 & 697 \\
\hline 1956 & April 26 & 30.25 & 2,070 & 1985 & May 16 & 27.73 & 680 \\
\hline 1986 & April 19 & 30.22 & 2,050 & 1964 & June 28 & 27.51 & 679 \\
\hline 1953 & July 16 & 29.40 & 1,980 & 1989 & April 4 & 27.60 & 642 \\
\hline 1974 & April 21 & 30.02 & 1,940 & 1957 & September 4 & 27.50 & 598 \\
\hline 1982 & April 12 & 30.01 & 1,930 & 1980 & April 3 & 27.38 & 516 \\
\hline 1983 & March 9 & 29.91 & 1,910 & 1981 & April 3 & 27.37 & 493 \\
\hline 1971 & April 20 & 29.75 & 1,830 & 1952 & August 29 & -- & 400 \\
\hline 1984 & April 3 & 29.50 & 1,790 & 1958 & July 17 & -- & 240 \\
\hline 1955 & April 13 & 28.86 & 1,640 & 1958 & November 18 & 26.83 & 190 \\
\hline 1975 & April 29 & 29.20 & 1,640 & 1988 & January 19 & 26.84 & 146 \\
\hline 1994 & April 2 & 29.28 & 1,630 & 1990 & September 13 & 26.75 & 123 \\
\hline 1960 & April 17 & 29.24 & 1,570 & 1973 & March 29 & 26.50 & 90.0 \\
\hline 1967 & April 9 & 29.00 & 1,560 & 1991 & July 25 & 26.60 & 82.0 \\
\hline 1978 & April 7 & 28.55 & 1,370 & 1963 & November 15 & 26.30 & 56.0 \\
\hline 1951 & April 19 & 28.00 & 1,270 & 1976 & October 1 & 26.16 & 37.0 \\
\hline
\end{tabular}




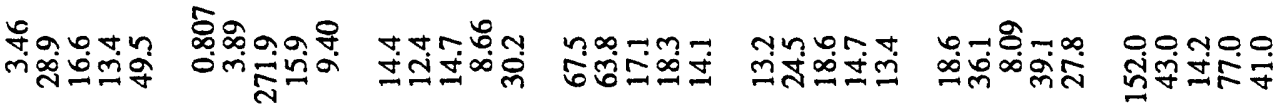

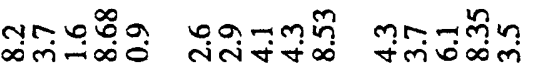

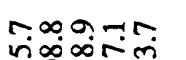

พฺพฺฺ

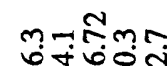

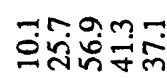

naoun rarom

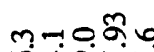

츄-

ทำำำ

ทักั้ํำ

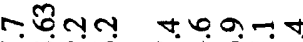

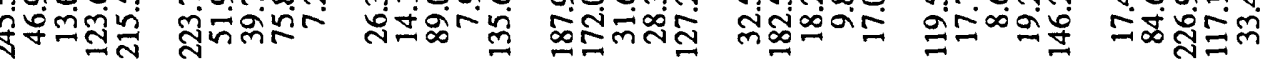

\section{บุทบฺฺ์}

aronn

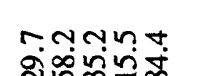

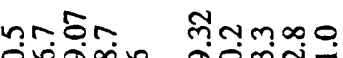
Nㅡ는 ลิ츔ำ

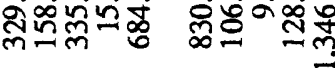

बำฝึंज्ञ

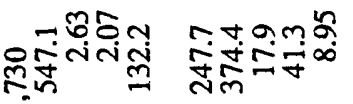

영융ㅎㅇ용

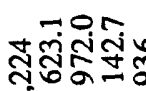

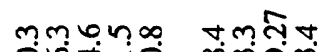

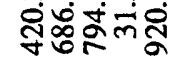

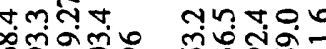

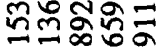

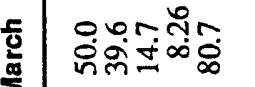

कొmon

क99.5

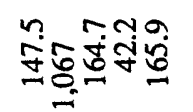

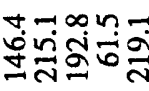

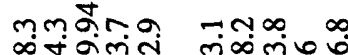

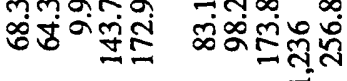

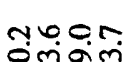

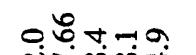

$m r+0.00$

ํํㅇํำ

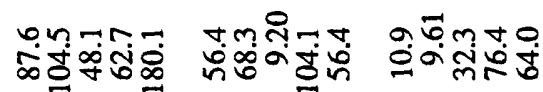

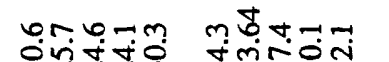

ก⿻ำ

กับสท

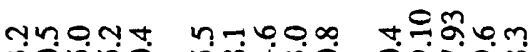

ㅇำำ

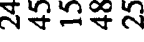

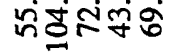

กิำiñ

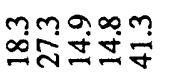

กี่

ㅇํำำำ

mํำ?

otinon

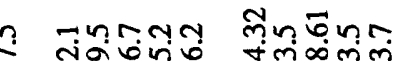

สำn

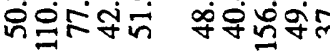

กิ่อำ

अ

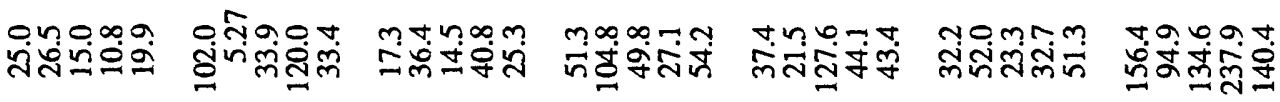

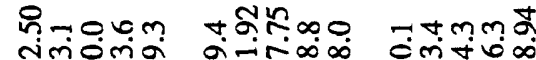

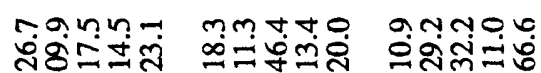

mann

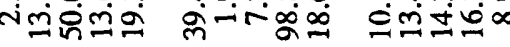
믐

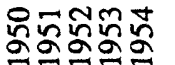




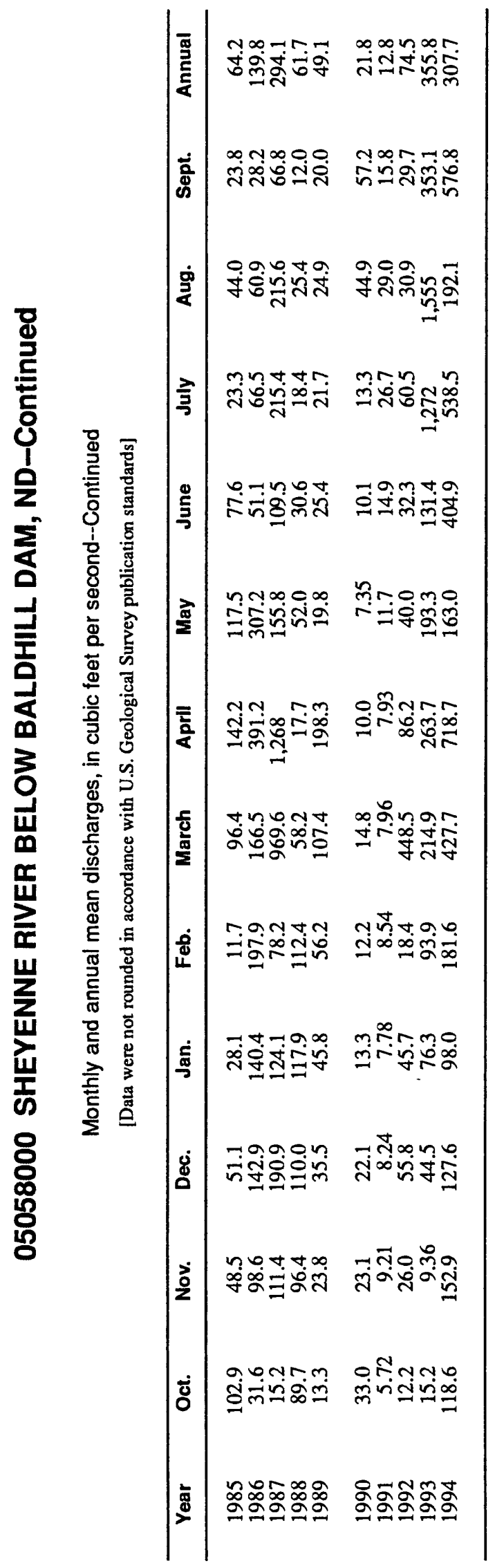




\section{SHEYENNE RIVER AT VALLEY CITY, ND}

\section{Station Description}

LOCATION.--Lat $46^{\circ} 54^{\prime} 50^{\prime \prime}$, long 98 $00^{\prime} 30^{\prime \prime}$, in SE $1 / 4 \mathrm{NW}^{1} / 4$ sec. 28, T.140 N., R.58 W., Barnes County, Hydrologic Unit 09020204, on left bank $100 \mathrm{ft}$ downstream from College Dam in Valley City, and at mile 253.0.

DRAINAGE AREA.--7,810 $\mathrm{mi}^{2}$, approximately, of which about $5,700 \mathrm{mi}^{2}$ is probably noncontributing, includes $3,800 \mathrm{mi}^{2}$ in closed basins.

PERIOD OF RECORD.--March to August 1919, March to June 1938, August to September 1975; October 1979 to current year (gage heights and annual maximum discharge since 1979). Records for July 1938, published in WSP 855, have been found to be unreliable and should not be used.

GAGE.--Water-stage recorder and concrete control. Datum of gage is $1,199.27 \mathrm{ft}$ above sea level. March to August 1919, nonrecording gage at site $0.5 \mathrm{mi}$ upstream at different datum. March to Oct. 13, 1938, nonrecording gage at present site and datum.

EXTREMES FOR PERIOD OF RECORD.--Maximum discharge, 4,580 $\mathrm{ft}^{3} / \mathrm{s}$, Apr. 28, 1948, gage height, $17.51 \mathrm{ft}$; maximum gage height, $18.05 \mathrm{ft}$, July 16 1993; no flow for several periods during 1938-41.

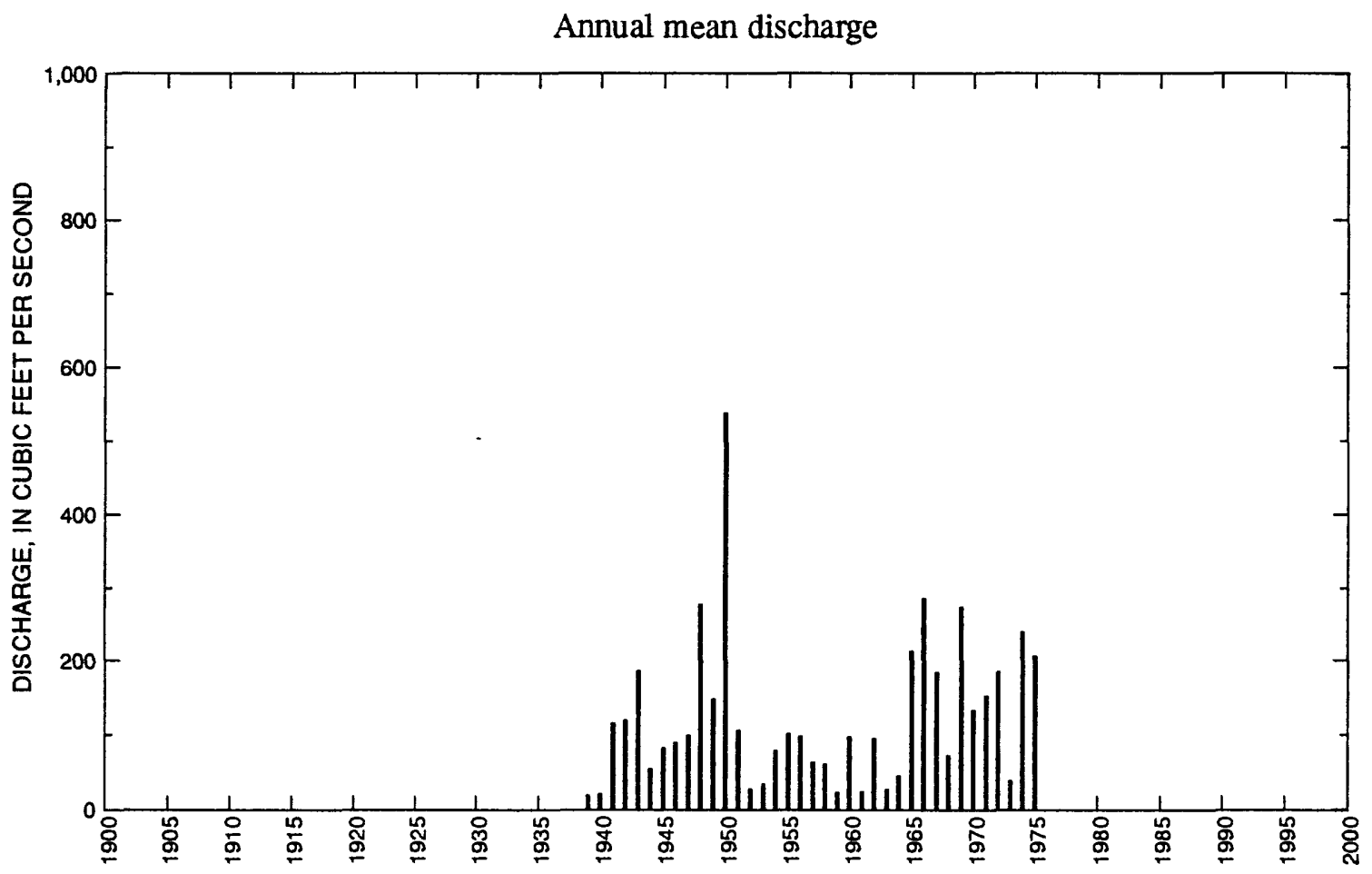


05058500 SHEYENNE RIVER AT VALLEY CITY, ND--Continued

Pre-regulation period

Statistics of monthly and annual mean discharges, pre-regulation period

[m, more than 1 year of occurrence]

\begin{tabular}{|c|c|c|c|c|c|c|c|c|}
\hline \multirow[b]{2}{*}{ Month } & \multicolumn{2}{|c|}{ Maximum } & \multicolumn{2}{|c|}{ Minimum } & \multicolumn{4}{|c|}{ Mean } \\
\hline & $\begin{array}{c}\text { Discharge } \\
\left(\mathrm{ft}^{3} / \mathbf{s}\right)\end{array}$ & $\begin{array}{c}\text { Water year } \\
\text { of } \\
\text { occurrence }\end{array}$ & $\begin{array}{c}\text { Discharge } \\
\left(\mathrm{ft}^{3} / \mathrm{s}\right)\end{array}$ & $\begin{array}{l}\text { Water year } \\
\text { of } \\
\text { occurrence }\end{array}$ & $\begin{array}{c}\text { Discharge } \\
\left(\mathrm{ft}^{3} / \mathbf{s}\right)\end{array}$ & $\begin{array}{l}\text { Standard } \\
\text { deviation } \\
\left(\mathrm{ft}^{3} / \mathrm{s}\right)\end{array}$ & $\begin{array}{l}\text { Coeffi- } \\
\text { cient of } \\
\text { variation }\end{array}$ & $\begin{array}{l}\text { Percentage } \\
\text { of annuai } \\
\text { discharge }\end{array}$ \\
\hline October & 51.9 & 1942 & 0.048 & $\mathrm{~m}$ & 15.5 & 17.4 & 1.12 & 1.18 \\
\hline November & 54.7 & 1945 & 0 & $\mathrm{~m}$ & 19.8 & 17.0 & 0.86 & 1.50 \\
\hline December & 21.6 & 1942 & 0 & 1940 & 11.1 & 9.43 & 0.85 & 0.85 \\
\hline January & 15.3 & 1947 & 0 & $\mathrm{~m}$ & 6.18 & 5.03 & 0.81 & 0.47 \\
\hline February & 20.0 & 1943 & 0 & $\mathrm{~m}$ & 6.21 & 5.95 & 0.96 & 0.47 \\
\hline March & 470 & 1943 & 0 & 1940 & 168 & 187 & 1.11 & 12.8 \\
\hline April & 2,080 & 1948 & 45.3 & 1938 & 702 & 637 & 0.91 & 53.4 \\
\hline May & 960 & 1948 & 21.1 & 1939 & 177 & 256 & 1.44 & 13.5 \\
\hline June & 407 & 1943 & 8.43 & 1938 & 109 & 109 & 1.00 & 8.29 \\
\hline July & 131 & 1943 & 0.316 & 1940 & 52.2 & 38.6 & 0.74 & 3.97 \\
\hline August & 74.5 & 1944 & 0.026 & 1939 & 26.7 & 23.5 & 0.88 & 2.03 \\
\hline September & 100 & 1944 & 0.010 & 1939 & 20.3 & 29.9 & 1.47 & 1.55 \\
\hline Annual & 277 & 1948 & 18.4 & 1939 & 110 & 74.9 & 0.68 & 100 \\
\hline
\end{tabular}

Annual flow duration, pre-regulation period

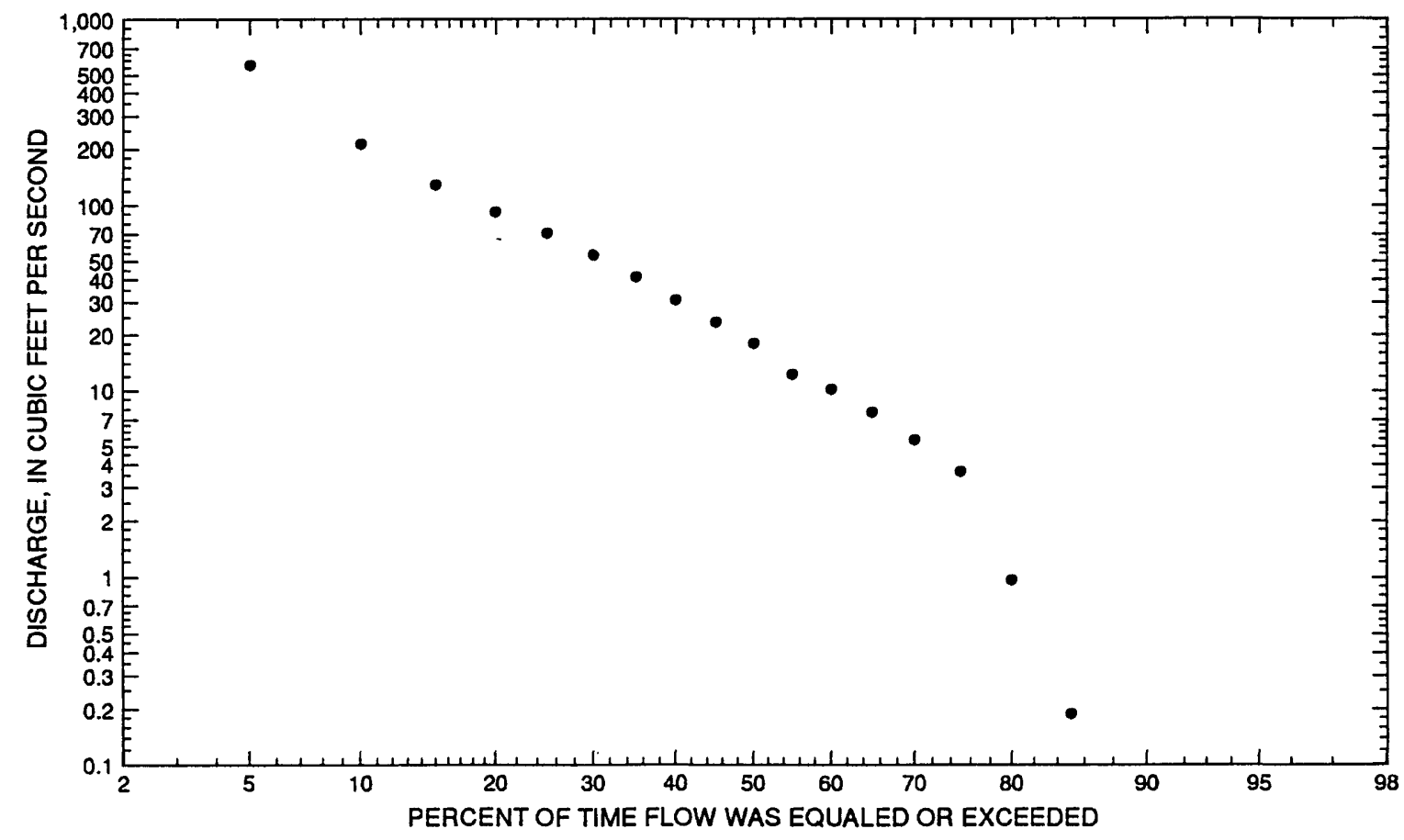




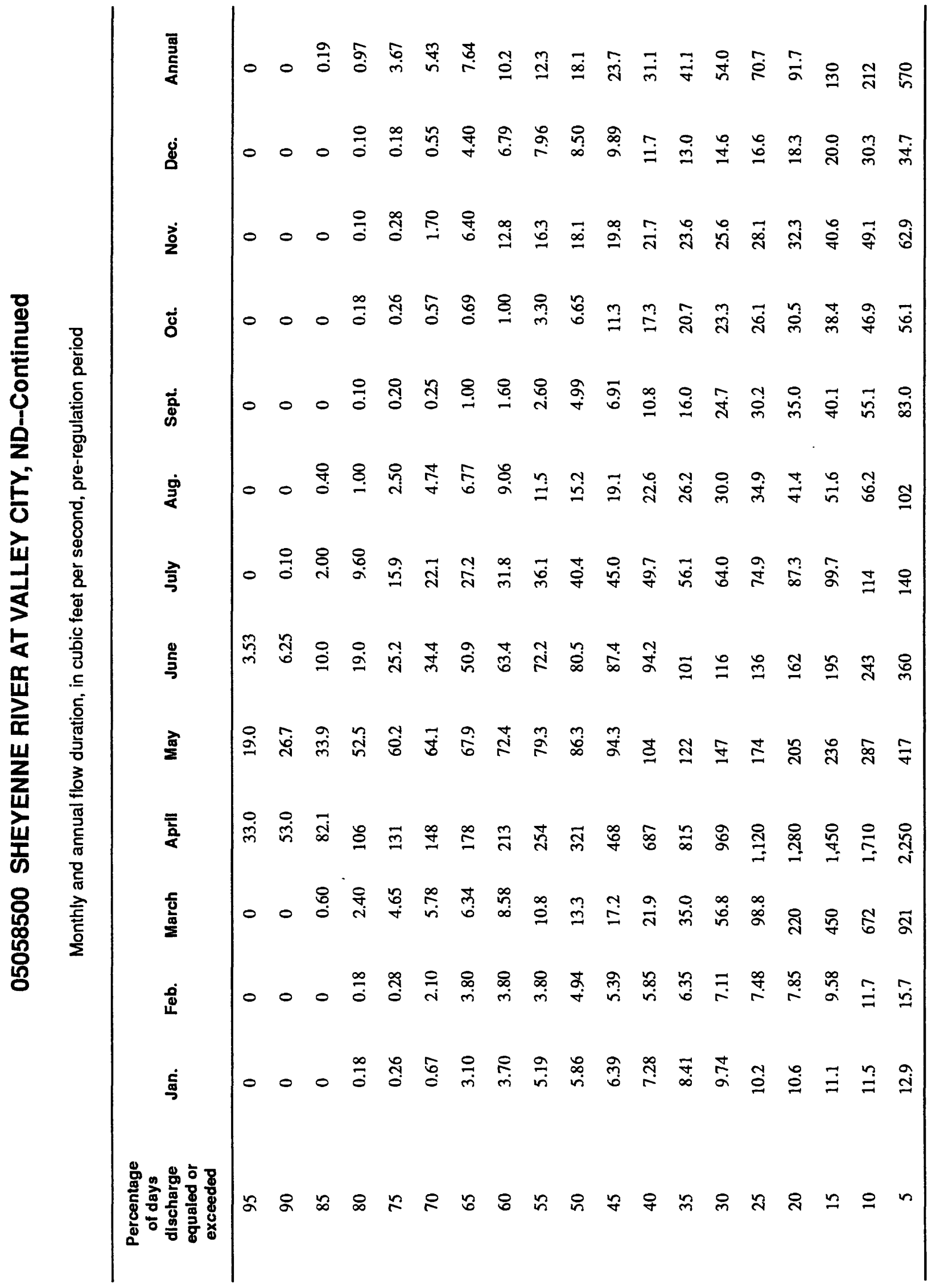




\section{SHEYENNE RIVER AT VALLEY CITY, ND--Continued}

Probability of annual high discharges, pre-regulation period

\begin{tabular}{lcccccc}
\hline & & & \multicolumn{4}{c}{$\begin{array}{c}\text { Maximum average discharge } \\
\left(\mathbf{f t}^{3} / \mathbf{s}\right)\end{array}$} \\
\cline { 5 - 7 } $\begin{array}{c}\text { Exceedance } \\
\text { probability }\end{array}$ & $\begin{array}{c}\text { Recurrence } \\
\text { interval } \\
\text { (years) }\end{array}$ & $\begin{array}{c}\text { Maximum } \\
\text { instantanoous } \\
\left(\mathbf{f t}^{3} / \mathbf{s}\right)\end{array}$ & 3-day period & 7-day period & 15-day period & 30-day period \\
\hline 0.99 & 1.01 & 113 & 127 & 96.5 & 81.0 & 56.8 \\
0.95 & 1.05 & 239 & 252 & 205 & 174 & 124 \\
0.90 & 1.11 & 349 & 358 & 299 & 254 & 184 \\
0.80 & 1.25 & 540 & 538 & 462 & 396 & 291 \\
0.50 & 2 & 1,170 & 1,120 & 1,000 & 863 & 651 \\
0.20 & 5 & 2,350 & 2,200 & 2,020 & 1,730 & 1,340 \\
0.10 & 10 & 3,280 & 3,060 & 2,830 & 2,420 & 1,900 \\
0.04 & 25 & 4,580 & 4,270 & 3,950 & 3,370 & 2,690 \\
0.02 & 50 & 5,620 & 5,260 & 4,850 & 4,130 & 3,320 \\
0.01 & 100 & 6,710 & 6,300 & 5,790 & 4,910 & 3,990 \\
0.005 & 200 & 7,840 & 7,400 & 6,770 & 5,720 & 4,680 \\
\hline
\end{tabular}

Probability of annual low discharges, pre-regulation period

\begin{tabular}{|c|c|c|c|c|c|c|c|c|c|c|}
\hline \multirow[b]{3}{*}{$\begin{array}{l}\text { Non- } \\
\text { exceed- } \\
\text { ance } \\
\text { prob- } \\
\text { ability }\end{array}$} & \multirow[b]{3}{*}{$\begin{array}{l}\text { Recur- } \\
\text { rence } \\
\text { inter- } \\
\text { val } \\
\text { (years) }\end{array}$} & \multicolumn{9}{|c|}{ Minimum average discharge $\left(\mathrm{ft}^{3} / \mathrm{s}\right)$} \\
\hline & & \multicolumn{9}{|c|}{ Number of consecutive days } \\
\hline & & 1 & 3 & 7 & 14 & 30 & 60 & 90 & 120 & 183 \\
\hline 0.05 & 20 & 0 & 0 & 0 & 0 & 0 & 0 & 0 & 0 & 0 \\
\hline 0.10 & 10 & 0 & 0 & 0 & 0 & 0 & 0 & 0 & 0 & 0 \\
\hline 0.20 & 5 & 0 & 0 & 0 & 0 & 0 & 0 & 0 & 0 & 0.559 \\
\hline 0.50 & 2 & 0.553 & 0.685 & 0.903 & 1.34 & 2.57 & 5.76 & 7.01 & 9.74 & 13.6 \\
\hline
\end{tabular}




\section{SHEYENNE RIVER AT VALLEY CITY, ND-Continued}

Probability of seasonal low discharges, pre-regulation period

\begin{tabular}{|c|c|c|c|c|c|c|c|c|c|}
\hline \multirow[b]{3}{*}{$\begin{array}{c}\text { Non- } \\
\text { exceedance } \\
\text { probability }\end{array}$} & \multirow[b]{3}{*}{$\begin{array}{l}\text { Recurrence } \\
\text { Interval } \\
\text { (years) }\end{array}$} & \multicolumn{8}{|c|}{ Minimum average discharge $\left(\mathrm{ft}^{3} / \mathrm{s}\right)$} \\
\hline & & \multicolumn{8}{|c|}{ Number of consecutive days } \\
\hline & & 1 & 7 & 14 & 30 & 1 & 7 & 14 & 30 \\
\hline & & \multicolumn{4}{|c|}{ December-January-February } & \multicolumn{4}{|c|}{ March-April-May } \\
\hline 0.05 & 20 & $\mathbf{0}$ & $\mathbf{0}$ & $\mathbf{0}$ & 0 & 0 & $\mathbf{0}$ & $\mathbf{0}$ & 0 \\
\hline 0.10 & 10 & 0 & 0 & $\mathbf{0}$ & 0 & $\mathbf{0}$ & 0 & $\mathbf{0}$ & 8.12 \\
\hline 0.20 & 5 & 0 & 0.056 & 0.066 & 0.086 & 0.493 & 0.914 & 2.12 & 18.9 \\
\hline \multirow[t]{2}{*}{0.50} & 2 & 0.875 & 1.16 & 2.58 & 4.54 & 3.38 & 4.76 & 8.51 & 43.1 \\
\hline & & \multicolumn{4}{|c|}{ June-July-August } & \multicolumn{4}{|c|}{ September-October-November } \\
\hline 0.05 & 20 & 0 & 0 & 0 & 0.036 & 0 & 0 & 0 & 0 \\
\hline 0.10 & 10 & 0 & 0 & 0.002 & 0.197 & 0 & 0 & 0 & 0 \\
\hline 0.20 & 5 & 0.135 & 0.349 & 0.503 & 1.14 & 0 & 0 & 0 & 0.049 \\
\hline 0.50 & 2 & 3.36 & 6.53 & 9.91 & 13.6 & 0.865 & 1.53 & 1.98 & 3.11 \\
\hline
\end{tabular}




\section{SHEYENNE RIVER AT VALLEY CITY, ND--Continued}

\section{Post-regulation period}

Statistics of monthly and annual mean discharges, post-regulation period

[m, more than 1 year of occurrence]

\begin{tabular}{|c|c|c|c|c|c|c|c|c|}
\hline \multirow[b]{2}{*}{ Month } & \multicolumn{2}{|c|}{ Maximum } & \multicolumn{2}{|c|}{ Minimum } & \multicolumn{4}{|c|}{ Mean } \\
\hline & $\begin{array}{c}\text { Discharge } \\
\left(\mathrm{ft}^{3} / \mathrm{s}\right)\end{array}$ & $\begin{array}{c}\text { Water year } \\
\text { of } \\
\text { occurrence }\end{array}$ & $\begin{array}{c}\text { Discharge } \\
\left(\mathrm{ft}^{3} / \mathrm{s}\right)\end{array}$ & $\begin{array}{c}\text { Water year } \\
\text { of } \\
\text { occurrence }\end{array}$ & $\begin{array}{c}\text { Discharge } \\
\left(\mathrm{ft}^{3} / \mathrm{s}\right)\end{array}$ & $\begin{array}{c}\text { Standard } \\
\text { deviation } \\
\left(\mathrm{ft}^{3} / \mathrm{s}\right)\end{array}$ & $\begin{array}{l}\text { Coeffi- } \\
\text { cient of } \\
\text { variation }\end{array}$ & $\begin{array}{l}\text { Percentage } \\
\text { of annuail } \\
\text { discharge }\end{array}$ \\
\hline October & 116 & 1983 & 1.29 & 1956 & 30.5 & 33.0 & 1.08 & 1.83 \\
\hline November & 192 & 1983 & 2.19 & 1956 & 48.9 & 45.0 & 0.92 & 2.93 \\
\hline December & 155 & 1972 & 6.27 & 1956 & 49.2 & 31.5 & 0.64 & 2.94 \\
\hline January & 119 & 1972 & 2.66 & 1956 & 50.8 & 29.3 & 0.58 & 3.04 \\
\hline February & 300 & 1994 & 7.17 & 1956 & 70.1 & 6,801 & 0.97 & 4.20 \\
\hline March & 1,230 & 1966 & 10.0 & 1964 & 164 & 233 & 1.42 & 9.81 \\
\hline April & 2,090 & 1969 & 3.19 & 1953 & 547 & 569 & 1.04 & 32.8 \\
\hline May & 2,980 & 1950 & 9.77 & 1959 & 302 & 570 & 1.89 & 18.1 \\
\hline June & 1,230 & 1950 & 8.49 & 1958 & 201 & 236 & 1.17 & 12.0 \\
\hline July & 501 & 1994 & 10.2 & 1963 & 112 & 110 & 0.98 & 6.69 \\
\hline August & 216 & 1966 & 7.94 & 1963 & 38.9 & 47.6 & 1.22 & 2.33 \\
\hline September & 507 & 1994 & 2.29 & $\mathrm{~m}$ & 55.7 & 109 & 1.96 & 3.34 \\
\hline Annual & 537 & 1950 & 22.5 & 1959 & 130 & 115 & 0.88 & 100 \\
\hline
\end{tabular}

Annual flow duration, post-regulation period

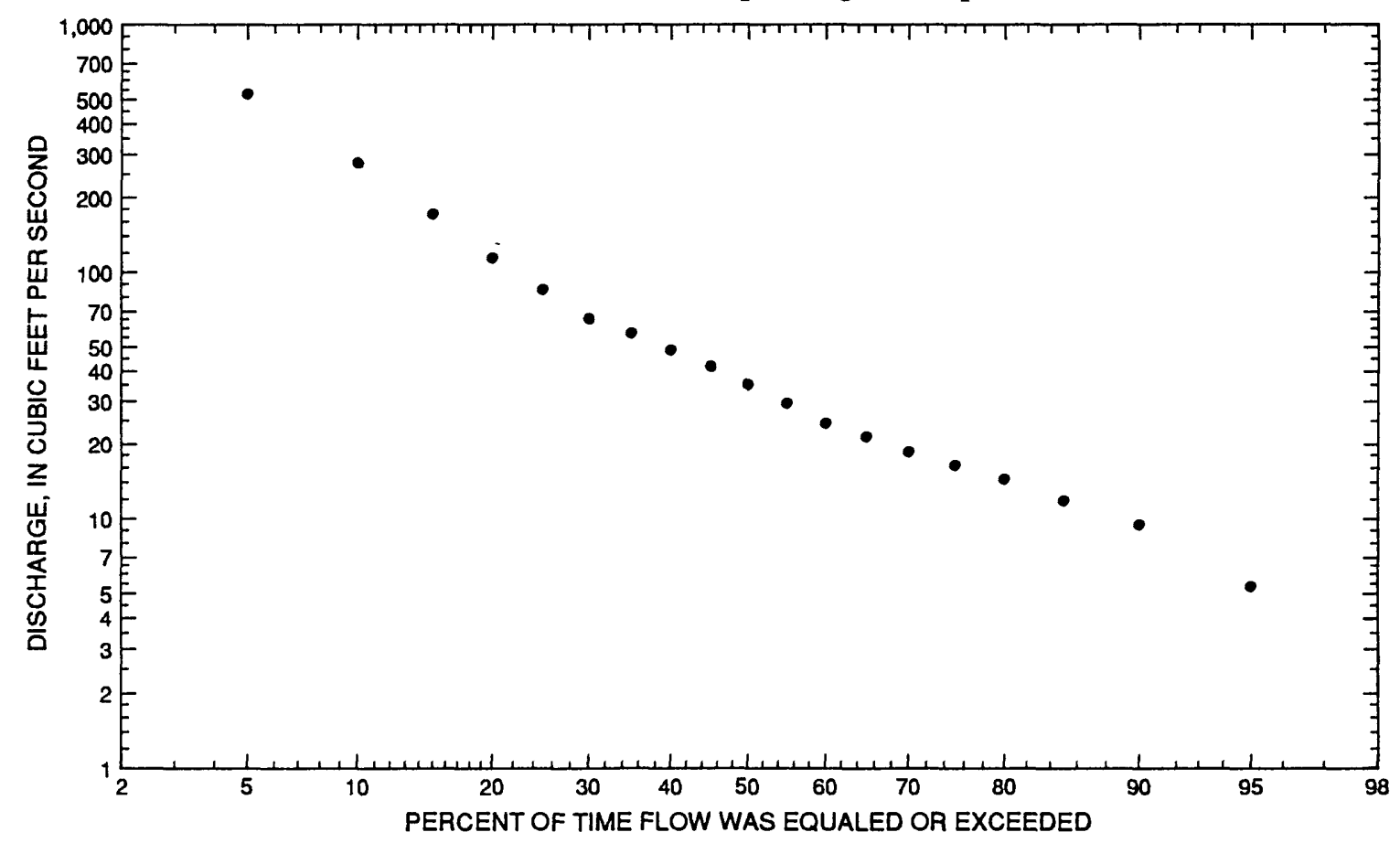




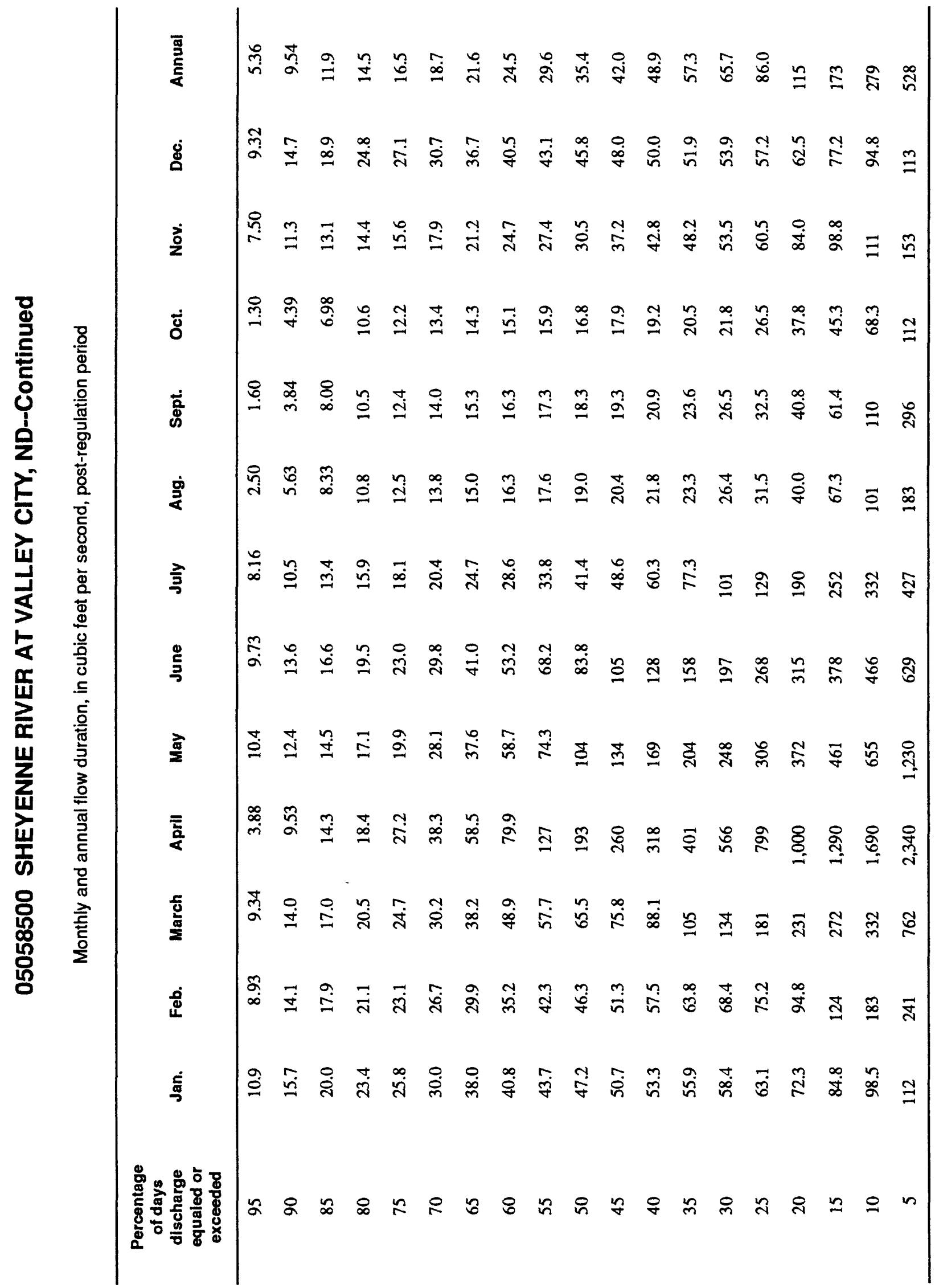




\section{SHEYENNE RIVER AT VALLEY CITY, ND--Continued}

Probability of annual high discharges, post-regulation period

[ng, statistic not given]

\begin{tabular}{lcccccc}
\hline & & & \multicolumn{5}{c}{$\begin{array}{c}\text { Maximum average discharge } \\
\left(\mathbf{f t}^{3} / \mathbf{s}\right)\end{array}$} \\
\cline { 5 - 7 } $\begin{array}{c}\text { Exceedance } \\
\text { probability }\end{array}$ & $\begin{array}{c}\text { Recurrence } \\
\text { interval } \\
\text { (years) }\end{array}$ & $\begin{array}{c}\text { Maximum } \\
\text { instantaneous } \\
\left(\mathbf{f t}^{3} / \mathbf{s}\right)\end{array}$ & 3-day period & 7-day period & 15-day period & 30-day period \\
\hline 0.99 & 1.01 & ng & 35.2 & 24.3 & 17.7 & 16.3 \\
0.95 & 1.05 & 201 & 108 & 78.3 & 56.9 & 47.5 \\
0.90 & 1.11 & 316 & 185 & 138 & 101 & 80.5 \\
0.80 & 1.25 & 523 & 337 & 262 & 193 & 147 \\
0.50 & 2 & 1,200 & 911 & 773 & 586 & 415 \\
0.20 & 5 & 2,320 & 2,040 & 1,900 & 1,500 & 1,020 \\
0.10 & 10 & 3,100 & 2,900 & 2,840 & 2,310 & 1,550 \\
0.04 & 25 & 4,030 & 4,120 & 4,160 & 3,490 & 2,330 \\
0.02 & 50 & 4,680 & 4,830 & 5,180 & 4,440 & 2,960 \\
0.01 & 100 & 5,280 & 5,620 & 6,220 & 5,450 & 3,640 \\
0.005 & 200 & 5,830 & 6,370 & 7,260 & 6,480 & 4,340 \\
\hline
\end{tabular}

Probability of annual low discharges, post-regulation period

\begin{tabular}{|c|c|c|c|c|c|c|c|c|c|c|}
\hline \multirow[b]{3}{*}{$\begin{array}{l}\text { Non- } \\
\text { exceed- } \\
\text { ance } \\
\text { prob- } \\
\text { ability }\end{array}$} & \multirow[b]{3}{*}{$\begin{array}{c}\text { Recur- } \\
\text { rence } \\
\text { inter- } \\
\text { val } \\
\text { (years) }\end{array}$} & \multicolumn{9}{|c|}{ Minimum average discharge $\left(\mathrm{ft}^{3} / \mathrm{s}\right)$} \\
\hline & & \multicolumn{9}{|c|}{ Number of consecutive days } \\
\hline & & 1 & 3 & 7 & 14 & 30 & 60 & 90 & 120 & 183 \\
\hline 0.05 & 20 & ${ }^{1} 0.190$ & 0.309 & 0.810 & 1.35 & 2.32 & 3.30 & 4.31 & 5.43 & 7.01 \\
\hline 0.10 & 10 & ${ }^{1} 0.200$ & 0.605 & 1.41 & 2.18 & 3.61 & 4.76 & 6.14 & 7.44 & 9.96 \\
\hline 0.20 & 5 & ${ }^{1} 0.800$ & 1.27 & 2.58 & 3.70 & 5.76 & 7.20 & 9.15 & 10.7 & 14.8 \\
\hline 0.50 & 2 & ${ }^{1} 1.80$ & 4.24 & 6.66 & 8.52 & 11.7 & 14.4 & 17.9 & 20.2 & 28.9 \\
\hline
\end{tabular}

${ }^{1}$ Graphical interpretation. 


\section{SHEYENNE RIVER AT VALLEY CITY, ND--Continued}

Probability of seasonal low discharges, post-regulation period

\begin{tabular}{|c|c|c|c|c|c|c|c|c|c|}
\hline \multirow[b]{3}{*}{$\begin{array}{c}\text { Non- } \\
\text { exceedance } \\
\text { probability }\end{array}$} & \multirow[b]{3}{*}{$\begin{array}{l}\text { Recurrence } \\
\text { interval } \\
\text { (years) }\end{array}$} & \multicolumn{8}{|c|}{ Minimum average discharge $\left(\mathrm{ft}^{3} / \mathrm{s}\right)$} \\
\hline & & \multicolumn{8}{|c|}{ Number of consecutive days } \\
\hline & & 1 & 7 & 14 & 30 & 1 & 7 & 14 & 30 \\
\hline & & \multicolumn{4}{|c|}{ December-January-February } & \multicolumn{4}{|c|}{ March-April-May } \\
\hline 0.05 & 20 & 3.76 & 4.64 & 5.97 & 7.30 & 0.687 & 1.76 & 2.86 & 4.06 \\
\hline 0.10 & 10 & 6.74 & 7.88 & 9.59 & 12.1 & 1.48 & 3.10 & 4.67 & 7.38 \\
\hline 0.20 & 5 & 12.2 & 13.6 & 15.7 & 20.1 & 3.49 & 5.96 & 8.39 & 14.7 \\
\hline \multirow[t]{2}{*}{0.50} & 2 & 27.6 & 29.8 & 32.3 & 39.8 & 14.5 & 18.9 & 25.0 & 48.4 \\
\hline & & \multicolumn{4}{|c|}{ June-July-August } & \multicolumn{4}{|c|}{ September-October-November } \\
\hline 0.05 & 20 & 0.339 & 1.84 & 3.05 & 6.77 & 0.373 & 1.18 & 1.81 & 2.70 \\
\hline 0.10 & 10 & 0.792 & 2.83 & 4.02 & 7.72 & 0.744 & 2.13 & 3.01 & 4.19 \\
\hline 0.20 & 5 & 1.90 & 4.53 & 5.64 & 9.40 & 1.60 & 3.99 & 5.24 & 6.82 \\
\hline 0.50 & 2 & 6.49 & 9.79 & 10.8 & 15.7 & 5.62 & 10.4 & 12.5 & 15.4 \\
\hline
\end{tabular}




\section{SHEYENNE RIVER AT VALLEY CITY, ND--Continued}

Annual peak discharge and corresponding gage height, period of record

\begin{tabular}{|c|c|c|c|c|c|c|c|}
\hline $\begin{array}{l}\text { Water } \\
\text { year }\end{array}$ & Date & $\begin{array}{c}\text { Gage } \\
\text { height } \\
\text { (feet) }\end{array}$ & $\begin{array}{c}\text { Peak } \\
\text { discharge } \\
\left(\mathrm{ft}^{3} / \mathrm{s}\right)\end{array}$ & $\begin{array}{l}\text { Water } \\
\text { year }\end{array}$ & Date & $\begin{array}{c}\text { Gage } \\
\text { height } \\
\text { (feet) }\end{array}$ & $\begin{array}{c}\text { Peak } \\
\text { discharge } \\
\left(\mathrm{ft}^{3} / \mathrm{s}\right)\end{array}$ \\
\hline \multicolumn{8}{|c|}{ Annual peak discharge, by year, and corresponding gage height } \\
\hline 1919 & April 18 & 14.90 & 2,750 & 1964 & June 28 & 6.56 & 643 \\
\hline 1938 & March 29 & 3.90 & 244 & 1965 & April 16 & 14.93 & 3,080 \\
\hline 1939 & April 6 & 4.34 & 342 & 1966 & March 28 & 14.27 & 3,350 \\
\hline 1940 & April 8 & 5.14 & 525 & 1967 & April 10 & 9.03 & 1,570 \\
\hline 1941 & April 19 & 9.10 & 1,590 & 1968 & June 13 & 8.48 & 1,220 \\
\hline 1942 & April 17 & 7.72 & 1,190 & 1969 & April 19 & 17.62 & 4,520 \\
\hline 1943 & March 27 & 10.78 & 1,900 & 1970 & April 20 & 8.47 & 1,370 \\
\hline 1944 & June 3 & 4.50 & 399 & 1971 & April 21 & 10.27 & 1,820 \\
\hline 1945 & March 24 & 7.06 & 1,020 & 1972 & April 16 & 7.23 & 1,070 \\
\hline 1946 & March 26 & 7.56 & 1,160 & 1973 & March 14 & 6.32 & 761 \\
\hline 1947 & April 11 & 10.19 & 1,910 & 1974 & April 22 & 10.74 & 2,160 \\
\hline 1948 & April 28 & 17.51 & 4,580 & 1975 & June 30 & 10.27 & 1,960 \\
\hline 1949 & April 21 & 10.90 & 2,120 & 1980 & August 29 & 7.00 & 765 \\
\hline 1950 & May 5 & 14.60 & 3,050 & 1981 & April 2 & 5.91 & 542 \\
\hline 1951 & April 19 & 8.08 & 1,270 & 1982 & April 14 & 10.95 & 1,990 \\
\hline 1952 & March 31 & 6.02 & 650 & 1983 & March 13 & 11.26 & 2,030 \\
\hline 1953 & July 17 & 8.11 & 1,230 & 1984 & April 6 & 10.43 & 1,950 \\
\hline 1954 & July 13 & 6.29 & 726 & 1985 & May 17 & 6.37 & 680 \\
\hline 1955 & April 14 & 9.04 & 1,490 & 1986 & April 19 & 11.04 & 2,100 \\
\hline 1956 & April 28 & 10.58 & 1,870 & 1987 & April 13 & 11.43 & 2,300 \\
\hline 1957 & September 4 & 6.48 & 627 & 1988 & February 28 & 4.80 & 150 \\
\hline 1958 & July 17 & 4.21 & 248 & 1989 & March 30 & 8.38 & 850 \\
\hline 1959 & June 27 & 7.02 & 988 & 1990 & July 11 & 4.59 & 278 \\
\hline 1960 & April 17 & 9.52 & 1,570 & 1991 & August 24 & 3.37 & 77.0 \\
\hline 1961 & March 2 & 4.01 & 251 & 1992 & March 8 & 8.59 & 1,260 \\
\hline 1962 & July 7 & 9.71 & 1,270 & 1993 & July 28 & 17.30 & 3,830 \\
\hline 1963 & July 26 & 3.39 & 78.0 & 1994 & April 9 & 9.00 & 1,530 \\
\hline \multicolumn{8}{|c|}{ Annual peak discharge, from highest to lowest, and corresponding gage height } \\
\hline 1948 & April 28 & 17.51 & 4,580 & 1943 & March 27 & 10.78 & 1,900 \\
\hline 1969 & April 19 & 17.62 & 4,520 & 1956 & April 28 & 10.58 & 1,870 \\
\hline 1993 & July 28 & 17.30 & 3,830 & 1971 & April 21 & 10.27 & 1,820 \\
\hline 1966 & March 28 & 14.27 & 3,350 & 1941 & April 19 & 9.10 & 1,590 \\
\hline 1965 & April 16 & 14.93 & 3,080 & 1960 & April 17 & 9.52 & 1,570 \\
\hline 1950 & May 5 & 14.60 & 3,050 & 1967 & April 10 & 9.03 & 1,570 \\
\hline 1919 & April 18 & 14.90 & 2,750 & 1994 & April 9 & 9.00 & 1,530 \\
\hline 1987 & April 13 & 11.43 & 2,300 & 1955 & April 14 & 9.04 & 1,490 \\
\hline 1974 & April 22 & 10.74 & 2,160 & 1970 & April 20 & 8.47 & 1,370 \\
\hline 1949 & April 21 & 10.90 & 2,120 & 1951 & April 19 & 8.08 & 1,270 \\
\hline 1986 & April 19 & 11.04 & 2,100 & 1962 & July 7 & 9.71 & 1,270 \\
\hline 1983 & March 13 & 11.26 & 2,030 & 1992 & March 8 & 8.59 & 1,260 \\
\hline 1982 & April 14 & 10.95 & 1,990 & 1953 & July 17 & 8.11 & 1,230 \\
\hline 1975 & June 30 & 10.27 & 1,960 & 1968 & June 13 & 8.48 & 1,220 \\
\hline 1984 & April 6 & 10.43 & 1,950 & 1942 & April 17 & 7.72 & 1,190 \\
\hline 1947 & April 11 & 10.19 & 1,910 & 1946 & March 26 & 7.56 & 1,160 \\
\hline
\end{tabular}




\section{SHEYENNE RIVER AT VALLEY CITY, ND--Continued}

Annual peak discharge and corresponding gage height, period of record--Continued

\begin{tabular}{|c|c|c|c|c|c|c|c|}
\hline $\begin{array}{l}\text { Water } \\
\text { year }\end{array}$ & Date & $\begin{array}{c}\text { Gage } \\
\text { height } \\
\text { (feet) }\end{array}$ & $\begin{array}{c}\text { Peak } \\
\text { discharge } \\
\left(\mathrm{ft}^{3} / \mathrm{s}\right)\end{array}$ & $\begin{array}{c}\text { Water } \\
\text { year }\end{array}$ & Date & $\begin{array}{c}\text { Gage } \\
\text { height } \\
\text { (feet) }\end{array}$ & $\begin{array}{c}\text { Peak } \\
\text { discharge } \\
\left(\mathrm{ft}^{3} / \mathrm{s}\right)\end{array}$ \\
\hline \multicolumn{8}{|c|}{ Annual peak discharge, from highest to lowest, and corresponding gage height--Continued } \\
\hline 1972 & April 16 & 7.23 & 1,070 & 1981 & April 2 & 5.91 & 542 \\
\hline 1945 & March 24 & 7.06 & 1,020 & 1940 & April 8 & 5.14 & 525 \\
\hline 1959 & June 27 & 7.02 & 988 & 1944 & June 3 & 4.50 & 399 \\
\hline 1989 & March 30 & 8.38 & 850 & 1939 & April 6 & 4.34 & 342 \\
\hline 1980 & August 29 & 7.00 & 765 & 1990 & July 11 & 4.59 & 278 \\
\hline 1973 & March 14 & 6.32 & 761 & 1961 & March 2 & 4.01 & 251 \\
\hline 1954 & July 13 & 6.29 & 726 & 1958 & July 17 & 4.21 & 248 \\
\hline 1985 & May 17 & 6.37 & 680 & 1938 & March 29 & 3.90 & 244 \\
\hline 1952 & March 31 & 6.02 & 650 & 1988 & February 28 & 4.80 & 150 \\
\hline 1964 & June 28 & 6.56 & 643 & 1963 & July 26 & 3.39 & 78.0 \\
\hline 1957 & September 4 & 6.48 & 627 & 1991 & August 24 & 3.37 & 77.0 \\
\hline
\end{tabular}




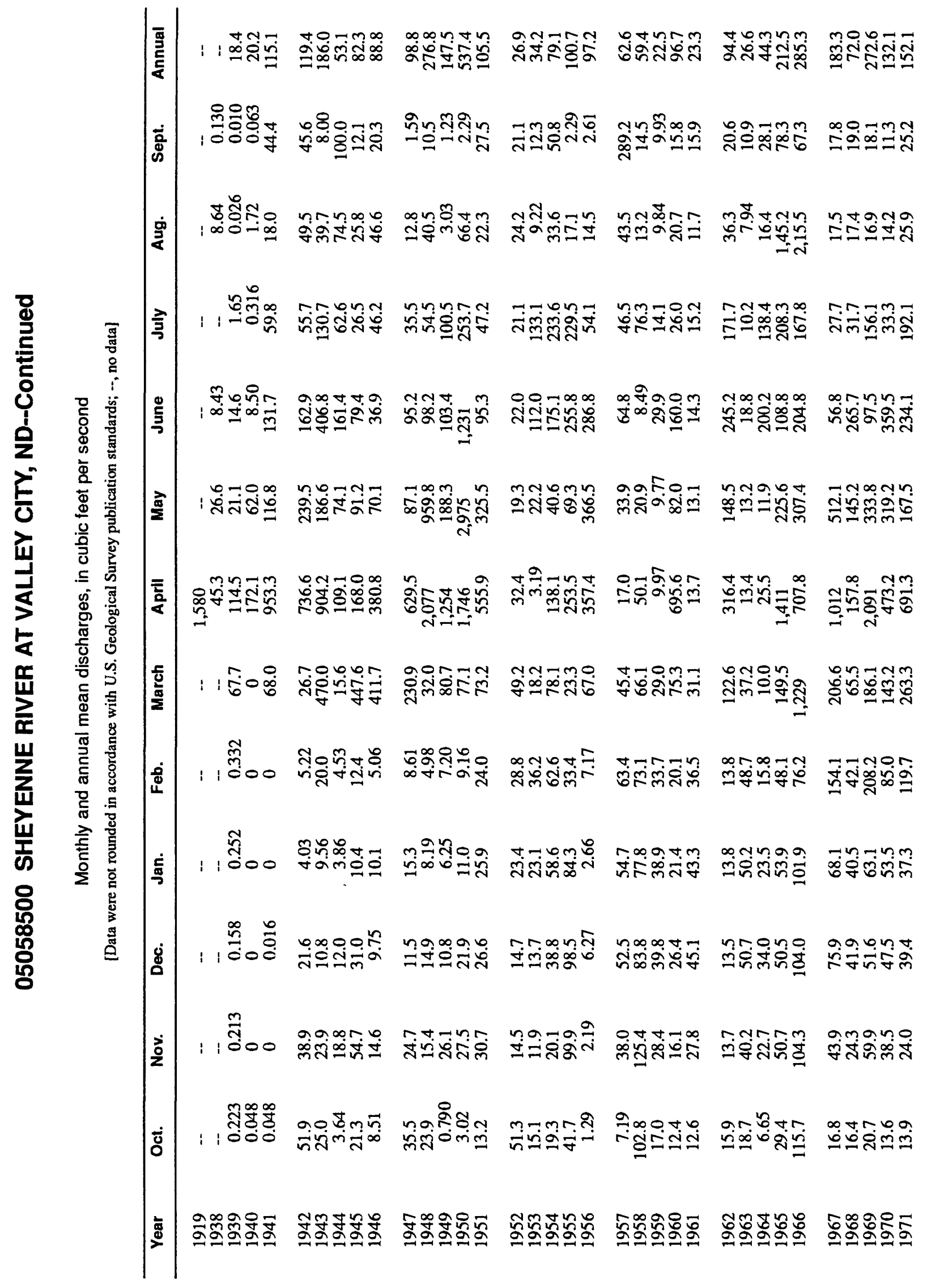




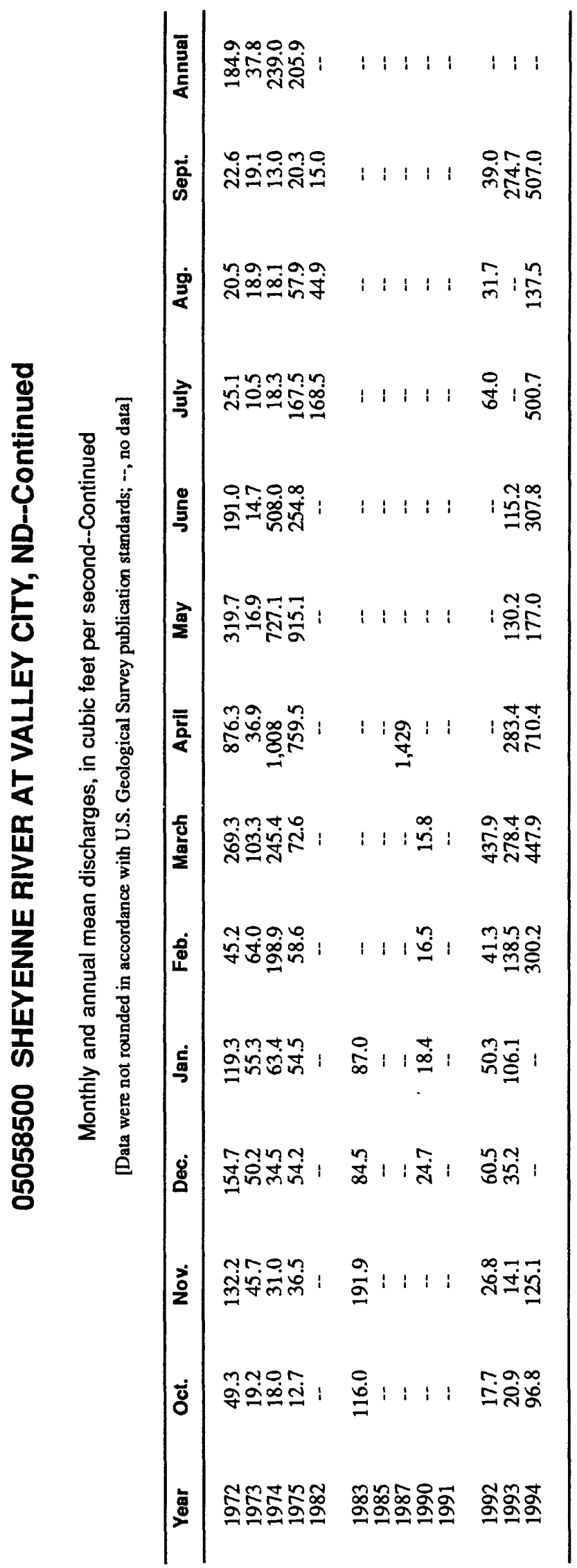




\section{SHEYENNE RIVER AT LISBON, ND}

\section{Station Description}

LOCATION.--Lat $46^{\circ} 26^{\prime} 49^{\prime \prime}$, long 97 $40^{\prime} 44^{\prime \prime}$, on line between secs.1 and 2, T.134 N., R.56 W., Ransom County, Hydrologic Unit 09020204, on left bank $150 \mathrm{ft}$ downstream from dam at State Fish Hatchery at north edge of city of Lisbon, $3 \mathrm{mi}$ upstream from Timber Coulee, and at mile 162.1.

DRAINAGE AREA.--8,190 $\mathrm{mi}^{2}$, approximately, of which about $5,700 \mathrm{mi}^{2}$ is probably noncontributing, including $3,800 \mathrm{mi}^{2}$ in closed basins.

PERIOD OF RECORD.--September 1956 to current year.

GAGE.--Water-stage recorder. Datum of gage is $1,066.46 \mathrm{ft}$ above sea level.

EXTREMES FOR PERIOD OF RECORD.--Maximum discharge, 5,270 $\mathrm{ft}^{3} / \mathrm{s}$, July 1, 1975 (gage height, $19.04 \mathrm{ft}$ ); minimum discharge, no flow Sept. 19-21 and Oct. 23-24, 1956, and Aug. 16, 1961 (gage height, $1.53 \mathrm{ft}$ ).

Annual mean discharge

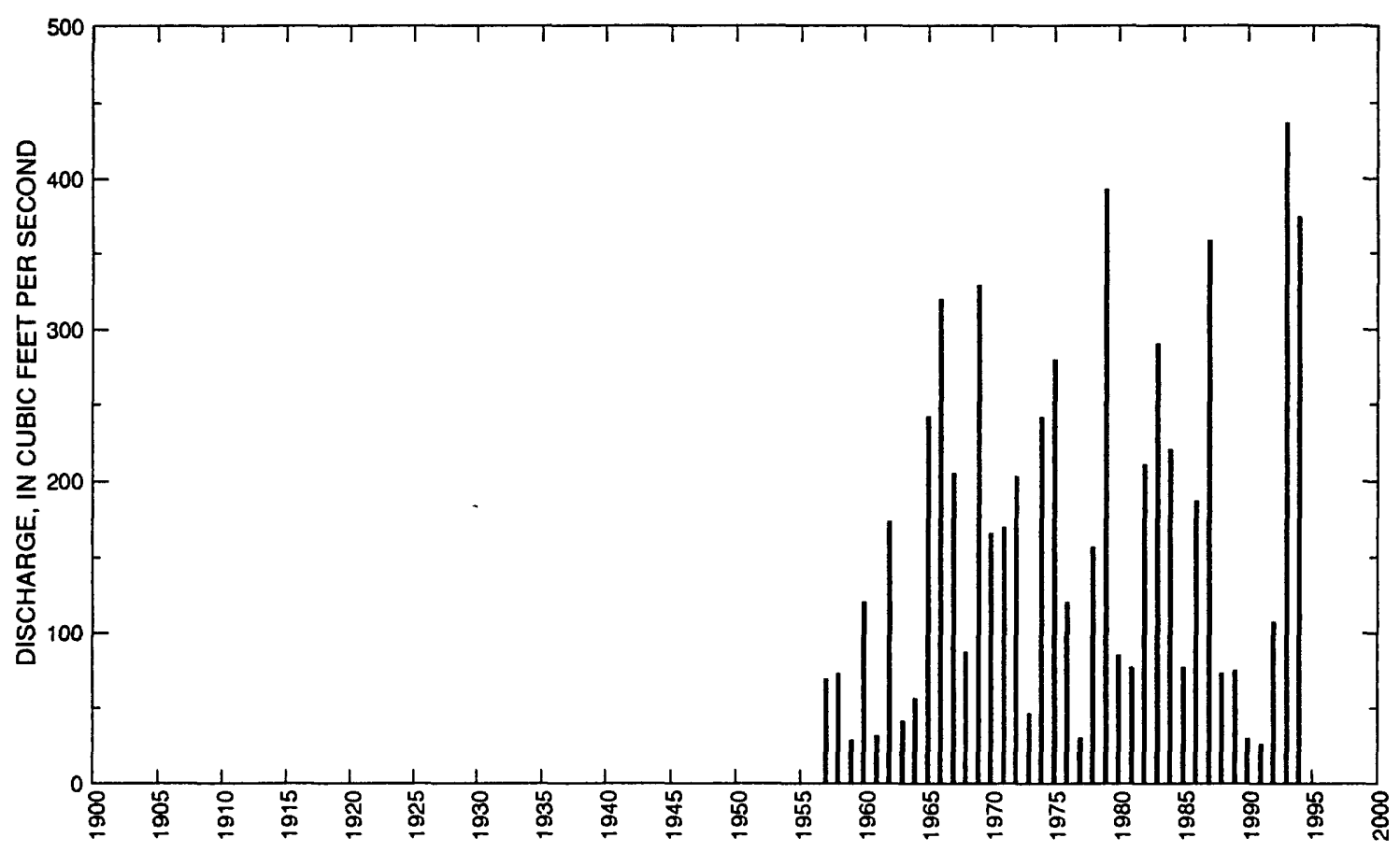




\section{SHEYENNE RIVER AT LISBON, ND-Continued}

Statistics of monthly and annual mean discharges

\begin{tabular}{|c|c|c|c|c|c|c|c|c|}
\hline \multirow[b]{2}{*}{ Month } & \multicolumn{2}{|c|}{ Maximum } & \multicolumn{2}{|c|}{ Minimum } & \multicolumn{4}{|c|}{ Mean } \\
\hline & $\begin{array}{c}\text { Discharge } \\
\left(\mathrm{ft}^{3} / \mathrm{s}\right)\end{array}$ & $\begin{array}{c}\text { Water year } \\
\text { of } \\
\text { occurrence }\end{array}$ & $\begin{array}{c}\text { Discharge } \\
\left(\mathrm{ft}^{3} / \mathrm{s}\right)\end{array}$ & $\begin{array}{c}\text { Water year } \\
\text { of } \\
\text { occurrence }\end{array}$ & $\begin{array}{c}\text { Discharge } \\
\left(\mathrm{ft}^{3} / \mathrm{s}\right)\end{array}$ & $\begin{array}{c}\text { Standard } \\
\text { deviation } \\
\left(\mathrm{ft}^{3} / \mathbf{s}\right)\end{array}$ & $\begin{array}{l}\text { Coeffl- } \\
\text { clent of } \\
\text { variation }\end{array}$ & $\begin{array}{l}\text { Percentage } \\
\text { of annual } \\
\text { discharge }\end{array}$ \\
\hline October & 150 & 1994 & 7.66 & 1957 & 49.2 & 42.8 & 0.87 & 2.51 \\
\hline November & 195 & 1983 & 12.2 & 1991 & 66.2 & 50.2 & 0.76 & 3.38 \\
\hline December & 191 & 1994 & 8.69 & 1991 & 64.0 & 48.0 & 0.75 & 3.27 \\
\hline January & 140 & 1986 & 8.15 & 1991 & 59.9 & 39.3 & 0.66 & 3.06 \\
\hline February & 202 & 1969 & 10.7 & 1991 & 72.6 & 53.6 & 0.74 & 3.71 \\
\hline March & 1,330 & 1987 & 30.9 & 1990 & 294 & 325 & 1.11 & 15.0 \\
\hline April & 2,550 & 1969 & 20.3 & 1991 & 606 & 598 & 0.99 & 31.0 \\
\hline May & 1,990 & 1979 & 17.5 & 1959 & 261 & 359 & 1.38 & 13.3 \\
\hline June & 555 & 1974 & 14.8 & 1961 & 157 & 139 & 0.88 & 8.03 \\
\hline July & 1,420 & 1993 & 6.07 & 1973 & 160 & 273 & 1.70 & 8.18 \\
\hline August & 1,940 & 1993 & 6.54 & 1961 & 103 & 314 & 3.05 & 5.26 \\
\hline September & 561 & 1994 & 5.25 & 1959 & 65.5 & 113 & 1.72 & 3.34 \\
\hline Annual & 437 & 1993 & 25.9 & 1991 & 163 & 117 & 0.72 & 100 \\
\hline
\end{tabular}

Annual flow duration

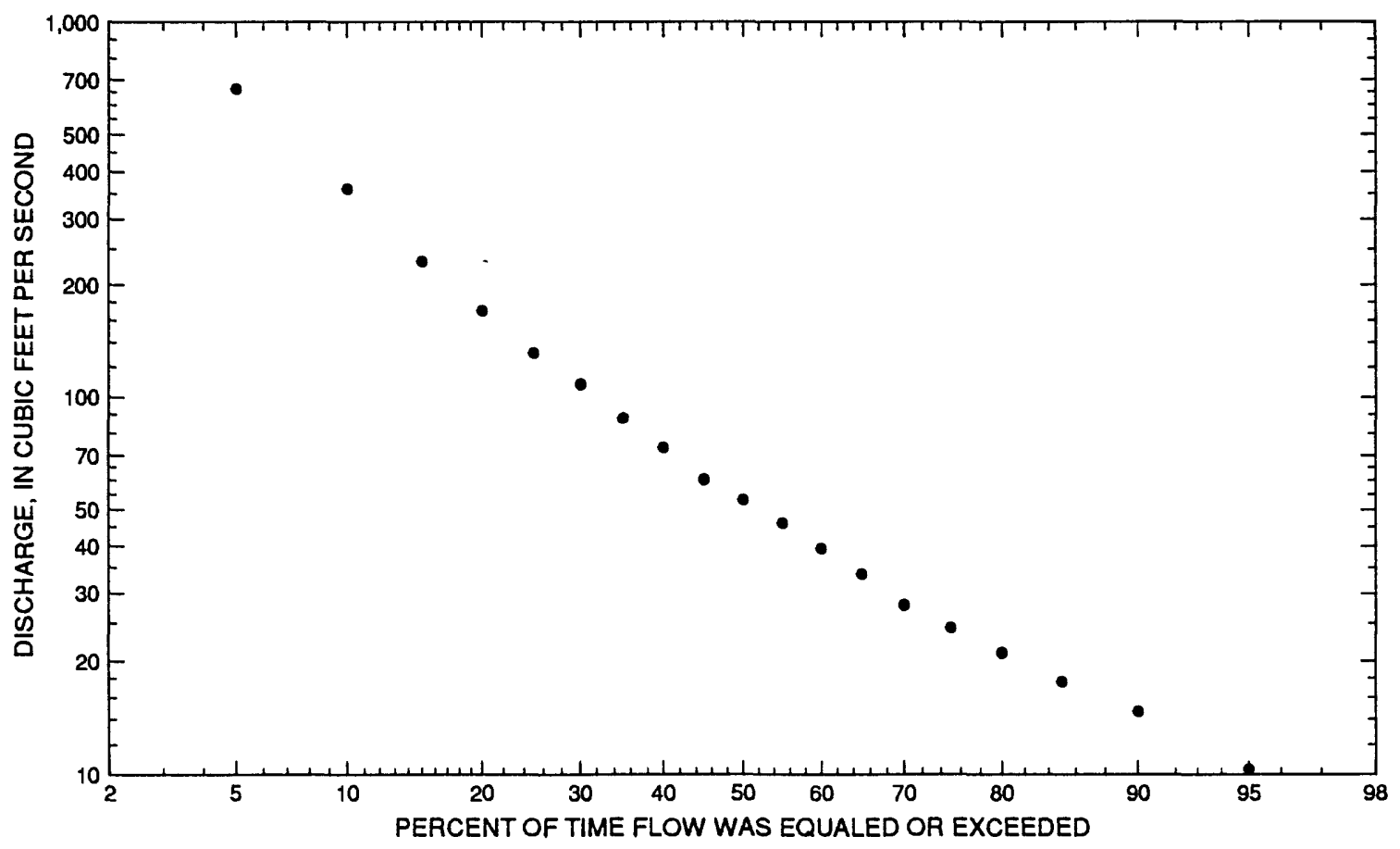




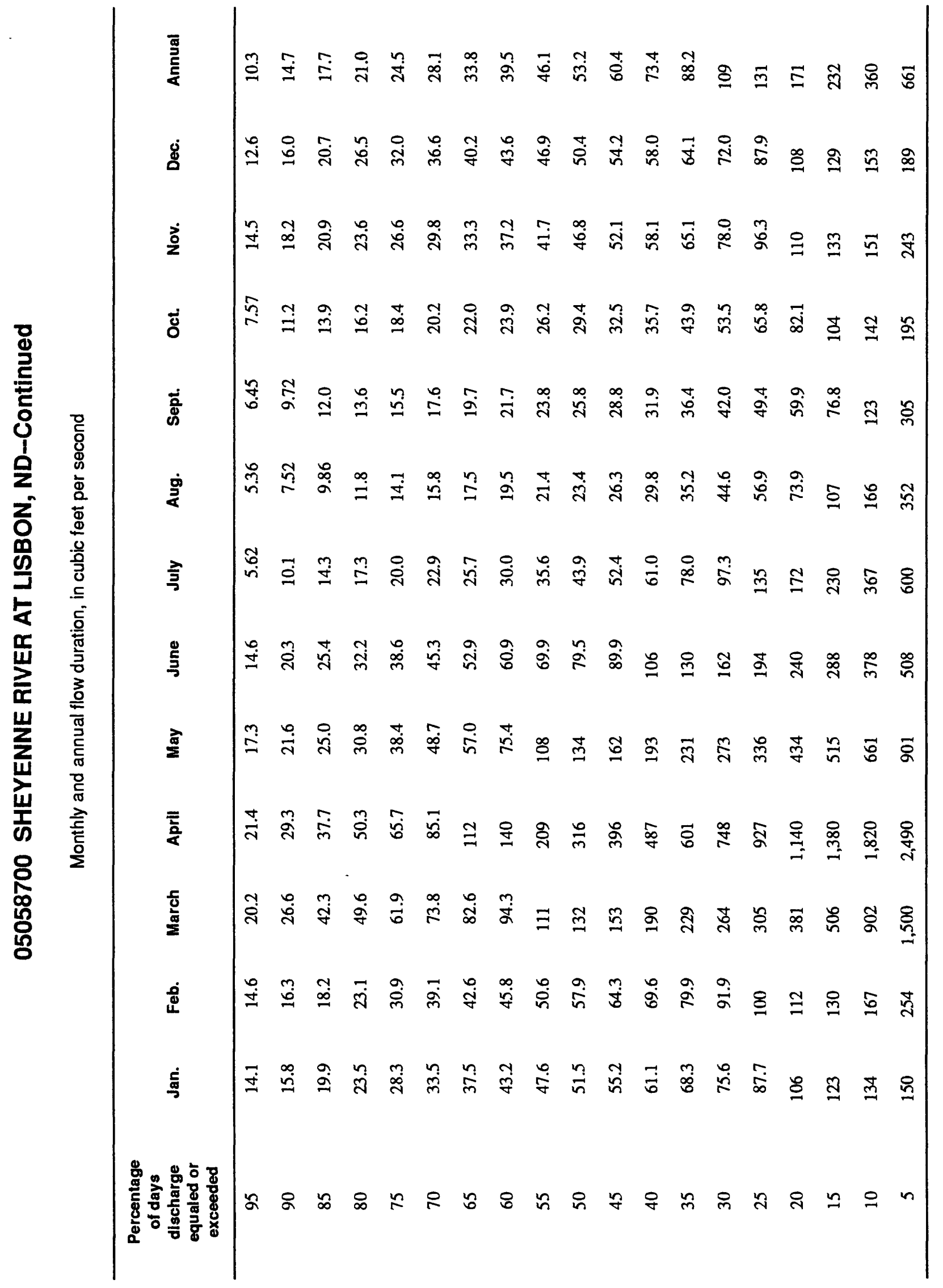




\section{SHEYENNE RIVER AT LISBON, ND-Continued}

Probability of annual high discharges

[ng, statistic not given]

\begin{tabular}{|c|c|c|c|c|c|c|}
\hline \multirow[b]{2}{*}{$\begin{array}{l}\text { Exceedance } \\
\text { probability }\end{array}$} & \multirow[b]{2}{*}{$\begin{array}{l}\text { Recurrence } \\
\text { interval } \\
\text { (years) }\end{array}$} & \multirow[b]{2}{*}{$\begin{array}{c}\text { Maximum } \\
\text { instantaneous } \\
\left(\mathrm{ft}^{3} / \mathbf{s}\right)\end{array}$} & \multicolumn{4}{|c|}{$\begin{array}{l}\text { Maximum average discharge } \\
\qquad\left(\mathrm{ft}^{3} / \mathrm{s}\right)\end{array}$} \\
\hline & & & 3-day period & 7-day period & 15-day period & 30-day period \\
\hline 0.99 & 1.01 & 131 & 87.1 & 49.3 & 30.2 & 22.6 \\
\hline 0.95 & 1.05 & 273 & 196 & 129 & 87.1 & 65.0 \\
\hline 0.90 & 1.11 & 396 & 295 & 209 & 147 & 110 \\
\hline 0.80 & 1.25 & 609 & 475 & 363 & 267 & 200 \\
\hline 0.50 & 2 & 1,320 & 1,110 & 948 & 753 & 568 \\
\hline 0.20 & 5 & 2,660 & 2,410 & 2,210 & 1,850 & 1,420 \\
\hline 0.10 & 10 & 3,740 & 3,510 & 3,280 & 2,800 & 2,180 \\
\hline 0.04 & 25 & 5,280 & 5,130 & 4,850 & 4,200 & 3,330 \\
\hline 0.02 & 50 & 6,520 & 6,480 & 6,140 & 5,360 & 4,290 \\
\hline 0.01 & 100 & 7,840 & 7,930 & 7,510 & 6,580 & 5,320 \\
\hline 0.005 & 200 & 9,220 & 9,480 & 8,940 & 7,860 & 6,420 \\
\hline 0.002 & 500 & 11,200 & ng & ng & $\mathrm{ng}$ & ng \\
\hline
\end{tabular}

Probability of annual low discharges

\begin{tabular}{|c|c|c|c|c|c|c|c|c|c|c|}
\hline \multirow{3}{*}{$\begin{array}{l}\text { Non- } \\
\text { exceed- } \\
\text { ance } \\
\text { prob- } \\
\text { ability }\end{array}$} & \multirow{3}{*}{$\begin{array}{c}\text { Recur- } \\
\text { rence } \\
\text { Inter- } \\
\text { val } \\
\text { (years) }\end{array}$} & \multicolumn{9}{|c|}{ Minimum average discharge $\left(\mathrm{ft}^{3} / \mathrm{s}\right)$} \\
\hline & & \multicolumn{9}{|c|}{ Number of consecutive days } \\
\hline & & 1 & 3 & 7 & 14 & 30 & 60 & 90 & 120 & 183 \\
\hline 0.05 & 20 & 0.044 & 0.389 & 1.80 & 3.36 & 5.73 & 8.20 & 9.37 & 10.4 & 14.6 \\
\hline 0.10 & 10 & 0.305 & 0.998 & 2.65 & 4.26 & 6.74 & 9.44 & 11.0 & 12.5 & 17.9 \\
\hline 0.20 & 5 & 1.42 & 2.57 & 4.14 & 5.75 & 8.40 & 11.5 & 13.6 & 16.0 & 23.1 \\
\hline 0.50 & 2 & 8.05 & 9.17 & ${ }^{1} 9.88$ & 10.6 & 13.9 & 18.6 & 22.6 & 27.2 & 38.8 \\
\hline
\end{tabular}

${ }^{1}$ Graphical interpretation. 


\section{SHEYENNE RIVER AT LISBON, ND-Continued}

Probability of seasonal low discharges

\begin{tabular}{|c|c|c|c|c|c|c|c|c|c|}
\hline \multirow[b]{3}{*}{$\begin{array}{c}\text { Non- } \\
\text { exceedance } \\
\text { probability }\end{array}$} & \multirow[b]{3}{*}{$\begin{array}{c}\text { Recurrence } \\
\text { Intervai } \\
\text { (years) }\end{array}$} & \multicolumn{8}{|c|}{ Minimum average discharge $\left(\mathrm{ft}^{3} / \mathrm{s}\right)$} \\
\hline & & \multicolumn{8}{|c|}{ Number of consecutive days } \\
\hline & & 1 & 7 & 14 & 30 & 1 & 7 & 14 & 30 \\
\hline & & \multicolumn{4}{|c|}{ December-January-February } & \multicolumn{4}{|c|}{ March-April-May } \\
\hline 0.05 & 20 & 4.80 & 8.51 & 9.60 & 11.6 & 8.25 & 10.1 & 11.4 & 14.8 \\
\hline 0.10 & 10 & 7.76 & 11.6 & 13.0 & 15.6 & 11.4 & 13.9 & 16.1 & 22.9 \\
\hline 0.20 & 5 & 13.1 & 16.6 & 18.4 & 22.0 & 16.9 & 20.5 & 24.4 & 38.1 \\
\hline \multirow[t]{2}{*}{0.50} & 2 & 30.3 & 32.1 & 34.9 & 40.6 & 35.2 & 43.3 & 54.7 & 94.3 \\
\hline & & \multicolumn{4}{|c|}{ June-July-August } & \multicolumn{4}{|c|}{ September-October-November } \\
\hline 0.05 & 20 & 1.58 & 2.47 & 3.68 & 5.52 & 0.224 & 2.77 & 4.88 & 8.26 \\
\hline 0.10 & 10 & 2.42 & 3.47 & 4.81 & 7.11 & 0.837 & 4.10 & 6.41 & 10.0 \\
\hline 0.20 & 5 & 3.85 & 5.33 & 6.86 & 10.0 & 2.99 & 6.43 & 8.98 & 12.9 \\
\hline 0.50 & 2 & 9.54 & 12.7 & 15.0 & 21.6 & 13.9 & 14.3 & 17.5 & 22.2 \\
\hline
\end{tabular}




\section{SHEYENNE RIVER AT LISBON, ND-Continued}

Annual peak discharge and corresponding gage height, period of record

$$
[-, \text { no data }]
$$

\begin{tabular}{|c|c|c|c|c|c|c|c|}
\hline $\begin{array}{l}\text { Water } \\
\text { year }\end{array}$ & Date & $\begin{array}{c}\text { Gage } \\
\text { height } \\
\text { (feet) }\end{array}$ & $\begin{array}{c}\text { Peak } \\
\text { discharge } \\
\left(\mathrm{ft}^{3} / \mathbf{s}\right)\end{array}$ & $\begin{array}{l}\text { Water } \\
\text { year }\end{array}$ & Date & $\begin{array}{c}\text { Gage } \\
\text { height } \\
\text { (feet) }\end{array}$ & $\begin{array}{c}\text { Peak } \\
\text { discharge } \\
\left(\mathrm{ft}^{3} / \mathbf{s}\right)\end{array}$ \\
\hline \multicolumn{8}{|c|}{ Annual peak discharge, by year, and corresponding gage height } \\
\hline 1957 & September 7 & 5.20 & 612 & 1976 & April 13 & 6.29 & 984 \\
\hline 1958 & April 7 & -- & 490 & 1977 & May 5 & 7.35 & 1,230 \\
\hline 1959 & June 29 & 4.28 & 451 & 1978 & March 23 & 11.73 & 1,410 \\
\hline 1960 & April 11 & 9.81 & 1,980 & 1979 & May 2 & 17.58 & 4,880 \\
\hline 1961 & March 6 & 4.61 & 482 & 1980 & March 23 & 7.01 & 760 \\
\hline 1962 & July 7 & 10.55 & 2,210 & 1981 & April 5 & 4.30 & 460 \\
\hline 1963 & June 8 & 3.02 & 207 & 1982 & April 13 & 11.44 & 2,600 \\
\hline 1964 & June 30 & 4.96 & 634 & 1983 & March 17 & 13.75 & 2,370 \\
\hline 1965 & April 14 & 16.02 & 3,630 & 1984 & April 6 & 9.79 & 2,000 \\
\hline 1966 & March 30 & 16.23 & 4,260 & 1985 & May 19 & 5.13 & 642 \\
\hline 1967 & April 15 & 8.52 & 1,580 & 1986 & April 21 & 10.75 & 2,110 \\
\hline 1968 & June 16 & 7.63 & 1,300 & 1987 & March 23 & 14.07 & 3,000 \\
\hline 1969 & April 24 & 16.54 & 4,380 & 1988 & March 26 & 3.63 & 284 \\
\hline 1970 & May 29 & 8.70 & 1,500 & 1989 & April 4 & 8.36 & 1,000 \\
\hline 1971 & April 28 & 9.67 & 1,850 & 1990 & June 4 & 3.74 & 311 \\
\hline 1972 & March 16 & 10.33 & 1,450 & 1991 & July 2 & 3.49 & 261 \\
\hline 1973 & March 17 & 5.29 & 677 & 1992 & March 11 & 9.18 & 1,500 \\
\hline 1974 & April 25 & 10.25 & 2,020 & 1993 & August 2 & 16.34 & 3,790 \\
\hline 1975 & July 1 & 19.04 & 5,270 & 1994 & April 5 & 10.70 & 1,980 \\
\hline \multicolumn{8}{|c|}{ Annual peak discharge, from highest to lowest, and corresponding gage height } \\
\hline 1975 & July 1 & 19.04 & 5,270 & 1972 & March 16 & 10.33 & 1,450 \\
\hline 1979 & May 2 & 17.58 & 4,880 & 1978 & March 23 & 11.73 & 1,410 \\
\hline 1969 & April 24 & 16.54 & 4,380 & 1968 & June 16 & 7.63 & 1,300 \\
\hline 1966 & March 30 & 16.23 & 4,260 & 1977 & May 5 & 7.35 & 1,230 \\
\hline 1993 & August 2 & 16.34 & 3,790 & 1989 & April 4 & 8.36 & 1,000 \\
\hline 1965 & April 14 & 16.02 & 3,630 & 1976 & April 13 & 6.29 & 984 \\
\hline 1987 & March 23 & 14.07 & 3,000 & 1980 & March 23 & 7.01 & 760 \\
\hline 1982 & April 13 & 11.44 & 2,600 & 1973 & March 17 & 5.29 & 677 \\
\hline 1983 & March 17 & 13.75 & 2,370 & 1985 & May 19 & 5.13 & 642 \\
\hline 1962 & July 7 & 10.55 & 2,210 & 1964 & June 30 & 4.96 & 634 \\
\hline 1986 & April 21 & 10.75 & 2,110 & 1957 & September 7 & 5.20 & 612 \\
\hline 1974 & April 25 & 10.25 & 2,020 & 1958 & April 7 & -- & 490 \\
\hline 1984 & April 6 & 9.79 & 2,000 & 1961 & March 6 & 4.61 & 482 \\
\hline 1960 & April 11 & 9.81 & 1,980 & 1981 & April 5 & 4.30 & 460 \\
\hline 1994 & April 5 & 10.70 & 1,980 & 1959 & June 29 & 4.28 & 451 \\
\hline 1971 & April 28 & 9.67 & 1,850 & 1990 & June 4 & 3.74 & 311 \\
\hline 1967 & April 15 & 8.52 & 1,580 & 1988 & March 26 & 3.63 & 284 \\
\hline 1970 & May 29 & 8.70 & 1,500 & 1991 & July 2 & 3.49 & 261 \\
\hline 1992 & March 11 & 9.18 & 1,500 & 1963 & June 8 & 3.02 & 207 \\
\hline
\end{tabular}




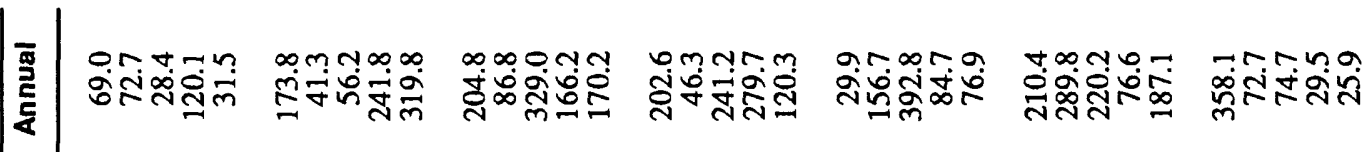

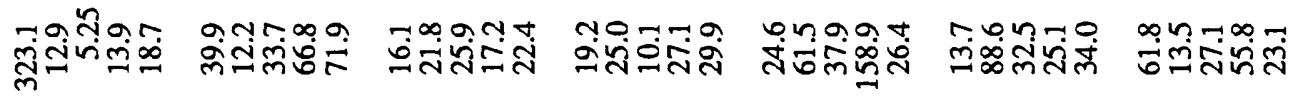

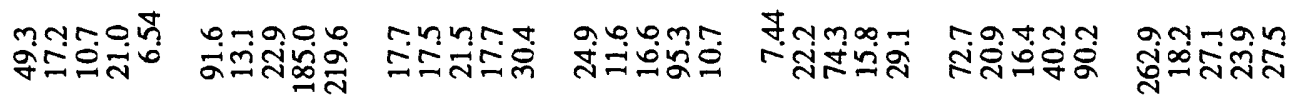

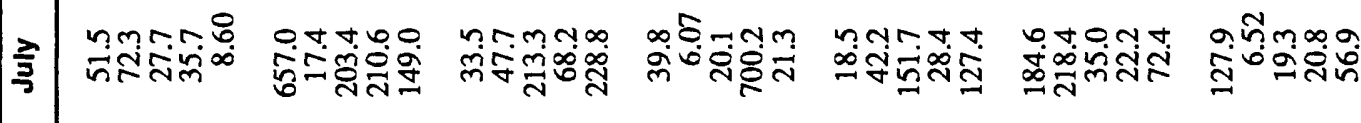

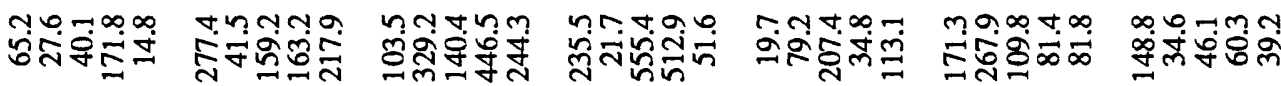

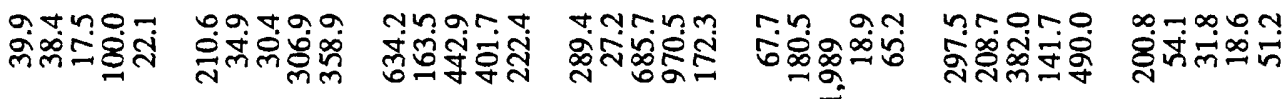

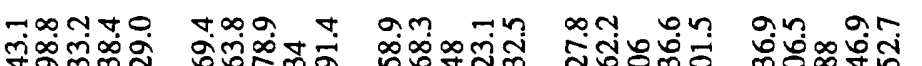

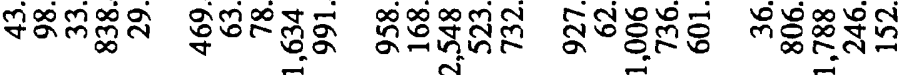

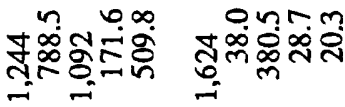

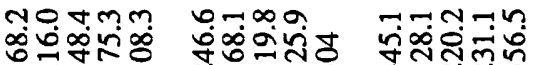

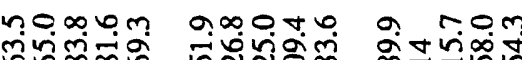

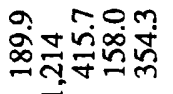

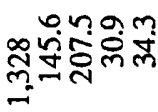

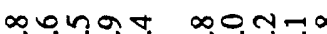

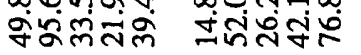

omma-

मn.

orrono whor otrino

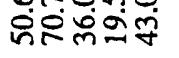

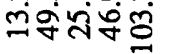

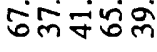

whroen-

$\ln \rightarrow \infty \infty$

o. atro

boor

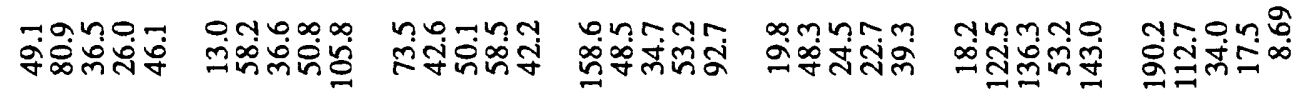

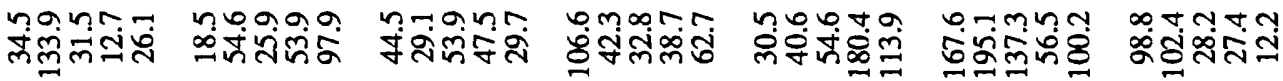

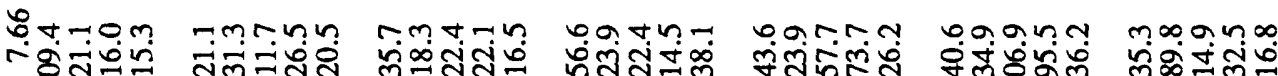

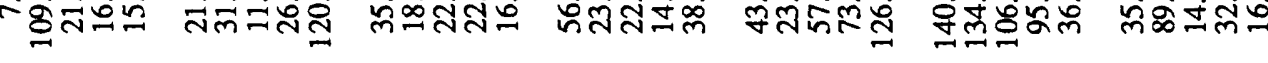

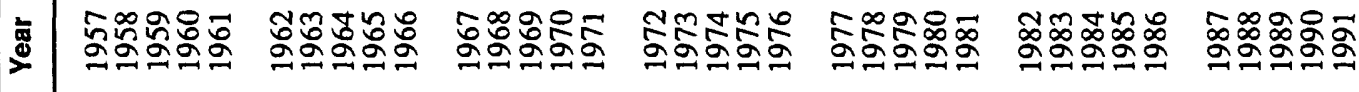




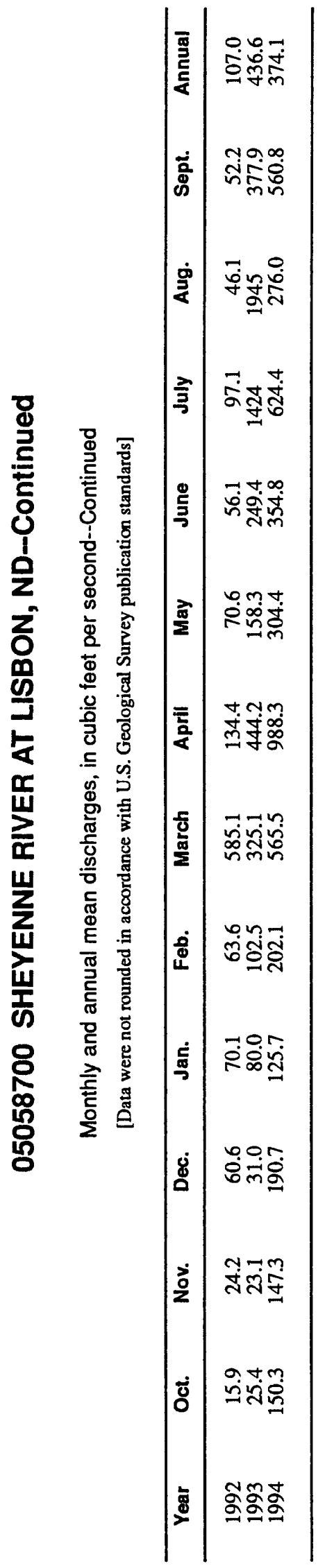




\section{SHEYENNE RIVER NEAR KINDRED, ND}

\section{Station Description}

LOCATION.--Lat 46³7'54", long 97 $00^{\prime} 01^{\prime \prime}$, in SE $1 / 4 \mathrm{SE}^{1} / 4 \mathrm{SW}^{1} / 4$ sec.33, T.137 N., R.50 W., Cass County, Hydrologic Unit 09020204, on left bank $100 \mathrm{ft}$ downstream from North Dakota State Highway 46 bridge crossing, $1.5 \mathrm{mi}$ southeast of Kindred, and at mile 67.9.

DRAINAGE AREA.--8,800 $\mathrm{mi}^{2}$, approximately, of which about $5,780 \mathrm{mi}^{2}$ is probably noncontributing, including $3,800 \mathrm{mi}^{2}$ in closed basins.

PERIOD OF RECORD.--July 1949 to current year.

GAGE.--Water-stage recorder. Datum of gage is $925.55 \mathrm{ft}$ above sea level. From Oct. 1, 1962, to Sept. 30, 1989 , gage was located at site $1,500 \mathrm{ft}$ upstream. July 1949 to Sept. 30, 1962, non recording gage at same site and datum.

EXTREMES FOR PERIOD OF RECORD.--Maximum discharge, 4,690 $\mathrm{ft}^{3} / \mathrm{s}$, Apr. 15, 1969; maximum gage height, $21.66 \mathrm{ft}$, July 6, 1975; minimum daily discharge, $9.2 \mathrm{ft}^{3} / \mathrm{s}$, Aug. 16, 1990.

EXTREMES OUTSIDE PERIOD OF RECORD.--Spring flood in 1947 or 1948 reached a stage of $22.1 \mathrm{ft}$ from floodmarks, discharge about $3,600 \mathrm{ft}^{3} / \mathrm{s}$.

Annual mean discharge

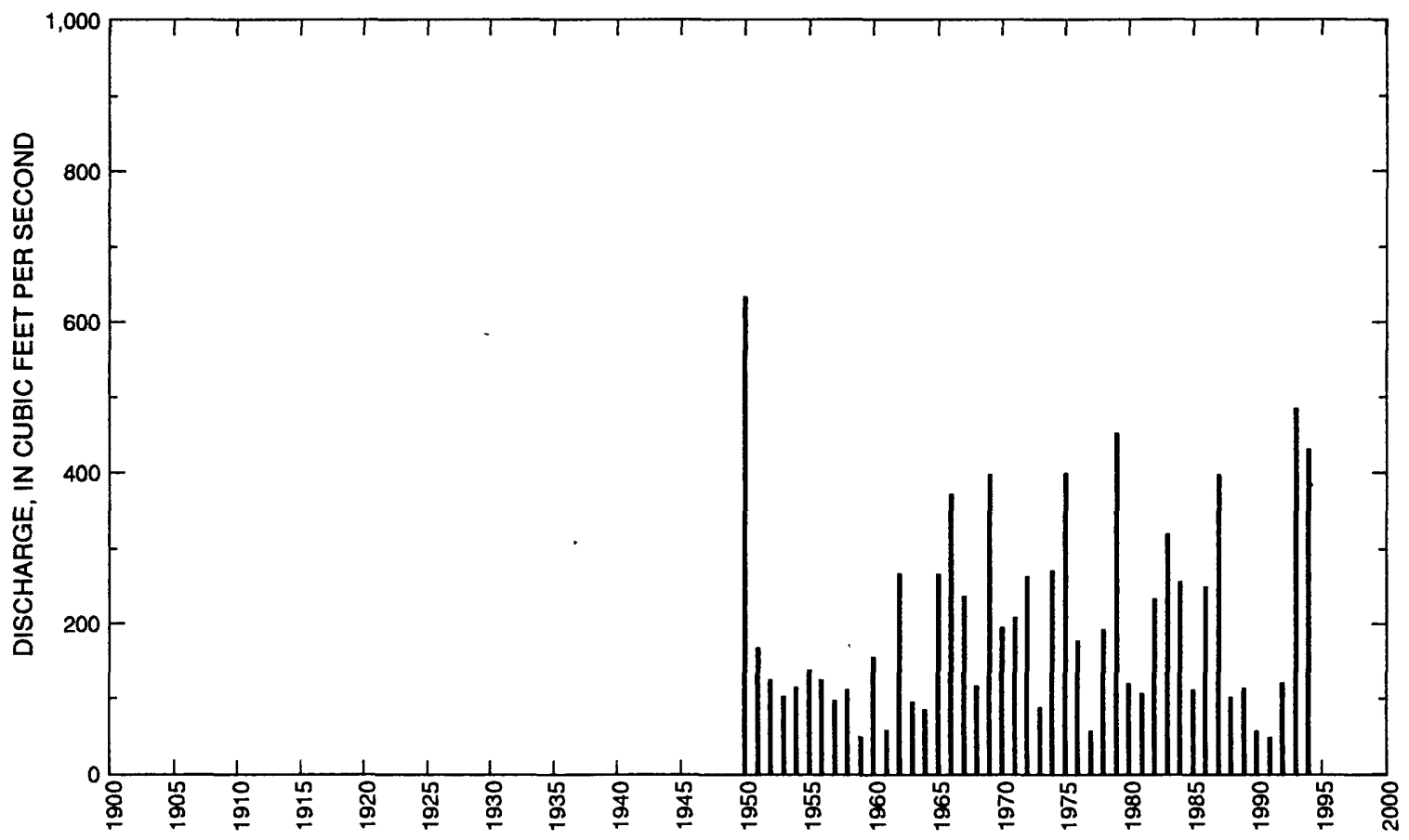




\section{SHEYENNE RIVER NEAR KINDRED, ND--Continued}

Statistics of monthly and annual mean discharges

\begin{tabular}{|c|c|c|c|c|c|c|c|c|}
\hline \multirow[b]{2}{*}{ Month } & \multicolumn{2}{|c|}{ Maximum } & \multicolumn{2}{|c|}{ Minimum } & \multicolumn{4}{|c|}{ Mean } \\
\hline & $\begin{array}{c}\text { Discharge } \\
\left(\mathrm{ft}^{3} / \mathrm{s}\right)\end{array}$ & $\begin{array}{c}\text { Water year } \\
\text { of } \\
\text { occurrence }\end{array}$ & $\begin{array}{c}\text { Discharge } \\
\left(\mathrm{ft}^{3} / \mathbf{s}\right)\end{array}$ & $\begin{array}{c}\text { Water year } \\
\text { of } \\
\text { occurrence }\end{array}$ & $\begin{array}{c}\text { Discharge } \\
\left(\mathrm{ft}^{3} / \mathrm{s}\right)\end{array}$ & $\begin{array}{c}\text { Standard } \\
\text { deviation } \\
\left(\mathrm{ft}^{3} / \mathrm{s}\right)\end{array}$ & $\begin{array}{l}\text { Coeffl- } \\
\text { cient of } \\
\text { variation }\end{array}$ & $\begin{array}{l}\text { Percentage } \\
\text { of annual } \\
\text { discharge }\end{array}$ \\
\hline October & 224 & 1994 & 24.6 & 1957 & 71.2 & 44.2 & 0.62 & 2.92 \\
\hline November & 232 & 1980 & 22.7 & 1956 & 82.2 & 51.0 & 0.62 & 3.37 \\
\hline December & 247 & 1994 & 17.6 & 1956 & 75.3 & 51.1 & 0.68 & 3.09 \\
\hline January & 170 & 1987 & 17.5 & 1991 & 69.3 & 42.5 & 0.61 & 2.85 \\
\hline February & 221 & 1976 & 26.6 & 1991 & 77.8 & 48.9 & 0.63 & 3.19 \\
\hline March & 1,260 & 1987 & 35.1 & 1956 & 277 & 284 & 1.02 & 11.4 \\
\hline April & 2,460 & 1969 & 71.7 & 1991 & 673 & 567 & 0.84 & 27.6 \\
\hline May & 3,050 & 1950 & 53.6 & 1990 & 410 & 567 & 1.38 & 16.8 \\
\hline June & 1,940 & 1950 & 48.4 & 1961 & 254 & 297 & 1.17 & 10.4 \\
\hline July & 1,470 & 1975 & 26.7 & 1988 & 228 & 312 & 1.37 & 9.34 \\
\hline August & 2,230 & 1993 & 22.5 & 1977 & 134 & 329 & 2.46 & 5.49 \\
\hline September & 483 & 1993 & 25.1 & 1959 & 83.8 & 98.6 & 1.18 & 3.44 \\
\hline Annual & 633 & 1950 & 48.0 & 1991 & 203 & 137 & 0.67 & 100 \\
\hline
\end{tabular}

Annual flow duration

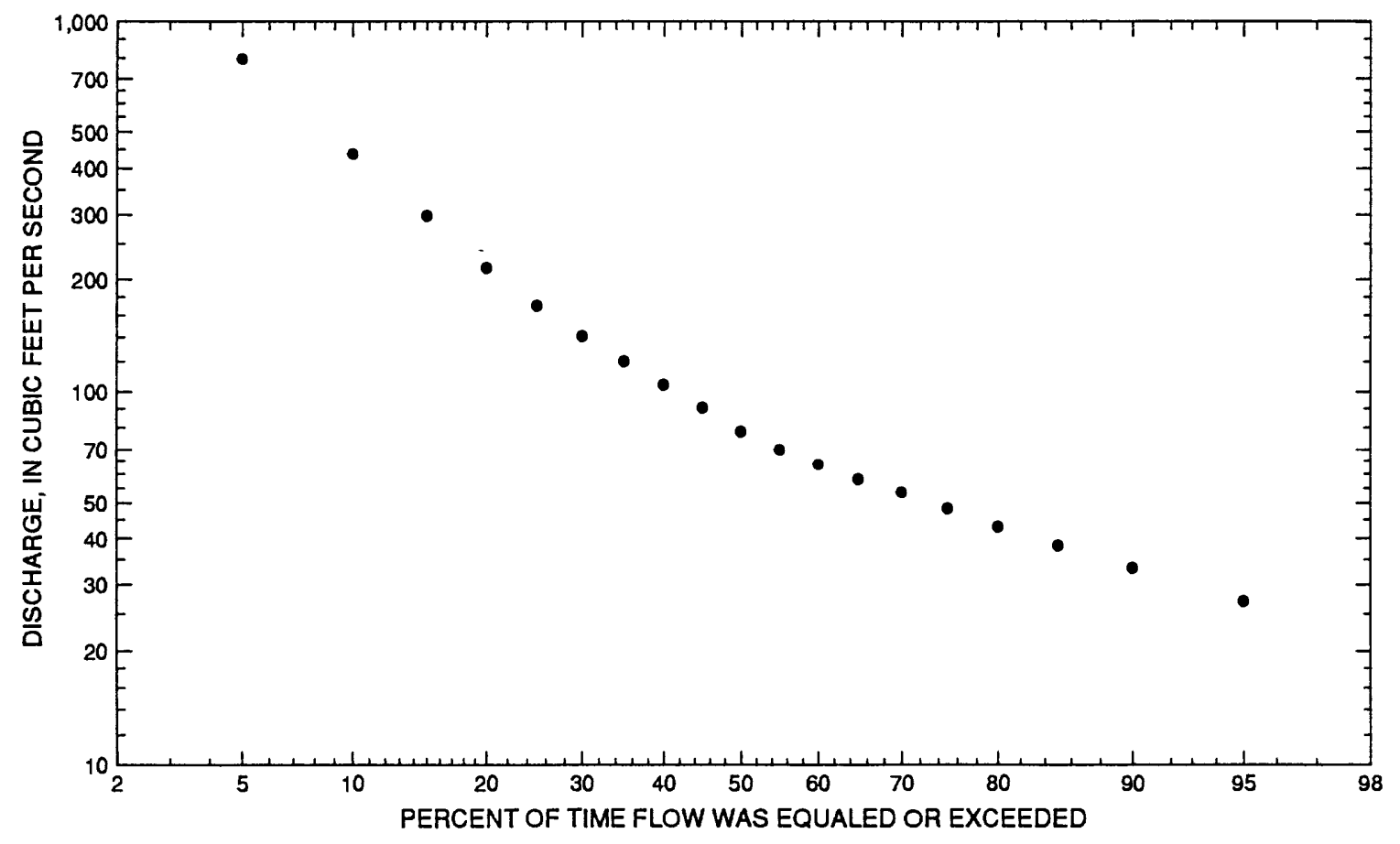




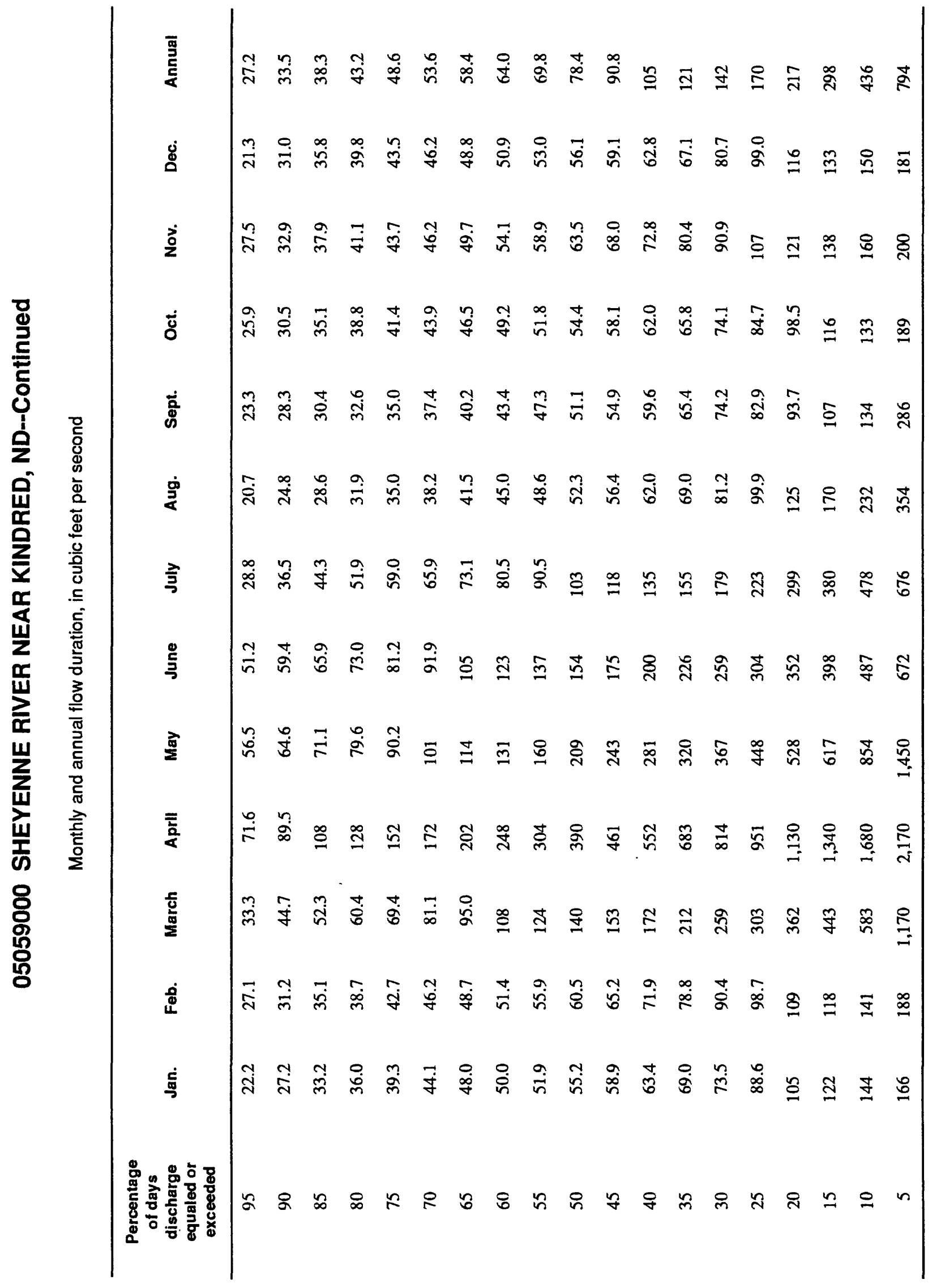




\section{SHEYENNE RIVER NEAR KINDRED, ND--Continued}

Probability of annual high discharges

[ng, statistic not given]

\begin{tabular}{|c|c|c|c|c|c|c|}
\hline \multirow[b]{2}{*}{$\begin{array}{l}\text { Exceedance } \\
\text { probability }\end{array}$} & \multirow[b]{2}{*}{$\begin{array}{l}\text { Recurrence } \\
\text { intervai } \\
\text { (years) }\end{array}$} & \multirow[b]{2}{*}{$\begin{array}{c}\text { Maximum } \\
\text { instantaneous } \\
\left(\mathrm{ft}^{3} / \mathbf{s}\right)\end{array}$} & \multicolumn{4}{|c|}{$\begin{array}{c}\text { Maximum average discharge } \\
\left(\mathrm{ft}^{3} / \mathrm{s}\right)\end{array}$} \\
\hline & & & 3-day period & 7-day period & 15-day period & 30-day period \\
\hline 0.99 & 1.01 & 137 & 104 & 79.7 & 68.7 & 61.4 \\
\hline 0.95 & 1.05 & 276 & 225 & 184 & 155 & 128 \\
\hline 0.90 & 1.11 & 392 & 332 & 279 & 233 & 187 \\
\hline 0.80 & 1.25 & 591 & 519 & 450 & 374 & 293 \\
\hline 0.50 & 2 & 1,220 & 1,140 & 1,040 & 866 & 668 \\
\hline 0.20 & 5 & 2,380 & 2,300 & 2,160 & 1,844 & 1,460 \\
\hline 0.10 & 10 & 3,270 & 3,200 & 3,050 & 2,650 & 2,150 \\
\hline 0.04 & 25 & 4,510 & 4,460 & 4,290 & 3,820 & 3,220 \\
\hline 0.02 & 50 & 5,500 & 5,450 & 5,270 & 4,770 & 4,140 \\
\hline 0.01 & 100 & 6,520 & 6,480 & 6,280 & 5,780 & 5,180 \\
\hline 0.005 & 200 & 7,590 & 7,540 & 7,310 & 6,840 & 6,320 \\
\hline 0.002 & 500 & 9,050 & $\mathrm{ng}$ & $\mathrm{ng}$ & $\mathrm{ng}$ & $\mathrm{ng}$ \\
\hline
\end{tabular}

Probability of annual low discharges

\begin{tabular}{|c|c|c|c|c|c|c|c|c|c|c|}
\hline \multirow[b]{3}{*}{$\begin{array}{l}\text { Non- } \\
\text { exceed- } \\
\text { ance } \\
\text { prob- } \\
\text { ability }\end{array}$} & \multirow[b]{3}{*}{$\begin{array}{c}\text { Recur- } \\
\text { rence } \\
\text { Inter- } \\
\text { val } \\
\text { (years) }\end{array}$} & \multicolumn{9}{|c|}{ Minimum average discharge $\left(\mathrm{ft}^{3} / \mathrm{s}\right)$} \\
\hline & & \multicolumn{9}{|c|}{ Number of consecutive days } \\
\hline & & 1 & 3 & 7 & 14 & 30 & 60 & 90 & 120 & 183 \\
\hline 0.05 & 20 & 10.9 & 12.8 & 14.2 & 16.3 & 18.4 & 21.2 & 23.4 & 25.6 & 28.6 \\
\hline 0.10 & 10 & 13.0 & 14.8 & 16.1 & 18.1 & 20.6 & 24.1 & 26.6 & 29.3 & 33.1 \\
\hline 0.20 & 5 & 16.3 & 17.9 & 19.0 & 21.0 & 24.1 & 28.4 & 31.6 & 35.1 & 40.0 \\
\hline 0.50 & 2 & 25.3 & 26.6 & 27.9 & 30.0 & 34.6 & 41.1 & 46.0 & 51.2 & 59.2 \\
\hline
\end{tabular}




\section{SHEYENNE RIVER NEAR KINDRED, ND--Continued}

Probability of seasonal low discharges

\begin{tabular}{|c|c|c|c|c|c|c|c|c|c|}
\hline \multirow[b]{3}{*}{$\begin{array}{c}\text { Non- } \\
\text { exceedance } \\
\text { probability }\end{array}$} & \multirow[b]{3}{*}{$\begin{array}{c}\text { Recurrence } \\
\text { Interval } \\
\text { (years) }\end{array}$} & \multicolumn{8}{|c|}{ Minimum average dlscharge $\left(\mathrm{ft}^{3} / \mathrm{s}\right)$} \\
\hline & & \multicolumn{8}{|c|}{ Number of consecutive days } \\
\hline & & 1 & 7 & 14 & 30 & 1 & 7 & 14 & 30 \\
\hline & & \multicolumn{4}{|c|}{ December-January-February } & \multicolumn{4}{|c|}{ March-April-May } \\
\hline 0.05 & 20 & 13.3 & 16.0 & 18.0 & 20.8 & 24.3 & 26.3 & 28.7 & 41.4 \\
\hline 0.10 & 10 & 16.7 & 19.9 & 22.1 & 25.0 & 29.2 & 31.8 & 34.9 & 51.8 \\
\hline 0.20 & 5 & 22.2 & 26.0 & 28.2 & 31.4 & 36.8 & 40.6 & 45.1 & 68.9 \\
\hline \multirow[t]{2}{*}{0.50} & 2 & 37.9 & 43.1 & 45.8 & 49.3 & 59.4 & 68.2 & 78.1 & 125 \\
\hline & & \multicolumn{4}{|c|}{ June-July-August } & \multicolumn{4}{|c|}{ September-October-November } \\
\hline 0.05 & 20 & 13.5 & 16.4 & 18.1 & 20.4 & 12.1 & 17.3 & 19.0 & 23.0 \\
\hline 0.10 & 10 & 16.2 & 19.3 & 21.3 & 24.6 & 14.9 & 19.9 & 21.8 & 26.1 \\
\hline 0.20 & 5 & 20.7 & 24.2 & 26.6 & 31.5 & 19.0 & 24.0 & 26.2 & 30.8 \\
\hline 0.50 & 2 & 34.9 & 39.4 & 43.8 & 54.5 & 30.5 & 35.7 & 39.0 & 44.7 \\
\hline
\end{tabular}




\section{SHEYENNE RIVER NEAR KINDRED, ND--Continued}

Annual peak discharge and corresponding gage height, period of record

$[-$, no data $]$

\begin{tabular}{|c|c|c|c|c|c|c|c|}
\hline $\begin{array}{l}\text { Water } \\
\text { year }\end{array}$ & Date & $\begin{array}{c}\text { Gage } \\
\text { height } \\
\text { (feet) }\end{array}$ & $\begin{array}{c}\text { Peak } \\
\text { discharge } \\
\left(\mathrm{ft}^{3} / \mathrm{s}\right)\end{array}$ & $\begin{array}{l}\text { Water } \\
\text { year }\end{array}$ & Date & $\begin{array}{c}\text { Gage } \\
\text { helght } \\
\text { (feet) }\end{array}$ & $\begin{array}{c}\text { Peak } \\
\text { discharge } \\
\left(\mathrm{ft}^{3} / \mathrm{s}\right)\end{array}$ \\
\hline \multicolumn{8}{|c|}{ Annual peak discharge, by year, and corresponding gage height } \\
\hline 1947 & - & 22.10 & 3,600 & 1972 & March 19 & 13.54 & 1,530 \\
\hline 1950 & May 13 & 20.50 & 3,210 & 1973 & March 22 & 6.19 & 710 \\
\hline 1951 & May 5 & 7.70 & 1,010 & 1974 & April 29 & 12.29 & 1,940 \\
\hline 1952 & April 8 & 17.80 & 2,240 & 1975 & July 6 & 21.66 & 4,640 \\
\hline 1953 & July 3 & 6.44 & 679 & 1976 & April 16 & 8.75 & 925 \\
\hline 1954 & July 6 & 6.17 & 631 & 1977 & May 8 & 6.05 & 570 \\
\hline 1955 & April 19 & 8.80 & 1,120 & 1978 & March 28 & 16.43 & 1,410 \\
\hline 1956 & May 4 & 10.82 & 1,460 & 1979 & May 7 & 20.75 & 4,160 \\
\hline 1957 & September 12 & 5.70 & 547 & 1980 & April 4 & 7.92 & 750 \\
\hline 1958 & March 5 & 6.95 & 480 & 1981 & April 10 & 4.77 & 435 \\
\hline 1959 & July 3 & 4.13 & 204 & 1982 & April 16 & 12.84 & 2,040 \\
\hline 1960 & April 13 & 11.76 & 1,820 & 1983 & April 21 & 17.64 & 2,060 \\
\hline 1961 & March 11 & 5.10 & 350 & 1984 & April 11 & 11.51 & 1,810 \\
\hline 1962 & July 11 & 15.14 & 2,310 & 1985 & March 18 & 7.06 & 555 \\
\hline 1963 & April 6 & 4.99 & 430 & 1986 & April 24 & 11.13 & 1,740 \\
\hline 1964 & July 3 & - & 600 & 1987 & March 26 & 18.26 & 3,000 \\
\hline 1965 & April 18 & 17.30 & 2,760 & 1988 & March 7 & 5.46 & 460 \\
\hline 1966 & April 3 & 19.15 & 3,380 & 1989 & April 3 & 15.01 & 1,430 \\
\hline 1967 & April 18 & 10.48 & 1,460 & 1990 & June 7 & 3.42 & 286 \\
\hline 1968 & June 18 & 7.67 & 1,010 & 1991 & May 29 & 3.11 & 184 \\
\hline 1969 & April 15 & 21.03 & 4,690 & 1992 & March 15 & 11.44 & 1,400 \\
\hline 1970 & April 27 & 9.59 & 1,230 & 1993 & August 9 & 19.84 & 3,550 \\
\hline 1971 & April 30 & 11.46 & 1,750 & 1994 & April 7 & 12.87 & 2,030 \\
\hline \multicolumn{8}{|c|}{ Annual peak discharge, from highest to lowest, and corresponding gage height } \\
\hline 1969 & April 15 & 21.03 & 4,690 & 1978 & March 28 & 16.43 & 1,410 \\
\hline 1975 & July 6 & 21.66 & 4,640 & 1992 & March 15 & 11.44 & 1,400 \\
\hline 1979 & May 7 & 20.75 & 4,160 & 1970 & April 27 & 9.59 & 1,230 \\
\hline 1947 & -- & 22.10 & 3,600 & 1955 & April 19 & 8.80 & 1,120 \\
\hline 1993 & August 9 & 19.84 & $-3,550$ & 1951 & May 5 & 7.70 & 1,010 \\
\hline 1966 & April 3 & 19.15 & 3,380 & 1968 & June 18 & 7.67 & 1,010 \\
\hline 1950 & May 13 & 20.50 & 3,210 & 1976 & April 16 & 8.75 & 925 \\
\hline 1987 & March 26 & 18.26 & 3,000 & 1980 & April 4 & 7.92 & 750 \\
\hline 1965 & April 18 & 17.30 & 2,760 & 1973 & March 22 & 6.19 & 710 \\
\hline 1962 & July 11 & 15.14 & 2,310 & 1953 & July 3 & 6.44 & 679 \\
\hline 1952 & April 8 & 17.80 & 2,240 & 1954 & July 6 & 6.17 & 631 \\
\hline 1983 & April 21 & 17.64 & 2,060 & 1964 & July 3 & - & 600 \\
\hline 1982 & April 16 & 12.84 & 2,040 & 1977 & May 8 & 6.05 & 570 \\
\hline 1994 & April 7 & 12.87 & 2,030 & 1985 & March 18 & 7.06 & 555 \\
\hline 1974 & April 29 & 12.29 & 1,940 & 1957 & September 12 & 5.70 & 547 \\
\hline 1960 & April 13 & 11.76 & 1,820 & 1958 & March 5 & 6.95 & 480 \\
\hline 1984 & April 11 & 11.51 & 1,810 & 1988 & March 7 & 5.46 & 460 \\
\hline 1971 & April 30 & 11.46 & 1,750 & 1981 & April 10 & 4.77 & 435 \\
\hline 1986 & April 24 & 11.13 & 1,740 & 1963 & April 6 & 4.99 & 430 \\
\hline 1972 & March 19 & 13.54 & 1,530 & 1961 & March 11 & 5.10 & 350 \\
\hline 1967 & April 18 & 10.48 & 1,460 & 1990 & June 7 & 3.42 & 286 \\
\hline 1956 & May 4 & 10.82 & 1,460 & 1959 & July 3 & 4.13 & 204 \\
\hline 1989 & April 3 & 15.01 & 1,430 & 1991 & May 29 & 3.11 & 184 \\
\hline
\end{tabular}




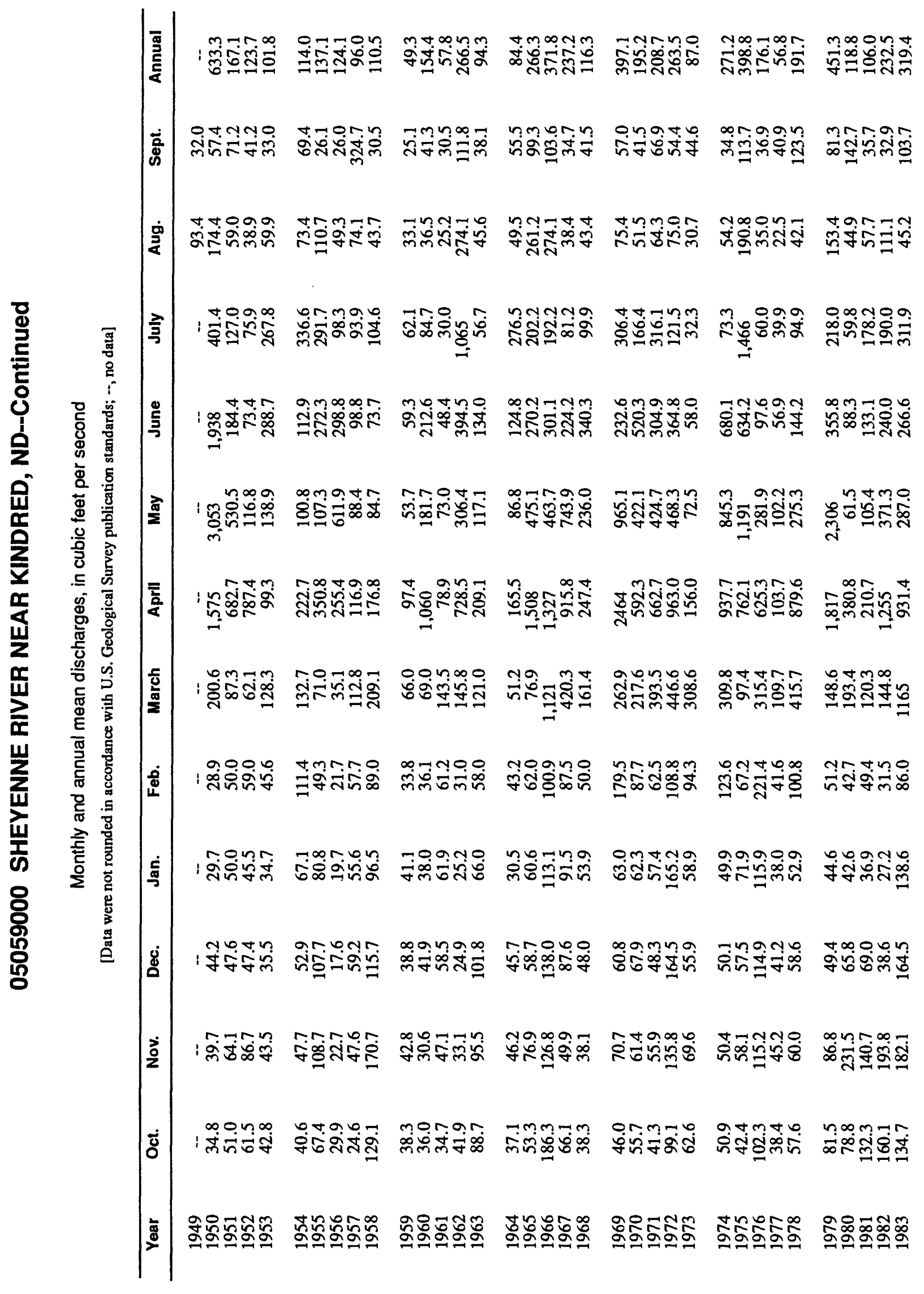




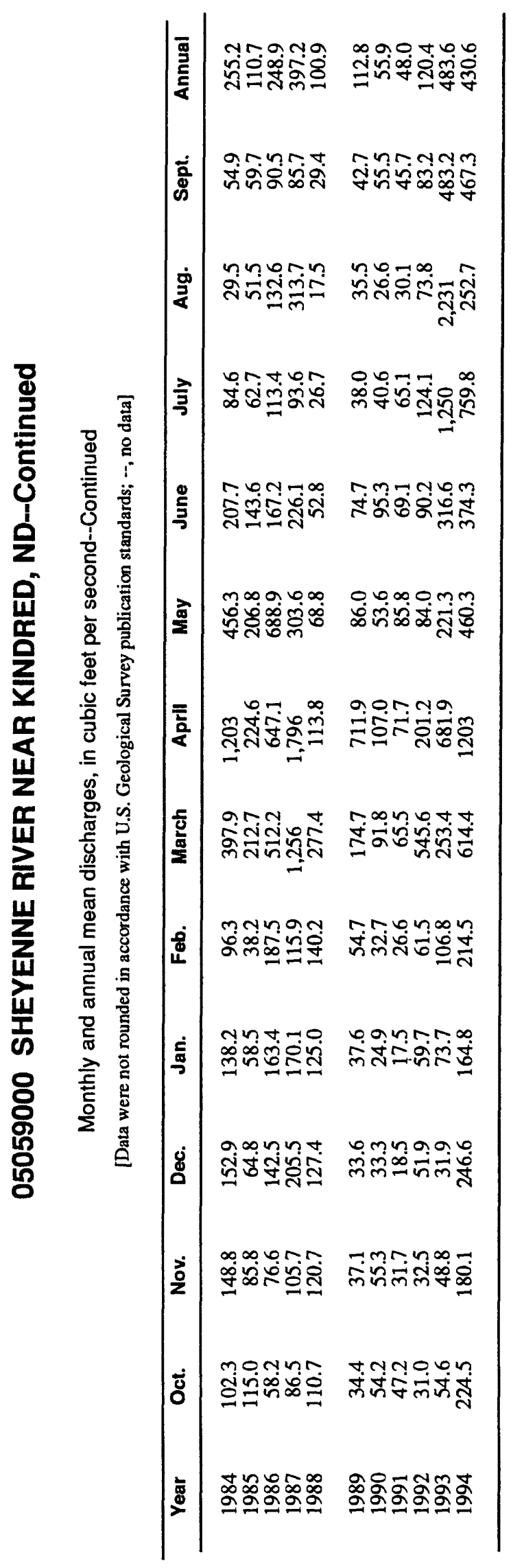




\section{SHEYENNE RIVER AT WEST FARGO, ND}

\section{Station Description}

LOCATION.--Lat 46 53'28', long 9654'24', in SE $1 / 4 \mathrm{SE}^{1 / 4} \mathrm{sec} .31$, T.140 N., R.49 W., Cass County, Hydrologic Unit 09020204, on right bank at downstream side of county highway bridge, 1 mi north of West Fargo, 3 mi upstream from Maple River, and at mile 24.5.

DRAINAGE AREA.--8,870 $\mathrm{mi}^{2}$, approximately, of which about $5,780 \mathrm{mi}^{2}$ is probably noncontributing, including $3,800 \mathrm{mi}^{2}$ in closed basins.

PERIOD OF RECORD.--March to November 1902 (gage heights only), April 1903 to October 1905, March to August 1919, September 1929 to current year. Published as "at or near Haggart" 1902-7, 1919. Records for March to November 1902 and November 1905 to June 1907, published in WSP $100,171,207$, and 245 , have been found to be unreliable and should not be used. Monthly discharge only for some periods, published in WSP 1308.

GAGE.--Water-stage recorder. Datum of gage is $877.19 \mathrm{ft}$ above sea level. June 27,1933 , to September 1969 on left bank about $600 \mathrm{ft}$ downstream on unimproved channel at same datum. See WSP 1728 or 1913 for history of changes prior to June $27,1933$.

EXTREMES FOR PERIOD OF RECORD.--Maximum discharge, $3,480 \mathrm{ft}^{3} / \mathrm{s}$, Apr. 21, 1979; maximum gage height, $22.25 \mathrm{ft}$, July 5, 1975; minimum daily discharge, $1.0 \mathrm{ft}^{3} / \mathrm{s}$, Sept. 23, 1976.

Annual mean discharge

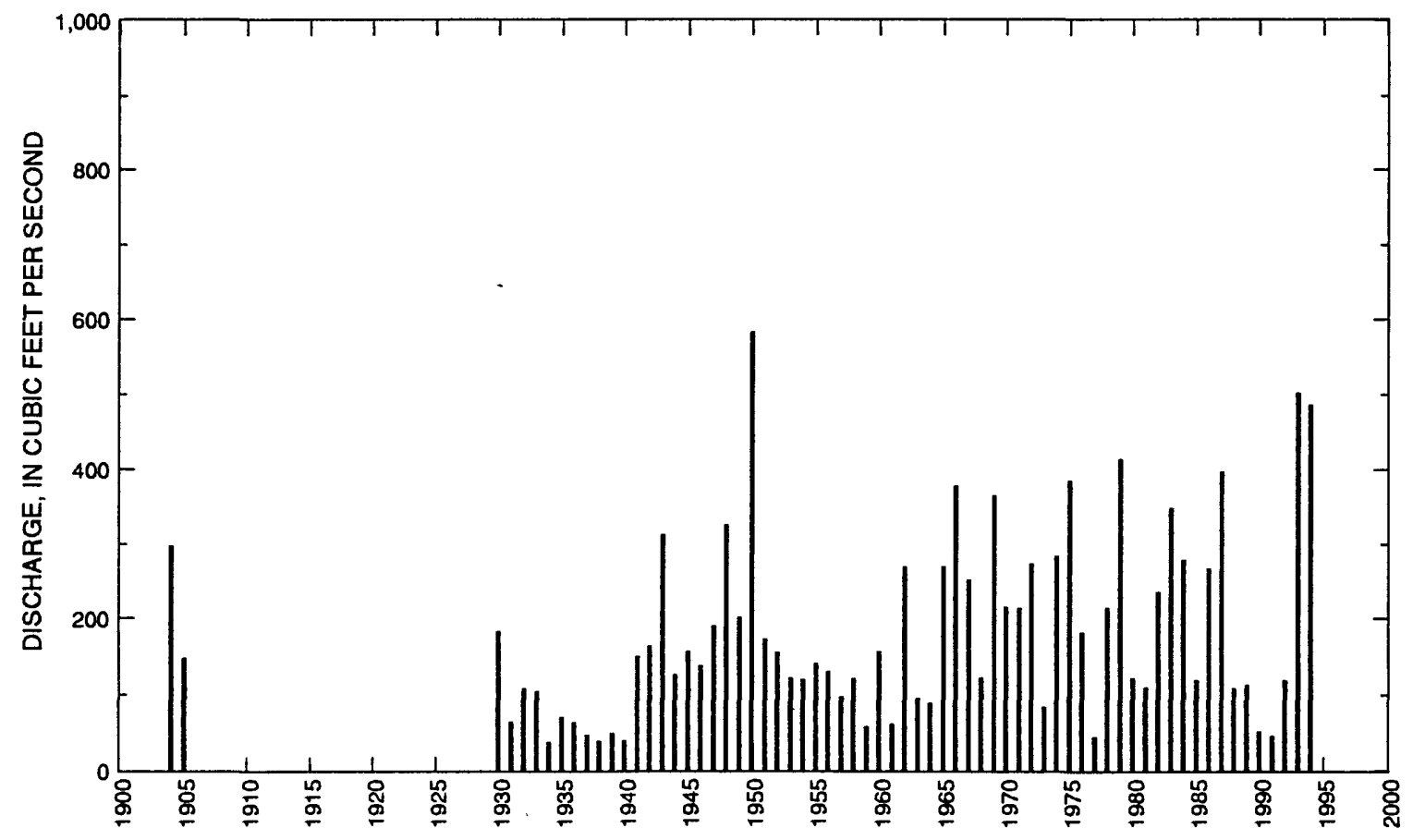




\section{SHEYENNE RIVER AT WEST FARGO, ND--Continued}

\section{Pre-regulation period}

Statistics of monthly and annual mean discharges, pre-regulation period

\begin{tabular}{|c|c|c|c|c|c|c|c|c|}
\hline \multirow[b]{2}{*}{ Month } & \multicolumn{2}{|c|}{ Maximum } & \multicolumn{2}{|c|}{ Minimum } & \multicolumn{4}{|c|}{ Mean } \\
\hline & $\begin{array}{c}\text { Discharge } \\
\left(\mathrm{ft}^{3} / \mathbf{s}\right)\end{array}$ & $\begin{array}{c}\text { Water year } \\
\text { of } \\
\text { occurrence }\end{array}$ & $\begin{array}{c}\text { Discharge } \\
\left(\mathrm{ft}^{3} / \mathbf{s}\right)\end{array}$ & $\begin{array}{c}\text { Water year } \\
\text { of } \\
\text { occurrence }\end{array}$ & $\begin{array}{c}\text { Discharge } \\
\left(\mathrm{ft}^{3} / \mathrm{s}\right)\end{array}$ & $\begin{array}{c}\text { Standard } \\
\text { deviation } \\
\left(\mathrm{ft}^{3} / \mathrm{s}\right)\end{array}$ & $\begin{array}{l}\text { Coeffi- } \\
\text { cient of } \\
\text { varlation }\end{array}$ & $\begin{array}{l}\text { Percentage } \\
\text { of annual } \\
\text { dlscharge }\end{array}$ \\
\hline October & 93.2 & 1905 & 9.88 & 1937 & 39.2 & 27.8 & 0.71 & 2.29 \\
\hline November & 92.5 & 1945 & 12.4 & 1937 & 42.2 & 25.8 & 0.61 & 2.46 \\
\hline December & 60.7 & 1945 & 7.48 & 1937 & 30.2 & 17.8 & 0.59 & 1.77 \\
\hline January & 44.5 & 1945 & 6.37 & 1940 & 22.4 & 12.0 & 0.53 & 1.31 \\
\hline February & 61.7 & 1930 & 5.47 & 1937 & 25.7 & 15.3 & 0.60 & 1.50 \\
\hline March & 838 & 1930 & 6.76 & 1940 & 171 & 203 & 1.19 & 10.0 \\
\hline April & 1,660 & 1943 & 116 & 1938 & 654 & 515 & 0.79 & 38.2 \\
\hline May & 1,630 & 1948 & 56.2 & 1934 & 330 & 362 & 1.10 & 19.2 \\
\hline June & 769 & 1943 & 25.2 & 1934 & 186 & 160 & 0.86 & 10.8 \\
\hline July & 272 & 1943 & 14.7 & 1934 & 96.6 & 71.0 & 0.74 & 5.64 \\
\hline August & 303 & 1905 & 7.46 & 1936 & 68.7 & 66.2 & 0.96 & 4.01 \\
\hline September & 185 & 1944 & 8.37 & 1936 & 46.7 & 44.2 & 0.95 & 2.73 \\
\hline Annual & 326 & 1948 & 37.1 & 1934 & 136 & 88.4 & 0.65 & 100 \\
\hline
\end{tabular}

Annual flow duration, pre-regulation period

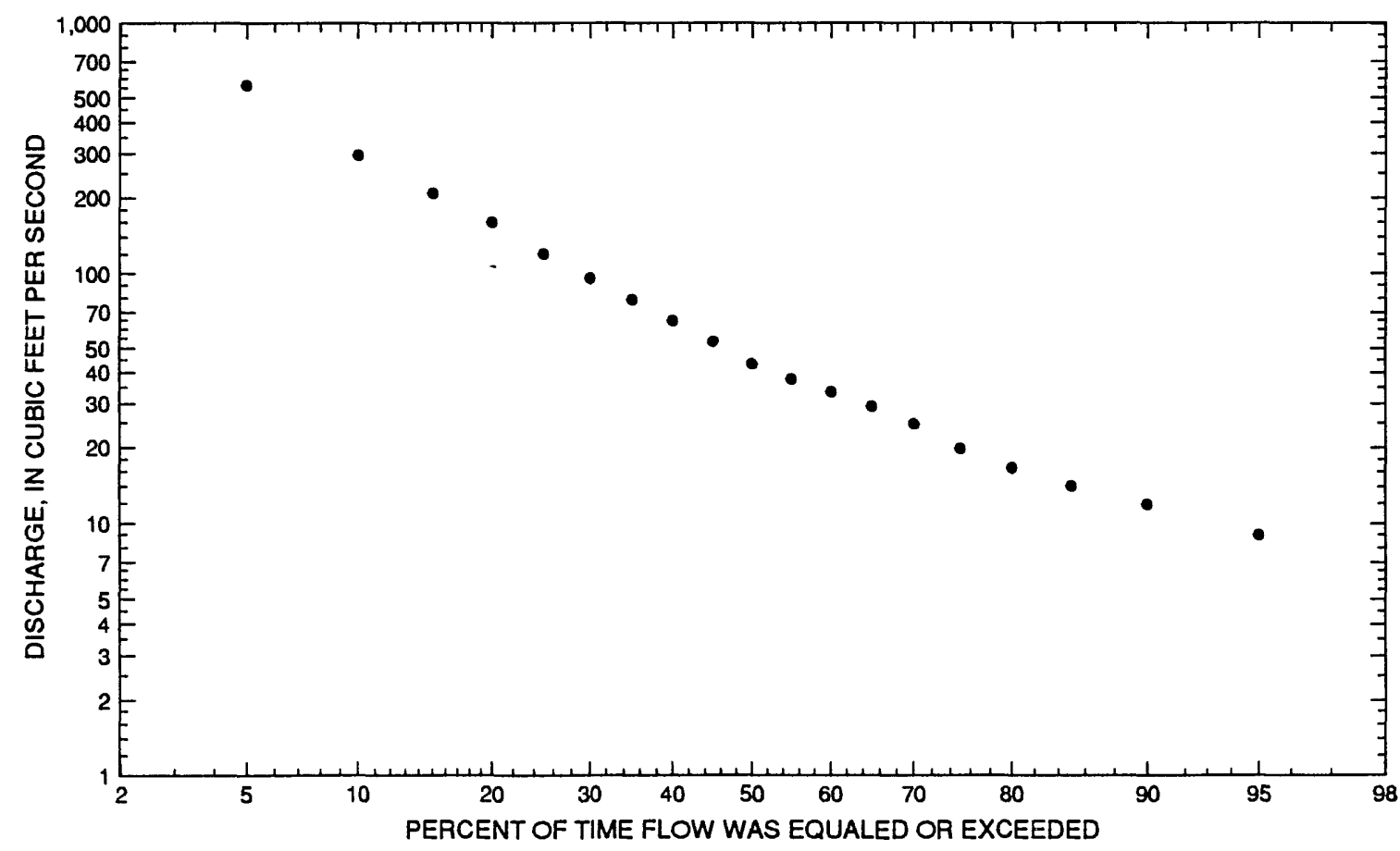




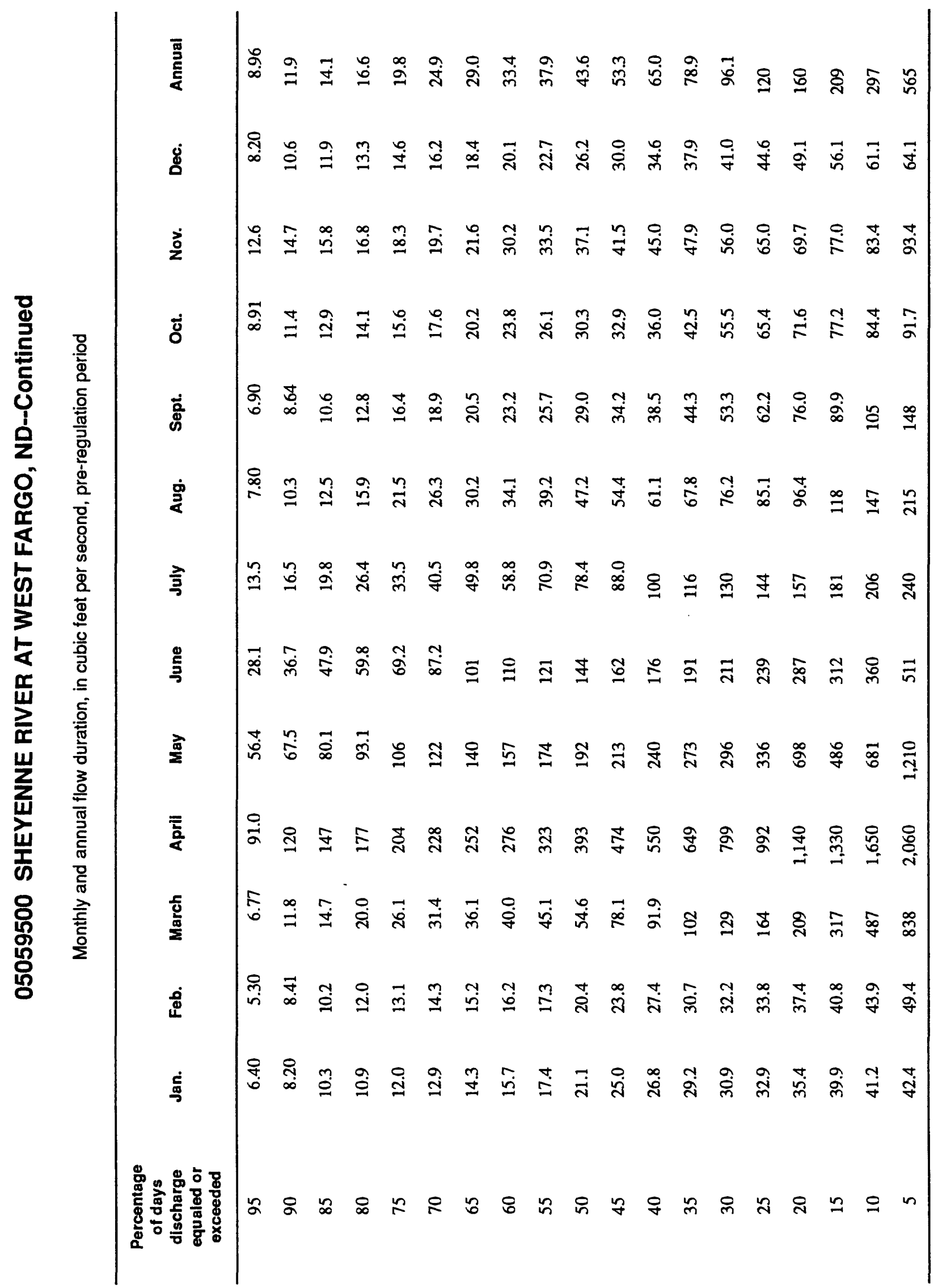




\section{SHEYENNE RIVER AT WEST FARGO, ND--Continued}

Probability of annual high discharges, pre-regulation period

[ng, statistic not given]

\begin{tabular}{lcccccc}
\hline & & & \multicolumn{5}{c}{$\begin{array}{c}\text { Maximum average discharge } \\
\left(\mathbf{f t}^{3} / \mathbf{s}\right)\end{array}$} \\
\cline { 5 - 7 } $\begin{array}{c}\text { Exceedance } \\
\text { probability }\end{array}$ & $\begin{array}{c}\text { Recurrence } \\
\text { interval } \\
\text { (years) }\end{array}$ & $\begin{array}{c}\text { Maximum } \\
\text { instantaneous } \\
\left(\mathbf{f t}^{3} / \mathbf{s}\right)\end{array}$ & 3-day period & 7-day period & 15-day period & 30-day period \\
\hline 0.99 & 1.01 & 163 & 136 & 123 & 103 & 90.5 \\
0.95 & 1.05 & 295 & 240 & 213 & 177 & 151 \\
0.90 & 1.11 & 398 & 324 & 284 & 237 & 199 \\
0.80 & 1.25 & 564 & 461 & 403 & 339 & 281 \\
0.50 & 2 & 1,040 & 887 & 784 & 673 & 552 \\
0.20 & 5 & 1,820 & 1,660 & 1,520 & 1,350 & 1,110 \\
0.10 & 10 & 2,390 & 2,280 & 2,130 & 1,940 & 1,610 \\
0.04 & 25 & 3,130 & 3,180 & 3,070 & 2,880 & 2,420 \\
0.02 & 50 & 3,690 & 3,920 & 3,880 & 3,710 & 3,160 \\
0.01 & 100 & 4,260 & 4,720 & 4,780 & 4,680 & 4,030 \\
0.005 & 200 & 4,840 & 5,580 & 5,790 & 5,780 & 5,050 \\
0.002 & 500 & 5,600 & ng & ng & ng & ng \\
\hline
\end{tabular}

Probability of annual low discharges, pre-regulation period

\begin{tabular}{|c|c|c|c|c|c|c|c|c|c|c|}
\hline \multirow{3}{*}{$\begin{array}{l}\text { Non- } \\
\text { exceed- } \\
\text { ance } \\
\text { prob- } \\
\text { ability }\end{array}$} & \multirow{3}{*}{$\begin{array}{l}\text { Recur- } \\
\text { rence } \\
\text { inter- } \\
\text { val } \\
\text { (years) }\end{array}$} & \multicolumn{9}{|c|}{ Minimum average discharge $\left(\mathrm{ft}^{3} / \mathrm{s}\right)$} \\
\hline & & \multicolumn{9}{|c|}{ Number of consecutive days } \\
\hline & & 1 & 3 & 7 & 14 & 30 & 60 & 90 & 120 & 183 \\
\hline 0.05 & 20 & 3.07 & 3.49 & 3.76 & 4.28 & 5.12 & 5.96 & 6.67 & 7.54 & 8.21 \\
\hline 0.10 & 10 & 4.30 & 4.82 & 5.14 & 5.72 & 6.68 & 7.68 & 8.60 & 9.64 & 10.5 \\
\hline 0.20 & 5 & 6.34 & 7.00 & 7.38 & 8.00 & 9.14 & 10.3 & 11.6 & 12.9 & 14.0 \\
\hline 0.50 & 2 & 12.6 & 13.4 & 13.9 & 14.6 & 16.1 & 17.8 & 19.8 & 22.2 & 24.6 \\
\hline
\end{tabular}




\section{SHEYENNE RIVER AT WEST FARGO, ND--Continued}

Probability of seasonal low discharges, pre-regulation period

\begin{tabular}{|c|c|c|c|c|c|c|c|c|c|}
\hline \multirow[b]{3}{*}{$\begin{array}{c}\text { Non- } \\
\text { exceedance } \\
\text { probability }\end{array}$} & \multirow[b]{3}{*}{$\begin{array}{l}\text { Recurrence } \\
\text { interval } \\
\text { (years) }\end{array}$} & \multicolumn{8}{|c|}{ Minimum average dlscharge $\left(\mathrm{ft}^{3} / \mathrm{s}\right)$} \\
\hline & & \multicolumn{8}{|c|}{ Number of consecutive days } \\
\hline & & 1 & 7 & 14 & 30 & 1 & 7 & 14 & 30 \\
\hline & & \multicolumn{4}{|c|}{ December-January-February } & \multicolumn{4}{|c|}{ March-April-May } \\
\hline 0.05 & 20 & 3.73 & 4.41 & 4.90 & 5.97 & 6.27 & 6.34 & 6.49 & 15.3 \\
\hline 0.10 & 10 & 5.23 & 6.02 & 6.55 & 7.70 & 8.63 & 8.97 & 9.48 & 23.7 \\
\hline 0.20 & 5 & 7.69 & 8.59 & 9.14 & 10.4 & 12.7 & 13.6 & 14.9 & 38.3 \\
\hline \multirow[t]{2}{*}{0.50} & 2 & 14.8 & 15.8 & 16.4 & 47.7 & 26.5 & 30.4 & 34.7 & 82.3 \\
\hline & & \multicolumn{4}{|c|}{ June-July-August } & \multicolumn{4}{|c|}{ September-October-November } \\
\hline 0.05 & 20 & 4.97 & 5.97 & 6.48 & 8.27 & 4.35 & 5.11 & 5.47 & 5.98 \\
\hline 0.10 & 10 & 7.29 & 8.76 & 9.53 & 12.3 & 6.01 & 6.83 & 7.29 & 8.00 \\
\hline 0.20 & 5 & 11.4 & 13.7 & 14.9 & 19.4 & 8.75 & 9.72 & 10.3 & 11.4 \\
\hline 0.50 & 2 & 25.1 & 30.2 & 33.6 & 43.7 & 17.2 & 19.1 & 20.3 & 22.7 \\
\hline
\end{tabular}




\section{SHEYENNE RIVER AT WEST FARGO, ND--Continued}

\section{Post-regulation period}

Statistics of monthly and annual mean discharges, post-regulation period

\begin{tabular}{|c|c|c|c|c|c|c|c|c|}
\hline \multirow[b]{2}{*}{ Month } & \multicolumn{2}{|c|}{ Maximum } & \multicolumn{2}{|c|}{ Minimum } & \multicolumn{4}{|c|}{ Mean } \\
\hline & $\begin{array}{c}\text { Discharge } \\
\left(\mathrm{ft}^{3} / \mathrm{s}\right)\end{array}$ & $\begin{array}{c}\text { Water year } \\
\text { of } \\
\text { occurrence }\end{array}$ & $\begin{array}{c}\text { Dlscharge } \\
\left(\mathrm{ft}^{3} / \mathrm{s}\right)\end{array}$ & $\begin{array}{c}\text { Water year } \\
\text { of } \\
\text { occurrence }\end{array}$ & $\begin{array}{c}\text { Discharge } \\
\left(\mathrm{ft}^{3} / \mathbf{s}\right)\end{array}$ & $\begin{array}{l}\text { Standard } \\
\text { deviation } \\
\left(\mathrm{ft}^{3} / \mathrm{s}\right)\end{array}$ & $\begin{array}{l}\text { Coeffi- } \\
\text { clent of } \\
\text { variation }\end{array}$ & $\begin{array}{l}\text { Percentage } \\
\text { of annual } \\
\text { discharge }\end{array}$ \\
\hline October & 246 & 1994 & 18.5 & 1992 & 73.4 & 48.1 & 0.66 & 2.95 \\
\hline November & 241 & 1980 & 18.9 & 1977 & 84.3 & 55.8 & 0.66 & 3.38 \\
\hline December & 227 & 1987 & 9.15 & 1977 & 76.5 & 52.2 & 0.68 & 3.07 \\
\hline January & 176 & 1987 & 8.40 & 1977 & 69.8 & 43.8 & 0.63 & 2.80 \\
\hline February & 223 & 1994 & 16.4 & 1977 & 78.0 & 48.1 & 0.62 & 3.13 \\
\hline March & 1,120 & 1987 & 34.0 & 1956 & 252 & 255 & 1.01 & 10.1 \\
\hline April & 1,790 & 1969 & 65.2 & 1991 & 692 & 525 & 0.76 & 27.8 \\
\hline May & 2,650 & 1950 & 54.0 & 1959 & 424 & 513 & 1.21 & 17.0 \\
\hline June & 1,780 & 1950 & 40.2 & 1977 & 269 & 284 & 1.05 & 10.8 \\
\hline July & 1,360 & 1975 & 26.8 & 1988 & 246 & 320 & 1.30 & 9.87 \\
\hline August & 2,220 & 1993 & 21.1 & 1988 & 141 & 328 & 2.33 & 5.65 \\
\hline September & 532 & 1993 & 7.43 & 1976 & 85.3 & 104 & 1.22 & 3.42 \\
\hline Annual & 584 & 1950 & 44.5 & 1977 & 208 & 134 & 0.65 & 100 \\
\hline
\end{tabular}

Annual flow duration, post-regulation period

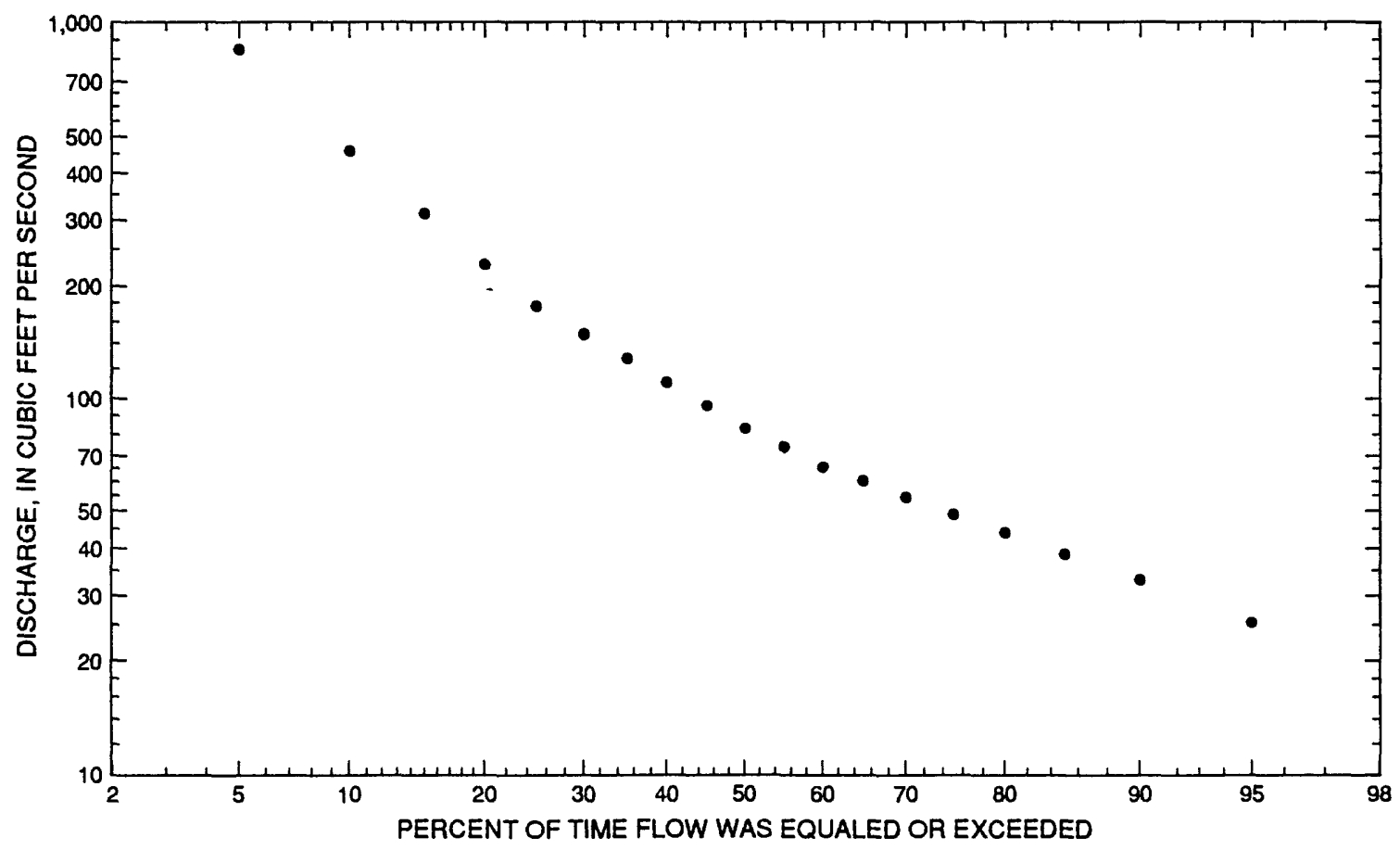




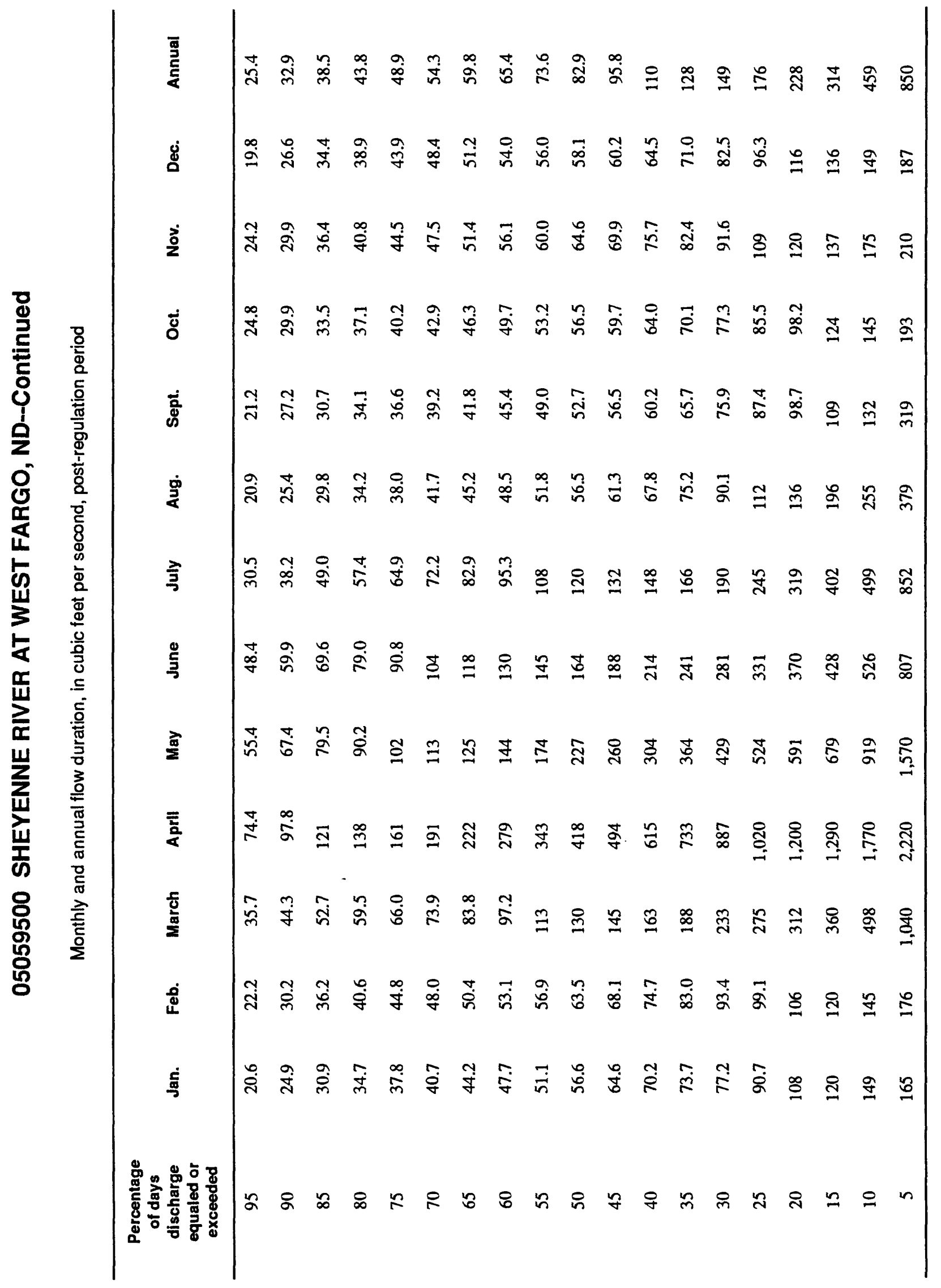




\section{SHEYENNE RIVER AT WEST FARGO, ND--Continued}

Probability of annual high discharges, post-regulation period

[ng, statistic not given]

\begin{tabular}{lcccccc}
\hline & & & \multicolumn{5}{c}{$\begin{array}{c}\text { Maximum average discharge } \\
\left(\mathbf{f t}^{3} / \mathbf{s}\right)\end{array}$} \\
\cline { 5 - 7 } $\begin{array}{c}\text { Exceedance } \\
\text { probability }\end{array}$ & $\begin{array}{c}\text { Recurrence } \\
\text { interval } \\
\text { (years) }\end{array}$ & $\begin{array}{c}\text { Maximum } \\
\text { instantaneous } \\
\left(\mathrm{ft}^{3} / \mathbf{s}\right)\end{array}$ & 3-day period & 7-day period & 15-day period & 30-day period \\
\hline 0.99 & 1.01 & 124 & 85.6 & 75.4 & 67.2 & 57.2 \\
0.95 & 1.05 & 264 & 210 & 186 & 158 & 128 \\
0.90 & 1.11 & 383 & 324 & 287 & 242 & 191 \\
0.80 & 1.25 & 586 & 526 & 470 & 391 & 305 \\
0.50 & 2 & 1,220 & 1,180 & 1,070 & 902 & 698 \\
0.20 & 5 & 2,310 & 2,270 & 2,130 & 1,860 & 1,470 \\
0.10 & 10 & 3,100 & 3,030 & 2,890 & 2,600 & 2,110 \\
0.04 & 25 & 4,130 & 3,960 & 3,860 & 3,600 & 3,020 \\
0.02 & 50 & 4,900 & 4,610 & 4,560 & 4,370 & 3,760 \\
0.01 & 100 & 5,650 & 5,220 & 5,230 & 5,160 & 4,550 \\
0.005 & 200 & 6,400 & 5,800 & 5,880 & 5,950 & 5,380 \\
0.002 & 500 & 7,370 & $n g$ & $n g$ & $n g$ & ng \\
\hline
\end{tabular}

Probability of annual low discharges, post-regulation period

\begin{tabular}{|c|c|c|c|c|c|c|c|c|c|c|}
\hline \multirow{3}{*}{$\begin{array}{l}\text { Non- } \\
\text { exceed- } \\
\text { ance } \\
\text { prob- } \\
\text { abllity }\end{array}$} & \multirow{3}{*}{$\begin{array}{l}\text { Recur- } \\
\text { rence } \\
\text { inter- } \\
\text { val } \\
\text { (years) }\end{array}$} & \multicolumn{9}{|c|}{ Minimum average dlscharge $\left(\mathrm{ft}^{3} / \mathrm{s}\right)$} \\
\hline & & \multicolumn{9}{|c|}{ Number of consecutlve days } \\
\hline & & 1 & 3 & 7 & 14 & 30 & 60 & 90 & 120 & 183 \\
\hline 0.05 & 20 & 5.25 & 6.23 & 7.81 & 9.27 & 14.5 & 17.1 & 19.6 & 21.9 & 24.9 \\
\hline 0.10 & 10 & 8.61 & 9.76 & 11.6 & 13.4 & 17.9 & 21.0 & 23.9 & 26.5 & 30.4 \\
\hline 0.20 & 5 & 14.3 & 15.6 & 17.6 & 19.8 & 23.0 & 27.0 & 30.4 & 33.6 & 38.6 \\
\hline 0.50 & 2 & 28.7 & 30.9 & 32.8 & 35.8 & 36.8 & 43.1 & 48.1 & 52.8 & 61.2 \\
\hline
\end{tabular}


05059500 SHEYENNE RIVER AT WEST FARGO, ND-Continued

Probability of seasonal low discharges, post-regulation period

\begin{tabular}{|c|c|c|c|c|c|c|c|c|c|}
\hline \multirow[b]{3}{*}{$\begin{array}{l}\text { Non- } \\
\text { exceedance } \\
\text { probability }\end{array}$} & \multirow[b]{3}{*}{$\begin{array}{c}\text { Recurrence } \\
\text { interval } \\
\text { (years) }\end{array}$} & \multicolumn{8}{|c|}{ Minimum average discharge $\left(\mathrm{ft}^{3} / \mathrm{s}\right)$} \\
\hline & & \multicolumn{8}{|c|}{ Number of consecutive days } \\
\hline & & 1 & 7 & 14 & 30 & 1 & 7 & 14 & 30 \\
\hline & & \multicolumn{4}{|c|}{ December-January-February } & \multicolumn{4}{|c|}{ March-April-May } \\
\hline 0.05 & 20 & 13.0 & 14.1 & 14.9 & 16.4 & 20.8 & 27.0 & 31.1 & 41.6 \\
\hline 0.10 & 10 & 17.0 & 18.4 & 19.7 & 21.6 & 26.6 & 32.4 & 37.2 & 51.9 \\
\hline 0.20 & 5 & 23.1 & 25.3 & 27.1 & 29.6 & 35.6 & 40.9 & 47.0 & 68.8 \\
\hline \multirow[t]{2}{*}{0.50} & 2 & 39.9 & 44.5 & 47.5 & 51.4 & 61.6 & 66.4 & 78.0 & 124 \\
\hline & & \multicolumn{4}{|c|}{ June-July-August } & \multicolumn{4}{|c|}{ September-October-November } \\
\hline 0.05 & 20 & 11.9 & 14.2 & 17.2 & 20.6 & 6.30 & 9.52 & 10.6 & 17.2 \\
\hline 0.10 & 10 & 15.6 & 18.0 & 21.1 & 25.4 & 10.5 & 14.6 & 15.9 & 22.0 \\
\hline 0.20 & 5 & 21.7 & 24.3 & 27.3 & 33.3 & 17.7 & 22.8 & 24.4 & 29.1 \\
\hline 0.50 & 2 & 40.6 & 43.9 & 47.8 & 59.8 & 35.6 & 42.6 & 45.5 & 48.2 \\
\hline
\end{tabular}




\section{SHEYENNE RIVER AT WEST FARGO, ND--Continued}

Annual peak discharge and corresponding gage height, period of record

$$
[-, \text { no data }]
$$

\begin{tabular}{|c|c|c|c|c|c|c|c|}
\hline $\begin{array}{l}\text { Water } \\
\text { year }\end{array}$ & Date & $\begin{array}{c}\text { Gage } \\
\text { height } \\
\text { (feet) }\end{array}$ & $\begin{array}{c}\text { Peak } \\
\text { discharge } \\
\left(\mathrm{ft}^{3} / \mathrm{s}\right)\end{array}$ & $\begin{array}{c}\text { Water } \\
\text { year }\end{array}$ & Date & $\begin{array}{c}\text { Gage } \\
\text { height } \\
\text { (feet) }\end{array}$ & $\begin{array}{c}\text { Peak } \\
\text { discharge } \\
\left(\mathrm{ft}^{3} / \mathrm{s}\right)\end{array}$ \\
\hline \multicolumn{8}{|c|}{ Annual peak discharge, by year, and corresponding gage height } \\
\hline 1903 & April 11 & 14.70 & 1,570 & 1960 & April 14 & 14.75 & 1,720 \\
\hline 1904 & April 25 & 8.70 & 1,950 & 1961 & March 14 & 8.08 & 255 \\
\hline 1905 & May 13 & 9.80 & 814 & 1962 & July 13 & 18.77 & 2,420 \\
\hline 1906 & April 16 & 11.70 & 1,060 & 1963 & April 8 & 8.63 & 490 \\
\hline 1919 & April 28 & 16.80 & 2,220 & 1964 & July 6 & 8.99 & 542 \\
\hline 1930 & April 5 & 14.90 & 1,780 & 1965 & April 24 & - & 2,530 \\
\hline 1931 & April 7 & 7.84 & 390 & 1966 & April 4 & - & 3,110 \\
\hline 1932 & April 13 & 11.84 & 1,110 & 1967 & April 21 & 14.92 & 1,830 \\
\hline 1933 & March 11 & 11.82 & 680 & 1968 & June 20 & 11.46 & 1,020 \\
\hline 1934 & April 13 & 7.22 & 336 & 1969 & April 22 & 21.20 & 3,060 \\
\hline 1935 & March 29 & 7.32 & 362 & 1970 & June 2 & 14.83 & 1,120 \\
\hline 1936 & April 21 & 9.74 & 718 & 1971 & May 1 & 14.63 & 1,800 \\
\hline 1937 & April 18 & 7.40 & 485 & 1972 & March 20 & 18.28 & 1,560 \\
\hline 1938 & March 27 & 5.50 & 249 & 1973 & March 17 & 13.14 & 500 \\
\hline 1939 & April 3 & - & 600 & 1974 & April 29 & 16.36 & 2,060 \\
\hline 1940 & April 18 & 7.88 & 555 & 1975 & July 3 & 21.75 & 2,850 \\
\hline 1941 & April 26 & 12.72 & 1,340 & 1976 & April 17 & 11.46 & 963 \\
\hline 1942 & April 27 & 11.02 & 1,040 & 1977 & May 9 & 8.54 & 475 \\
\hline 1943 & April 7 & - & 2,400 & 1978 & March 29 & 21.04 & 1,660 \\
\hline 1944 & May 22 & 10.10 & 861 & 1979 & April 21 & 22.12 & 3,480 \\
\hline 1945 & March 22 & 13.38 & 1,360 & 1980 & April 8 & 11.55 & 825 \\
\hline 1946 & March 31 & 15.01 & 1,690 & 1981 & April 11 & 7.99 & 460 \\
\hline 1947 & April 18 & 20.53 & 2,800 & 1982 & April 17 & 17.12 & 2,040 \\
\hline 1948 & May 7 & 18.46 & 2,650 & 1983 & March 22 & 19.82 & 2,100 \\
\hline 1949 & April 29 & 16.19 & 1,980 & 1984 & April 12 & 15.60 & 2,070 \\
\hline 1950 & May 22 & 19.99 & 2,810 & 1985 & May 23 & 8.84 & 608 \\
\hline 1951 & April 5 & 13.25 & 1,020 & 1986 & April 25 & 14.97 & 1,720 \\
\hline 1952 & April 12 & 20.28 & $.2,510$ & 1987 & March 29 & 20.35 & 2,940 \\
\hline 1953 & June 17 & - & 1,300 & 1988 & March 10 & 9.28 & 450 \\
\hline 1954 & July 7 & 9.11 & 565 & 1989 & April 4 & 20.53 & 1,420 \\
\hline 1955 & April 21 & 11.68 & 1,110 & 1990 & June 9 & 6.16 & 155 \\
\hline 1956 & May 8 & 13.01 & 1,450 & 1991 & July 1 & 11.67 & 270 \\
\hline 1957 & September 14 & 7.86 & 442 & 1992 & March 17 & 14.39 & 1,070 \\
\hline 1958 & March 7 & 8.84 & 470 & 1993 & August 11 & - & 3,250 \\
\hline 1959 & June 12 & 6.70 & 246 & 1994 & March 30 & - & 2,840 \\
\hline \multicolumn{8}{|c|}{ Annual peak discharge, from highest to lowest, and corresponding gage height } \\
\hline 1979 & April 21 & 22.12 & 3,480 & 1947 & April 18 & 20.53 & 2,800 \\
\hline 1993 & August 11 & - & 3,250 & 1948 & May 7 & 18.46 & 2,650 \\
\hline 1966 & April 4 & - & 3,110 & 1965 & April 24 & - & 2,530 \\
\hline 1969 & April 22 & 21.20 & 3,060 & 1952 & April 12 & 20.28 & 2,510 \\
\hline 1987 & March 29 & 20.35 & 2,940 & 1962 & July 13 & 18.77 & 2,420 \\
\hline 1975 & July 3 & 21.75 & 2,850 & 1943 & April 7 & - & 2,400 \\
\hline 1994 & March 30 & - & 2,840 & 1919 & April 28 & 16.80 & 2,220 \\
\hline 1950 & May 22 & 19.99 & 2,810 & 1983 & March 22 & 19.82 & 2,100 \\
\hline
\end{tabular}




\section{SHEYENNE RIVER AT WEST FARGO, ND--Continued}

Annual peak discharge and corresponding gage height, period of record--Continued

$$
[--, \text { no data }]
$$

\begin{tabular}{|c|c|c|c|c|c|c|c|}
\hline $\begin{array}{l}\text { Water } \\
\text { year }\end{array}$ & Date & $\begin{array}{c}\text { Gage } \\
\text { height } \\
\text { (feet) }\end{array}$ & $\begin{array}{c}\text { Peak } \\
\text { discharge } \\
\left(\mathrm{ft}^{3} / \mathrm{s}\right)\end{array}$ & $\begin{array}{l}\text { Water } \\
\text { year }\end{array}$ & Date & $\begin{array}{c}\text { Gage } \\
\text { height } \\
\text { (feet) }\end{array}$ & $\begin{array}{c}\text { Peak } \\
\text { discharge } \\
\left(\mathrm{ft}^{3} / \mathrm{s}\right)\end{array}$ \\
\hline \multicolumn{8}{|c|}{ Annual peak discharge, from highest to lowest, and corresponding gage height--Continued } \\
\hline 1984 & April 12 & 15.60 & 2,070 & 1976 & April 17 & 11.46 & 963 \\
\hline 1974 & April 29 & 16.36 & 2,060 & 1944 & May 22 & 10.10 & 861 \\
\hline 1982 & April 17 & 17.12 & 2,040 & 1980 & April 8 & 11.55 & 825 \\
\hline 1949 & April 29 & 16.19 & 1,980 & 1905 & May 13 & 9.80 & 814 \\
\hline 1904 & April 25 & 8.70 & 1,950 & 1936 & April 21 & 9.74 & 718 \\
\hline 1967 & April 21 & 14.92 & 1,830 & 1933 & March 11 & 11.82 & 680 \\
\hline 1971 & May 1 & 14.63 & 1,800 & 1985 & May 23 & 8.84 & 608 \\
\hline 1930 & April 5 & 14.90 & 1,780 & 1939 & April 3 & - & 600 \\
\hline 1960 & April 14 & 14.75 & 1,720 & 1954 & July 7 & 9.11 & 565 \\
\hline 1986 & April 25 & 14.97 & 1,720 & 1940 & April 18 & 7.88 & 555 \\
\hline 1946 & March 31 & 15.01 & 1,690 & 1964 & July 6 & 8.99 & 542 \\
\hline 1978 & March 29 & 21.04 & 1,660 & 1973 & March 17 & 13.14 & 500 \\
\hline 1903 & April 11 & 14.70 & 1,570 & 1963 & April 8 & 8.63 & 490 \\
\hline 1972 & March 20 & 18.28 & 1,560 & 1937 & April 18 & 7.40 & 485 \\
\hline 1956 & May 8 & 13.01 & 1,450 & 1977 & May 9 & 8.54 & 475 \\
\hline 1989 & April 4 & 20.53 & 1,420 & 1958 & March 7 & 8.84 & 470 \\
\hline 1945 & March 22 & 13.38 & 1,360 & 1981 & April 11 & 7.99 & 460 \\
\hline 1941 & April 26 & 12.72 & 1,340 & 1988 & March 10 & 9.28 & 450 \\
\hline 1953 & June 17 & - & 1,300 & 1957 & September 14 & 7.86 & 442 \\
\hline 1970 & June 2 & 14.83 & 1,120 & 1931 & April 7 & 7.84 & 390 \\
\hline 1932 & April 13 & 11.84 & 1,110 & 1935 & March 29 & 7.32 & 362 \\
\hline 1955 & April 21 & 11.68 & 1,110 & 1934 & April 13 & 7.22 & 336 \\
\hline 1992 & March 17 & 14.39 & 1,070 & 1991 & July 1 & 11.67 & 270 \\
\hline 1906 & April 16 & 11.70 & 1,060 & 1961 & March 14 & 8.08 & 255 \\
\hline 1942 & April 27 & 11.02 & 1,040 & 1938 & March 27 & 5.50 & 249 \\
\hline 1951 & April 5 & 13.25 & 1,020 & 1959 & June 12 & 6.70 & 246 \\
\hline 1968 & June 20 & 11.46 & 1,020 & 1990 & June 9 & 6.16 & 155 \\
\hline
\end{tabular}




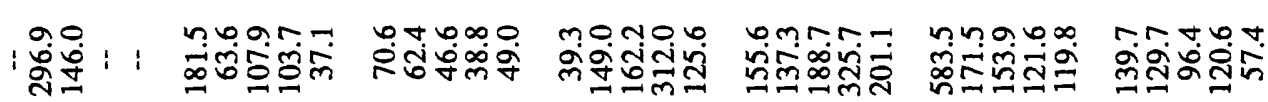

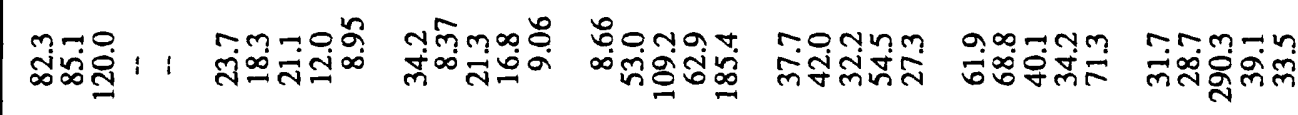

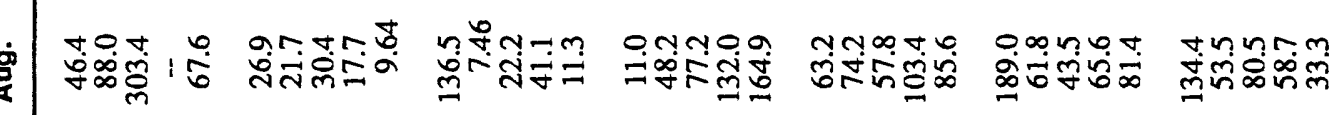

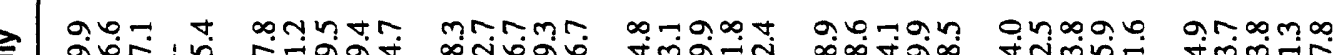

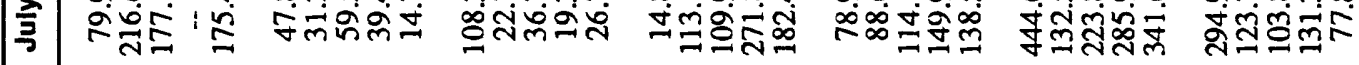

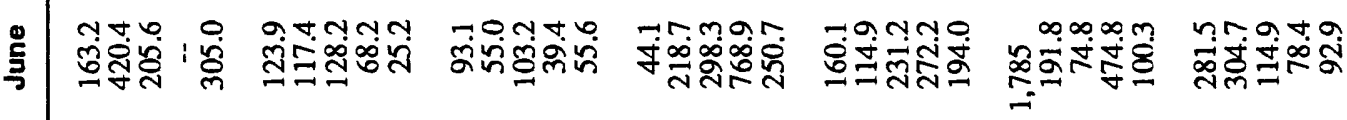

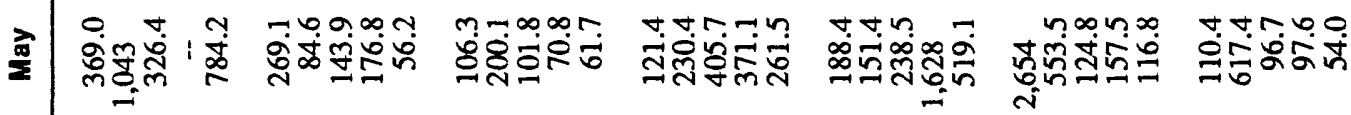

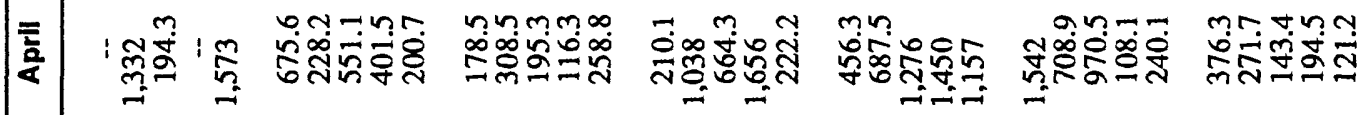

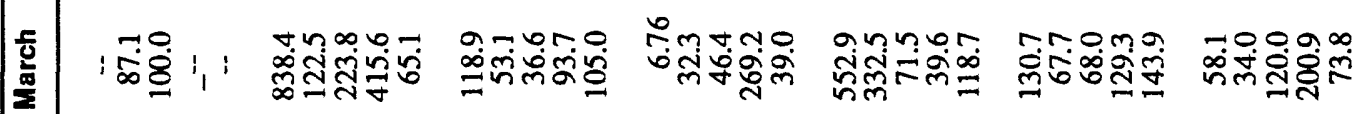

:

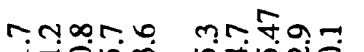

Rotono कृ.

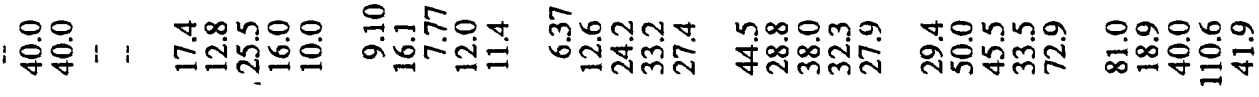

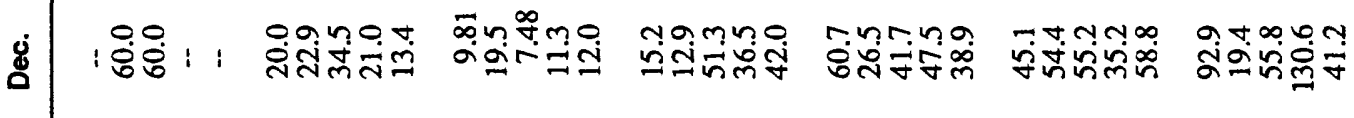

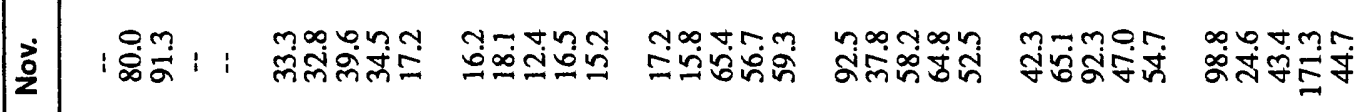

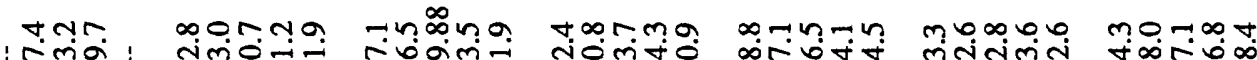

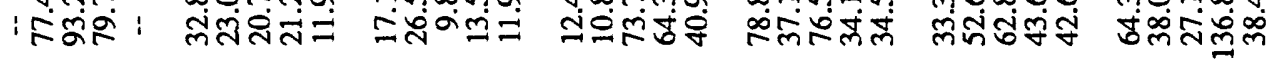

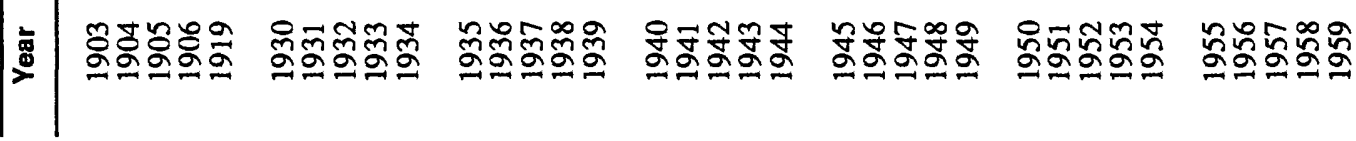




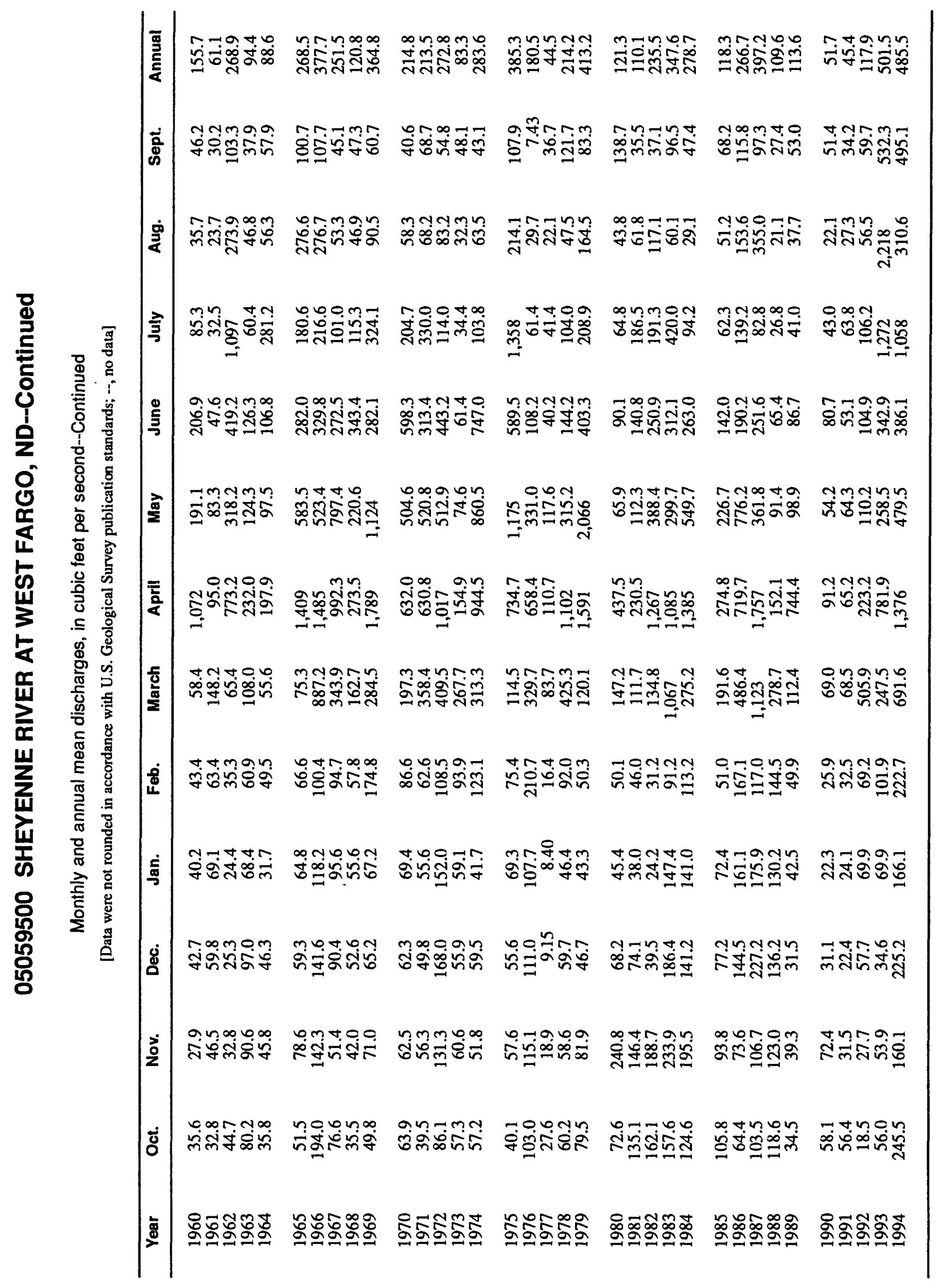




\section{Station Description}

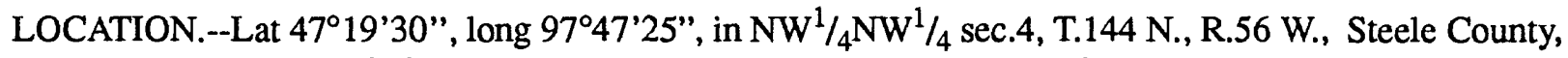
Hydrologic Unit $09020205,100 \mathrm{ft}$ downstream from box culvert on State Highway 38, $500 \mathrm{ft}$ east of the intersection of State Highway 32 and 38, and $3 \mathrm{mi}$ west of Hope.

DRAINAGE AREA.--20.2 $\mathrm{mi}^{2}$, of which about $2.8 \mathrm{mi}^{2}$ is probably noncontributing.

PERIOD OF RECORD.--October 1964 to current year (seasonal records only since 1983).

GAGE.--Water-stage recorder. Datum of gage is $1,296.62 \mathrm{ft}$ above sea level.

EXTREMES FOR PERIOD OF RECORD.--Maximum discharge, $900 \mathrm{ft}^{3} / \mathrm{s}$, Apr. 18, 1979; maximum gage height, $7.53 \mathrm{ft}$, July 24, 1993; minimum discharge, no flow for several months each year.

Annual mean discharge

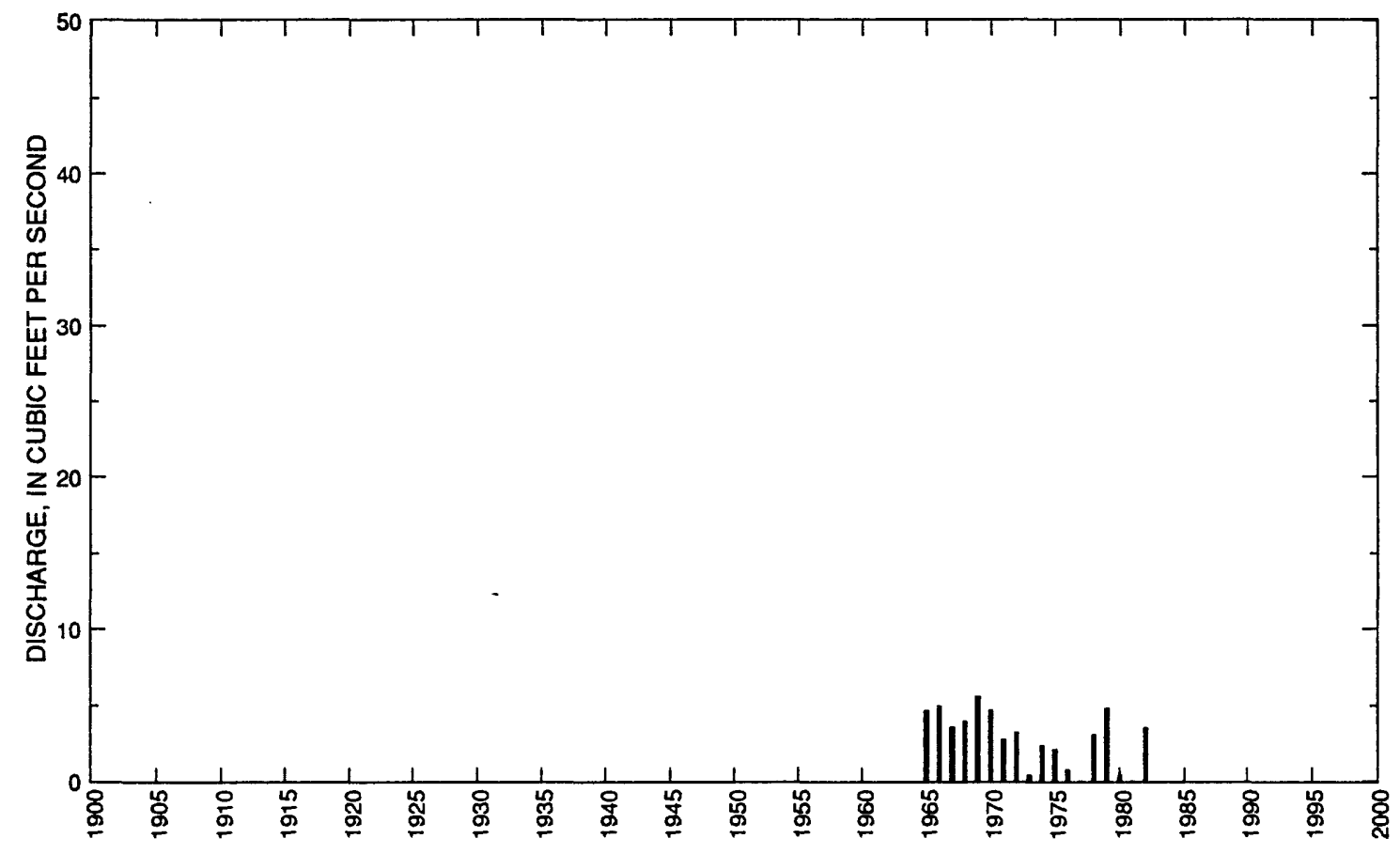




\section{MAPLE RIVER NEAR HOPE, ND--Continued}

Statistics of monthly and annual mean discharges

[ $\mathrm{m}$, more than 1 year of occurrence; ng, statistic not given]

\begin{tabular}{|c|c|c|c|c|c|c|c|c|}
\hline \multirow[b]{2}{*}{ Month } & \multicolumn{2}{|c|}{ Maximum } & \multicolumn{2}{|c|}{ Minimum } & \multicolumn{4}{|c|}{ Mean } \\
\hline & $\begin{array}{c}\text { Discharge } \\
\left(\mathrm{ft}^{3} / \mathbf{s}\right)\end{array}$ & $\begin{array}{c}\text { Water year } \\
\text { of } \\
\text { occurrence }\end{array}$ & $\begin{array}{c}\text { Discharge } \\
\left(\mathrm{ft}^{3} / \mathrm{s}\right)\end{array}$ & $\begin{array}{c}\text { Water year } \\
\text { of } \\
\text { occurrence }\end{array}$ & $\begin{array}{c}\text { Discharge } \\
\left(\mathrm{ft}^{3} / \mathbf{s}\right)\end{array}$ & $\begin{array}{c}\text { Standard } \\
\text { deviation } \\
\left(\mathrm{ft}^{3} / \mathrm{s}\right)\end{array}$ & $\begin{array}{l}\text { Coeffi- } \\
\text { cient of } \\
\text { variation }\end{array}$ & $\begin{array}{c}\text { Percentage } \\
\text { of annual } \\
\text { discharge }\end{array}$ \\
\hline October & 1.07 & 1966 & 0 & $\mathrm{~m}$ & 0.060 & 0.25 & 4.19 & 0.18 \\
\hline November & 0.054 & 1966 & 0 & $\mathrm{~m}$ & 0 & 0.01 & 3.76 & 0.01 \\
\hline December & 0 & $\mathrm{~m}$ & 0 & $\mathrm{~m}$ & 0 & 0 & ng & 0 \\
\hline January & 0 & $\mathrm{~m}$ & 0 & $\mathrm{~m}$ & 0 & 0 & ng & 0 \\
\hline February & 0.006 & 1981 & 0 & $\mathrm{~m}$ & 0 & 0 & 4.24 & 0 \\
\hline March & 41.8 & 1987 & 0 & $\mathrm{~m}$ & 9.12 & 10.6 & 1.17 & 26.7 \\
\hline April & 56.5 & 1969 & 0.007 & 1991 & 13.8 & 16.8 & 1.22 & 40.3 \\
\hline May & 11.9 & 1972 & 0 & $\mathrm{~m}$ & 2.13 & 2.84 & 1.33 & 6.23 \\
\hline June & 34.5 & 1968 & 0 & $\mathrm{~m}$ & 2.85 & 6.77 & 2.38 & 8.34 \\
\hline July & 65.3 & 1993 & 0 & $\mathrm{~m}$ & 5.06 & 12.9 & 2.55 & 14.8 \\
\hline August & 7.95 & 1993 & 0 & $\mathrm{~m}$ & 0.580 & 1.90 & 3.30 & 1.69 \\
\hline September & 15.3 & 1994 & 0 & $\mathrm{~m}$ & 0.590 & 2.80 & 4.74 & 1.73 \\
\hline Annual & 5.55 & 1969 & 0.002 & 1981 & 2.82 & 1.84 & 0.65 & 100 \\
\hline
\end{tabular}

Annual flow duration

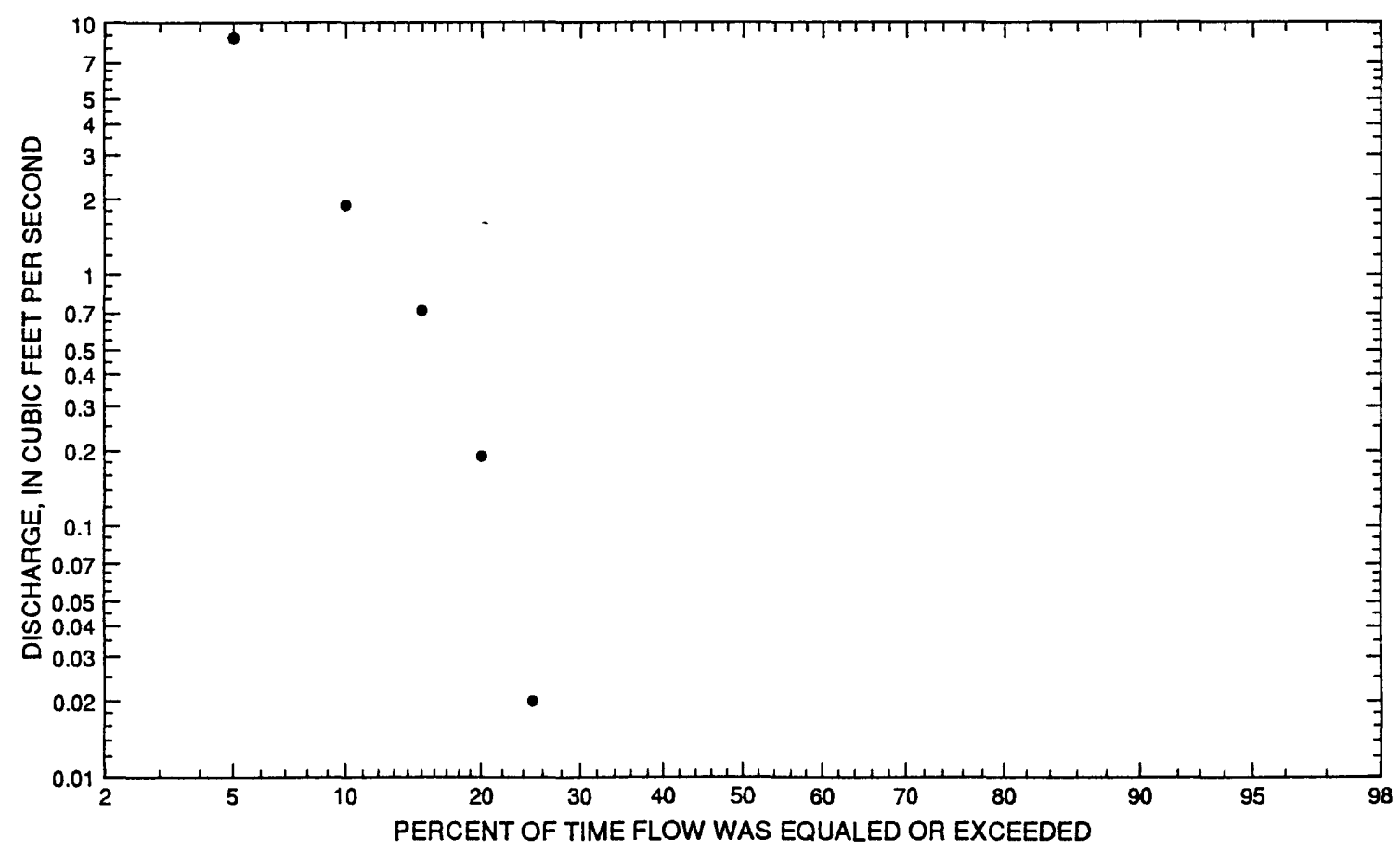




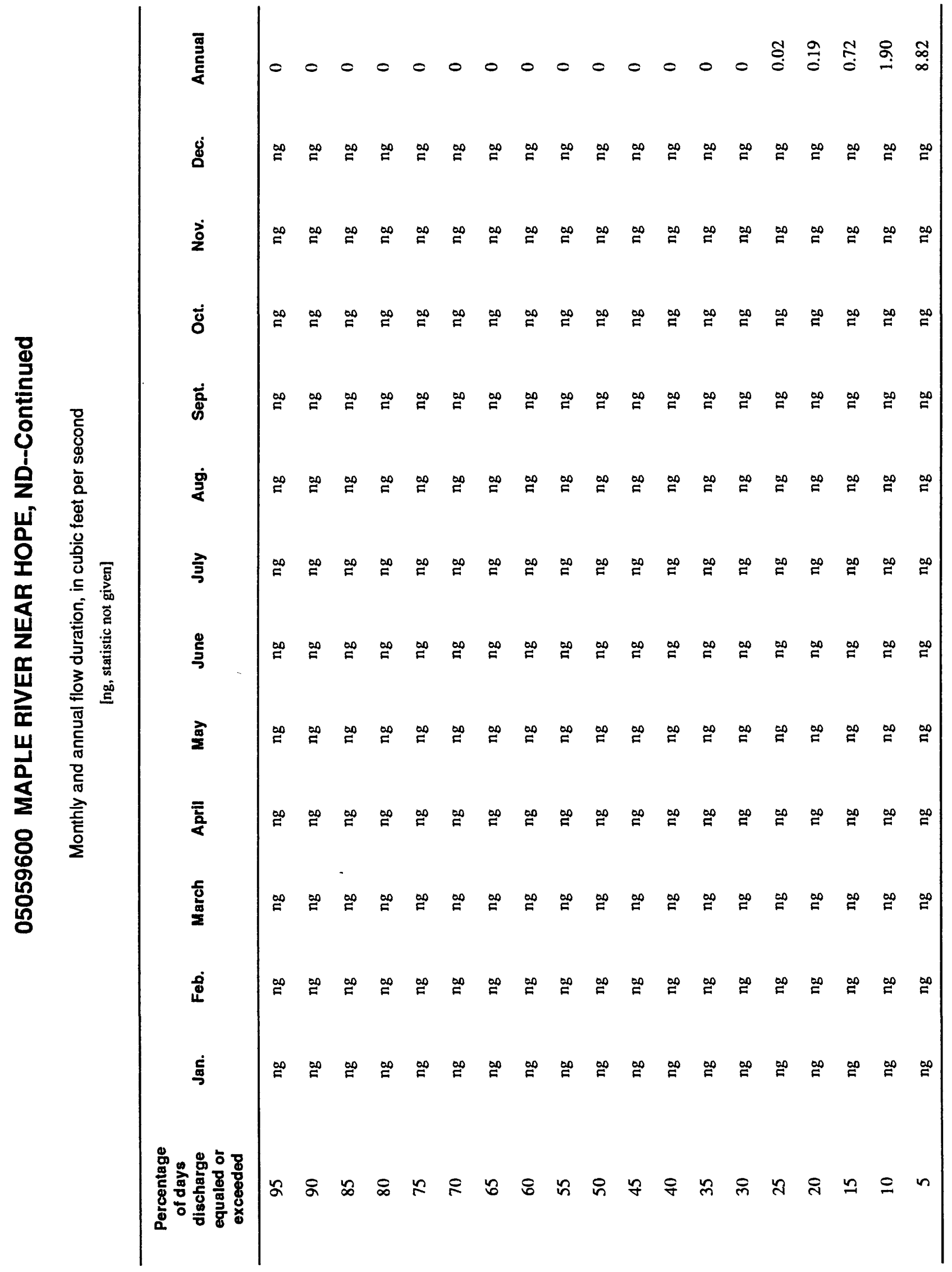




\section{MAPLE RIVER NEAR HOPE, ND--Continued}

Probability of annual high discharges

[ng, statistic not given]

\begin{tabular}{|c|c|c|c|c|c|c|}
\hline \multirow[b]{2}{*}{$\begin{array}{l}\text { Exceedance } \\
\text { probablity }\end{array}$} & \multirow[b]{2}{*}{$\begin{array}{l}\text { Recurrence } \\
\text { interval } \\
\text { (years) }\end{array}$} & \multirow[b]{2}{*}{$\begin{array}{c}\text { Maximum } \\
\text { instantaneous } \\
\left(\mathrm{ft}^{3} / \mathbf{s}\right)\end{array}$} & \multicolumn{4}{|c|}{$\begin{array}{l}\text { Maximum average discharge } \\
\qquad\left(\mathrm{f}^{3} / \mathrm{s}\right)\end{array}$} \\
\hline & & & 3-day period & 7-day period & 15-day period & 30-day period \\
\hline 0.99 & 1.01 & ng & 0.005 & 0.003 & 0.002 & 0.001 \\
\hline 0.95 & 1.05 & $\mathrm{ng}$ & 0.377 & 0.238 & 0.140 & 0.084 \\
\hline 0.90 & 1.11 & 25.5 & 2.29 & 1.44 & 0.838 & 0.498 \\
\hline 0.80 & 1.25 & 53.0 & 13.4 & 8.28 & 4.81 & 2.83 \\
\hline 0.50 & 2 & 177 & 122 & 73.6 & 42.5 & 24.6 \\
\hline 0.20 & 5 & 465 & 327 & 194 & 111 & 64.0 \\
\hline 0.10 & 10 & 705 & 400 & 236 & 135 & 77.5 \\
\hline 0.04 & 25 & 1,030 & 439 & 257 & 148 & 84.3 \\
\hline 0.02 & 50 & 1,280 & 449 & 263 & 151 & 86.1 \\
\hline 0.01 & 100 & 1,530 & 453 & 265 & 152 & 86.8 \\
\hline 0.005 & 200 & 1,760 & 455 & 266 & 152 & 87.1 \\
\hline 0.002 & 500 & 2,060 & $\mathrm{ng}$ & $\mathrm{ng}$ & $\mathrm{ng}$ & $\mathrm{ng}$ \\
\hline
\end{tabular}

Probability of annual low discharges

[ng, statistic not given]

Minimum average discharge $\left(\mathrm{ft}^{3} / \mathrm{s}\right)$

\begin{tabular}{|c|c|c|c|c|c|c|c|c|c|c|}
\hline \multirow{3}{*}{$\begin{array}{c}\text { Non- } \\
\text { exceed- } \\
\text { ance } \\
\text { prob- } \\
\text { ability }\end{array}$} & \multirow{3}{*}{$\begin{array}{l}\text { Recur- } \\
\text { rence } \\
\text { inter- } \\
\text { vai } \\
\text { (years) }\end{array}$} & \multicolumn{9}{|c|}{ Minimum average discharge $\left(\mathrm{ft}^{3} / \mathrm{s}\right)$} \\
\hline & & \multicolumn{9}{|c|}{ Number of consecutive days } \\
\hline & & 1 & 3 & 7 & 14 & 30 & 60 & 90 & 120 & 183 \\
\hline 0.05 & 20 & ng & ng & ng & ng & $\mathrm{ng}$ & $\mathrm{ng}$ & ng & ng & $\mathrm{ng}$ \\
\hline 0.10 & 10 & ng & ng & ng & ng & ng & $\mathrm{ng}$ & ng & ng & $\mathrm{ng}$ \\
\hline 0.20 & 5 & ng & $\mathrm{ng}$ & ng & ng & ng & ng & ng & ng & ng \\
\hline 0.50 & 2 & $\mathrm{ng}$ & $\mathrm{ng}$ & ng & $\mathbf{n g}$ & $\mathrm{ng}$ & $\mathrm{ng}$ & ng & ng & ng \\
\hline
\end{tabular}




\section{MAPLE RIVER NEAR HOPE, ND--Continued}

Probability of seasonal low discharges

[ng, statistic not given]

\begin{tabular}{|c|c|c|c|c|c|c|c|c|c|}
\hline \multirow[b]{3}{*}{$\begin{array}{c}\text { Non- } \\
\text { exceedance } \\
\text { probability }\end{array}$} & \multirow[b]{3}{*}{$\begin{array}{l}\text { Recurrence } \\
\text { Intervai } \\
\text { (years) }\end{array}$} & \multicolumn{8}{|c|}{ Minimum average discharge $\left(\mathrm{ft}^{3} / \mathrm{s}\right)$} \\
\hline & & \multicolumn{8}{|c|}{ Number of consecutive days } \\
\hline & & 1 & 7 & 14 & 30 & 1 & 7 & 14 & 30 \\
\hline & & \multicolumn{4}{|c|}{ December-January-February } & \multicolumn{4}{|c|}{ March-April-May } \\
\hline 0.05 & 20 & ng & $\mathrm{ng}$ & ng & ng & ng & $\mathrm{ng}$ & 0 & 0 \\
\hline 0.10 & 10 & ng & $\mathrm{ng}$ & $\mathrm{ng}$ & ng & ng & $\mathrm{ng}$ & 0 & 0 \\
\hline 0.20 & 5 & $\mathrm{ng}$ & $\mathrm{ng}$ & ng & ng & $\mathrm{ng}$ & ng & 0 & 0 \\
\hline \multirow[t]{2}{*}{0.50} & 2 & $\mathrm{ng}$ & $\mathrm{ng}$ & ng & ng & $\mathrm{ng}$ & $\mathrm{ng}$ & 0 & 0.123 \\
\hline & & \multicolumn{4}{|c|}{ June-July-August } & \multicolumn{4}{|c|}{ September-October-November } \\
\hline 0.05 & 20 & $\mathrm{ng}$ & 0 & 0 & 0 & $\mathrm{ng}$ & $\mathrm{ng}$ & $\mathrm{ng}$ & $\mathrm{ng}$ \\
\hline 0.10 & 10 & $\mathrm{ng}$ & 0 & 0 & 0 & $\mathrm{ng}$ & $\mathrm{ng}$ & $\mathrm{ng}$ & $\mathrm{ng}$ \\
\hline 0.20 & 5 & $\mathrm{ng}$ & 0 & 0 & 0 & $\mathrm{ng}$ & $\mathrm{ng}$ & $\mathrm{ng}$ & $\mathrm{ng}$ \\
\hline 0.50 & 2 & $\mathrm{ng}$ & 0 & 0 & 0 & $\mathrm{ng}$ & $\mathrm{ng}$ & $\mathrm{ng}$ & $\mathrm{ng}$ \\
\hline
\end{tabular}




\section{MAPLE RIVER NEAR HOPE, ND--Continued}

Annual peak discharge and corresponding gage height, period of record

\begin{tabular}{|c|c|c|c|c|c|c|c|}
\hline $\begin{array}{l}\text { Water } \\
\text { year }\end{array}$ & Date & $\begin{array}{c}\text { Gage } \\
\text { height } \\
\text { (feet) }\end{array}$ & $\begin{array}{c}\text { Peak } \\
\text { discharge } \\
\left(\mathrm{ft}^{3} / \mathrm{s}\right)\end{array}$ & $\begin{array}{l}\text { Water } \\
\text { year }\end{array}$ & Date & $\begin{array}{c}\text { Gage } \\
\text { helght } \\
\text { (feet) }\end{array}$ & $\begin{array}{c}\text { Peak } \\
\text { discharge } \\
\left(\mathrm{ft}^{3} / \mathrm{s}\right)\end{array}$ \\
\hline \multicolumn{8}{|c|}{ Annual peak discharge, by year, and corresponding gage height } \\
\hline 1965 & April 10 & 4.43 & 575 & 1980 & March 29 & 3.18 & 33.0 \\
\hline 1966 & March 14 & 3.18 & 150 & 1981 & June 21 & 1.88 & 0.980 \\
\hline 1967 & April 20 & 3.88 & 248 & 1982 & April 1 & 5.56 & 850 \\
\hline 1968 & June 10 & 4.78 & 734 & 1983 & March 13 & 4.29 & 160 \\
\hline 1969 & April 9 & 4.50 & 540 & 1984 & March 24 & 4.81 & 300 \\
\hline 1970 & April 25 & 4.03 & 349 & 1985 & March 12 & 3.29 & 73.0 \\
\hline 1971 & April 2 & 4.52 & 145 & 1986 & March 17 & 3.69 & 100 \\
\hline 1972 & May 27 & 4.65 & 465 & 1987 & March 21 & 6.47 & 360 \\
\hline 1973 & March 14 & 2.92 & 59.0 & 1988 & March 24 & 3.78 & 75.0 \\
\hline 1974 & April 10 & 3.80 & 232 & 1989 & April 5 & 4.11 & 217 \\
\hline 1975 & April 18 & 3.48 & 122 & 1990 & June 13 & 3.08 & 4.10 \\
\hline 1976 & March 24 & 3.38 & 104 & 1991 & May 24 & 3.33 & 19.0 \\
\hline 1977 & July 5 & 1.70 & 0.910 & 1992 & March 4 & 4.11 & 100 \\
\hline 1978 & March 26 & 4.19 & 175 & 1993 & July 24 & 7.53 & 585 \\
\hline 1979 & April 18 & 5.86 & 900 & 1994 & July 8 & 5.30 & 234 \\
\hline \multicolumn{8}{|c|}{ Annual peak discharge, from highest to lowest, and corresponding gage height } \\
\hline 1979 & April 18 & 5.86 & 900 & 1983 & March 13 & 4.29 & 160 \\
\hline 1982 & April 1 & 5.56 & 850 & 1966 & March 14 & 3.18 & 150 \\
\hline 1968 & June 10 & 4.78 & 734 & 1971 & April 2 & 4.52 & 145 \\
\hline 1993 & July 24 & 7.53 & 585 & 1975 & April 18 & 3.48 & 122 \\
\hline 1965 & April 10 & 4.43 & 575 & 1976 & March 24 & 3.38 & 104 \\
\hline 1969 & April 9 & 4.50 & 540 & 1986 & March 17 & 3.69 & 100 \\
\hline 1972 & May 27 & 4.65 & 465 & 1992 & March 4 & 4.11 & 100 \\
\hline 1987 & March 21 & 6.47 & 360 & 1988 & March 24 & 3.78 & 75.0 \\
\hline 1970 & April 25 & 4.03 & 349 & 1985 & March 12 & 3.29 & 73.0 \\
\hline 1984 & March 24 & 4.81 & 300 & 1973 & March 14 & 2.92 & 59.0 \\
\hline 1967 & April 20 & 3.88 & 248 & 1980 & March 29 & 3.18 & 33.0 \\
\hline 1994 & July 8 & 5.30 & 234 & 1991 & May 24 & 3.33 & 19.0 \\
\hline 1974 & April 10 & 3.80 & 232 & 1990 & June 13 & 3.08 & 4.10 \\
\hline 1989 & April 5 & 4.11 & 217 & 1981 & June 21 & 1.88 & 0.980 \\
\hline 1978 & March 26 & 4.19 & 175 & 1977 & July 5 & 1.70 & 0.910 \\
\hline
\end{tabular}




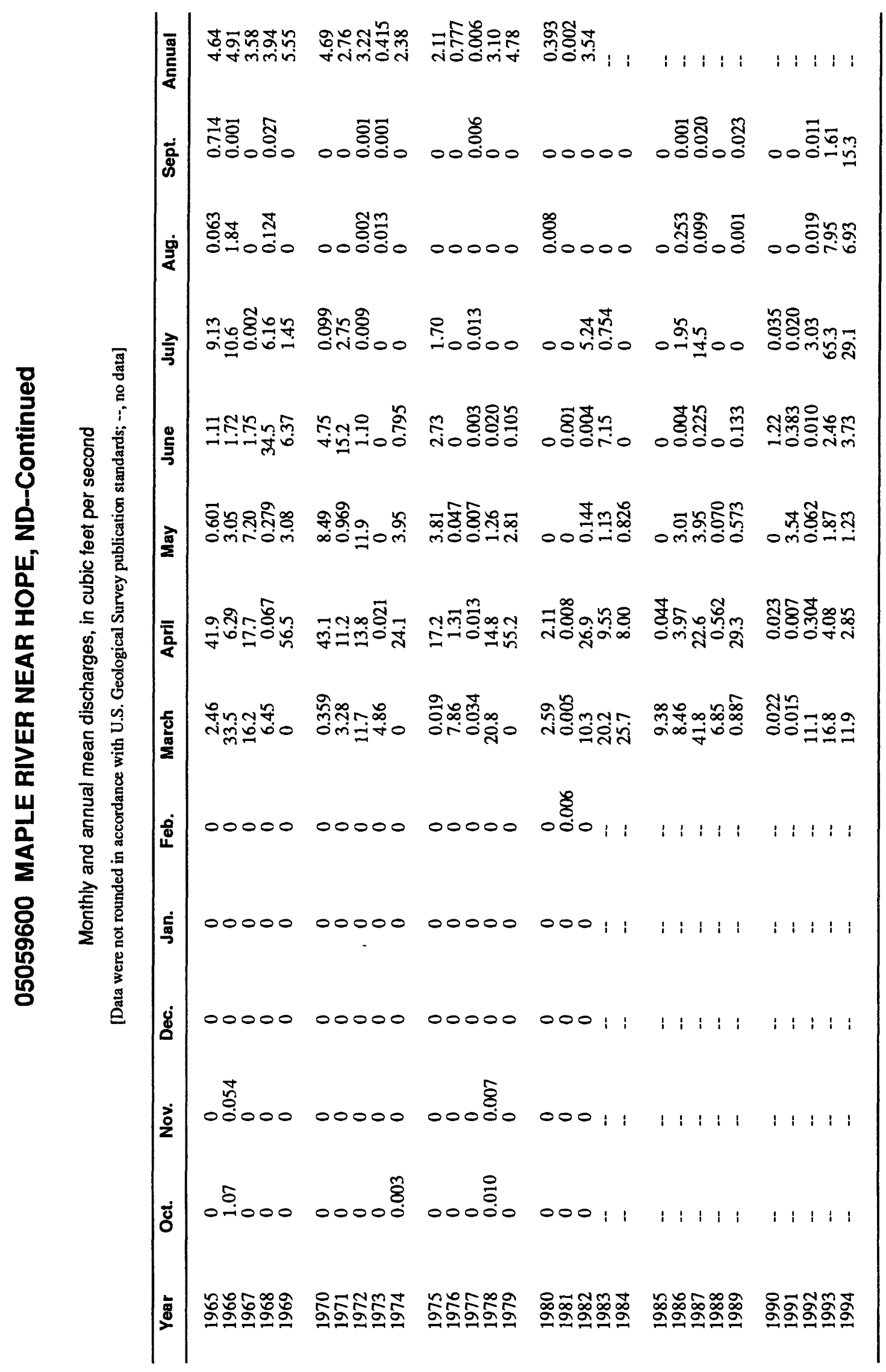




\section{MAPLE RIVER NEAR ENDERLIN, ND}

\section{Station Description}

LOCATION.--Lat $46^{\circ} 37^{\prime} 18^{\prime \prime}$, long $97^{\circ} 34^{\prime} 25^{\prime \prime}$, on west line sec.2, T.136 N., R.55 W., Ransom County, Hydrologic Unit 09020205 , on left bank $25 \mathrm{ft}$ downstream from county highway bridge, $1 \mathrm{mi}$ downstream from South Branch, and $1.2 \mathrm{mi}$ east of Enderlin.

DRAINAGE AREA.--843 $\mathrm{mi}^{2}$, of which about $47 \mathrm{mi}^{2}$ is probably noncontributing.

PERIOD OF RECORD.--May 1956 to current year.

GAGE.--Water-stage recorder. Datum of gage is $1,056.72 \mathrm{ft}$ above sea level. Sept. 21,1956 , to June 9,1969 , recording gage on right bank at same datum. Prior to Sept. 20, 1956, nonrecording gage at site $25 \mathrm{ft}$ upstream at same datum.

EXTREMES FOR PERIOD OF RECORD.--Maximum discharge, 7,610 $\mathrm{ft}^{3} / \mathrm{s}$, June 30, 1975; maximum gage height, $15.41 \mathrm{ft}$, June 30,1975 ; minimum daily discharge, $0.1 \mathrm{ft}^{3} / \mathrm{s}$, Dec. 7-9, 1963; minimum gage height, $1.90 \mathrm{ft}$, Oct. $5,1956$.

Annual mean discharge

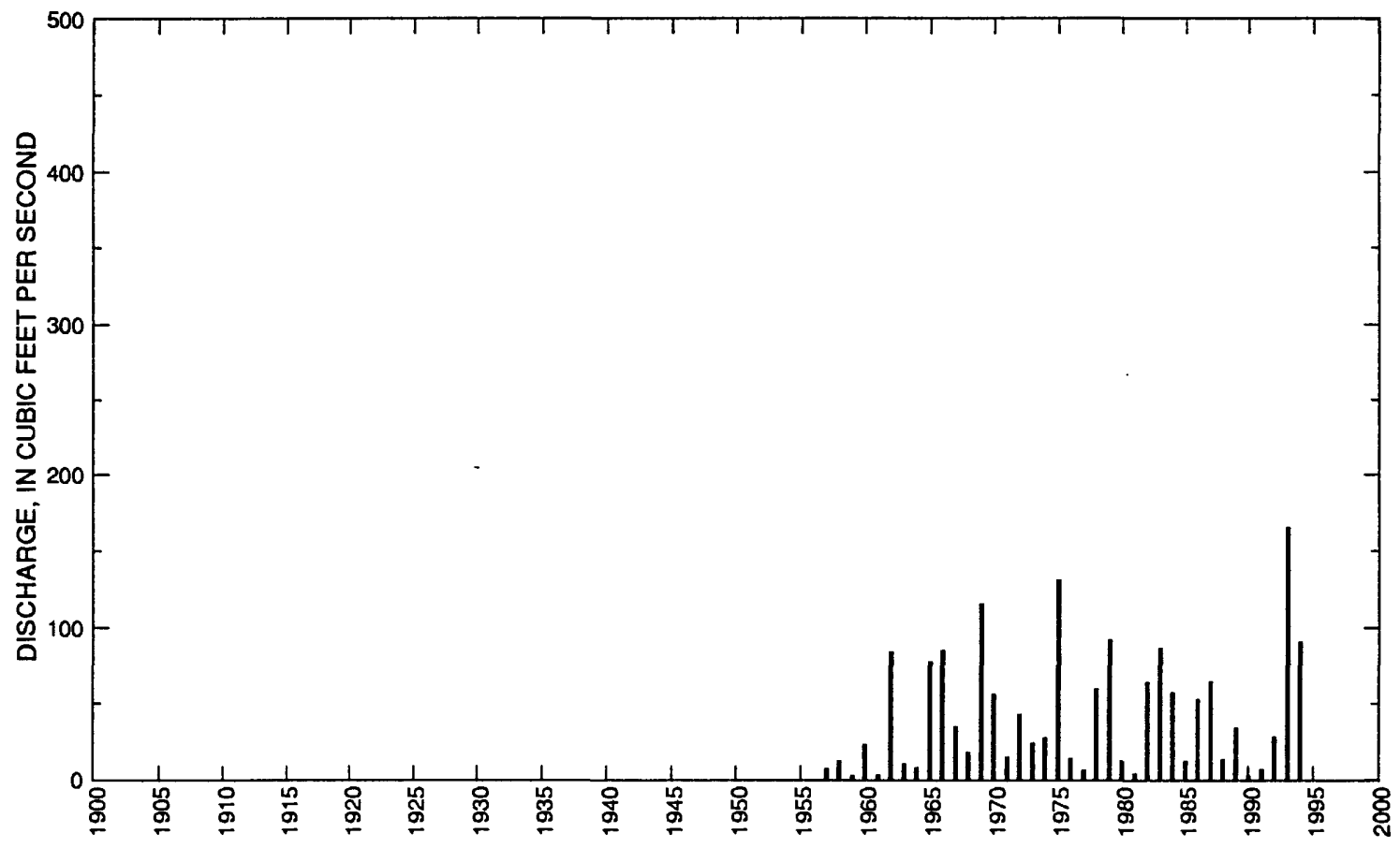




\section{MAPLE RIVER NEAR ENDERLIN, ND-Continued}

Statistics of monthly and annual mean discharges

\begin{tabular}{|c|c|c|c|c|c|c|c|c|}
\hline \multirow[b]{2}{*}{ Month } & \multicolumn{2}{|c|}{ Maximum } & \multicolumn{2}{|c|}{ Minimum } & \multicolumn{4}{|c|}{ Mean } \\
\hline & $\begin{array}{c}\text { Discharge } \\
\left(\mathrm{ft}^{3} / \mathrm{s}\right)\end{array}$ & $\begin{array}{c}\text { Water year } \\
\text { of } \\
\text { occurrence }\end{array}$ & $\begin{array}{c}\text { Discharge } \\
\left(\mathrm{ft}^{3} / \mathrm{s}\right)\end{array}$ & $\begin{array}{c}\text { Water year } \\
\text { of } \\
\text { occurrence }\end{array}$ & $\begin{array}{c}\text { Discharge } \\
\left(\mathrm{ft}^{3} / \mathrm{s}\right)\end{array}$ & $\begin{array}{c}\text { Standard } \\
\text { deviation } \\
\left(\mathrm{ft}^{3} / \mathrm{s}\right)\end{array}$ & $\begin{array}{l}\text { Coeffi- } \\
\text { cient of } \\
\text { variation }\end{array}$ & $\begin{array}{l}\text { Percentage } \\
\text { of annual } \\
\text { discharge }\end{array}$ \\
\hline October & 16.6 & 1966 & 1.52 & 1993 & 4.16 & 3.31 & 0.80 & 0.81 \\
\hline November & 13.3 & 1958 & 1.49 & 1961 & 4.12 & 2.76 & 0.67 & 0.80 \\
\hline December & 5.87 & 1958 & 1.32 & 1961 & 2.97 & 1.24 & 0.42 & 0.58 \\
\hline January & 4.05 & 1976 & 1.21 & 1969 & 2.37 & 0.69 & 0.29 & 0.46 \\
\hline February & 11.9 & 1976 & 1.30 & 1969 & 2.94 & 2.05 & 0.70 & 0.57 \\
\hline March & 622 & 1966 & 2.10 & 1969 & 113 & 157 & 1.39 & 22.0 \\
\hline April & 1,230 & 1969 & 2.06 & 1991 & 207 & 273 & 1.32 & 40.3 \\
\hline May & 191 & 1970 & 2.19 & 1992 & 40.4 & 48.8 & 1.21 & 7.88 \\
\hline June & 424 & 1975 & 2.26 & 1988 & 39.3 & 87.1 & 2.22 & 7.65 \\
\hline July & 874 & 1993 & 1.44 & 1961 & 64.6 & 168 & 2.60 & 12.6 \\
\hline August & 506 & 1993 & 1.33 & 1961 & 23.5 & 83.4 & 3.55 & 4.58 \\
\hline September & 111 & 1994 & 1.28 & 1984 & 9.27 & 19.5 & 2.10 & 1.81 \\
\hline Annual & 165 & 1993 & 2.14 & 1990 & 43.1 & 40.6 & 0.94 & 100 \\
\hline
\end{tabular}

Annual flow duration

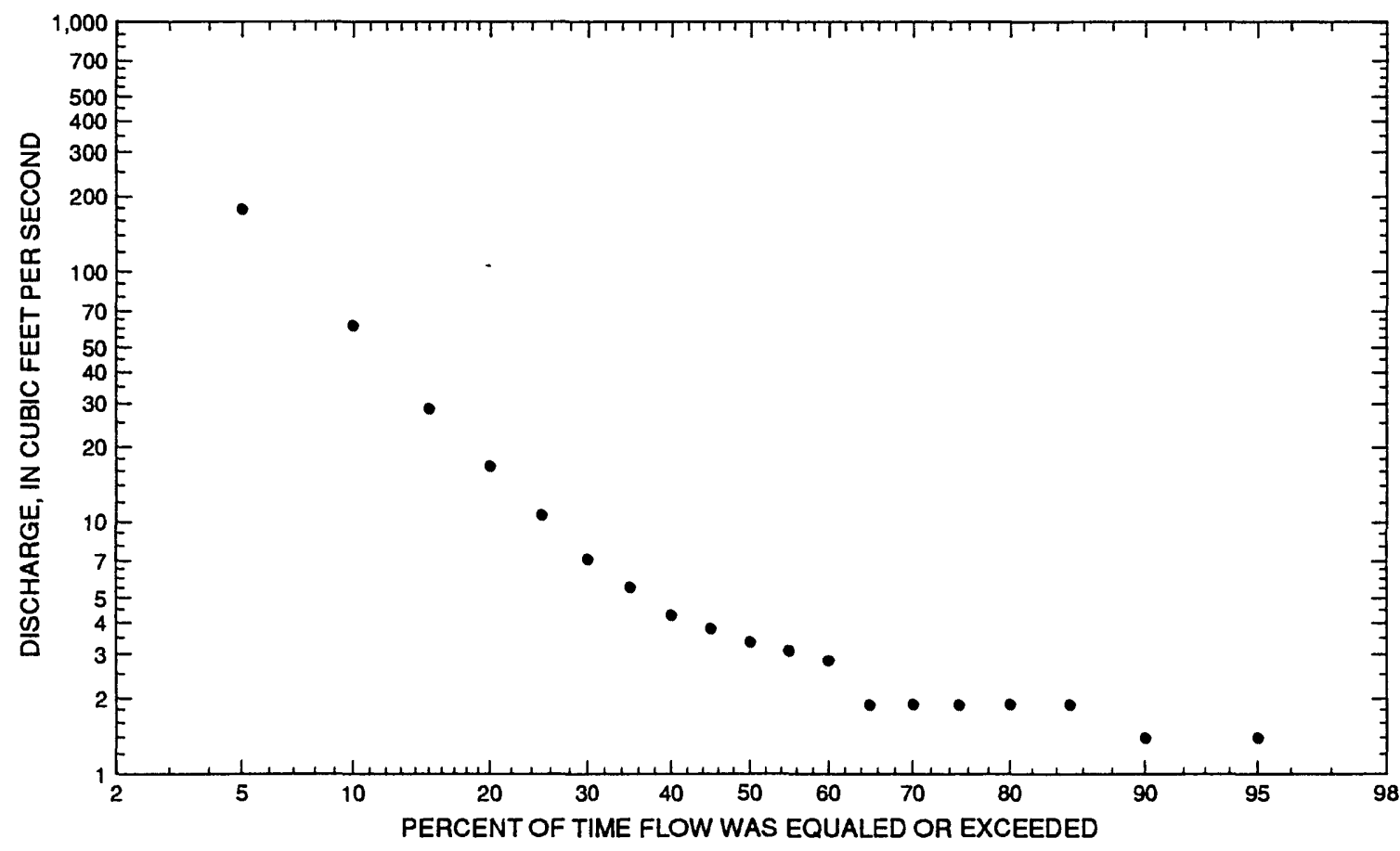




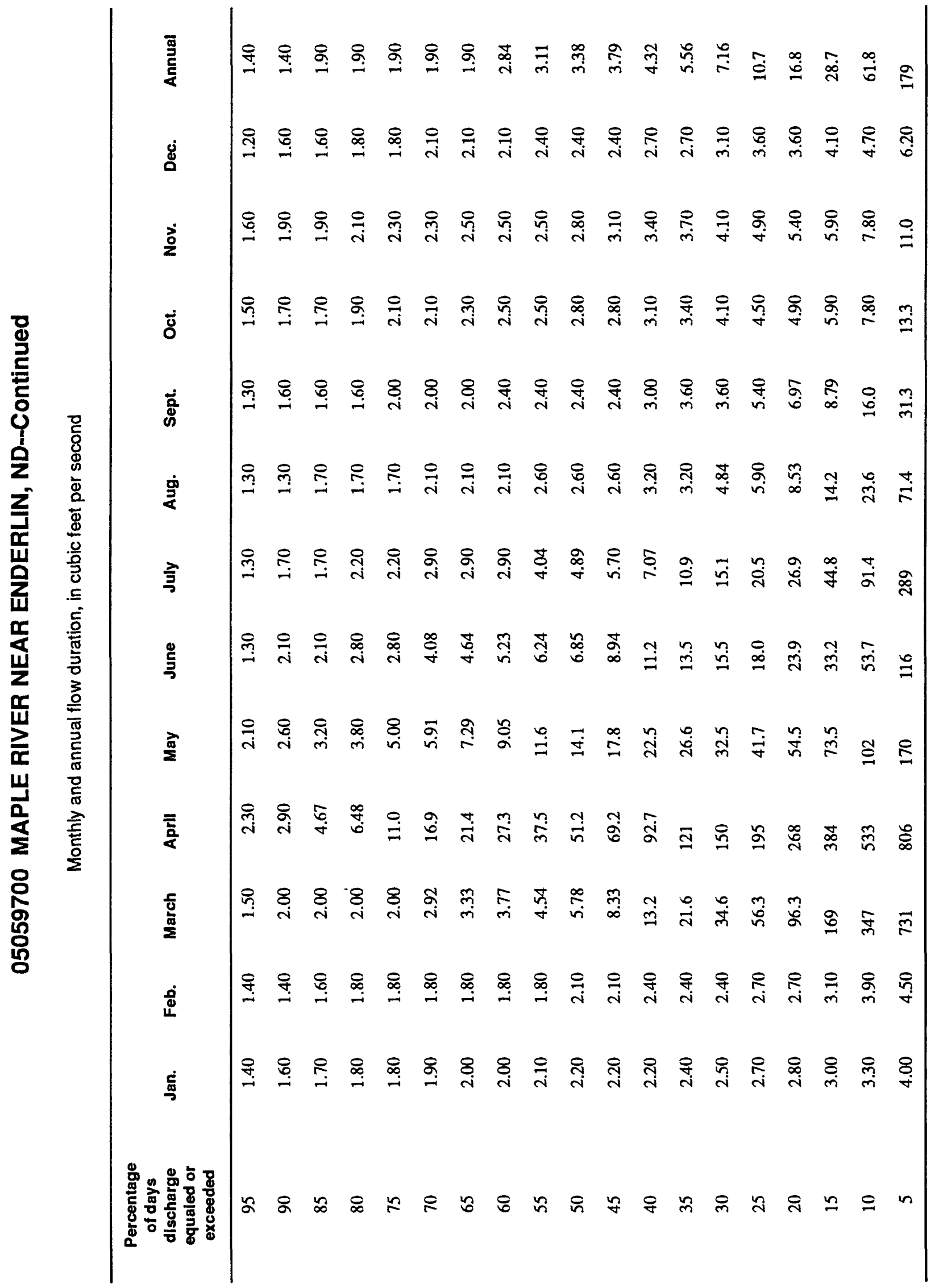




\section{MAPLE RIVER NEAR ENDERLIN, ND--Continued}

Probability of annual high discharges

[ng, statistic not given]

\begin{tabular}{lcccccc}
\hline & & & \multicolumn{5}{c}{$\begin{array}{c}\text { Maximum average discharge } \\
\left(\mathbf{f t}^{3} / \mathbf{s}\right)\end{array}$} \\
\cline { 5 - 7 } $\begin{array}{c}\text { Exceedance } \\
\text { probability }\end{array}$ & $\begin{array}{c}\text { Recurrence } \\
\text { interval } \\
\text { (years) }\end{array}$ & $\begin{array}{c}\text { Maximum } \\
\text { instantaneous } \\
\left(\mathrm{ft}^{3} / \mathbf{s}\right)\end{array}$ & 3-day period & 7-day period & 15-day period & 30-day period \\
\hline 0.99 & 1.01 & 11.8 & 3.88 & 2.67 & 1.98 & 1.58 \\
0.95 & 1.05 & 44.3 & 20.8 & 15.4 & 11.3 & 8.40 \\
0.90 & 1.11 & 85.9 & 46.7 & 35.6 & 25.9 & 18.7 \\
0.80 & 1.25 & 184 & 115 & 89.9 & 64.8 & 45.2 \\
0.50 & 2 & 700 & 514 & 410 & 290 & 192 \\
0.20 & 5 & 2,300 & 1,730 & 1,370 & 947 & 602 \\
0.10 & 10 & 4,040 & 2,950 & 2,290 & 1,570 & 981 \\
0.04 & 25 & 7,080 & 4,830 & 3,660 & 2,470 & 1,530 \\
0.02 & 50 & 9,950 & 6,400 & 4,760 & 3,190 & 1,960 \\
0.01 & 100 & 13,300 & 8,040 & 5,870 & 3,910 & 2,390 \\
0.005 & 200 & 17,200 & 9,730 & 6,970 & 4,610 & 2,810 \\
0.002 & 500 & 23,000 & $\mathrm{ng}$ & $\mathrm{ng}$ & $\mathrm{ng}$ & $\mathrm{ng}$ \\
\hline
\end{tabular}

Probability of annual low discharges

\begin{tabular}{|c|c|c|c|c|c|c|c|c|c|c|}
\hline \multirow[b]{3}{*}{$\begin{array}{l}\text { Non- } \\
\text { exceed- } \\
\text { ance } \\
\text { prob- } \\
\text { ability }\end{array}$} & \multirow[b]{3}{*}{$\begin{array}{l}\text { Recur- } \\
\text { rence } \\
\text { inter- } \\
\text { val } \\
\text { (years) }\end{array}$} & \multicolumn{9}{|c|}{ Minimum average discharge $\left(\mathrm{ft}^{3} / \mathrm{s}\right)$} \\
\hline & & \multicolumn{9}{|c|}{ Number of consecutive days } \\
\hline & & 1 & 3 & 7 & 14 & 30 & 60 & 90 & 120 & 183 \\
\hline 0.05 & 20 & 0.44 & 0.48 & 0.92 & 1.14 & 127 & 1.33 & 1.41 & 1.49 & 1.54 \\
\hline 0.10 & 10 & 0.69 & 0.77 & 1.06 & 1.24 & 1.36 & 1.45 & 1.55 & 1.65 & 1.70 \\
\hline 0.20 & 5 & 1.06 & 1.17 & 1.24 & 1.37 & 1.49 & 1.62 & 1.76 & 1.88 & 1.97 \\
\hline 0.50 & 2 & 1.72 & 1.81 & 1.65 & 1.72 & 1.84 & 2.03 & 2.28 & 2.52 & 2.78 \\
\hline
\end{tabular}


05059700 MAPLE RIVER NEAR ENDERLIN, ND--Continued

Probability of seasonal low discharges

[ng, statistic not given]

\begin{tabular}{|c|c|c|c|c|c|c|c|c|c|}
\hline \multirow[b]{3}{*}{$\begin{array}{c}\text { Non- } \\
\text { excoedance } \\
\text { probability }\end{array}$} & \multirow[b]{3}{*}{$\begin{array}{c}\text { Recurrence } \\
\text { interval } \\
\text { (years) }\end{array}$} & \multicolumn{8}{|c|}{ Minimum average discharge $\left(\mathrm{ft}^{3} / \mathrm{s}\right)$} \\
\hline & & \multicolumn{8}{|c|}{ Number of consecutive days } \\
\hline & & 1 & 7 & 14 & 30 & 1 & 7 & 14 & 30 \\
\hline & & \multicolumn{4}{|c|}{ December-January-February } & \multicolumn{4}{|c|}{ March-April-May } \\
\hline 0.05 & 20 & ng & 1.05 & 1.27 & 1.34 & 0.58 & 1.49 & ${ }^{1} 1.52$ & 1.70 \\
\hline 0.10 & 10 & ng & 1.20 & 1.36 & 1.44 & 0.97 & 1.61 & 1.65 & 2.30 \\
\hline 0.20 & 5 & $\mathrm{ng}$ & 1.40 & 1.50 & 1.59 & 1.54 & 1.82 & 2.00 & 3.41 \\
\hline \multirow[t]{2}{*}{0.50} & 2 & $\mathrm{ng}$ & 1.85 & 1.87 & 1.98 & 2.50 & 2.53 & 3.35 & 8.00 \\
\hline & & \multicolumn{4}{|c|}{ June-July-August } & \multicolumn{4}{|c|}{ September-October-November } \\
\hline 0.05 & 20 & 0.88 & 1.09 & 1.20 & 1.37 & 0.94 & 1.18 & 1.30 & 1.41 \\
\hline 0.10 & 10 & 1.02 & 1.22 & 1.30 & 1.50 & 1.06 & 1.29 & 1.42 & 1.54 \\
\hline 0.20 & 5 & 1.25 & 1.43 & 1.51 & 1.78 & 1.25 & 1.47 & 1.60 & 1.75 \\
\hline 0.50 & 2 & 1.96 & 2.16 & 2.32 & 3.04 & 1.84 & 2.05 & 2.23 & 2.48 \\
\hline
\end{tabular}

${ }^{1}$ Graphical interpretation. 


\section{MAPLE RIVER NEAR ENDERLIN, ND--Continued}

Annual peak discharge and corresponding gage height, period of record

\begin{tabular}{|c|c|c|c|c|c|c|c|}
\hline $\begin{array}{l}\text { Water } \\
\text { year }\end{array}$ & Date & $\begin{array}{c}\text { Gage } \\
\text { height } \\
\text { (feet) }\end{array}$ & $\begin{array}{c}\text { Peak } \\
\text { discharge } \\
\left(\mathrm{ft}^{3} / \mathrm{s}\right)\end{array}$ & $\begin{array}{l}\text { Water } \\
\text { year }\end{array}$ & Date & $\begin{array}{c}\text { Gage } \\
\text { height } \\
\text { (feet) }\end{array}$ & $\begin{array}{c}\text { Peak } \\
\text { discharge } \\
\left(\mathrm{ft}^{3} / \mathrm{s}\right)\end{array}$ \\
\hline \multicolumn{8}{|c|}{ Annual peak discharge, by year, and corresponding gage height } \\
\hline 1956 & June 7 & 4.15 & 92.0 & 1976 & March 23 & 5.72 & 246 \\
\hline 1957 & August 13 & 4.89 & 150 & 1977 & July 5 & 5.32 & 208 \\
\hline 1958 & April 6 & 5.34 & 202 & 1978 & $\operatorname{March} 24$ & 10.19 & 2,350 \\
\hline 1959 & November 18 & 3.03 & 34.0 & 1979 & April 20 & 11.50 & 3,200 \\
\hline 1960 & April 8 & 7.26 & 621 & 1980 & March 19 & 6.07 & 250 \\
\hline 1961 & March 4 & 3.78 & 39.0 & 1981 & July 14 & 5.84 & 172 \\
\hline 1962 & August 11 & 9.54 & 1,630 & 1982 & April 2 & 11.72 & 3,400 \\
\hline 1963 & April 6 & 5.85 & 280 & 1983 & June 23 & 12.23 & 2,930 \\
\hline 1964 & June 9 & 5.32 & 209 & 1984 & March 28 & 10.95 & 2,590 \\
\hline 1965 & April 12 & 11.05 & 3,390 & 1985 & May 12 & 6.39 & 357 \\
\hline 1966 & March 18 & 10.39 & 2,100 & 1986 & March 24 & 8.48 & 1,090 \\
\hline 1967 & April 23 & 7.31 & 702 & 1987 & March 22 & 10.41 & 2,220 \\
\hline 1968 & June 19 & 6.05 & 325 & 1988 & March 9 & 7.84 & 302 \\
\hline 1969 & April 11 & 13.55 & 5,750 & 1989 & March 31 & 8.42 & 981 \\
\hline 1970 & May 29 & 11.06 & 3,260 & 1990 & June 2 & 3.37 & 15.0 \\
\hline 1971 & March 24 & 5.58 & 207 & 1991 & May 27 & 5.25 & 178 \\
\hline 1972 & March 18 & 7.48 & 742 & 1992 & March 10 & 7.32 & 710 \\
\hline 1973 & March 17 & 9.03 & 1,400 & 1993 & July 17 & 12.72 & 3,770 \\
\hline 1974 & April 14 & 7.22 & 594 & 1994 & March 22 & 11.50 & 3,040 \\
\hline 1975 & June 30 & 15.41 & 7,610 & & & & \\
\hline \multicolumn{8}{|c|}{ Annual peak discharge, from highest to lowest, and corresponding gage height } \\
\hline 1975 & June 30 & 15.41 & 7,610 & 1960 & April 8 & 7.26 & 621 \\
\hline 1969 & April 11 & 13.55 & 5,750 & 1974 & April 14 & 7.22 & 594 \\
\hline 1993 & July 17 & 12.72 & 3,770 & 1985 & May 12 & 6.39 & 357 \\
\hline 1982 & April 2 & 11.72 & 3,400 & 1968 & June 19 & 6.05 & 325 \\
\hline 1965 & April 12 & 11.05 & 3,390 & 1988 & March 9 & 7.84 & 302 \\
\hline 1970 & May 29 & 11.06 & 3,260 & 1963 & April 6 & 5.85 & 280 \\
\hline 1979 & April 20 & 11.50 & 3,200 & 1980 & March 19 & 6.07 & 250 \\
\hline 1994 & March 22 & 11.50 & 3,040 & 1976 & March 23 & 5.72 & 246 \\
\hline 1983 & June 23 & 12.23 & 2,930 & 1964 & June 9 & 5.32 & 209 \\
\hline 1984 & March 28 & 10.95 & 2,590 & 1977 & July 5 & 5.32 & 208 \\
\hline 1978 & March 24 & 10.19 & 2,350 & 1971 & March 24 & 5.58 & 207 \\
\hline 1987 & March 22 & 10.41 & 2,220 & 1958 & April 6 & 5.34 & 202 \\
\hline 1966 & March 18 & 10.39 & 2,100 & 1991 & May 27 & 5.25 & 178 \\
\hline 1962 & August 11 & 9.54 & 1,630 & 1981 & July 14 & 5.84 & 172 \\
\hline 1973 & March 17 & 9.03 & 1,400 & 1957 & August 13 & 4.89 & 150 \\
\hline 1986 & March 24 & 8.48 & 1,090 & 1956 & June 7 & 4.15 & 92.0 \\
\hline 1989 & March 31 & 8.42 & 981 & 1961 & March 4 & 3.78 & 39.0 \\
\hline 1972 & March 18 & 7.48 & 742 & 1959 & November 18 & 3.03 & 34.0 \\
\hline 1992 & March 10 & 7.32 & 710 & 1990 & June 2 & 3.37 & 15.0 \\
\hline 1967 & April 23 & 7.31 & 702 & & & & \\
\hline
\end{tabular}




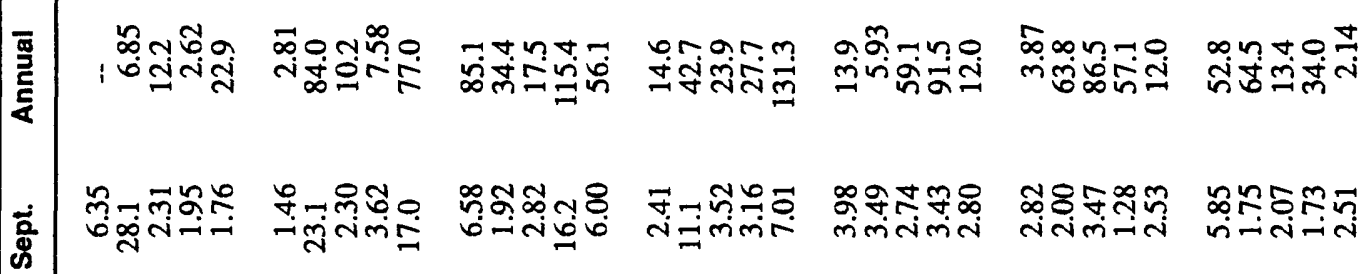

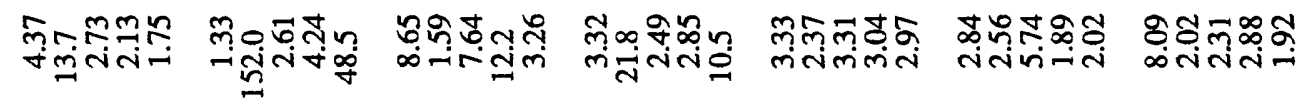

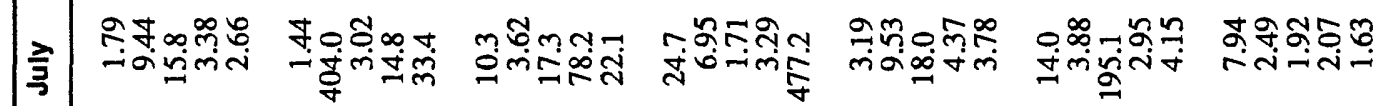

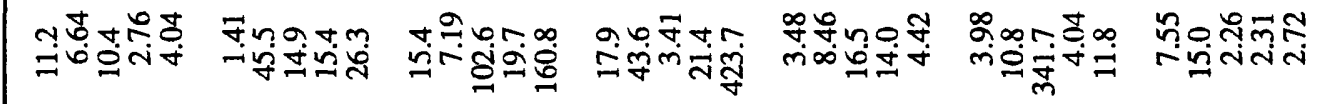

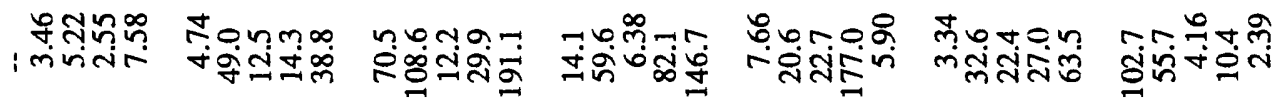

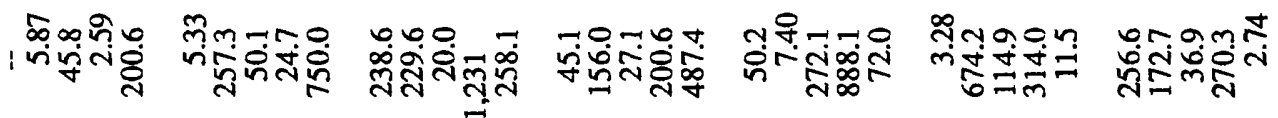

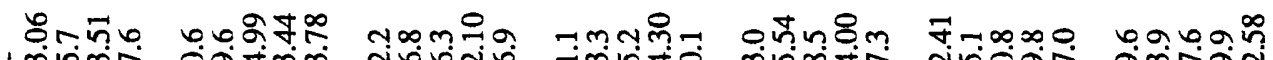

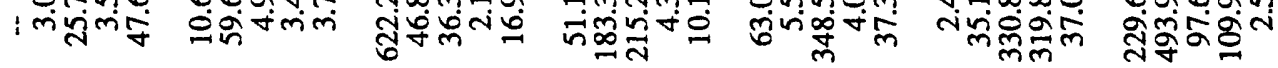

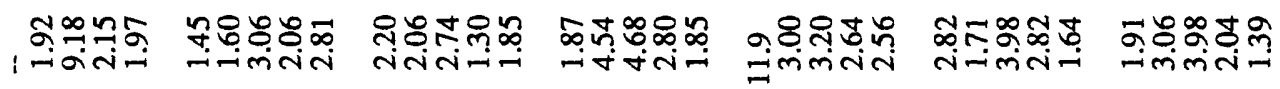

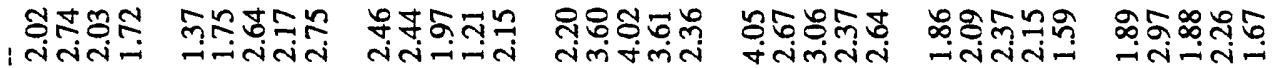

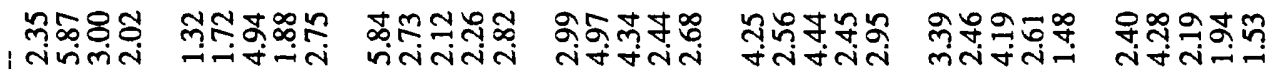

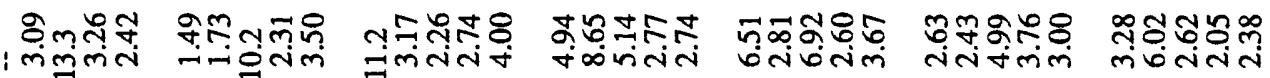

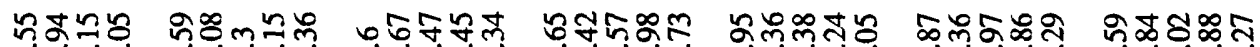

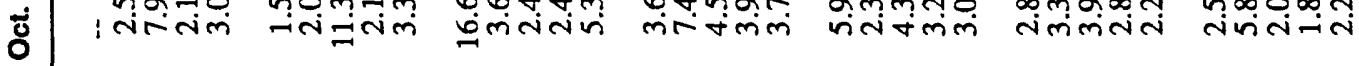

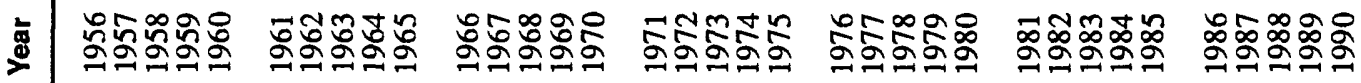




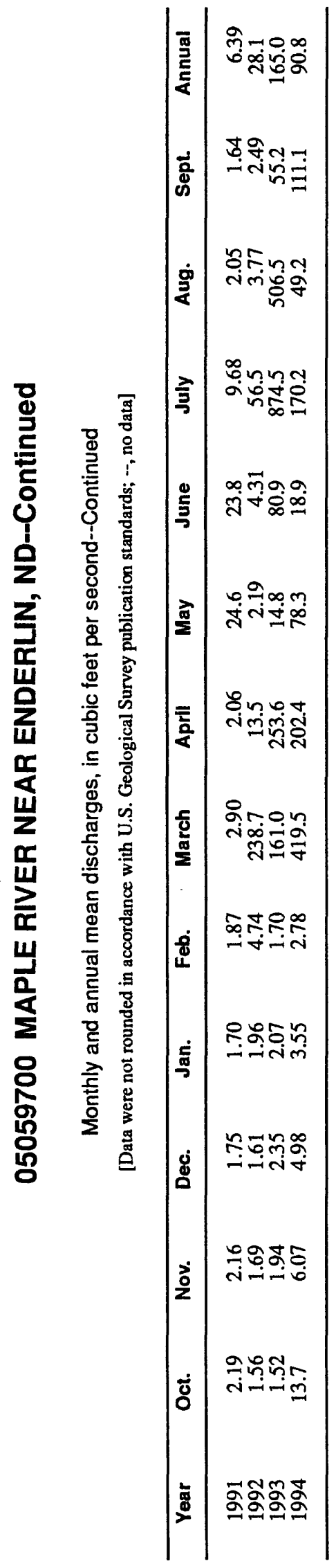




\section{MAPLE RIVER NEAR MAPLETON, ND}

\section{Station Description}

LOCATION.--Lat $46^{\circ} 51^{\prime} 40^{\prime \prime}$, long $97^{\circ} 06^{\prime} 10^{\prime \prime}$, in SW $1 / 4 \mathrm{SE}^{1 / 4} \mathrm{sec} .10$, T.139 N., R.51 W., Cass County, on left bank $25 \mathrm{ft}$ upstream from dam, $3 \mathrm{mi}$ southwest of Mapleton, and $20 \mathrm{mi}$ upstream from mouth.

DRAINAGE AREA.--1,450 $\mathrm{mi}^{2}$, of which about $71 \mathrm{mi}^{2}$, is probably noncontributing.

PERIOD OF RECORD.--April 1944 to September 1975. Prior to October 1958, published as "at Mapleton".

GAGE.--Water-stage recorder and rubble masonry dam. Datum of gage is $893.53 \mathrm{ft}$ above mean sea level (levels by Soil Conservation Service). Prior to Oct. 1, 1958, nonrecording gage at site $7 \mathrm{mi}$ downstream at different datum.

EXTREMES since Oct. 1, 1958.--Maximum discharge, $11,600 \mathrm{ft}^{3} / \mathrm{s}$, July 2, 1975 (gage height, $15.03 \mathrm{ft}$ ); maximum gage height, $18.9 \mathrm{ft}$, Apr. 6, 1952; no flow for many days.

Annual mean discharge

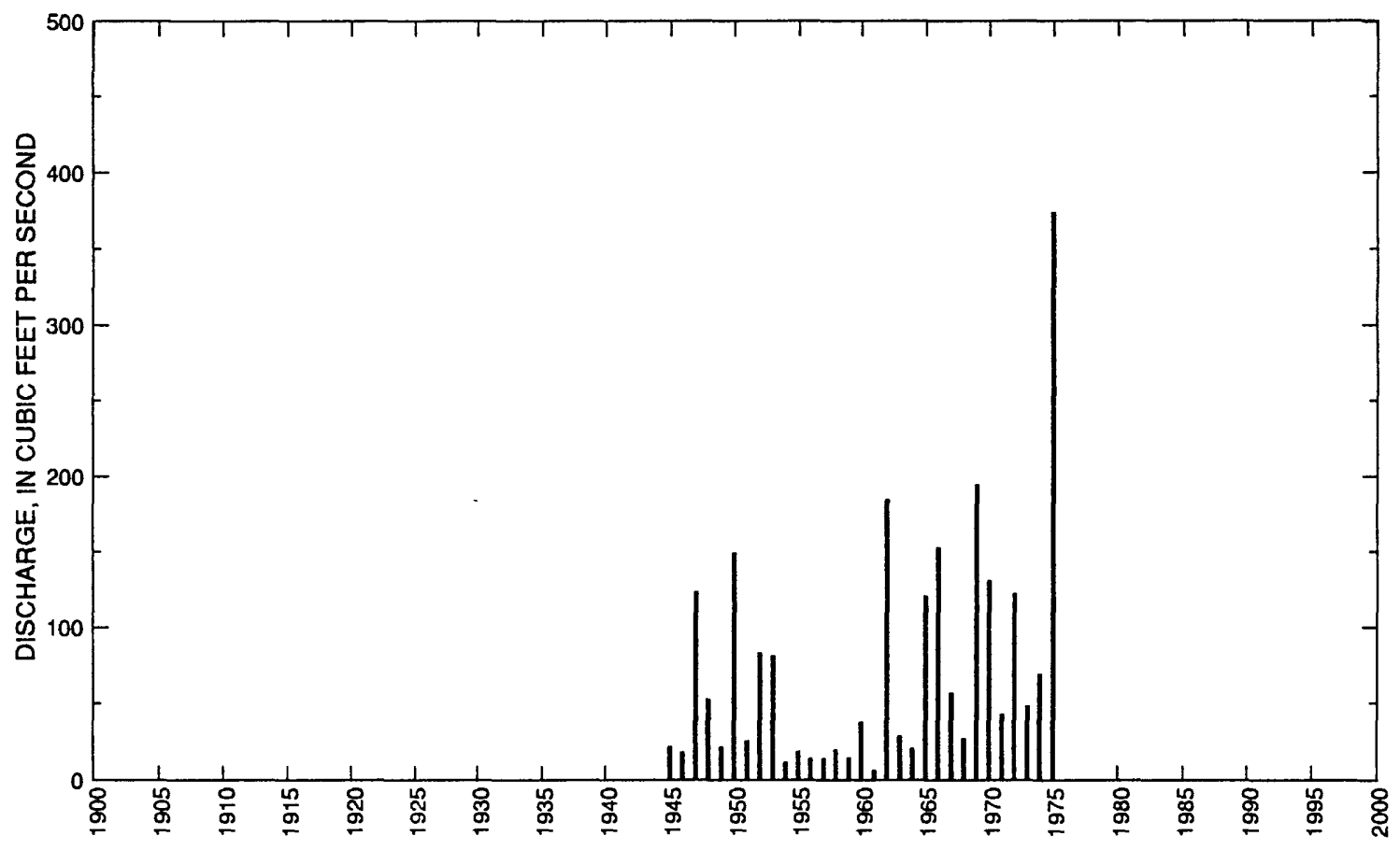




\section{MAPLE RIVER NEAR MAPLETON, ND--Continued}

Statistics of monthly and annual mean discharges

[m, more than 1 year of occurrence]

\begin{tabular}{|c|c|c|c|c|c|c|c|c|}
\hline \multirow[b]{2}{*}{ Month } & \multicolumn{2}{|c|}{ Maximum } & \multicolumn{2}{|c|}{ Minimum } & \multicolumn{4}{|c|}{ Mean } \\
\hline & $\begin{array}{c}\text { Discharge } \\
\left(\mathrm{ft}^{3} / \mathrm{s}\right)\end{array}$ & $\begin{array}{c}\text { Water year } \\
\text { of } \\
\text { occurrence }\end{array}$ & $\begin{array}{c}\text { Discharge } \\
\left(\mathrm{ft}^{3} / \mathrm{s}\right)\end{array}$ & $\begin{array}{c}\text { Water year } \\
\text { of } \\
\text { occurrence }\end{array}$ & $\begin{array}{c}\text { Discharge } \\
\left(\mathrm{ft}^{3} / \mathrm{s}\right)\end{array}$ & $\begin{array}{c}\text { Standard } \\
\text { deviation } \\
\left(\mathrm{ft}^{3} / \mathrm{s}\right)\end{array}$ & $\begin{array}{l}\text { Coeffi- } \\
\text { cient of } \\
\text { variation }\end{array}$ & $\begin{array}{l}\text { Percentage } \\
\text { of annual } \\
\text { discharge }\end{array}$ \\
\hline October & 49.1 & 1972 & 0 & $\mathrm{~m}$ & 7.94 & 11.8 & 1.49 & 0.92 \\
\hline November & 36.2 & 1972 & 0 & 1961 & 8.84 & 8.28 & 0.94 & 1.02 \\
\hline December & 12.2 & 1963 & 0 & 1961 & 3.70 & 3.32 & 0.90 & 0.43 \\
\hline January & 4.30 & 1973 & 0 & $\mathrm{~m}$ & 1.12 & 1.25 & 1.12 & 0.13 \\
\hline February & 4.85 & 1973 & 0 & $\mathrm{~m}$ & 0.620 & 1.12 & 1.81 & 0.07 \\
\hline March & 1,040 & 1966 & 0 & $\mathbf{m}$ & 95.1 & 208 & 2.18 & 11.0 \\
\hline April & 1,710 & 1969 & 13.9 & 1959 & 385 & 435 & 1.13 & 44.6 \\
\hline May & 620 & 1950 & 6.30 & 1955 & 104 & 145 & 1.40 & 12.0 \\
\hline June & 353 & 1947 & 1.71 & 1961 & 94.2 & 160 & 1.70 & 10.9 \\
\hline July & 2,380 & 1975 & 0 & 1961 & 132 & 434 & 3.29 & 15.3 \\
\hline August & 267 & 1962 & 0 & $\mathrm{~m}$ & 20.8 & 48.1 & 2.31 & 2.41 \\
\hline September & 65.8 & 1962 & 0 & $\mathbf{m}$ & 11.0 & 15.0 & 1.35 & 1.28 \\
\hline Annual & 374 & 1975 & 11.1 & 1954 & 73.5 & 78.8 & 1.07 & 100 \\
\hline
\end{tabular}

Annual flow duration

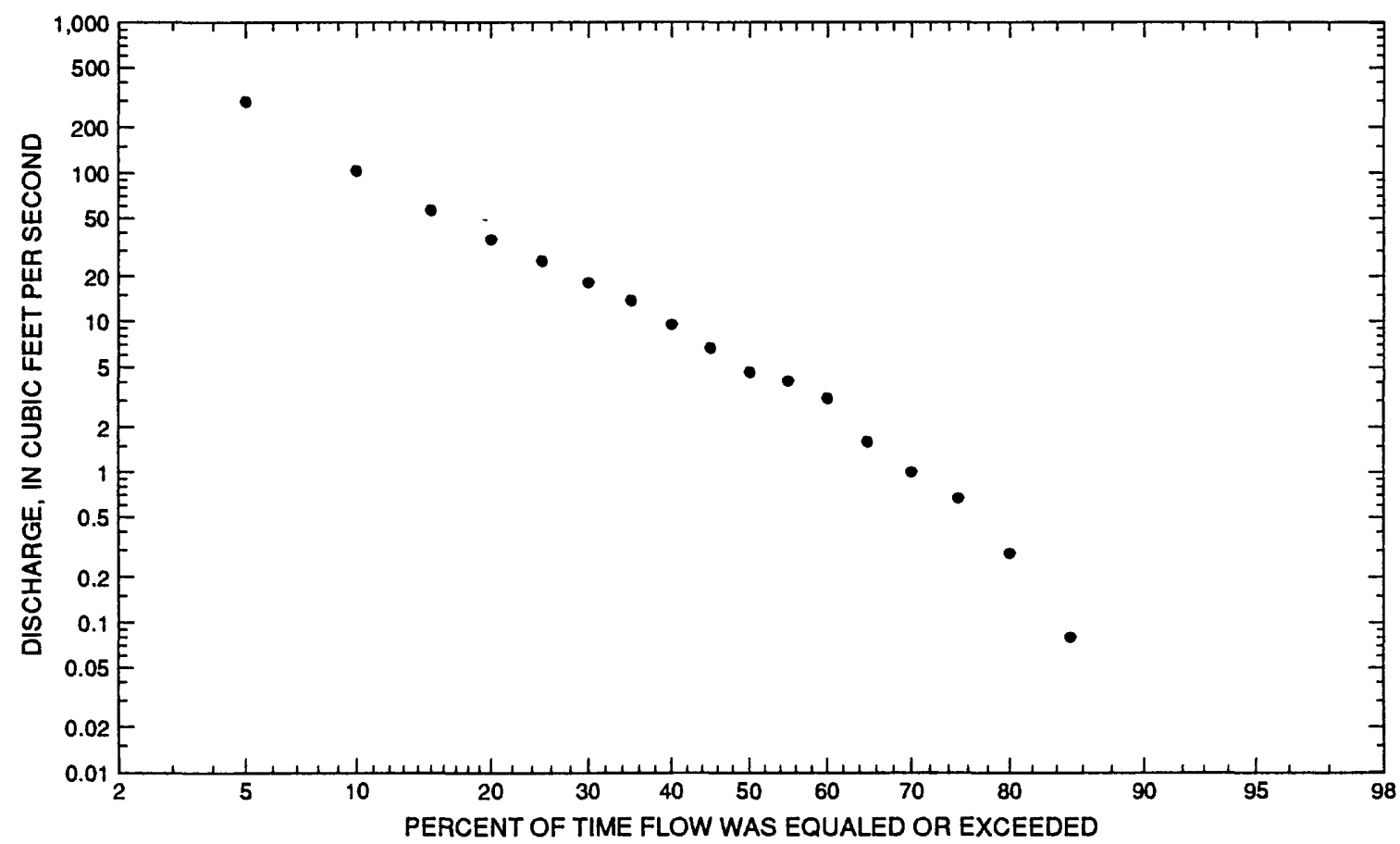




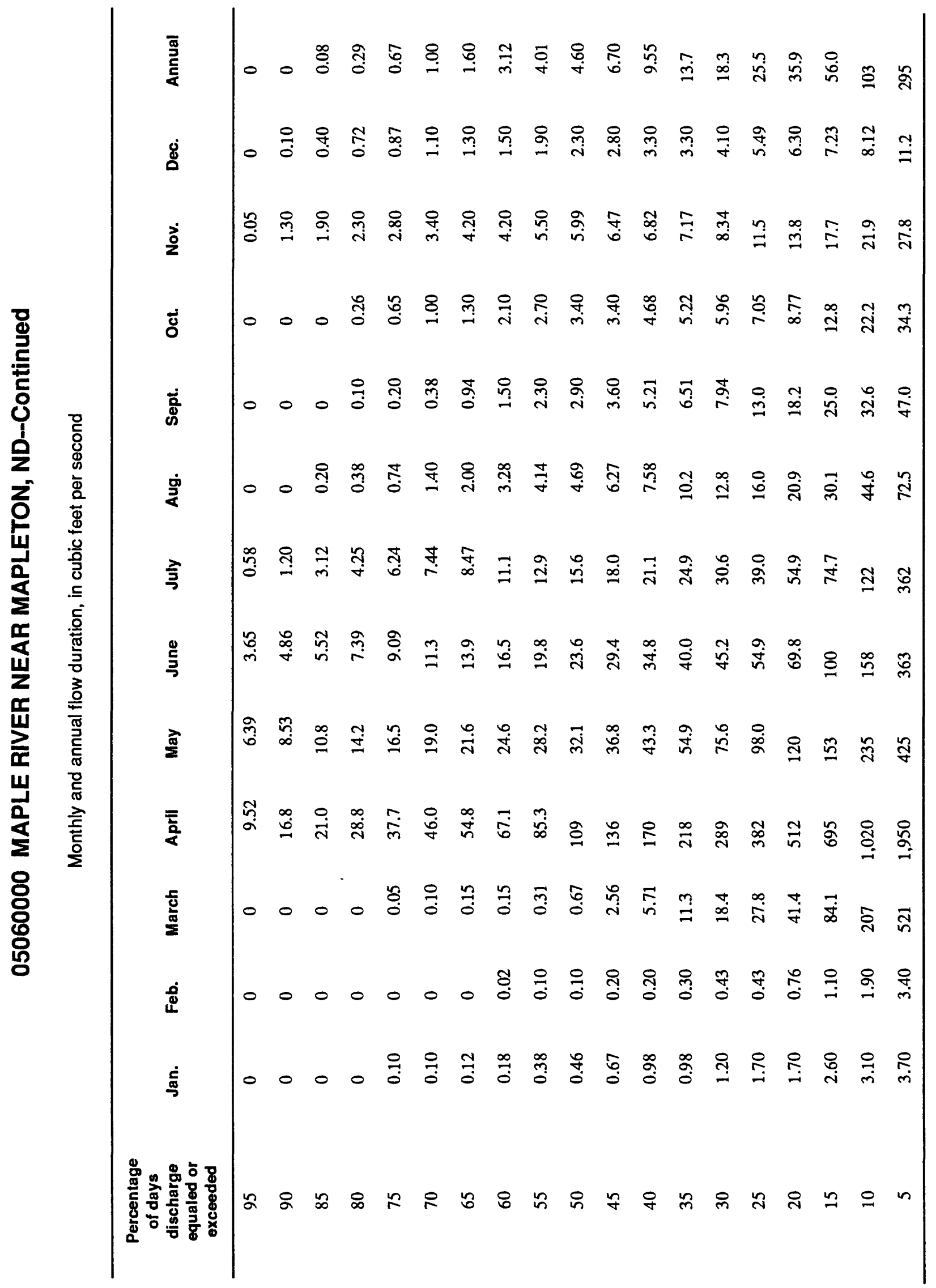




\section{MAPLE RIVER NEAR MAPLETON, ND--Continued}

Probability of annual high discharges

[ng, statistic not given]

\begin{tabular}{lcccccc}
\hline & & & \multicolumn{5}{c}{$\begin{array}{c}\text { Maximum average discharge } \\
\left(\mathrm{ft}^{3} / \mathbf{s}\right)\end{array}$} \\
\cline { 6 - 7 } $\begin{array}{c}\text { Exceedance } \\
\text { probability }\end{array}$ & $\begin{array}{c}\text { Recurrence } \\
\text { interval } \\
\text { (years) }\end{array}$ & $\begin{array}{c}\text { Maximum } \\
\text { instantaneous } \\
\left(\mathrm{ft}^{3} / \mathbf{s}\right)\end{array}$ & 3-day period & 7-day period & 15-day period & 30-day period \\
\hline 0.99 & 1.01 & 47.6 & 43.5 & 35.3 & 26.6 & 22.5 \\
0.95 & 1.05 & 131 & 119 & 91.7 & 66.6 & 50.2 \\
0.90 & 1.11 & 219 & 197 & 150 & 107 & 76.7 \\
0.80 & 1.25 & 396 & 355 & 267 & 187 & 127 \\
0.50 & 2 & 1,140 & 1,020 & 771 & 524 & 329 \\
0.20 & 5 & 2,980 & 2,650 & 2,090 & 1,390 & 832 \\
0.10 & 10 & 4,740 & 4,220 & 3,420 & 2,260 & 1,340 \\
0.04 & 25 & 7,560 & 6,740 & 5,700 & 3,730 & 2,200 \\
0.02 & 50 & 10,100 & 8,990 & 7,850 & 5,120 & 3,030 \\
0.01 & 100 & 12,900 & 11,500 & 10,400 & 6,770 & 4,030 \\
0.005 & 200 & 16,100 & 14,400 & 13,400 & 8,700 & 5,220 \\
0.002 & 500 & 20,700 & ng & ng & ng & ng \\
\hline
\end{tabular}

Probability of annual low discharges

\begin{tabular}{|c|c|c|c|c|c|c|c|c|c|c|}
\hline \multirow[b]{3}{*}{$\begin{array}{l}\text { Non- } \\
\text { exceed- } \\
\text { ance } \\
\text { prob- } \\
\text { abiiity }\end{array}$} & \multirow[b]{3}{*}{$\begin{array}{c}\text { Recur- } \\
\text { rence } \\
\text { inter- } \\
\text { val } \\
\text { (years) }\end{array}$} & \multicolumn{9}{|c|}{ Minimum average discharge $\left(\mathrm{ft}^{3} / \mathrm{s}\right)$} \\
\hline & & \multicolumn{9}{|c|}{ Number of consecutive days } \\
\hline & & 1 & 3 & 7 & 14 & 30 & 60 & 90 & 120 & 183 \\
\hline 0.05 & 20 & 0 & 0 & $\mathbf{0}$ & 0 & 0 & 0 & 0 & 0.165 & 0.450 \\
\hline 0.10 & 10 & 0 & 0 & 0 & 0 & 0 & 0 & 0 & 0.303 & 0.738 \\
\hline 0.20 & 5 & 0 & 0 & 0 & 0 & 0 & 0 & 0.044 & 0.525 & 1.18 \\
\hline 0.50 & 2 & 0 & 0 & 0 & 0 & 0 & 0.103 & 0.436 & 1.35 & 2.74 \\
\hline
\end{tabular}




\section{MAPLE RIVER NEAR MAPLETON, ND--Continued}

Probability of seasonal low discharges

\begin{tabular}{|c|c|c|c|c|c|c|c|c|c|}
\hline \multirow[b]{3}{*}{$\begin{array}{c}\text { Non- } \\
\text { exceedance } \\
\text { probability }\end{array}$} & \multirow[b]{3}{*}{$\begin{array}{l}\text { Recurrence } \\
\text { interval } \\
\text { (years) }\end{array}$} & \multicolumn{8}{|c|}{ Minimum average discharge $\left(\mathrm{ft}^{3} / \mathrm{s}\right)$} \\
\hline & & \multicolumn{8}{|c|}{ Number of consecutive days } \\
\hline & & 1 & 7 & 14 & 30 & 1 & 7 & 14 & 30 \\
\hline & & \multicolumn{4}{|c|}{ December-January-February } & \multicolumn{4}{|c|}{ March-April-May } \\
\hline 0.05 & 20 & 0 & 0 & 0 & 0 & 0 & 0 & 0 & 0 \\
\hline 0.10 & 10 & 0 & 0 & 0 & 0 & 0 & 0 & 0 & 0.09 \\
\hline 0.20 & 5 & 0 & 0 & 0 & 0 & 0 & 0 & 0 & 0.71 \\
\hline \multirow[t]{2}{*}{0.50} & 2 & 0 & 0.02 & 0.08 & 0.16 & 0 & 0.14 & 0.50 & 8.09 \\
\hline & & \multicolumn{4}{|c|}{ June-July-August } & \multicolumn{4}{|c|}{ September-October-November } \\
\hline 0.05 & 20 & 0 & 0 & 0 & 0 & 0 & 0 & 0 & 0 \\
\hline 0.10 & 10 & 0 & 0 & 0 & 0.15 & 0 & 0 & 0 & 0 \\
\hline 0.20 & 5 & 0.02 & 0.15 & 0.24 & 0.68 & 0 & 0 & 0 & 0.18 \\
\hline 0.50 & 2 & 1.42 & 2.04 & 2.66 & 4.90 & 0.46 & 0.64 & 0.83 & 1.87 \\
\hline
\end{tabular}




\section{MAPLE RIVER NEAR MAPLETON, ND--Continued}

Annual peak discharge and corresponding gage height, period of record

$$
[-, \text { no data }]
$$

\begin{tabular}{|c|c|c|c|c|c|c|c|}
\hline $\begin{array}{l}\text { Water } \\
\text { year }\end{array}$ & Date & $\begin{array}{c}\text { Gage } \\
\text { helght } \\
\text { (feet) }\end{array}$ & $\begin{array}{c}\text { Peak } \\
\text { discharge } \\
\left(\mathrm{ft}^{3} / \mathrm{s}\right)\end{array}$ & $\begin{array}{l}\text { Water } \\
\text { year }\end{array}$ & Date & $\begin{array}{c}\text { Gage } \\
\text { height } \\
\text { (feet) }\end{array}$ & $\begin{array}{c}\text { Peak } \\
\text { dlscharge } \\
\left(\mathrm{ft}^{3} / \mathrm{s}\right)\end{array}$ \\
\hline \multicolumn{8}{|c|}{ Annual peak discharge, by year, and corresponding gage height } \\
\hline 1944 & April 8 & 8.16 & 177 & 1961 & March 10 & 1.42 & 49.0 \\
\hline 1945 & April 12 & 8.59 & 373 & 1962 & April 5 & 12.48 & 2,740 \\
\hline 1946 & March 21 & 13.15 & 550 & 1963 & April 8 & 7.19 & 779 \\
\hline 1947 & April 14 & 18.04 & 3,880 & 1964 & April 15 & 5.65 & 314 \\
\hline 1948 & April 7 & 17.28 & 1,500 & 1965 & April 12 & 13.22 & 3,210 \\
\hline 1949 & April 3 & 14.75 & 850 & 1966 & March 17 & 13.46 & 3,610 \\
\hline 1950 & April 2 & 17.73 & 1,980 & 1967 & April 21 & 9.29 & 1,420 \\
\hline 1951 & April 7 & 12.50 & 750 & 1968 & June 23 & 5.20 & 302 \\
\hline 1952 & April 6 & 18.90 & 3,850 & 1969 & April 11 & 14.00 & 7,000 \\
\hline 1953 & June 17 & 18.62 & 4,840 & 1970 & May 30 & 12.39 & 3,340 \\
\hline 1954 & March 21 & 8.62 & 200 & 1971 & March 19 & 6.82 & 778 \\
\hline 1955 & April 2 & 12.40 & 500 & 1972 & March 17 & 12.27 & 2,430 \\
\hline 1956 & April 14 & 11.51 & 630 & 1973 & March 15 & - & 1,300 \\
\hline 1957 & June 14 & 8.52 & 430 & 1974 & April 13 & 10.30 & 1,970 \\
\hline 1958 & April 10 & 7.57 & 195 & 1975 & July 2 & 15.03 & 11,600 \\
\hline 1959 & June 12 & 5.58 & 1,160 & 1976 & March 27 & 7.50 & 1,000 \\
\hline 1960 & April 7 & 5.65 & 1,220 & & & & \\
\hline \multicolumn{8}{|c|}{ Annual peak discharge, from highest to lowest, and corresponding gage height } \\
\hline 1975 & July 2 & 15.03 & 11,600 & 1976 & March 27 & 7.50 & 1,000 \\
\hline 1969 & April 11 & 14.00 & 7,000 & 1949 & April 3 & 14.75 & 850 \\
\hline 1953 & June 17 & 18.62 & 4,840 & 1963 & April 8 & 7.19 & 779 \\
\hline 1947 & April 14 & 18.04 & 3,880 & 1971 & March 19 & 6.82 & 778 \\
\hline 1952 & April 6 & 18.90 & 3,850 & 1951 & April 7 & 12.50 & 750 \\
\hline 1966 & March 17 & 13.46 & 3,610 & 1956 & April 14 & 11.51 & 630 \\
\hline 1970 & May 30 & 12.39 & 3,340 & 1946 & March 21 & 13.15 & 550 \\
\hline 1965 & April 12 & 13.22 & 3,210 & 1955 & April 2 & 12.40 & 500 \\
\hline 1962 & April 5 & 12.48 & 2,740 & 1957 & June 14 & 8.52 & 430 \\
\hline 1972 & March 17 & 12.27 & 2,430 & 1945 & April 12 & 8.59 & 373 \\
\hline 1950 & April 2 & 17.73 & 1,980 & 1964 & April 15 & 5.65 & 314 \\
\hline 1974 & April 13 & 10.30 & 1,970 & 1968 & June 23 & 5.20 & 302 \\
\hline 1948 & April 7 & 17.28 & 1,500 & 1954 & March 21 & 8.62 & 200 \\
\hline 1967 & April 21 & 9.29 & 1,420 & 1958 & April 10 & 7.57 & 195 \\
\hline 1973 & March 15 & - & 1,300 & 1944 & April 8 & 8.16 & 177 \\
\hline 1960 & April 7 & 5.65 & 1,220 & 1961 & March 10 & 1.42 & 49.0 \\
\hline 1959 & June 12 & 5.58 & 1,160 & & & & \\
\hline
\end{tabular}




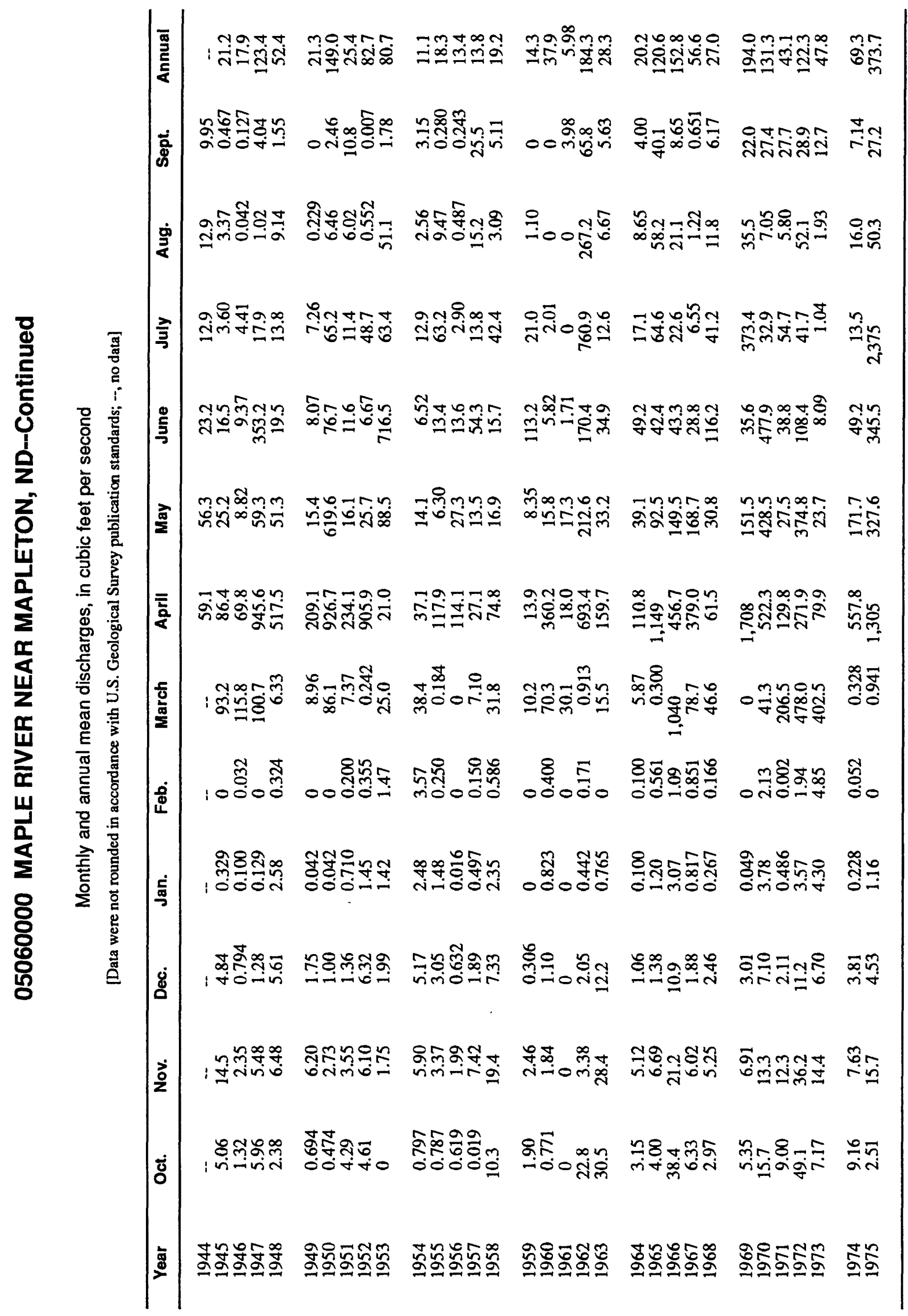




\section{RUSH RIVER AT AMENIA, ND}

\section{Station Description}

LOCATION.--Lat $47^{\circ} 01^{\prime} 00^{\prime}$, long $97^{\circ} 12^{\prime} 50^{\prime \prime}$, in SE $1 / 4 \mathrm{NW}^{1} / 4 \mathrm{sec} .24, \mathrm{~T} .141$ N., R.52 W., Cass County, Hydrologic Unit 09020204 , on left bank downstream side of bridge on State Highway 18, and $0.6 \mathrm{mi}$ north of Amenia.

DRAINAGE AREA.--116 $\mathrm{mi}^{2}$.

PERIOD OF RECORD.--July 1946 to current year.

GAGE.--Water-stage recorder. Datum of gage is $943 \mathrm{ft}$ above sea level, from topographic map. See WSP 1913 for history of changes prior to June 10, 1961.

EXTREMES FOR PERIOD OF RECORD.--Maximum discharge, 3,490 ft 3 /s, Apr. 19, 1979, maximum gage height, $11.6 \mathrm{ft}$, Apr. 11, 1965, and Apr. 18, 1975; no flow for periods each year.

Annual mean discharge

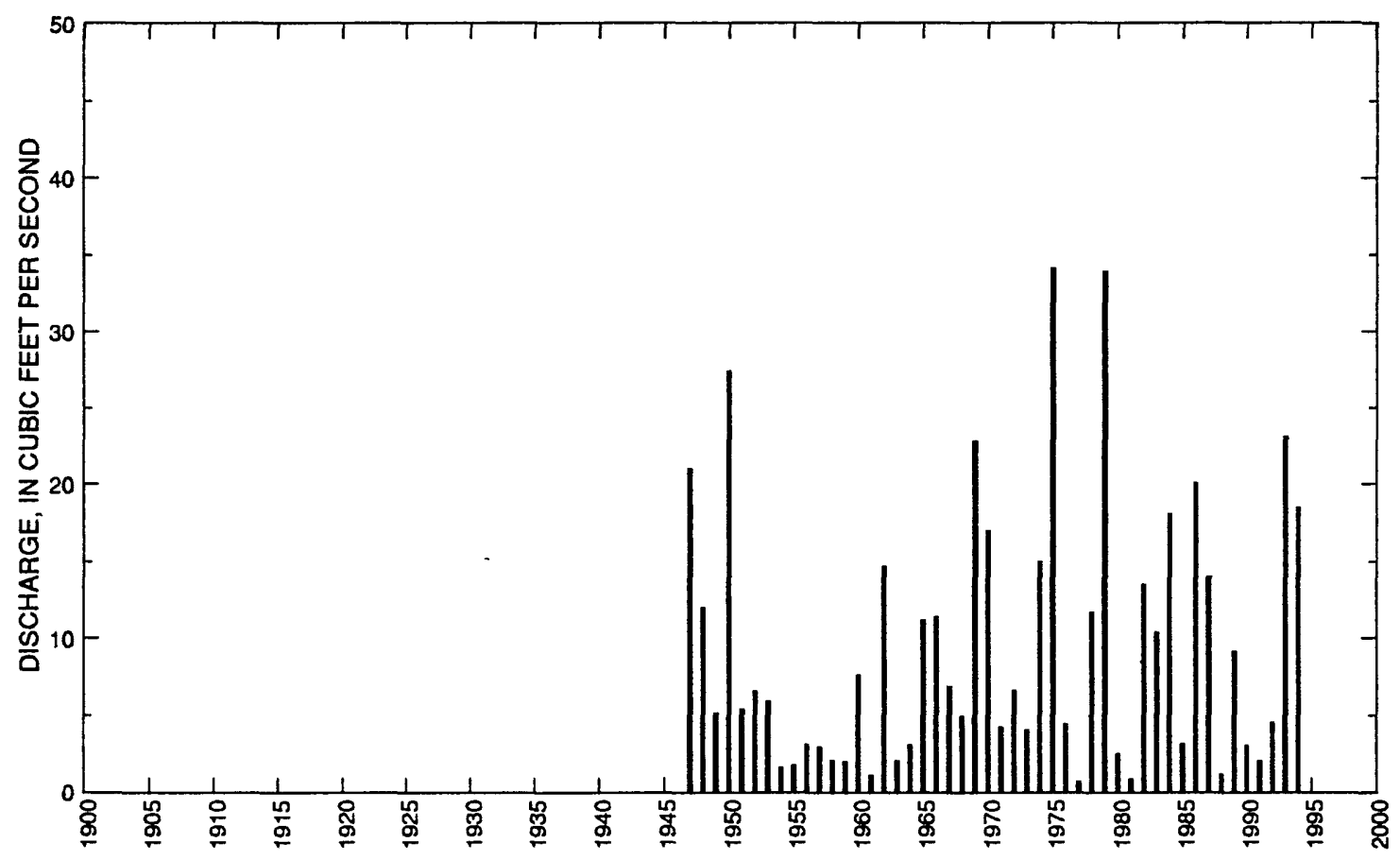


05060500 RUSH RIVER AT AMENIA, ND--Continued

Statistics of monthly and annual mean discharges

[m, more than 1 year of occurrence]

\begin{tabular}{|c|c|c|c|c|c|c|c|c|}
\hline \multirow[b]{2}{*}{ Month } & \multicolumn{2}{|c|}{ Maximum } & \multicolumn{2}{|c|}{ Minimum } & \multicolumn{4}{|c|}{ Mean } \\
\hline & $\begin{array}{c}\text { Discharge } \\
\left(\mathrm{ft}^{3} / \mathrm{s}\right)\end{array}$ & $\begin{array}{c}\text { Water year } \\
\text { of } \\
\text { occurrence }\end{array}$ & $\begin{array}{c}\text { Discharge } \\
\left(\mathrm{ft}^{3} / \mathrm{s}\right)\end{array}$ & $\begin{array}{c}\text { Water year } \\
\text { of } \\
\text { occurrence }\end{array}$ & $\begin{array}{c}\text { Discharge } \\
\left(\mathrm{ft}^{3} / \mathrm{s}\right)\end{array}$ & $\begin{array}{c}\text { Standard } \\
\text { deviation } \\
\left(\mathrm{ft}^{3} / \mathrm{s}\right)\end{array}$ & $\begin{array}{l}\text { Coeffi- } \\
\text { cient of } \\
\text { variation }\end{array}$ & $\begin{array}{l}\text { Percentage } \\
\text { of annual } \\
\text { discharge }\end{array}$ \\
\hline October & 11.4 & 1983 & 0 & $\mathrm{~m}$ & 0.660 & 1.86 & 2.83 & 0.57 \\
\hline November & 3.50 & 1983 & 0 & $\mathrm{~m}$ & 0.570 & 0.88 & 1.55 & 0.50 \\
\hline December & 1.24 & 1963 & 0 & $\mathrm{~m}$ & 0.130 & 0.27 & 2.03 & 0.12 \\
\hline January & 0.448 & 1987 & 0 & $\mathrm{~m}$ & 0.010 & 0.07 & 4.85 & 0.01 \\
\hline February & 2.21 & 1976 & 0 & m & 0.130 & 0.44 & 3.42 & 0.11 \\
\hline March & 136 & 1984 & 0 & $\mathrm{~m}$ & 20.8 & 28.0 & 1.34 & 18.2 \\
\hline April & 382 & 1979 & 1.12 & 1981 & 59.9 & 82.0 & 1.37 & 52.2 \\
\hline May & 81.3 & 1949 & 0.119 & 1955 & 10.7 & 15.5 & 1.45 & 9.33 \\
\hline June & 48.9 & 1953 & 0.009 & 1988 & 9.13 & 12.3 & 1.34 & 7.95 \\
\hline July & 168 & 1993 & 0 & $\mathrm{~m}$ & 10.1 & 26.5 & 2.62 & 8.82 \\
\hline August & 22.3 & 1993 & 0 & $\mathrm{~m}$ & 1.25 & 4.43 & 3.55 & 1.09 \\
\hline September & 40.1 & 1994 & 0 & $\mathrm{~m}$ & 1.26 & 5.84 & 4.62 & 1.10 \\
\hline Annual & 34.1 & 1975 & 0.678 & 1977 & 9.55 & 8.69 & 0.91 & 100 \\
\hline
\end{tabular}

Annual flow duration

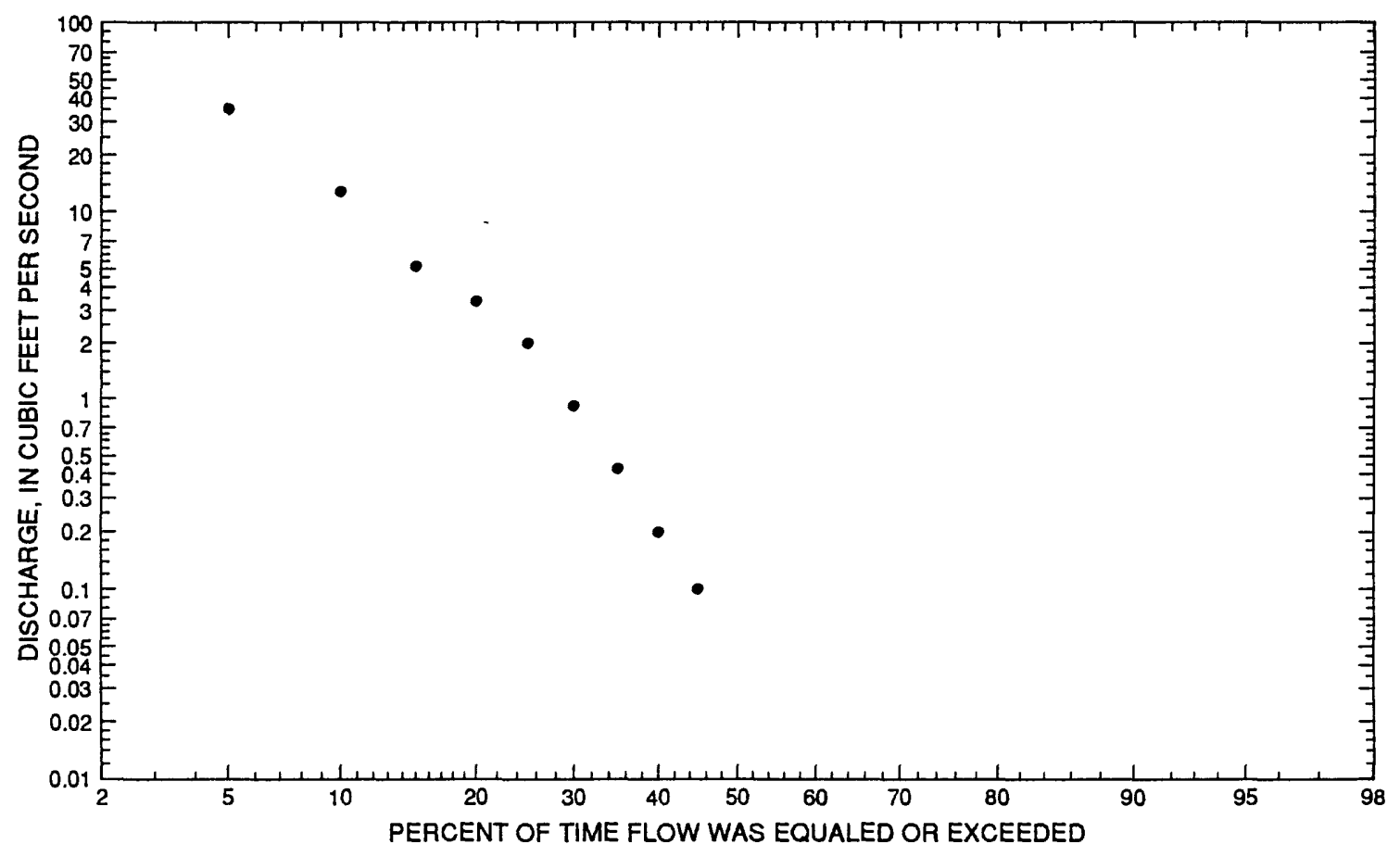




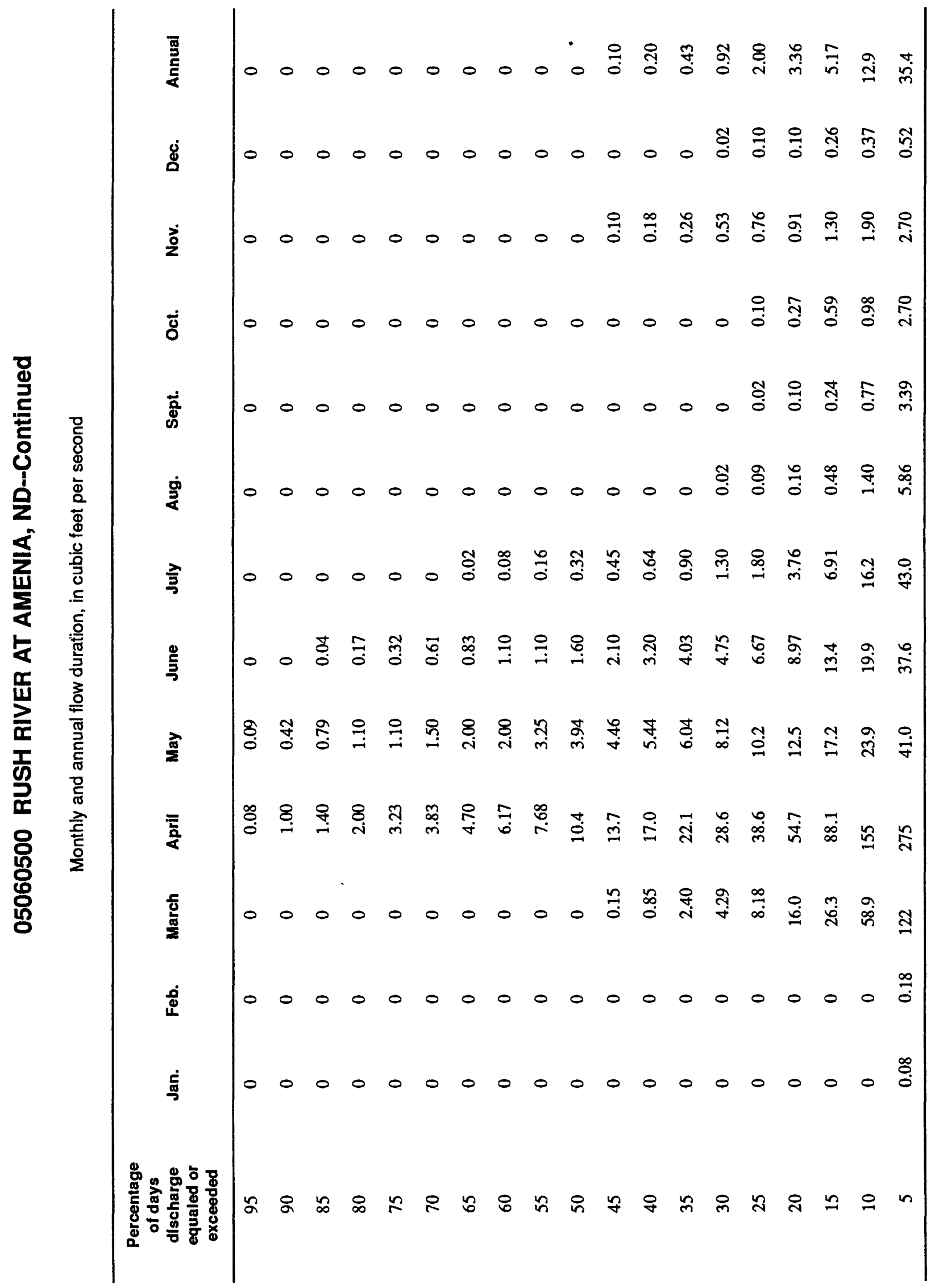




\section{RUSH RIVER AT AMENIA, ND--Continued}

Probability of annual high discharges

[ng, statistic not given]

\begin{tabular}{lcccccc}
\hline & & & \multicolumn{5}{c}{$\begin{array}{c}\text { Maximum average discharge } \\
\left(\mathrm{ft}^{3} / \mathbf{s}\right)\end{array}$} \\
\cline { 5 - 7 } $\begin{array}{c}\text { Exceedance } \\
\text { probability }\end{array}$ & $\begin{array}{c}\text { Recurrence } \\
\text { Interval } \\
\text { (years) }\end{array}$ & $\begin{array}{c}\text { Maximum } \\
\text { instantaneous } \\
\left(\mathrm{ft}^{3} / \mathbf{s}\right)\end{array}$ & 3-day period & 7-day period & 15-day period & 30-day period \\
\hline 0.99 & 1.01 & 12.9 & 8.39 & 5.40 & 3.56 & 2.92 \\
0.95 & 1.05 & 33.9 & 21.2 & 14.2 & 9.59 & 7.08 \\
0.90 & 1.11 & 55.8 & 34.5 & 23.4 & 15.9 & 11.2 \\
0.80 & 1.25 & 100 & 61.3 & 42.2 & 28.6 & 19.1 \\
0.50 & 2 & 293 & 179 & 124 & 82.7 & 50.9 \\
0.20 & 5 & 806 & 501 & 344 & 220 & 128 \\
0.10 & 10 & 1,330 & 844 & 571 & 355 & 202 \\
0.04 & 25 & 2,240 & 1,450 & 964 & 578 & 324 \\
0.02 & 50 & 3,100 & 2,060 & 1,340 & 781 & 435 \\
0.01 & 100 & 4,130 & 2,790 & 1,790 & 1,020 & 564 \\
0.005 & 200 & 5,340 & 3,680 & 2,320 & 1,280 & 712 \\
0.002 & 500 & 7,240 & ng & ng & ng & ng \\
\hline
\end{tabular}

Probability of annual low discharges

[ng, statistic not given]

\begin{tabular}{|c|c|c|c|c|c|c|c|c|c|c|}
\hline \multirow[b]{3}{*}{$\begin{array}{l}\text { Non- } \\
\text { exceed- } \\
\text { ance } \\
\text { prob- } \\
\text { ability }\end{array}$} & \multirow[b]{3}{*}{$\begin{array}{c}\text { Recur- } \\
\text { rence } \\
\text { Inter- } \\
\text { val } \\
\text { (years) }\end{array}$} & \multicolumn{9}{|c|}{ Minimum average dlscharge $\left(\mathrm{ft}^{3} / \mathrm{s}\right)$} \\
\hline & & \multicolumn{9}{|c|}{ Number of consecutive days } \\
\hline & & 1 & 3 & 7 & 14 & 30 & 60 & 90 & 120 & 183 \\
\hline 0.05 & 20 & ng & ng & ng & ng & ng & ng & 0 & 0 & 0 \\
\hline 0.10 & 10 & ng & ng & ng & ng & ng & ng & 0 & 0 & 0 \\
\hline 0.20 & 5 & ng & ng & ng & ng & ng & ng & 0 & 0 & 0 \\
\hline 0.50 & 2 & $\mathrm{ng}$ & ng & $\mathrm{ng}$ & ng & ng & ng & 0 & 0 & 0.044 \\
\hline
\end{tabular}




\section{RUSH RIVER AT AMENIA, ND--Continued}

Probability of seasonal low discharges

[ng, statistic not given]

\begin{tabular}{|c|c|c|c|c|c|c|c|c|c|}
\hline \multirow[b]{3}{*}{$\begin{array}{l}\text { Non- } \\
\text { exceedance } \\
\text { probability }\end{array}$} & \multirow[b]{3}{*}{$\begin{array}{c}\text { Recurrence } \\
\text { interval } \\
\text { (years) }\end{array}$} & \multicolumn{8}{|c|}{ Minimum average discharge $\left(\mathrm{ft}^{3} / \mathrm{s}\right)$} \\
\hline & & \multicolumn{8}{|c|}{ Number of consecutive days } \\
\hline & & 1 & 7 & 14 & 30 & 1 & 7 & 14 & 30 \\
\hline & & \multicolumn{4}{|c|}{ December-January-February } & \multicolumn{4}{|c|}{ March-April-May } \\
\hline 0.05 & 20 & ng & ng & $\mathrm{ng}$ & ng & 0 & 0 & 0 & 0 \\
\hline 0.10 & 10 & ng & ng & ng & $\mathrm{ng}$ & 0 & 0 & 0 & 0 \\
\hline 0.20 & 5 & $\mathrm{ng}$ & $\mathrm{ng}$ & ng & $\mathrm{ng}$ & 0 & 0 & 0 & 0.232 \\
\hline \multirow[t]{2}{*}{0.50} & 2 & $\mathrm{ng}$ & ng & $\mathrm{ng}$ & ng & 0 & 0 & 0 & 1.90 \\
\hline & & \multicolumn{4}{|c|}{ June-July-August } & \multicolumn{4}{|c|}{ September-October-November } \\
\hline 0.05 & 20 & 0 & 0 & 0 & 0 & $\mathrm{ng}$ & 0 & 0 & 0 \\
\hline 0.10 & 10 & 0 & 0 & 0 & 0 & $\mathrm{ng}$ & 0 & 0 & 0 \\
\hline 0.20 & 5 & 0 & 0 & 0 & 0 & $\mathrm{ng}$ & 0 & 0 & 0 \\
\hline 0.50 & 2 & 0 & 0 & 0 & 0 & ng & 0 & 0 & 0 \\
\hline
\end{tabular}




\section{RUSH RIVER AT AMENIA, ND--Continued}

Annual peak discharge and corresponding gage height, period of record $[-$, no data $]$

\begin{tabular}{|c|c|c|c|c|c|c|c|}
\hline $\begin{array}{l}\text { Water } \\
\text { year }\end{array}$ & Date & $\begin{array}{c}\text { Gage } \\
\text { height } \\
\text { (feet) }\end{array}$ & $\begin{array}{c}\text { Peak } \\
\text { discharge } \\
\left(\mathrm{ft}^{3} / \mathrm{s}\right)\end{array}$ & $\begin{array}{l}\text { Water } \\
\text { year }\end{array}$ & Date & $\begin{array}{c}\text { Gage } \\
\text { height } \\
\text { (feet) }\end{array}$ & $\begin{array}{c}\text { Peak } \\
\text { discharge } \\
\left(\mathrm{f}^{3} / \mathrm{s}\right)\end{array}$ \\
\hline \multicolumn{8}{|c|}{ Annual peak discharge, by year, and corresponding gage height } \\
\hline 1947 & April 14 & 8.90 & 1,230 & 1971 & April 2 & 6.20 & 97.0 \\
\hline 1948 & April 11 & - & 590 & 1972 & April 14 & 7.05 & 252 \\
\hline 1949 & March 31 & - & 400 & 1973 & March 15 & 8.47 & 200 \\
\hline 1950 & April 7 & -- & 620 & 1974 & April 12 & 9.27 & 790 \\
\hline 1951 & March 28 & 8.60 & 368 & 1975 & April 18 & 11.62 & 2,550 \\
\hline 1952 & April 1 & 9.70 & 600 & 1976 & March 24 & 9.93 & 150 \\
\hline 1953 & June 16 & 8.63 & 1,050 & 1977 & May 31 & 5.02 & 41.0 \\
\hline 1954 & April 6 & 4.90 & 120 & 1978 & March 29 & 8.99 & 375 \\
\hline 1955 & March 31 & 9.00 & 200 & 1979 & April 19 & 10.37 & 3,490 \\
\hline 1956 & April 13 & - & 250 & 1980 & March 24 & -- & 63.0 \\
\hline 1957 & June 24 & 5.58 & 115 & 1981 & May 27 & 5.14 & 22.0 \\
\hline 1958 & July 5 & 5.40 & 77.0 & 1982 & April 3 & 10.55 & 710 \\
\hline 1959 & June 10 & 6.25 & 100 & 1983 & June 22 & 8.07 & 428 \\
\hline 1960 & April 6 & 8.09 & 437 & 1984 & March 29 & -- & 987 \\
\hline 1961 & March 6 & 6.06 & 25.0 & 1985 & March 18 & - & 164 \\
\hline 1962 & April 5 & 10.30 & 450 & 1986 & July 11 & 10.12 & 767 \\
\hline 1963 & April 7 & 5.30 & 68.0 & 1987 & March 20 & 7.98 & 475 \\
\hline 1964 & April 5 & 6.70 & 100 & 1988 & April 1 & - & 30.0 \\
\hline 1965 & April 11 & 11.60 & 900 & 1989 & April 2 & -- & 602 \\
\hline 1966 & March 31 & 9.16 & 300 & 1990 & June 4 & 6.01 & 64.0 \\
\hline 1967 & April 17 & 7.82 & 384 & 1991 & May 25 & - & 43.0 \\
\hline 1968 & June 8 & 7.07 & 190 & 1992 & July 1 & 7.82 & 255 \\
\hline 1969 & April 10 & 11.41 & 1,690 & 1993 & July 17 & - & 2,970 \\
\hline 1970 & April 6 & 9.41 & 380 & 1994 & March 19 & 9.47 & 470 \\
\hline \multicolumn{8}{|c|}{ Annual peak discharge, from highest to lowest, and corresponding gage height } \\
\hline 1979 & April 19 & 10.37 & 3,490 & 1983 & June 22 & 8.07 & 428 \\
\hline 1993 & July 17 & - & 2,970 & 1949 & March 31 & -- & 400 \\
\hline 1975 & April 18 & 11.62 & 2,550 & 1967 & April 17 & 7.82 & 384 \\
\hline 1969 & April 10 & 11.41 & 1,690 & 1970 & April 6 & 9.41 & 380 \\
\hline 1947 & April 14 & 8.90 & 1,230 & 1978 & March 29 & 8.99 & 375 \\
\hline 1953 & June 16 & 8.63 & 1,050 & 1951 & March 28 & -- & 368 \\
\hline 1984 & March 29 & -- & 987 & 1966 & March 31 & 9.16 & 300 \\
\hline 1965 & April 11 & 11.60 & 900 & 1992 & July 1 & 7.82 & 255 \\
\hline 1974 & April 12 & 9.27 & 790 & 1972 & April 14 & 7.05 & 252 \\
\hline 1986 & July 11 & 10.12 & 767 & 1956 & April 13 & -- & 250 \\
\hline 1982 & April 3 & 10.55 & 710 & 1955 & March 31 & 9.00 & 200 \\
\hline 1950 & April 7 & - & 620 & 1973 & March 15 & 8.47 & 200 \\
\hline 1989 & April 2 & - & 602 & 1968 & June 8 & 7.07 & 190 \\
\hline 1952 & April 1 & 9.70 & 600 & 1985 & March 18 & -- & 164 \\
\hline 1948 & April 11 & - & 590 & 1976 & March 24 & 9.93 & 150 \\
\hline 1987 & March 20 & 7.98 & 475 & 1954 & April 6 & - & 120 \\
\hline 1994 & March 19 & 9.47 & 470 & 1957 & June 24 & 5.58 & 115 \\
\hline 1962 & April 5 & 10.30 & 450 & 1959 & June 10 & 6.25 & 100 \\
\hline 1960 & April 6 & 8.09 & 437 & 1964 & April 5 & 6.70 & 100 \\
\hline
\end{tabular}




\section{RUSH RIVER AT AMENIA, ND--Continued}

Annual peak discharge and corresponding gage height, period of record--Continued $[-$, no data $]$

\begin{tabular}{cccccccc}
\hline $\begin{array}{c}\text { Water } \\
\text { year }\end{array}$ & Date & $\begin{array}{c}\text { Gage } \\
\text { height } \\
\text { (feet) }\end{array}$ & $\begin{array}{c}\text { Peak } \\
\text { discharge } \\
\left(\mathbf{f t}^{3} / \mathbf{s}\right)\end{array}$ & $\begin{array}{c}\text { Water } \\
\text { year }\end{array}$ & Date & $\begin{array}{c}\text { Gage } \\
\text { height } \\
\text { (feet) }\end{array}$ & $\begin{array}{c}\text { Peak } \\
\text { discharge } \\
\left(\mathrm{ft}^{3} / \mathbf{s}\right)\end{array}$ \\
\hline \multicolumn{7}{c}{ Annual peak discharge, from highest to lowest, and corresponding gage helght..Continued } \\
1971 & April 2 & 6.20 & 97.0 & 1991 & May 25 & - & 43.0 \\
1958 & July 5 & 5.40 & 77.0 & 1977 & May 31 & 5.02 & 41.0 \\
1963 & April 7 & 5.30 & 68.0 & 1988 & April 1 & - & 30.0 \\
1990 & June 4 & 6.01 & 64.0 & 1961 & March 6 & 6.06 & 25.0 \\
1980 & April 1 & - & 63.0 & 1981 & May 27 & 5.14 & 22.0 \\
\hline
\end{tabular}




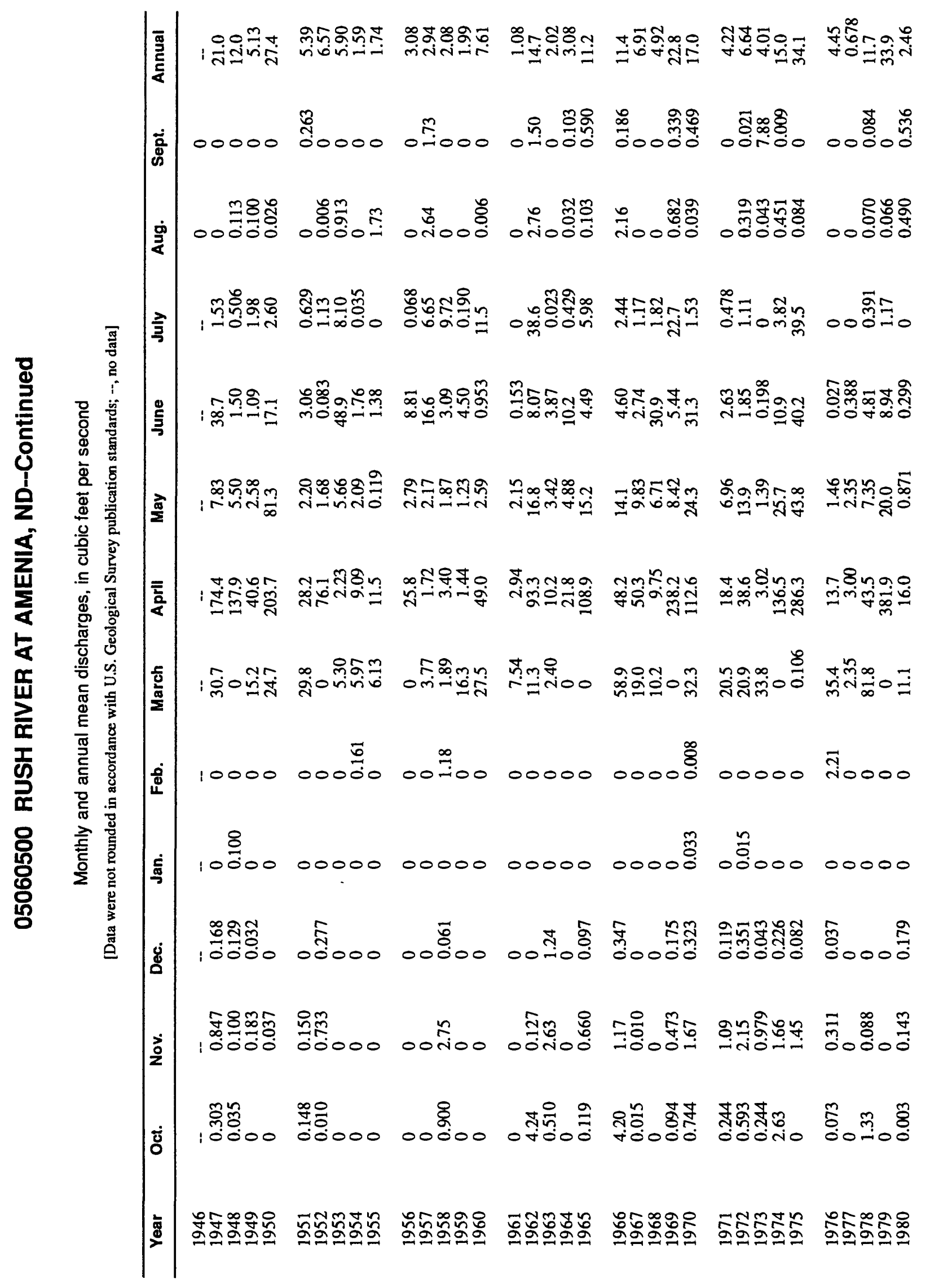




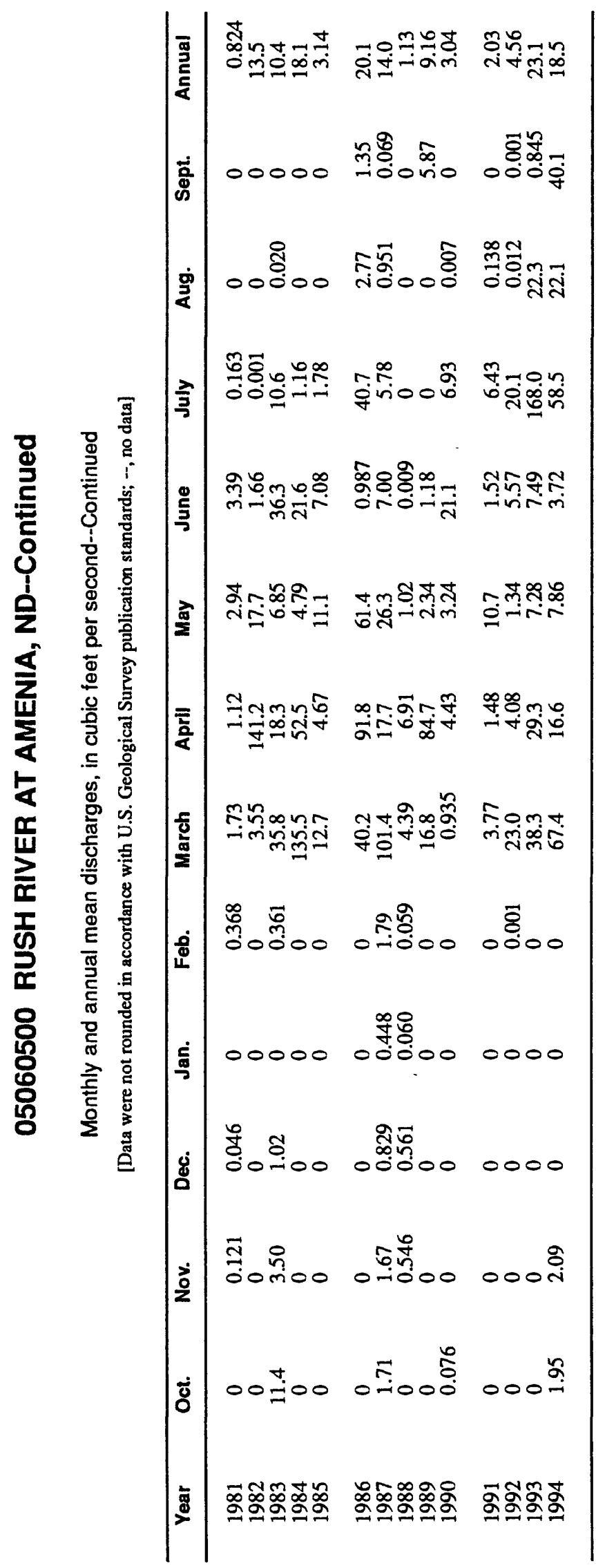




\section{BUFFALO RIVER NEAR HAWLEY, MN}

\section{Station Description}

LOCATION.--Lat $46^{\circ} 51^{\prime} 00^{\prime \prime}$, long $96^{\circ} 19^{\prime} 45^{\prime \prime}$, in NW $1 / 4 \mathrm{SE}^{1 / 4} \mathrm{sec} .14, \mathrm{~T} .139 \mathrm{~N}$., R.45 W., Clay County, Hydrologic Unit 09020106, near left downstream end of bridge on farm lane, 2 mi southwest of Hawley.

DRAINAGE AREA.--322 $\mathrm{mi}^{2}$.

PERIOD OF RECORD.--March 1945 to current year, WY 1981 (annual maximum only), March 1982 to September 1985 (no winter records).

GAGE.--Water-stage recorder. Datum of gage is $1,111.91 \mathrm{ft}$ above sea level. Prior to Jan. 29, 1953, nonrecording gage at bridge $1,800 \mathrm{ft}$ upstream at datum $3.17 \mathrm{ft}$ lower.

EXTREMES FOR PERIOD OF RECORD.--Maximum discharge, 2,050 ft $3 / \mathrm{s}$, July 1, 1975; maximum gage height, $10.40 \mathrm{ft}$, July 18, 1993; minimum discharge, $2.8 \mathrm{ft}^{3} / \mathrm{s}$, Aug. 26, 1977; minimum gage height, $3.15 \mathrm{ft}$, June 27 and July 26, 1977.

EXTREMES OUTSIDE PERIOD OF RECORD.--Maximum stage known, about $11.3 \mathrm{ft}$, present datum, spring of 1921, from information by local resident.

Annual mean discharge

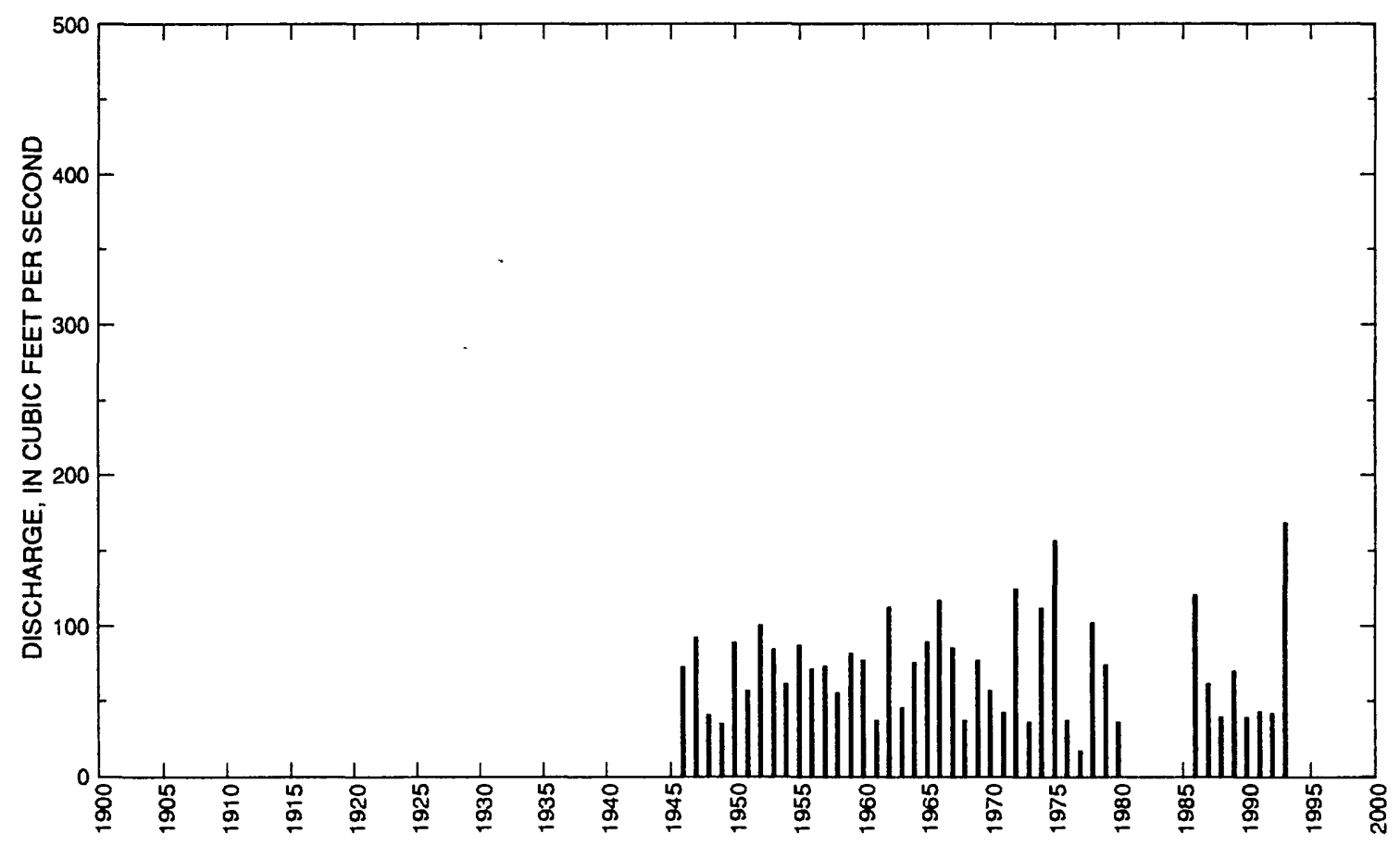




\section{BUFFALO RIVER NEAR HAWLEY, MN--Continued}

Statistics of monthly and annual mean discharges

\begin{tabular}{|c|c|c|c|c|c|c|c|c|}
\hline \multirow[b]{2}{*}{ Month } & \multicolumn{2}{|c|}{ Maximum } & \multicolumn{2}{|c|}{ Minimum } & \multicolumn{4}{|c|}{ Mean } \\
\hline & $\begin{array}{c}\text { Discharge } \\
\left(\mathrm{ft}^{3} / \mathrm{s}\right)\end{array}$ & $\begin{array}{c}\text { Water year } \\
\text { of } \\
\text { occurrence }\end{array}$ & $\begin{array}{c}\text { Discharge } \\
\left(\mathrm{ft}^{3} / \mathrm{s}\right)\end{array}$ & $\begin{array}{l}\text { Water year } \\
\text { of } \\
\text { occurrence }\end{array}$ & $\begin{array}{c}\text { Discharge } \\
\left(\mathrm{ft}^{3} / \mathbf{s}\right)\end{array}$ & $\begin{array}{l}\text { Standard } \\
\text { deviation } \\
\left(\mathrm{ft}^{3} / \mathrm{s}\right)\end{array}$ & $\begin{array}{l}\text { Coeffi- } \\
\text { cient of } \\
\text { variation }\end{array}$ & $\begin{array}{l}\text { Percentage } \\
\text { of annual } \\
\text { discharge }\end{array}$ \\
\hline October & 151 & 1974 & 11.6 & 1979 & 36.3 & 29.3 & 0.81 & 4.14 \\
\hline November & 176 & 1972 & 12.2 & 1977 & 33.8 & 26.4 & 0.78 & 3.86 \\
\hline December & 63.8 & 1972 & 10.6 & 1977 & 23.4 & 11.2 & 0.48 & 2.67 \\
\hline January & 54.7 & 1981 & 9.94 & 1962 & 19.8 & 9.16 & 0.46 & 2.26 \\
\hline February & 99.6 & 1981 & 9.87 & 1949 & 20.7 & 13.8 & 0.67 & 2.36 \\
\hline March & 434 & 1966 & 15.0 & 1969 & 80.7 & 81.2 & 1.01 & 9.22 \\
\hline April & 792 & 1978 & 33.3 & 1981 & 251 & 176 & 0.70 & 28.6 \\
\hline May & 372 & 1985 & 21.5 & 1977 & 123 & 85.2 & 0.69 & 14.0 \\
\hline June & 530 & 1962 & 12.7 & 1977 & 98.3 & 95.5 & 0.97 & 11.2 \\
\hline July & 784 & 1993 & 10.1 & 1976 & 99.0 & 154 & 1.56 & 11.3 \\
\hline August & 472 & 1955 & 5.87 & 1976 & 52.4 & 92.4 & 1.76 & 5.99 \\
\hline September & 182 & 1957 & 8.52 & 1976 & 37.9 & 34.8 & 0.92 & 4.33 \\
\hline Annual & 168 & 1993 & 16.7 & 1977 & 72.7 & 34.0 & 0.47 & 100 \\
\hline
\end{tabular}

Annual flow duration

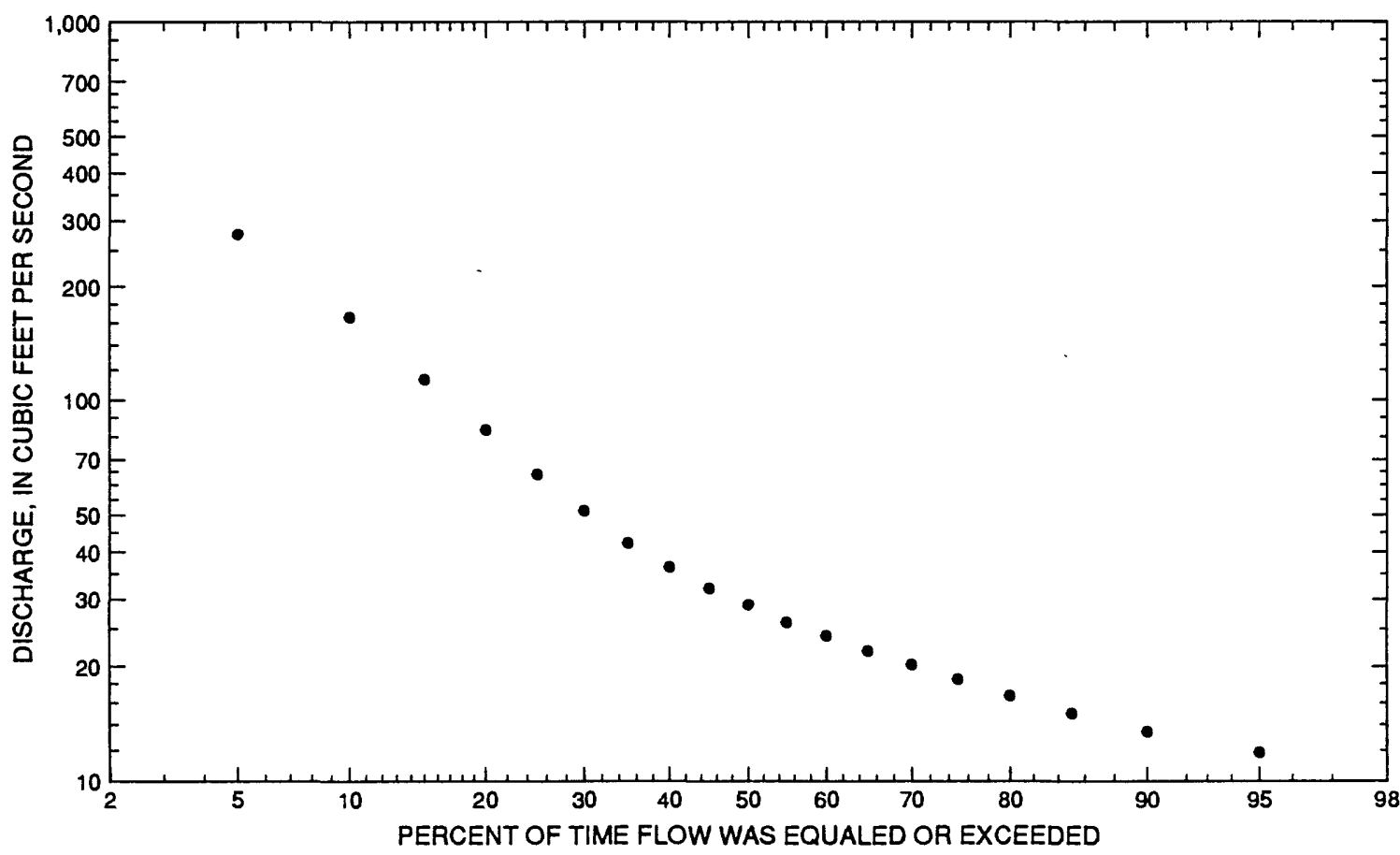




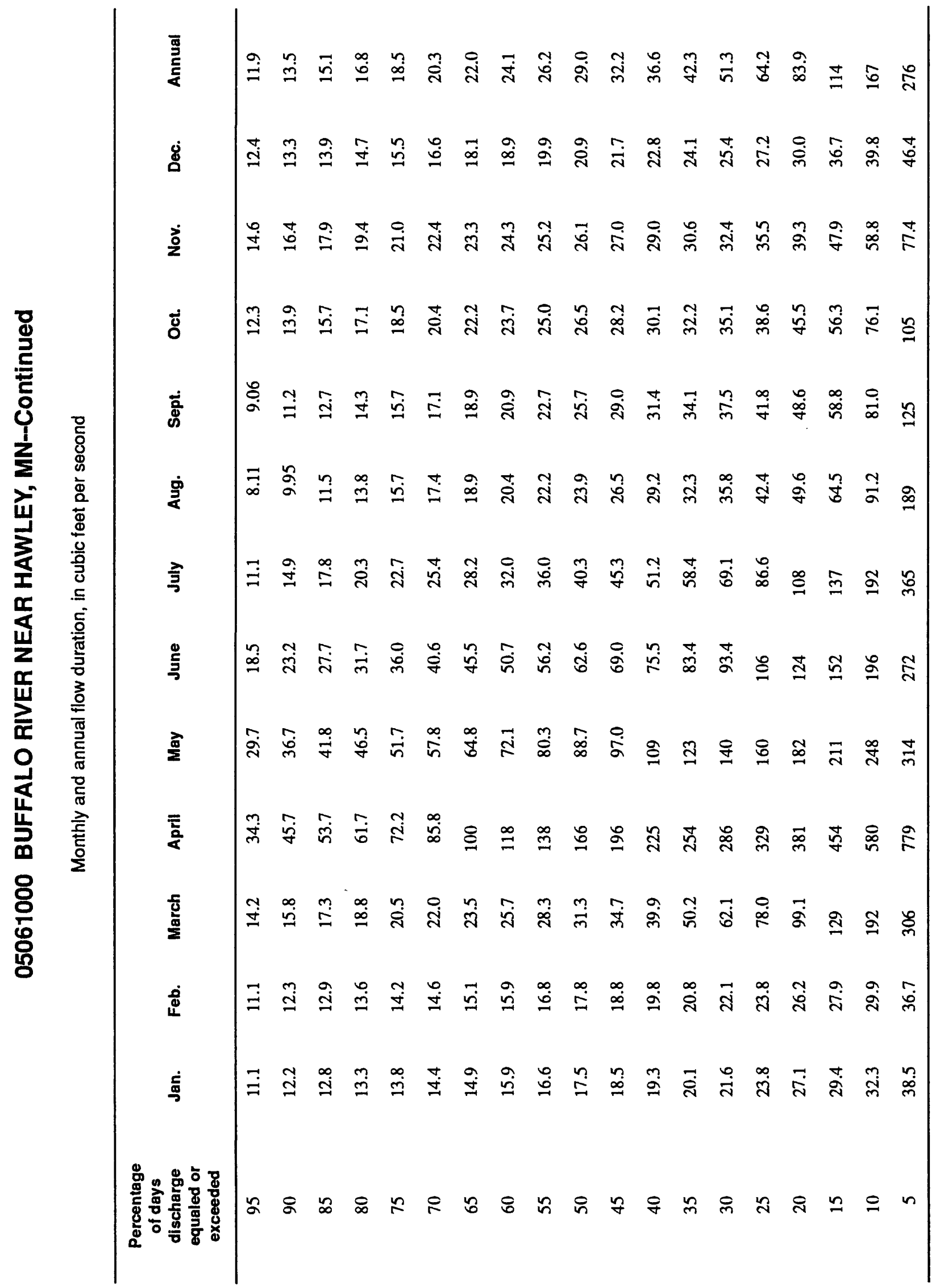




\section{BUFFALO RIVER NEAR HAWLEY, MN--Continued}

Probability of annual high discharges]

[ng, statistic not given]

\begin{tabular}{lcccccc}
\hline & & & \multicolumn{5}{c}{$\begin{array}{c}\text { Maximum average discharge } \\
\left(\mathbf{f t}^{3} / \mathbf{s}\right)\end{array}$} \\
\cline { 5 - 7 } $\begin{array}{c}\text { Exceedance } \\
\text { probability }\end{array}$ & $\begin{array}{c}\text { Recurrence } \\
\text { interval } \\
\text { (years) }\end{array}$ & $\begin{array}{c}\text { Maximum } \\
\text { instantaneous } \\
\left(\mathbf{f t}^{3} / \mathbf{s}\right)\end{array}$ & 3-day period & 7-day period & 15-day period & 30-day period \\
\hline 0.99 & 1.01 & 76.6 & 67.5 & 57.0 & 49.3 & 44.4 \\
0.95 & 1.05 & 154 & 133 & 114 & 95.2 & 81.6 \\
0.90 & 1.11 & 218 & 187 & 161 & 133 & 111 \\
0.80 & 1.25 & 326 & 278 & 241 & 195 & 158 \\
0.50 & 2 & 661 & 562 & 489 & 388 & 297 \\
0.20 & 5 & 1,240 & 1,060 & 923 & 723 & 525 \\
0.10 & 10 & 1,680 & 1,440 & 1,250 & 977 & 690 \\
0.04 & 25 & 2,270 & 1,950 & 1,690 & 1,320 & 907 \\
0.02 & 50 & 2,720 & 2,350 & 2,040 & 1,590 & 1,070 \\
0.01 & 100 & 3,180 & 2,770 & 2,390 & 1,870 & 1,240 \\
0.005 & 200 & 3,640 & 3,190 & 2,740 & 2,160 & 1,410 \\
0.002 & 500 & 4,310 & ng & ng & ng & ng \\
\hline
\end{tabular}

Probability of annual low discharges

\begin{tabular}{|c|c|c|c|c|c|c|c|c|c|c|}
\hline \multirow[b]{3}{*}{$\begin{array}{l}\text { Non- } \\
\text { exceed- } \\
\text { ance } \\
\text { prob- } \\
\text { ability }\end{array}$} & \multirow[b]{3}{*}{$\begin{array}{c}\text { Recur- } \\
\text { rence } \\
\text { inter- } \\
\text { val } \\
\text { (years) }\end{array}$} & \multicolumn{9}{|c|}{ Minimum average discharge $\left(\mathrm{ft}^{3} / \mathrm{s}\right)$} \\
\hline & & \multicolumn{9}{|c|}{ Number of consecutive days } \\
\hline & & 1 & 3 & 7 & 14 & 30 & 60 & 90 & 120 & 183 \\
\hline 0.05 & 20 & 5.26 & 5.62 & 6.28 & 6.83 & 7.46 & 8.59 & 9.75 & 11.1 & 12.2 \\
\hline 0.10 & 10 & 6.43 & 6.78 & 7.35 & 7.87 & 8.52 & 9.67 & 10.9 & 12.3 & 13.6 \\
\hline 0.20 & 5 & 8.10 & 8.43 & 8.87 & 9.35 & 10.0 & 11.2 & 12.6 & 14.0 & 15.6 \\
\hline 0.50 & 2 & 12.1 & 12.4 & 12.7 & 13.1 & 13.9 & 152 & 16.8 & 18.5 & 21.3 \\
\hline
\end{tabular}




\section{BUFFALO RIVER NEAR HAWLEY, MN--Continued}

Probability of seasonal low discharges

\begin{tabular}{|c|c|c|c|c|c|c|c|c|c|}
\hline \multirow[b]{3}{*}{$\begin{array}{c}\text { Non- } \\
\text { excoedance } \\
\text { probabllity }\end{array}$} & \multirow[b]{3}{*}{$\begin{array}{c}\text { Recurrence } \\
\text { Interval } \\
\text { (years) }\end{array}$} & \multicolumn{8}{|c|}{ Minimum average discharge $\left(\mathrm{ft}^{3} / \mathrm{s}\right)$} \\
\hline & & \multicolumn{8}{|c|}{ Number of consecutlve days } \\
\hline & & 1 & 7 & 14 & 30 & 1 & 7 & 14 & 30 \\
\hline & & \multicolumn{4}{|c|}{ December-January-February } & \multicolumn{4}{|c|}{ March-April-May } \\
\hline 0.05 & 20 & 9.10 & 9.23 & 9.31 & 9.64 & 11.0 & 11.9 & 13.3 & 16.2 \\
\hline 0.10 & 10 & 9.95 & 10.1 & 10.2 & 10.6 & 12.3 & 13.3 & 14.8 & 19.3 \\
\hline 0.20 & 5 & 11.2 & 11.4 & 11.6 & 12.0 & 14.2 & 15.4 & 17.1 & 24.3 \\
\hline \multirow[t]{2}{*}{0.50} & 2 & 14.6 & 14.9 & 15.2 & 15.7 & 18.9 & 21.1 & 23.4 & 40.2 \\
\hline & & \multicolumn{4}{|c|}{ June-July-August } & \multicolumn{4}{|c|}{ September-October-November } \\
\hline 0.05 & 20 & 5.51 & 6.53 & 7.08 & 8.12 & 6.55 & 7.59 & 8.37 & 10.1 \\
\hline 0.10 & 10 & 6.98 & 8.03 & 8.68 & 10.0 & 7.96 & 9.13 & 9.97 & 11.6 \\
\hline 0.20 & 5 & 9.27 & 10.4 & 11.2 & 13.1 & 10.1 & 11.5 & 12.4 & 14.1 \\
\hline 0.50 & 2 & 15.8 & 17.2 & 18.9 & 22.4 & 15.6 & 18.0 & 19.2 & 21.2 \\
\hline
\end{tabular}




\section{BUFFALO RIVER NEAR HAWLEY, MN--Continued}

Annual peak discharge and corresponding gage height, period of record

$$
[-, \text { no data }]
$$

\begin{tabular}{|c|c|c|c|c|c|c|c|}
\hline $\begin{array}{l}\text { Water } \\
\text { year }\end{array}$ & Date & $\begin{array}{c}\text { Gage } \\
\text { height } \\
\text { (foet) }\end{array}$ & $\begin{array}{c}\text { Peak } \\
\text { discharge } \\
\left(\mathrm{ft}^{3} / \mathrm{s}\right)\end{array}$ & $\begin{array}{l}\text { Water } \\
\text { year }\end{array}$ & Date & $\begin{array}{c}\text { Gage } \\
\text { helght } \\
\text { (feet) }\end{array}$ & $\begin{array}{c}\text { Peak } \\
\text { discharge } \\
\left(\mathrm{ft}^{3} / \mathrm{s}\right)\end{array}$ \\
\hline \multicolumn{8}{|c|}{ Annual peak discharge, by year, and corresponding gage height } \\
\hline 1945 & March 16 & 9.52 & 894 & 1970 & April 10 & 6.98 & 498 \\
\hline 1946 & March 19 & 8.49 & 452 & 1971 & April 2 & 6.45 & 382 \\
\hline 1947 & April 13 & 9.42 & 878 & 1972 & March 25 & 8.35 & 1,310 \\
\hline 1948 & April 13 & - & 297 & 1973 & March 18 & 4.88 & 172 \\
\hline 1949 & April 7 & 6.67 & 256 & 1974 & April 13 & 8.64 & 1,280 \\
\hline 1950 & April 7 & 9.32 & 841 & 1975 & July 1 & 9.76 & 2,050 \\
\hline 1951 & April 7 & 9.00 & 792 & 1976 & March 31 & 6.49 & 408 \\
\hline 1952 & April 8 & 9.10 & 880 & 1977 & July 4 & 5.31 & 121 \\
\hline 1953 & June 17 & 7.47 & 598 & 1978 & April 7 & 9.44 & 2,000 \\
\hline 1954 & April 12 & 7.36 & 630 & 1979 & April 17 & 8.67 & 980 \\
\hline 1955 & August 5 & 9.31 & 1,590 & 1980 & April 3 & 7.42 & 619 \\
\hline 1956 & April 11 & 8.50 & 1,020 & 1981 & July 31 & 4.47 & 74.0 \\
\hline 1957 & March 26 & 6.61 & 456 & 1982 & April 2 & 7.22 & 468 \\
\hline 1958 & July 5 & 4.86 & 195 & 1983 & July 5 & 8.96 & 1,080 \\
\hline 1959 & July 11 & 7.04 & 551 & 1984 & June 10 & 8.10 & 846 \\
\hline 1960 & April 27 & 7.54 & 660 & 1985 & May 13 & 8.58 & 1,060 \\
\hline 1961 & May 16 & 5.28 & 246 & 1986 & May 13 & 8.11 & 866 \\
\hline 1962 & June 10 & 9.11 & 1,430 & 1987 & March 25 & 6.06 & 330 \\
\hline 1963 & August 12 & 5.48 & 253 & 1988 & April 2 & 6.12 & 350 \\
\hline 1964 & April 19 & 8.46 & 1,000 & 1989 & April 5 & 9.11 & 1,510 \\
\hline 1965 & April 11 & 8.87 & 1,250 & 1990 & March 31 & 7.18 & 562 \\
\hline 1966 & March 18 & 10.42 & 1,520 & 1991 & May 6 & -- & 248 \\
\hline 1967 & March 30 & 7.57 & 669 & 1992 & July 11 & 5.66 & 237 \\
\hline 1968 & March 28 & 4.97 & 202 & 1993 & July 18 & 10.40 & 2,010 \\
\hline 1969 & April 9 & 9.07 & 1,880 & 1994 & March 23 & 7.88 & 540 \\
\hline \multicolumn{8}{|c|}{ Annual peak discharge, from highest to lowest, and corresponding gage height } \\
\hline 1975 & July 1 & 9.76 & 2,050 & 1947 & April 13 & 9.42 & 878 \\
\hline 1993 & July 18 & 10.40 & 2,010 & 1986 & May 13 & 8.11 & 866 \\
\hline 1978 & April 7 & 9.44 & 2,000 & 1984 & June 10 & 8.10 & 846 \\
\hline 1969 & April 9 & 9.07 & 1,880 & 1950 & April 7 & 9.32 & 841 \\
\hline 1955 & August 5 & 9.31 & 1,590 & 1951 & April 7 & 9.00 & 792 \\
\hline 1966 & March 18 & 10.42 & 1,520 & 1967 & March 30 & 7.57 & 669 \\
\hline 1989 & April 5 & 9.11 & 1,510 & 1960 & April 27 & 7.54 & 660 \\
\hline 1962 & June 10 & 9.11 & 1,430 & 1954 & April 12 & 7.36 & 630 \\
\hline 1972 & March 25 & 8.35 & 1,310 & 1980 & April 3 & 7.42 & 619 \\
\hline 1974 & April 13 & 8.64 & 1,280 & 1953 & June 17 & 7.47 & 598 \\
\hline 1965 & April 11 & 8.87 & 1,250 & 1990 & March 31 & 7.18 & 562 \\
\hline 1983 & July 5 & 8.96 & 1,080 & 1959 & July 11 & 7.04 & 551 \\
\hline 1985 & May 13 & 8.58 & 1,060 & 1994 & March 23 & 7.88 & 540 \\
\hline 1956 & April 11 & 8.50 & 1,020 & 1970 & April 10 & 6.98 & 498 \\
\hline 1964 & April 19 & 8.46 & 1,000 & 1982 & April 2 & 7.22 & 468 \\
\hline 1979 & April 17 & 8.67 & 980 & 1957 & March 26 & 6.61 & 456 \\
\hline 1945 & March 16 & 9.52 & 894 & 1946 & March 19 & 8.49 & 452 \\
\hline 1952 & April 8 & 9.10 & 880 & 1976 & March 31 & 6.49 & 408 \\
\hline
\end{tabular}




\section{BUFFALO RIVER NEAR HAWLEY, MN--Continued}

Annual peak discharge and corresponding gage height, period of record--Continued $[-$, no data $]$

\begin{tabular}{|c|c|c|c|c|c|c|c|}
\hline $\begin{array}{l}\text { Water } \\
\text { year }\end{array}$ & Date & $\begin{array}{c}\text { Gage } \\
\text { height } \\
\text { (feet) }\end{array}$ & $\begin{array}{c}\text { Peak } \\
\text { discharge } \\
\left(\mathrm{ft}^{3} / \mathrm{s}\right)\end{array}$ & $\begin{array}{c}\text { Water } \\
\text { year }\end{array}$ & Date & $\begin{array}{c}\text { Gage } \\
\text { height } \\
\text { (feet) }\end{array}$ & $\begin{array}{c}\text { Peak } \\
\text { discharge } \\
\left(\mathrm{ft}^{3} / \mathrm{s}\right)\end{array}$ \\
\hline \multicolumn{8}{|c|}{ Annual peak discharge, from highest to lowest, and corresponding gage height.-Continued } \\
\hline 1971 & April 2 & 6.45 & 382 & 1961 & May 16 & 5.28 & 246 \\
\hline 1988 & April 2 & 6.12 & 350 & 1992 & July 11 & 5.66 & 237 \\
\hline 1987 & March 25 & 6.06 & 330 & 1968 & March 28 & 4.97 & 202 \\
\hline 1948 & April 13 & - & 297 & 1958 & July 5 & 4.86 & 195 \\
\hline 1949 & April 7 & 6.67 & 256 & 1973 & March 18 & 4.88 & 172 \\
\hline 1963 & August 12 & 5.48 & 253 & 1977 & July 4 & 5.31 & 121 \\
\hline 1991 & May 6 & - & 248 & 1981 & July 31 & 4.47 & 74.0 \\
\hline
\end{tabular}




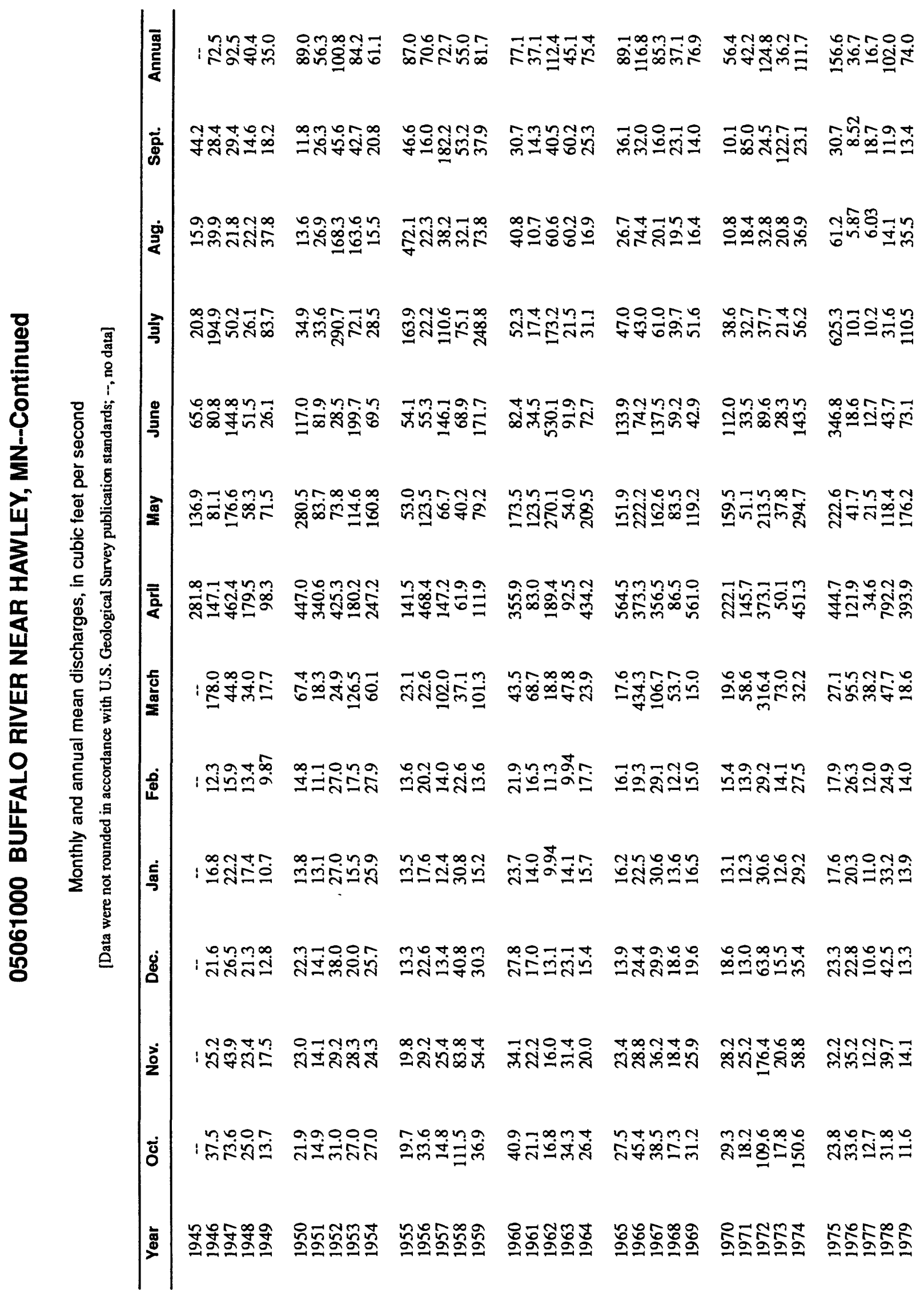




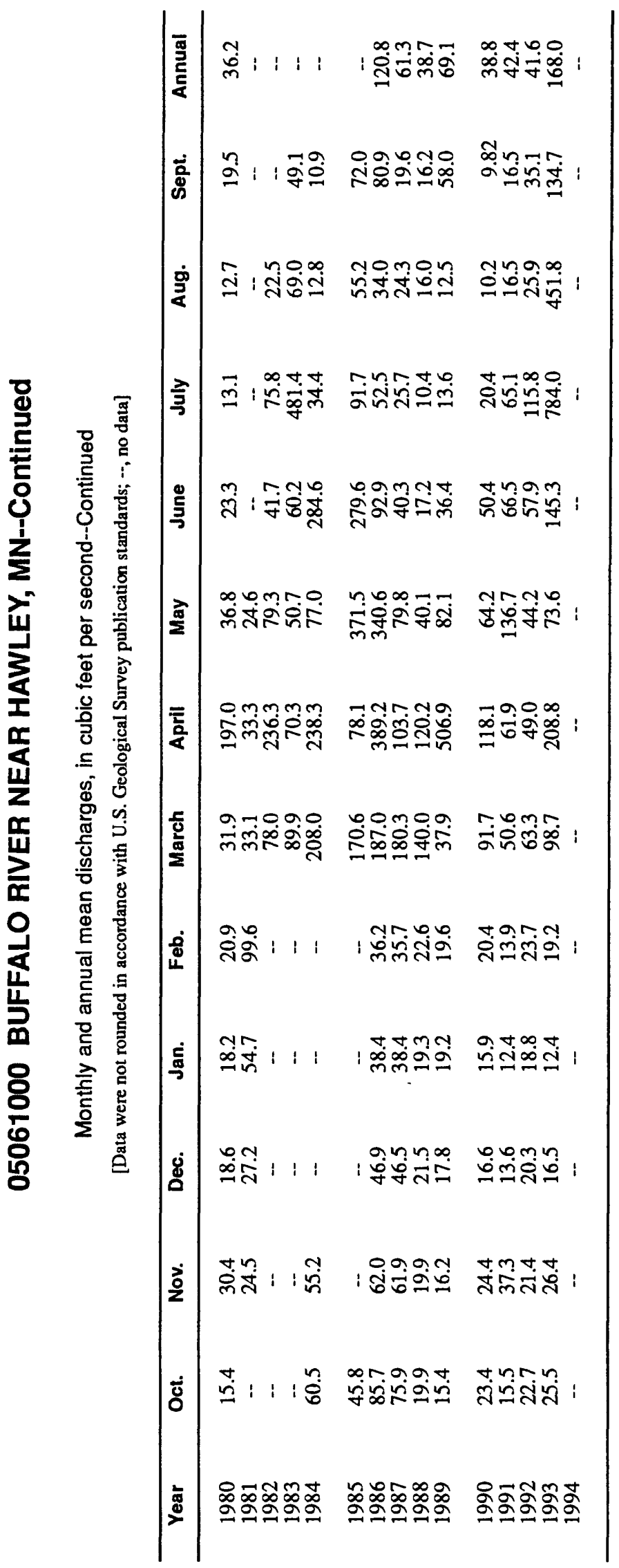




\section{SOUTH BRANCH BUFFALO RIVER AT SABIN, MN}

\section{Station Description}

LOCATION.--Lat 4646'20", long 96³7'40”, in SW $1 / 4 \mathrm{SW}^{1} / 4$ sec.9, T.138 N., R.47 W., Clay County, Hydrologic Unit 09020106, near center of span on downstream side of highway bridge, $0.3 \mathrm{mi}$ downstream from Stony Creek and $1 \mathrm{mi}$ east of Sabin.

DRAINAGE AREA.--522 $\mathrm{mi}^{2}$.

PERIOD OF RECORD.--March 1945 to current year, WY 1981 (annual maximum only), March 1982 to September 1985 (no winter records).

GAGE.--Water-stage recorder. Datum of gage is $902.39 \mathrm{ft}$ above sea level (levels by Soil Conservation Service). Prior to Aug. 17, 1948, nonrecording gage at site $1 \mathrm{mi}$ downstream at different datum. Aug. 17, 1948, to Oct. 4,1989 , nonrecording gage at present site and datum.

EXTREMES FOR PERIOD OF RECORD.--Maximum discharge, 8,500 $\mathrm{ft}^{3} / \mathrm{s}$, July 2, 1975 (gage height, $19.9 \mathrm{ft}$ ); no flow for many days most years.

Annual mean discharge

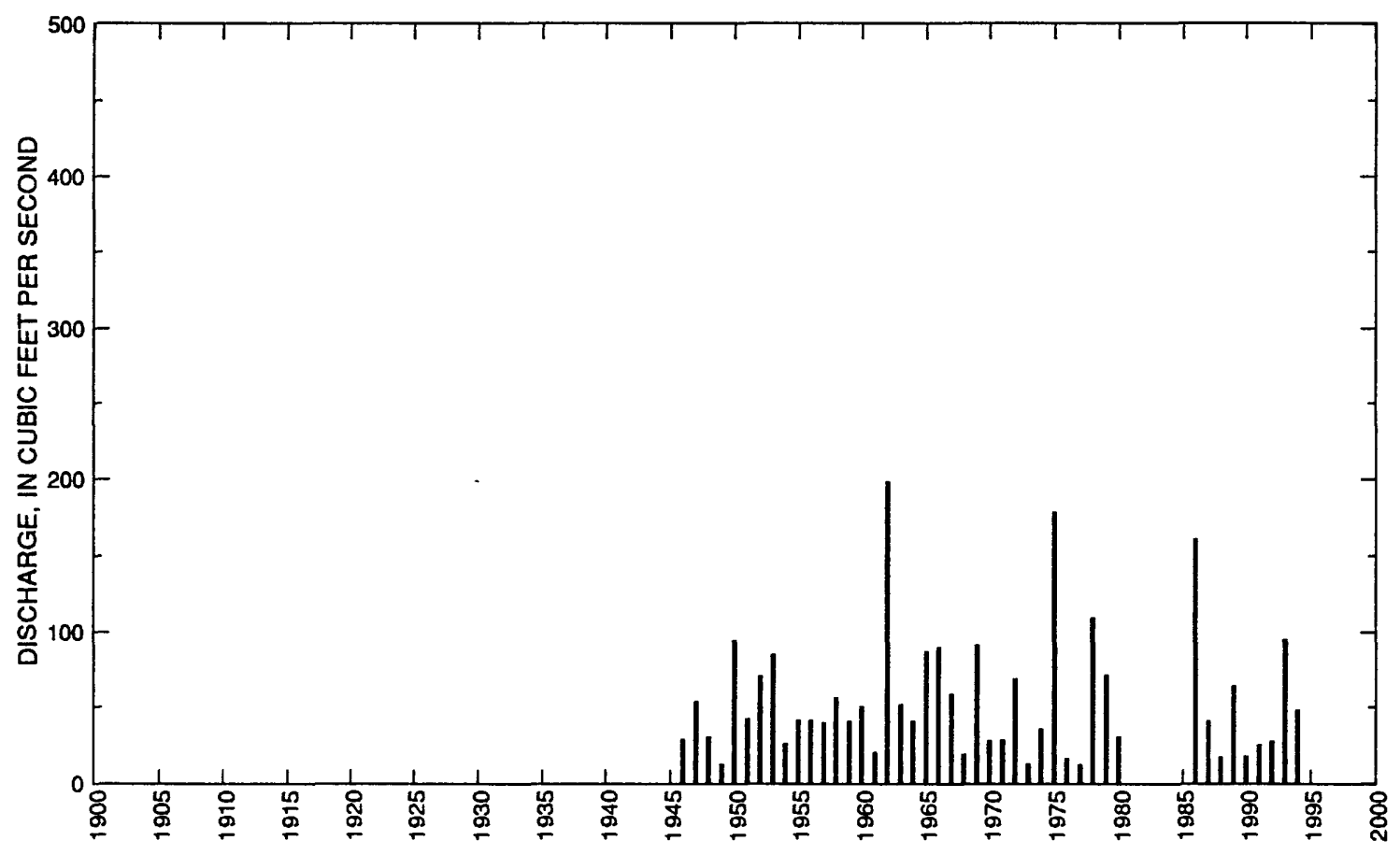




\section{SOUTH BRANCH BUFFALO RIVER AT SABIN, MN--Continued}

Statistics of monthly and annual mean discharges

[m, more than 1 year of occurrence]

\begin{tabular}{|c|c|c|c|c|c|c|c|c|}
\hline \multirow[b]{2}{*}{ Month } & \multicolumn{2}{|c|}{ Maximum } & \multicolumn{2}{|c|}{ Minimum } & \multicolumn{4}{|c|}{ Mean } \\
\hline & $\begin{array}{c}\text { Discharge } \\
\left(\mathrm{ft}^{3 / 3} / \mathbf{s}\right)\end{array}$ & $\begin{array}{c}\text { Water year } \\
\text { of } \\
\text { occurrence }\end{array}$ & $\begin{array}{c}\text { Discharge } \\
\left(\mathrm{ft}^{3} / \mathbf{s}\right)\end{array}$ & $\begin{array}{c}\text { Water year } \\
\text { of } \\
\text { occurrence }\end{array}$ & $\begin{array}{c}\text { Discharge } \\
\left(\mathrm{ft}^{3} / \mathrm{s}\right)\end{array}$ & $\begin{array}{c}\text { Standard } \\
\text { deviation } \\
\left(\mathrm{ft}^{3} / \mathrm{s}\right)\end{array}$ & $\begin{array}{l}\text { Coeffi- } \\
\text { cient of } \\
\text { variation }\end{array}$ & $\begin{array}{l}\text { Percentage } \\
\text { of annual } \\
\text { discharge }\end{array}$ \\
\hline October & 51.1 & 1978 & 0.023 & 1977 & 13.9 & 13.7 & 0.98 & 2.09 \\
\hline November & 76.7 & 1972 & 2.05 & 1977 & 14.2 & 13.1 & 0.92 & 2.12 \\
\hline December & 23.5 & 1978 & 0.006 & 1961 & 4.95 & 4.90 & 0.99 & 0.74 \\
\hline January & 13.1 & 1978 & 0 & $\mathrm{~m}$ & 1.67 & 2.59 & 1.55 & 0.25 \\
\hline February & 14.0 & 1987 & 0 & $\mathrm{~m}$ & 1.62 & 2.63 & 1.63 & 0.24 \\
\hline March & 581 & 1966 & 0 & $\mathrm{~m}$ & 104 & 137 & 1.32 & 15.6 \\
\hline April & 928 & 1969 & 27.9 & 1973 & 254 & 233 & 0.92 & 38.0 \\
\hline May & 580 & 1962 & 8.28 & 1980 & 76.7 & 98.5 & 1.28 & 11.5 \\
\hline June & 1,070 & 1962 & 1.30 & 1976 & 93.1 & 184 & 1.97 & 14.0 \\
\hline July & 1,110 & 1975 & 0 & 1988 & 77.4 & 182 & 2.35 & 11.6 \\
\hline August & 152 & 1993 & 0 & 1976 & 12.1 & 25.3 & 2.09 & 1.81 \\
\hline September & 174 & 1986 & 0 & 1976 & 13.9 & 29.3 & 2.11 & 2.09 \\
\hline Annual & 198 & 1962 & 12.2 & 1977 & 55.8 & 42.5 & 0.76 & 100 \\
\hline
\end{tabular}

Annual flow duration

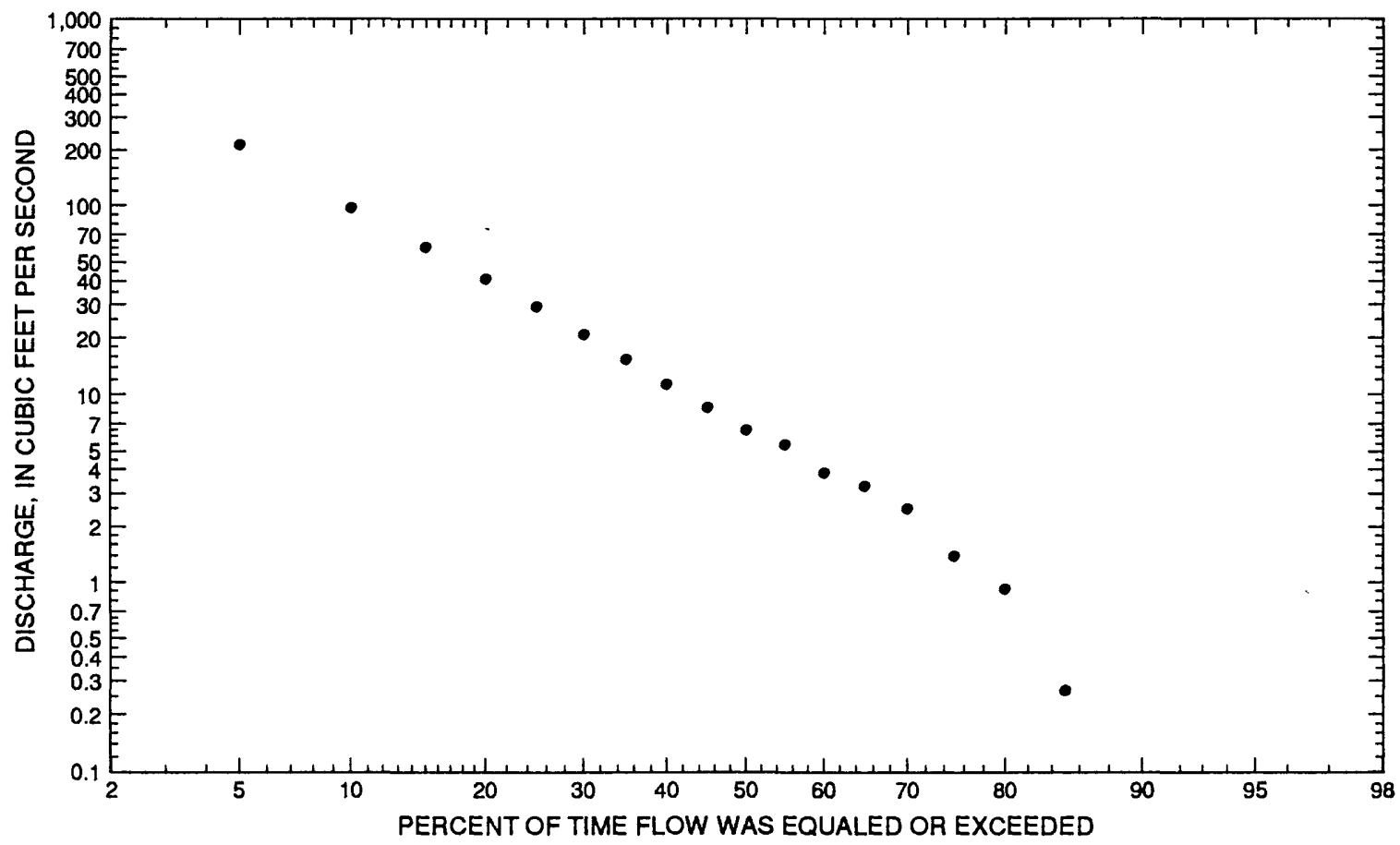




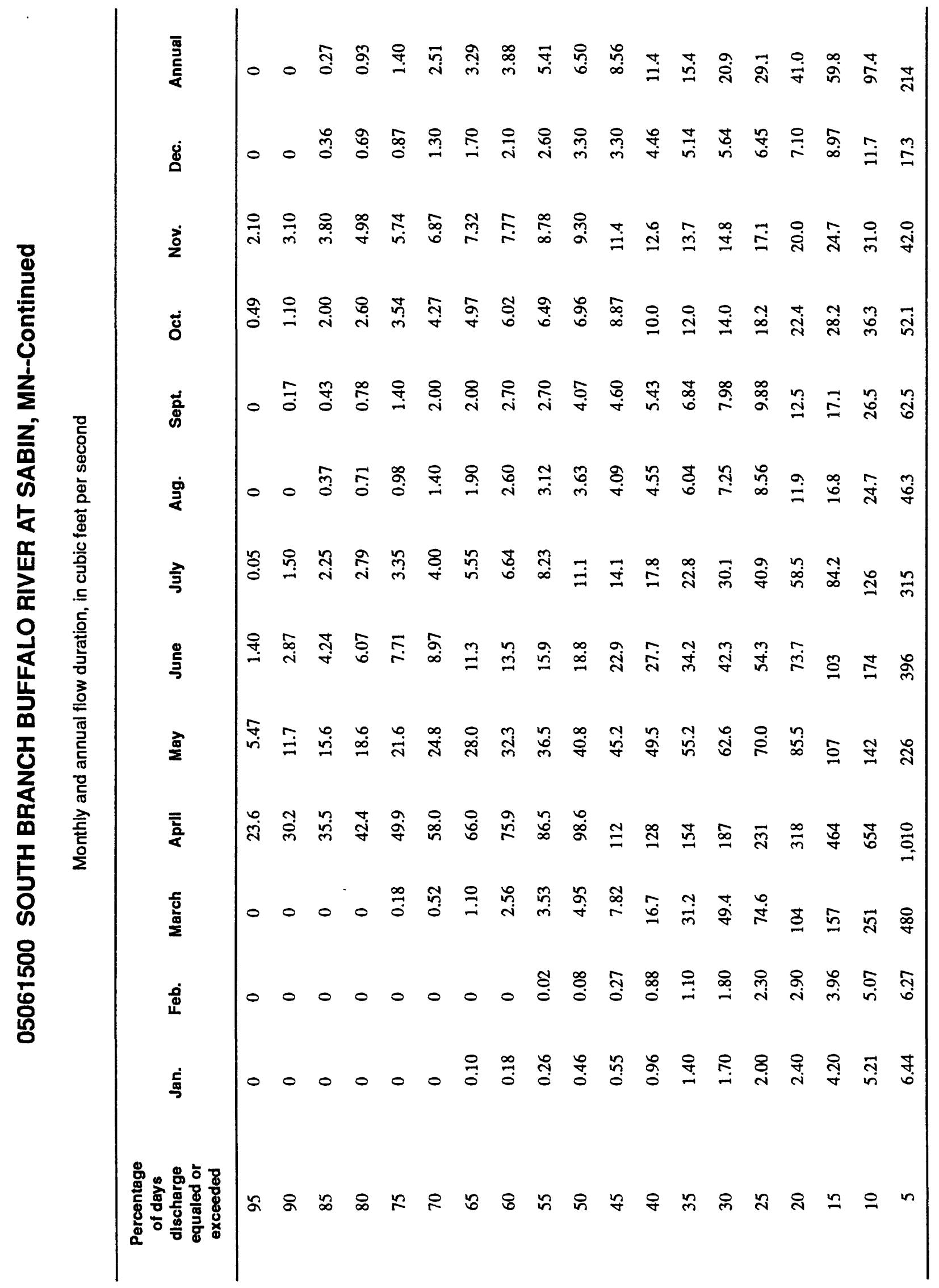




\section{SOUTH BRANCH BUFFALO RIVER AT SABIN, MN--Continued}

Probability of annual high discharges

[ng, statistic not given]

\begin{tabular}{lcccccc}
\hline & & & \multicolumn{5}{c}{$\begin{array}{c}\text { Maximum average discharge } \\
\left(\mathrm{ft}^{3} / \mathbf{s}\right)\end{array}$} \\
\cline { 6 - 7 } $\begin{array}{c}\text { Exceedance } \\
\text { probability }\end{array}$ & $\begin{array}{c}\text { Recurrence } \\
\text { interval } \\
\text { (years) }\end{array}$ & $\begin{array}{c}\text { Maximum } \\
\text { instantaneous } \\
\left(\mathrm{ft}^{3} / \mathbf{s}\right)\end{array}$ & 3-day period & 7-day period & 15-day period & 30-day period \\
\hline 0.99 & 1.01 & 83.7 & 66.0 & 54.8 & 37.9 & 30.1 \\
0.95 & 1.05 & 185 & 144 & 117 & 81.8 & 61.4 \\
0.90 & 1.11 & 279 & 217 & 175 & 121 & 88.7 \\
0.80 & 1.25 & 452 & 354 & 280 & 194 & 137 \\
0.50 & 2 & 1,100 & 882 & 673 & 454 & 302 \\
0.20 & 5 & 2,540 & 2,140 & 1,570 & 1,010 & 637 \\
0.10 & 10 & 3,880 & 3,370 & 2,410 & 1,510 & 924 \\
0.04 & 25 & 5,990 & 5,430 & 3,770 & 2,280 & 1,350 \\
0.02 & 50 & 7,870 & 7,350 & 5,010 & 2,950 & 1,720 \\
0.01 & 100 & 10,000 & 9,620 & 6,450 & 3,700 & 2,120 \\
0.005 & 200 & 12,400 & 12,300 & 8,100 & 4,540 & 2,570 \\
0.002 & 500 & 16,100 & ng & ng & ng & ng \\
\hline
\end{tabular}

Probability of annual low discharges

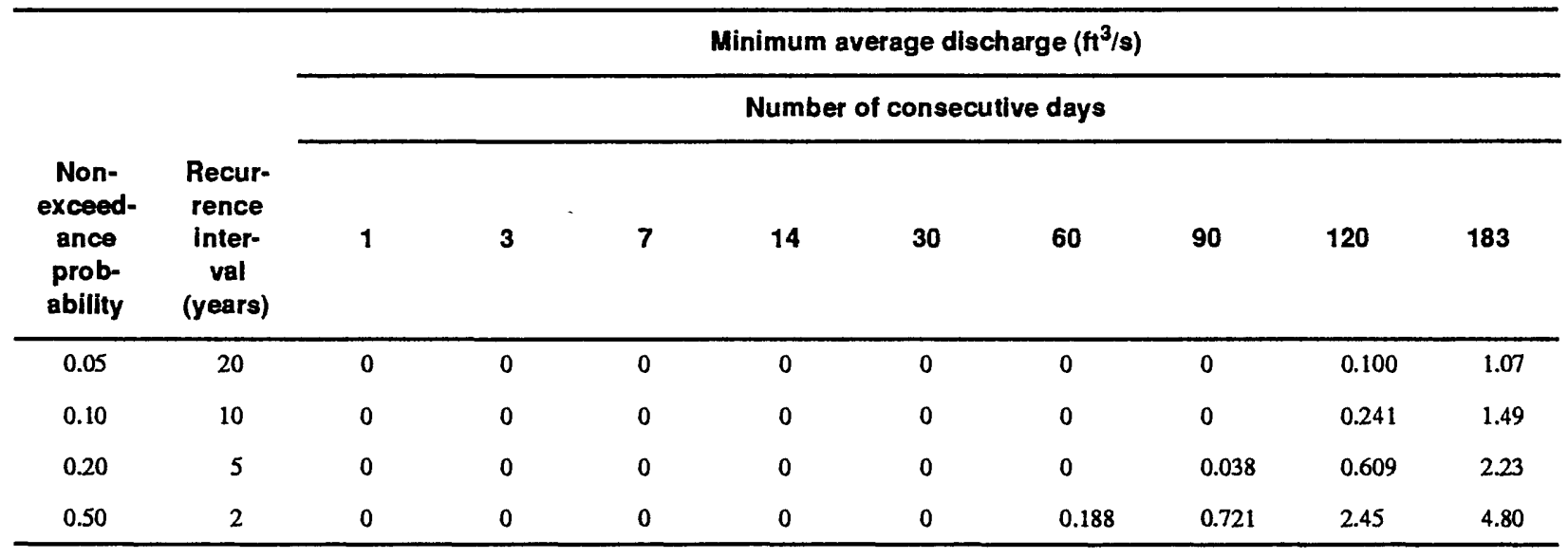


05061500 SOUTH BRANCH BUFFALO RIVER AT SABIN, MN--Continued

Probability of seasonal low discharges

\begin{tabular}{|c|c|c|c|c|c|c|c|c|c|}
\hline \multirow[b]{3}{*}{$\begin{array}{c}\text { Non- } \\
\text { exceedance } \\
\text { probability }\end{array}$} & \multirow[b]{3}{*}{$\begin{array}{c}\text { Recurrence } \\
\text { interval } \\
\text { (years) }\end{array}$} & \multicolumn{8}{|c|}{ Minimum average discharge $\left(\mathrm{ft}^{3} / \mathrm{s}\right)$} \\
\hline & & \multicolumn{8}{|c|}{ Number of consecutive days } \\
\hline & & 1 & 7 & 14 & 30 & 1 & 7 & 14 & 30 \\
\hline & & \multicolumn{4}{|c|}{ December-January-February } & \multicolumn{4}{|c|}{ March-April-May } \\
\hline 0.05 & 20 & 0 & 0 & 0 & 0 & 0 & 0 & 0 & 0 \\
\hline 0.10 & 10 & 0 & 0 & 0 & 0 & 0 & 0 & 0 & 0.308 \\
\hline 0.20 & 5 & 0 & 0 & 0 & 0 & 0 & 0 & 0 & 2.34 \\
\hline \multirow[t]{2}{*}{0.50} & 2 & 0.068 & 0.068 & 0.070 & 0.100 & 0.312 & 0.550 & 1.90 & 20.9 \\
\hline & & \multicolumn{4}{|c|}{ June-July-August } & \multicolumn{4}{|c|}{ September-October-November } \\
\hline 0.05 & 20 & 0 & 0 & 0 & 0 & 0 & 0 & 0 & 0.297 \\
\hline 0.10 & 10 & 0 & 0 & 0 & 0.073 & 0.032 & 0.058 & 0.190 & 0.610 \\
\hline 0.20 & 5 & 0.151 & 0.264 & 0.529 & 0.647 & 0.201 & 0.357 & 0.679 & 1.24 \\
\hline 0.50 & 2 & 0.951 & 1.39 & 1.75 & 4.21 & 0.966 & 1.64 & 2.26 & 3.87 \\
\hline
\end{tabular}


05061500 SOUTH BRANCH BUFFALO RIVER AT SABIN, MN-Continued

Annual peak discharge and corresponding gage height, period of record

$$
[--, \text { no data }]
$$

\begin{tabular}{|c|c|c|c|c|c|c|c|}
\hline $\begin{array}{l}\text { Water } \\
\text { year }\end{array}$ & Date & $\begin{array}{c}\text { Gage } \\
\text { height } \\
\text { (feet) }\end{array}$ & $\begin{array}{c}\text { Peak } \\
\text { discharge } \\
\left(\mathrm{ft}^{3} / \mathrm{s}\right)\end{array}$ & $\begin{array}{c}\text { Water } \\
\text { year }\end{array}$ & Date & $\begin{array}{c}\text { Gage } \\
\text { height } \\
\text { (feet) }\end{array}$ & $\begin{array}{c}\text { Peak } \\
\text { discharge } \\
\left(\mathrm{ft}^{3} / \mathrm{s}\right)\end{array}$ \\
\hline \multicolumn{8}{|c|}{ Annual peak discharge, by year, and corresponding gage height } \\
\hline 1945 & March 18 & 14.69 & 1,480 & 1970 & April 9 & 10.70 & 432 \\
\hline 1946 & March 18 & 14.34 & 955 & 1971 & September 6 & 9.32 & 326 \\
\hline 1947 & April 12 & 13.82 & 1,230 & 1972 & March 17 & 14.39 & 2,140 \\
\hline 1948 & April 4 & - & 730 & 1973 & March 16 & 7.33 & 132 \\
\hline 1949 & April 5 & 9.08 & 324 & 1974 & April 13 & 12.76 & 1,330 \\
\hline 1950 & April 1 & - & 1,460 & 1975 & July 2 & 19.90 & 8,500 \\
\hline 1951 & April 6 & 13.39 & 1,640 & 1976 & March 26 & 11.10 & 369 \\
\hline 1952 & April 8 & 15.37 & 3,400 & 1977 & June 3 & 9.05 & 234 \\
\hline 1953 & May 31 & 15.38 & 3,410 & 1978 & March 31 & 15.70 & 3,440 \\
\hline 1954 & March 24 & 10.90 & 390 & 1979 & April 14 & 15.33 & 2,800 \\
\hline 1955 & April 2 & 12.28 & 862 & 1980 & April 2 & 12.99 & 1,260 \\
\hline 1956 & April 5 & 15.18 & 2,410 & 1981 & May 23 & 11.33 & 500 \\
\hline 1957 & April 21 & 12.53 & 982 & 1982 & April 1 & 13.12 & 1,350 \\
\hline 1958 & July 7 & 14.60 & 1,520 & 1983 & July 5 & 14.03 & 1,500 \\
\hline 1959 & June 20 & 11.18 & 572 & 1984 & March 26 & 13.94 & 2,090 \\
\hline 1960 & April 27 & 12.34 & 1,020 & 1985 & June 2 & 13.69 & 1,660 \\
\hline 1961 & May 18 & 9.09 & 248 & 1986 & March 25 & 14.86 & 2,550 \\
\hline 1962 & June 9 & 17.04 & 6,340 & 1987 & March 26 & 11.34 & 689 \\
\hline 1963 & June 4 & 13.20 & 1,420 & 1988 & March 9 & 10.64 & 175 \\
\hline 1964 & April 17 & 11.72 & 796 & 1989 & April 4 & 16.30 & 3,800 \\
\hline 1965 & April 11 & 15.96 & 4,130 & 1990 & April 1 & 9.22 & 178 \\
\hline 1966 & March 15 & 16.78 & 3,310 & 1991 & May 4 & 10.30 & 285 \\
\hline 1967 & March 27 & 12.95 & 1,240 & 1992 & June 20 & 10.94 & 414 \\
\hline 1968 & April 10 & 8.25 & 230 & 1993 & March 30 & 14.57 & 1,910 \\
\hline 1969 & April 10 & 18.12 & 6,410 & 1994 & March 24 & 12.79 & 730 \\
\hline \multicolumn{8}{|c|}{ Annual peak discharge, from highest to lowest, and corresponding gage height } \\
\hline 1975 & July 2 & 19.90 & 8,500 & 1983 & July 5 & 14.03 & 1,500 \\
\hline 1969 & April 10 & 18.12 & $-6,410$ & 1945 & March 18 & 14.69 & 1,480 \\
\hline 1962 & June 9 & 17.04 & 6,340 & 1950 & April 1 & - & 1.460 \\
\hline 1965 & April 11 & 15.96 & 4,130 & 1963 & June 4 & 13.20 & 1,420 \\
\hline 1989 & April 4 & 16.30 & 3,800 & 1982 & April 1 & 13.12 & 1,350 \\
\hline 1978 & March 31 & 15.70 & 3,440 & 1974 & April 13 & 12.76 & 1,330 \\
\hline 1953 & May 31 & 15.38 & 3,410 & 1980 & April 2 & 12.99 & 1,260 \\
\hline 1952 & April 8 & 15.37 & 3,400 & 1967 & March 27 & 12.95 & 1,240 \\
\hline 1966 & March 15 & 16.78 & 3,310 & 1947 & April 12 & 13.82 & 1,230 \\
\hline 1979 & April 14 & 15.33 & 2,800 & 1960 & April 27 & 12.34 & 1,020 \\
\hline 1986 & March 25 & 14.86 & 2,550 & 1957 & April 21 & 12.53 & 982 \\
\hline 1956 & April 5 & 15.18 & 2,410 & 1946 & March 18 & 14.34 & 955 \\
\hline 1972 & March 17 & 14.39 & 2,140 & 1955 & April 2 & 12.28 & 862 \\
\hline 1984 & March 26 & 13.94 & 2,090 & 1964 & April 17 & 11.72 & 796 \\
\hline 1993 & March 30 & 14.57 & 1,910 & 1948 & April 4 & - & 730 \\
\hline 1985 & June 2 & 13.69 & 1,660 & 1994 & March 24 & 12.79 & 730 \\
\hline 1951 & April 6 & 13.39 & 1,640 & 1987 & March 26 & 11.34 & 689 \\
\hline 1958 & July 7 & 14.60 & 1,520 & 1959 & June 20 & 11.18 & 572 \\
\hline
\end{tabular}


05061500 SOUTH BRANCH BUFFALO RIVER AT SABIN, MN-Continued

Annual peak discharge and corresponding gage height, period of record--Continued $[-$, no data $]$

\begin{tabular}{llclllrl}
\hline $\begin{array}{l}\text { Water } \\
\text { year }\end{array}$ & \multicolumn{1}{c}{ Date } & $\begin{array}{c}\text { Gage } \\
\text { height } \\
(\mathrm{feet})\end{array}$ & $\begin{array}{c}\text { Peak } \\
\text { discharge } \\
\left(\mathrm{ft}^{3} / \mathbf{s}\right)\end{array}$ & $\begin{array}{c}\text { Water } \\
\text { year }\end{array}$ & Date & $\begin{array}{c}\text { Gage } \\
\text { height } \\
\text { (feet) }\end{array}$ & $\begin{array}{c}\text { Peak } \\
\text { discharge } \\
\left(\mathbf{f t}^{3} / \mathbf{s}\right)\end{array}$ \\
\hline \multicolumn{7}{c}{ Annual peak discharge, from highest to lowest, and corresponding gage height--Continued } \\
1981 & May 23 & 11.33 & 500 & 1991 & May 4 & 10.30 & 285 \\
1970 & April 9 & 10.70 & 432 & 1961 & May 18 & 9.09 & 248 \\
1992 & June 20 & 10.94 & 414 & 1977 & June 3 & 9.05 & 234 \\
1954 & March 24 & 10.90 & 390 & 1968 & April 10 & 8.25 & 230 \\
1976 & March 26 & 11.10 & 369 & 1990 & April 1 & 9.22 & 178 \\
1971 & September 6 & 9.32 & 326 & 1988 & March 9 & 10.64 & 175 \\
1949 & April 5 & 9.08 & 324 & 1973 & March 16 & 7.33 & 132 \\
\hline
\end{tabular}




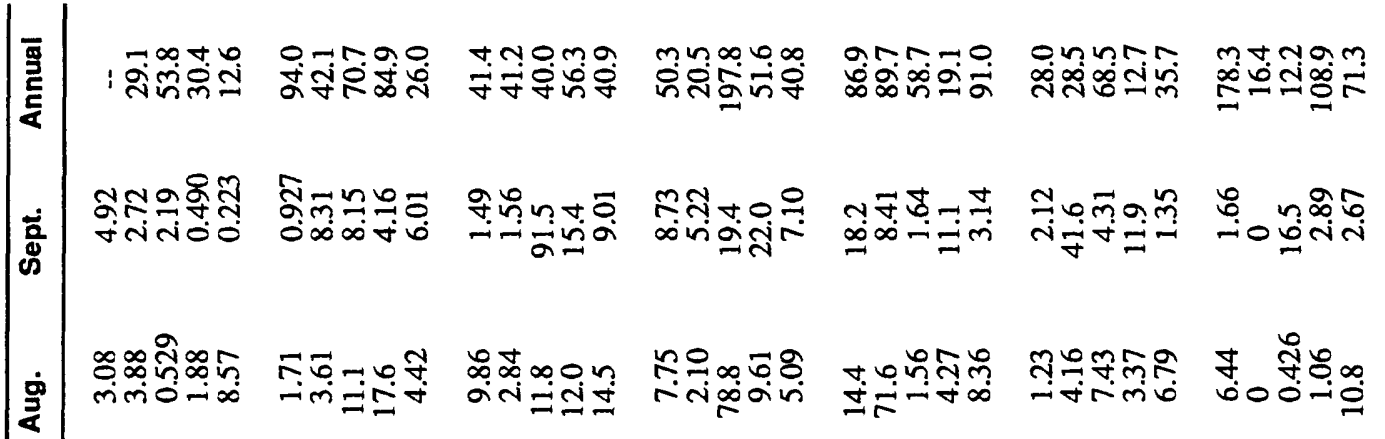

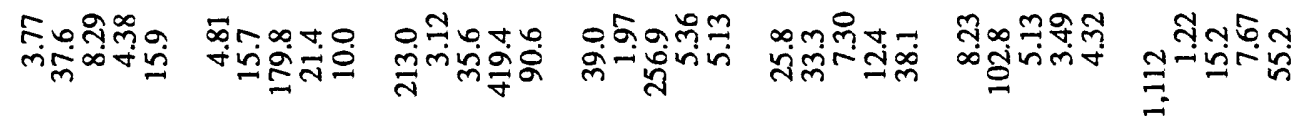

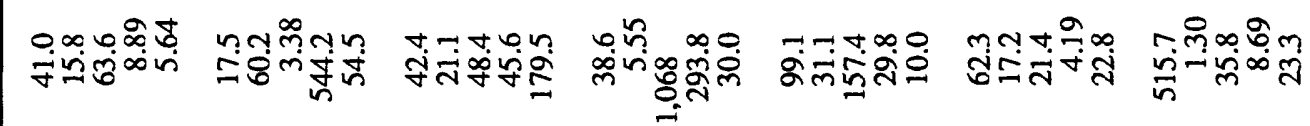

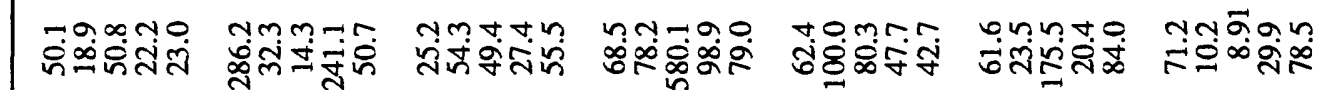

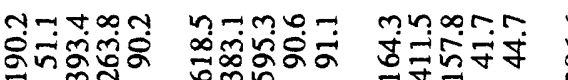

bry-a-7 $0-\infty \rightarrow \infty$

ringa-

$0 m a 00$

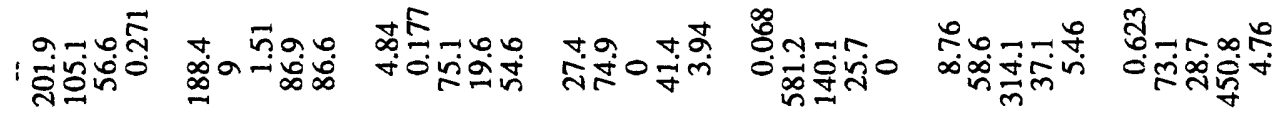

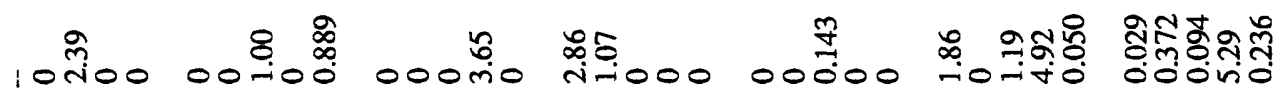

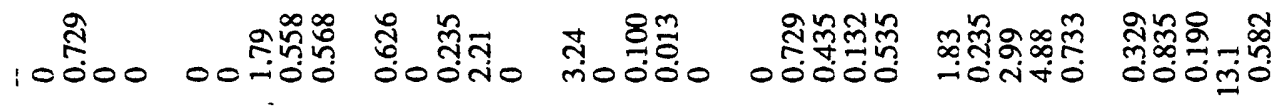

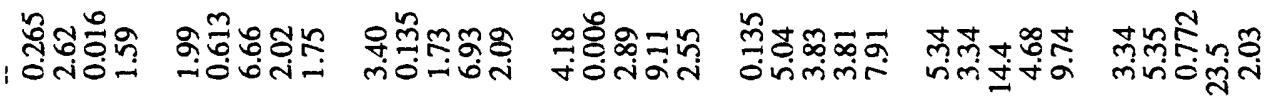

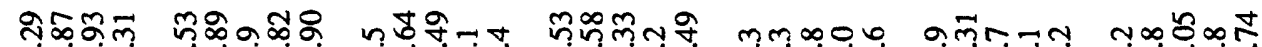
inain

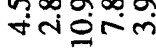
อलiviğ

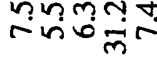

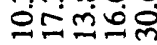
ำำต

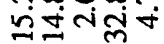

ॠ 


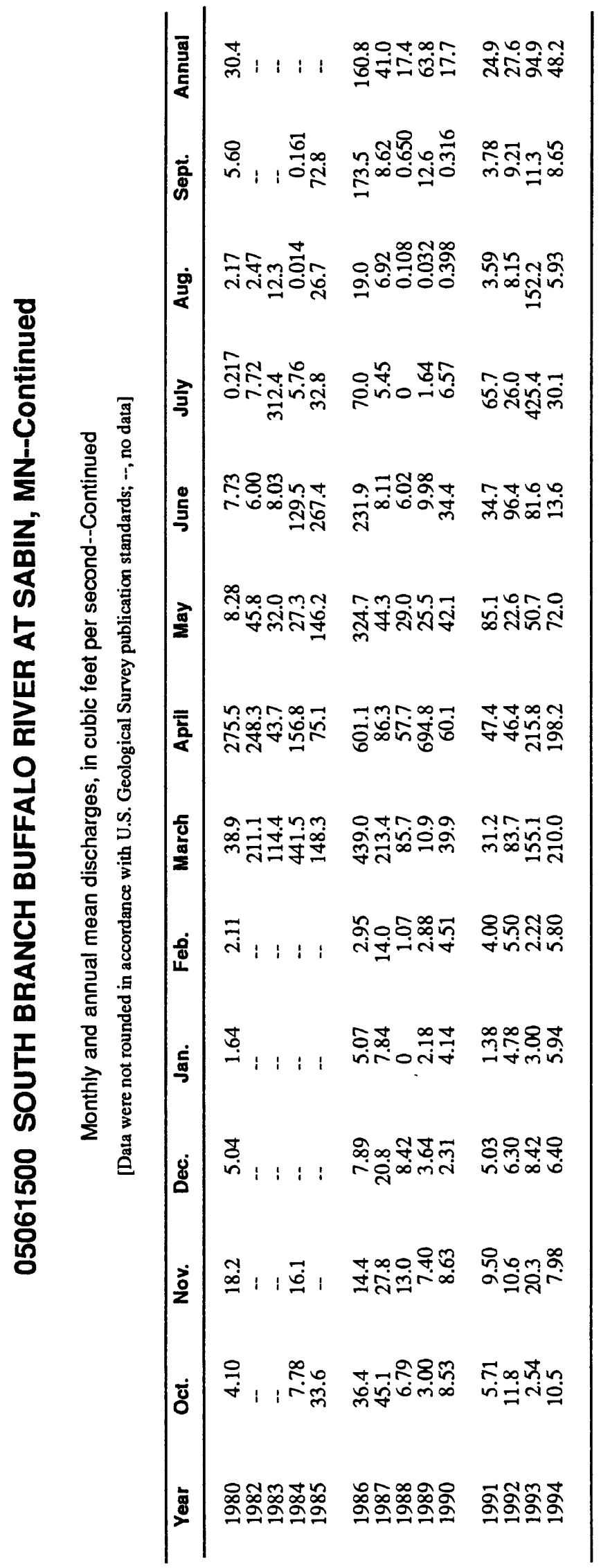




\section{BUFFALO RIVER NEAR DILWORTH, MN}

\section{Station Description}

LOCATION.--Lat $46^{\circ} 57^{\prime} 40^{\prime \prime}$, long 96 $39^{\prime} 40^{\prime \prime}$, in SW $1 / 4 \mathrm{SE}^{1} / 4$ sec.6, T.140 N., R.47 W., Clay County, Hydrologic Unit 09020106, on left bank $4.5 \mathrm{mi}$ southeast of Kragnes, $6.5 \mathrm{mi}$ northeast of Dilworth, and $9 \mathrm{mi}$ downstream from South Branch.

DRAINAGE AREA.--1,040 $\mathrm{mi}^{2}$, approximately.

PERIOD OF RECORD.--March 1931 to current year. Monthly discharge only for some periods, published in WSP 1308.

GAGE.--Water-stage recorder. Datum of gage is $878.31 \mathrm{ft}$ above sea level (levels by U.S. Army Corps of Engineers). Prior to Apr. 5, 1937, nonrecording gage at same site and datum.

EXTREMES FOR PERIOD OF RECORD.--Maximum discharge, 13,600 $\mathrm{ft}^{3} / \mathrm{s}$, July 2, 1975 (gage height, $27.1 \mathrm{ft}$ ); no flow at times in 1936.

Annual mean discharge

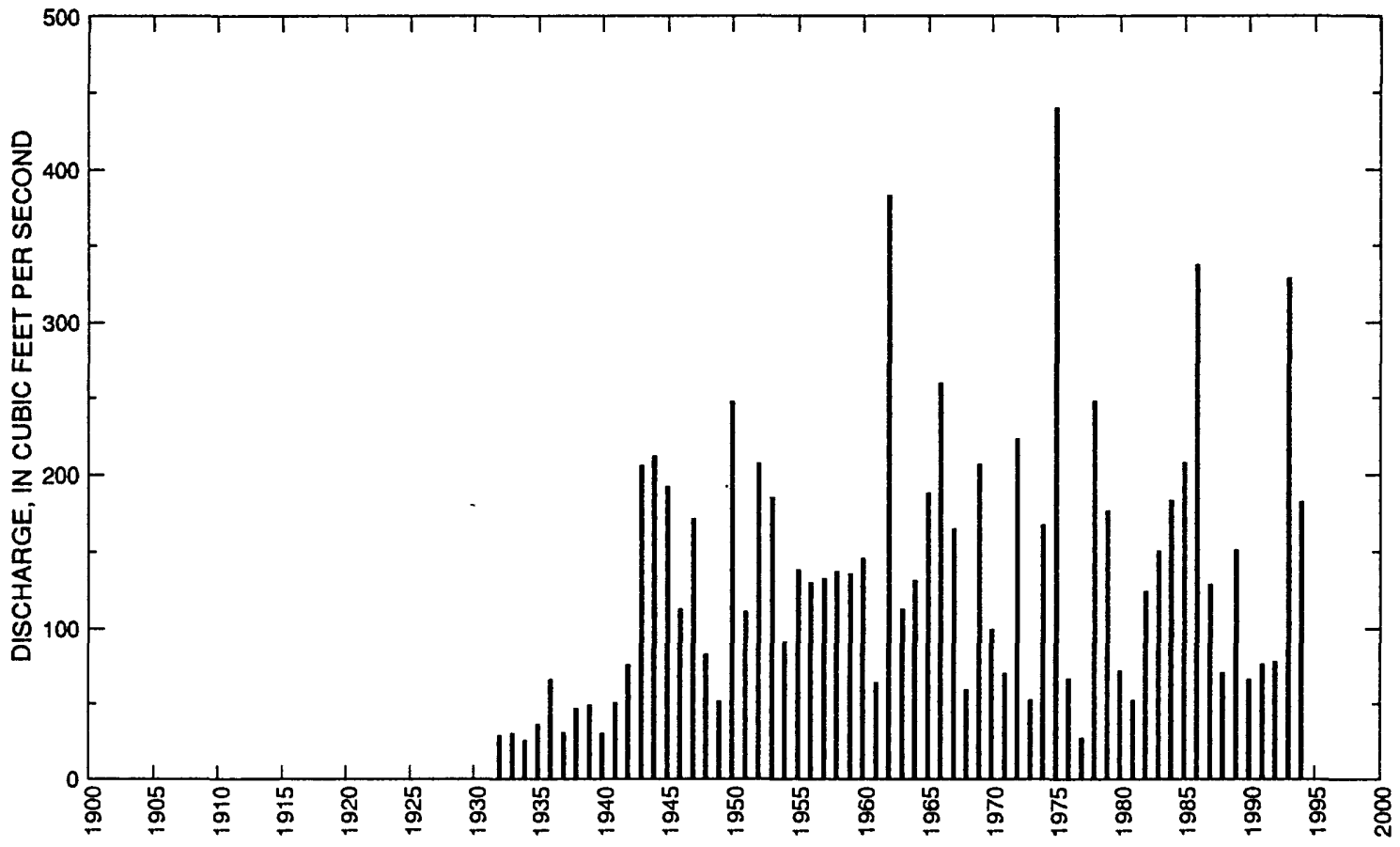




\section{BUFFALO RIVER NEAR DILWORTH, MN--Continued}

Statistics of monthly and annual mean discharges

[m, more than 1 year of occurrence]

\begin{tabular}{|c|c|c|c|c|c|c|c|c|}
\hline \multirow[b]{2}{*}{ Month } & \multicolumn{2}{|c|}{ Maximum } & \multicolumn{2}{|c|}{ Minimum } & \multicolumn{4}{|c|}{ Mean } \\
\hline & $\begin{array}{c}\text { Discharge } \\
\left(\mathrm{ft}^{3} / \mathrm{s}\right)\end{array}$ & $\begin{array}{c}\text { Water year } \\
\text { of } \\
\text { occurrence }\end{array}$ & $\begin{array}{c}\text { Discharge } \\
\left(\mathrm{ft}^{3} / \mathrm{s}\right)\end{array}$ & $\begin{array}{c}\text { Water year } \\
\text { of } \\
\text { occurrence }\end{array}$ & $\begin{array}{c}\text { Discharge } \\
\left(\mathrm{ft}^{3} / \mathrm{s}\right)\end{array}$ & $\begin{array}{c}\text { Standard } \\
\text { deviation } \\
\left(\mathrm{ft}^{3} / \mathrm{s}\right)\end{array}$ & $\begin{array}{l}\text { Coeffi- } \\
\text { clent of } \\
\text { variation }\end{array}$ & $\begin{array}{l}\text { Percentage } \\
\text { of annual } \\
\text { discharge }\end{array}$ \\
\hline October & 186 & 1958 & 5.48 & 1940 & 50.5 & 46.4 & 0.92 & 3.15 \\
\hline November & 305 & 1972 & 8.74 & 1937 & 48.0 & 44.2 & 0.92 & 3.00 \\
\hline December & 97.0 & 1972 & 4.75 & 1938 & 29.2 & 19.4 & 0.66 & 1.82 \\
\hline January & 53.5 & $\mathrm{~m}$ & 0.868 & 1940 & 19.3 & 12.1 & 0.63 & 1.21 \\
\hline February & 61.1 & 1984 & 0.759 & 1940 & 19.4 & 12.7 & 0.65 & 1.21 \\
\hline March & 1,310 & 1966 & 2.26 & 1940 & 176 & 239 & 1.36 & 11.0 \\
\hline April & 1,980 & 1978 & 33.5 & 1931 & 535 & 478 & 0.89 & 33.4 \\
\hline May & 909 & 1986 & 27.2 & 1931 & 216 & 194 & 0.90 & 13.5 \\
\hline June & 2,140 & 1962 & 15.1 & 1934 & 197 & 310 & 1.58 & 12.3 \\
\hline July & 2,810 & 1975 & 2.23 & 1936 & 185 & 412 & 2.23 & 11.5 \\
\hline August & 910 & 1993 & 0 & 1936 & 72.9 & 151 & 2.07 & 4.55 \\
\hline September & 518 & 1944 & 0.790 & 1936 & 54.1 & 81.6 & 1.51 & 3.38 \\
\hline Annual & 440 & 1975 & 25.6 & 1934 & 135 & 90.1 & 0.67 & 100 \\
\hline
\end{tabular}

Annual flow duration

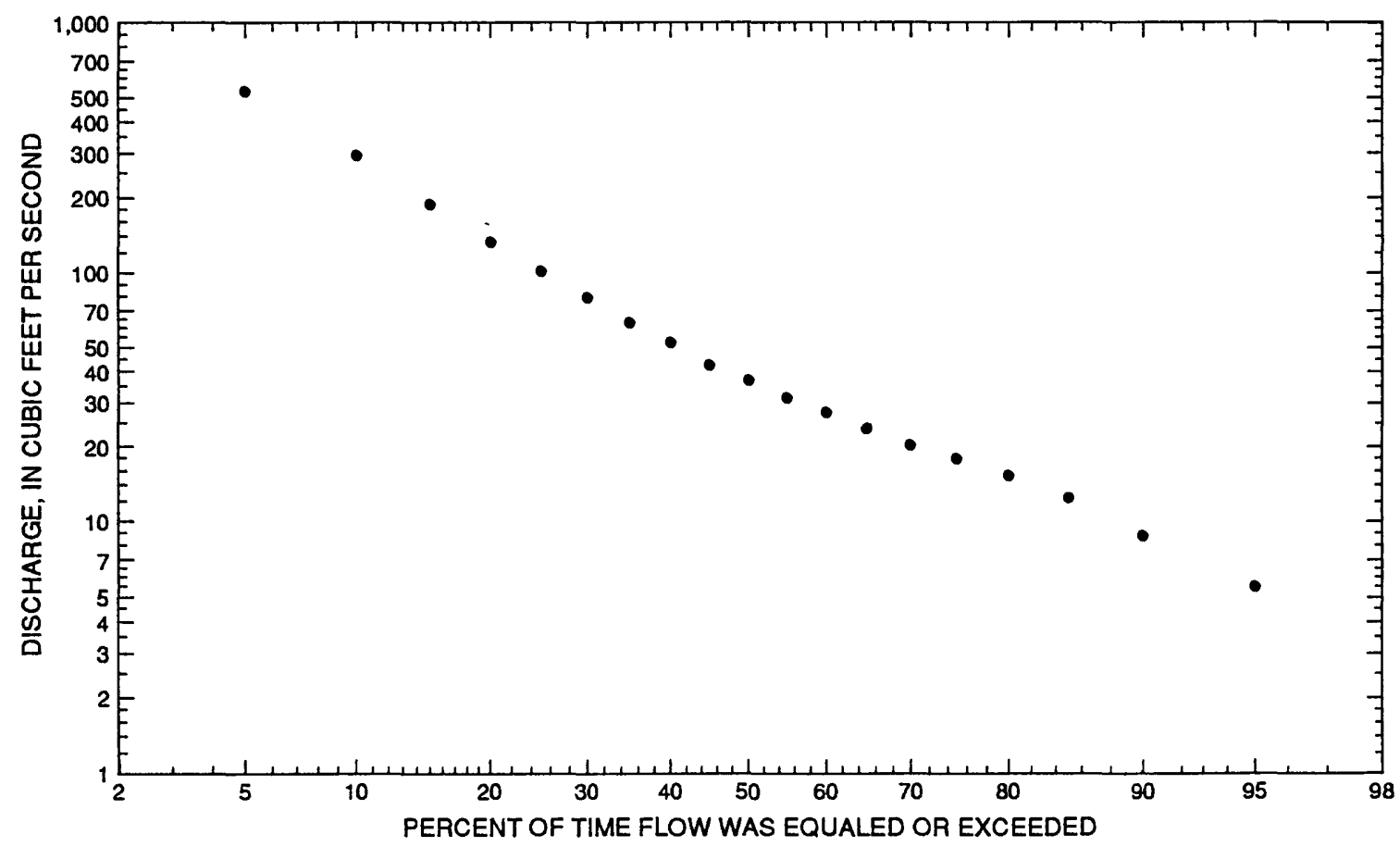




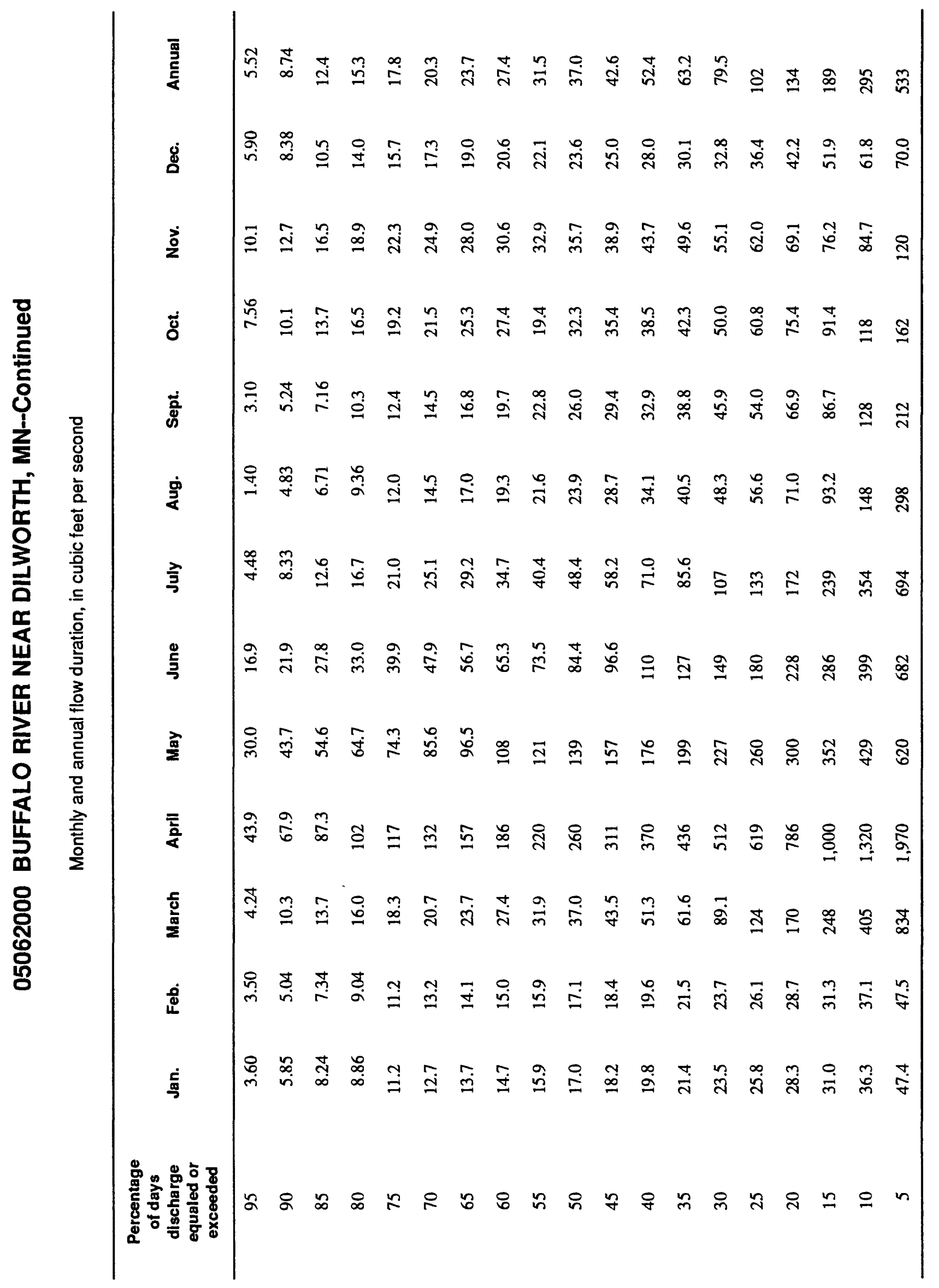




\section{BUFFALO RIVER NEAR DILWORTH, MN--Continued}

Probability of annual high discharges

[ng, statistic not given]

\begin{tabular}{|c|c|c|c|c|c|c|}
\hline \multirow[b]{2}{*}{$\begin{array}{l}\text { Exceedance } \\
\text { probability }\end{array}$} & \multirow[b]{2}{*}{$\begin{array}{l}\text { Recurrence } \\
\text { interval } \\
\text { (years) }\end{array}$} & \multirow[b]{2}{*}{$\begin{array}{c}\text { Maximum } \\
\text { instantaneous } \\
\left(\mathrm{ft}^{3} / \mathrm{s}\right)\end{array}$} & \multicolumn{4}{|c|}{$\begin{array}{l}\text { Maximum average discharge } \\
\qquad\left(\mathrm{ft}^{3} / \mathrm{s}\right)\end{array}$} \\
\hline & & & 3-day period & 7-day period & 15-day period & 30-day period \\
\hline 0.99 & 1.01 & $\mathrm{ng}$ & 135 & 114 & 90.2 & 69.2 \\
\hline 0.95 & 1.05 & 258 & 260 & 225 & 176 & 134 \\
\hline 0.90 & 1.11 & 375 & 368 & 322 & 250 & 189 \\
\hline 0.80 & 1.25 & 586 & 563 & 493 & 379 & 283 \\
\hline 0.50 & 2 & 1,360 & 1,270 & 1,100 & 825 & 595 \\
\hline 0.20 & 5 & 3,120 & 2,870 & 2,390 & 1,750 & 1,210 \\
\hline 0.10 & 10 & 4,780 & 4,400 & 3,560 & 2,570 & 1,720 \\
\hline 0.04 & 25 & 7,480 & 6,960 & 5,420 & 3,840 & 2,490 \\
\hline 0.02 & 50 & 9,970 & 9,350 & 7,080 & 4,960 & 3,130 \\
\hline 0.01 & 100 & 12,900 & 12,200 & 8,980 & 6,220 & 3,850 \\
\hline 0.005 & 200 & 16,300 & 15,600 & 11,100 & 7,640 & 4,620 \\
\hline 0.002 & 500 & 21,600 & $\mathrm{ng}$ & $\mathrm{ng}$ & $\mathrm{ng}$ & $\mathrm{ng}$ \\
\hline
\end{tabular}

Probability of annual low discharges

\begin{tabular}{|c|c|c|c|c|c|c|c|c|c|c|}
\hline \multirow[b]{3}{*}{$\begin{array}{l}\text { Non- } \\
\text { exceed- } \\
\text { ance } \\
\text { prob- } \\
\text { ability }\end{array}$} & \multirow[b]{3}{*}{$\begin{array}{c}\text { Recur- } \\
\text { rence } \\
\text { Inter- } \\
\text { val } \\
\text { (years) }\end{array}$} & \multicolumn{9}{|c|}{ Minimum average discharge $\left(\mathrm{ft}^{3} / \mathrm{s}\right)$} \\
\hline & & \multicolumn{9}{|c|}{ Number of consecutlve days } \\
\hline & & 1 & 3 & 7 & 14 & 30 & 60 & 90 & 120 & 183 \\
\hline 0.05 & 20 & 0.401 & 0.478 & 0.547 & 0.679 & 0.990 & 1.25 & 277 & 4.76 & 6.70 \\
\hline 0.10 & 10 & 1.07 & 1.23 & 1.38 & 1.68 & 2.23 & 2.84 & 4.54 & 6.84 & 9.16 \\
\hline 0.20 & 5 & 2.70 & 3.00 & 3.32 & 3.91 & 4.77 & 6.29 & 7.70 & 10.3 & 13.2 \\
\hline 0.50 & 2 & 9.38 & 9.93 & 10.6 & 11.8 & 13.1 & 16.7 & 17.1 & 20.2 & 25.5 \\
\hline
\end{tabular}




\section{BUFFALO RIVER NEAR DILWORTH, MN--Continued}

Probability of seasonal low discharges

\begin{tabular}{|c|c|c|c|c|c|c|c|c|c|}
\hline \multirow[b]{3}{*}{$\begin{array}{c}\text { Non- } \\
\text { exceedance } \\
\text { probability }\end{array}$} & \multirow[b]{3}{*}{$\begin{array}{c}\text { Recurrence } \\
\text { interval } \\
\text { (years) }\end{array}$} & \multicolumn{8}{|c|}{ Minimum average discharge $\left(\mathrm{ft}^{3} / \mathrm{s}\right)$} \\
\hline & & \multicolumn{8}{|c|}{ Number of consecutive days } \\
\hline & & 1 & 7 & 14 & 30 & 1 & 7 & 14 & 30 \\
\hline & & \multicolumn{4}{|c|}{ December-January-February } & \multicolumn{4}{|c|}{ March-April-May } \\
\hline 0.05 & 20 & 1.64 & 1.70 & 1.78 & 2.07 & 3.38 & 3.69 & 4.03 & 9.30 \\
\hline 0.10 & 10 & 3.38 & 3.52 & 3.70 & 4.06 & 5.34 & 5.77 & 6.51 & 14.9 \\
\hline 0.20 & 5 & 6.87 & 7.16 & 7.50 & 7.84 & 8.74 & 9.45 & 11.0 & 25.4 \\
\hline \multirow[t]{2}{*}{0.50} & 2 & 16.5 & 17.1 & 17.6 & 17.9 & 18.8 & 21.0 & 25.4 & 63.3 \\
\hline & & \multicolumn{4}{|c|}{ June-July-August } & \multicolumn{4}{|c|}{ September-October-November } \\
\hline 0.05 & 20 & 0.702 & 1.08 & 1.55 & 2.37 & 1.42 & 2.29 & ${ }^{1} 2.84$ & 3.40 \\
\hline 0.10 & 10 & 1.71 & 2.38 & 3.07 & 4.34 & 2.68 & 3.78 & ${ }^{1} 4.65$ & 5.53 \\
\hline 0.20 & 5 & 4.12 & 5.23 & 6.23 & 8.24 & 5.10 & 6.46 & 7.13 & 9.50 \\
\hline 0.50 & 2 & 14.8 & 17.0 & 19.0 & 23.8 & 13.5 & 15.9 & 20.3 & 23.4 \\
\hline
\end{tabular}

${ }^{1}$ Graphical interpretation. 


\section{BUFFALO RIVER NEAR DILWORTH, MN--Continued}

Annual peak discharge and corresponding gage height, period of record $[-$, no data $]$

\begin{tabular}{|c|c|c|c|c|c|c|c|}
\hline $\begin{array}{l}\text { Water } \\
\text { year }\end{array}$ & Date & $\begin{array}{c}\text { Gage } \\
\text { height } \\
\text { (foet) }\end{array}$ & $\begin{array}{c}\text { Peak } \\
\text { discharge } \\
\left(\mathrm{f}^{3} / \mathbf{s}\right)\end{array}$ & $\begin{array}{l}\text { Water } \\
\text { year }\end{array}$ & Date & $\begin{array}{c}\text { Gage } \\
\text { height } \\
\text { (feet) }\end{array}$ & $\begin{array}{c}\text { Peak } \\
\text { discharge } \\
\left(\mathrm{f}^{3} / \mathrm{s}\right)\end{array}$ \\
\hline \multicolumn{8}{|c|}{ Annual peak discharge, by year, and corresponding gage height } \\
\hline 1931 & April 10 & - & 46.0 & 1963 & June 7 & 14.76 & 1,300 \\
\hline 1932 & April 12 & 8.83 & 311 & 1964 & April 22 & 16.40 & 1,740 \\
\hline 1933 & April 3 & 9.02 & 269 & 1965 & April 11 & 23.37 & 5,960 \\
\hline 1934 & April 10 & 9.02 & 374 & 1966 & March 18 & 23.31 & 5,000 \\
\hline 1935 & March 21 & 9.09 & 311 & 1967 & March 30 & 16.68 & 1,820 \\
\hline 1936 & April 16 & 14.59 & 1,460 & 1968 & March 30 & 8.92 & 406 \\
\hline 1937 & April 14 & 9.00 & 390 & 1969 & April 11 & 25.55 & 10,400 \\
\hline 1938 & May 5 & 8.78 & 550 & 1970 & April 12 & 12.85 & 802 \\
\hline 1939 & March 29 & 15.77 & 1,350 & 1971 & April 10 & 9.81 & 493 \\
\hline 1940 & April 7 & 10.29 & 510 & 1972 & March 19 & 19.36 & 2,590 \\
\hline 1941 & April 4 & 12.71 & 800 & 1973 & March 18 & - & 205 \\
\hline 1942 & May 6 & 10.94 & 762 & 1974 & April 14 & 17.97 & 2,130 \\
\hline 1943 & April 2 & 22.60 & 4,530 & 1975 & July 2 & 27.10 & 13,600 \\
\hline 1944 & August 20 & 12.81 & 998 & 1976 & April 1 & 13.50 & 1,000 \\
\hline 1945 & March 20 & 19.38 & 2,660 & 1977 & June 5 & 5.99 & 163 \\
\hline 1946 & March 22 & 17.05 & 1,670 & 1978 & March 31 & 22.76 & 5,420 \\
\hline 1947 & April 13 & 20.26 & 3,380 & 1979 & April 16 & 21.60 & 4,380 \\
\hline 1948 & April 8 & 14.44 & 1,310 & 1980 & April 4 & 16.78 & 1,850 \\
\hline 1949 & April 8 & 11.17 & 602 & 1981 & May 23 & 13.02 & 1,090 \\
\hline 1950 & April 7 & - & 2,600 & 1982 & April 2 & 17.80 & 2,210 \\
\hline 1951 & April 7 & 17.47 & 2,230 & 1983 & July 9 & 16.75 & 1,990 \\
\hline 1952 & April 10 & 21.24 & 4,310 & 1984 & March 29 & 19.27 & 2,940 \\
\hline 1953 & June 20 & 18.43 & 2,430 & 1985 & June 5 & 15.53 & 1,940 \\
\hline 1954 & April 12 & 11.54 & 686 & 1986 & March 28 & 20.50 & 3,440 \\
\hline 1955 & April 4 & 14.85 & 1,260 & 1987 & March 13 & 13.19 & 954 \\
\hline 1956 & April 12 & 18.37 & 2,410 & 1988 & April 4 & 10.55 & 648 \\
\hline 1957 & April 24 & 13.70 & 1,080 & 1989 & April 6 & 22.80 & 5,380 \\
\hline 1958 & July 11 & 15.70 & 1,540 & 1990 & April 3 & 12.40 & 600 \\
\hline 1959 & June 23 & 11.81 & 699 & 1991 & May 6 & 10.66 & 576 \\
\hline 1960 & April 30 & 15.12 & 1,390 & 1992 & June 22 & - & 492 \\
\hline 1961 & May 19 & 9.90 & 480 & 1993 & July 20 & 22.88 & 3,450 \\
\hline 1962 & June 11 & 23.56 & 6,140 & 1994 & March 24 & 20.82 & 3,270 \\
\hline \multicolumn{8}{|c|}{ Annual peak discharge, from highest to lowest, and corresponding gage height } \\
\hline 1975 & July 2 & 27.10 & 13,600 & 1986 & March 28 & 20.50 & 3,440 \\
\hline 1969 & April 11 & 25.55 & 10,400 & 1947 & April 13 & 20.26 & 3,380 \\
\hline 1962 & June 11 & 23.56 & 6,140 & 1994 & March 24 & 20.82 & 3,270 \\
\hline 1965 & April 11 & 23.37 & 5,960 & 1984 & March 29 & 19.27 & 2,940 \\
\hline 1978 & March 31 & 22.76 & 5,420 & 1945 & March 20 & 19.38 & 2,660 \\
\hline 1989 & April 6 & 22.80 & 5,380 & 1950 & April 7 & - & 2,600 \\
\hline 1966 & March 18 & 23.31 & 5,000 & 1972 & March 19 & 19.36 & 2,590 \\
\hline 1943 & April 2 & 22.60 & 4,530 & 1953 & June 20 & 18.43 & 2,430 \\
\hline 1979 & April 16 & 21.60 & 4,380 & 1956 & April 12 & 18.37 & 2,410 \\
\hline 1952 & April 10 & 21.24 & 4,310 & 1951 & April 7 & 17.47 & 2,230 \\
\hline 1993 & July 20 & 22.88 & 3,450 & 1982 & April 2 & 17.80 & 2,210 \\
\hline
\end{tabular}


05062000 BUFFALO RIVER NEAR DILWORTH, MN--Continued

Annual peak discharge and corresponding gage height, period of record--Continued

$$
[--, \text { no data }]
$$

\begin{tabular}{|c|c|c|c|c|c|c|c|}
\hline $\begin{array}{l}\text { Water } \\
\text { year }\end{array}$ & Date & $\begin{array}{c}\text { Gage } \\
\text { height } \\
\text { (feet) }\end{array}$ & $\begin{array}{c}\text { Peak } \\
\text { discharge } \\
\left(\mathrm{ft}^{3} / \mathrm{s}\right)\end{array}$ & $\begin{array}{l}\text { Water } \\
\text { year }\end{array}$ & Date & $\begin{array}{c}\text { Gage } \\
\text { height } \\
\text { (feet) }\end{array}$ & $\begin{array}{c}\text { Peak } \\
\text { discharge } \\
\left(\mathrm{ft}^{3} / \mathrm{s}\right)\end{array}$ \\
\hline \multicolumn{8}{|c|}{ Annual peak discharge, from highest to lowest, and corresponding gage height-.-Continued } \\
\hline 1974 & April 14 & 17.97 & 2,130 & 1942 & May 6 & 10.94 & 762 \\
\hline 1983 & July 9 & 16.75 & 1,990 & 1959 & June 23 & 11.81 & 699 \\
\hline 1985 & June 5 & 15.53 & 1,940 & 1954 & April 12 & 11.54 & 686 \\
\hline 1980 & April 4 & 16.78 & 1,850 & 1988 & April 4 & 10.55 & 648 \\
\hline 1967 & March 30 & 16.68 & 1,820 & 1949 & April 8 & 11.17 & 602 \\
\hline 1964 & April 22 & 16.40 & 1,740 & 1990 & April 3 & 12.40 & 600 \\
\hline 1946 & $\operatorname{March} 22$ & 17.05 & 1,670 & 1991 & May 6 & 10.66 & 576 \\
\hline 1958 & July 11 & 15.70 & 1,540 & 1938 & May 5 & 8.78 & 550 \\
\hline 1936 & April 16 & 14.59 & 1,460 & 1940 & April 7 & 10.29 & 510 \\
\hline 1960 & April 30 & 15.12 & 1,390 & 1971 & April 10 & 9.81 & 493 \\
\hline 1939 & March 29 & 15.77 & 1,350 & 1992 & June 22 & -- & 492 \\
\hline 1948 & April 8 & 14.44 & 1,310 & 1961 & May 19 & 9.90 & 480 \\
\hline 1963 & June 7 & 14.76 & 1,300 & 1968 & March 30 & 8.92 & 406 \\
\hline 1955 & April 4 & 14.85 & 1,260 & 1937 & April 14 & 9.00 & 390 \\
\hline 1981 & May 23 & 13.02 & 1,090 & 1934 & April 10 & 9.02 & 374 \\
\hline 1957 & April 24 & 13.70 & 1,080 & 1932 & April 12 & 8.83 & 311 \\
\hline 1976 & April 1 & 13.50 & 1,000 & 1935 & March 21 & 9.09 & 311 \\
\hline 1944 & August 20 & 12.81 & 998 & 1933 & April 3 & 9.02 & 269 \\
\hline 1987 & March 13 & 13.19 & 954 & 1973 & March 18 & -- & 205 \\
\hline 1970 & April 12 & 12.85 & 802 & 1977 & June 5 & 5.99 & 163 \\
\hline 1941 & April 4 & 12.71 & 800 & 1931 & April 10 & - & 46.0 \\
\hline
\end{tabular}




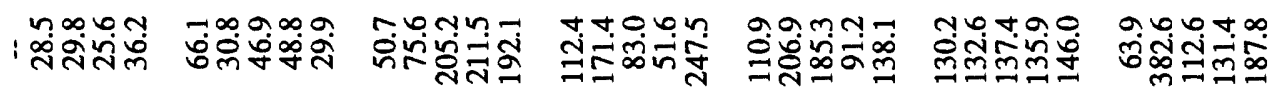

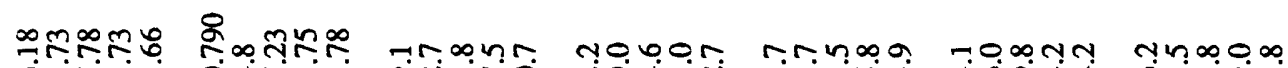

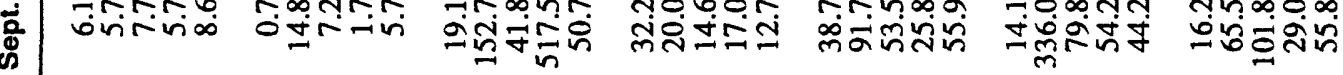

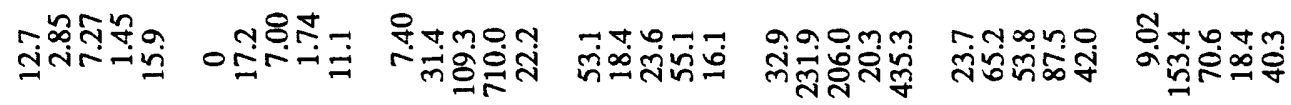

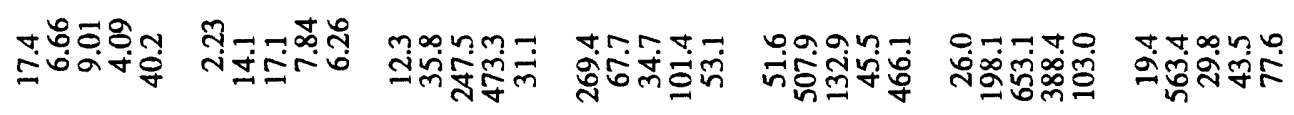

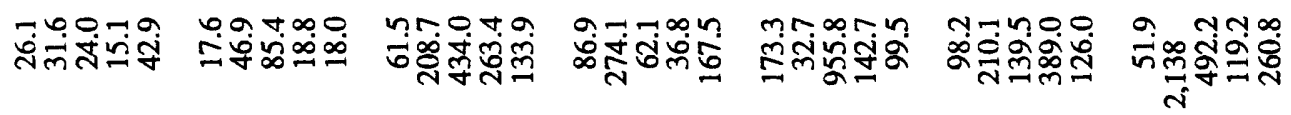

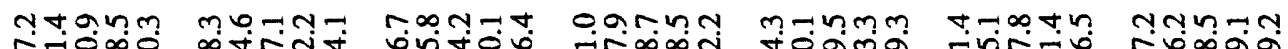

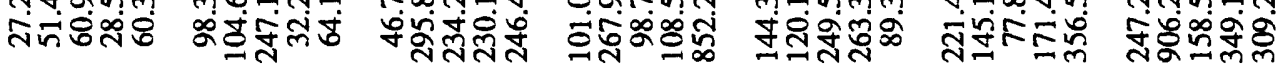

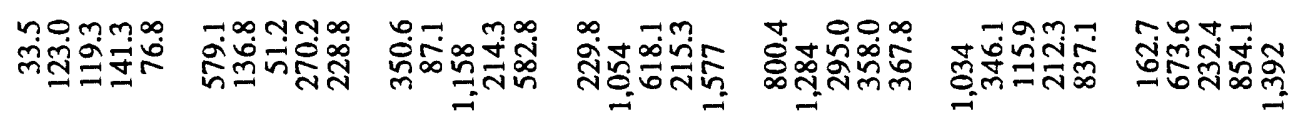

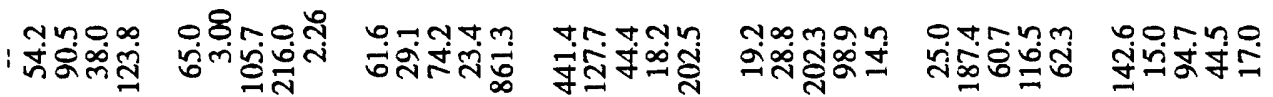

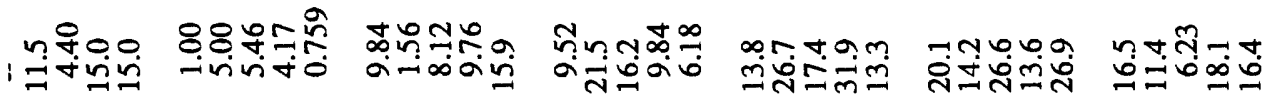

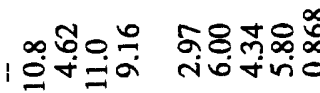

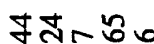

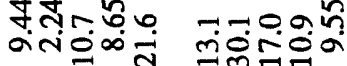

ตุกี่ำ

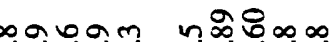

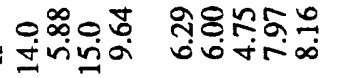

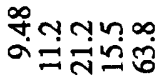

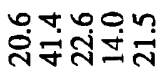

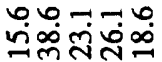

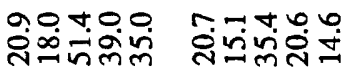

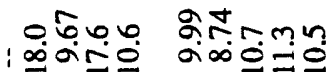

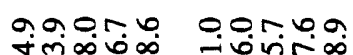

$2 n=0$

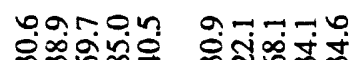

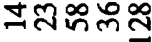

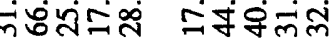
mino

लेखिएल

renoro arn-0 th-nom

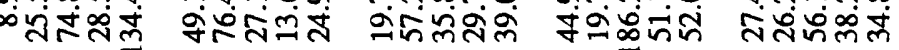




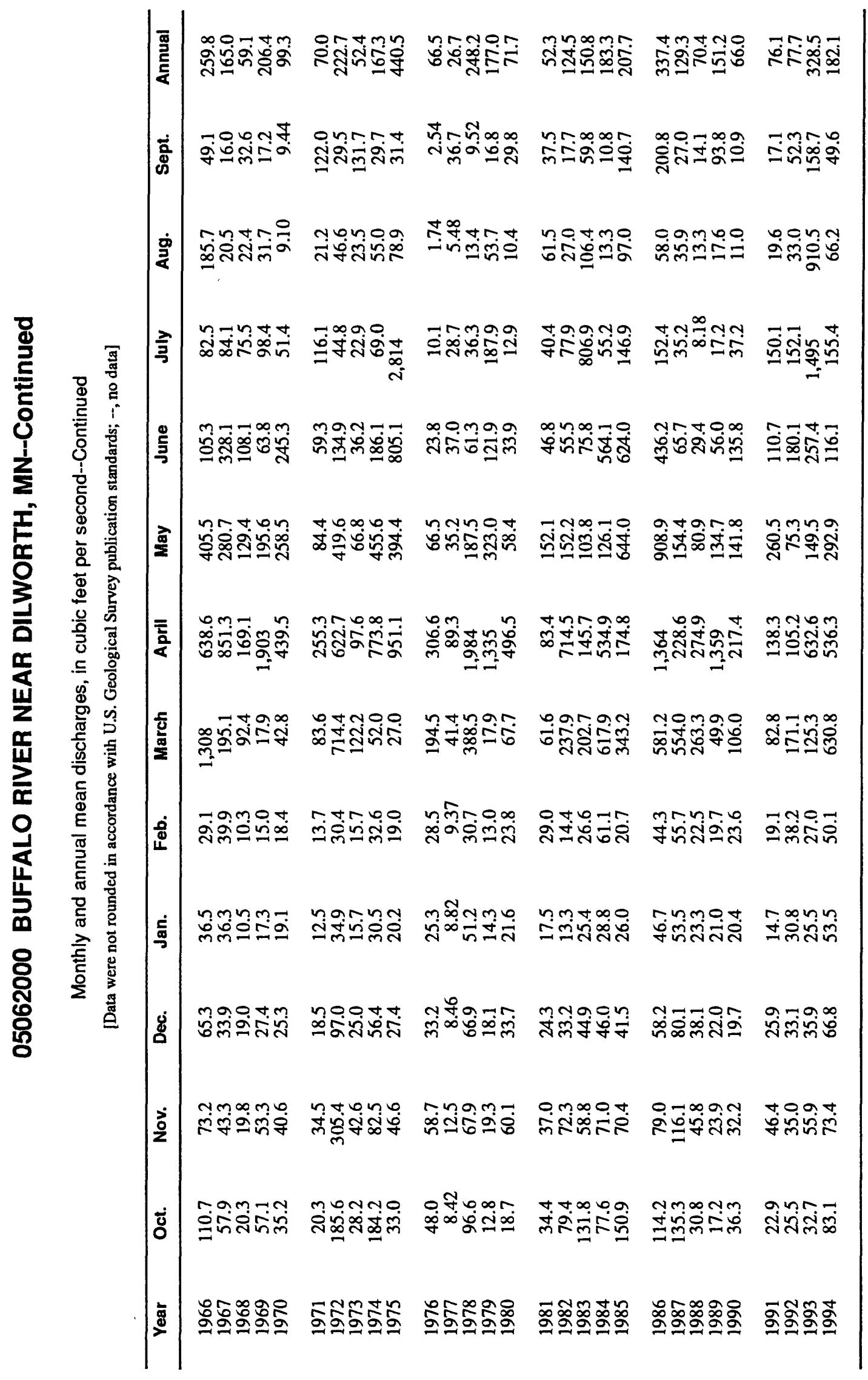




\title{
05062200 ELM RIVER NEAR KELSO, ND
}

\author{
Station Description
}

LOCATION.--Lat $47^{\circ} 17^{\prime} 30^{\prime \prime}$, long 97 06'50', in sec.23, T.144 N., R.51 W., Traill County, Hydrologic Unit 09020107 , on left bank $50 \mathrm{ft}$ upstream from county road, $4.0 \mathrm{mi}$ south and $3.4 \mathrm{mi}$ west of Kelso.

DRAINAGE AREA.--199 $\mathrm{mi}^{2}$.

PERIOD OF RECORD.--December 1955 to September 1963, 1964-73 (annual maximum only), October 1980 to August 1986 (seasonal records only since 1982).

GAGE.--Water-stage recorder. Elevation of gage is $893 \mathrm{ft}$ above National Geodetic Vertical Datum of 1929, from topographic map. Prior to September 1973, gage located at site 1 mile upstream at datum of $887.60 \mathrm{ft}$ above National Geodetic Vertical Datum of 1929, Emerson-Crookston supplementary adjustment of 1941 .

EXTREMES FOR PERIOD OF RECORD.--Maximum discharge, 1,000 $\mathrm{ft}^{3} / \mathrm{s}$, occurred during Mar. 1966; maximum gage height, $13.75 \mathrm{ft}$, Mar. 6, 1983, from floodmark, backwater from ice; no flow at times each year.

EXTREMES OUTSIDE PERIOD OF RECORD.--Maximum stage known since 1925, about $14 \mathrm{ft}$ in 1950 , from information by local resident.

Annual mean discharge

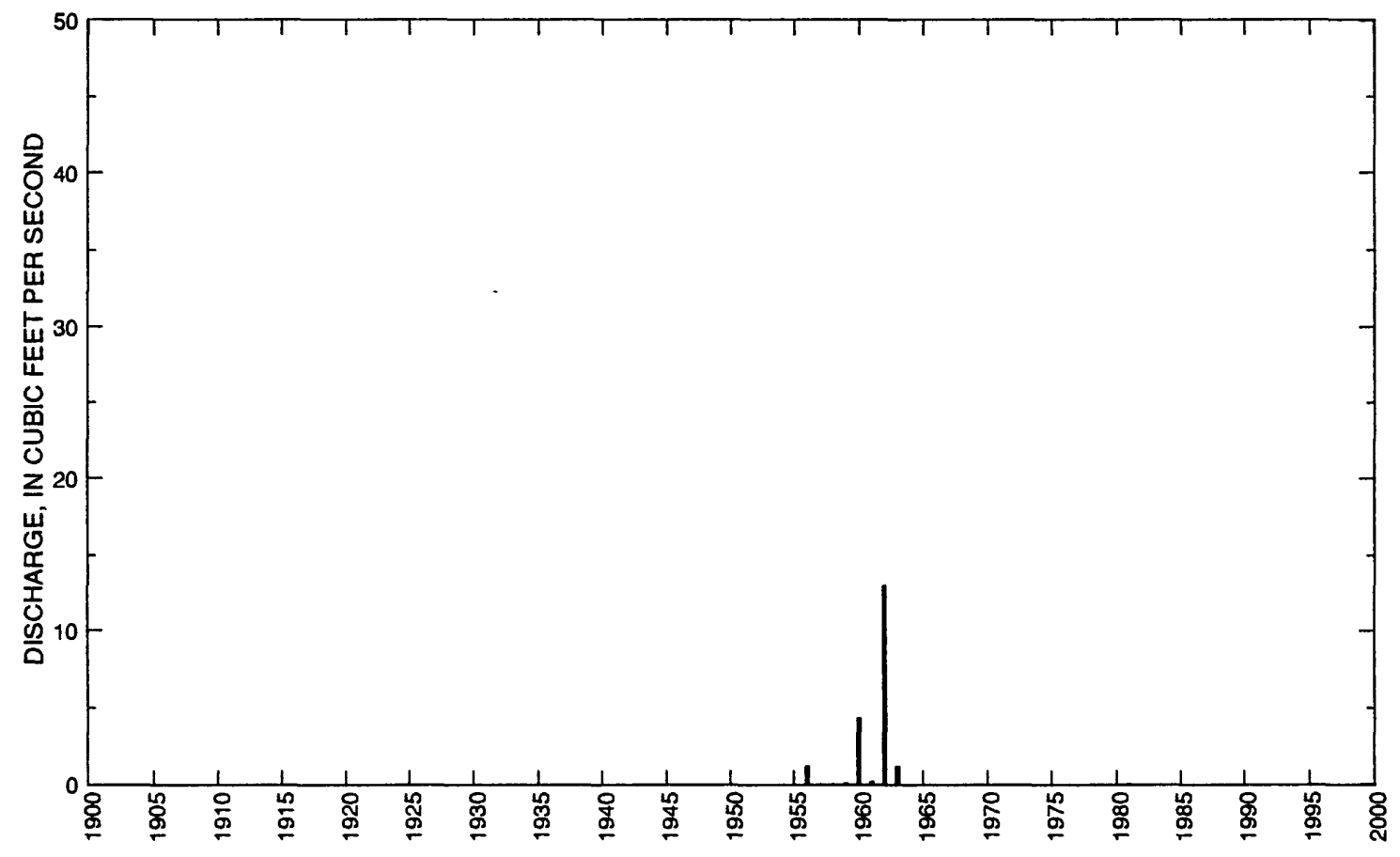


05062200 ELM RIVER NEAR KELSO, ND-Continued

Statistics of monthly and annual mean discharges

[m, more than 1 year of occurrence]

\begin{tabular}{|c|c|c|c|c|c|c|c|c|}
\hline \multirow[b]{2}{*}{ Month } & \multicolumn{2}{|c|}{ Maximum } & \multicolumn{2}{|c|}{ Minimum } & \multicolumn{4}{|c|}{ Mean } \\
\hline & $\begin{array}{c}\text { Discharge } \\
\left(\mathrm{ft}^{3} / \mathrm{s}\right)\end{array}$ & $\begin{array}{c}\text { Water year } \\
\text { of } \\
\text { occurrence }\end{array}$ & $\begin{array}{c}\text { Discharge } \\
\left(\mathrm{ft}^{3} / \mathrm{s}\right)\end{array}$ & $\begin{array}{c}\text { Water year } \\
\text { of } \\
\text { occurrence }\end{array}$ & $\begin{array}{c}\text { Discharge } \\
\left(\mathrm{ft}^{3} / \mathrm{s}\right)\end{array}$ & $\begin{array}{c}\text { Standard } \\
\text { deviation } \\
\left(\mathrm{ft}^{3} / \mathrm{s}\right)\end{array}$ & $\begin{array}{l}\text { Coeffi- } \\
\text { cient of } \\
\text { variation }\end{array}$ & $\begin{array}{l}\text { Percentage } \\
\text { of annual } \\
\text { discharge }\end{array}$ \\
\hline October & 1.11 & 1963 & 0 & $\mathrm{~m}$ & 0.150 & 0.37 & 2.40 & 0.15 \\
\hline November & 0.590 & 1963 & 0 & $\mathrm{~m}$ & 0.070 & 0.20 & 3.00 & 0.06 \\
\hline December & 0.500 & 1963 & 0 & $\mathrm{~m}$ & 0.060 & 0.17 & 3.00 & 0.05 \\
\hline January & 0.026 & 1963 & 0 & $\mathrm{~m}$ & 0 & 0.01 & 3.00 & 0 \\
\hline February & 0.009 & 1981 & 0 & $\mathrm{~m}$ & 0 & 0 & 3.00 & 0 \\
\hline March & 147 & 1984 & 0 & $\mathrm{~m}$ & 30.6 & 52.2 & 1.71 & 29.8 \\
\hline April & 240 & 1982 & 0 & 1958 & 45.0 & 66.4 & 1.48 & 43.8 \\
\hline May & 24.5 & 1986 & 0 & $\mathrm{~m}$ & 5.26 & 7.42 & 1.41 & 5.12 \\
\hline June & 111 & 1983 & 0 & $\mathrm{~m}$ & 15.9 & 37.0 & 2.32 & 15.5 \\
\hline July & 37.6 & 1962 & 0 & $\mathrm{~m}$ & 4.61 & 10.9 & 2.37 & 4.49 \\
\hline August & 4.00 & 1983 & 0 & $\mathrm{~m}$ & 0.42 & 1.10 & 2.60 & 0.41 \\
\hline September & 5.19 & 1962 & 0 & $\mathrm{~m}$ & 0.58 & 1.73 & 3.00 & 0.56 \\
\hline Annual & 13.0 & 1962 & 0 & 1958 & 2.22 & 4.27 & 1.93 & 100 \\
\hline
\end{tabular}

Annual flow duration

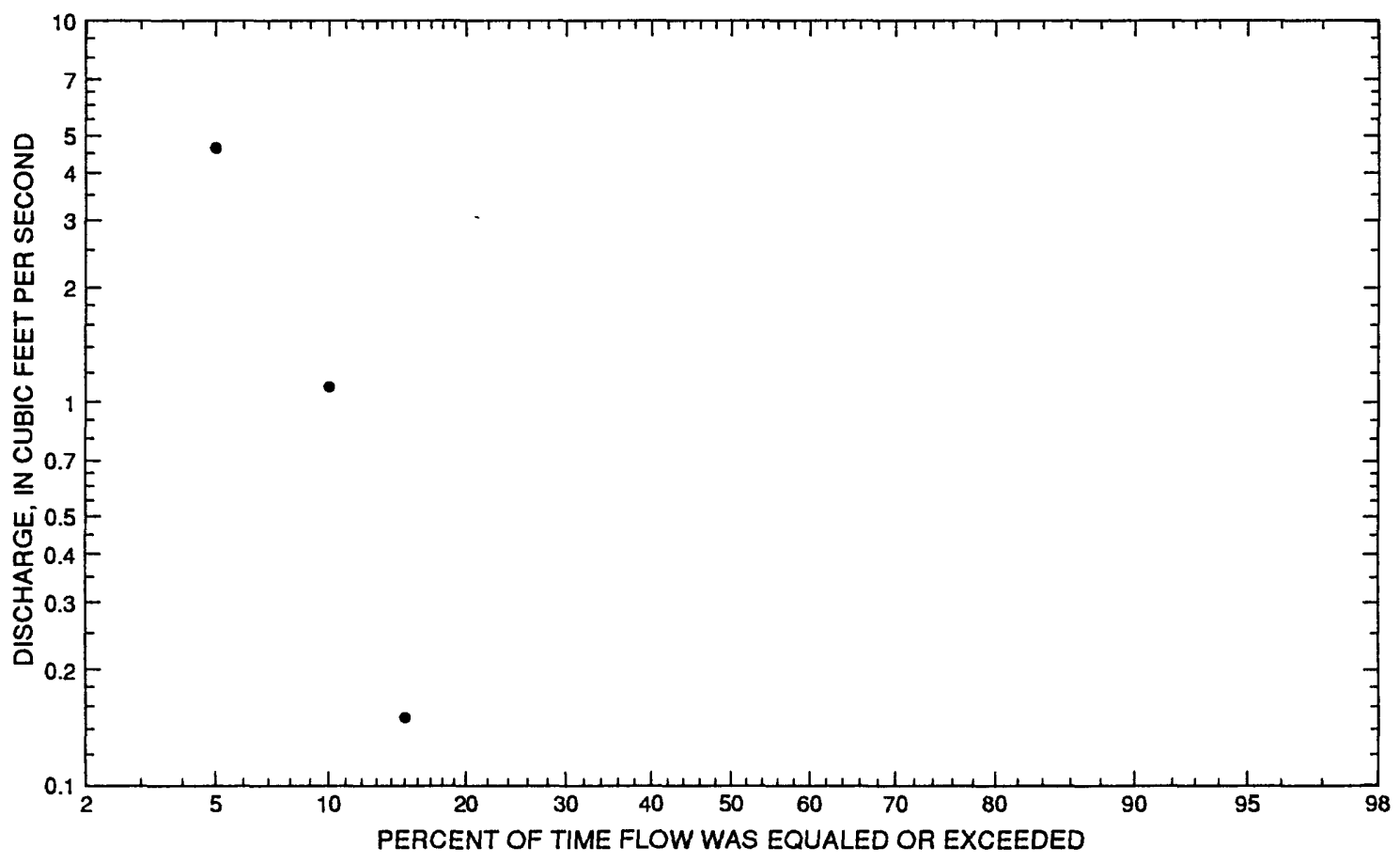




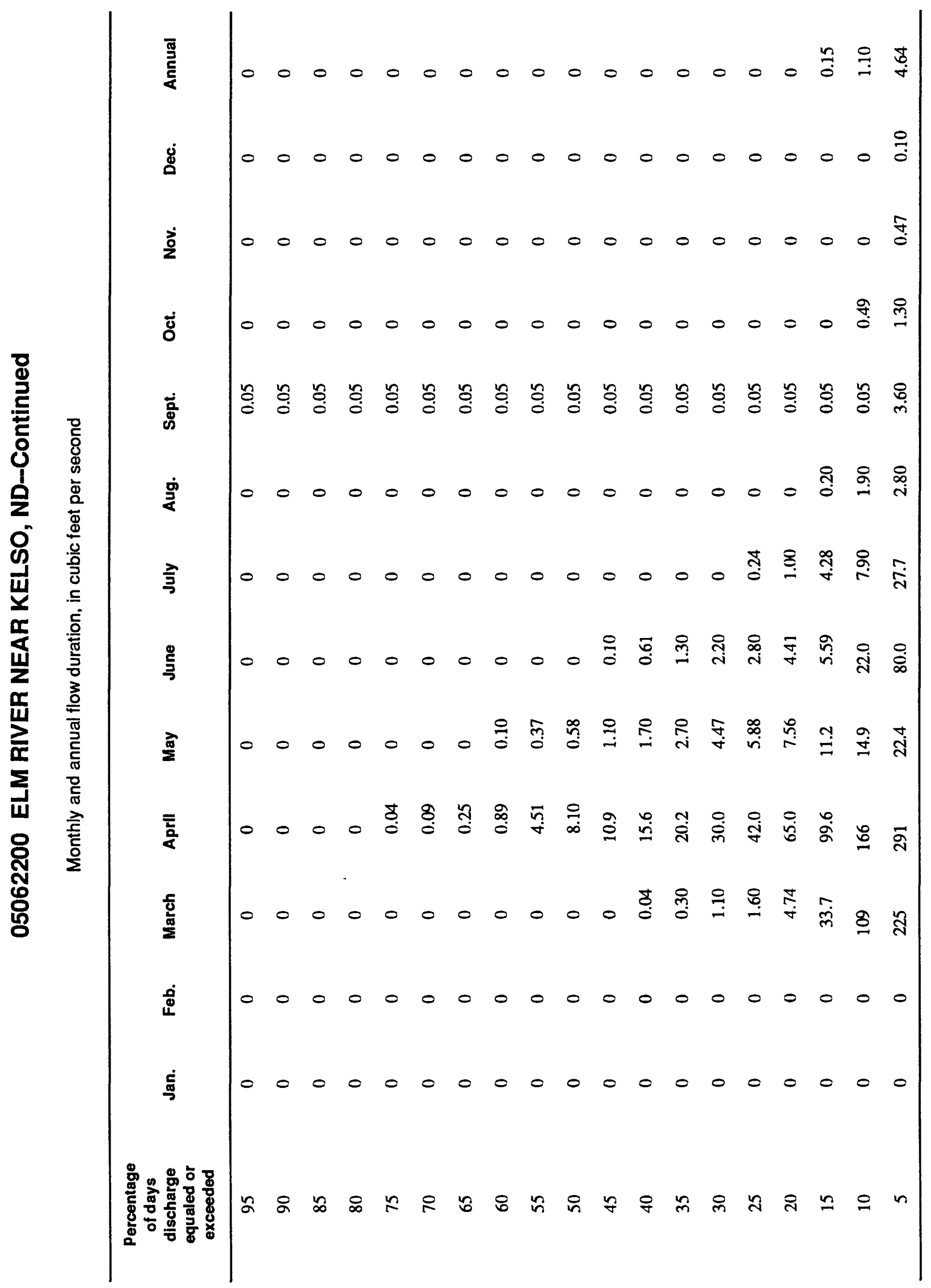




\section{ELM RIVER NEAR KELSO, ND-Continued}

Probability of annual high discharges

[ng, statistic not given]

\begin{tabular}{|c|c|c|c|c|c|c|}
\hline \multirow[b]{2}{*}{$\begin{array}{l}\text { Exceedance } \\
\text { probability }\end{array}$} & \multirow[b]{2}{*}{$\begin{array}{l}\text { Recurrence } \\
\text { interval } \\
\text { (years) }\end{array}$} & \multirow[b]{2}{*}{$\begin{array}{c}\text { Maximum } \\
\text { instantaneous } \\
\left(\mathrm{ft}^{3} / \mathbf{s}\right)\end{array}$} & \multicolumn{4}{|c|}{$\begin{array}{c}\text { Maximum average dlscharge } \\
\left(\mathrm{ft}^{3} / \mathrm{s}\right)\end{array}$} \\
\hline & & & 3-day period & 7-day period & 15-day period & 30-day period \\
\hline 0.99 & 1.01 & $\mathrm{ng}$ & 0 & 0 & 0 & 0 \\
\hline 0.95 & 1.05 & $\mathrm{ng}$ & 0 & 0 & 0 & 0 \\
\hline 0.90 & 1.11 & 9.00 & 0 & 0 & 0 & 0 \\
\hline 0.80 & 1.25 & 26.5 & 0.202 & 0.158 & 0.115 & 0.072 \\
\hline 0.50 & 2 & 158 & 7.67 & 5.79 & 4.22 & 2.55 \\
\hline 0.20 & 5 & 660 & 83.3 & 64.2 & 47.2 & 27.4 \\
\hline 0.10 & 10 & 1,220 & 229 & 182 & 134 & 76.1 \\
\hline 0.04 & 25 & 2,160 & 570 & 468 & 348 & 193 \\
\hline 0.02 & 50 & 2,980 & 970 & 819 & 611 & 334 \\
\hline 0.01 & 100 & 3,860 & 1,520 & 1,320 & 988 & 532 \\
\hline 0.005 & 200 & 4,780 & 2,180 & 1,950 & 1,470 & 779 \\
\hline 0.002 & 500 & 6,020 & $\mathrm{ng}$ & $\mathrm{ng}$ & ng & $\mathrm{ng}$ \\
\hline
\end{tabular}

Probability of annual low discharges

[ng, statistic not given]

\begin{tabular}{|c|c|c|c|c|c|c|c|c|c|c|}
\hline \multirow{3}{*}{$\begin{array}{l}\text { Non- } \\
\text { exceed- } \\
\text { ance } \\
\text { prob- } \\
\text { ability }\end{array}$} & \multirow{3}{*}{$\begin{array}{l}\text { Recur- } \\
\text { rence } \\
\text { Inter- } \\
\text { val } \\
\text { (years) }\end{array}$} & \multicolumn{9}{|c|}{ Minimum average discharge $\left(\mathrm{ft}^{3} / \mathrm{s}\right)$} \\
\hline & & \multicolumn{9}{|c|}{ Number of consecutive days } \\
\hline & & 1 & 3 & 7 & 14 & 30 & 60 & 90 & 120 & 183 \\
\hline 0.05 & 20 & ng & ng & $\mathrm{ng}$ & ng & ng & ng & ng & ng & ng \\
\hline 0.10 & 10 & $\mathrm{ng}$ & ng & ng & ng & ng & ng & $\mathrm{ng}$ & $\mathbf{n g}$ & ng \\
\hline 0.20 & 5 & $\mathbf{n g}$ & ng & ng & ng & $\mathrm{ng}$ & ng & ng & ng & ng \\
\hline 0.50 & 2 & ng & ng & $\mathrm{ng}$ & ng & $\mathrm{ng}$ & $\mathbf{n g}$ & $\mathrm{ng}$ & ng & ng \\
\hline
\end{tabular}




\section{ELM RIVER NEAR KELSO, ND-Continued}

Probability of seasonal low discharges

[ng, statistic not given]

\begin{tabular}{|c|c|c|c|c|c|c|c|c|c|}
\hline \multirow[b]{3}{*}{$\begin{array}{c}\text { Non- } \\
\text { exceedance } \\
\text { probability }\end{array}$} & \multirow[b]{3}{*}{$\begin{array}{l}\text { Recurrence } \\
\text { Interval } \\
\text { (years) }\end{array}$} & \multicolumn{8}{|c|}{ Minimum average discharge $\left(\mathrm{ft}^{3} / \mathrm{s}\right)$} \\
\hline & & \multicolumn{8}{|c|}{ Number of consecutive days } \\
\hline & & 1 & 7 & 14 & 30 & 1 & 7 & 14 & 30 \\
\hline & & \multicolumn{4}{|c|}{ December-January-February } & \multicolumn{4}{|c|}{ March-April-May } \\
\hline 0.05 & 20 & ng & ng & ng & ng & $\mathrm{ng}$ & ng & 0 & 0 \\
\hline 0.10 & 10 & ng & ng & ng & $\mathrm{ng}$ & ng & ng & 0 & 0 \\
\hline 0.20 & 5 & ng & ng & ng & $\mathrm{ng}$ & ng & ng & 0 & 0 \\
\hline \multirow[t]{2}{*}{0.50} & 2 & ng & ng & ng & ng & ng & ng & 0 & 0.083 \\
\hline & & \multicolumn{4}{|c|}{ June-July-August } & \multicolumn{4}{|c|}{ September-October-November } \\
\hline 0.05 & 20 & ng & ng & 0 & 0 & ng & ng & ng & $\mathrm{ng}$ \\
\hline 0.10 & 10 & ng & $\mathrm{ng}$ & 0 & 0 & $\mathrm{ng}$ & $\mathrm{ng}$ & ng & $\mathrm{ng}$ \\
\hline 0.20 & 5 & $\mathrm{ng}$ & ng & 0 & 0 & ng & $\mathrm{ng}$ & ng & $\mathrm{ng}$ \\
\hline 0.50 & 2 & $\mathrm{ng}$ & ng & 0 & 0 & $\mathrm{ng}$ & ng & $\mathrm{ng}$ & ng \\
\hline
\end{tabular}




\section{ELM RIVER NEAR KELSO, ND-Continued}

Annual peak discharge and corresponding gage height, period of record

$$
[--, \text { no data }]
$$

\begin{tabular}{|c|c|c|c|c|c|c|c|}
\hline $\begin{array}{l}\text { Water } \\
\text { year }\end{array}$ & Date & $\begin{array}{c}\text { Gage } \\
\text { height } \\
\text { (feet) }\end{array}$ & $\begin{array}{c}\text { Peak } \\
\text { discharge } \\
\left(\mathrm{ft}^{3} / \mathrm{s}\right)\end{array}$ & $\begin{array}{l}\text { Water } \\
\text { year }\end{array}$ & Date & $\begin{array}{c}\text { Gage } \\
\text { height } \\
\text { (feet) }\end{array}$ & $\begin{array}{c}\text { Peak } \\
\text { discharge } \\
\left(f^{3} / s\right)\end{array}$ \\
\hline \multicolumn{8}{|c|}{ Annual peak discharge, by year, and corresponding gage height } \\
\hline 1956 & June 4 & 6.80 & 102 & 1968 & -- & 6.73 & 117 \\
\hline 1957 & March 24 & 4.75 & 3.00 & 1969 & - & 12.16 & 930 \\
\hline 1958 & -- & -- & 0 & 1970 & -- & 9.58 & 452 \\
\hline 1959 & March 20 & 4.55 & 3.20 & 1971 & $\operatorname{March}^{1}$ & 7.28 & 140 \\
\hline 1960 & April 1 & 7.76 & 165 & 1972 & April 14 & 10.60 & 635 \\
\hline 1961 & March 18 & 4.81 & 9.90 & 1973 & $\operatorname{March}^{1}$ & 8.58 & 265 \\
\hline 1962 & April 8 & 9.51 & 440 & 1981 & February 27 & - & 0.050 \\
\hline 1963 & April 7 & 5.80 & 53.0 & 1982 & April 2 & 12.93 & 500 \\
\hline 1964 & -- & 5.14 & 20.0 & 1983 & March 17 & 10.10 & 310 \\
\hline 1965 & April $^{1}$ & 11.73 & 850 & 1984 & March 26 & 13.75 & 747 \\
\hline 1966 & $\operatorname{March}^{1}$ & 12.48 & 1,000 & 1985 & March 17 & 8.95 & 255 \\
\hline 1967 & Apriil ${ }^{1}$ & 8.72 & 300 & 1986 & March 23 & 10.22 & 362 \\
\hline \multicolumn{8}{|c|}{ Annual peak discharge, from highest to lowest, and corresponding gage height } \\
\hline 1966 & $\operatorname{March}^{1}$ & 12.48 & 1,000 & 1985 & March 17 & 8.95 & 255 \\
\hline 1969 & -- & 12.16 & 930 & 1960 & April 1 & 7.76 & 165 \\
\hline 1965 & Apriil ${ }^{1}$ & 11.73 & 850 & 1971 & $\operatorname{March}^{1}$ & 7.28 & 140 \\
\hline 1984 & March 26 & 13.72 & 747 & 1968 & -- & 6.73 & 117 \\
\hline 1972 & April 14 & 10.60 & 635 & 1956 & June 4 & 6.80 & 102 \\
\hline 1982 & April 2 & 12.93 & 500 & 1963 & April 7 & 5.80 & 53.0 \\
\hline 1970 & -- & 9.58 & 452 & 1964 & -- & 5.14 & 20.0 \\
\hline 1962 & April 8 & 9.51 & 440 & 1961 & March 18 & 4.81 & 9.90 \\
\hline 1986 & March 23 & 10.22 & 362 & 1959 & March 20 & 4.55 & 3.20 \\
\hline 1983 & March 17 & 10.10 & 310 & 1957 & March 24 & 4.75 & 3.00 \\
\hline 1967 & April $^{1}$ & 8.72 & 300 & 1981 & February 27 & - & 0.050 \\
\hline 1973 & March $^{1}$ & 8.58 & 265 & 1958 & -- & - & 0 \\
\hline
\end{tabular}

${ }^{1}$ Day of month unknown. 


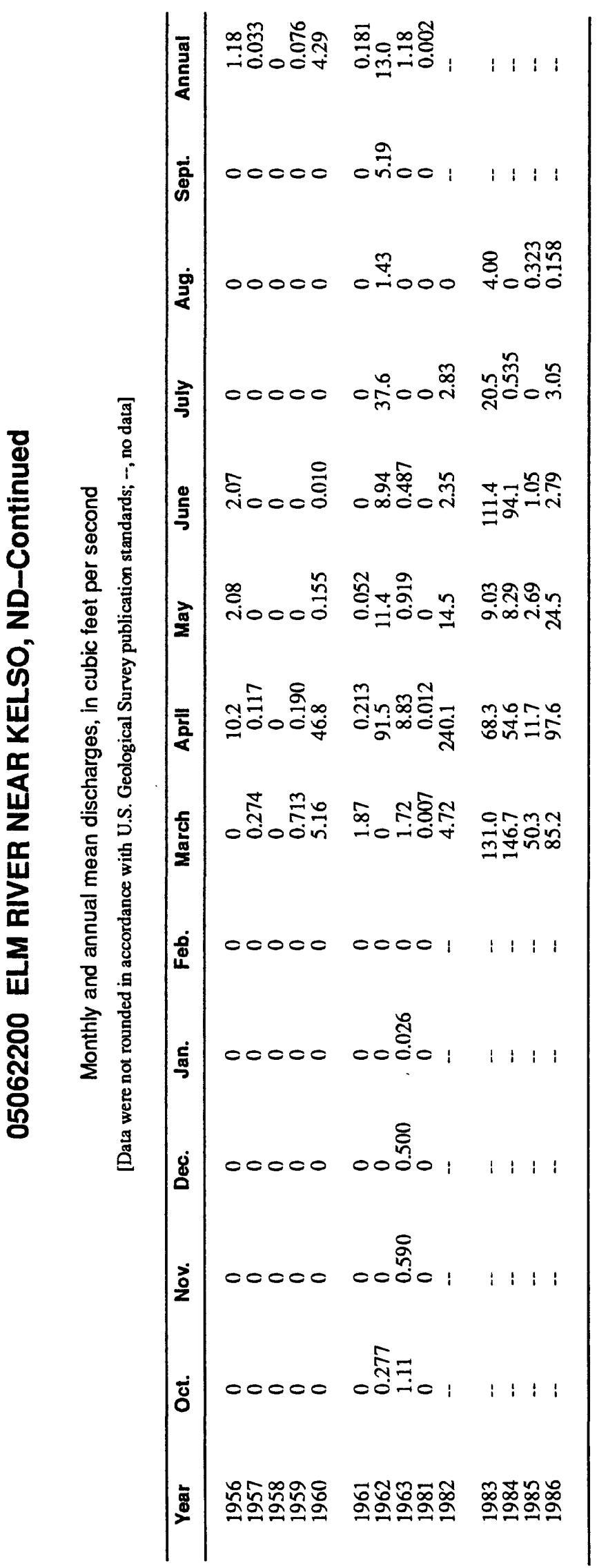




\section{WILD RICE RIVER AT TWIN VALLEY, MN}

\section{Station Description}

LOCATION.--Lat $47^{\circ} 16^{\prime} 00^{\prime \prime}$, long $96^{\circ} 14^{\prime} 40^{\prime \prime}$, in NW $1 /{ }^{1} \mathrm{NE}^{1} / 4$ sec. 27, T.144 N., R.44 W., Norman County, Hydrologic Unit 09020108 , on left bank $100 \mathrm{ft}$ upstream from highway bridge, $0.8 \mathrm{mi}$ northeast of Twin Valley, and 2 mi upstream from small tributary.

DRAINAGE AREA.--888 $\mathrm{mi}^{2}$.

PERIOD OF RECORD.--June 1909 to September 1917, July 1930 to September 1983, October 1989 to current year. Monthly discharge only for some periods, published in WSP 1308 . October 1983 to September 1989, annual maximums only.

GAGE.--Water-stage recorder. Datum of gage is $1,008.16 \mathrm{ft}$ above mean sea level (U.S. Army Corps of Engineers bench mark). June 1909 to September 1917, nonrecording gage at site 0.2 mi downstream at different datum. July 23,1930 , to Nov. 24,1934 , nonrecording gage at highway bridge $100 \mathrm{ft}$ downstream from present site at present datum. Nov. 25, 1934, to Aug. 2, 1950, water-stage recorder $80 \mathrm{ft}$ upstream from present site at present datum.

EXTREMES FOR PERIOD OF RECORD.--Maximum discharge, 9,200 ft $\mathrm{ft}^{3} / \mathrm{s}$, July 22, 1909 (gage height, $20.0 \mathrm{ft}$, from rating curve extended above $3,300 \mathrm{ft}^{3} / \mathrm{s}$ ); minimum discharge, $0.5 \mathrm{ft}^{3} / \mathrm{s}$, Nov. 4,1939 .

Annual mean discharge

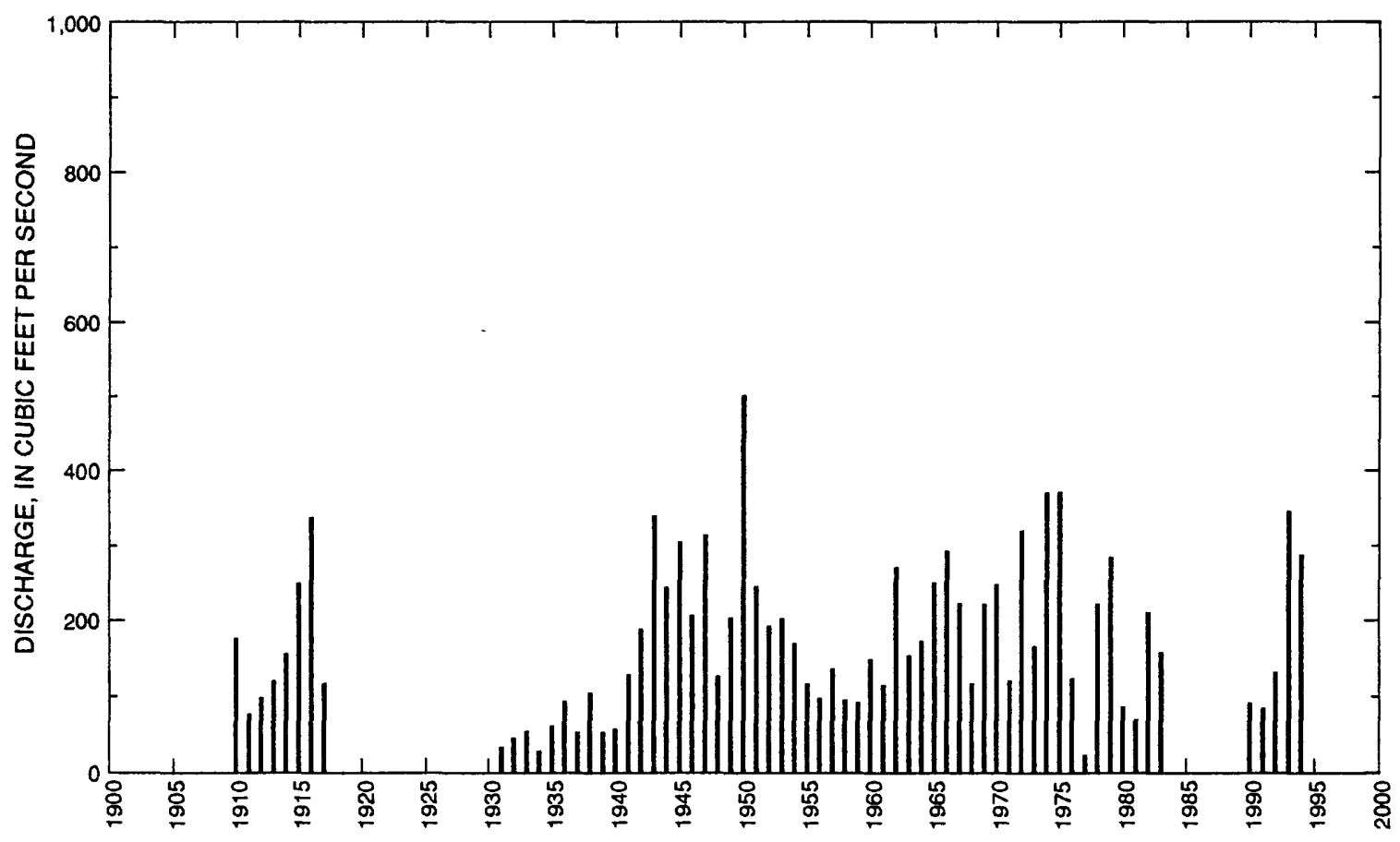




\section{WILD RICE RIVER AT TWIN VALLEY, MN-Continued}

Statistics of monthly and annual mean discharges

\begin{tabular}{|c|c|c|c|c|c|c|c|c|}
\hline \multirow[b]{2}{*}{ Month } & \multicolumn{2}{|c|}{ Maximum } & \multicolumn{2}{|c|}{ Minimum } & \multicolumn{4}{|c|}{ Mean } \\
\hline & $\begin{array}{c}\text { Discharge } \\
\left(\mathrm{ft}^{3} / \mathbf{s}\right)\end{array}$ & $\begin{array}{c}\text { Water year } \\
\text { of } \\
\text { occurrence }\end{array}$ & $\begin{array}{c}\text { Discharge } \\
\left(\mathrm{ft}^{3} / \mathrm{s}\right)\end{array}$ & $\begin{array}{c}\text { Water year } \\
\text { of } \\
\text { occurrence }\end{array}$ & $\begin{array}{c}\text { Discharge } \\
\left(\mathrm{ft}^{3} / \mathbf{s}\right)\end{array}$ & $\begin{array}{l}\text { Standard } \\
\text { deviation } \\
\left(\mathrm{ft}^{3} / \mathrm{s}\right)\end{array}$ & $\begin{array}{l}\text { Coeffi- } \\
\text { clent of } \\
\text { variation }\end{array}$ & $\begin{array}{l}\text { Percentage } \\
\text { of annual } \\
\text { discharge }\end{array}$ \\
\hline October & 614 & 1974 & 6.10 & 1933 & 84.2 & 95.9 & 1.14 & 3.97 \\
\hline November & 488 & 1972 & 9.31 & 1933 & 73.5 & 68.6 & 0.93 & 3.46 \\
\hline December & 123 & 1972 & 6.00 & 1933 & 49.8 & 31.9 & 0.64 & 2.35 \\
\hline January & 100 & 1910 & 4.00 & 1933 & 38.3 & 23.0 & 0.60 & 1.81 \\
\hline February & 90.8 & 1994 & 4.00 & 1933 & 35.5 & 20.2 & 0.57 & 1.67 \\
\hline March & 747 & 1945 & 12.8 & 1940 & 129 & 141 & 1.09 & 6.08 \\
\hline April & 1,540 & 1979 & 73.8 & 1931 & 562 & 419 & 0.75 & 26.5 \\
\hline May & 2,260 & 1950 & 30.9 & 1977 & 414 & 338 & 0.82 & 19.5 \\
\hline June & 1,560 & 1943 & 26.4 & 1977 & 306 & 294 & 0.96 & 14.4 \\
\hline July & 1,920 & 1909 & 8.04 & 1934 & 236 & 332 & 1.40 & 11.1 \\
\hline August & 1,020 & 1993 & 3.02 & 1932 & 107 & 180 & 1.69 & 5.02 \\
\hline September & 788 & 1973 & 2.96 & 1936 & 86.7 & 123 & 1.42 & 4.08 \\
\hline Annual & 500 & 1950 & 22.7 & 1977 & 174 & 102 & 0.59 & 100 \\
\hline
\end{tabular}

Annual flow duration

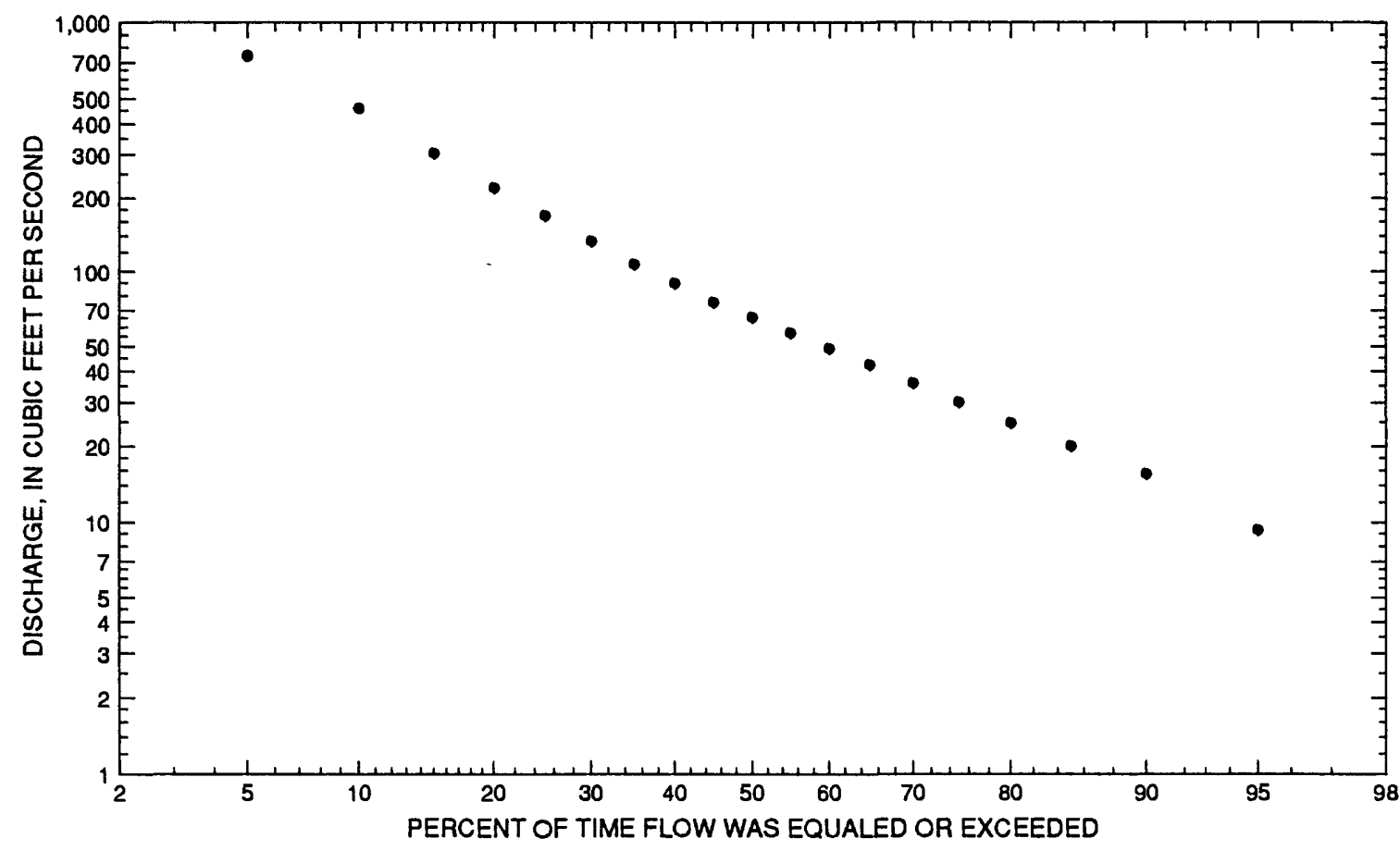




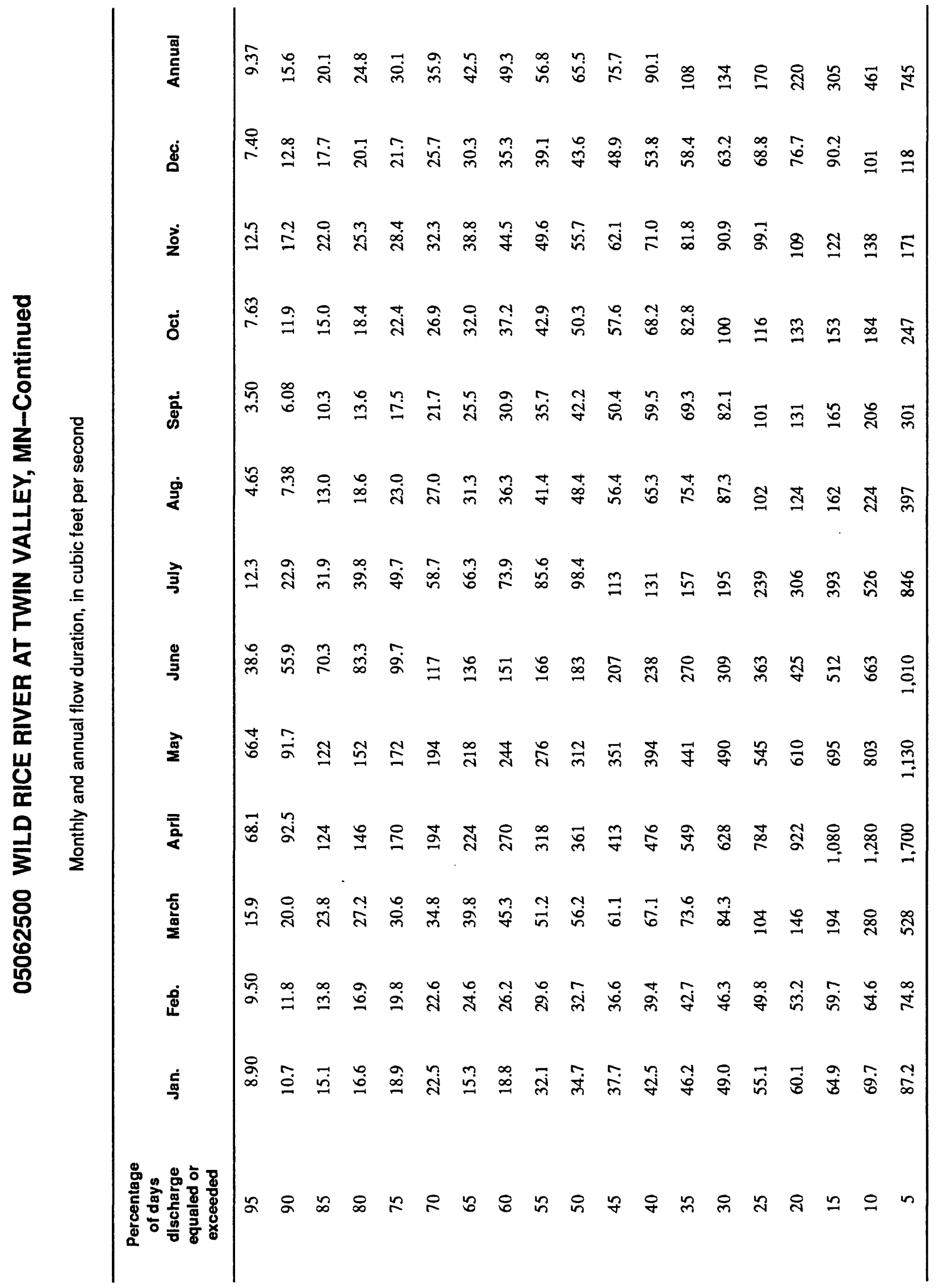




\section{WILD RICE RIVER AT TWIN VALLEY, MN-Continued}

Probability of annual high discharges

[ng, statistic not given]

\begin{tabular}{lcccccc}
\hline & & & \multicolumn{5}{c}{$\begin{array}{c}\text { Maximum average discharge } \\
\left(\mathrm{ft}^{3} / \mathbf{s}\right)\end{array}$} \\
\cline { 6 - 7 } $\begin{array}{c}\text { Exceedance } \\
\text { probability }\end{array}$ & $\begin{array}{c}\text { Recurrence } \\
\text { interval } \\
\text { (years) }\end{array}$ & $\begin{array}{c}\text { Maximum } \\
\text { instantaneous } \\
\left(\mathbf{f t}^{3} / \mathbf{s}\right)\end{array}$ & 3-day period & 7-day period & 15-day period & 30-day period \\
\hline 0.99 & 1.01 & 126 & 101 & 88.9 & 80.4 & 70.3 \\
0.95 & 1.05 & 265 & 218 & 197 & 174 & 148 \\
0.90 & 1.11 & 386 & 321 & 291 & 254 & 214 \\
0.80 & 1.25 & 599 & 498 & 452 & 388 & 322 \\
0.50 & 2 & 1,310 & 1,070 & 960 & 797 & 649 \\
0.20 & 5 & 2,680 & 2,090 & 1,820 & 1,460 & 1,170 \\
0.10 & 10 & 3,790 & 2,870 & 2,430 & 1,910 & 1,530 \\
0.04 & 25 & 5,390 & 3,910 & 3,210 & 2,480 & 1,980 \\
0.02 & 50 & 6,690 & 4,710 & 3,790 & 2,880 & 2,290 \\
0.01 & 100 & 8,060 & 5,520 & 4,350 & 3,260 & 2,600 \\
0.005 & 200 & 9,520 & 6,330 & 4,890 & 3,630 & 2,890 \\
0.002 & 500 & 11,600 & ng & ng & ng & ng \\
\hline
\end{tabular}

Probability of annual low discharges

\begin{tabular}{|c|c|c|c|c|c|c|c|c|c|c|}
\hline \multirow{3}{*}{$\begin{array}{l}\text { Non- } \\
\text { exceed- } \\
\text { ance } \\
\text { prob- } \\
\text { ability }\end{array}$} & \multirow{3}{*}{$\begin{array}{c}\text { Recur- } \\
\text { rence } \\
\text { inter- } \\
\text { val } \\
\text { (years) }\end{array}$} & \multicolumn{9}{|c|}{ Minimum average discharge $\left(\mathrm{ft}^{3} / \mathrm{s}\right)$} \\
\hline & & \multicolumn{9}{|c|}{ Number of consecutive days } \\
\hline & & 1 & 3 & 7 & 14 & 30 & 60 & 90 & 120 & 183 \\
\hline 0.05 & 20 & 2.46 & 2.74 & 2.99 & 3.42 & 4.10 & 5.20 & 6.79 & 8.35 & 9.66 \\
\hline 0.10 & 10 & 3.76 & 4.22 & 4.58 & 5.15 & 6.07 & 7.61 & 9.70 & 11.9 & 13.9 \\
\hline 0.20 & 5 & 6.12 & 6.86 & 7.39 & 8.17 & 9.46 & 11.7 & 14.5 & 17.6 & 21.1 \\
\hline 0.50 & 2 & 14.1 & 15.6 & 16.6 & 17.9 & 20.2 & 242 & 28.6 & 34.1 & 43.2 \\
\hline
\end{tabular}


05062500 WILD RICE RIVER AT TWIN VALLEY, MN-Continued

Probability of seasonal low discharges

\begin{tabular}{|c|c|c|c|c|c|c|c|c|c|}
\hline \multirow[b]{3}{*}{$\begin{array}{c}\text { Non- } \\
\text { exceedance } \\
\text { probability }\end{array}$} & \multirow[b]{3}{*}{$\begin{array}{c}\text { Recurrence } \\
\text { interval } \\
\text { (years) }\end{array}$} & \multicolumn{8}{|c|}{ Minimum average discharge $\left(\mathrm{ft}^{3} / \mathrm{s}\right)$} \\
\hline & & \multicolumn{8}{|c|}{ Number of consecutive days } \\
\hline & & 1 & 7 & 14 & 30 & 1 & 7 & 14 & 30 \\
\hline & & \multicolumn{4}{|c|}{ December-January-February } & \multicolumn{4}{|c|}{ March-April-May } \\
\hline 0.05 & 20 & 5.88 & 6.59 & 6.89 & 7.28 & 9.98 & 11.1 & 12.3 & 19.9 \\
\hline 0.10 & 10 & 8.31 & 9.09 & 9.46 & 10.0 & 13.6 & 14.9 & 16.3 & 26.1 \\
\hline 0.20 & 5 & 12.4 & 13.2 & 13.7 & 14.5 & 19.3 & 20.8 & 22.5 & 36.5 \\
\hline \multirow[t]{2}{*}{0.50} & 2 & 24.7 & 25.5 & 26.1 & 27.6 & 34.1 & 36.9 & 40.4 & 72.1 \\
\hline & & \multicolumn{4}{|c|}{ June-July-August } & \multicolumn{4}{|c|}{ September-October-November } \\
\hline 0.05 & 20 & 3.21 & 4.02 & 4.58 & 6.09 & 2.90 & 3.69 & 4.21 & 5.19 \\
\hline 0.10 & 10 & 5.11 & 6.53 & 7.49 & 9.97 & 4.50 & 5.78 & 6.60 & 8.16 \\
\hline 0.20 & 5 & 8.86 & 11.4 & 13.2 & 17.5 & 7.48 & 9.67 & 11.1 & 13.7 \\
\hline 0.50 & 2 & 24.2 & 30.5 & 35.3 & 46.7 & 18.7 & 24.0 & 27.3 & 33.6 \\
\hline
\end{tabular}


05062500 WILD RICE RIVER AT TWIN VALLEY, MN-Continued

Annual peak discharge and corresponding gage height, period of record

$[--$, no data $]$

\begin{tabular}{|c|c|c|c|c|c|c|c|}
\hline $\begin{array}{l}\text { Water } \\
\text { year }\end{array}$ & Date & $\begin{array}{c}\text { Gage } \\
\text { height } \\
\text { (foet) }\end{array}$ & $\begin{array}{c}\text { Peak } \\
\text { discharge } \\
\left(\mathrm{ft}^{3} / \mathbf{s}\right)\end{array}$ & $\begin{array}{l}\text { Water } \\
\text { year }\end{array}$ & Date & $\begin{array}{c}\text { Gage } \\
\text { height } \\
\text { (feet) }\end{array}$ & $\begin{array}{c}\text { Peak } \\
\text { discharge } \\
\left(\mathrm{ft}^{3} / \mathbf{s}\right)\end{array}$ \\
\hline \multicolumn{8}{|c|}{ Annual peak discharge, by year, and corresponding gage height } \\
\hline 1909 & July 22 & 20.00 & 9,200 & 1959 & May 6 & 3.73 & 451 \\
\hline 1910 & April 26 & 9.60 & 1,610 & 1960 & April 15 & 4.80 & 716 \\
\hline 1911 & April 22 & 6.70 & 473 & 1961 & May 17 & 5.29 & 847 \\
\hline 1912 & May 12 & 7.55 & 758 & 1962 & June 9 & 9.83 & 2,760 \\
\hline 1913 & April 2 & 9.60 & 1,610 & 1963 & May 30 & 8.00 & 1,680 \\
\hline 1914 & June 10 & 8.50 & 1,120 & 1964 & April 17 & 7.68 & 1,640 \\
\hline 1915 & June 29 & 11.10 & 2,340 & 1965 & April 12 & 10.48 & 3,160 \\
\hline 1916 & June 1 & -- & 1,670 & 1966 & April 2 & 8.90 & 2,120 \\
\hline 1917 & April 3 & - & 719 & 1967 & April 1 & 9.09 & 1,710 \\
\hline 1931 & May 21 & -- & 112 & 1968 & March 30 & 4.33 & 594 \\
\hline 1932 & April 9 & 3.59 & 358 & 1969 & April 10 & 11.83 & 4,850 \\
\hline 1933 & May 23 & 3.27 & 450 & 1970 & April 30 & 7.97 & 1,740 \\
\hline 1934 & April 12 & 2.60 & 266 & 1971 & April 10 & 6.01 & 1,060 \\
\hline 1935 & July 14 & -- & 216 & 1972 & March 21 & 9.66 & 2,220 \\
\hline 1936 & April 14 & 9.27 & 2,490 & 1973 & September 4 & 7.75 & 1,670 \\
\hline 1937 & May 3 & 3.32 & 301 & 1974 & April 12 & 11.03 & 3,890 \\
\hline 1938 & May 12 & 5.82 & 836 & 1975 & July 1 & 11.29 & 3,660 \\
\hline 1939 & March 30 & 4.44 & 459 & 1976 & March 29 & 7.10 & 1,250 \\
\hline 1940 & April 9 & 7.28 & 1,100 & 1977 & April 21 & 2.29 & 146 \\
\hline 1941 & April 3 & 6.11 & 828 & 1978 & April 7 & 13.64 & 6,470 \\
\hline 1942 & May 3 & 8.15 & 1,550 & 1979 & April 18 & 12.93 & 6,010 \\
\hline 1943 & June 4 & 11.74 & 4,120 & 1980 & April 3 & 6.08 & 1,080 \\
\hline 1944 & July 8 & 7.86 & 1,560 & 1981 & September 6 & 3.45 & 295 \\
\hline 1945 & April 2 & 8.15 & 1,520 & 1982 & April 19 & 6.51 & 1,200 \\
\hline 1946 & March 24 & 7.60 & 1,490 & 1983 & March 7 & 6.44 & 635 \\
\hline 1947 & April 15 & 9.47 & 2,510 & 1984 & June 11 & 6.96 & 1,370 \\
\hline 1948 & April 9 & 5.65 & 916 & 1985 & May 13 & 11.42 & 4,100 \\
\hline 1949 & July 8 & 7.94 & 1,610 & 1986 & May 13 & 8.38 & 1,960 \\
\hline 1950 & June 26 & 12.02 & 4,380 & 1987 & July 24 & 6.91 & 1,280 \\
\hline 1951 & April 9 & 8.31 & 1,820 & 1988 & April 5 & 6.28 & 711 \\
\hline 1952 & April 8 & 8.27 & 1,810 & 1989 & April 5 & 13.65 & 5,260 \\
\hline 1953 & July 4 & 6.44 & 1,170 & 1990 & April 3 & 6.31 & 1,090 \\
\hline 1954 & April 10 & 7.09 & 1,390 & 1991 & May 6 & 4.96 & 682 \\
\hline 1955 & April 4 & 5.57 & 927 & 1992 & August 25 & -- & 791 \\
\hline 1956 & April 12 & 6.99 & 1,380 & 1993 & July 28 & 11.74 & 3,980 \\
\hline 1957 & April 21 & 5.17 & 814 & 1994 & June 21 & 8.37 & 1,810 \\
\hline 1958 & July 7 & 3.00 & 294 & & & & \\
\hline \multicolumn{8}{|c|}{ Annual peak discharge, from highest to lowest, and corresponding gage height } \\
\hline 1909 & July 22 & 20.00 & 9,200 & 1943 & June 4 & 11.74 & 4,120 \\
\hline 1978 & April 7 & 13.64 & 6,470 & 1985 & May 13 & 11.42 & 4,100 \\
\hline 1979 & April 18 & 12.93 & 6,010 & 1993 & July 28 & 11.74 & 3,980 \\
\hline 1989 & April 5 & 13.65 & 5,260 & 1974 & April 12 & 11.03 & 3,890 \\
\hline 1969 & April 10 & 11.83 & 4,850 & 1975 & July 1 & 11.29 & 3,660 \\
\hline
\end{tabular}




\section{WILD RICE RIVER AT TWIN VALLEY, MN-Continued}

Annual peak discharge and corresponding gage height, period of record--Continued

$$
[-, \text { no data }]
$$

\begin{tabular}{|c|c|c|c|c|c|c|c|}
\hline $\begin{array}{l}\text { Water } \\
\text { year }\end{array}$ & Date & $\begin{array}{c}\text { Gage } \\
\text { height } \\
\text { (feet) }\end{array}$ & $\begin{array}{c}\text { Peak } \\
\text { discharge } \\
\left(\mathrm{ft}^{3} / \mathrm{s}\right)\end{array}$ & $\begin{array}{l}\text { Water } \\
\text { year }\end{array}$ & Date & $\begin{array}{c}\text { Gage } \\
\text { height } \\
\text { (feet) }\end{array}$ & $\begin{array}{c}\text { Peak } \\
\text { discharge } \\
\left(\mathrm{ft}^{3} / \mathrm{s}\right)\end{array}$ \\
\hline 1950 & June 26 & 12.02 & 4,380 & 1965 & April 12 & 10.48 & 3,160 \\
\hline \multicolumn{8}{|c|}{ Annual peak discharge, from highest to lowest, and corresponding gage height-.Continued } \\
\hline 1962 & June 9 & 9.83 & 2.760 & 1990 & April 3 & 6.31 & 1,090 \\
\hline 1947 & April 15 & 9.47 & 2,510 & 1980 & April 3 & 6.08 & 1,080 \\
\hline 1936 & April 14 & 9.27 & 2,490 & 1971 & April 10 & 6.01 & 1,060 \\
\hline 1915 & June 29 & 11.10 & 2,340 & 1955 & April 4 & 5.57 & 927 \\
\hline 1972 & March 21 & 9.66 & 2,220 & 1948 & April 9 & 5.65 & 916 \\
\hline 1966 & April 2 & 8.90 & 2,120 & 1961 & May 17 & 5.29 & 847 \\
\hline 1986 & May 13 & 8.38 & 1,960 & 1938 & May 12 & 5.82 & 836 \\
\hline 1951 & April 9 & 8.31 & 1,820 & 1941 & April 3,June 6 & 6.11 & 828 \\
\hline 1952 & April 8 & 8.27 & 1,810 & 1957 & April 21 & 5.17 & 814 \\
\hline 1994 & June 21 & 8.37 & 1,810 & 1992 & August 25 & - & 791 \\
\hline 1970 & April 30 & 7.97 & 1,740 & 1912 & May 12 & 7.55 & 758 \\
\hline 1967 & April 1 & 9.09 & 1,710 & 1917 & April 3 & - & 719 \\
\hline 1963 & May 30 & 8.00 & 1,680 & 1960 & April 15 & 4.80 & 716 \\
\hline 1916 & June 1 & - & 1,670 & 1988 & April 5 & 6.28 & 711 \\
\hline 1973 & September 4 & 7.75 & 1,670 & 1991 & May 6 & 4.96 & 682 \\
\hline 1964 & April 17 & 7.68 & 1,640 & 1983 & March 7 & 6.44 & 635 \\
\hline 1910 & April 26 & 9.60 & 1,610 & 1968 & March 30 & 4.33 & 594 \\
\hline 1913 & April 2 & 9.60 & 1,610 & 1911 & April 22 & 6.70 & 473 \\
\hline 1949 & July 8 & 7.94 & 1,610 & 1939 & March 30 & 4.44 & 459 \\
\hline 1944 & July 8 & 7.86 & 1,560 & 1959 & May 6 & 3.73 & 451 \\
\hline 1942 & May 3 & 8.15 & 1,550 & 1933 & May 23 & 3.27 & 450 \\
\hline 1945 & April 2 & 8.15 & 1,520 & 1932 & April 9 & 3.59 & 358 \\
\hline 1946 & March 24 & 7.60 & 1,490 & 1937 & May 3 & 3.32 & 301 \\
\hline 1954 & April 10 & 7.09 & 1,390 & 1981 & September 6 & 3.45 & 295 \\
\hline 1956 & April 12 & 6.99 & 1,380 & 1958 & July 7 & 3.00 & 294 \\
\hline 1984 & June 11 & 6.96 & 1,370 & 1934 & April 12 & 2.60 & 266 \\
\hline 1987 & July 24 & 6.91 & $.1,280$ & 1935 & July 14 & - & 216 \\
\hline 1976 & March 29 & 7.10 & 1,250 & 1977 & April 21 & 2.29 & 146 \\
\hline 1982 & April 19 & 6.51 & 1,200 & 1914 & June 10 & 8.50 & 112 \\
\hline 1953 & July 4 & 6.44 & 1,170 & 1931 & May 21 & - & 112 \\
\hline 1940 & April 9 & 7.28 & 1,100 & & & & \\
\hline
\end{tabular}




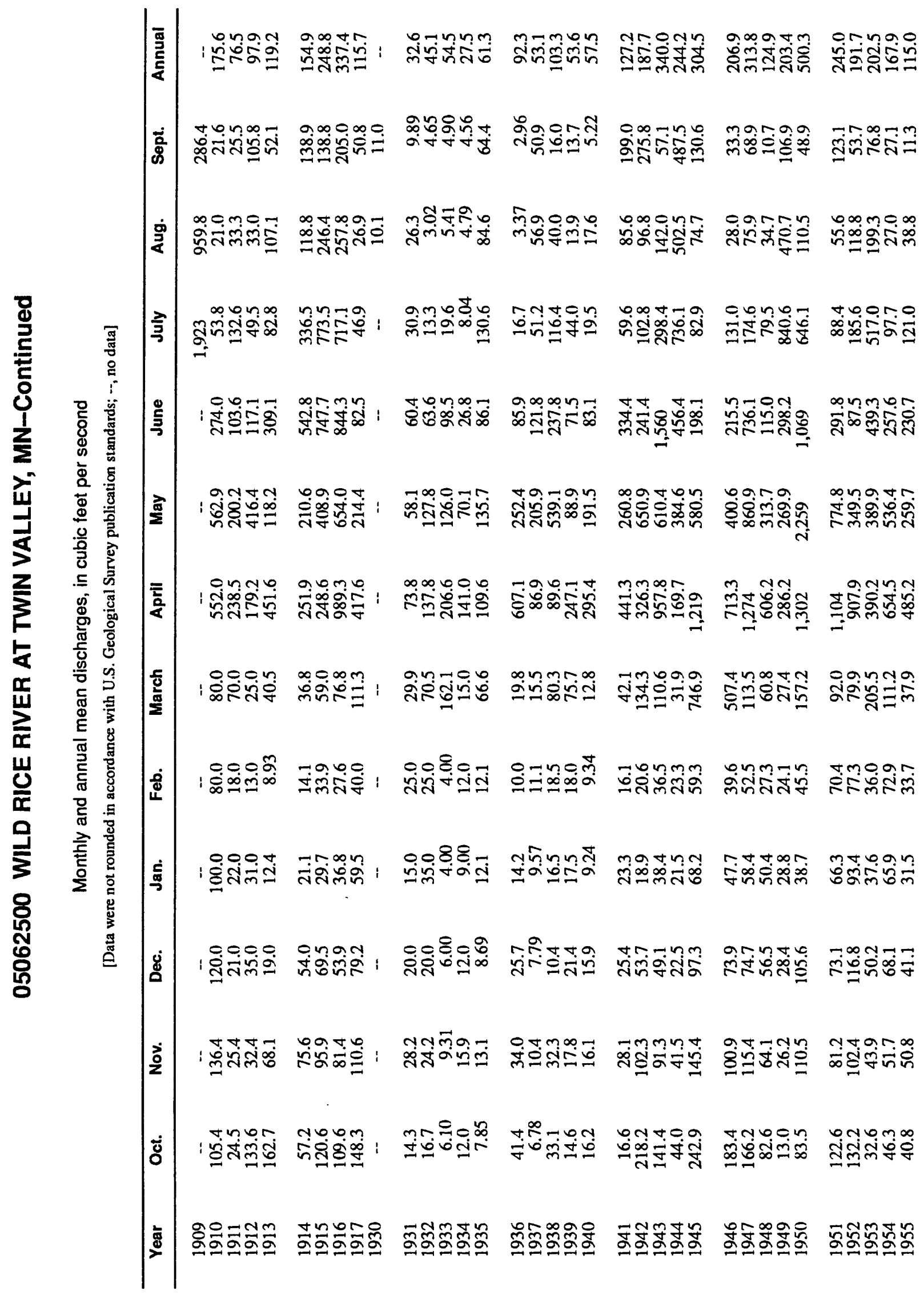




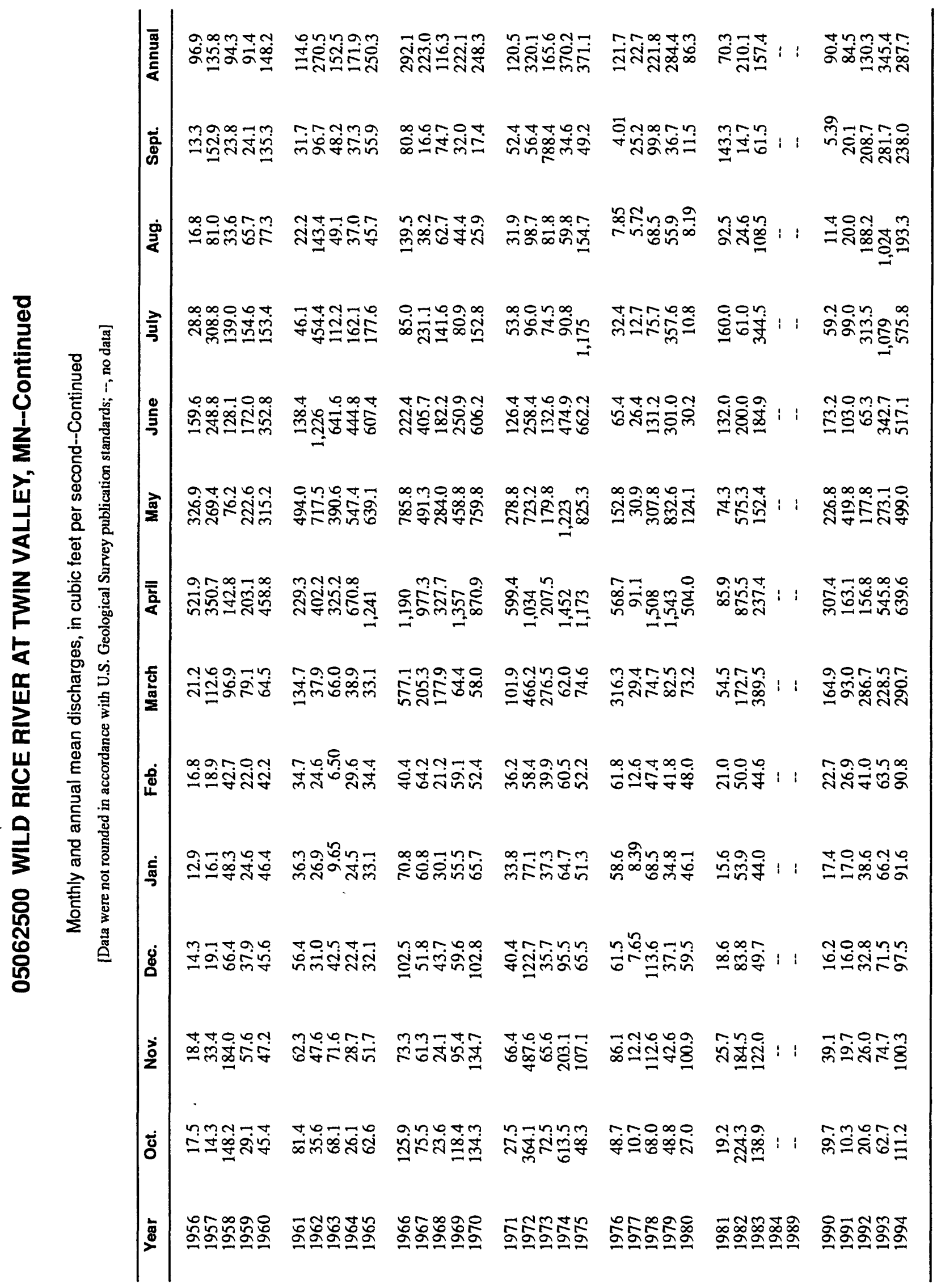




\section{WILD RICE RIVER AT HENDRUM, MN}

\section{Station Description}

LOCATION.--Lat $47^{\circ} 16^{\prime} 05^{\prime \prime}$, long $96^{\circ} 47^{\prime} 50^{\prime \prime}$, in SE $1 / 4 \mathrm{SE}^{1} / 4 \mathrm{sec} .19, \mathrm{~T} .144$ N., R.48 W., Norman County, Hydrologic Unit 09020108 , on right bank $30 \mathrm{ft}$ downstream from highway bridge, $0.5 \mathrm{mi}$ east of Hendrum and 4 mi upstream from mouth.

DRAINAGE AREA.--1,600 $\mathrm{mi}^{2}$, approximately.

PERIOD OF RECORD.--March 1944 to September 1984 and May 1985 to current year. Operated as a high-flow partial-record station October 1984 to April 1985.

GAGE.--Water-stage recorder. Datum of gage is $836.75 \mathrm{ft}$ above mean sea level (levels by U.S. Army Corps of Engineers). Prior to July 18, 1989, nonrecording gage at same site and datum.

EXTREMES FOR PERIOD OF RECORD.--Maximum discharge, 9,350 $\mathrm{ft}^{3} / \mathrm{s}$, Apr. 10, 1978 (gage height, $31.4 \mathrm{ft}$ ); maximum gage height, $32.3 \mathrm{ft}$, Apr. 21, 1979; minimum discharge, $0 \mathrm{ft}^{3} / \mathrm{s}$ for some days in $1948-49$.

Annual mean discharge

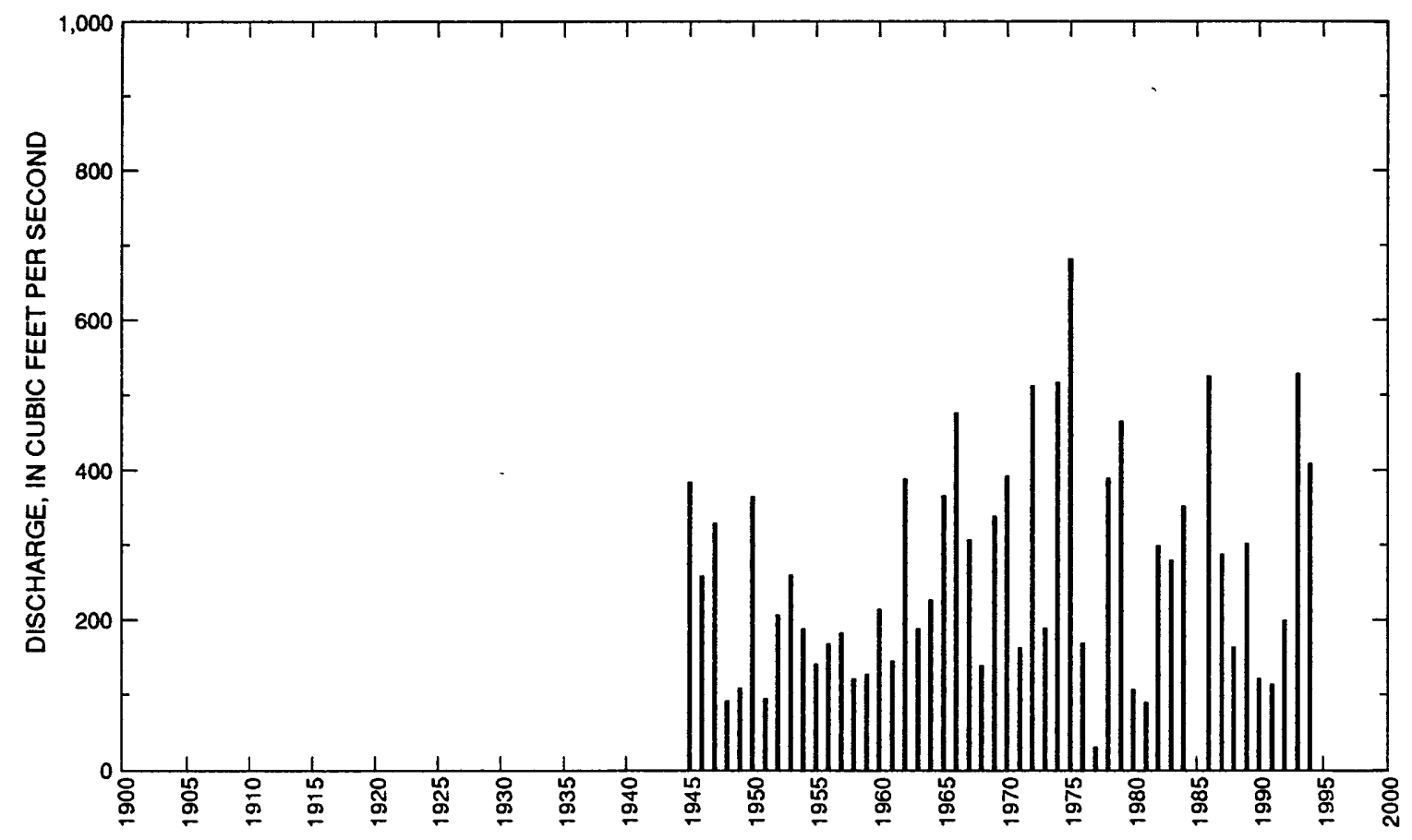




\section{WILD RICE RIVER AT HENDRUM, MN-Continued}

\section{Pre-regulation period}

Statistics of monthly and annual mean discharges, pre-regulation period

\begin{tabular}{|c|c|c|c|c|c|c|c|c|}
\hline \multirow[b]{2}{*}{ Month } & \multicolumn{2}{|c|}{ Maximum } & \multicolumn{2}{|c|}{ Minimum } & \multicolumn{4}{|c|}{ Mean } \\
\hline & $\begin{array}{c}\text { Discharge } \\
\left(\mathrm{ft}^{3} / \mathrm{s}\right)\end{array}$ & $\begin{array}{c}\text { Water year } \\
\text { of } \\
\text { occurrence }\end{array}$ & $\begin{array}{c}\text { Discharge } \\
\left(\mathrm{ft}^{3} / \mathrm{s}\right)\end{array}$ & $\begin{array}{c}\text { Water year } \\
\text { of } \\
\text { occurrence }\end{array}$ & $\begin{array}{c}\text { Discharge } \\
\left(\mathrm{ft}^{3} / \mathrm{s}\right)\end{array}$ & $\begin{array}{c}\text { Standard } \\
\text { deviation } \\
\left(\mathrm{ft}^{3} / \mathbf{s}\right)\end{array}$ & $\begin{array}{l}\text { Coeffi- } \\
\text { cient of } \\
\text { variation }\end{array}$ & $\begin{array}{l}\text { Percentage } \\
\text { of annual } \\
\text { discharge }\end{array}$ \\
\hline October & 299 & 1945 & 0.435 & 1949 & 79.8 & 92.1 & 1.15 & 3.18 \\
\hline November & 228 & 1945 & 3.32 & 1949 & 70.3 & 66.9 & 0.95 & 2.80 \\
\hline December & 152 & 1952 & 1.73 & 1949 & 51.3 & 42.9 & 0.84 & 2.04 \\
\hline January & 99.2 & 1952 & 0.800 & 1949 & 35.4 & 27.8 & 0.79 & 1.41 \\
\hline February & 83.1 & 1952 & 0.400 & 1949 & 32.6 & 24.5 & 0.75 & 1.30 \\
\hline March & 1,080 & 1945 & 0.461 & 1949 & 191 & 285 & 1.49 & 7.61 \\
\hline April & 1,790 & 1947 & 173 & 1958 & 799 & 507 & 0.63 & 31.8 \\
\hline May & 1,630 & 1950 & 99.0 & 1949 & 451 & 346 & 0.77 & 17.9 \\
\hline June & 888 & 1953 & 9.15 & 1952 & 321 & 239 & 0.74 & 12.8 \\
\hline July & 1,120 & 1944 & 8.82 & 1951 & 246 & 263 & 1.07 & 9.80 \\
\hline August & 960 & 1944 & 3.93 & 1948 & 132 & 222 & 1.68 & 5.24 \\
\hline September & 762 & 1944 & 0.183 & 1948 & 104 & 185 & 1.78 & 4.12 \\
\hline Annual & 384 & 1945 & 90.4 & 1948 & 198 & 92.0 & 0.46 & 100 \\
\hline
\end{tabular}

Annual flow duration, pre-regulation period

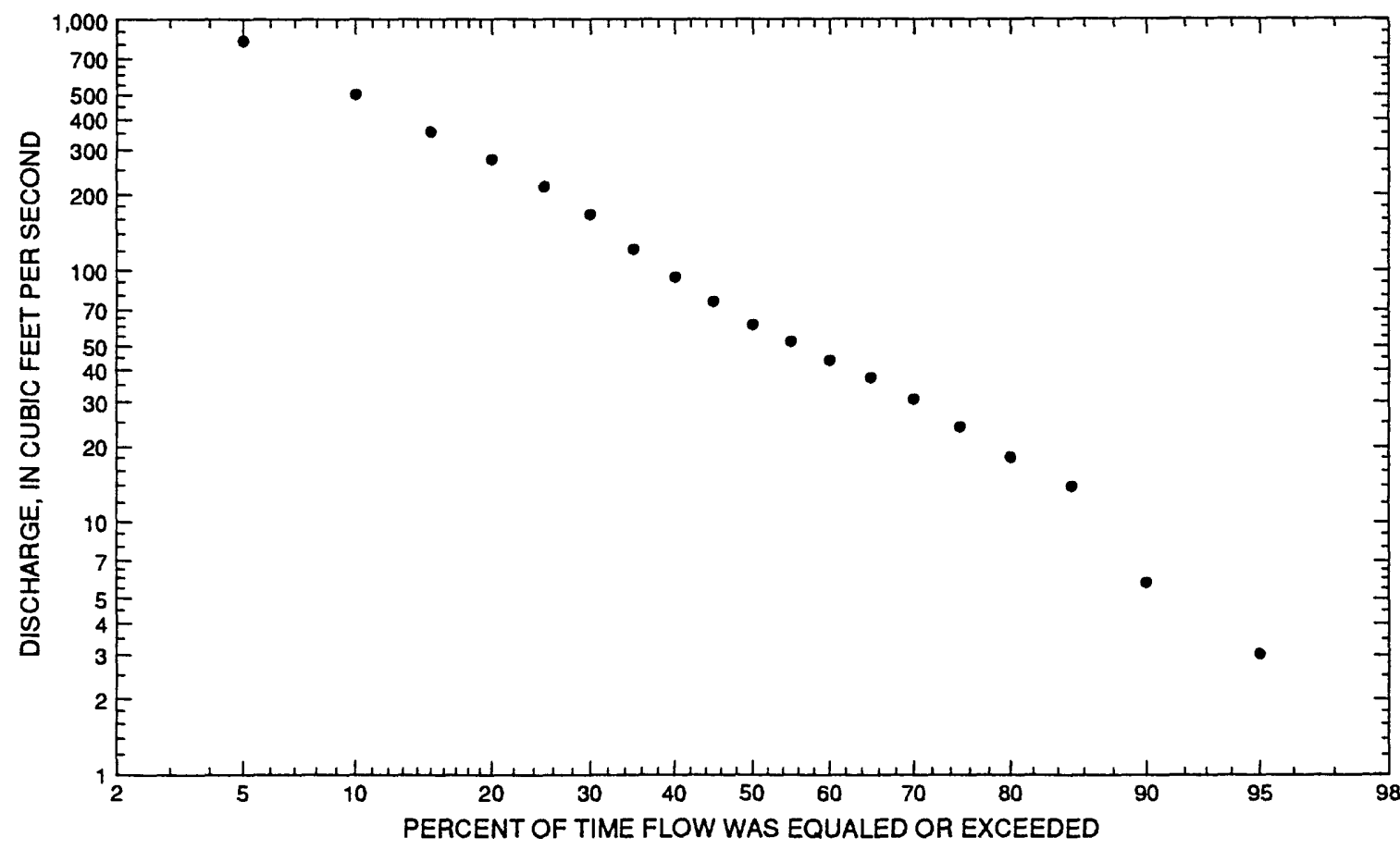




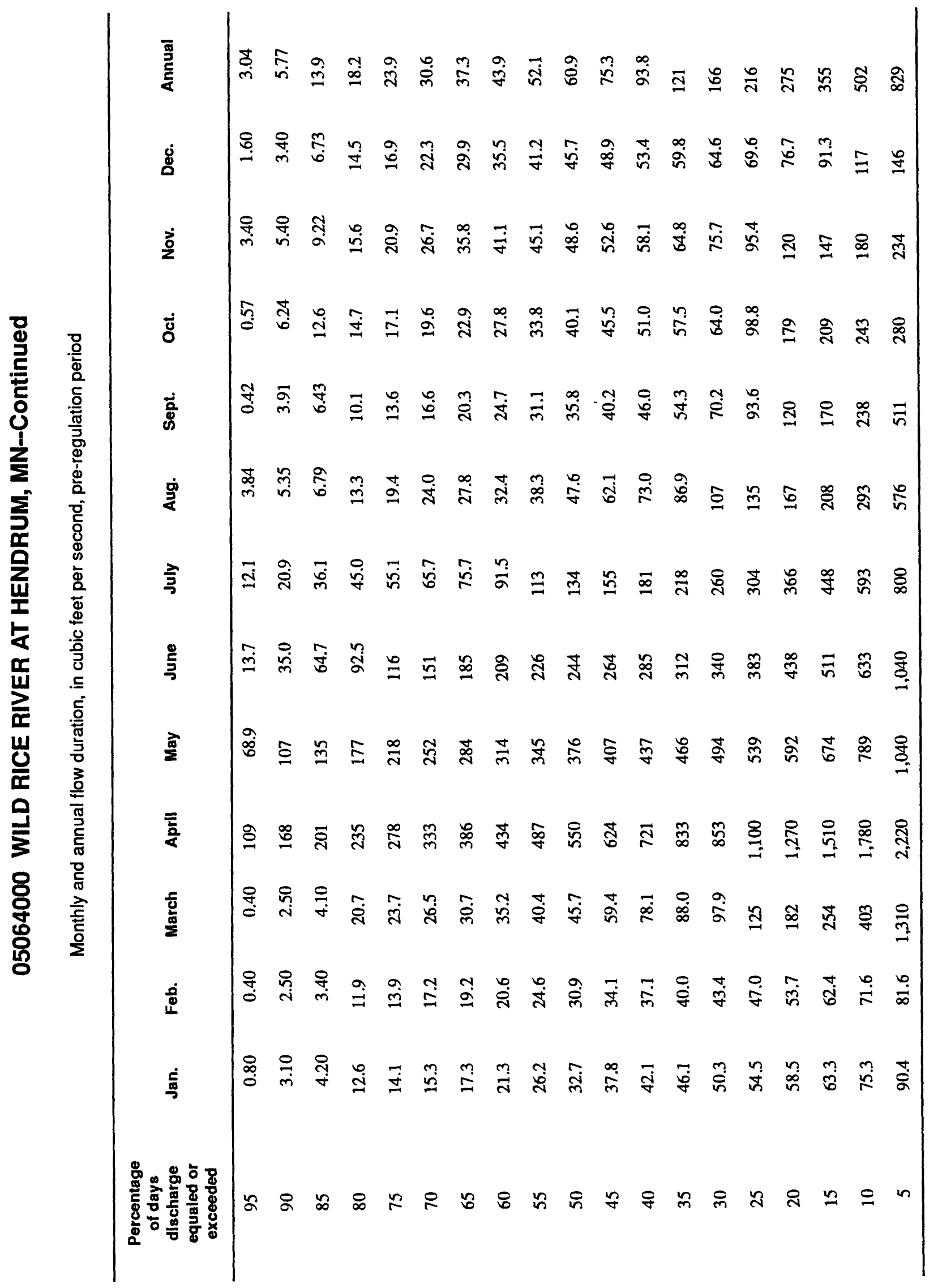


05064000 WILD RICE RIVER AT HENDRUM, MN-Continued

Probability of annual high discharges, pre-regulation period [ng, statistic not given]

\begin{tabular}{lcccccc}
\hline & & & \multicolumn{5}{c}{$\begin{array}{c}\text { Maximum average discharge } \\
\left(\mathrm{ft}^{3} / \mathbf{s}\right)\end{array}$} \\
\cline { 5 - 7 } $\begin{array}{c}\text { Exceedance } \\
\text { probability }\end{array}$ & $\begin{array}{c}\text { Recurrence } \\
\text { interval } \\
\text { (years) }\end{array}$ & $\begin{array}{c}\text { Maximum } \\
\text { instantaneous } \\
\left(\mathbf{f t}^{3} / \mathbf{s}\right)\end{array}$ & 3-day period & 7-day period & 15-day period & 30-day period \\
\hline 0.99 & 1.01 & 355 & 312 & 279 & 233 & 167 \\
0.95 & 1.05 & 615 & 543 & 482 & 394 & 293 \\
0.90 & 1.11 & 808 & 716 & 632 & 512 & 386 \\
0.80 & 1.25 & 1,100 & 983 & 685 & 692 & 528 \\
0.50 & 2 & 1,890 & 1,720 & 1,500 & 1,170 & 901 \\
0.20 & 5 & 3,030 & 2,810 & 2,430 & 1,850 & 1,420 \\
0.10 & 10 & 3,770 & 3,550 & 3,050 & 2,300 & 1,750 \\
0.04 & 25 & 4,670 & 4,480 & 3,830 & 2,850 & 2,130 \\
0.02 & 50 & 5,320 & 5,150 & 4,390 & 3,240 & 2,400 \\
0.01 & 100 & 5,930 & 5,800 & 4,940 & 3,610 & 2,650 \\
0.005 & 200 & 6,520 & 6,440 & 5,470 & 3,970 & 2,880 \\
0.002 & 500 & 7,260 & ng & ng & ng & ng \\
\hline
\end{tabular}

Probability of annual low discharges, pre-regulation period

\begin{tabular}{|c|c|c|c|c|c|c|c|c|c|c|}
\hline \multirow[b]{3}{*}{$\begin{array}{l}\text { Non- } \\
\text { exceed- } \\
\text { ance } \\
\text { prob- } \\
\text { ability }\end{array}$} & \multirow[b]{3}{*}{$\begin{array}{l}\text { Recur- } \\
\text { rence } \\
\text { inter- } \\
\text { val } \\
\text { (years) }\end{array}$} & \multicolumn{9}{|c|}{ Minimum average discharge $\left(\mathrm{ft}^{3} / \mathrm{s}\right)$} \\
\hline & & \multicolumn{9}{|c|}{ Number of consecutive days } \\
\hline & & 1 & 3 & 7 & 14 & 30 & 60 & 90 & 120 & 183 \\
\hline 0.05 & 20 & 0 & 0 & 0 & 0 & 0.440 & 0.846 & 1.50 & 1.93 & 2.52 \\
\hline 0.10 & 10 & 0.800 & 1.13 & 1.31 & 2.11 & ${ }^{1} 2.20$ & 2.28 & 3.35 & 4.12 & 5.21 \\
\hline 0.20 & 5 & 2.36 & 3.23 & 3.66 & 4.78 & ${ }^{1} 5.50$ & 6.20 & 7.72 & 9.17 & 11.4 \\
\hline 0.50 & 2 & 9.52 & 11.9 & 13.2 & 14.1 & 20.2 & 23.6 & 25.9 & 30.2 & 38.4 \\
\hline
\end{tabular}

${ }^{\mathfrak{I} G r a p h i c a l}$ interpretation. 


\section{WILD RICE RIVER AT HENDRUM, MN-Continued}

Probability of seasonal low discharges, pre-regulation period

\begin{tabular}{|c|c|c|c|c|c|c|c|c|c|}
\hline \multirow[b]{3}{*}{$\begin{array}{c}\text { Non- } \\
\text { exceedance } \\
\text { probabllity }\end{array}$} & \multirow[b]{3}{*}{$\begin{array}{c}\text { Recurrence } \\
\text { interval } \\
\text { (years) }\end{array}$} & \multicolumn{8}{|c|}{ Minimum average discharge $\left(\mathrm{ft}^{3} / \mathrm{s}\right)$} \\
\hline & & \multicolumn{8}{|c|}{ Number of consecutive days } \\
\hline & & 1 & 7 & 14 & 30 & 1 & 7 & 14 & 30 \\
\hline & & \multicolumn{4}{|c|}{ December-January-February } & \multicolumn{4}{|c|}{ March-April-May } \\
\hline 0.05 & 20 & 1.16 & 1.18 & 1.19 & 1.27 & 1.09 & 1.10 & 1.16 & 1.67 \\
\hline 0.10 & 10 & 2.67 & 2.75 & 2.79 & 2.96 & 2.72 & 2.76 & 2.97 & 5.00 \\
\hline 0.20 & 5 & 6.40 & 6.62 & 6.77 & 7.15 & 6.98 & 7.18 & 8.00 & 15.8 \\
\hline \multirow[t]{2}{*}{0.50} & 2 & 22.7 & 23.5 & 24.2 & 25.4 & 26.4 & 28.6 & 34.7 & 86.9 \\
\hline & & \multicolumn{4}{|c|}{ June-July-August } & \multicolumn{4}{|c|}{ September-October-November } \\
\hline 0.05 & 20 & 1.24 & 2.02 & 2.87 & 4.08 & 0 & 0 & 0 & 0.519 \\
\hline 0.10 & 10 & 2.27 & 3.39 & 4.59 & 6.69 & 1.13 & 1.39 & 2.33 & 1.86 \\
\hline 0.20 & 5 & 4.67 & 6.36 & 8.19 & 12.2 & 3.41 & 4.26 & 5.81 & 6.75 \\
\hline 0.50 & 2 & 18.0 & 21.5 & 25.6 & 38.8 & 14.8 & 19.4 & 21.9 & 38.3 \\
\hline
\end{tabular}


05064000 WILD RICE RIVER AT HENDRUM, MN-Continued

Post-regulation period

Statistics of monthly and annual mean discharges, post-regulation period

$[\mathrm{m}$, more than 1 year of occurrence]

\begin{tabular}{|c|c|c|c|c|c|c|c|c|}
\hline \multirow[b]{2}{*}{ Month } & \multicolumn{2}{|c|}{ Maximum } & \multicolumn{2}{|c|}{ Minimum } & \multicolumn{4}{|c|}{ Mean } \\
\hline & $\begin{array}{c}\text { Discharge } \\
\left(\mathrm{ft}^{3} / \mathrm{s}\right)\end{array}$ & $\begin{array}{l}\text { Water year } \\
\text { of } \\
\text { occurrence }\end{array}$ & $\begin{array}{c}\text { Discharge } \\
\left(\mathrm{ft}^{3} / \mathrm{s}\right)\end{array}$ & $\begin{array}{c}\text { Water year } \\
\text { of } \\
\text { occurrence }\end{array}$ & $\begin{array}{c}\text { Discharge } \\
\left(\mathrm{ft}^{3} / \mathrm{s}\right)\end{array}$ & $\begin{array}{c}\text { Standard } \\
\text { deviation } \\
\left(\mathrm{ft}^{3} / \mathrm{s}\right)\end{array}$ & $\begin{array}{l}\text { Coeffi- } \\
\text { clent of } \\
\text { variation }\end{array}$ & $\begin{array}{l}\text { Percentage } \\
\text { of annual } \\
\text { discharge }\end{array}$ \\
\hline October & 744 & 1972 & 7.68 & 1977 & 133 & 165 & 1.24 & 3.58 \\
\hline November & 784 & 1972 & 10.3 & 1977 & 122 & 137 & 1.12 & 3.27 \\
\hline December & 160 & 1972 & 1.08 & 1977 & 69.2 & 43.5 & 0.63 & 1.85 \\
\hline January & 121 & 1986 & 0.092 & 1977 & 51.4 & 28.4 & 0.55 & 1.38 \\
\hline February & 124 & 1984 & 0.219 & 1977 & 50.5 & 27.6 & 0.55 & 1.35 \\
\hline March & 1,480 & 1966 & 23.4 & 1965 & 314 & 344 & 1.10 & 8.41 \\
\hline April & 3,260 & 1978 & 106 & 1981 & 1,240 & 916 & 0.74 & 33.2 \\
\hline May & 2,070 & 1985 & 56.1 & 1977 & 625 & 483 & 0.77 & 16.8 \\
\hline June & 1,780 & 1962 & 36.7 & 1977 & 472 & 440 & 0.93 & 12.7 \\
\hline July & 3,140 & 1975 & 11.9 & 1980 & 379 & 600 & 1.58 & 10.2 \\
\hline August & 1,830 & 1993 & 1.07 & 1977 & 156 & 319 & 2.05 & 4.17 \\
\hline September & 824 & 1973 & 0.722 & 1976 & 120 & 164 & 1.37 & 3.22 \\
\hline Annual & 682 & 1975 & 28.9 & 1977 & 303 & 159 & 0.53 & 100 \\
\hline
\end{tabular}

Annual flow duration, post-regulation period

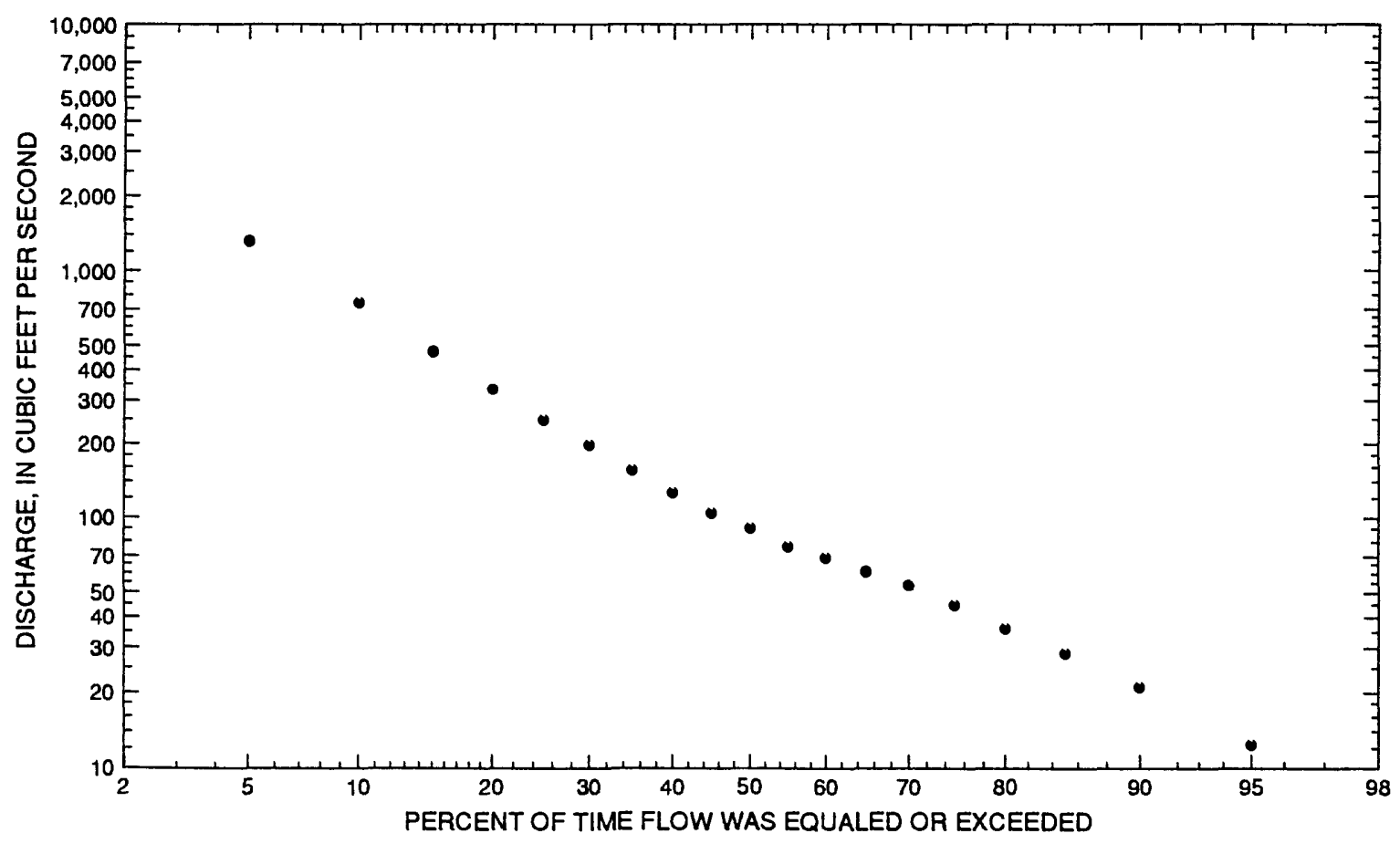




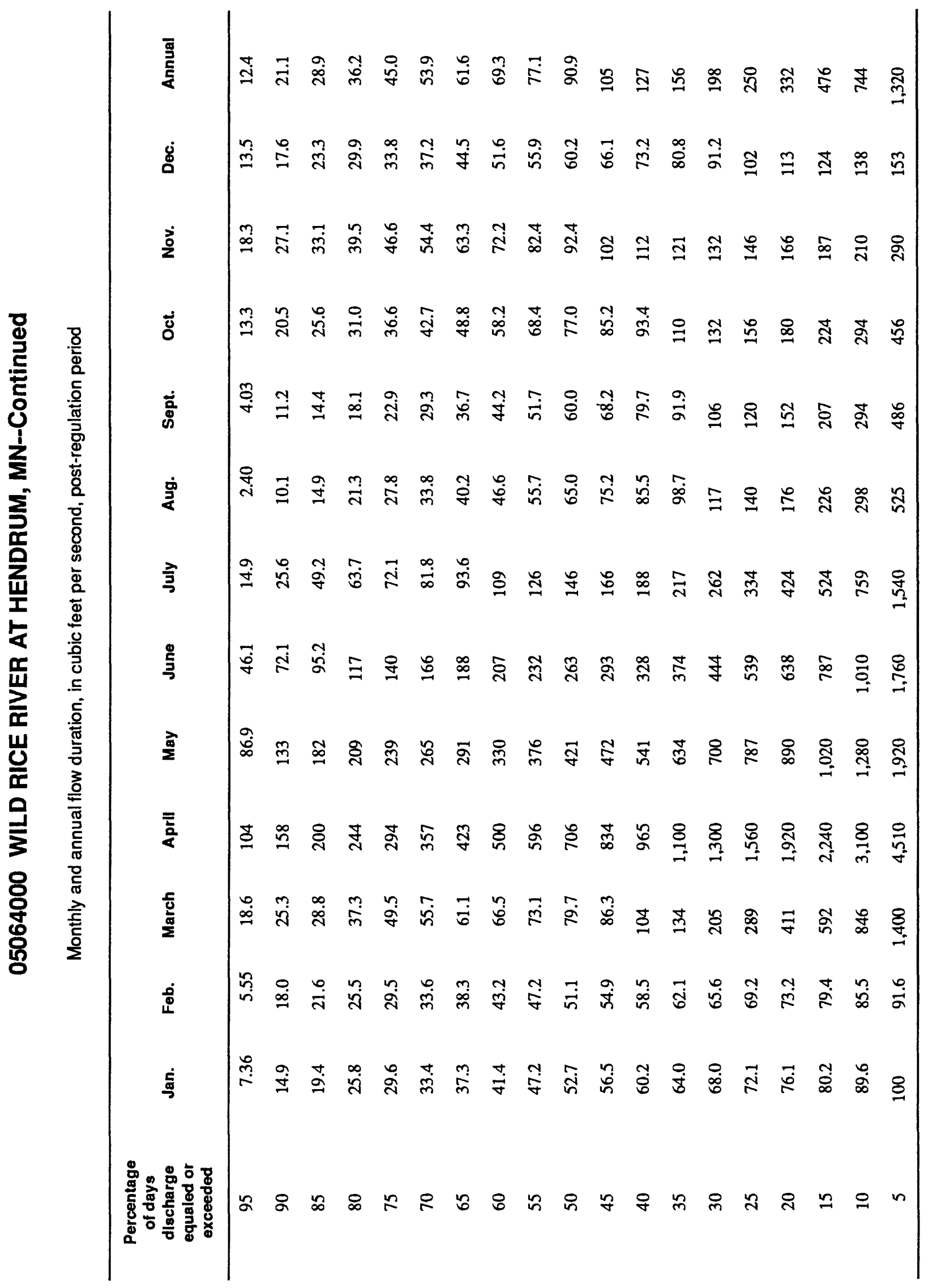




\section{WILD RICE RIVER AT HENDRUM, MN-Continued}

Probability of annual high discharges, post-regulation period

[ng, statistic not given]

\begin{tabular}{|c|c|c|c|c|c|c|}
\hline \multirow[b]{2}{*}{$\begin{array}{l}\text { Exceedance } \\
\text { probability }\end{array}$} & \multirow[b]{2}{*}{$\begin{array}{c}\text { Recurrence } \\
\text { interval } \\
\text { (years) }\end{array}$} & \multirow[b]{2}{*}{$\begin{array}{c}\text { Maximum } \\
\text { instantaneous } \\
\left(\mathrm{ft}^{3 / / 8}\right)\end{array}$} & \multicolumn{4}{|c|}{$\begin{array}{l}\text { Maximum average discharge } \\
\qquad\left(\mathrm{ft}^{3} / \mathrm{s}\right)\end{array}$} \\
\hline & & & 3-day period & 7-day period & 15-day period & 30-day perlod \\
\hline 0.99 & 1.01 & $\mathrm{ng}$ & 250 & 202 & 165 & 133 \\
\hline 0.95 & 1.05 & 870 & 562 & 465 & 372 & 299 \\
\hline 0.90 & 1.11 & 1,160 & 833 & 697 & 552 & 442 \\
\hline 0.80 & 1.25 & 1,620 & 1,300 & 1,100 & 861 & 682 \\
\hline 0.50 & 2 & 2,980 & 2,750 & $2, \overline{380}$ & 1,830 & 1,400 \\
\hline 0.20 & 5 & 5,270 & 5,140 & 4,550 & 3,430 & 2,500 \\
\hline 0.10 & 10 & 6,990 & 6,800 & 6,090 & 4,550 & 3,220 \\
\hline 0.04 & 25 & 9,340 & 8,870 & 8,040 & 5,960 & 4,070 \\
\hline 0.02 & 50 & 11,200 & 10,400 & 9,450 & 6,960 & 4,650 \\
\hline 0.01 & 100 & 13,100 & 11,800 & 10,800 & 7,930 & 5,180 \\
\hline 0.005 & 200 & 15,100 & 13,100 & 12,100 & 8,850 & 5,670 \\
\hline 0.002 & 500 & 17,900 & ng & $\mathrm{ng}$ & ng & $\mathrm{ng}$ \\
\hline
\end{tabular}

Probability of annual low discharges, post-regulation period

\begin{tabular}{|c|c|c|c|c|c|c|c|c|c|c|}
\hline \multirow[b]{3}{*}{$\begin{array}{l}\text { Non- } \\
\text { exceed- } \\
\text { ance } \\
\text { prob- } \\
\text { ability }\end{array}$} & \multirow[b]{3}{*}{$\begin{array}{c}\text { Recur- } \\
\text { rence } \\
\text { Inter- } \\
\text { val } \\
\text { (years) }\end{array}$} & \multicolumn{9}{|c|}{ Minimum average discharge $\left(\mathrm{ft}^{3} / \mathrm{s}\right)$} \\
\hline & & \multicolumn{9}{|c|}{ Number of consecutive days } \\
\hline & & 1 & 3 & 7 & 14 & 30 & 60 & 90 & 120 & 183 \\
\hline 0.05 & 20 & 0.336 & 0.384 & 0.434 & 0.596 & 1.01 & 1.90 & 3.13 & 6.78 & 10.5 \\
\hline 0.10 & 10 & 1.18 & 1.31 & 1.47 & 1.88 & 2.92 & 5.18 & 7.56 & 12.6 & 17.1 \\
\hline 0.20 & 5 & 4.14 & 4.50 & 4.96 & 5.92 & 8.40 & 13.5 & 17.7 & 23.4 & 28.8 \\
\hline 0.50 & 2 & 22.0 & 23.1 & 25.0 & 27.3 & 32.8 & 42.2 & 49.2 & 54.3 & 64.5 \\
\hline
\end{tabular}




\section{WILD RICE RIVER AT HENDRUM, MN-Continued}

Probability of seasonal low discharges, post-regulation period

[ng, statistic not given]

\begin{tabular}{|c|c|c|c|c|c|c|c|c|c|}
\hline \multirow[b]{3}{*}{$\begin{array}{c}\text { Non- } \\
\text { exceedance } \\
\text { probability }\end{array}$} & \multirow[b]{3}{*}{$\begin{array}{c}\text { Recurrence } \\
\text { interval } \\
\text { (years) }\end{array}$} & \multicolumn{8}{|c|}{ Minimum average discharge $\left(\mathrm{ft}^{3} / \mathrm{s}\right)$} \\
\hline & & \multicolumn{8}{|c|}{ Number of consecutive days } \\
\hline & & 1 & 7 & 14 & 30 & 1 & 7 & 14 & 30 \\
\hline & & \multicolumn{4}{|c|}{ December-January-February } & \multicolumn{4}{|c|}{ March-April-May } \\
\hline 0.05 & 20 & $\mathrm{ng}$ & ng & $\mathrm{ng}$ & $\mathrm{ng}$ & 5.02 & 5.46 & 6.70 & 28.0 \\
\hline 0.10 & 10 & ng & $\mathrm{ng}$ & $\mathrm{ng}$ & $\mathrm{ng}$ & 11.5 & 12.0 & 13.5 & 38.6 \\
\hline 0.20 & 5 & $\mathrm{ng}$ & ng & ng & $\mathrm{ng}$ & 25.1 & 25.5 & 27.4 & 57.2 \\
\hline \multirow[t]{2}{*}{0.50} & 2 & ng & $\mathrm{ng}$ & $\mathrm{ng}$ & $\mathrm{ng}$ & 59.8 & 64.7 & 70.7 & 123 \\
\hline & & \multicolumn{4}{|c|}{ June-July-August } & \multicolumn{4}{|c|}{ September-October-November } \\
\hline 0.05 & 20 & 0.873 & 1.09 & 1.66 & 3.99 & 1.07 & 1.44 & 1.86 & 4.11 \\
\hline 0.10 & 10 & 2.63 & 3.26 & 4.43 & 8.38 & 3.28 & 4.10 & 5.00 & 8.52 \\
\hline 0.20 & 5 & 8.11 & 9.89 & 12.2 & 18.7 & 9.70 & 11.5 & 13.3 & 18.2 \\
\hline 0.50 & 2 & 38.7 & 45.2 & 50.9 & 65.2 & 36.7 & 43.2 & 47.2 & 54.6 \\
\hline
\end{tabular}




\section{WILD RICE RIVER AT HENDRUM, MN-Continued}

Annual peak discharge and correspoinding gage height, period of record

$[--$, no data]

\begin{tabular}{|c|c|c|c|c|c|c|c|}
\hline $\begin{array}{l}\text { Water } \\
\text { year }\end{array}$ & Date & $\begin{array}{c}\text { Gage } \\
\text { height } \\
\text { (feet) }\end{array}$ & $\begin{array}{c}\text { Peak } \\
\text { discharge } \\
\left(\mathrm{ft}^{3} / \mathrm{s}\right)\end{array}$ & $\begin{array}{l}\text { Water } \\
\text { year }\end{array}$ & Date & $\begin{array}{c}\text { Gage } \\
\text { height } \\
\text { (foet) }\end{array}$ & $\begin{array}{c}\text { Peak } \\
\text { discharge } \\
\left(f^{3} / s\right)\end{array}$ \\
\hline \multicolumn{8}{|c|}{ Annual peak dlscharge, by year, and corresponding gage height } \\
\hline 1944 & July 15 & 16.07 & 2,260 & 1970 & June 20 & 22.41 & 3,940 \\
\hline 1945 & March 18 & - & 2,200 & 1971 & April 10 & 13.20 & 1,500 \\
\hline 1946 & March 23 & 20.56 & 2,600 & 1972 & April 15 & 23.24 & 4,550 \\
\hline 1947 & April 15 & 27.70 & 4,200 & 1973 & September 6 & 13.03 & 1,630 \\
\hline 1948 & April 8 & 20.13 & 2,200 & 1974 & April 16 & 26.21 & 5,590 \\
\hline 1949 & July 11 & - & 738 & 1975 & July 5 & 30.91 & 7,660 \\
\hline 1950 & May 10 & - & 3,000 & 1976 & March 30 & 20.22 & 2,120 \\
\hline 1951 & April 7 & - & 2,570 & 1977 & April 11 & 5.55 & 245 \\
\hline 1952 & April 11 & - & 2,860 & 1978 & April 10 & 31.42 & 9,350 \\
\hline 1953 & June 18 & 16.88 & 1,650 & 1979 & April 21 & 32.30 & 8,800 \\
\hline 1954 & April 12 & 15.26 & 1,940 & 1980 & April 5 & 17.36 & 1,800 \\
\hline 1955 & April 5 & 15.99 & 1,850 & 1981 & May 24 & 14.50 & 1,840 \\
\hline 1956 & April 14 & 24.26 & 4,660 & 1982 & April 3 & 22.51 & 3,280 \\
\hline 1957 & September 4 & - & 1,250 & 1983 & July 5 & 15.12 & 2,290 \\
\hline 1958 & July 7 & 8.16 & 633 & 1984 & June 11 & 25.00 & 5,400 \\
\hline 1959 & April 6 & 8.92 & 540 & 1985 & May 16 & 25.14 & 5,230 \\
\hline 1960 & April 8 & 16.48 & 1,600 & 1986 & March 31 & 23.10 & 3,850 \\
\hline 1961 & May 17 & 10.66 & 1,080 & 1987 & July 25 & 14.26 & 1,500 \\
\hline 1962 & June 13 & 22.26 & 3,680 & 1988 & April 8 & 11.30 & 1,190 \\
\hline 1963 & June 3 & 13.89 & 1,670 & 1989 & April 7 & 29.60 & 5,480 \\
\hline 1964 & April 23 & 17.55 & 2,690 & 1990 & April 1 & 14.26 & 1,100 \\
\hline 1965 & April 14 & 29.44 & 6,800 & 1991 & May 6 & 10.27 & 952 \\
\hline 1966 & March 31 & 28.30 & 4,120 & 1992 & July 3 & 14.70 & 1,950 \\
\hline 1967 & April 1 & 20.57 & 3,250 & 1993 & August 1 & - & 3,680 \\
\hline 1968 & March 29 & 11.26 & 726 & 1994 & April 3 & 20.30 & 2,600 \\
\hline 1969 & April 15 & 31.42 & 8,300 & & & & \\
\hline \multicolumn{8}{|c|}{ Annual peak discharge, from highest to lowest, and corresponding gage height } \\
\hline 1978 & April 10 & 31.42 & 9,350 & 1982 & April 3 & 22.51 & 3,280 \\
\hline 1979 & April 21 & 32.30 & 8,800 & 1967 & April 1 & 20.57 & 3,250 \\
\hline 1969 & April 15 & 31.42 & 8,300 & 1950 & May 10 & -- & 3,000 \\
\hline 1975 & July 5 & 30.91 & 7,660 & 1952 & April 11 & -- & 2,860 \\
\hline 1965 & April 14 & 29.44 & 6,800 & 1964 & April 23 & 17.55 & 2,690 \\
\hline 1974 & April 16 & 26.21 & 5,590 & 1946 & March 23 & 20.56 & 2,600 \\
\hline 1989 & April 7 & 29.60 & 5,480 & 1994 & April 3 & 20.30 & 2,600 \\
\hline 1984 & June 11 & 25.00 & 5,400 & 1951 & April 7 & - & 2,570 \\
\hline 1985 & May 16 & 25.14 & 5,230 & 1983 & July 5 & 15.12 & 2,290 \\
\hline 1956 & April 14 & 24.26 & 4,660 & 1944 & July 15 & 16.07 & 2,260 \\
\hline 1972 & April 15 & 23.24 & 4,550 & 1945 & March 18 & -- & 2,200 \\
\hline 1947 & April 15 & 27.70 & 4,200 & 1948 & April 8 & 20.13 & 2,200 \\
\hline 1966 & March 31 & 28.30 & 4,120 & 1976 & March 30 & 20.22 & 2,120 \\
\hline 1970 & June 20 & 22.41 & 3,940 & 1992 & July 3 & 14.70 & 1,950 \\
\hline 1986 & March 31 & 23.10 & 3,850 & 1954 & April 12 & 15.26 & 1,940 \\
\hline 1962 & June 13 & 22.26 & 3,680 & 1955 & April 5 & 15.99 & 1,850 \\
\hline 1993 & August 1 & - & 3,680 & 1981 & May 24 & 14.50 & 1,840 \\
\hline
\end{tabular}


05064000 WILD RICE RIVER AT HENDRUM, MN-Continued

Annual peak discharge and correspoinding gage height, period of record--Continued $[--$, no data $]$

\begin{tabular}{llllllll}
\hline $\begin{array}{l}\text { Water } \\
\text { year }\end{array}$ & Date & $\begin{array}{c}\text { Gage } \\
\text { height } \\
\text { (feet) }\end{array}$ & $\begin{array}{c}\text { Peak } \\
\text { discharge } \\
\left(\mathbf{f t}^{3} / \mathbf{s}\right)\end{array}$ & $\begin{array}{c}\text { Water } \\
\text { year }\end{array}$ & Date & $\begin{array}{c}\text { Gage } \\
\text { height } \\
\text { (feet) }\end{array}$ & $\begin{array}{c}\text { Peak } \\
\text { discharge } \\
\left(\mathbf{f t}^{3} / \mathbf{s}\right)\end{array}$ \\
\hline \multicolumn{7}{c}{ Annual peak discharge, from highest to lowest, and corresponding gage height-.Continued } \\
1980 & April 5 & 17.36 & 1,800 & 1990 & April 1 & 14.26 & 1,100 \\
1963 & June 3 & 13.89 & 1,670 & 1961 & May 17 & 10.66 & 1,080 \\
1953 & June 18 & 16.88 & 1,650 & 1991 & May 6 & 10.27 & 952 \\
1973 & September 6 & 13.03 & 1,630 & 1949 & July 11 & - & 738 \\
1960 & April 8 & 16.48 & 1,600 & 1968 & March 29 & 11.26 & 726 \\
1971 & April 10 & 13.20 & 1,500 & 1958 & July 7 & 8.16 & 633 \\
1987 & July 25 & 14.26 & 1,500 & 1959 & April 6 & 8.92 & 540 \\
1957 & September 4 & - & 1,250 & 1977 & April 11 & 5.55 & 245 \\
1988 & April 8 & 11.30 & 1,190 & & & & \\
\hline
\end{tabular}




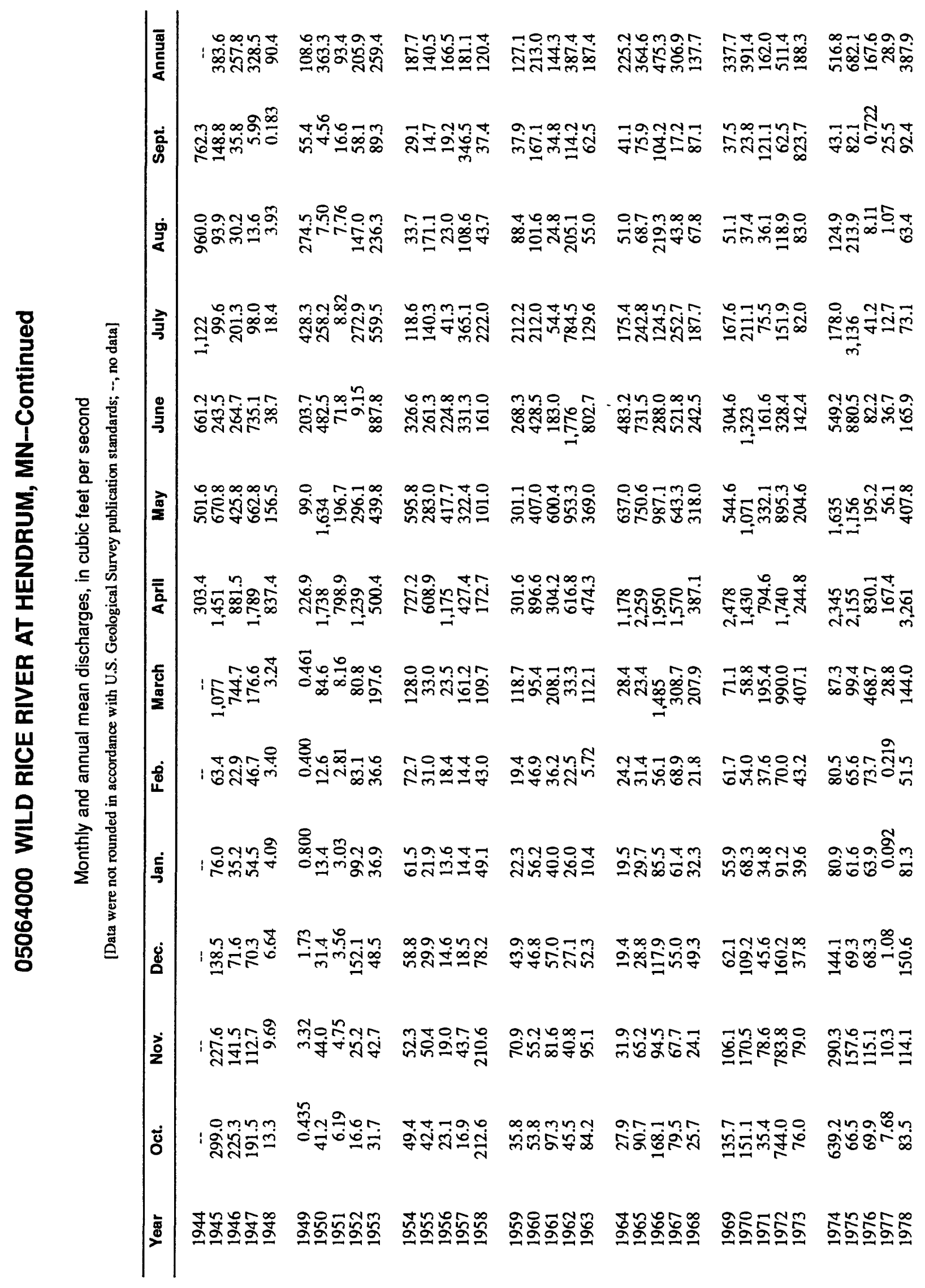




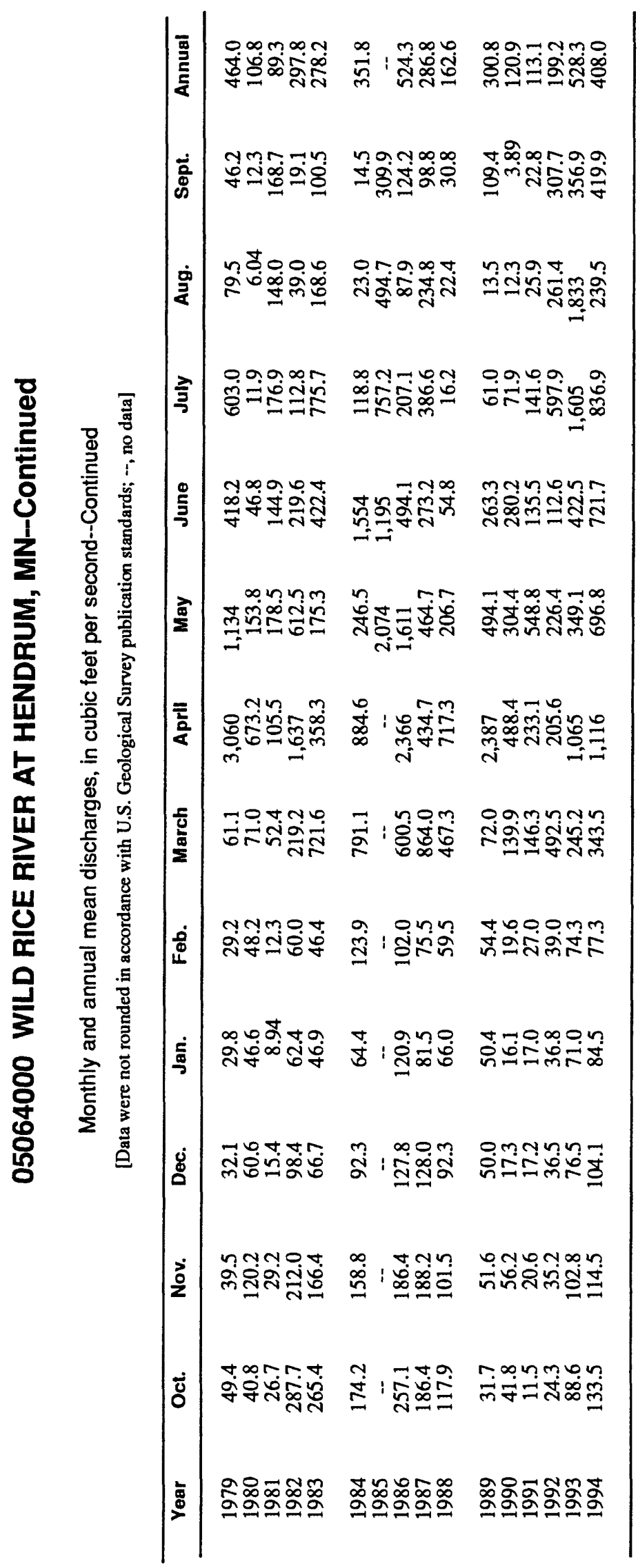




\section{RED RIVER OF THE NORTH AT HALSTAD, MN}

\section{Station Description}

LOCATION.--Lat $47^{\circ} 21^{\prime} 10^{\prime \prime}$, long 96 $50^{\prime} 50^{\prime \prime}$, on line between secs.24 and 25, T.145 N., R.49 W., Traill County, Hydrologic Unit 09020107, on left bank on upstream side of highway bridge, $0.5 \mathrm{mi}$ west of Halstad, $2.5 \mathrm{mi}$ downstream from Wild Rice River, and at mile 375.2.

DRAINAGE AREA.--21,800 $\mathrm{mi}^{2}$, approximately, including $3,800 \mathrm{mi}^{2}$ in closed basins.

PERIOD OF RECORD.--April 1936 to June 1937 (no winter records), April 1942 to September 1960 (spring and summer months only), May 1961 to current year.

GAGE.--Water-stage recorder. Datum of gage is $826.65 \mathrm{ft}$ above sea level. Prior to July 17, 1961, nonrecording gage at present site and datum.

EXTREMES FOR PERIOD OF RECORD.--Maximum discharge, 42,000 $\mathrm{ft}^{3} / \mathrm{s}$, Apr. 22, 1979 (gage height, $39.0 \mathrm{ft}$ ); minimum discharge, $5.4 \mathrm{ft}^{3} / \mathrm{s}$, Oct. 8, 9, and 12-14, 1936.

EXTREMES OUTSIDE PERIOD OF RECORD.--Flood in 1897 reached a stage of about $38.5 \mathrm{ft}$.

Annual mean discharge

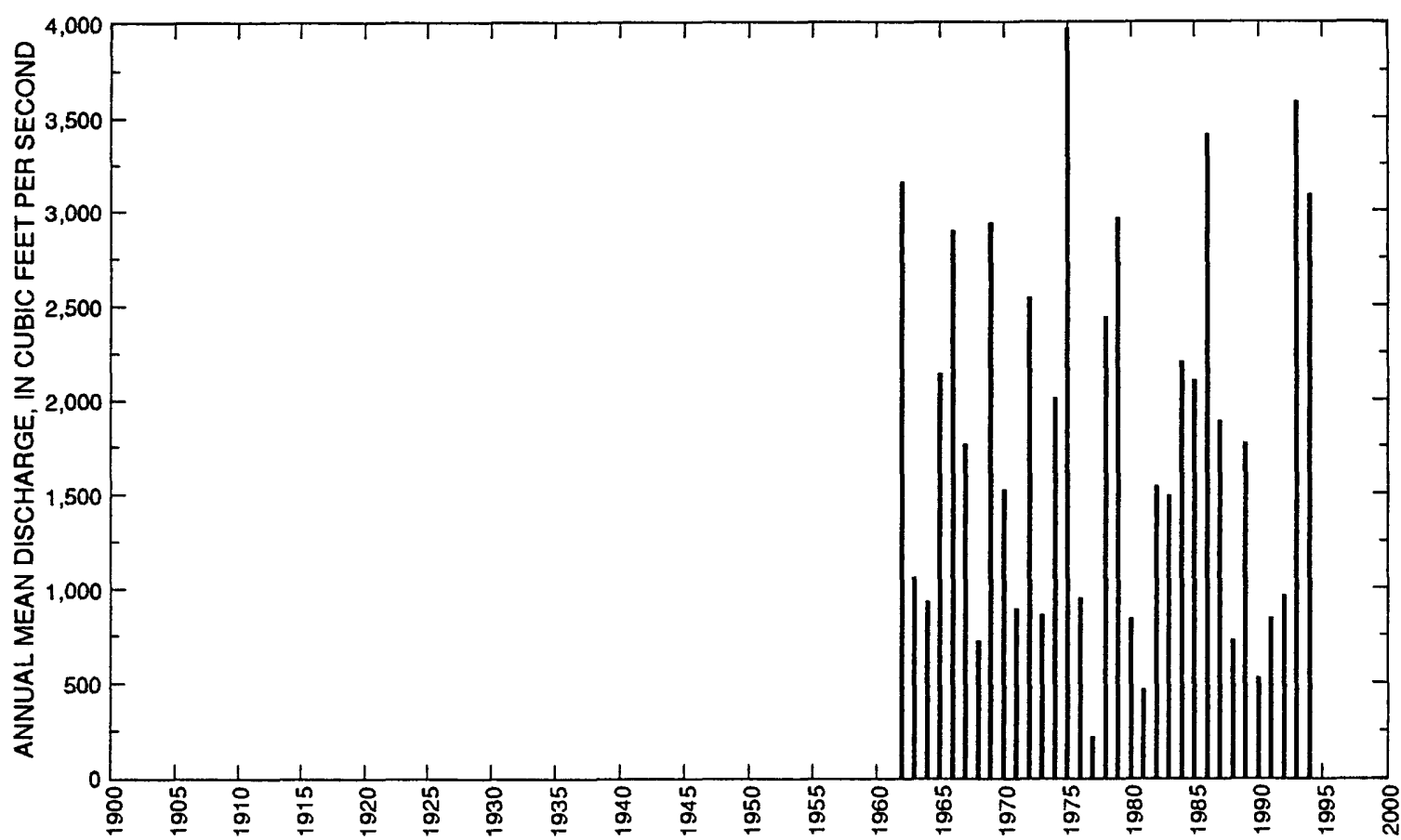


05064500 RED RIVER OF THE NORTH AT HALSTAD, MN--Continued

Statistics of monthly and annual mean discharges

\begin{tabular}{|c|c|c|c|c|c|c|c|c|}
\hline \multirow[b]{2}{*}{ Month } & \multicolumn{2}{|c|}{ Maximum } & \multicolumn{2}{|c|}{ Minimum } & \multicolumn{4}{|c|}{ Mean } \\
\hline & $\begin{array}{c}\text { Discharge } \\
\left(\mathrm{ft}^{3} / \mathrm{s}\right)\end{array}$ & $\begin{array}{c}\text { Water year } \\
\text { of } \\
\text { occurrence }\end{array}$ & $\begin{array}{c}\text { Discharge } \\
\left(\mathrm{ft}^{3 / \mathrm{s}}\right)\end{array}$ & $\begin{array}{c}\text { Water year } \\
\text { of } \\
\text { occurrence }\end{array}$ & $\begin{array}{c}\text { Discharge } \\
\left(\mathrm{ft}^{3} / \mathrm{s}\right)\end{array}$ & $\begin{array}{c}\text { Standard } \\
\text { deviation } \\
\left(\mathrm{ft}^{3} / \mathrm{s}\right)\end{array}$ & $\begin{array}{l}\text { Coeffl- } \\
\text { cient of } \\
\text { variation }\end{array}$ & $\begin{array}{l}\text { Percentage } \\
\text { of annual } \\
\text { discharge }\end{array}$ \\
\hline October & 2,190 & 1987 & 61.5 & 1977 & 704 & 540 & 0.77 & 3.29 \\
\hline November & 1,770 & 1972 & 92.3 & 1977 & 650 & 402 & 0.62 & 3.04 \\
\hline December & 1,250 & 1987 & 51.2 & 1977 & 513 & 325 & 0.63 & 2.40 \\
\hline January & 1,020 & 1987 & 32.1 & 1977 & 432 & 275 & 0.64 & 2.02 \\
\hline February & 1,050 & 1987 & 45.9 & 1977 & 445 & 252 & 0.57 & 2.08 \\
\hline March & 9,430 & 1966 & 249 & 1962 & 2,050 & 2,060 & 1.00 & 9.60 \\
\hline April & 20,100 & 1969 & 705 & 1981 & 6,620 & 5,280 & 0.80 & 31.0 \\
\hline May & 8,990 & 1979 & 449 & 1977 & 3,070 & 2,270 & 0.74 & 14.3 \\
\hline June & 10,300 & 1962 & 242 & 1977 & 2,530 & 1,990 & 0.79 & 11.8 \\
\hline July & 20,100 & 1975 & 153 & 1988 & 2,550 & 3,890 & 1.52 & 11.9 \\
\hline August & 11,700 & 1993 & 59.5 & 1977 & 1,110 & 2,010 & 1.81 & 5.19 \\
\hline September & 3,360 & 1993 & 38.4 & 1976 & 712 & 702 & 0.99 & 3.33 \\
\hline Annual & 3,970 & 1975 & 214 & 1977 & 1,800 & 1,030 & 0.57 & 100 \\
\hline
\end{tabular}

Annual flow duration

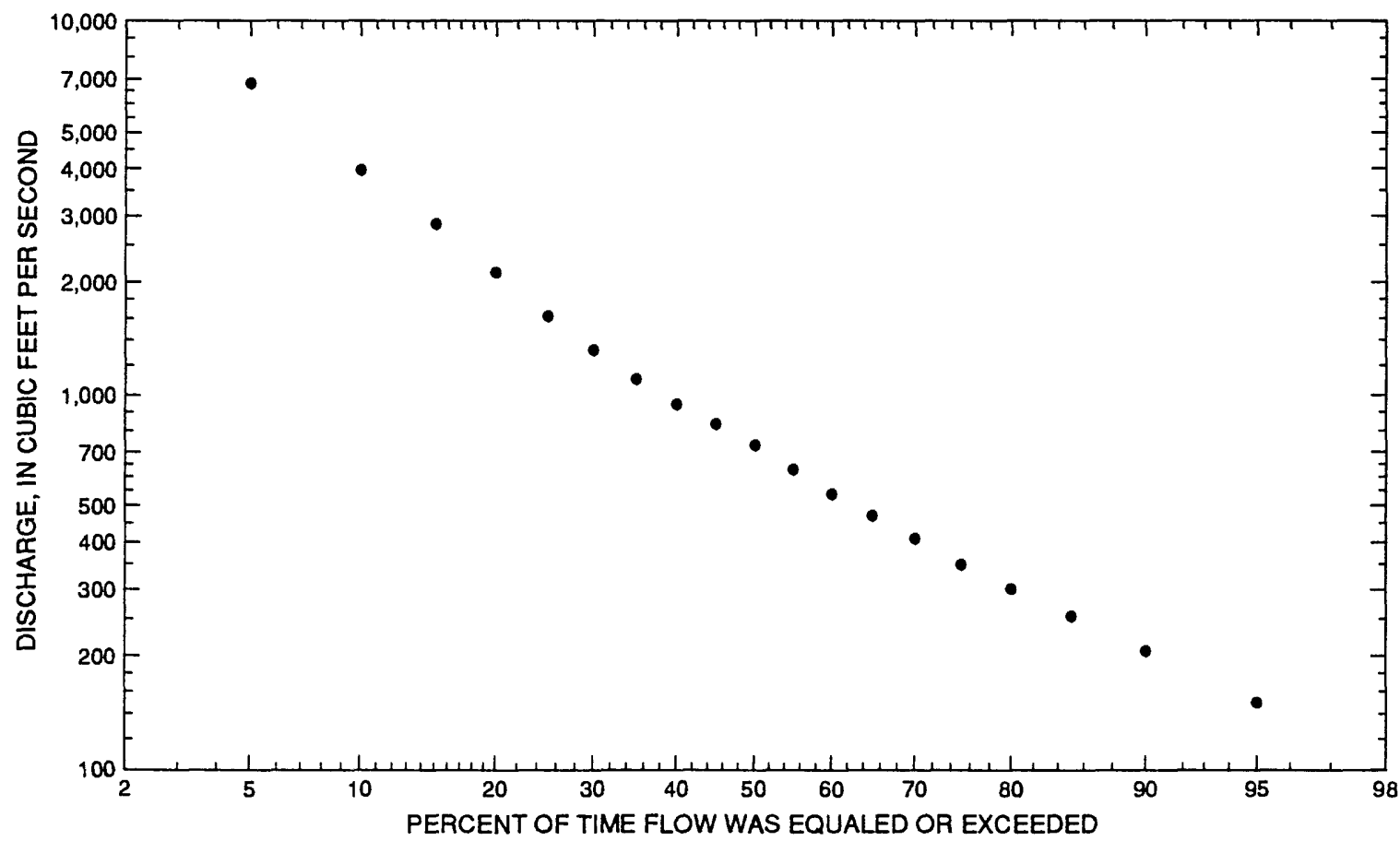




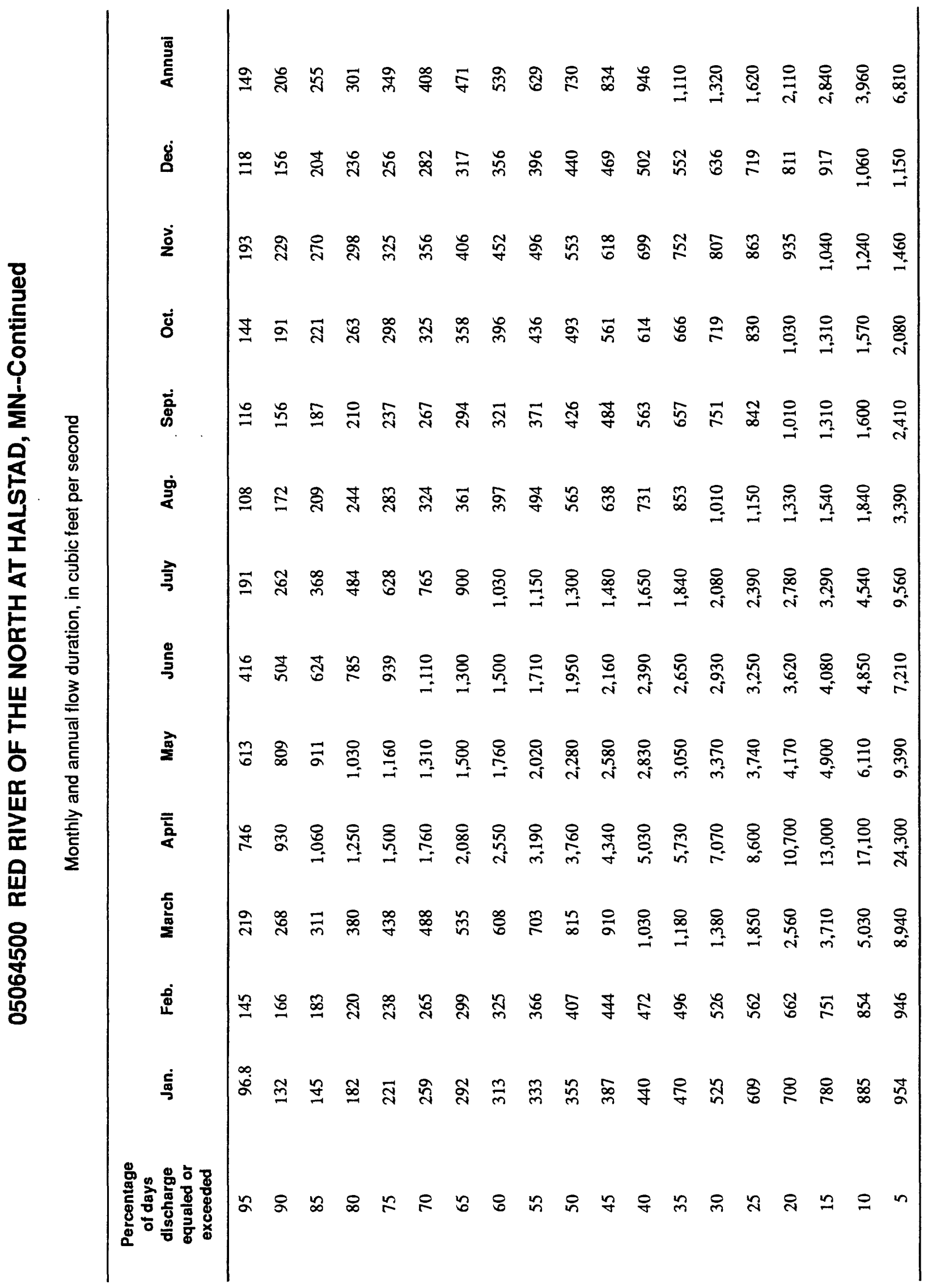


05064500 RED RIVER OF THE NORTH AT HALSTAD, MN--Continued

Probability of annual high discharges

[ng, statistic not given]

\begin{tabular}{|c|c|c|c|c|c|c|}
\hline \multirow[b]{2}{*}{$\begin{array}{l}\text { Exceedance } \\
\text { probability }\end{array}$} & \multirow[b]{2}{*}{$\begin{array}{l}\text { Recurrence } \\
\text { interval } \\
\text { (years) }\end{array}$} & \multirow[b]{2}{*}{$\begin{array}{c}\text { Maximum } \\
\text { Instantaneous } \\
\left(\mathrm{ft}^{3} / \mathrm{s}\right)\end{array}$} & \multicolumn{4}{|c|}{$\begin{array}{l}\text { Maximum average discharge } \\
\left(\mathrm{ft}^{3} / \mathrm{s}\right)\end{array}$} \\
\hline & & & 3-day perlod & 7-day perlod & 15-day period & 30-day period \\
\hline 0.99 & 1.01 & 1,420 & 1,090 & 841 & 691 & 546 \\
\hline 0.95 & 1.05 & 2,690 & 2,300 & 1,910 & 1,570 & 1,260 \\
\hline 0.90 & 1.11 & 3,720 & 3,350 & 2,870 & 2,370 & 1,910 \\
\hline 0.80 & 1.25 & 5,430 & 5,170 & 4,580 & 3,790 & 3,050 \\
\hline 0.50 & 2 & 10,700 & 11,100 & 10,400 & 8,710 & 6,840 \\
\hline 0.20 & 5 & 20,000 & 22,100 & 21,200 & 18,200 & 13,700 \\
\hline 0.10 & 10 & 27,000 & 30,600 & 29,700 & 25,900 & 18,900 \\
\hline 0.04 & 25 & 36,700 & 42,400 & 41,300 & 36,700 & 25,800 \\
\hline 0.02 & 50 & 44,400 & 51,700 & 50,400 & 45,300 & 31,000 \\
\hline 0.01 & 100 & 52,400 & 61,300 & 59,800 & 54,300 & 36,200 \\
\hline 0.005 & 200 & 60,600 & 71,200 & 69,300 & 63,700 & 41,400 \\
\hline 0.002 & 500 & 71,900 & $\mathrm{ng}$ & $\mathrm{ng}$ & $\mathrm{ng}$ & $\mathrm{ng}$ \\
\hline
\end{tabular}

Probability of annual low discharges

\begin{tabular}{|c|c|c|c|c|c|c|c|c|c|c|}
\hline \multirow{3}{*}{$\begin{array}{l}\text { Non- } \\
\text { exceed- } \\
\text { ance } \\
\text { prob- } \\
\text { ablity }\end{array}$} & \multirow{3}{*}{$\begin{array}{l}\text { Recur- } \\
\text { rence } \\
\text { inter- } \\
\text { val } \\
\text { (years) }\end{array}$} & \multicolumn{9}{|c|}{ Minimum average discharge $\left(\mathrm{ft}^{3} / \mathrm{s}\right)$} \\
\hline & & \multicolumn{9}{|c|}{ Number of consecutive days } \\
\hline & & 1 & 3 & 7 & 14 & 30 & 60 & 90 & 120 & 183 \\
\hline 0.05 & 20 & 34.3 & 37.1 & 44.3 & 53.2 & 65.0 & 81.4 & 97.8 & 112 & 124 \\
\hline 0.10 & 10 & 56.1 & 59.2 & 67.3 & 77.7 & 92.5 & 114 & 137 & 156 & 172 \\
\hline 0.20 & 5 & 95.8 & 98.6 & 107 & 119 & 138 & 167 & 199 & 225 & 250 \\
\hline 0.50 & 2 & 223 & 225 & 233 & 247 & 273 & 318 & 370 & 413 & 468 \\
\hline
\end{tabular}


05064500 RED RIVER OF THE NORTH AT HALSTAD, MN--Continued

Probability of seasonal low discharges

\begin{tabular}{|c|c|c|c|c|c|c|c|c|c|}
\hline \multirow[b]{3}{*}{$\begin{array}{c}\text { Non- } \\
\text { exceedance } \\
\text { probability }\end{array}$} & \multirow[b]{3}{*}{$\begin{array}{c}\text { Recurrence } \\
\text { interval } \\
\text { (years) }\end{array}$} & \multicolumn{8}{|c|}{ Minimum average discharge $\left(\mathrm{ft}^{3} / \mathrm{s}\right)$} \\
\hline & & \multicolumn{8}{|c|}{ Number of consecutive days } \\
\hline & & 1 & 7 & 14 & 30 & 1 & 7 & 14 & 30 \\
\hline & & \multicolumn{4}{|c|}{ December-January-February } & \multicolumn{4}{|c|}{ March-April-May } \\
\hline 0.05 & 20 & 66.6 & 68.5 & 69.8 & 78.4 & 128 & 136 & 160 & 273 \\
\hline 0.10 & 10 & 98.9 & 102 & 105 & 115 & 177 & 190 & 217 & 347 \\
\hline 0.20 & 5 & 153 & 159 & 165 & 176 & 253 & 274 & 306 & 472 \\
\hline \multirow[t]{2}{*}{0.50} & 2 & 307 & 322 & 335 & 348 & 454 & 501 & 546 & 891 \\
\hline & & \multicolumn{4}{|c|}{ June-July-August } & \multicolumn{4}{|c|}{ September-October-November } \\
\hline 0.05 & 20 & 45.5 & 66.0 & 82.2 & 108 & 44.9 & 69.8 & 79.0 & 97.1 \\
\hline 0.10 & 10 & 78.8 & 103 & 123 & 159 & 72.9 & 97.3 & 108 & 134 \\
\hline 0.20 & 5 & 145 & 172 & 196 & 249 & 123 & 144 & 158 & 193 \\
\hline 0.50 & 2 & 394 & 421 & 458 & 568 & 282 & 292 & 313 & 373 \\
\hline
\end{tabular}


05064500 RED RIVER OF THE NORTH AT HALSTAD, MN-Continued

Annual peak discharge and corresponding gage height, period of record

$[--$, no data]

\begin{tabular}{|c|c|c|c|c|c|c|c|}
\hline $\begin{array}{l}\text { Water } \\
\text { year }\end{array}$ & Date & $\begin{array}{c}\text { Gage } \\
\text { height } \\
\text { (feet) }\end{array}$ & $\begin{array}{c}\text { Peak } \\
\text { discharge } \\
\left(\mathrm{ft}^{3} / \mathrm{s}\right)\end{array}$ & $\begin{array}{l}\text { Water } \\
\text { year }\end{array}$ & Date & $\begin{array}{c}\text { Gage } \\
\text { helght } \\
\text { (feet) }\end{array}$ & $\begin{array}{c}\text { Peak } \\
\text { discharge } \\
\left(\mathrm{ft}^{3} / \mathrm{s}\right)\end{array}$ \\
\hline \multicolumn{8}{|c|}{ Annual peak discharge, by year, and corresponding gage height } \\
\hline 1936 & April 15 & 16.33 & 7,670 & 1968 & June 19 & 7.80 & 2,350 \\
\hline 1937 & April 15 & 9.39 & 2,660 & 1969 & April 18 & 38.29 & 35,700 \\
\hline 1942 & May 5 & 12.86 & 5,060 & 1970 & April 10 & 22.36 & 11,600 \\
\hline 1943 & April 11 & 31.31 & 21,800 & 1971 & April 1 & 15.62 & 5,480 \\
\hline 1944 & July 13 & 15.79 & 7,200 & 1972 & $\operatorname{March} 24$ & 28.96 & 16,200 \\
\hline 1945 & March 23 & 23.60 & 13,300 & 1973 & March 18 & 17.71 & 6,200 \\
\hline 1946 & March 29 & 19.50 & 10,000 & 1974 & April 16 & 26.72 & 17,800 \\
\hline 1947 & April 16 & 33.50 & 24,500 & 1975 & July 10 & 38.55 & 39,900 \\
\hline 1948 & April 10 & - & 16,000 & 1976 & March 31 & 23.30 & 9,950 \\
\hline 1949 & April 7 & 16.53 & 7,710 & 1977 & May 7 & 7.50 & 2,050 \\
\hline 1950 & May 11 & - & 18,700 & 1978 & April 9 & 37.61 & 28,800 \\
\hline 1951 & April 10 & 22.43 & 12,900 & 1979 & April 22 & 39.00 & 42,000 \\
\hline 1952 & April 18 & 29.78 & 20,700 & 1980 & April 5 & 21.98 & 12,900 \\
\hline 1953 & June 22 & 22.78 & 13,600 & 1981 & May 25 & 10.57 & 3,920 \\
\hline 1954 & April 13 & 11.44 & 4,660 & 1982 & April 9 & 27.13 & 13,200 \\
\hline 1955 & April 6 & 19.28 & 7,200 & 1983 & July 6 & 14.98 & 7,800 \\
\hline 1956 & April 15 & 23.67 & 12,900 & 1984 & April 1 & 29.99 & 21,900 \\
\hline 1957 & June 24 & 12.20 & 4,980 & 1985 & May 13 & 19.07 & 10,400 \\
\hline 1958 & July 8 & 11.31 & 4,420 & 1986 & March 31 & 25.89 & 17,400 \\
\hline 1959 & June 13 & 10.13 & 3,780 & 1987 & March 30 & 21.43 & 9,860 \\
\hline 1960 & April 10 & 21.66 & 8,600 & 1988 & March 28 & 12.42 & 5,010 \\
\hline 1961 & May 22 & 6.96 & 1,900 & 1989 & April 9 & 35.65 & 26,000 \\
\hline 1962 & June 16 & 24.70 & 15,900 & 1990 & April 10 & 8.55 & 2,880 \\
\hline 1963 & June 16 & 13.14 & 5,850 & 1991 & July 8 & 9.99 & 3,700 \\
\hline 1964 & April 23 & 15.27 & 7,820 & 1992 & March 9 & 15.64 & 5,200 \\
\hline 1965 & April 17 & 35.27 & 25,600 & 1993 & August 2 & 30.56 & 22,500 \\
\hline 1966 & March 27 & 35.35 & 26,800 & 1994 & April 3 & - & 16,600 \\
\hline 1967 & April 23 & 22.71 & 13,800 & & & & \\
\hline \multicolumn{8}{|c|}{ Annual peak discharge, from highest to lowest, and corresponding gage height } \\
\hline 1979 & April 22 & 39.00 & 42,000 & 1946 & March 29 & 19.50 & 10,000 \\
\hline 1975 & July 10 & 38.55 & 39,900 & 1976 & March 31 & 23.30 & 9,950 \\
\hline 1969 & April 18 & 38.29 & 35,700 & 1987 & March 30 & 21.43 & 9,860 \\
\hline 1978 & April 9 & 37.61 & 28,800 & 1960 & April 10 & 21.66 & 8,600 \\
\hline 1966 & March 27 & 35.35 & 26,800 & 1964 & April 23 & 15.27 & 7,820 \\
\hline 1989 & April 9 & 35.65 & 26,000 & 1983 & July 6 & 14.98 & 7,800 \\
\hline 1965 & April 17 & 35.27 & 25,600 & 1949 & April 7 & 16.53 & 7,710 \\
\hline 1947 & April 16 & 33.50 & 24,500 & 1936 & April 15 & 16.33 & 7,670 \\
\hline 1993 & August 2 & 30.56 & 22,500 & 1944 & July 13 & 15.79 & 7,200 \\
\hline 1984 & April 1 & 29.99 & 21,900 & 1955 & April 6 & 19.28 & 7,200 \\
\hline 1943 & April 11 & 31.31 & 21,800 & 1973 & March 18 & 17.71 & 6,200 \\
\hline 1952 & April 18 & 29.78 & 20,700 & 1963 & June 16 & 13.14 & 5,850 \\
\hline 1950 & May 11 & - & 18,700 & 1971 & April 1 & 15.62 & 5,480 \\
\hline 1974 & April 16 & 26.72 & 17,800 & 1992 & March 9 & 15.64 & 5,200 \\
\hline 1986 & March 31 & 25.89 & 17,400 & 1942 & May 5 & 12.86 & 5,060 \\
\hline
\end{tabular}




\section{RED RIVER OF THE NORTH AT HALSTAD, MN--Continued}

Annual peak discharge and corresponding gage height, period of record--Continued

$[-$, no data $]$

\begin{tabular}{|c|c|c|c|c|c|c|c|}
\hline $\begin{array}{l}\text { Water } \\
\text { year }\end{array}$ & Date & $\begin{array}{c}\text { Gage } \\
\text { helght } \\
\text { (feet) }\end{array}$ & $\begin{array}{c}\text { Peak } \\
\text { discharge } \\
\left(\mathrm{ft}^{3} / \mathrm{s}\right)\end{array}$ & $\begin{array}{l}\text { Water } \\
\text { year }\end{array}$ & Date & $\begin{array}{c}\text { Gage } \\
\text { height } \\
\text { (feet) }\end{array}$ & $\begin{array}{c}\text { Peak } \\
\text { discharge } \\
\left(\mathrm{ft}^{3} / \mathrm{s}\right)\end{array}$ \\
\hline \multicolumn{8}{|c|}{ Annual peak discharge, from highest to lowest, and corresponding gage height--Continued } \\
\hline 1994 & April 3 & - & 16,600 & 1988 & $\operatorname{March} 28$ & 12.42 & 5,010 \\
\hline 1972 & March 24 & 28.96 & 16,200 & 1957 & June 24 & 12.20 & 4,980 \\
\hline 1948 & April 10 & - & 16,000 & 1954 & April 13 & 11.44 & 4,660 \\
\hline 1962 & June 16 & 24.70 & 15,900 & 1958 & July 8 & 11.31 & 4,420 \\
\hline 1967 & April 23 & 22.71 & 13,800 & 1981 & May 25 & 10.57 & 3,920 \\
\hline 1953 & June 22 & 22.78 & 13,600 & 1959 & June 13 & 10.13 & 3,780 \\
\hline 1945 & March 23 & 23.60 & 13,300 & 1991 & July 8 & 9.99 & 3,700 \\
\hline 1982 & April 9 & 27.13 & 13,200 & 1990 & April 10 & 8.55 & 2,880 \\
\hline 1951 & April 10 & 22.43 & 12,900 & 1937 & April 15 & 9.39 & 2,660 \\
\hline 1956 & April 15 & 23.67 & 12,900 & 1968 & June 19 & 7.80 & 2,350 \\
\hline 1980 & April 5 & 21.98 & 12,900 & 1977 & May 7 & 7.50 & 2,050 \\
\hline 1970 & April 10 & 22.36 & 11,600 & 1961 & May 22 & 6.96 & 1,900 \\
\hline 1985 & May 13 & 19.07 & 10,400 & & & & \\
\hline
\end{tabular}




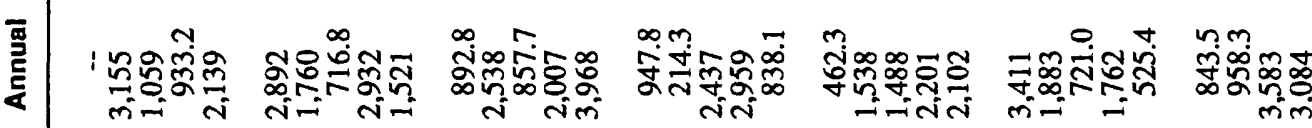

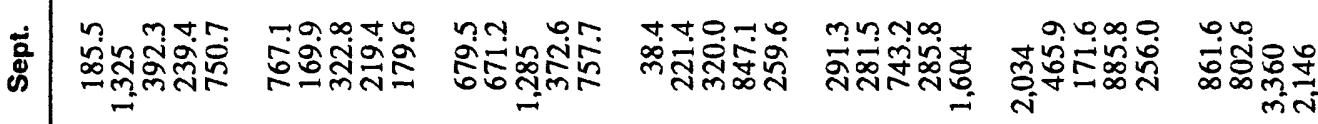

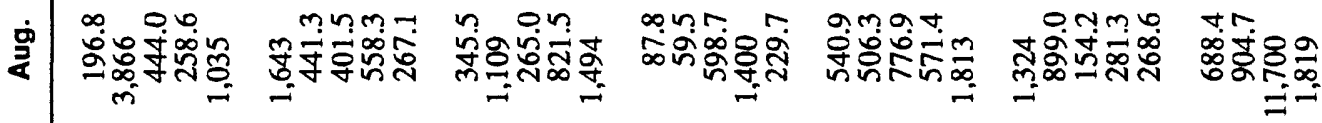

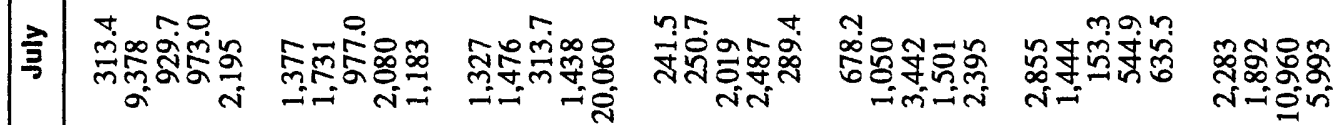

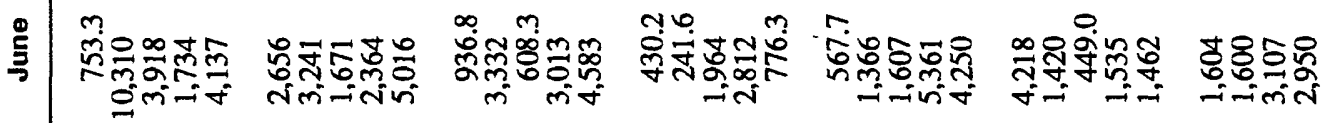

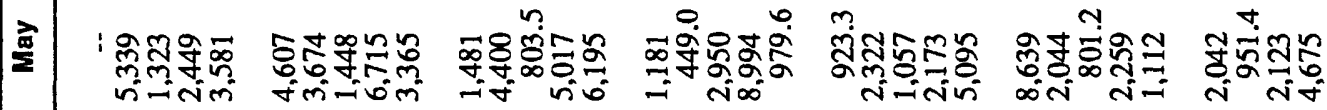

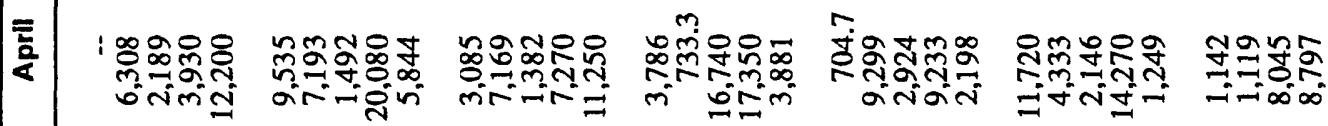

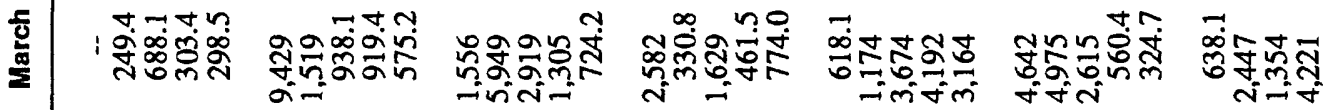

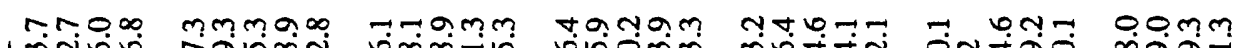

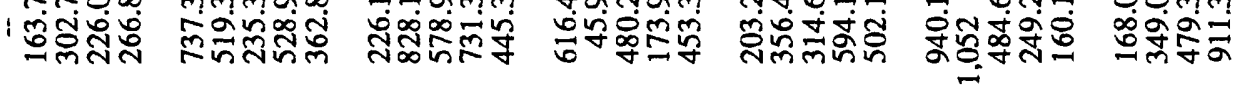

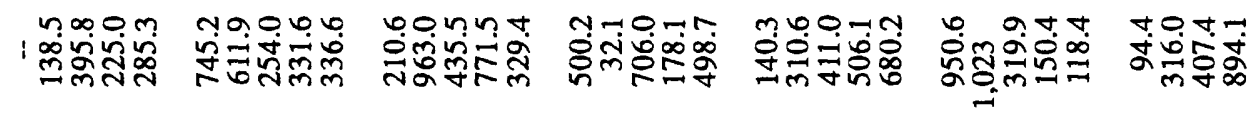

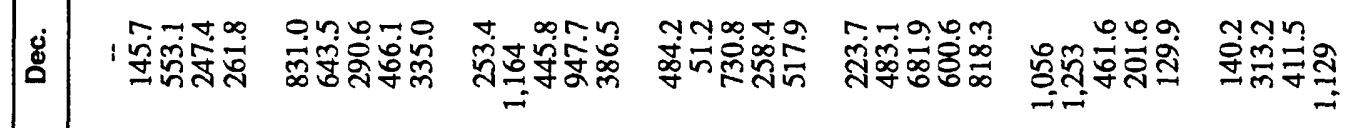

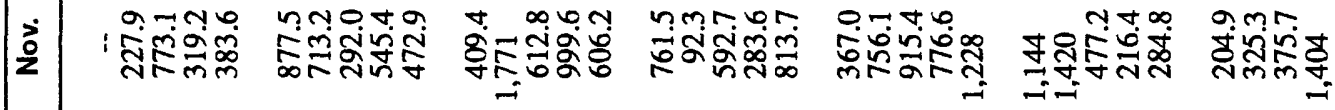

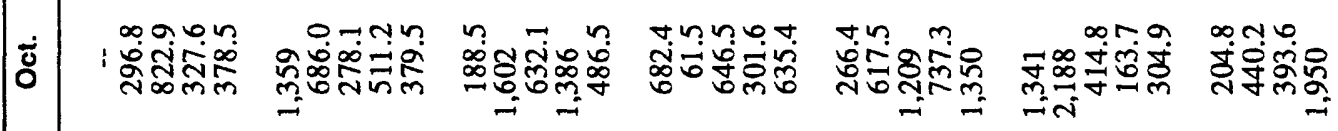

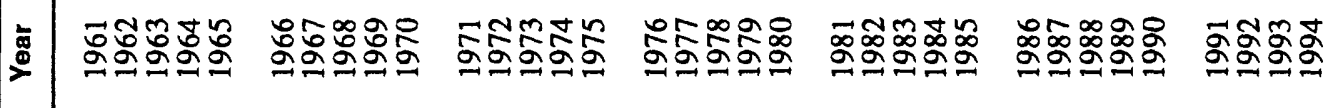

